\title{
A ROBUST APROACH FOR ROAD USERS CLASSIFICATION USING MOTION CUES
}

\author{
by
}

Haider Talib

A thesis submitted to the Faculty of Graduate and Postdoctoral Affairs in partial fulfillment of the requirements for the degree of

Master of Applied Science

in

Civil Engineering

Carleton University

Ottawa, Ontario

(C)2015

Haider Talib 


\begin{abstract}
Video monitoring of traffic is a common practice in major cities. The data generated by video monitoring has practical uses such as traffic analysis for city planning. However, the usefulness of video monitoring of traffic is limited unless there is also a reliable way to automatically classify road users. This thesis presents a framework that is designed to classify road users into vehicles, cyclists and pedestrians by using the motion cues obtained from their tracks. The road user tracks are obtained using a tracker system such as computer vision techniques. As such, this classification technique does not require additional video or image analysis alongside obtained road user tracks. The separate pieces of information are gained from these motion cues are hereafter called Classifiers. There are nineteen classifiers included in this framework. These classifiers include: average and maximum speeds; average and maximum acceleration; average and maximum deceleration; average and maximum direction; average of change in direction; average of cosine of change in direction; average and maximum area; average and maximum length; average and maximum width; peaks in speed; effective frequency; and the effective weighted average frequency. After obtaining the classifiers' values from the tracked objects' tracks, the information from these classifiers will be assessed and integrated using fuzzy membership approach, which in turn requires prior configurations to be available. This will lead to the final classification of the tracked object. The performance of this framework demonstrated very promising results under different measures. An important contribution of this study is the creation of a robust approach that can integrate different motion cues using fuzzy membership framework. The developed approach also uses parametric classifiers, which do not
\end{abstract}


depend on the geometry or specific traffic operation of the intersection. This is a key advantage because it enables transferability and improves the practicality and usefulness of the approach. 


\section{Acknowledgements}

First and foremost, all thanks are due to my God for giving me strength and the ability to complete this thesis. My thanks are first owed to my thesis supervisor and mentor, Dr. Karim Ismail. He spent substantial and valued time supporting my work in a variety of ways for which I am indebted - introducing me to new techniques that were invaluable to my research, helping me to improve my reading and writing skills, and offering considerable knowledge to this project, especially in traffic safety. I would also like to express my gratitude to Dr. Ali Kassim for sharing the data sets that enabled this study, as well as for elucidating the video tracking system that was so essential to its methodology. I would also like to thank the members of examination committee, Dr. Andy Alder and Dr. David Lau for the valuable comments and suggestions. Special thanks are also due to the data programmer for the project, Ahmed Rassem. My gratitude goes out to each of these supporters, without whom my thesis would not have come to fruition. 


\section{Table of Contents}

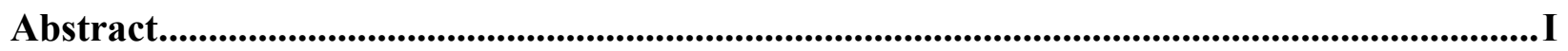

Acknowledgements ..........................................................................................................................III

List of Tables .........................................................................................................................................VIII

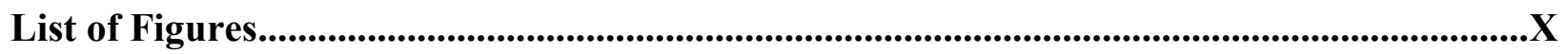

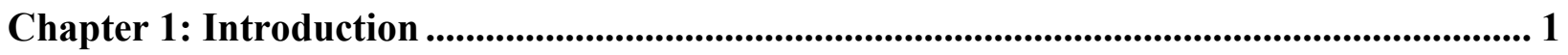

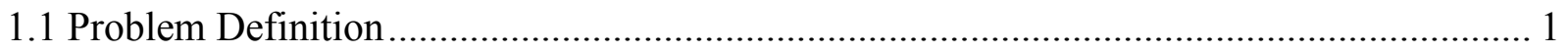

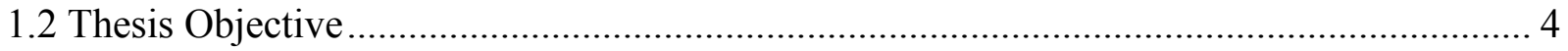

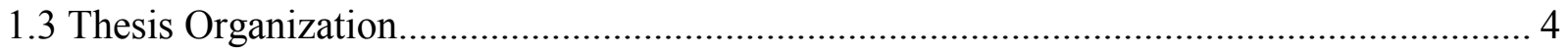

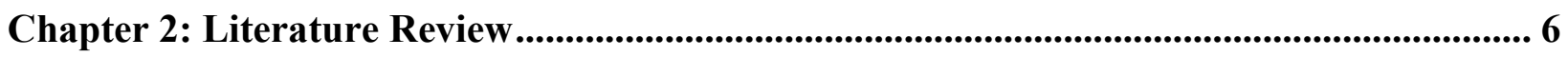

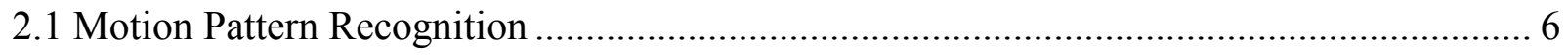

2.2 Movement Paths (Movement Trajectories) ........................................................... 9

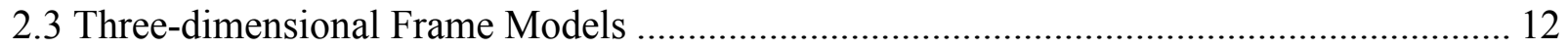

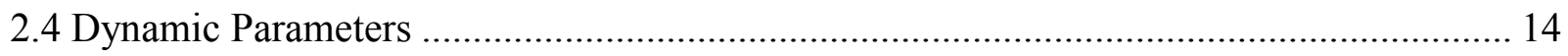

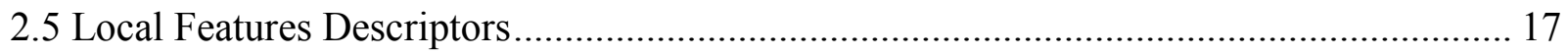

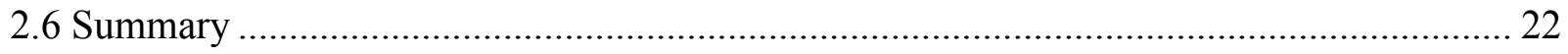

Chapter 3: Development of a Framework for Object Classification ...................................... 24

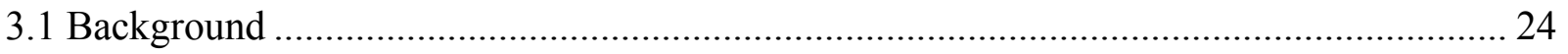

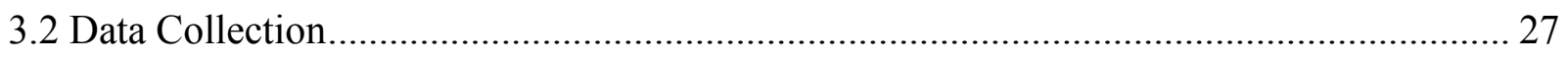

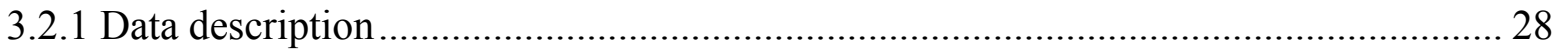




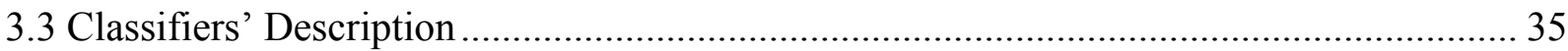

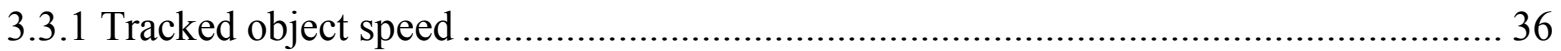

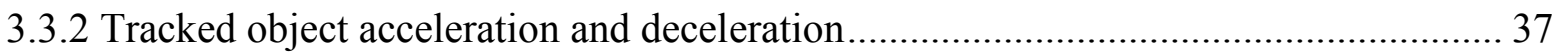

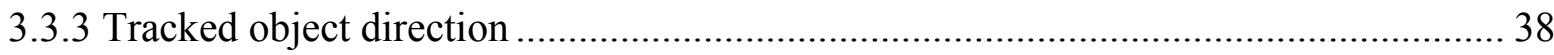

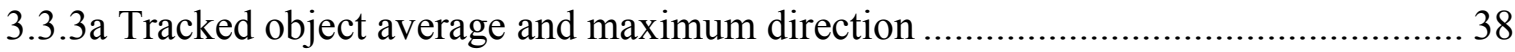

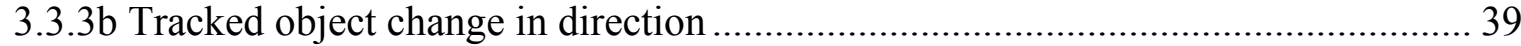

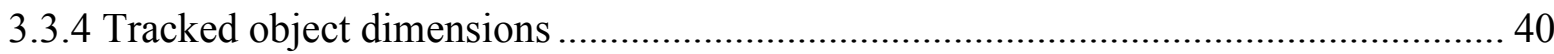

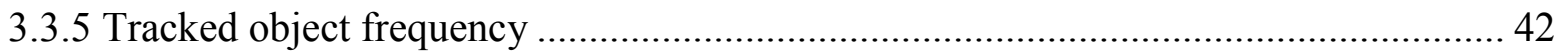

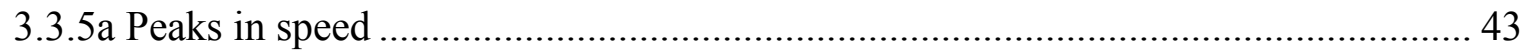

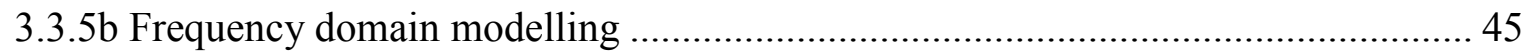

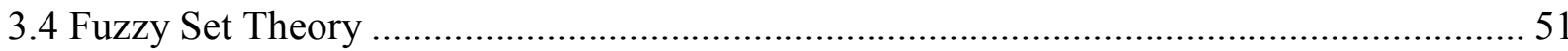

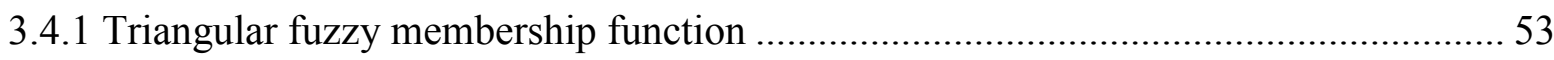

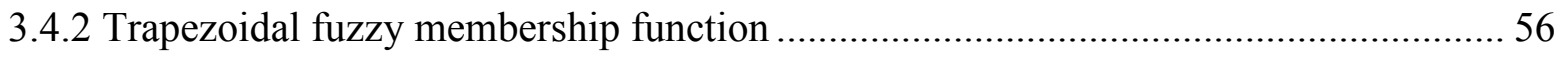

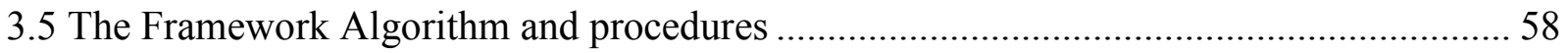

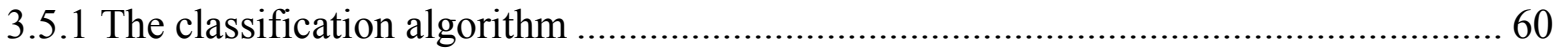

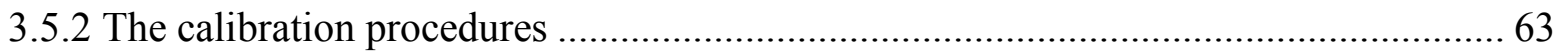

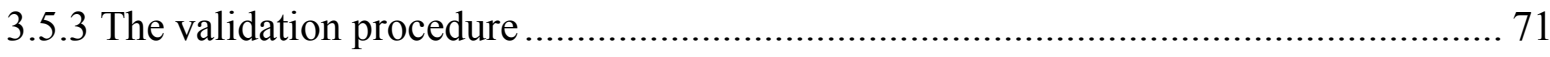

Chapter 4: Results and Discussions ......................................................................................... 76

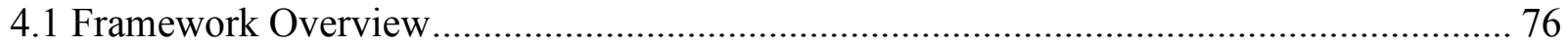




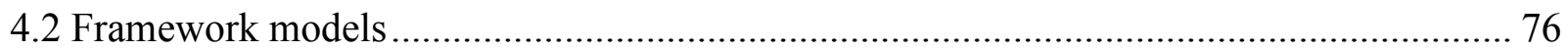

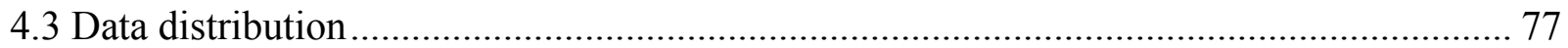

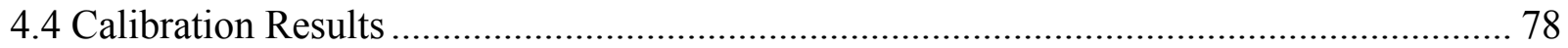

4.4.1 Obtain the initial limits for the fuzzy membership .................................................... 78

4.4.2 Obtain the calibrated limits for the fuzzy membership ............................................ 81

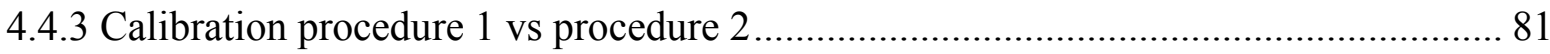

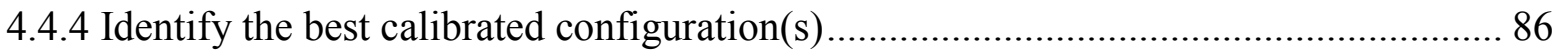

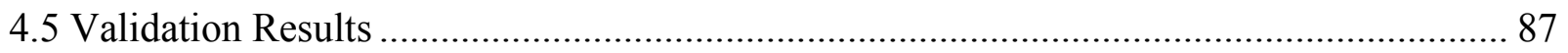

4.5.1 Effect of the minimum duration of tracked objects appearance $\boldsymbol{\varphi g}$ on results ............ 87

4.5.2 Effect of the fuzzy membership function $\boldsymbol{\Lambda} \boldsymbol{l}$ on results........................................... 87

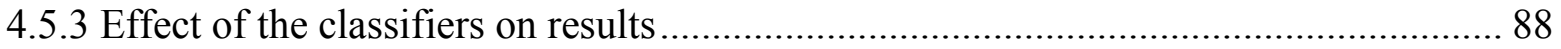

4.4.3a Validation results: procedure 1 as compared with procedure 2 ............................ 89

4.4.3b Tracked object speed........................................................................................ 93

4.4.3c Acceleration and decelerations .......................................................................... 97

4.4.3d Tracked object direction ................................................................................. 98

4.4.3d Tracked object dimensions .......................................................................... 98

4.4.3e Tracked object frequency .............................................................................. 99

Chapter 5: The Effect of Filtration ...................................................................................... 104

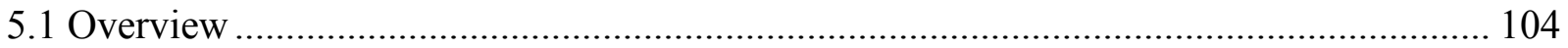

5.2 The Moving Average Filter......................................................................................... 105 
5.3 Tracked Object Acceleration and Deceleration

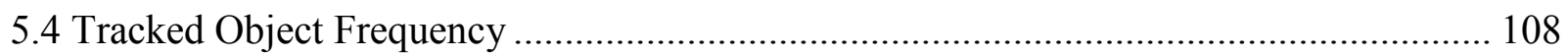

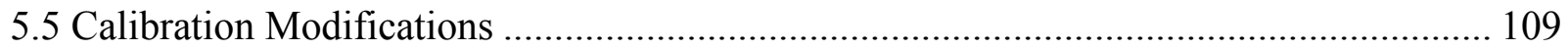

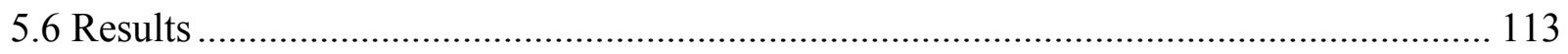

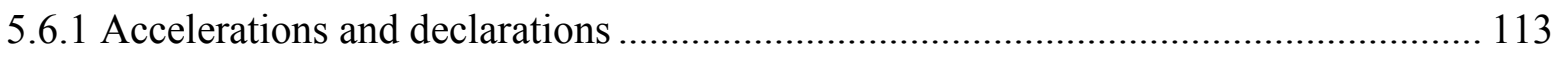

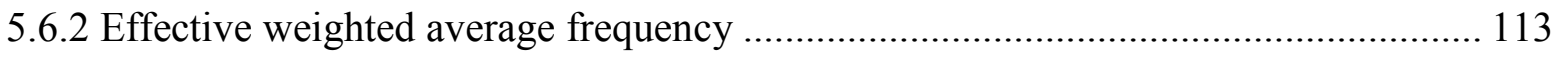

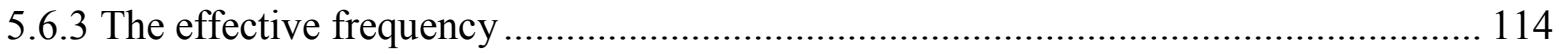

Chapter 6: Summary and Conclusion ............................................................................................. 116

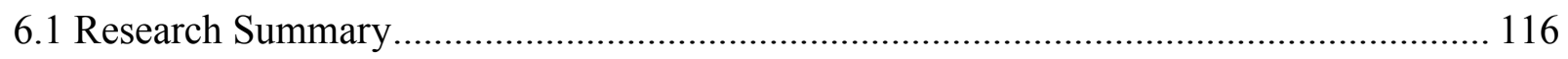

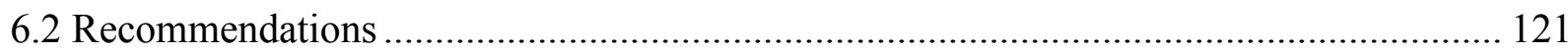

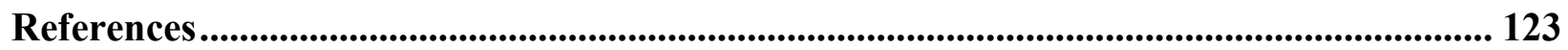

Appendix A......................................................................................................................................... 131

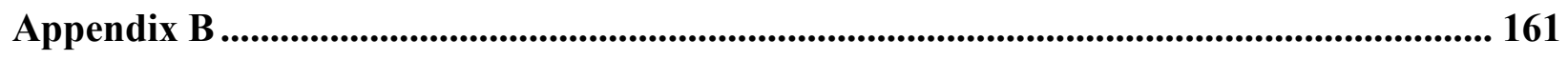

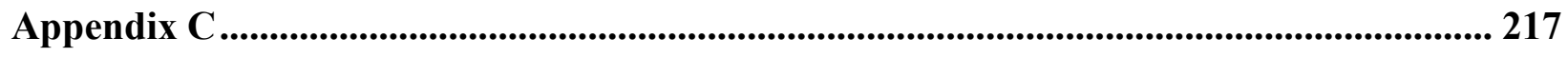

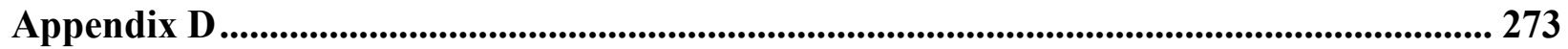

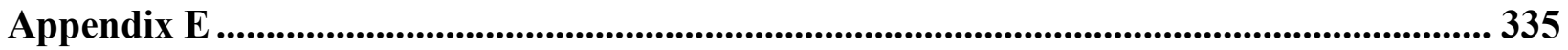




\section{List of Tables}

Table 2. 1 The levels type and stages in Gupte et al., (2000) classification system.................. 15

Table 3. 1 Directions of movement of the tracked object....................................................... 38

Table 3. 2 Fuzzy set- Triangular membership function equations........................................ 53

Table 3. 3 Fuzzy set- Trapezoidal membership function equations ..................................... 56

Table 3. 4 The used durations of the tracked object's appearance......................................... 59

Table 3. 5 The used types of fuzzy membership functions................................................ 59

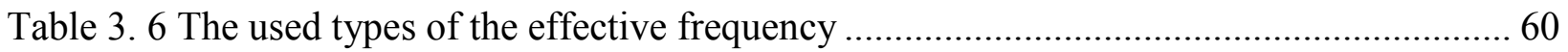

Table 3.7 The used types of the effective weighted average frequency................................ 60

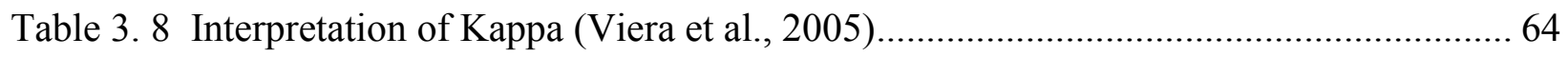

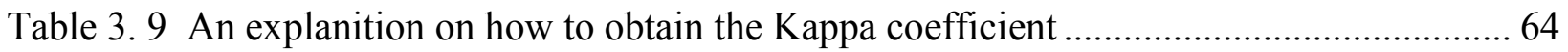

Table 4. 1 Data distribution shows the number of tracked objects used in calibration and

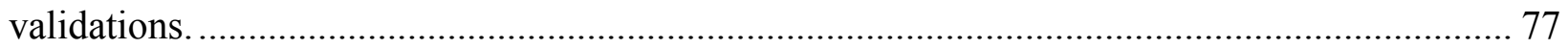

Table 4. 2 The initial values obtained from the tracked objects. ........................................ 79

Table 4. 3 Coefficient of determination for the four models ............................................ 85

Table 4. 4 The effectiveness of all the classifiers using calibration procedure 2 for the four

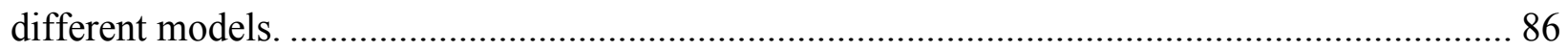

Table 4. 5 The accuracy rate of classification using the percent correct value. The results are for

the successful configurations only in the three validations. ............................................ 91

Table 4. 6 The accuracy rate of classification using the percent correct value. The results are for the successful configurations only in the three validations.

Table 4. 7 The accuracy for the rate of classification, using a percent correct value. These results are based on the three validations. 96 
Table 4. 8 The accuracy rate of classification using a percent correct value. The results are for the successful configurations only in the three validations.

Table 4. 9 The accuracy rate of classification using a percent correct value. The results are for the successful configurations only in the three validations. 103 Table 5. 1 The accuracy rate of classification using a percentage correct value. The results are for the successful configurations only in the three validations. 114 Table 5. 2 The accuracy rate of classification using a percentage correct value. The results are for the successful configurations only in the three validations. 115 


\section{List of Figures}

Figure 3. 1 A basic description of the classification process................................................. 27

Figure 3. 2 A video sequence analyised using a feature-based tracking system. ...................... 30

Figure 3. 3 The output text of tracker system. ................................................................. 31

Figure 3. 4 An explanation of partial tracks. ................................................................. 33

Figure 3. 5 A sample output text of the tracker system explains the partial tracks of an object.

Missing tracks are presented with the "?" symbol. ................................................................. 34

Figure 3. 6 An explanation of the linear interpolation...................................................... 34

Figure 3. 7 An example of the change in the size of the bounding box for both object 5 and

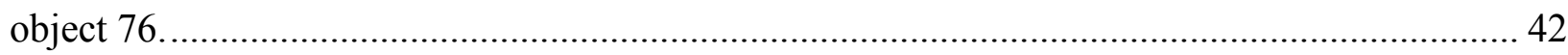

Figure 3. 9 The variation in speed profiles of a vehicle, a cyclist, and a pedestrian ................ 45

Figure 3. 10 a) An example of three different sine waves; b) combining the three different sine

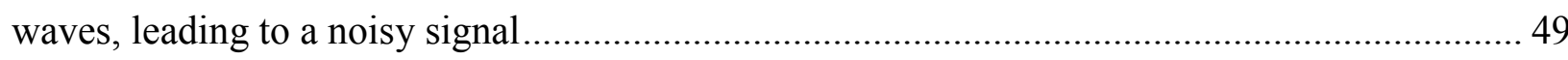

Figure 3. 11 The frequency domain obtained from the speed profile of a) pedestrian, b) cyclists,

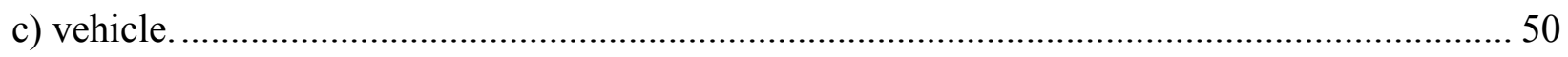

Figure 3. 12 Three cases of the triangular fuzzy membership function. a) where the $\delta=0.25$; b)

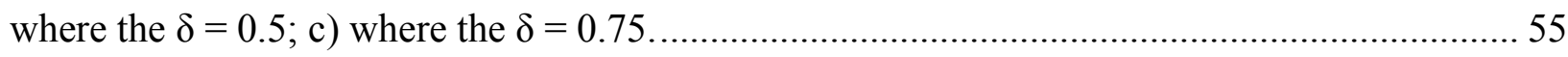

Figure 3.13 The trapezoidal fuzzy membership function ............................................. 57

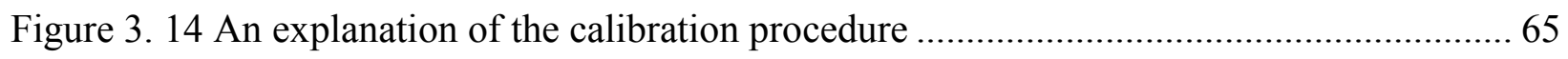

Figure 3. 15 A detailed explanation of the framework mechanism..................................... 72

Figure 4. 2 The observed average speed histogram for cyclists ........................................ 80

Figure 4. 3 The observed average speed histogram for pedestrians .................................... 80 
Figure 4. 4 a) Cumulative curves show the improvement of Kappa coefficient using calibration procedure $1 \mathrm{~b}$ ) Cumulative curves show the improvement of Kappa value using calibration

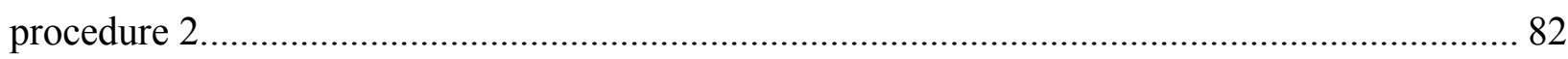

Figure 4. 5 Cumulative curves show the improvement of Kappa coefficient with calibration for

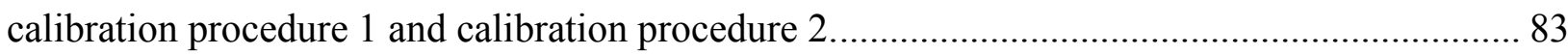

Figure 4. 6 Relationship between calibration procedure 1 and calibration procedure 2. a) for the first model; b) for the second model; c) for the third model; d) for the fourth model. 84

Figure 4. 7 Cumulative curves show the improvement of Kappa value with calibration using different kind of fuzzy membership functions. 88

Figure 4. 8 Receiver operating characteristic for the first model illustrate the validation results for vehicles using validation type 1 and 2 . 92

Figure 4. 9 Receiver operating characteristic for the first model illustrate the validation results for cyclists using validation type 1 and 2 92 Figure 4. 10 Receiver operating characteristic for the first model illustrate the validation results for pedestrians using validation type 1 and 2 . 93

Figure 5. 1 A speed profile of a pedestrian under different window sizes. The window sizes are represented as a percentage value. 106

Figure 5. 2 The observed average acceleration histogram for vehicles.

Figure 5. 3 The observed average acceleration histogram for cyclists. 112

Figure 5. 4 The observed average acceleration histogram for pedestrians 112 


\section{Chapter 1: Introduction}

\subsection{Problem Definition}

Road Safety is a global concern that has been growing over the years. World Health Organization (WHO) recognized traffic injuries as the eighth cause of death globally with over 1.24 million deaths per year (WHO, 2013). WHO, (2009) estimated the total number of road traffic deaths and injuries worldwide to be increase by $65 \%$ between 2000 and 2020 . The Ministry of Transportation of Canada has reported that the average number for injuries and fatalities are 188,862 and 2,634 respectively for the period between 2005 and 2009 (The Ministry of Transportation of Canada, 2011). The National Highway Traffic Safety Administration (NHTSA) has reported 1,530,000 injuries and 29,757 fatalities as a result of motor vehicle traffic crashes in 2011. Hence, it has motivated the governments and local authorities to develop new techniques to improve traffic safety such as video monitoring.

Video monitoring of traffic is a common practice in major cities in order to meet practical needs such as exploring traffic conditions, identifying traffic violations, and detecting incidents (Ismail et al., 2011). Using tracking systems such as computer vision (CV) techniques, which enable the automated analysis of video data, is also growing in popularity. Recently, numerous applications have been developed for traffic data collection, traffic conflict analysis, and detection of non-conformist traffic behavior. In these applications, the paths of road users are tracked in a sequence of video frames. A track is composed of a sequence of positions that represent the movement of a road user within the field of view recorded in one frame after another. From this sequence of positions, valuable information can be extracted regarding the 
road users such as speed, acceleration, the direction of movement, and variations thereof. Manually analyzing traffic data to track road users would consume tremendous effort and time, while being error-prone. These practical limitations have motivated important advances in developing and adopting $\mathrm{CV}$ techniques to meet growing demands for accurate and large-scale traffic data collection.

While tracking road users is an important task, it does not completely meet all practical needs for traffic studies. Traffic facilities in urban environments are typically used by a mixture of road users. For instance, within a monitored roadway or intersection at any given time there are likely to be varied types of road users traveling such as pedestrians, cyclists, and vehicles. Therefore, it is important to associate the extracted track with corresponding types of road user. This becomes particularly important if information is needed for a specific road user or for interactions between specific pairs of road users. Without using automated classification, having a human observer review these tracks manually, along with the traffic videos, is necessary before further analysis becomes possible. While certainly less laborious than manual tracking, this still can be time-consuming if a large quantity of video data are available. Furthermore, another possibility is that a feedback mechanism may exist between tracking and classification. That is, it is possible that tracking accuracy can be refined if we know what type of road user is actually being tracked. Various automated classification techniques have therefore been developed with the purpose of identifying or constructing distinctive features of various road users as they appear or move in video sequence. Utilizing these features classification can be performed, which refines the process of monitoring traffic conditions and use.

Several studies have attempted to use tracking systems to classify tracked objects in traffic environment, but thus far, many attempts at classification have been proven insufficient in some 
way. Some relatively simple techniques, e.g., based on road user speed, can be easy to apply, but may not provide adequate accuracy. Other more complex techniques, such as relying on motion patterns (Ismail et al., 2010), can yield adequate accuracy but lack generality and transferability from one intersection to another. In addition, image-processing (particularly, the processing of still images) techniques for road user detection and recognition can be adopted for classification. While promising, it is likely that image-processing techniques will be computationally expensive in a video sequence, and will require extensive development to be integrated with existing tracking systems.

This thesis attempts to use motion cues obtained from road user tracks. As such, this classification technique does not require additional video or image analysis alongside obtained road user tracks. An important contribution of this study is the creation of a robust approach that can integrate different motion cues using fuzzy membership framework. The developed approach also uses parametric classifiers, which do not depend on the geometry or specific traffic operation of the intersection. This is a key advantage because it enables transferability and improves the practicality and usefulness of the approach.

Subsequent parts of this thesis achieve the following: [i] provide a review of previous research in the area of classifying road users; $[i i]$ describe the proposed classification approach; [iii] describe motion cues and how they are measured and used in a fuzzy membership approach; and present the calibration procedure used to find optimal classification thresholds and membership function specifications, as well as performing validation using different datasets. The transferability of this classification approach was tested using: a new (different from calibration) dataset from the intersection employed during calibration, another dataset from a different intersection, and, lastly, the data from both intersections combined. Overall, the 
performance of the proposed classification approach is satisfactory based on its simplicity of application, transferability, and successful validation results. However, a number of shortcomings are also noted in this thesis, and future work is also recommended in the concluding chapter.

\subsection{Thesis Objective}

The thesis objective is to create a classification framework which utilizes motion cues (dynamical parameters or characteristics) obtained from road user tracks in order to classify different kinds of road users. The thesis also aims at examining the accuracy of the classification algorithm by comparing its performance to manual (observer-based) classification of the road users in the same video sequence. While a multitude of road user classification methods already exist, the advantage of this study is that it utilizes the regularity of road user movements that are typically present in a real-world traffic situation at a negligible computational cost. The three road user categories identified in the study are vehicles, cyclists, and walking pedestrians. All classification work was focused on movements within urban traffic intersections.

Key questions proposed in this thesis are the following:

1. Can we reliably classify road users based on motion cues?

2. What are the motion cues that are most useful for categorizing tracked objects?

3. Which of the three targeted road user categories can be classified with the highest accuracy?

\subsection{Thesis Organization}

In Chapter Two, a literature review presents important work that has been conducted in this field thus far, and discusses current developments in the tracking system utilized in this study, road user recognition research, and its implications. The chapter also reviews older models that 
laid the groundwork for techniques presented and utilized in this thesis. Chapter Three presents the main research methodology, with detailed explanations of the algorithm underlying the road user classification system and theory-based justifications for the decisions that were made. In Chapter Four, the study results are presented and discussed. This includes both the numerical outputs of the study and an explanation of what the results entail. In Chapter Five, the effect of noise filtration on the classification process is examined. Chapter Six concludes the study; providing a summary of what was accomplished and discovered. Recommendations for future research are also made in the same chapter. 


\section{Chapter 2: Literature Review}

Traffic analysis is essential to planning of traffic in urban environment. For instance, to determine the proper placement and timing of traffic lights, the widths of sidewalks and the need for amenities such as bike lanes and crosswalks, traffic patterns need to be observed and analysed. The manual classification of traffic patterns and categorization of traffic users is tedious, error-prone, and time-consuming. As a result, researchers have created different approaches and techniques for developing different systems of road users' classification. This includes using thermal sensors, speed detectors and loop detectors. In recent years, identification of road users using video cameras has become increasingly popular. Video cameras are easy to use and install, the acquisition of the images is cheap, and are non-intrusive. Hence a single video camera can record the view for an entire location (Saunier \& Sayed, 2006). Analysing video sequence can provide rich description of the targeted intersection such as the traffic flow and the motion trajectories (movement paths) of road user. This has motivated many researchers to develop new methods to classify road users captured either on static images or in a sequence of images (video). Each of these two methods has its own distinctive features. However, these methods share key technical stages: the detection stage (identify the object hypothesis or the region of interest in the image), the tracking stage (only in image sequence) and the verification stage (objects classification). This chapter provides an overview of several different automated systems which were designed to observe and classify road users.

\subsection{Motion Pattern Recognition}

By the year 2000, the study of automated traffic recognition software was still relatively new and had not reached the sophistication that it has subsequently. Nevertheless, effective 
algorithms were being designed to recognize different kinds of road users for city planning and traffic control purposes. Notably, a method was developed for pedestrian recognition that involved tracking the semi-rigid torso of a pedestrian while analysing motion patterns in the legs to distinguish the pedestrian from the fully-rigid vehicles that were also caught on surveillance (Curio et al., 2000).

The study by Curio et al., (2000), presented an image processing approach to detect, track, and classify walking pedestrians captured on a video sequence using a moving observer. The moving observer was a video camera installed on a vehicle. The basic distinction is that cars and other motorized vehicles had completely static shapes as they travelled within the field of view, while pedestrians move their arms and legs. This study aimed at developing a system that could classify objects based on the human limb motion.

In their study, Curio et al., (2000) divided the identification process into three main stages: the initial detections of object hypothesis, tracking of object hypothesis and finally classify the moving objects. The initial detections object hypothesis attempts to identify the region of interest (ROI) in the image. This is to eliminate the search outside these regions. The initial detection was accomplished by calculating the local image entropy, matching different walking models based on contour information, and applying inverse perspective mapping (IPM) (Bertozzi et al., 1998). Local image entropy, also called local image texture, makes the subsequent processing to concentrate on the discrete regions in the image. Curio et al., (2000) were able to match different walking models based contour information using the Hausdorff-Distance algorithm (Huttenlocher et al., 1993). IPM was used to detect any object within a short distance to the observer. Finally, the system made the final decision about the ROI by integrating all the previous information. Curio et al., (2000) created a human walking model order to distinguish 
between pedestrians and other objects during the initial detection stage. They then exploited this feature "in particular the lower limbs movement" to identify the ROI. They used data concerning the angles between hips and knees, and thighs and calves, as well as the frequency with which different parts of the legs move and the rate at which people typically walk. They furthermore took into account the possibility of people wearing skirts, measuring only the outside lines of the body.

After identifying the ROI in the image, tracking of object hypothesis was performed. This is because Curio et al., (2000) used a moving observer. Classifying a moving object in front of a moving background is likely to be difficult. Therefore, they developed an algorithm that search for similar features between successive frames. Curio et al., (2000) also applied a Kalman filter (Kalman, 1960) to account for the change in speed of the object hypothesis and to "reduce search space between successive frames" (Curio et al., 2000). The tracking of object hypothesis provided a dynamic verification of the initial detection of object hypothesis. From the tracking of the object hypothesis, they used the periodic motion of the gait (Ran et al., 2006) to distinguish between pedestrians and other objects. Combining the results of the previous step led to the final classifcation of pedestrains.

The aim of Curio et al., (2000) study was simply to determine if it was possible to create an algorithm that could accurately identify pedestrians on the street, not to analyse pedestrians' gaits, as would occur later (Zaki \& Sayed, 2014). The study had certain definitive drawbacks, such as the difficulty of separating objects from noisy backgrounds and that of separating multiple pedestrians walking in groups. Nevertheless, as a seminal work in road user identification, the Curio et al., (2000) study laid the foundation for many others using more 
sophisticated technology, which incrementally increased the robustness and accuracy of the field as a whole.

In a study by Pai et al., (2003), a system was developed to classify walking pedestrians using image processing techniques. The classifcation process goes into two steps: [i] create pedestrians model and [ii] test the walking rhythm of pedestrians. The pedestrians model was developed to identify any "possible pedestrians" in video sequence, this to eliminate any other moving objects from the classifcation process. The walking rhythm test was developed to confirm the "possible pedestrians" are actually pedestrians. This approach is tested on a dataset of 46 walking pedestrians. 40 out of the 46 were idntified as a "possible pedestrians", and 39 were idntified as "confirmed pedestrians".

\subsection{Movement Paths (Movement Trajectories)}

Ismail et al., (2010) developed methods for binary classification of the tracked objects captured on a video sequence using a commercial-grade video camera in Oakland, California. These methods were designed to discriminate between pedestrians and motorized vehicles by utilizing their movement paths. The study took place in the city of Vancouver, British Columbia. The movement paths of the tracked objects were collected using a feature-based tracking system (Saunier \& Sayed, 2006).

The feature-based tracking system uses the Kanade-Lucas-Tomasi (KLT) algorithm to detect and track features (salient points) in video sequence (Lucas \& Kanade, 1981). These features are distinguishable points on the moving object, such as bumper edges and window corners. In addition to tracking, these features are subsequently grouped into one object (Beymer et al., 1997; Saunier \& Sayed, 2006). This is because tracked objects usually have multiple 
features and the tracking system must be able to decide which group of features belong together and are part of the same road user. Therefore, for each tracked object, features which belong to the same object will be linked together by edges in some network or a graph. The features and the edges will form a graph which will represent the track object. Ismail et al., (2010) used two classification methods: [i] Prototype-based classification and [ii] Speed-based classification.

In the prototype-based classification, Ismail et al., (2010) identified small subsets of tracked object movement paths called prototype movement paths based on the similarity of longest common subsequence (LCSS) (Vlachos et al., 2005). The prototype movement paths are labelled based on the type of road user. A road user class, e.g., pedestrian, is assigned to the tracked object based on the match between its movement paths and the closest prototype. If an object could not be classified using the prototype-based classification then it will be classified based on the speed thresholds. The speed threshold was assigned to be $3 \mathrm{~m} / \mathrm{s}$ for binary classification. Both methods were evaluated using a sample of 1,063 road users' movement path. For the speed thresholds, the classification accuracy was $85 \%$, while the prototype-based classification yielded an accuracy rate of $97 \%$. The accuracy rate is based on a percentage correct classification.

A study by Zaki \& Sayed (2013) presented a system that could classify tracked objects into one of three categories (similar to the system in this thesis): vehicles, cyclists, and pedestrians. This classification system "relies the motion pattern attributes associated with [the movement paths] of each road user" (Zaki \& Sayed, 2013). Zaki \& Sayed (2013) classification system was tested on three sets of video data collected from two different areas in the Greater Vancouver Area, British Columbia. The data sets included 620 vehicles, 222 cyclists, and 242 pedestrians. Zaki \& Sayed (2013) started their research by collecting the movement paths of the tracked 
objects. The movement paths collection was done using a feature-base tracking system. Discriminating features are required for effective classification. Therefore, Zaki \& Sayed (2013) used Singular Spectrum Analysis (SSA) (Golyandina, 2005) to extract the discriminating features from the speed profiles of different type of tracked objects. Zaki and Sayed (2013) proposed two classification procedures: [i] threshold-based classification and [ii] spectral clustering.

In threshold-based classification, a class is assigned to the tracked objects according to whether or not constraints on its features are deemed acceptable. In spectral clustering, the data are organized into groups based on their characteristics. A class is assigned to each group "through internal specifics to the clusters such as the centroid characteristics" (Zaki \& Sayed, 2013). The classification system was evaluated and produced an accuracy rate of $94.83 \%$ for motorized vs. the non-motorized moving objects. The system also produced an accuracy rate of $88.64 \%$ for the three type classification. The accuracy rate is based on a percentage correct classification. Zaki \& Sayed's primary contribution to the field was to recognize classification as a major and initial goal in the analysis of urban road user behaviour; likewise, their research provides an important groundwork for this thesis, given my own focus on streamlining object classification.

A subsequent study by Zaki et al., (2013) presented a classification system that could classify tracked objects into vehicles and pedestrians by relying on their movement paths, in particular their speed profiles. (Zaki et al., 2013) classification system was tested on traffic data collected using a commercial-grade video camera in Oakland, California, the data included 1,063 road users. The tracked objects trajectories were collected using a feature-base tracking system. 
The movement paths in Zaki et al., (2013) are presented in the time domain. Data presented in the time domain usually exhibits high variations (e.g., speed profile). Therefore, Zaki et al., (2013) used Singular Value Decomposition (SVD) (Golub \& Van Loan 1996) to identify these variations by decomposing the speed profile. This following step was to reconstruct the speed profile after excluding the variations that are below the assigned threshold. This mainly serves to reduce the amount of data produced, while prioritizing the primary relationships upon which the study wishes to concentrate. Then, the correlation coefficient between the original and reconstructed movement paths was measured. Based on the score of the correlation two classification methods were presented: [i] threshold-based classification and $[i i]$ Prototype-based classification.

In the threshold-based classification, a class is assigned to the tracked object based on the correlation value between the original track and the reconstructed track. The tracked object associated with the movement paths can be classified as a vehicle if the correlation value is higher than the assigned threshold. Otherwise, it is classified as a pedestrian. In the prototypebased classification, Zaki et al., (2013), applied eigenstructure to generate reconstructed tracks from each prototype class. A class is assigned to the tracked object based on the highest correlation value between the original track and the reconstructed track. The performance of the Zaki et al., system was evaluated. The classification accuracy rate for both methods was approximately $90 \%$. The accuracy rate is based on a percentage correct classification.

\subsection{Three-dimensional Frame Models}

A study by Messelodi et al., (2005), a system was developed to collect statisical traffic informations at road intersections. The system called SCOCA (System for Counting and Classifying Automatically vehicles). This system is capable of detecting, tracking, and 
classifying moving objects captured on a video sequence using a static camera installed on a high elevation. The study took place in the city of Trento, Italy. The classification process of the moving objects begins by acquiring some information about these objects. This information was obtained during the tracking stage.

The detection in the SCOCA system relies on subraction technqiues of each frame from a background image. the background image is an image of the trageted intersection without any road users. Using the well-known Kalman filter, Messelodi et al.,, (2005) developed a method for automatic upadate of the background imgae to to account for lighting conditions and the time of the day. The tracking in the SCOCA system relies on a hybird method that consists of region-based tracking and feature-based tracking (Saunier and Sayed, 2006). Messelodi et al., (2005) divided the classifcation process into two subsets: [i] model-based classifcation and [ii] post classifcation.

In the model-based classifcation, the moving objects are classified into three categores (vehicles, cyclists and pedestrains) by relying on a set of 3D models. Three models were introduced using the model-based classifier, the pedestrain model enables the system to classify the moving people as pedestrians, the bicycle model enables the system classify cyclists and motorcyclist, and the vehicle model enable the system to classify any type of vehicle as vehicles (e.g., passenger cars and buses). Post classifcation is intened to categorize the identified vehicles in the model-base classifcations into classes using a 3D model for each vehicle class. The classifcation performance was evaluated on a 45 minute video data obtained from two location, the data include 1,332 moving objects. The classifcation accuracy varied depend on the type of the object. However, the overal the classifcation accuracy was $92.5 \%$. The accuracy rate is based on a percentage correct classification. 
In a study by Buch et al., (2010), system was developed to detect, track, and classify moving objects captured on a video sequence using a stationary camera. Buch et al., (2010) study took place in Surry, UK. In Buch et al., (2010) study, the motion silhouettes of each tracked object was extracted and compared with the projected 3D models. The projected 3D models represent the object hypothesis. There were 5 type of 3D models (bus-lorry, van, car, motorcycle-bicycle, and pedestrain). The classification was achieved based on the match measure between the projected model and the extraced silhouettes. The match measure was performed using the Image Measurement (IM) based features method (Morris \& Trivedi, 2008). The type of object is assigned to the highest match measure between a projected model and an object silhouettes. The preformance of Buch et al., (2010) system was evaluated on a dataset of 782 moving objects. It provided an aceptable classifcation accuracy, it achieved a recal of $87 \%$ at a precision of $85.5 \%$.

\subsection{Dynamic Parameters}

Another relatively early method of classifying various road users involved establishing correspondences between vehicles and regions based on motion (Gupte et al., 2002). As these explained, the predominant method of measuring traffic activity was by using loop detectors. Traffic monitoring through videos was, at the time, far less common, and the researchers argued for the far greater effectiveness and convenience of using video monitoring systems for traffic surveillance.

The Gupte et al., (2002) system was designed to detect and classify vehicles captured on video sequence using a static camera. The main observation of this system was that it identified regions in traffic surveillance that stood out from the background by changing from frame to 
frame. These regions were identified as vehicles moving on the road only if the region size is within pre-assigned thresholds. There are three processing levels in Gupte et al., (2000) system and as in Table 2.1.

Table 2. 1 The levels type and stages in Gupte et al., (2000) classification system.

\begin{tabular}{|c|c|c|}
\hline Level number & Level type & Level stages \\
\hline Level 1 & Image level & Segmentation \\
\hline Level 2 & Regional level & Region tracking \\
\hline \multirow{2}{*}{ Level 3 } & \multirow{3}{*}{ Vehicle level } & Recovery of vehicle parameters \\
\cline { 3 - 3 } & & Vehicle identification \\
\cline { 3 - 3 } & & Vehicle tracking \\
\cline { 3 - 3 } & & Vehicle classification \\
\hline
\end{tabular}

The image level is the detection level where the system detects the object in each frame of the video sequence. Segmentation can be simply defined as the process of separating the objects "in this case the objects are vehicles" from the background image. Gupte et al., (2002) developed a simple method that can provide high-processing and adapts to different lighting condition. This method operates by subtracting the current image from the background image. The background image gets updated regularly throughout the video sequence.

The regional level is the tracking level, Region tracking can be simply defined as connecting the regions in the successive frames. Regions tracking prevent double-counting or data changes through the video sequence as well as it provide the velocity of the tracked region. Gupte et al., (2002) took in considerations some of uncontrolled conditions that could affect the 
tracking process such as momentary disappearances of cars-for example, from reflections on the glass or metal of the vehicle that could make the vehicle temporarily blend in with the street too much for the application to see it. Gupte et al., (2002) also recognized that multiple regional might merge e.g., two or more vehicles traveling next to each other, and therefore they conducted the tracking by creating an association graph between successive frames. The association graph that consists of two partitions, it includes vertices which correspond to a region from the previous frame and to a region the current frame. The region tracking was achieved by matching the graphs of successive frames using the maximal weight matching method.

The vehicular level is consisting of four synchronous steps as in Table 2.1. Recovery of vehicle parameters is the process of obtaining the dynamical parameters of the vehicles from the image coordinates and converting it to the world coordinates. The dynamical parameters include the speed, length and width of the vehicles. Obtaining the parameters was done through the camera calibration. Gupte et al., (2002) developed auto calibration technique that allows the system to calibrate itself by pointing at some points in the image through a user interface. Vehicle identification is the process of combining multiple regions to form a vehicle, Gupte et al., (2002) system operates under the assumption that a vehicle has multiple regions. The dimensions of new region assumed to the size of the vehicle. Vehicle tracking was required to obtain the speed of the tracked object. Using these dynamical parameters, the Gupte et al., (2002) method only identified vehicles as "cars" and "non-cars." This study successfully detected and tracked $90 \%$ of traffic vehicles; however, of these tracked vehicles, only $70 \%$ were correctly classified. The accuracy rate is based on a percentage correct classification.

A study by Hsieh et al., (2006) developed a system to detect, track, and classify vehicles, and then categorize them into classes. The classes were (cars, minivans, trucks, and van trucks) 
Hsieh et al., (2006) developed their classification system in an experimental setting on four sets of video surveillance data from Taiwanese highways. The used data was collected using one static camera. Hsieh et al., (2006) classification system consisted of two stages; the goal of the first stage was to detect the lane widths and lane-dividing lines and. This was accomplished by using an image subtraction technique to separate vehicles from the background in the image, creating a vehicle histogram, and finally analysing the histogram to obtain the lane width and the lane-dividing lines information. The goal of the second stage was to classify the vehicles and categorize them into classes; this was achieved by extracting some discriminative features and analysing them. Discriminative features extraction was done using a tracking system which relies on image subtraction techniques to detect the vehicles and Kalman filter algorithm (Kalman, 1960) to track the objects. An optimal classifier was later developed to classify and categorize the vehicles into classes based on the extracted features. The discriminative features include vehicle size and linearity. The linearity feature was used to discriminate between trucks and van trucks or buses. Although buses and trucks were approximately the same size, they had different upper-slanted edges because a truck consists of a tractor and a trailer. Hsieh et al., (2006) used the irregularity of the upper-slanted edges of the truck in order to categorize the vehicles into classes. Hsieh et al., (2006) conducted multiple classification experiments where the discriminative features were used individually and grouped together to classify the moving objects. The highest classification rate was $84.45 \%$ which was achieved by using all the features combined. The accuracy rate is also based on a percentage correct classification.

\subsection{Local Features Descriptors}

The most common approach in object classification relies on the features of the object in the images. These features are extracted using extraction techniques and be used to train a 
classifier. Histograms of Oriented Gradients (HOG) (Dalal \& Triggs, 2005) and Haar-like features (Bilgic et al., 2010) are the most common techniques for feature extractions. Adaboost algorithm (Viola et al., 2001) used to construct a strong classifier by combining the best weak classifiers. Support Vector Machine (SVM) (Schölkopf and Smola, 2002) are the most common methods for objects classification. These methods were used in many recent studies (Xu et al., 2011; Chen; 2013; Yang et al., 2013; Nigam et al., 2013; Somasundaram et al., 2013; Hong et al., 2015; Govardha \& Pati, 2014; Hariyono et al., 2014; Ng et al., 2014; Ballesteros \& Salgado, 2014; Liang \& Juang, 2014 Uijlings. 2014; Mishra \& Santoshi, 2015).

Messelodi et al., (2007), developed a technique to distinguish between bicycles and motorcycles based on differences in the appearance of their wheels. This study is a complement to Messelodi et al., (2005). The study took place in the city of Trento, Italy. Messelodi et al., (2007) noted that the area inside the wheels of a bicycle is very similar to the background, while the area inside the wheels of a motorcycle does not show the background as easily. Of course, this is only true when viewing a bicycle from the side. When viewed from the front or back, the noted difference was in the width, with bicycles typically having much narrower wheels than motorcycles. Messelodi et al., (2007) divided the classification approach into two stages: $[i]$ Model-based classification [ii] Feature based classification.

The model-based classification was designed to classify the tracked objects into vehicles, pedestrians and bicycle-like objects. The feature-based classification was designed to classify the bicycle -like objects into cyclists and motorcyclists. Messelodi et al., (2005) used SVMs to distinguish between the different wheel characteristics of bicycles and motorcycles when viewed from the side and from the front or back. SVMs are pattern-recognition algorithms that classify objects into two classes based on a given set of thresholds (Schölkopf and Smola, 2002). The 
performance of the classifier was evaluated on real-world traffic video sequence, with 78 bicycles and 197 motorcycles (Messelodi et al.,, 2007). This study yeilded an accuracy rate of $96.7 \%$. The accuracy rate is based on a percentage correct classification.

While surveillance video is useful for collecting data on traffic patterns and personal movements, it has a few downfalls. Non-object related items such as shadows can cause the video to change in appearance, and thus cause the data to be changed or skewed (Chen et al., 2008). Recognizing this need, Chen et al., (2008) designed a classification system distinguishing between pedestrians and vehicles captured on a video sequence using a static camera. The performance of the system was evaluated on dataset of 1,100 moving objects. This classification system depends on three elements: $[i]$ discriminative features, $[i i]$ an adaptation process, and [iii] an interactive interface. These elements were integrated together as probabilistic framework. Discriminative features include the object size, velocity, location and Differences of Histograms of Oriented Gradients (DHoG). "DHoG models the intra-object deformation in the temporal domain" (Chen et al., 2008) and was obtained from the difference of Histograms of Oriented Gradients (HoG) (Dalal \& Triggs, 2005) between successive frames. Chen et al., (2008) classification takes into account challenging conditions that happen frequently in the real world, including shadowy conditions, distortions in perspective, and large groups of people moving through traffic intersections. Furthermore, the researchers included an interactive user interface that allows users to manually correct perspective distortions and to select features and ROI for the program to focus on monitoring. The classification process was performed using as a Maximum-a-Posteriori (MAP) estimation by analyzing the extracted features. The system designed by Chen et al., scored a 97.7\% accuracy. 
A study Zhang et al., (2008) presented a classifcation system for vehicles and pedestrains. The dataset used in Zhang et al., (2008) study was collected using a static camera; the data include 58,958 vehicles, 56,942 pedestrians and 3,297 others objects identified as outliers. Outliers are all objects that are not vehicles neither pedestrians. The basic idea of Zhang et al., (2008) is to create a strong classifer by combining a set of weak classifiers. Zhang et al., (2008) system is a feature-based classification system which depened on local features decriptors. The local features decriptors are: [i] Histogram of Oriented Gradients (HOG) (Dalal \& Triggs, 2005), [ii] Scale-invariant feature transform (SIFT) (Lowe, 2004), [iii] Rotation -invariant feature transform (RIFT) (Lazebnik et al., 2005), and [iv] Spin Image (SPIN) (Lazebnik et al., 2005). Different classification experiments were conducted where features decriptors were used individually and grouped together to classify the moving objects. The highest classification result using one features decriptors was for the SIFT with $98.2 \%$ for vehicles classification and $99.3 \%$ for pedestrians classification. The highest classification result using a group of features decriptors was for the SIFT and SPIN with $98.8 \%$ for vehicle classification and $99.7 \%$ for pedestrian classification. These results are based on a percentage correct classification.

A study by Takahashi et al., (2012), proposed a system to detect, track and classify urban road users captured on a video sequence using a high resolution video camera. This classification process employed two phases. The first phase discriminated between pedestrians and cyclistslike objects. The second phase differentiated between cyclists and motorcyclists. Takahashi et al., (2012) tested their methods of classifying vulnerable road users in an experimental setting by comparing the results of their automated system with the results of manually identifying different kinds of road users in the same video sequence. The detection and tracking of the moving objects 
was done using the spatio-temporal Markov random field (S-T MRF) system (Kamijo et al., 2000).

In the first phase of their study, Takahashi et al., (2012) analysed two discriminative features using the Fisher's linear discriminant (Du, 2007; Du \& Younan 2008) to discriminate pedestrians from motorcyclists and cyclists. The features are: $[i](\mathrm{HoG})$ feature, and [ii] cooccurrence semantic (HoG). The co-occurrence semantic (HoG) "aims to make a meaningful non-linear space that is effective in decreasing false positive results of the linear classifier trained in the HOG space" (Takahashi et al., 2012). The first phase was tested by using 1,575 images of cyclists-like objects and 4,223 images of pedestrians. The Fisher's discriminant achieved accuracy rate of $90 \%$.

Cyclists move their legs in a pedalling motion while motorcyclists keep their legs still. Therefore, in the second phase of their study, Takahashi et al., (2012) used Hidden Markov Models (HMMs) (Baum \& Petrie, 1966) to distinguish between cyclists and motorcyclists based on the rider leg motions. The leg area was identified as the region of interest (ROI), various leg silhouettes of cyclists versus motorcyclists were used to distinguish between these two types of road users based on different leg positions. The second face is tested by using 2000 sequence of cyclists and motorcyclists. The system achieved accuracy rate of $88.9 \%$ in the second phase. The accuracy rate is based on a percentage correct classification.

A study by Møgelmose et al., (2012), presented a detection and identification system for pedestrian captured on static images. Møgelmose et al., (2012) system consisted of a detection stage and a verification stage. In the detection stage, the system searches for possible pedestrians by identifying the region of interest (ROI) in the input image. Identifying the ROI was achieved by extracting the Haar-like features (Bilgic et al., 2010) from the input images, then use these 
features in by the Adaboost algorithm (Viola et al., 2001) to construct a strong classifier for detection. Subsequently, the images with the identified ROI are sent to verification. Two types of verifications where introduced:

1. Individual part verification where the verification is based on upper or lower body part, and

2. The combined verification where the verification is based on the full body.

In the verification stage, the Histograms of Oriented Gradients was calculated and sent to SVM for the final identification. Møgelmose et al., (2012) system was tested on a dataset include 742 images. Comparing between the two types of verifications, Møgelmose et al., (2012) discovered that the combined verification performed better than the individual part verification.

A study by Cheng \& MaoJhan., (2012), presented a system for pedestrian detection and classification by combining the Adaboost and SVM into a cascade classifier. Cheng et al., (2012) study begin by segmenting the (ROI) in the image. The (ROI) include the "possible pedestrian". Segmentation was done using subtraction techniques of each frame from the background images. Then, features were extracted by applying three extraction techniques (edge detection, Haar-like features and histograms of oriented gradients). Finally, a cascade classifier was developed to classify the pedestrian based on the extracted features. The system was tested on 2 data sets where each set has 10,560 images captured in Wufong District, Taiwan. The system yielded an accuracy rate of $99.36 \%$. The accuracy rate is based on a percentage correct classification.

\subsection{Summary}

This chapter provided an overview of some of the developed methods of automated classification of road users. Each of these methods has its own uniqueness in some way. However, most of these methods share the same stages: the detection stage, the tracking stage and the verification stage. The classification methods were divided into five types: 
1. Motion Pattern: This method was used only used to classify detect and classify pedestrian. This method relies on the relative motion of human limbs.

2. Movement Paths: This method was used to classify road users based on motion information associated with their movement paths (Trajectories). The movement paths were collected using a feature-based tracking system.

3. Three-dimensional Frames Models: This method was used to classify road users into vehicles, cyclists and pedestrian based on a set of 3D models.

4. Dynamic Parameters: This method was used to classify road users based on their speed, dimensions and location.

5. Local Features Descriptors: This is the most common method. It was used to classify road users by extraction local features from the images using local feature descriptor such as (HoG, Haar-like feature).

Although the automated classification of road users has achieved advanced stages, there is still a need to develop a classification algorithm that is capable of performing the following:

1. Classify road users into classes and categorize them into subclasses, such a technique was developed for vehicles but not for the other types of road users.

2. Classify road user under different lighting condition. Lighting condition is one of the existing challenges that affects the classification accuracy.

3. Develop an approach that does not depend on the geometry or specific traffic operation of the intersection. This will allow transferability from on location to another and improves its practicality and usefulness.

4. Detection of traffic violations. 


\section{Chapter 3: Development of a Framework for Object Classification}

\subsection{Background}

Urban traffic involves the movements of road users using different modes. There are two main types of transportation modes in urban roads: motorized and non-motorized. Each mode includes multiple methods of travel. For example, motorized modes of transportation include passenger cars, trucks, buses, and motorcycles. Non-motorized modes of transportation include walking, cycling, and skateboarding. Each method of transportation has unique characteristics that can be used to distinguish it from other methods; for example, cyclists can travel at a higher speed than pedestrians. These unique characteristics can be also identified among road users of different transportation modes. Urban traffic often involves optimizing resources (e.g., space and signal time) to serve all these modes. Data collection is typically needed at a disaggregate level, thus distinguishing these categories of road users is required, preferably in an automated way.

This thesis presents a unique framework that is designed to classify different categories of tracked objects on the road, captured using a commercial-grade high-resolution video camera (1080 x 1920 at approximately 30 frames/second).Tracking an object in a video sequence is the process of locating the position of the tracked objects in each video frame (Prakash et al., 2012). A track is composed of a sequence of positions that represent the movement of the tracked object within the field of view recorded in one frame after another. Tracking an object can be performed using a tracking system such as computer vision application. The information of tracking is presented in world coordinates. The phrase "tracked objects" is carefully chosen to describe road users for the following reasons: 
1. The term "objects" is used because the tracking system used in this study is designed to detect and track the movement of any object, regardless of its type or category.

2. The term "tracked" is associated with the term "objects" because the tracking system can detect and track moving objects only. Stationary objects or objects which are not clearly distinguished from still background are not tracked.

The development of the classification framework went through three main stages: the calibration stage, the classification stage, and the validation stage. Each stage has multiple steps. Each of these stages is crucial for the classification of the tracked objects, and will be explained in detail later in this chapter. Before going into further details, three question need to be answered:

1. What are the types of tracked objects that this framework is targeting?

2. How many models are in this framework?

3. What kind of information does this framework need to classify different tracked objects?

To answer the first question, this framework targets three different types of tracked objects: motorized vehicles, cyclists, and pedestrians. For the vehicle category, this framework is designed to classify all motorized transportation modes as vehicles. Note that two-wheeled and three-wheeled motorized transportation modes are excluded, primarily because of their scarcity in the used video data. In the cyclist category, this framework is designed to classify all cyclists as such, regardless of what type of bicycle they are using, for example, two- or three-wheeled bicycles are placed together in the same category. In the pedestrian category, this framework is designed to classify only walking pedestrians as such. If a tracked object cannot be classified then it will be labelled as unknown. Unknown objects are all the tracked objects for which 
insufficient information was available to place it in any of these three categories (the classification system is simply stating that it does not know who is moving).

To answer the second question, there are four models in this framework. The first model tests the classification of the three categories: vehicles, cyclists and pedestrians. The second model tests the classification for vehicles and cyclists only. The third model tests the classification for vehicles and pedestrians only. The fourth model tests the classification for cyclists and pedestrians only.

To answer the third question, this framework uses the data obtained from the tracked object tracks, and uses it to classify the tracked object. The framework was not tested to include data which require separate video or image processing other than what is already available after tracking objects. The data includes the motion cues of the tracked objects. The separate pieces of information are gained from these motion cues are hereafter called Classifiers. There are nineteen classifiers included in this framework. These classifiers include: average and maximum speeds; average and maximum acceleration; average and maximum deceleration; average and maximum direction; average of change in direction; average of cosine of change in direction; average and maximum area; average and maximum length; average and maximum width; peaks in speed; the effective frequency; and the effective weighted average frequency.

After obtaining the classifiers' values from the tracked objects' tracks, the information from these classifiers will be assessed and integrated using fuzzy membership approach, which in turn requires prior configurations to be available. This will lead to the final classification of the tracked object. The fuzzy approach will be explained in section 3.4 of this chapter. The 
configuration used will be explained in section 3.5.1 of this chapter. Figure 3.1 is a flowchart illustrating a basic description of the classification process.

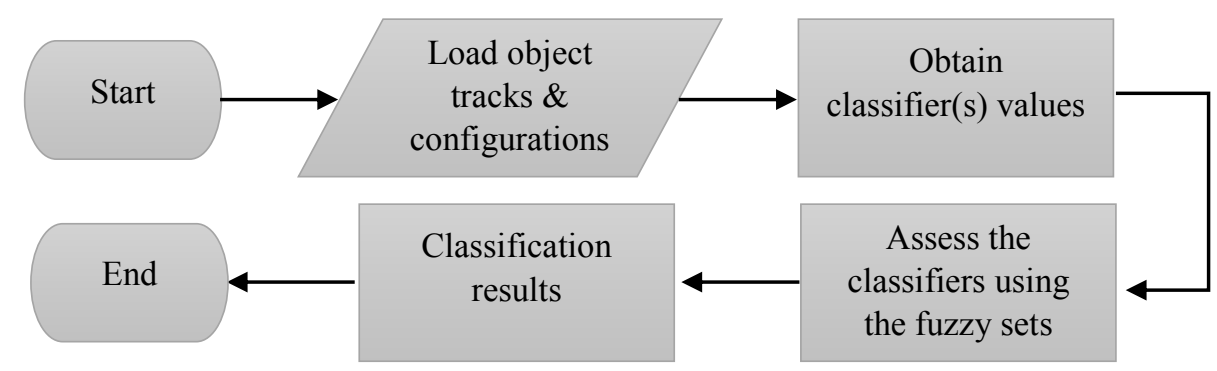

Figure 3. 1 A basic description of the classification process.

\subsection{Data Collection}

In order to build, calibrate, and test the framework, real-world traffic video data are required. This data was gratefully obtained from previous work (Kassim, 2014) in which the author constructed these datasets in separate research. Data were collected using a highresolution video camera installed at a high-elevation to cover the entirety of the monitored intersections. The recording resolution was set to $1080 \times 1920$ pixels. The frame rate is approximately 30 frames/second. The targeted intersections in this research are two four-legged signalized intersections in the Downtown area of Ottawa, Ontario (Kassim, 2014). The first intersection was located at Laurier Avenue West and Lyon Street; the video camera was located on a building facing this intersection. The second intersection was located at Laurier Avenue West and Bay Street; the video camera used was also installed on a building facing the targeted intersection. Video sequence were collected from various time periods covering the fall and summer seasons of the years 2011 and 2012, respectively. They show significant pedestrian and cyclist traffic, as well as vehicles traveling at different speeds and directions. Video subsequences were chosen to display the three road users co-existing in the intersections within 
reasonable time from each other. A total of 898 vehicles, 535 cyclists, and 314 pedestrians were observed and used in this thesis.

\subsubsection{Data description}

The data sets used in this research are the output of the tracker system that was used in study by (Kassim, 2014). The tracker system primarily uses the "Kanade-Lucas-Tomasi" algorithm (Lucas \& Kanade, 1981) to detect and track features (salient points) in video sequence. These features are subsequently grouped into one object based on an extensive set of rules to measure their similarity and conformance to a coherent body movement. The provided track dataset includes the following:

1. The positions of the vertices of the bounding box which encapsulates grouped features in world coordinates. The bounding box is the rectangular shape that contains the features of the tracked object. In this dataset, a feature is defined as "a salient point, or sub window, which can be tracked for an adequate number of frames while in motion" (Kassim, 2014). The bounding box has eight vertices. These vertices are Top-Left $(T L)$, Top (T), Top-Right $(T R)$, Bottom-Left $(B L)$, Bottom $(B)$, Bottom-Right $(B R)$, Centre-Left $(C L)$ and Centre-Right $(C R)$.

2. The sequence of the average displacement components $d x$ and $d y$. A frame is a still image of many distinct images that compose a video sequence. The displacement is the change in position of the tracked feature inside the bounding box between successive frames. The average displacement is the sum of changes in positions for these features divided by the number of these features. This therefore provides a robust estimate of the direction of 
movement of the tracked object based on constituent features. The average displacement is calculated for $x$-axis $(d x)$ and $y$-axis $(d y)$.

3. The visualization of the tracked objects in video sequence. The visualization is a by-product of the tracker system that shows the bounding box around the tracked object in the video sequence. The visualization also shows the unique ID of the tracked objects. The unique ID of a tracked object is a unique number (in each analysed video) assigned to each tracked object in a video sequence. The unique ID is important in this framework, because, with this ID, the tracked objects were manually classified so that the effectiveness of the framework could be empirically determined.

To illustrate the contents of this database, the following figures show set of features that represent the tracked objects. Figure 3.2 shows the bounding box, the vertices, the unique ID, the frame number and the mask in the tracker system. The mask is an assigned filter in the tracking system to exclude any movement fall within its boundaries from the tracking process. This mean moving object will not be tracked if they fall within the boundaries of the mask. This filter was added in earlier work by (Kassim, 2014) to exclude any object irrelevant to his research. In that, only one direction of cyclist movement was analysed. This direction involved possible interactions with right-turn vehicles. Because the cross-streets (crossing Laurier Avenue) are one-way, then the other cyclist movement direction was excluded, and thus masked as is shown in Figure 3.2.

Figure 3.3 shows a sample output text which includes the position of a tracked object in each frame. This position presented in $x$ and $y$ world coordinates. It includes, the unique ID of the tracked objects, the number of frame where the tracking began, the duration of tracking, the 
positions of vertices as well as the average displacement for each tracked object both in world coordinates.

(a)

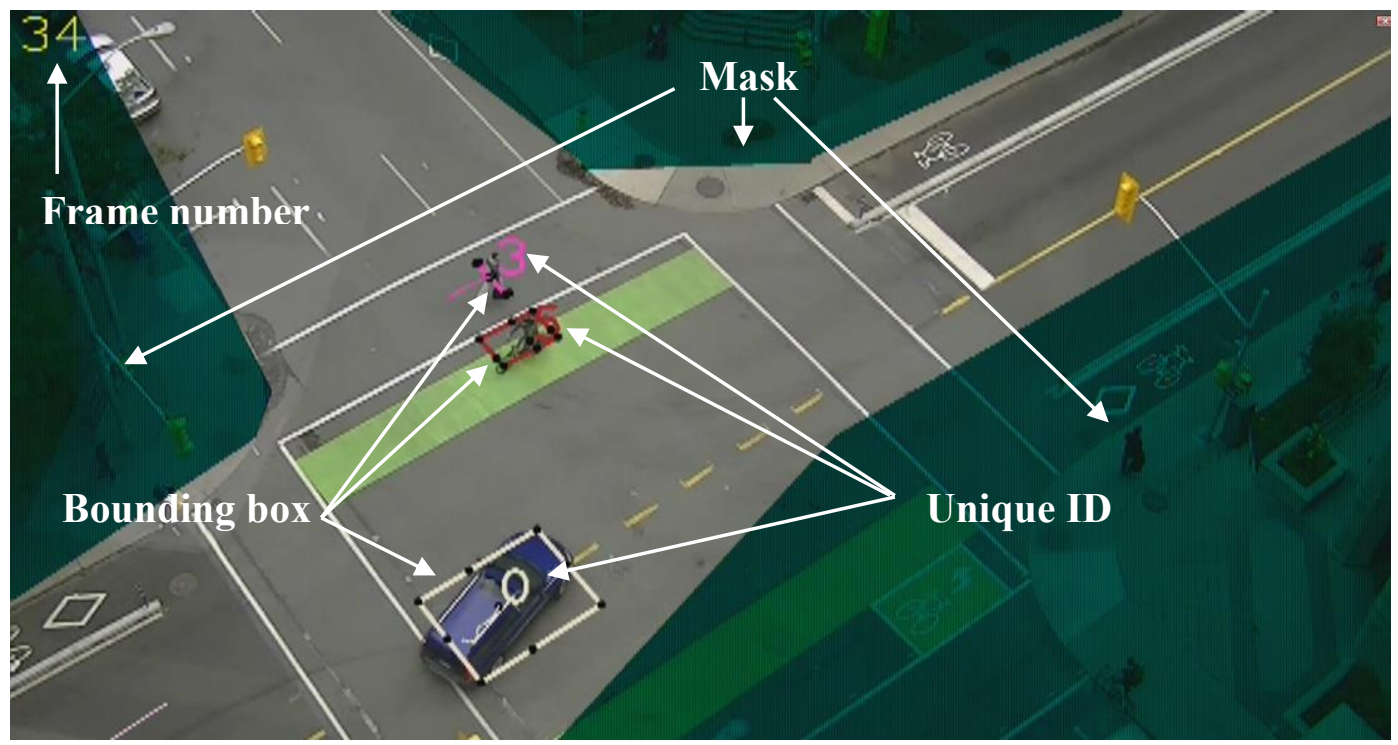

(b)

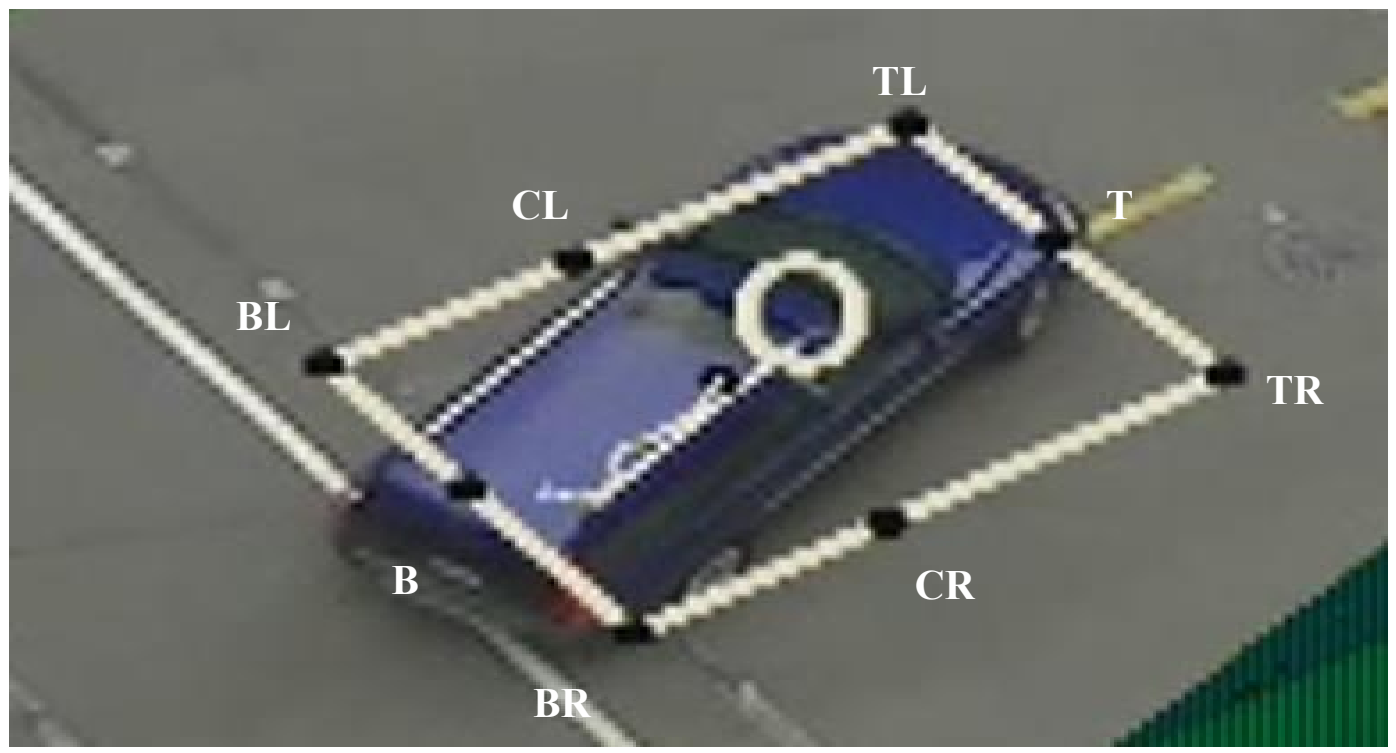

Figure 3. 2 A video sequence analyised using a feature-based tracking system.

a) Include the frame number, the mask, the bounding box, the vertices and the unique ID in the tracker system. Object (0) is a vehicle, object (5) is a cyclist and object (3) is a pedestrian. b) Shows the vertices of the bounding box for obejct (0). 


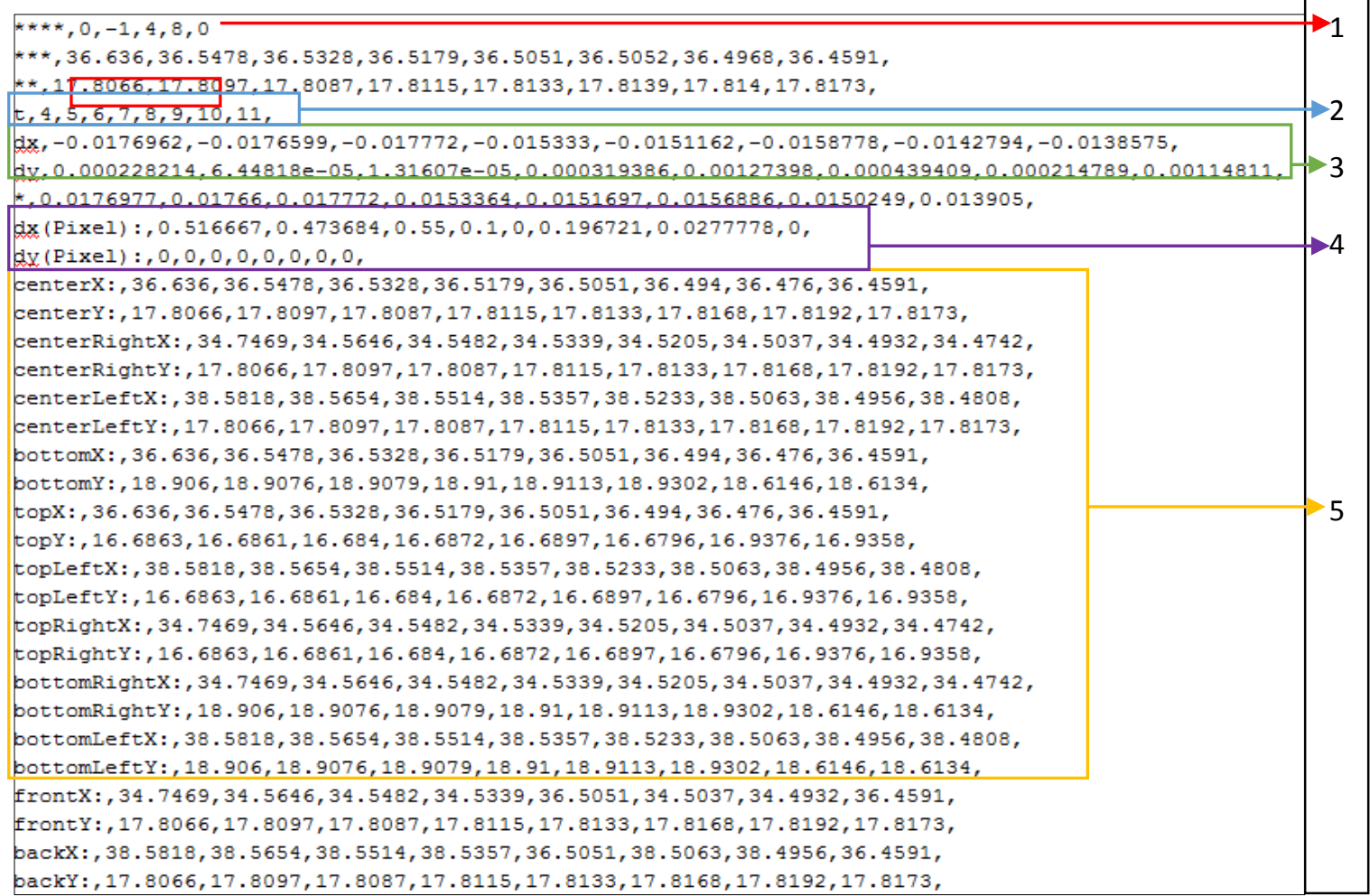

Figure 3. 3 The output text of tracker system.

1) The " 0 " is the tracked object ID, the " 4 " is the number of frame where the tracking began, the " 8 " the duration of tracking in unit frame, the " 0 " is the number of frames with missing tracks also known as partial tracks which will be explained in section 3.2 .2 of this thesis.

2) The numbers of tracked frames.

3) The average displacement in world coordinates.

4) The average displacement in image coordinates.

5) The coordinate of the vertices of the bounding box for the tracked object.

\subsubsection{Data correction}

The proposed framework uses the tracked object tracks to obtain the classifiers' values (e.g. average speed). The object tracks are presented in: [i] the position of the vertices of the bounding box in each frame; and [ii] the sequence of average displacement components of the tracked object in each frame. As a result, the proposed framework model can be affected by the 
tracking quality. Tracking systems are not perfect, there are some circumstances that can make them miss some tracks of an object. This is known as "partial tracks". Partial tracks occur when the number of features - that is, features in the bounding box — that are present within a specific frame is less than a threshold value in the tracking system. This threshold is precisely set to prevent the trackers from relying on too few features to constitute an object and thus be prone to produce unreliable tracks. Partial tracks can also occur due to uncontrolled circumstances such as light reflecting on the video camera, or from the shade of the surrounding buildings on the tracked object. Such circumstances make it difficult for the tracking system to detect and track objects. See figure 3.4 and figure 3.5.

There have been two proposed solutions to the problem of partial tracks. The solutions depend on the location of the missing tracks in the tracks series. The tracks series begins from the time that the tracked object enters the recording zone until it leaves it. If the missing tracks are in the beginning or the end of the track series, then these missing tracks will be ignored and removed from the tracked series. If there are tracks at the beginning and the end of the track series and the missing track is in between, then these missing positions are estimated using linear interpolation. This interpolation is intended to increase the information available for classification by providing complete tracks. It is acknowledged that linear interpolation is based on a simple assumption which may not be true. More sophisticated methods can be used, however, it was not considered in this thesis because abrupt change in movements within relatively short time is not a typical behaviour of road users in traffic scenes. Figure 3.6 illustrates linear interpolation on a speed profile of an object. 
a)

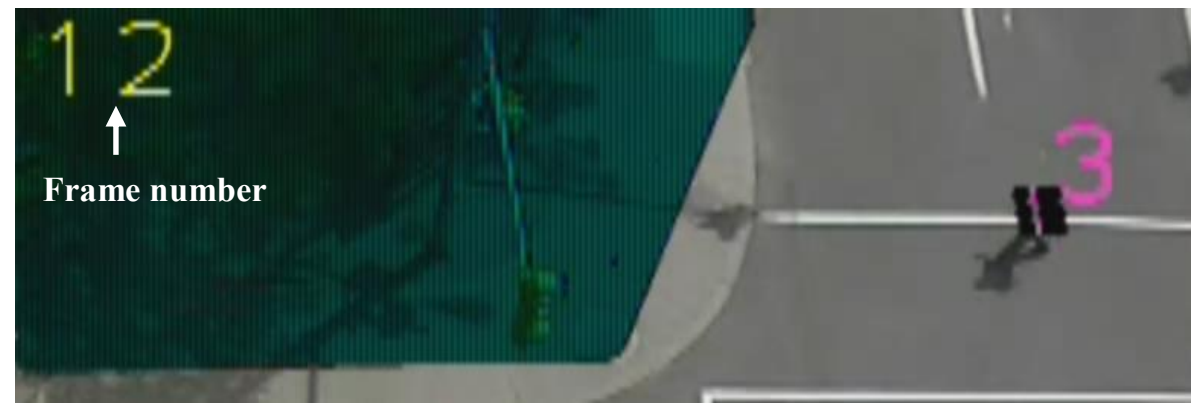

\section{Pedestrian}

tracked

b)

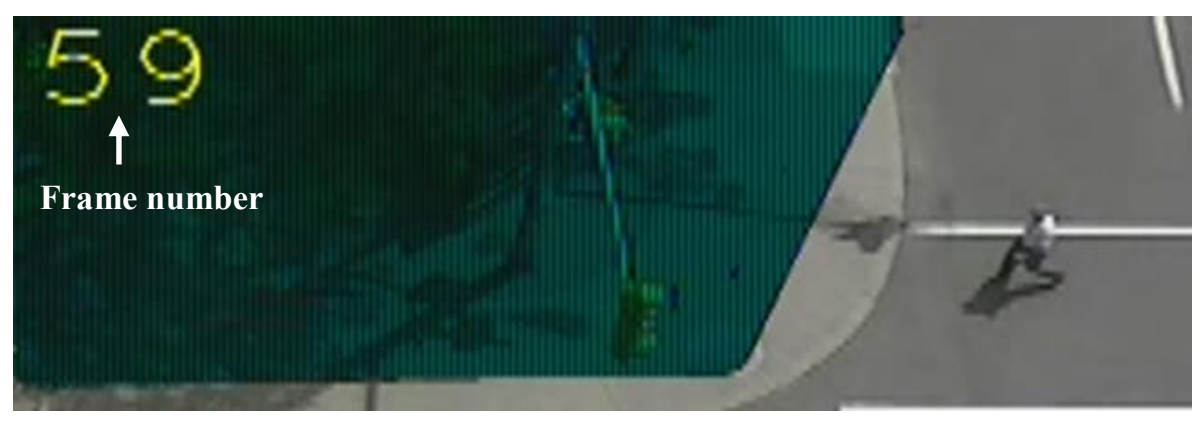

Pedestrian lost

c)

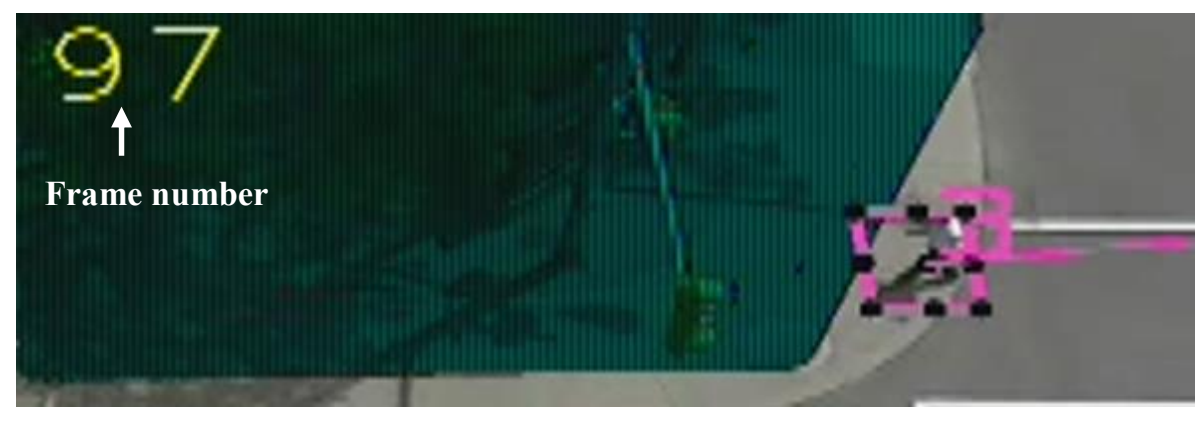

Pedestrian

tracked again

Figure 3. 4 An explanation of partial tracks. 
Figure 3.5 A sample output text of the tracker system explains the partial tracks of an

\title{
object. Missing tracks are presented with the "?" symbol.
}

(a)

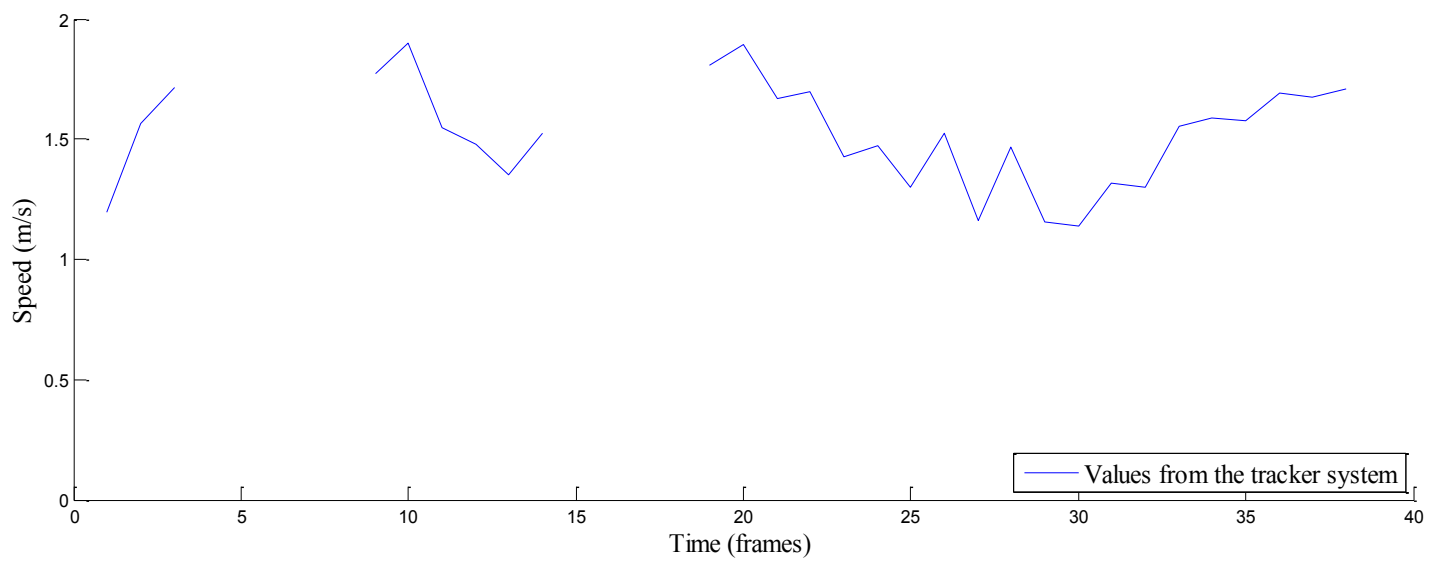

Figure 3. 6 An explanation of the linear interpolation.

* (a) Shows speed profile with missing tracks. 
(b)

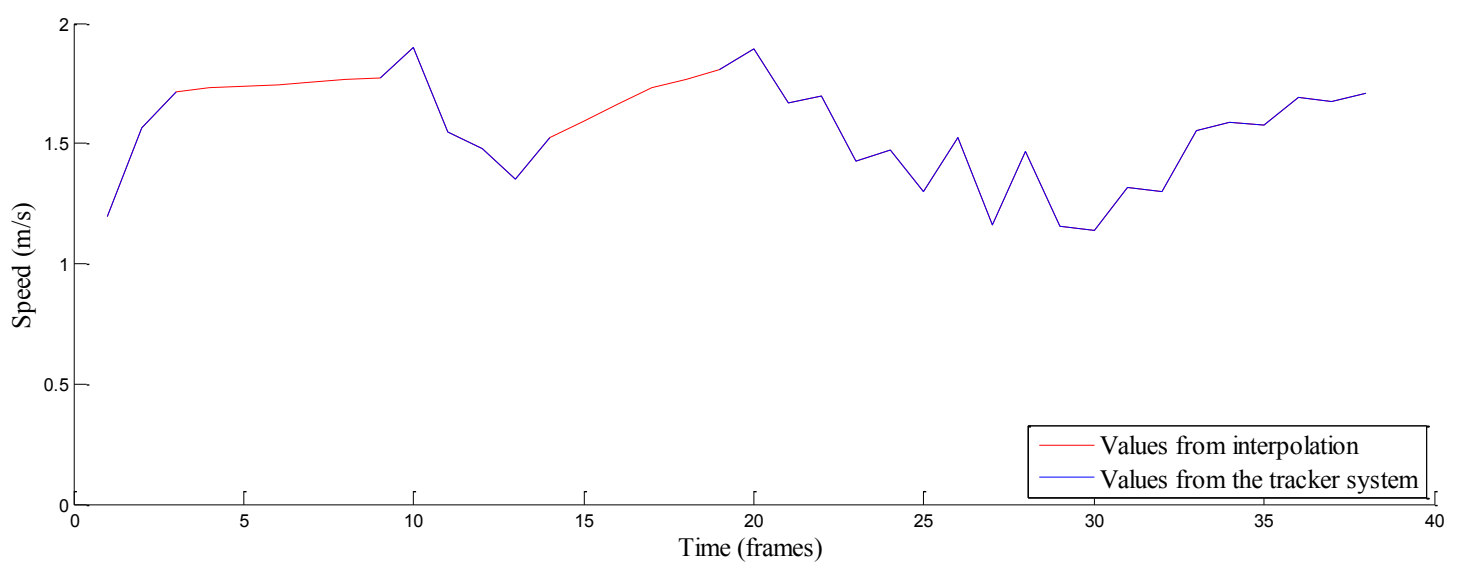

Figure 3. 6 (continue)

* (b) Substituting the missing tracks using liner interpolation.

\subsection{Classifiers' Description}

This framework is designed to extract information from the tracked object tracks and use this information to classify the object that associated with these tracks into: vehicles, cyclist and pedestrians. The difference sources of this information are called the classifiers. There are nineteen classifiers included in this thesis, and divided into five groups and as follows:

- Tracked object speed: include the average and maximum speed;

- Tracked object acceleration and deceleration: include the average and maximum acceleration; the average and maximum declaration;

- Tracked object direction: include the average and maximum direction; the average of change in direction; the average of cosine of change in direction;

- Tracked object dimensions: include the average and maximum length; the average and maximum width; the average and maximum area; 
- Tracked object frequency: include the peaks in speed; the effective frequency; the effective weighted average frequency.

Although the tracked object speed and dimensions were used in previous studies to classify tracked objects on the road, the way they were utilized in this framework is unique to this thesis. In addition, classifying road users based on their acceleration, direction and frequency is new and was never observed in previous studies.

\subsubsection{Tracked object speed}

Traditionally, the speed of any object is measured as the travelled distance divided by the travel time. In this framework, the speed of a tracked object is calculated from the average displacement of the features composing the tracked object in each pair of frames. The displacement of each tracked frame is then multiplied by the camera frame rates to convert the units to $(\mathrm{m} / \mathrm{s})$.

$$
S_{i}=\sqrt{d x_{i, f}^{2}+d y_{i, f}^{2}} * f_{r} ; f \in\left\{n_{1}, n_{2}\right\}
$$

where: $S_{i}$ is the speed in $(\mathrm{m} / \mathrm{s})$ for object $i ; n_{1}$ and $n_{2}=$ first and last frames between which the object $i$ is tracked, $d x_{i, f}$ and $d y_{i, f}=$ the average displacement of the object $i$ in frame $f$ defined in world coordinates, $f_{r}$ is the camera frame rate which set to 30 frames/sec.

The average speed of a tracked object is the sum of all the speed values for this object divided by the number of frames where this object was tracked in. The maximum speed of a tracked object is the highest speed value for this object within all the frames this object was tracked in. 


$$
\overline{S_{i}}=\frac{\sum s_{i, f}}{n_{f}} ; f \in\left\{f_{1}, \ldots, n_{f}\right\}
$$

where: $\overline{s_{i}}$ is the speed in $(\mathrm{m} / \mathrm{s})$ for object $i ; f_{1}$ is the first frame in the video sequence; $n_{f}$ is the number of frames sequence where object $i$ was tracked in.

\subsubsection{Tracked object acceleration and deceleration}

Acceleration (or conversely deceleration) of a tracked object is calculated by recording the change in speed between two consecutive frames. The initial speed of the tracked object is the speed at the (frame $j$ ) and the final speed of that object is the speed at the (frame $\left.j_{j+1}\right)$. If the subtracted result is a positive value, i.e., the tracked object is going faster, then this means that the tracked object is accelerating. If the subtracted result is a negative value, i.e., the tracked object is slowing down, then this means that the tracked object is decelerating. The result in each frame is multiplied by the video frame rates to convert the units to $\left(\mathrm{m} / \mathrm{s}^{2}\right)$.

$$
A_{i}=\left(S_{i, f_{j+1}}-S_{i, f_{j}}\right) ; * f_{r} ; f \in\left\{n_{1}, n_{2}\right\}
$$

where: $A$ is the acceleration or deceleration in $\left(\mathrm{m} / \mathrm{s}^{2}\right)$ for object $i ; S_{i, f_{j+1}}$ and $S_{i, f_{j}}$ are the speeds of object $i$ object in the successive frames.

The average acceleration for a tracked object is the sum of all the acceleration values of this object, divided by the total number of the acceleration for this object. The maximum acceleration of a tracked object is the highest acceleration value for this object within all the frames this object was tracked in.

The average deceleration for a tracked object is the sum of the absolute deceleration values for this object, divided by the total number of decelerations for this object. The maximum 
deceleration of a tracked object is the absolute highest deceleration value for this object within all the frames this object was tracked in.

\subsubsection{Tracked object direction}

\subsection{3a Tracked object average and maximum direction}

The direction of a tracked object represents the route that the object is taking while navigating the intersection. This can be measured using the deviation from a reference direction or azimuth. The azimuth measures the horizontal angle of the tracked object in each frame, from the North direction in a clockwise direction. To calculate the direction of a tracked object for each tracked frame, the sequence of the average displacement components $d_{x}$ and $d_{y}$ between successive frames is used. Table 3.1 is used to obtain the azimuth. For the purpose of this framework, the average and maximum directions are calculated. The average direction of a tracked object is the sum of all the direction values for this object divided by the number of frames where this object was tracked in. The maximum direction of a tracked object is the highest direction value for this object within all the frames this object was tracked in

Table 3. 1 Directions of movement of the tracked object

\begin{tabular}{|c|c|}
\hline Condition & Value of $\boldsymbol{\theta}$ \\
\hline$d y>0, d x=0$ & $\theta=0$ \\
\hline$d y<0, d x=0$ & $\theta=180$ \\
\hline$d y \geq 0, d x>0$ & $\theta=90-A B S\left(\tan ^{-1} \frac{d y}{d x}\right) * \frac{180}{\pi}$ \\
\hline$d y<0, d x>0$ & $\theta=90+A B S\left(\tan ^{-1} \frac{d y}{d x}\right) * \frac{180}{\pi}$ \\
\hline$d y<0, d x<0$ & $\theta=270-A B S\left(\tan ^{-1} \frac{d y}{d x}\right) * \frac{180}{\pi}$ \\
\hline$d y \geq 0, d x<0$ & $\theta=270+A B S\left(\tan ^{-1} \frac{d y}{d x}\right) * \frac{180}{\pi}$ \\
\hline
\end{tabular}


where: $\theta$ is the direction of the tracked object in degree;

$d x$ is the displacement of the tracker object in $x$-axis;

$d y$ is the displacement of the tracker object in $y$-axis.

\subsection{3b Tracked object change in direction}

Changes in direction of the tracked object are calculated in terms of the change in direction between two successive frames. The initial direction of a tracked object is the direction at the (frame $j$ ), and the final direction of that object is at the (frame $\left.{ }_{j+1}\right)$. For the purpose of this framework, the average of change in direction and the average of the cosines of change in direction are calculated.

The average of change in direction for a tracked object is the sum of the changes in direction values for this object, divided by the number of frames where this object was tracked in - one

$$
\overline{c_{d i}}=\frac{\sum c_{d, i, f}}{n_{f}-1} ; f \in\left\{f_{1}, \ldots, n_{f}\right\}
$$

where: $\overline{c_{d i}}$ is the average of change in direction of object $i$ in degrees;

$c_{d i}$ is the change in direction for object $i$ in degrees;

The average of the cosine of change in direction for a tracked object is calculated by obtaining the cosine value for the change in direction between successive frames for this object, sum the cosine values and divided by the total number tracked frames - one.

$$
\overline{\operatorname{co}_{d i}}=\frac{\sum \cos c_{d, i, f}}{n_{f}-1} ; f \in\left\{f_{1}, \ldots, n_{f}\right\}
$$

where: $\overline{C O_{d i}}$ is the average of cosine of change in direction of object $i$ in degrees. 


\subsubsection{Tracked object dimensions}

The physical dimensions of the tracked objects (vehicles, cyclists, and pedestrians) are noticeably different from each other. These differences could be used to discriminate between different tracked objects. Note that in some circumstances, the dimensions of some tracked objects are expected to overlap; for example, cyclists have roughly the same width as pedestrians. Thus this information is not solely relied on but is partially informative in a fuzzy framework.

The dimensions of tracked objects in each tracked frame were obtained from knowledge of the bounding box vertices. The tracked objects dimensions include the area, the length, and the width. The size of the bounding box should provide an estimate of the tracked object size. The size of the bounding box might change depending on the number of salient features inside the box. Please see Figure 3.7. A bounding box with a good amount of features should provide the closest size to the tracked object's size. In order to account for changes in the size of the bounding box, the average and maximum dimensions (which refers to the average and maximum length, the average and maximum width, and the average and maximum area) are taken.

The bounding box has eight vertices, and four of them are the corners of the bounding box. The corners' vertices are Top-Left $(T L)$, Top-Right $(T R)$, Bottom-Left $(B L)$ and Bottom-Right $(B R)$. The length of a tracked object is the distance measured from the Top-Left $(T L)$ corner to the Bottom-Left $(B L)$ corner of the bounding box. The average length of a tracked object is the sum of all the length values of this object in divided by the number frames where this object was tracked in. The maximum length of a tracked object is the maximum length value within all frames this object was tracked in. 


$$
L_{i, f}=\sqrt{\left(x_{2, i, f}-x_{1, i, f}\right)^{2}+\left(y_{2, i, f}-y_{1, i, f}\right)^{2}} ; f \in\left\{f_{1}, \ldots, n_{f}\right\}
$$

where: $L_{i}$ is the length of the object $i$ in frame $f$ in (m); $x_{2 i, f}, y_{2 i, f}$ are the Top-Left (TL) coordinates of the bounding box for object $i$ in frame $f ; x_{1, i, f}, y_{1, i, f}$ are the Bottom-Left $(B L)$ coordinates of the bounding box for object $i$ in frame $f$.

The width of a tracked object is the distance measured from the Bottom-Left $(B L)$ corner to the Bottom-Right $(B R)$ corner of the bounding box. The average width of a tracked object is the sum of all the width values of the bounding box associated with that object in divided by the number frames where this object was tracked in. The maximum width of a tracked object is the maximum width value within all frames this object was tracked in.

$$
W_{i, f}=\sqrt{\left(x_{2, i, f}-x_{1, i, f}\right)^{2}+\left(y_{2, i, f}-y_{1, i, f}\right)^{2}} ; f \in\left\{f_{1}, \ldots, n_{f}\right\}
$$

where: $W_{i}$ is the width of the object $i$ in frame $f$ in (m); $x_{2 i, f}, y_{2 i, f}$ are the Bottom-Right $(B R)$ coordinates of the bounding box for object $i$ in frame $f ; x_{1, i, f}, y_{1, i, f}$ are the Bottom-Left $(B L)$ coordinates of the bounding box for object $i$ in frame $f$.

The area of a tracked object is equal to the area of the bounding box, and can be simply calculated by multiplying the length by the width in each frame the object was tracked in. The average area of a tracked object is the sum of all area values of the bounding box associated with that object divided by the number frames where this object was tracked in. The maximum area of a tracked object is the maximum area value within all frames this object was tracked in. 
(a)

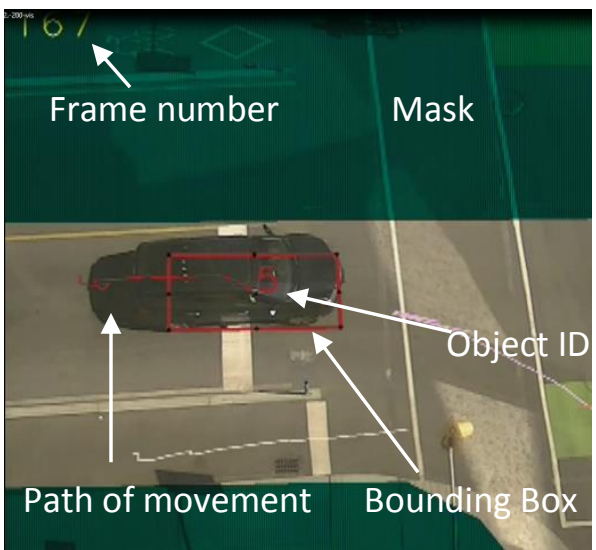

(c)

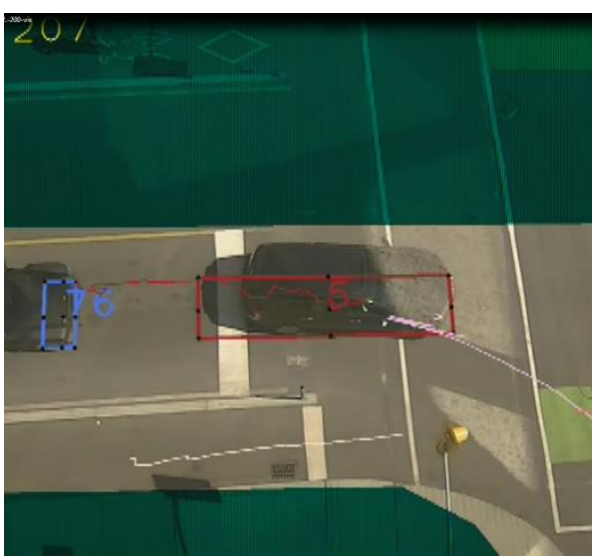

(b)

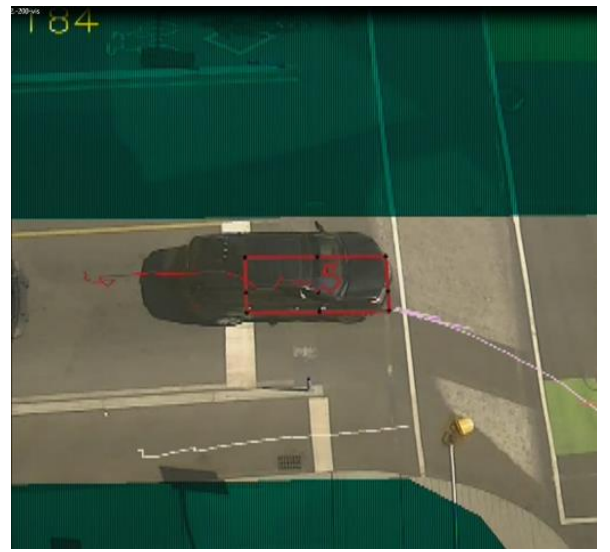

(d)

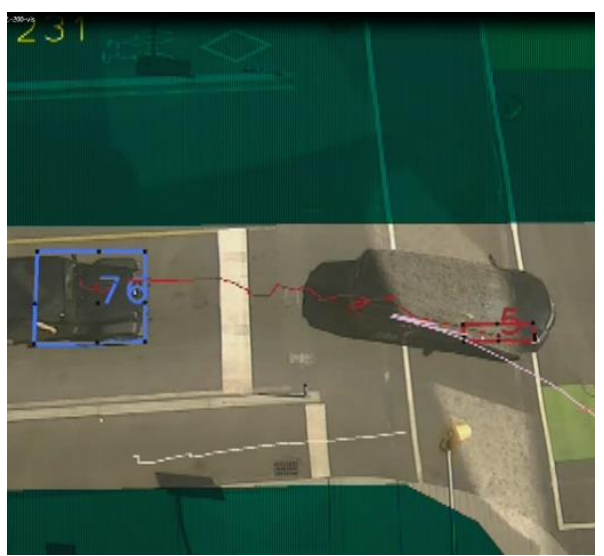

Figure 3. 7 An example of the change in the size of the bounding box for both object 5 and object 76.

\subsubsection{Tracked object frequency}

The idea behind using the frequency of changes in road user movements is to utilize the time dimension of tracking. Road users sometimes exhibit periodicity of changes in speed and/or direction while moving. It is imaginable that a pedestrian ambulatory movement may be discerned if speed tracking is accurate enough. Similarly, the torque applied by a cyclist while pedalling is not constant in time and, similarly, under accurate enough speed measurement, such periodicity or rhythmic movement can be discerned. Motorized traffic on the other hand is 
unlikely to exhibit such periodicity, at least in short time intervals, because of the engine and gearbox mechanical function. Thus, it is possible to utilize this periodicity in movement in classifying road users. Unfortunately, there is no universal measure of such periodicity and the following measures were used in this thesis.

\subsection{5a Peaks in speed}

Peaks in speed refer to the number of times per second that a tracked object passes its average speed, either by going from below the average speed to above the average speed, or by going from above the average speed to below the average speed. Figure 3.8 illustrates peaks in a speed profile of a pedestrian. Peaks in speed are calculated using the following procedure:

1. Calculate the speed deviation in each frame. The speed deviation is calculated by subtracting the speed of the tracked object in each frame from that object's average speed.

$$
S d_{i, f}=\left(S_{i, f}-\overline{S_{i}}\right) ; f \in\left\{f_{1}, \ldots, n_{f}\right\}
$$

Where: $S d_{i, f}$ refer to the speed deviation for object $i$ in frame $f$

2. Multiply the speed deviations between successive frames. If the result of is a negative value, then this value can be considered to be a peak.

$$
S d_{i, f} * S d_{i, f+1}<0 \rightarrow P_{k}=P_{k}+1 ; f \in\left\{f_{1}, \ldots, n_{f}\right\}
$$

Where: $P_{k}$ is the total number of peaks;

3. Peaks in speed are calculated per second using the following formula:

$$
P_{s}=\frac{P_{k} * f_{r}}{n_{f}}
$$

where: $P_{S}$ is the peaks in speed/seconds;

$f_{r}$ is the camera frame rate which is set to 30 frames/seconds; 
$n_{f}$ is number of frames sequence where the object was tracked in.

(a)

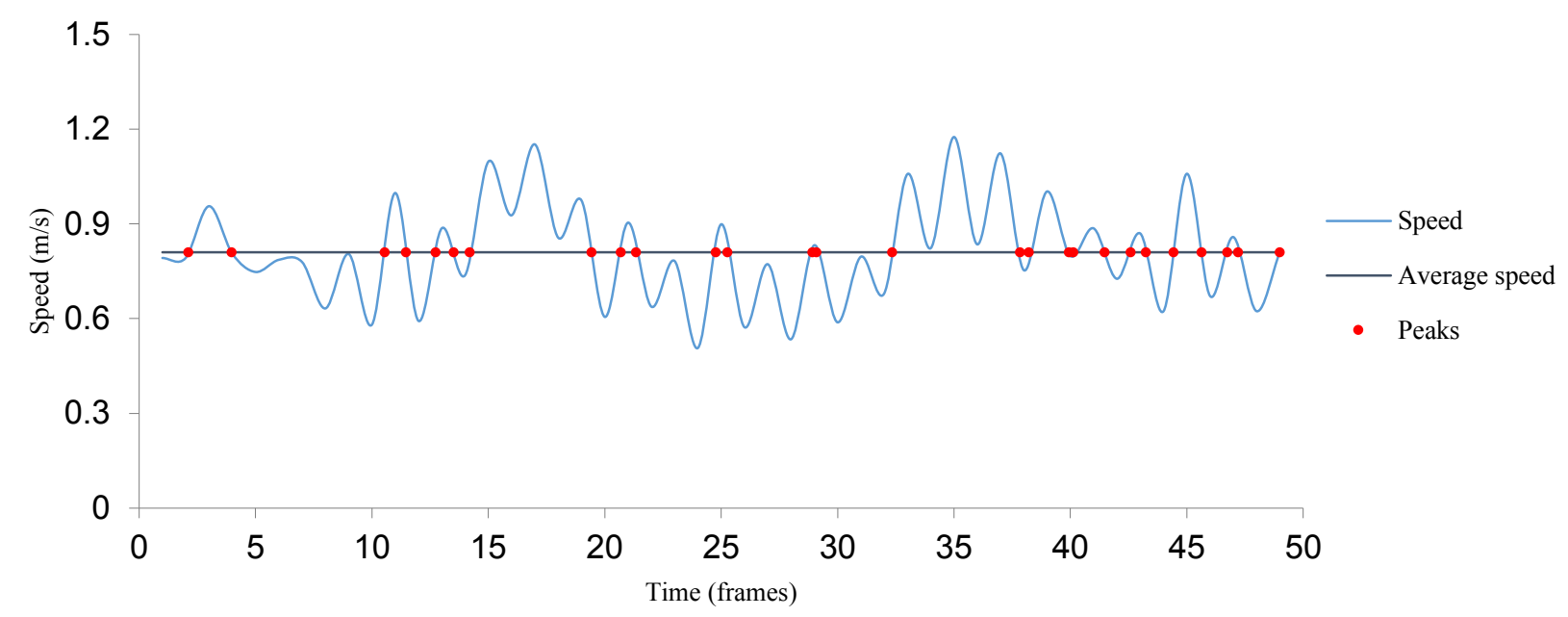

(b)

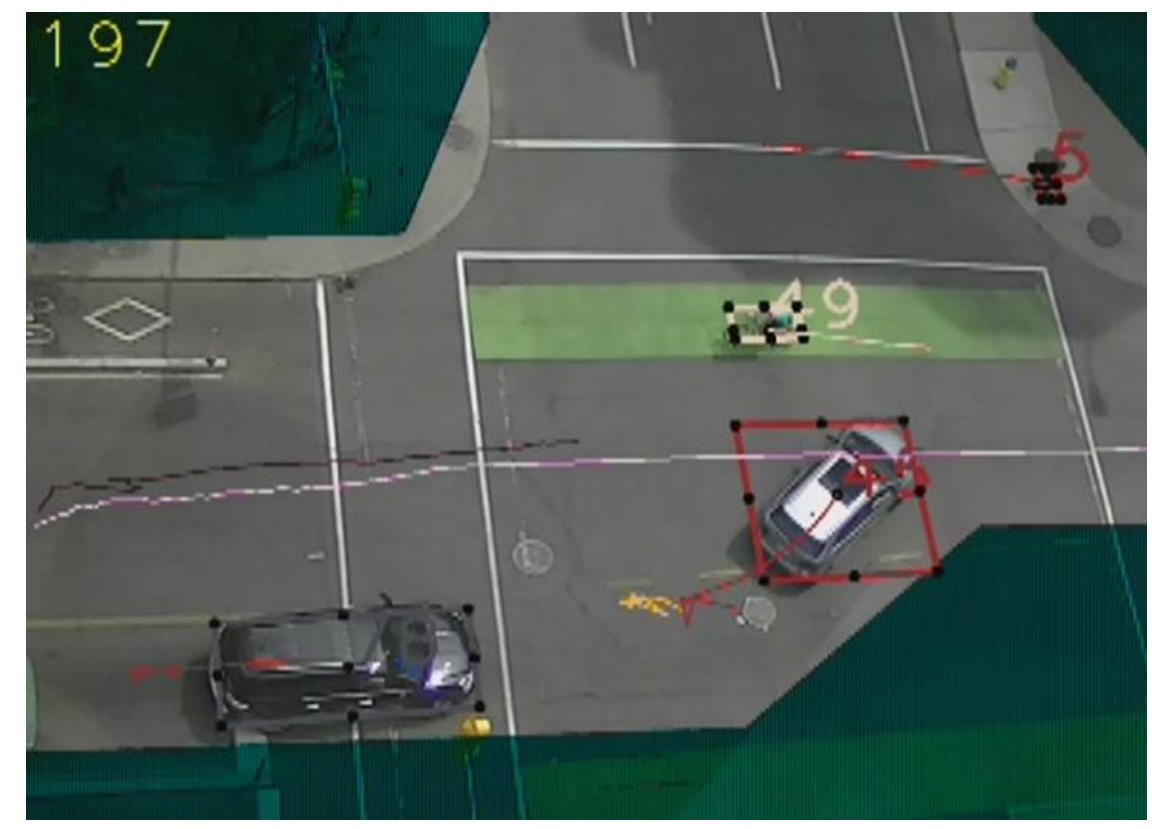

Figure 3. 8 An explanation of the peaks in a speed

a) Shows the peaks in a speed profile of a cyclist. b) The speed profile in (a) is belong to object 49 . 


\subsection{5b Frequency domain modelling}

From carefully examining the shape of the speed profile of different types of tracked objects, it was found that the tracked objects of different categories have different shapes in their speed profile. The speed profile for a pedestrian shows much peculiarity in comparison to a cyclist. The speed profile for vehicles is more uniform. Figure 3.9 include samples of speed profiles for individual road users of different categories. The discrepancy in the shape of the speed profile led to a question: can a frequency be used to classify tracked objects?

a)

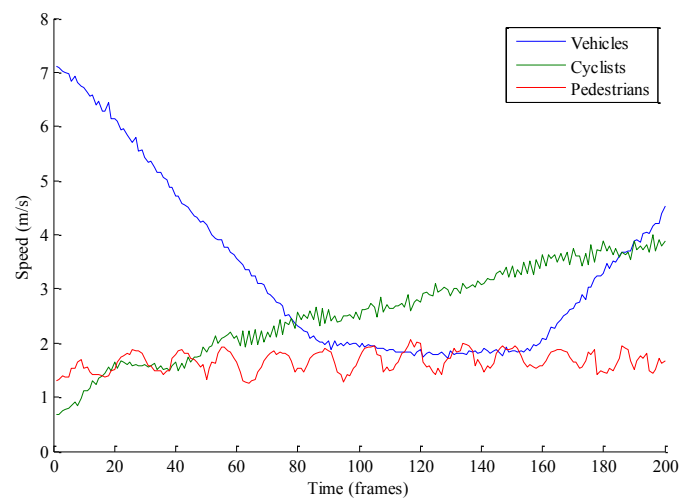

c)

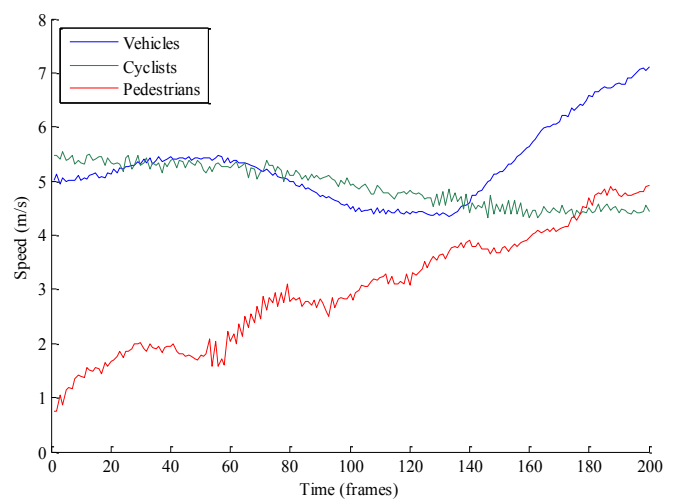

b)

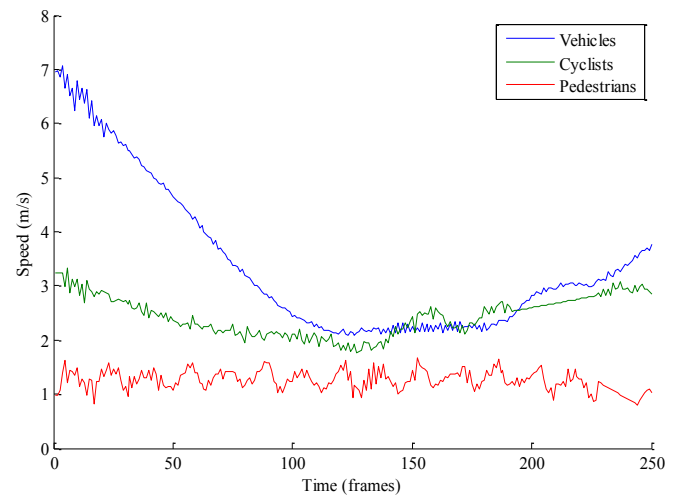

d)

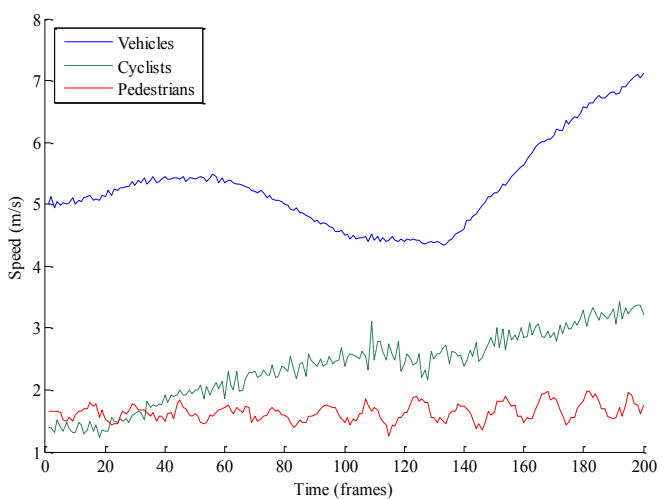

Figure 3.9 The variation in speed profiles of a vehicle, a cyclist, and a pedestrian 
Extracting information from a noisy signal such as a tracked object's speed is often difficult. This is because of the variation of speed over time. Any signal has two representations, a time domain representation and a frequency domain representation. A time domain representation is the original representation of a signal. A time domain represents the changes or progress of a signal over time- - for instance, the speed profile of a tracked object. A frequency domain represents the amount of the signal in each frequency. A transform needs to be performed in order to convert data from a time domain to a frequency domain. A transform is a two-way mapping between two domains. Although the information in each domain looks different, they are still exactly the same. A simple technique to achieve this objective is Fast Fourier transform (FFT) which can be used to convert the signal such as the speed from a time domain representation to a frequency domain representation by decomposing it into sinusoidal waves. Sinusoidal waves are described by their amplitude, frequency, and phase, which are the three types of information needed to describe a signal. In order to create a frequency domain representation of the signal, both amplitude and phase need to be plotted at every frequency, or across an entire spectrum. This transform is necessary to make it easier to work with such signals. A Fast Fourier transform (FFT) package (Wenykier, 2013) is used to obtain the phase and amplitude of the sinusoidal waves for the speed signal. The goal of using the Fast Fourier transform (FFT) "is to estimate the power spectrum of the time series" (Saunier et. al., 2011). The power of a signal is the power of that signal at each of its frequencies. The Frequency and the power are obtained as follows:

1. Perform a Fast Fourier transform (FFT) on the time series ( in this case it is the speed of the tracked objects). Let the FFT return the coefficient $\Omega$, the amount of $\Omega$ equal to the length of time series. The coefficient $\Omega$ is complex number which represents the amplitude 
and phase of the sinusoidal waves. a complex number consist of a real number and an imaginary numbers in the following format:

$$
C_{m}=R_{e}+I_{m} i
$$

where : $C_{m}$ represents the complex number, $R_{e}$ represent the real number and $I_{m}$ represents the imaginary number.

2. Calculate the power of a signal using the FFT coefficient $\Omega$ from step 1 , as in the following:

$$
P_{w, h}=\left(\frac{\Omega_{h}}{s_{l} / 2}\right)^{2} ; h \in\left\{1, \ldots, s_{l} / 2\right\}
$$

where: $P_{w}$ is the power of a signal in Watt/Hertz $(\mathrm{W} / \mathrm{Hz})$;

$s_{l}$ represents the length of the time series

3. Calculate the frequency for each power, as in:

$$
F=\frac{h * f_{r}}{s_{l}} ; h \in\left\{1, \ldots, s_{l} / 2\right\}
$$

where: $F$ is the frequency in Hertz;

Each frequency has its own power, and frequencies are sorted based on this power. A signal could have a number of frequencies and power. Not all the frequencies are good for classification. Therefore, this thesis aims to find the best frequency or group of frequencies for classification. The frequency that produce the highest classification accuracy will be re-labelled with term "the effective frequency", same apply for the group of frequencies that produce the highest classification accuracy. Two classifiers are created to classify road users based on their frequency: 
1. The effective frequency: is the frequency that can be used to classify tracked object with the highest possible classification accuracy. For the purpose of this thesis, only frequencies associated with highest 3 powers are tested.

2. The effective weighted average frequency: is the weighted average for a group of frequencies that can be used to classify tracked object with the highest possible classification accuracy. For the purpose of this thesis, six cases are studied: [i] the group of frequencies associated with the highest 5 powers, [ii] the group of frequencies associated with the highest 10 powers, [iii] the group of frequencies associated with the highest 15 powers, $[i v]$ the group of frequencies associated with the highest 20 powers, $[v]$ the group of frequencies associated with the highest 25 powers, and [vi] the group of frequencies associated with the highest 30 powers. 
(a)

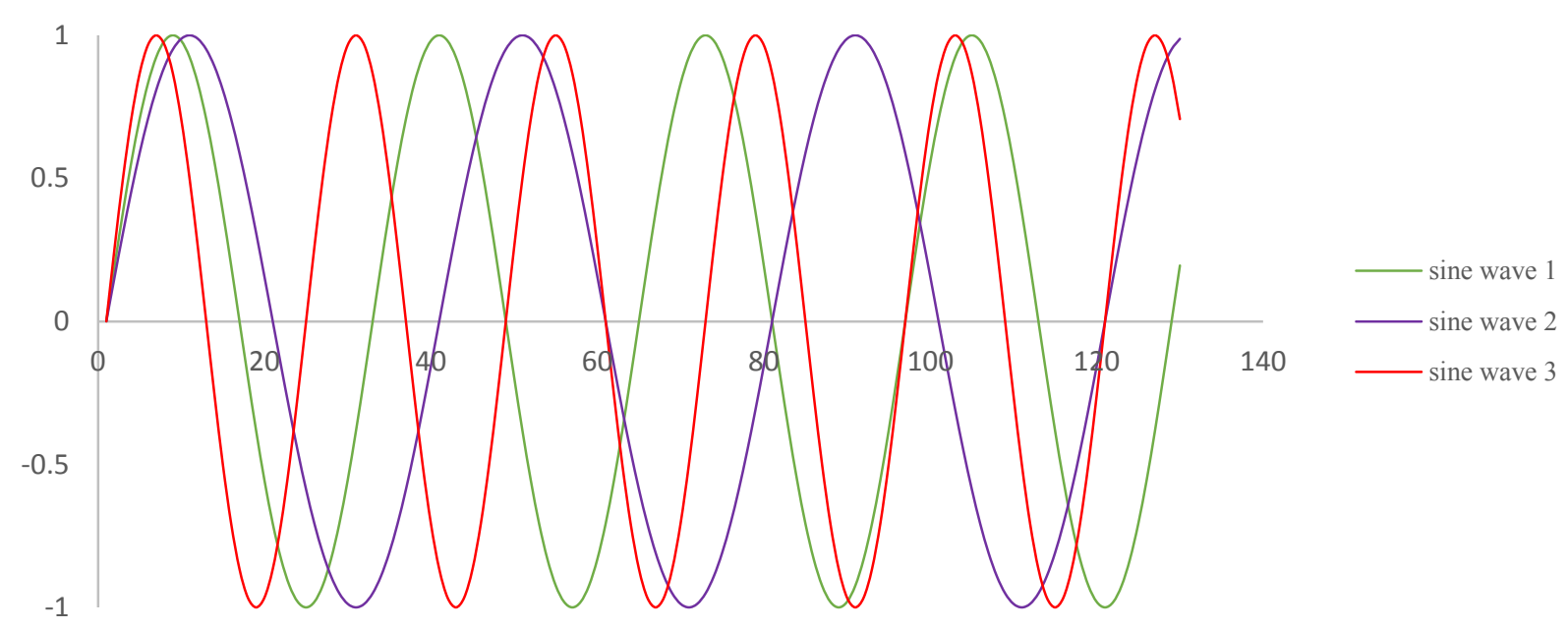

(b)

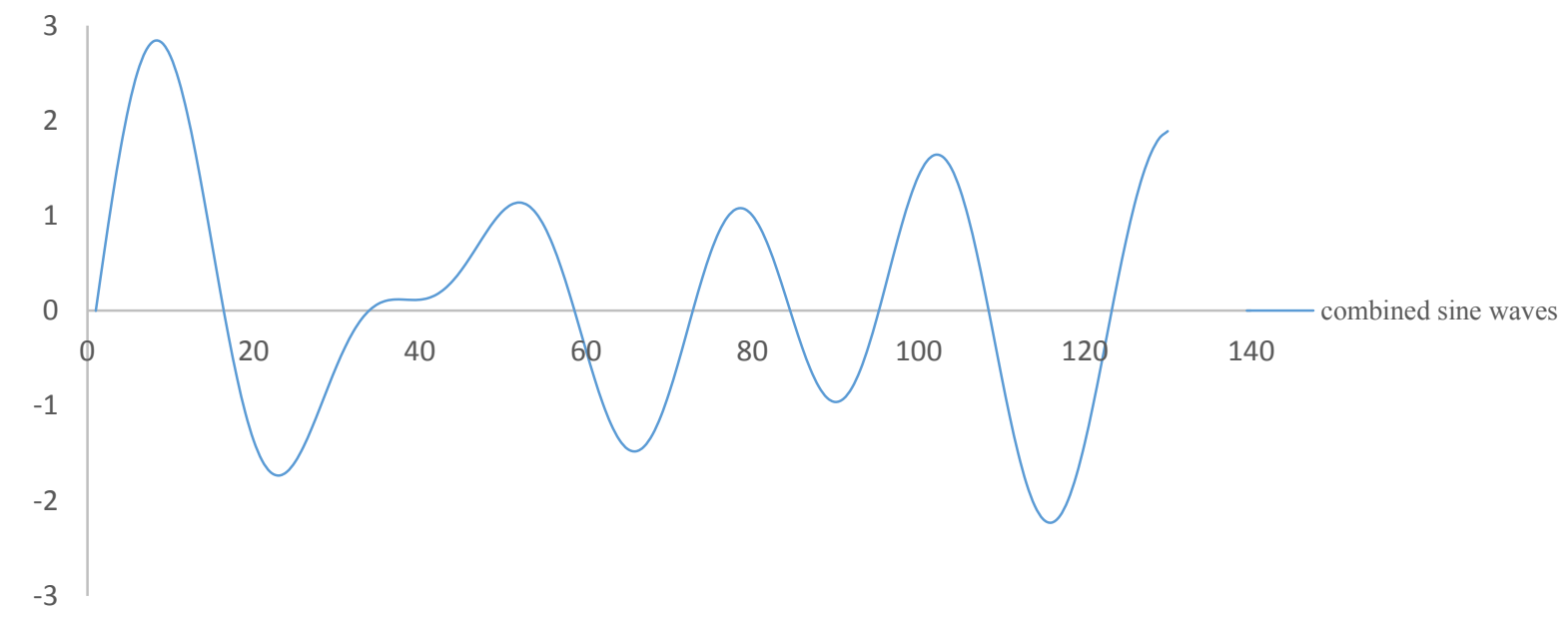

Figure 3. 10 a) An example of three different sine waves; b) combining the three different sine waves, leading to a noisy signal 
(a)

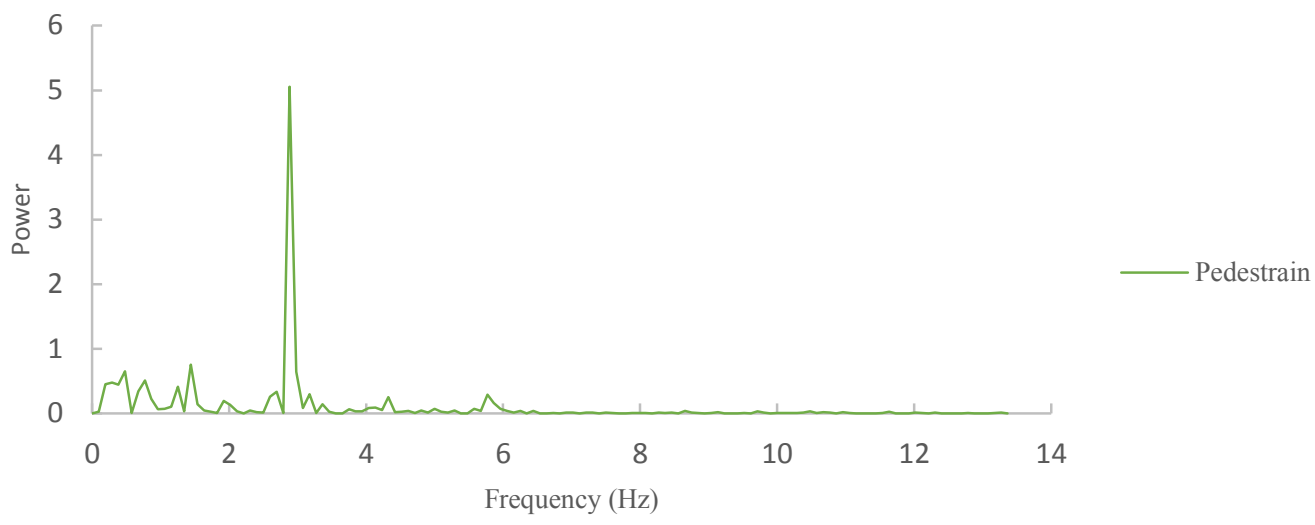

(b)

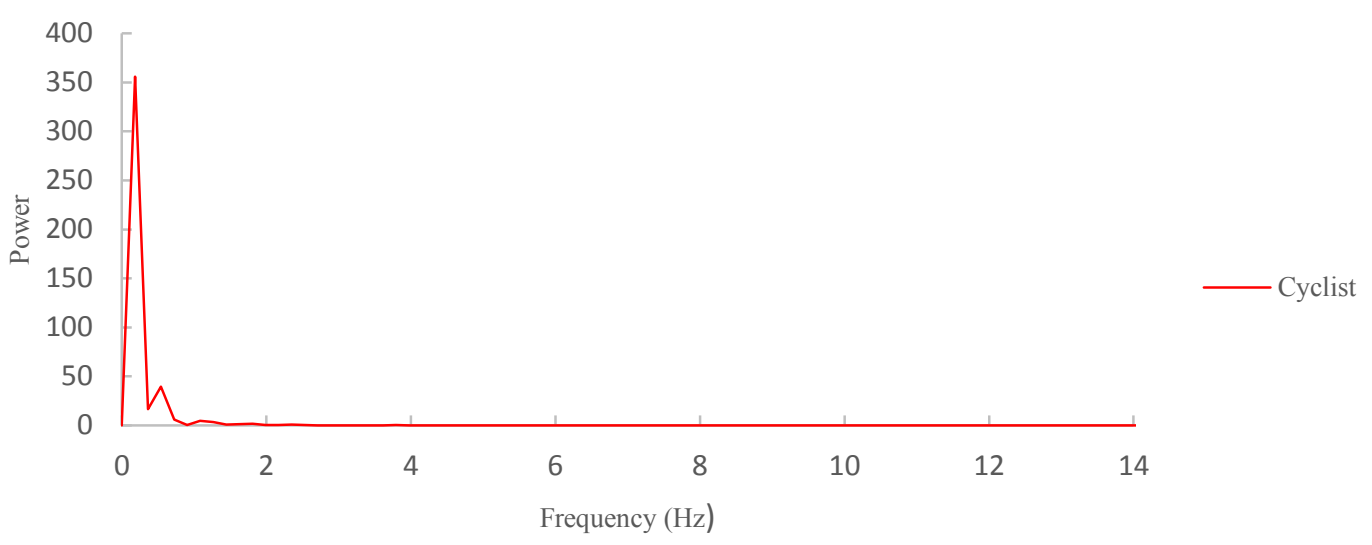

(c)

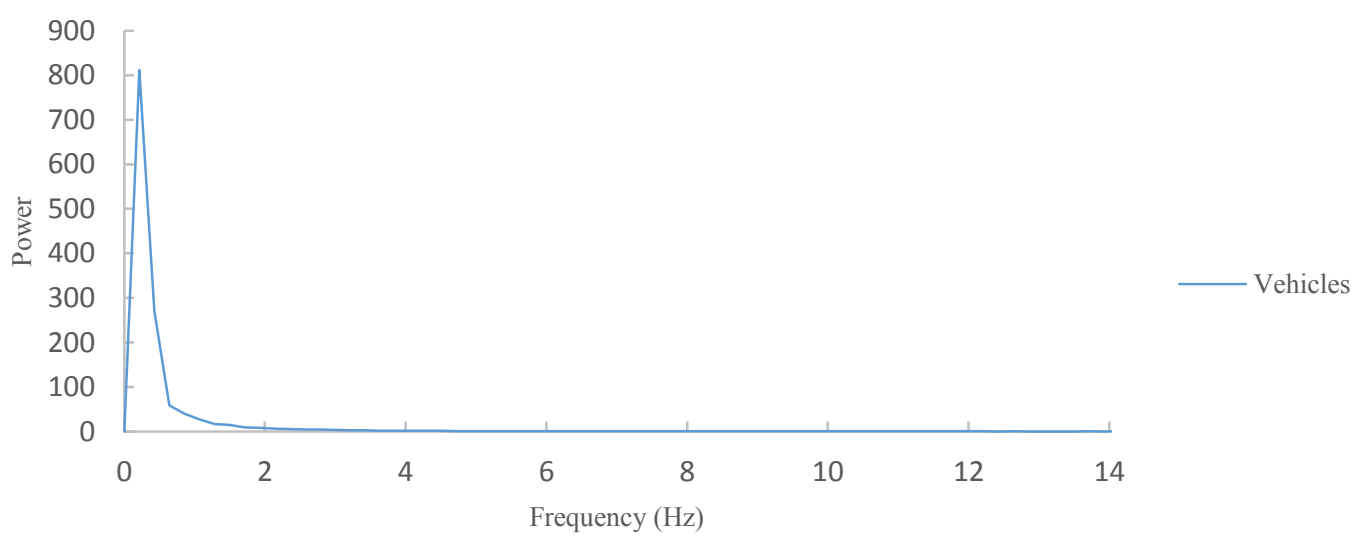

Figure 3. 11 The frequency domain obtained from the speed profile of a) pedestrian, b) cyclists, c) vehicle. 


\subsection{Fuzzy Set Theory}

Each category of tracked objects has some unique characterises that distinguish them from the other categories. For example, a tracked object traveling at a speed of $100 \mathrm{~km} / \mathrm{hr}$ is likely to be a vehicle. However, in some circumstances, two or more categories of tracked objects might share similar characterises, e.g., a vehicle is capable of traveling at the same speed as a cyclist or a pedestrian. This means there is an unavoidable level of uncertainty in classifying the tracked objects. Therefore, after obtaining the classifiers' values for the tracked objects, these values need to be assessed in order to utilize them to discriminate between the tracked objects. The term “classifiers values" refer to value of each classifier explained in section 3.3 (e.g., average speed). It will be described as $C v_{i, j}$ where it to the value of classifier $j$ for object $i$. The used approach for assessment is the Fuzzy Set theory.

Assessing a classifier of a tracked object is the process of finding the Fuzzy value for the tracked object in each category based on that classifier. The higher the assessment value for a classifier in a category, the more likely the tracked object that associated with the assessed classifier truly belongs to that category. Before going into the details of the assessment approach, it is important to establish in the first place why the Fuzzy set was selected.

The classifiers' values pertaining to different tracked objects are expected to overlap; for instance, a vehicle which has roughly the same length as a cyclist. Fuzzy set theory addresses this uncertainty by permitting "the gradual assessment of the membership of elements in a set, [which are] described with the aid of a membership function valued in the real unit interval (0, 1)" (Dhar, 2012). This means that the assessment value of an element in a set can range from 0 to 1 , where 1 indicates that an object is certainly part of that set and 0 indicates that an element does 
not belong to that set. The Fuzzy Set Theory attempts to model this uncertainty. As opposed to classical set theory, "[in which] the membership of elements is assessed in binary terms according to the bivalent condition, any given element either belongs to a given set, or it does not belong to that set" (Dhar, 2012). Therefore, in classical set theory, a tracked object possessing a certain data will certainly be considered to belong to a particular category, or else it will be excluded from that classification. In this thesis, it is proposed that this reasoning is not the most appropriate.

Assessing a classifier using fuzzy set theory is implemented through membership functions. Membership function is often expressed in terms of basic geometric functions such as: trapezoidal membership functions, triangular membership functions, Gaussian membership functions, generalised bell membership functions. These shape descriptions represent the basic form that the function takes on a graph, with the $x$-axis indicating the values of the category and the $y$-axis indicating the fuzzy membership value. The highest point in the shape represents the point at which the fuzzy value is 1 , while the sides of the shape represent the rate at which the fuzzy value drops down towards 0 . Each membership function requires number limits to assess a classifier. The number of limits depends on the type of membership function; for example, the triangular fuzzy membership functions require three limits (a lower limit, upper limit, and middle limit). In Section 3.4.1, a presented example illustrates how the limits are assigned for a membership function. The example also clarifies how the membership function assesses a classifier of a tracked object. In this research, two types of fuzzy membership functions were selected in order to classify the tracked objects: the triangular fuzzy membership function and the trapezoidal fuzzy membership function. 


\subsubsection{Triangular fuzzy membership function}

The triangular fuzzy membership function has three limits, as shown in Figure 3.12. The first limit is the lower limit. The lower limit represents the lowest value of a classifier to be included in the assessment. It is expressed as point $(a)$. The second limit is the middle limit. It is expressed as point $(m)$. The third limit is the upper limit. The upper limit represents the highest value of a classifier to be included in the assessment. It is expressed as point $(d)$. The location of the middle limit $(m)$ will be calculated depending on the value of $(\delta)$ assigned in the configuration. The fuzzy value for the triangular fuzzy membership function can be obtained using Table 3.2.

Table 3. 2 Fuzzy set- Triangular membership function equations

\begin{tabular}{|c|c|}
\hline Condition & Fuzzy Value $\boldsymbol{f z}$ \\
\hline$x \leq a$ or $x \geq d$ & $f_{z}=0$ \\
\hline$x=m$ & $f_{z}=1$ \\
\hline$a<x \leq m$ & $f_{z}=\frac{x-a}{m-a}$ \\
\hline$m<x \leq d$ & $f_{z}=\frac{d-x}{d-m}$ \\
\hline
\end{tabular}

where: $f_{z}$ is the fuzzy value;

$x$ is the classifier value for the tracked object;

$a$ is the lower limit;

$d$ is the upper limit;

$m$ is the middle limit such that $a<m<d$. 
The location of the middle limit (m) of the triangular fuzzy membership function was obtained using the following equation:

$$
m=a+\delta(d-a)
$$

where: $\delta$ is a selected value between 0 and 1 .

For this framework, the location of the middle limit for the triangular fuzzy membership function was assumed to be in three different positions, based on the $\delta$ value. The used $\delta$ values were $0.25,0.5$, or 0.75 . This results in three forms for the membership function, as shown in Figure 3.12.

To understand how to assign the limit's value for the triangular fuzzy membership function, let us assume that any object traveling on Highway 417 at a speed ranging between 70 and $100 \mathrm{~km} / \mathrm{hr}$ is to be identified as a vehicle. The limits for the triangular fuzzy membership function with $\delta=0.5$ will be as follows:

1. The lower limit (a) for the triangular fuzzy membership function is $70 \mathrm{~km} / \mathrm{hr}$. An object traveling on Highway 417 at a speed of $70 \mathrm{~km} / \mathrm{hr}$ or less will be given a fuzzy value $=0$.

2. The upper limit (b) for the triangular fuzzy membership function is $100 \mathrm{~km} / \mathrm{hr}$. An object traveling on Highway 417 at a speed of a $100 \mathrm{~km} / \mathrm{hr}$ or more will be given a fuzzy value $=0$.

3. Since the value of $\delta=0.5$, using Equation 3.10, the middle limit (m) for the triangular fuzzy membership function will equal to $85 \mathrm{~km} / \mathrm{hr}$. An object traveling on highway at a speed of $85 \mathrm{~km} / \mathrm{hr}$ will be given a fuzzy value $=1$.

4. An object traveling on Highway 417 at any speed between 70 and $85 \mathrm{~km} / \mathrm{hr}$, or at a speed between 85 and $100 \mathrm{~km} / \mathrm{hr}$, will be given a fuzzy value $>0$ and $<1$ based on Table 3.2. 
5. If an object is given a fuzzy value $=0$, this means that the classification system could not identify the object as a vehicle.

6. If an object is given a fuzzy value $=1$, this means that the classification system is certain that the object is a vehicle.

7. If an object is given a fuzzy value $>0$ and $<1$, this means that the classification system has some degree of certainty that the object is vehicle. The closer the fuzzy value to 1 , the higher the certainty that the object is a vehicle.

(a)

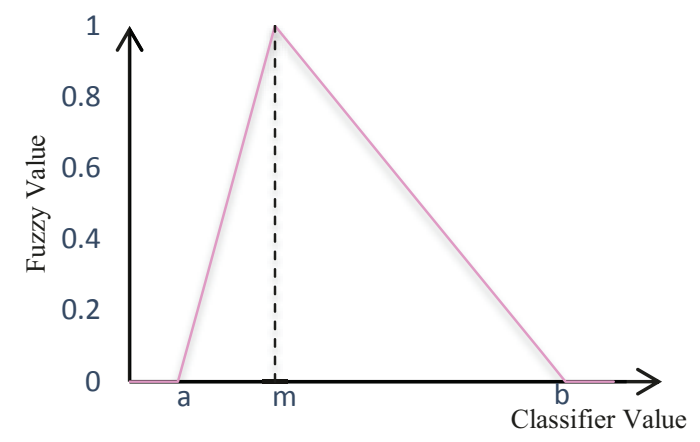

(b)

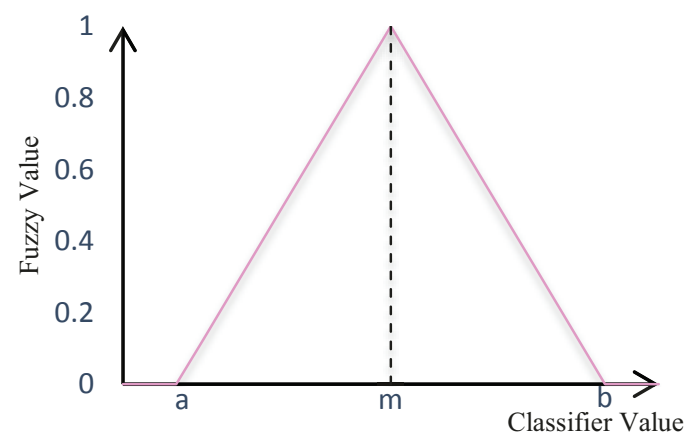

(c)

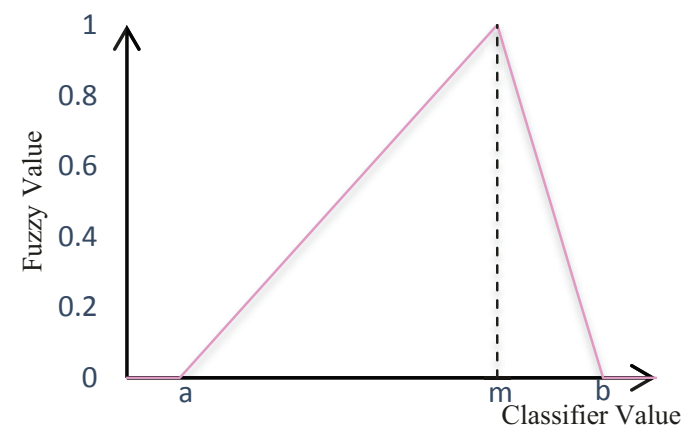

Figure 3. 12 Three cases of the triangular fuzzy membership function. a) where the $\delta=$ 0.25 ; b) where the $\delta=0.5 ;$ c) where the $\delta=0.75$. 


\subsubsection{Trapezoidal fuzzy membership function}

The trapezoidal fuzzy membership function has four limits, as shown in Figure 3.13. The first limit is the lower limit. The lower limit represents the lowest value of a classifier to be included in the assessment. It is expressed as point $(a)$. The second limit is expressed as point (b). The third limit is expressed as point $(c)$. The fourth limit is the upper limit. The upper limit represents the highest value of a classifier to be included in the assessment. It is expressed as point $(d)$. The location of the limits - $(b)$ and $(c)$-are assumed to have a fixed position in this framework. It is calculated depending on the lower and upper limits, as in Equation 3.12. In this definition, $a<b<c<d$. The fuzzy value for the triangular fuzzy membership function can be obtained using Table 3.3.

Table 3. 3 Fuzzy set- Trapezoidal membership function equations

\begin{tabular}{|c|c|}
\hline Condition & Fuzzy Value $\boldsymbol{f z}$ \\
\hline$x \leq a$ or $x \geq d$ & $f_{z}=0$ \\
\hline$b \leq x \leq c$ & $f_{z}=1$ \\
\hline$a \leq x<b$ & $f_{z}=\frac{x-a}{b-a}$ \\
\hline$c<x \leq d$ & $f_{z}=\frac{d-x}{d-c}$ \\
\hline
\end{tabular}

where: $f_{z}$ is the fuzzy value;

$x$ is the classifier value for the tracked object;

$a$ is the lower limit;

$b$ is the second limit; 
$c$ is the third limit;

$d$ is the upper limit.

The location of the second limit (b) of the trapezoidal fuzzy membership function is assumed to be in between lower limit (a) and $\left(\frac{a+d}{2}\right)$. The value of (b) is simply calculated using the following equation:

$$
b=a+\delta(d-a)
$$

where: $\delta$ is equal to 0.25 .

The location of the third limit (c) of the trapezoidal fuzzy membership function is assumed to be in between $\left(\frac{a+d}{2}\right)$ and the upper limit $(\mathrm{d})$. The value of $(\mathrm{c})$ is simply calculated using the equation 3.12 with $\delta$ is equal to 0.75 .

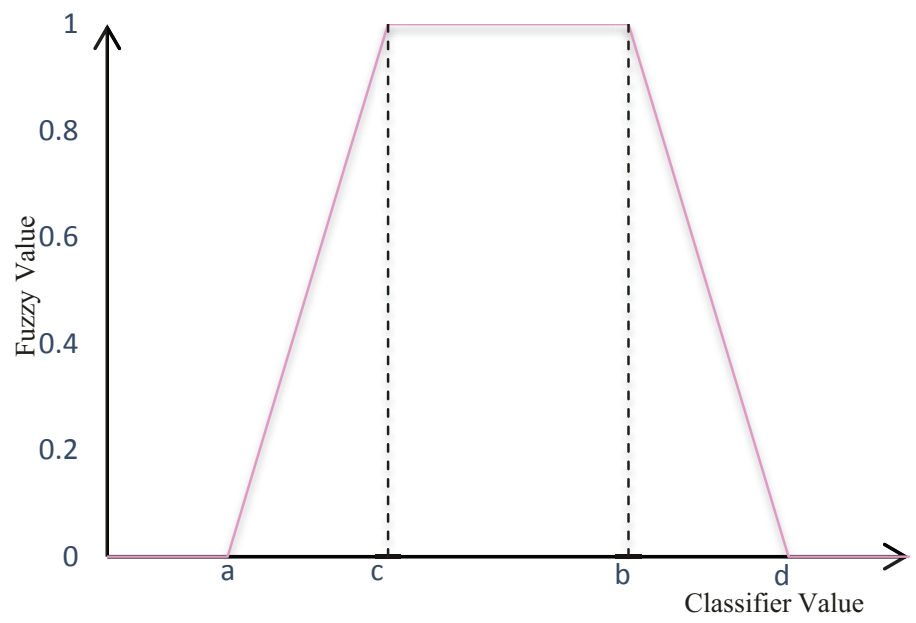

Figure 3. 13 The trapezoidal fuzzy membership function 


\subsection{The Framework Algorithm and procedures}

In this framework, an algorithm was created to classify tracked objects captured on a video sequence using a high-resolution video camera. The classification algorithm relies on different sources of information obtained from the object's track. The sources of information are called the classifiers. The value of each classifier will be assessed and given a fuzzy value for each category. The classifiers assessment is conducted for all the tracked objects. Based on the assessment, the tracked object will be assigned into: vehicles, cyclists, and pedestrians. A fourth category accounts for unknown tracked objects. The fourth category does not have any thresholds; a tracked object will be placed in this category if it cannot be classified as a vehicle, a cyclist, or a pedestrian. In addition to the classifiers, the classification process also relies on the configuration. The configuration consists of four elements. The elements divided into essential elements and optional elements and as follows:

1. The essential elements are mandatory for any classification. The essential elements consist of:

a. The duration of the tracked objects appearance in the video sequence: it is measured by the number of frames where the object was tracked in. Any object tracked in number of frames less than the assigned threshold will be excluded from the classification process. The minimum duration of the tracked objects appearance in the video sequence presented in $\varphi_{g}$, where $g \in\{1, \ldots, 5\}=$ the five groups representing the minimum duration of the tracked object's appearance that were considered in thesis and as in Table 3.4. 
Table 3. 4 The used durations of the tracked object's appearance.

\begin{tabular}{|c|l|}
\hline Group Number & \multicolumn{1}{|c|}{ Duration } \\
\hline 1 & all tracked objects are included \\
\hline 2 & all tracked objects appeared for at least one second (30 frames or more) \\
\hline 3 & all tracked objects appeared for at least two seconds (60 frames or more) \\
\hline 4 & all tracked objects appeared for at least three seconds (90 frames or more) \\
\hline 5 & all tracked objects appeared for at least four seconds (120 frames or more) \\
\hline
\end{tabular}

b. The fuzzy membership function $\Lambda_{l}$, where $l \in\{1, \ldots, 4\}=$ the four fuzzy membership

Table 3. 5 The used types of fuzzy membership functions

\begin{tabular}{|c|l|}
\hline Group Number & \multicolumn{1}{|c|}{ Type of Fuzzy membership function } \\
\hline 1 & Triangular membership function with $\delta=0.25$ \\
\hline 2 & Triangular membership function with $\delta=0.5$ \\
\hline 3 & Triangular membership function with $\delta=0.75$ \\
\hline 4 & Trapezoidal membership function \\
\hline
\end{tabular}

functions that studied in this thesis as in Table 3.5.

2. The optional elements are only required if the effective frequency and the effective weighted average frequency are used for classification. The classification system needs to know which frequency or group of frequencies shall use for classification.

a. The effective frequency $\eta_{m}$, where $m \in\{1, \ldots, 3\}=$ the frequencies associated with the highest 3 powers as in Table 3.6 
Table 3. 6 The used types of the effective frequency

\begin{tabular}{|c|l|}
\hline Group Number & \multicolumn{1}{c|}{ The effective frequency } \\
\hline 1 & The frequency associated with the highest power \\
\hline 2 & The frequency associated with the second highest power \\
\hline 3 & The frequency associated with the third highest power \\
\hline
\end{tabular}

b. The effective weighted average frequency $\Gamma_{n}$, where $n \in\{1, \ldots, 6\}=$ six groups representing the effective weighted average frequency are shown in Table 3.7.

Table 3.7 The used types of the effective weighted average frequency.

\begin{tabular}{|c|l|}
\hline Group Number & \multicolumn{1}{|c|}{ The effective weighted average frequency } \\
\hline 1 & Frequencies associated with the highest 5 powers \\
\hline 2 & Frequencies associated with the highest 10 powers \\
\hline 3 & Frequencies associated with the highest 15 powers \\
\hline 4 & Frequencies associated with the highest 20 powers \\
\hline 5 & Frequencies associated with the highest 25 powers \\
\hline 6 & Frequencies associated with the highest 30 powers \\
\hline
\end{tabular}

From these elements, a configuration can be created as follows:

$$
\left(\varphi_{g}, \Lambda_{l}, \eta_{m}, \Gamma_{n}\right)
$$

\subsubsection{The classification algorithm}

This section includes the classification algorithm that is used to classify different tracked objects. Note that at this stage of the thesis, this algorithm is not calibrated yet. The calibration procedure will be explained in section 3.5.2.

\section{Input:}

1. Tracks of the bounding box vertices: (TL, $B L$ and $B R)$; 
2. Sequence of displacement: $\left(d_{x}\right.$ and $\left.d_{y}\right)$;

3. The configuration (explained in section 3.5);

4. The membership limits a \& d;

5. Camera frame rate

\section{Output:}

- Classification of the tracked objects: as a vehicle $(V)$, a cyclist $(C)$, a pedestrian $(P)$, or an unknown $(U)$.

\section{Procedure:}

1. Obtain the value of each classifier(s) ${ }^{1} C v_{i, j}$ for each tracked object. where $C v_{i, j}$ is the value of classifier $j$ for object $i ; \mathrm{j} \in\{1, \ldots 19\}$.

2. Create a fuzzy membership function for each classifier in each category. Let $\Lambda_{l, j, q}$ is the fuzzy membership function for classifier $j$ in category $q$. where $l$ is the fuzzy membership type $l \in\{1, . ., 4\}$ as in Table $3.5 ; j \in\{1, \ldots 19\}$ which refers to the 19 classifiers as in section $3.3 ; q$ $\in\{1, . ., 3\}$ which refers to the classification categories (vehicles, cyclists and pedestrians).

3. Assess the value of each classifier(s) $C v_{i, j}$ in each category. A fuzzy value will be assigned to the tracked object in each category for each assessed classifier.

$$
V_{i, j}=\Lambda_{l}\left(C v_{i, j} \mid \boldsymbol{\beta}_{V, j}\right)
$$

where: $V_{i, j}$ if the fuzzy value for object $i$ in the vehicle category based on the assessment of classifier $j ; \boldsymbol{\beta}_{v, j}$ represent the limits for the fuzzy membership function in vehicle category $V$ for classifier $j$.

\footnotetext{
${ }^{1}$ Not necessarily all the 19 classifiers will be used. At this stage the algorithm is not calibrated yet therefore, there is a chance that some classifiers will be excluded.
} 


$$
C_{i, j}=\Lambda_{l}\left(C v_{i, j} \mid \boldsymbol{\beta}_{C, j}\right)
$$

where: $C_{i, j}$ if the fuzzy value for object $i$ in the cyclist category based on the assessment of classifier $j ; \boldsymbol{\beta}_{C, j}$ represent the limits for the fuzzy membership function in vehicle category $C$ for classifier $j$.

$$
P_{i, j}=\Lambda_{l}\left(C v_{i, j} \mid \boldsymbol{\beta}_{\boldsymbol{p}, \boldsymbol{j}}\right)
$$

where: $P_{i, j}$ if the fuzzy value for object $i$ in the pedestrian category based on the assessment of classifier $j ; \boldsymbol{\beta}_{\boldsymbol{p}, j}$ represent the limits for the fuzzy membership function in pedestrian category $P$ for classifier $j$.

4. Sum the fuzzy values for tracked object in each category:

$$
\begin{aligned}
& V_{i}=\sum_{j} V_{i, j} ; j \in\{1, \ldots, 19\} \\
& C_{i}=\sum_{j} C_{i, j} ; j \in\{1, \ldots, 19\} \\
& P_{i}=\sum_{j} P_{i, j} ; j \in\{1, \ldots, 19\}
\end{aligned}
$$

where:

$V_{i}$ is the summation of all fuzzy values for object $i$ in the vehicle category;

$C_{i}$ is the summation of all fuzzy values for object $i$ in the cyclist category;

$P_{i}$ is the summation of all fuzzy values for object $i$ in the pedestrian category.

5. Classify the tracked object as follows:
a. The tracked object $i$ is classified a vehicle if: $\operatorname{Max}\left\{V_{i}, C_{i}, P_{i}\right\}=V_{i}$;
b. The tracked object $i$ is classified a cyclist if: $\operatorname{Max}\left\{V_{i}, C_{i}, P_{i}\right\}=C_{i}$;
c. The tracked object $i$ is classified a pedestrian if: $\operatorname{Max}\left\{V_{i}, C_{i}, P_{i}\right\}=P_{i}$; 
d. The tracked object $i$ is classified as an unknown if: $V_{i}+C_{i}+P_{i}=0$;

e. The tracked object $i$ is classified as an unknown if: $V_{i}=C_{i}$, or $V_{i}=P_{i}$, or $C_{i}=P_{i}$.

\subsubsection{The calibration procedures}

After the classification algorithm is developed, it needs to be calibrated to provide the desirable accuracy in classifying different tracked objects. Calibration is the process of finding the calibrated limits for the fuzzy membership function for a specific configuration. In the case of multiple configurations, the calibration should be also able to identify the best configuration(s). The calibrated limits and the best configuration(s) are expected to be reliable and transferable between different intersections.

Two procedures were created for calibration. The first procedure will be called calibration procedure 1 , it is used to obtain the calibrated limits for the fuzzy membership in two cases: $[i]$ the calibration is being performed on one classifier only; or [ii] the calibration is being performed on more than one classifier, regardless of whether the new classifiers provide a significant improvement to the classification results or not. The second procedure will be called calibration procedure 2, it is used to obtain the calibrated limits for the fuzzy membership in two cases: $[i]$ the calibration is being performed on more than one classifier; and [ii] the required results are to keep the effective classifiers only. The effective classifiers are those that significantly improve the classification results. Both procedures will be detailed later in this section. Kappa statistic will be used in the calibration to measure the agreement between the manual and the automated classification. The Kappa statistic is "[a mathematical approach] measure the agreement between two or more [methods] should include a statistic that takes into account the fact that observers will sometimes agree or disagree simply by chance" (Viera and Garrett, 2005). The Kappa value is range between +1 and -1 . The closer the value to +1 , the 
higher the agreement is. Table 3.8 illustrate an interpretation of Kappa coefficient. Table 3.9 Explain how to obtain the Kappa coefficient.

Table 3. 8 Interpretation of Kappa (Viera et al., 2005)

\begin{tabular}{|c|l|}
\hline Kappa & \multicolumn{1}{|c|}{ Agreement } \\
\hline$<0$ & Less than chance of agreement \\
\hline $0.01-0.20$ & Slight agreement \\
\hline $0.21-0.40$ & Fair agreement \\
\hline $0.41-0.60$ & Moderate agreement \\
\hline $0.61-0.80$ & Substantial agreement \\
\hline $0.81-1$ & Almost prefect agreement \\
\hline
\end{tabular}

Table 3.9 An explanation on how to obtain the Kappa coefficient

\begin{tabular}{|c|c|c|c|c|c|}
\hline & \multicolumn{4}{|c|}{ Automated classification } \\
\hline & & Vehicles & Cyclists & Pedestrians & Total \\
\hline \multirow{4}{*}{ 窛 } & Vehicles & $a$ & $b$ & $c$ & $m_{2}$ \\
\hline & Cyclists & $d$ & $e$ & $f$ & $m_{1}$ \\
\hline & Pedestrians & $g$ & $h$ & $i$ & $m_{0}$ \\
\hline & Total & $n_{2}$ & $n_{1}$ & $n_{0}$ & $n$ \\
\hline
\end{tabular}

$$
\begin{gathered}
P_{e}=\left[\frac{n_{2}}{n} * \frac{m_{2}}{n}\right]+\left[\frac{n_{1}}{n} * \frac{m_{1}}{n}\right]+\left[\frac{n_{0}}{n} * \frac{m_{0}}{n}\right] \\
P_{o}=\frac{a+e+i}{n} \\
k=\frac{P_{o}-P_{e}}{1-P_{e}}
\end{gathered}
$$

Where: $k$ is the Kappa statistic or coefficient; $P_{o}$ is the observed agreement where both the automated and manual classification yield the same classification results; $P_{e}$ is the proportion of the agreement due to chance. $(a),(e)$ and $(i)$ is the agreement between the manual classification and the automated classification. $(n)$ is the sample size. 


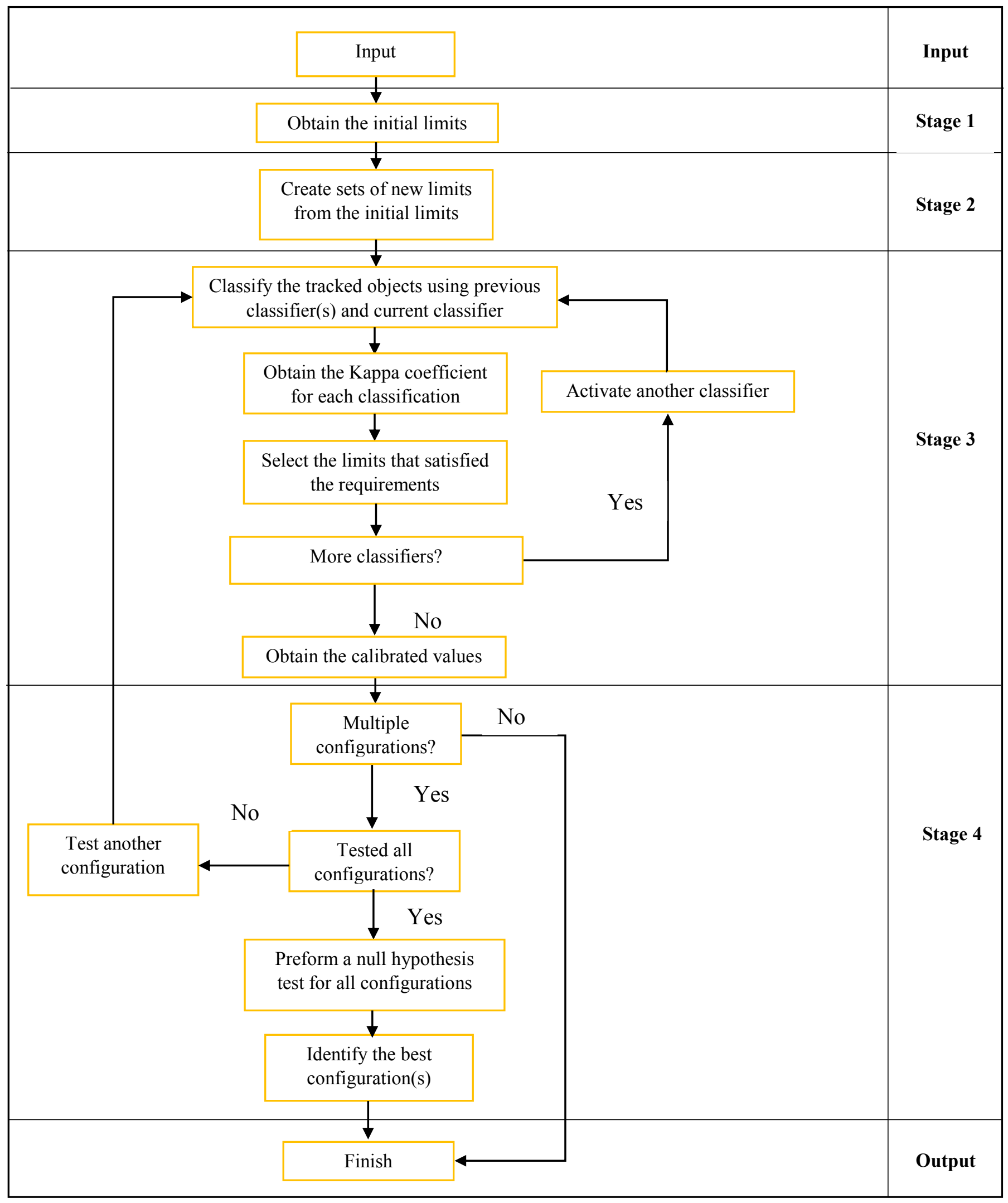

Figure 3. 14 An explanation of the calibration procedure 
The calibration procedure was divided into 4 stages as in Figure 3.14. The description of each stage as follows:

\section{Input:}

1. Tracks of the bounding box vertices: (TL, $B L$ and $B R)$;

2. Sequence of displacement: $\left(d_{x}\right.$ and $\left.d_{y}\right)$;

3. The configuration(s), (explained in section 3.5);

4. The manual classification of tracked objects;

5. Calibration procedure selection (calibration procedure 1, or calibration procedure 2);

6. Camera frame rate.

\section{Output:}

- The calibrate limits (in case of one configurations is only used);

- The best configuration(s) (in case of multiple configurations are used).

\section{Procedure:}

\section{Stage 1:}

In order to find the calibrated limits, the initial limits are required. The calibrated limits are calibrated values for the lower limit $\left(a_{j, q}\right)$ and upper limit $\left(d_{j, q}\right)$ of the fuzzy membership function $^{2}$. The initial limits are the initial values for lower limit $\left(a i_{j, q}\right)$ and upper limit $\left(d i_{j, q}\right)$ of the fuzzy membership function. To understand the importance of the initial limits, let assume that the lowest and highest speeds recorded on Highway 417 are 60 and $100 \mathrm{~km} / \mathrm{hr}$, respectively. This mean the initial lower limit for the fuzzy membership is $60 \mathrm{~km} / \mathrm{hr}$ and the initial upper limit for the fuzzy membership is $100 \mathrm{~km} / \mathrm{hr}$. The calibrated lower and upper limits should be between

\footnotetext{
${ }^{2}$ The limits are explained in section 3.4
} 
60 and $100 \mathrm{~km} / \mathrm{hr}$. This eliminates any speed less than $60 \mathrm{~km} / \mathrm{hr}$ and more than a $100 \mathrm{~km} / \mathrm{hr}$, which can save significant amount of time in the calibration process. The initial limits are obtained from the dataset that will be used in calibration as follows:

a) The tracked objects are manually observed and classified into vehicles, cyclists, and pedestrians;

b) Obtain the value of each classifier for each tracked object in each category; Let $C v_{i, j, q}$ is the value of classifier $j$ for object $i$ in category $q ; j \in\{1, \ldots 19\}$ which refers to the 19 classifiers as in section $3.3 ; q \in\{1, . ., 3\}$ which refers to the classification categories (vehicles, cyclists and pedestrians).

c) The lowest value recorded for a classifier $j$ in category $q$ is the initial lower limit for the fuzzy membership $l$ for classifier $j$ in this category $q$. The highest value recorded for a classifier $j$ in category $q$ is the value of the highest limit for the fuzzy membership $l$ for classifier $j$ in this category $q$.

\section{Stage 2:}

The aim of this stage is to create sets of new limits by utilizing the initial limits. One of these new limits is the calibrate limits. This can be done by decrementing the initial upper limit to the lower limit, and then increment the initial lower limit for each new upper limit and as follows:

a) Obtain the increment-decrement value. Let $I n_{j, q}$ is the increment value for classifier $j$ in category $q$.

$$
I n_{j, q}=\left(\frac{d i_{j, q}-a i_{j, q}}{100}\right)
$$


where: $d i_{j, q}$ is the initial upper limit for classifier $j$ in category $q$; $a i_{j, q}$ is the initial lower limit for classifier $j$ in category $q$.

b) Produce new upper limits. This can be done by reduce the initial upper limit $d i_{j, q}$ to the initial lower limit $a i_{j, q}+I n_{j, q}$ using the increment-decrement value $I n_{j, q}$.

$$
d n_{\gamma, j, q}=d i_{j, q}-\left(I n_{j, q} * \gamma\right) ; \gamma=\gamma+1
$$

where : $d n_{\gamma, j, q}$ is the is the new upper limit $\gamma$ for classifier $j$ in category $q$;

c) Produce new lower limits $a n_{\gamma, j, q}$ for each new upper limit $d n_{\gamma, j, q}$. This can be done by increase the initial lower limit $a i_{j, q}$ to each new upper limit $d n_{\gamma, j, q}-I n_{j, q}$ using the increment-decrement value $I n_{j, q}$.

$$
a n_{\gamma, j, q}=a i_{j, q}+\left(I n_{j, q} * \gamma\right) ; \gamma=\gamma+1
$$

d) From the previous step sets on thousands of new limits are created for classifier $j$ in category $q$. The calibrated limits are one of these new limits.

\section{Stage 3:}

The aim of this stage is to classify the tracked objects using all the new limits. The classification algorithm was explained in section 3.5.1. Two procedures are created and as follows:

\section{- Calibration procedure 1}

a) Classify the tracked objects using $\left(a n_{\gamma, j_{1}, q}, d n_{\gamma, j_{1}, q}\right)$; where $j_{1}$ is the first classifier.

b) Obtain the Kappa coefficient for each classification. 
c) The calibrate limits for the classifier $j$ in category $q$ must meet the following criteria: $[i]$ produced the highest Kappa coefficient; then [ii] Have the lowest upper limit value $d n_{\gamma, j, q}$; then $[i i i]$ Have the highest lower limit value $a n_{\gamma, j, q}$.

d) The previous steps will be repeated for the second classifier with one difference only, the classification will be performed using $\left(a n_{\gamma, j 2, q}, d n_{\gamma, j 2, q},\right)$ and the calibrated value for $j 1$ $\left(a_{j, q}, d_{j, q}\right)$

e) Two cases will be conducted, as follows:

i. $j_{2}$ will be terminated if:

$$
k_{j_{1}, j_{2}} \text { using the calibrate limits } \leq k_{j_{1}} \text { using the calibrate limits. }
$$

Where $k_{j_{1}, j_{2}}$ is the Kappa coefficient for classifier 1 and $2 ; k_{j_{1}}$ is the Kappa value for classifier 1.

ii. $j_{2}$ will be included if:

$$
k_{j_{1}, j_{2}} \text { using the calibrate limits }>k_{j_{1}} \text { using the calibrate limits. }
$$

f) The previous steps will be repeated until the calibrate limits for all the classifiers are found.

\section{- Calibration procedure 2}

This difference between calibration procedure 1 and calibration procedure 2 is only in step (e) and as follows:

1. $j 2$ will be terminated if:

$$
k_{j_{1}, j_{2}} \text { using the calibrate limits } \leq k_{j_{1}} \text { using the calibrate limits. }
$$

2. A null hypothesis $\left(H_{0}\right)$ test is performed if:

$$
k_{j_{1}, j_{2}} \text { using the calibrate limits }>k_{j_{1}} \text { using the calibrate limits. }
$$


The null hypothesis $\left(H_{0}\right)$ is performed using $t$-statistic at a significance level $\alpha=5 \%$ and as follows:

$$
H_{0:} k_{j_{1}, j_{2}}=k_{j_{1}}
$$

If the sample mean $k_{j_{1}, j_{2}}$ is significantly different from $k_{j_{1}}$ then the null hypothesis is rejected. This rejection means that $j_{2}$ in addition to $j_{1}$ do provide a significant improvement to the classification step and therefore, $j_{2}$ will be included as a classifier. Otherwise, the null hypothesis cannot be rejected and therefore, $j_{2}$ will be excluded and terminated.

\section{Stage 4:}

In case of multiple configurations, the calibration procedure should be able to identify the best configuration(s). Logically, the configuration with the highest Kappa coefficient is the best configuration. However, the following configuration(s) might provide similar performance. Therefore, to identify the best configuration(s) a null hypothesis $(H 0)$ is performed. The configurations are divided into five groups based on their assigned $\varphi_{g}$ in the configuration. $\varphi_{g}$ represents the minimum duration of tracked objects appearance and is explained in section $3.5 \&$ Table 3.4. For each $g$ a null hypothesis $\left(H_{0}\right)$ is performed between the configuration that yielded the highest Kappa and the other configuration(s) using $t$-statistic at a significance level $\alpha$ $=5 \%$ and as follows:

$$
H_{0:} k_{o, \varphi_{g}}=k_{z, \varphi_{g}}
$$

where: $k_{z, \varphi_{g}}$ is the Kappa coefficient for the configuration $z$ for group $g$ of $\varphi_{g} ; z$ is the configuration that yielded the highest Kappa. $k_{o, \varphi_{g}}$ is the Kappa coefficient for the configuration $o$ for group $g$ of $\varphi_{g} ; o$ is the other configuration(s). 
If the sample mean $k_{o, \varphi_{g}}$ is significantly different from $k_{z, \varphi_{g}}$ the null hypothesis is rejected. This rejection mean that the configuration associated with $k_{o, \varphi_{g}}$ did not provide the same performance as the configuration that yielded the highest Kappa $k_{z, \varphi_{g}}$ and therefore, the configuration associated with $k_{o, \varphi_{g}}$ will be excluded from further testing "validation". Otherwise the null hypothesis cannot be rejected and therefore the configuration associated with $k_{o, \varphi_{g}}$ did provide the same performance as $k_{z, \varphi_{g}}$ and therefore, it will be included in further testing "validation".

\subsubsection{The validation procedure}

After obtaining the best configuration(s) from the calibration, these configurations need to be validated. The validation is similar to classification algorithm with enhanced features: $[i]$ it automatically measures the Kappa coefficient between the manual classification and the automated classification, $[i i]$ it excludes any configuration that yields a Kappa coefficient of 0 or less $\left(k_{o, \varphi_{g}} \leq 0\right)$ during the validation, and $[i i i]$ it performs a null hypothesis $\left(H_{o}\right)$ on all validated configurations. The null hypothesis test is exactly as in stage 4 of section 3.5.2. 


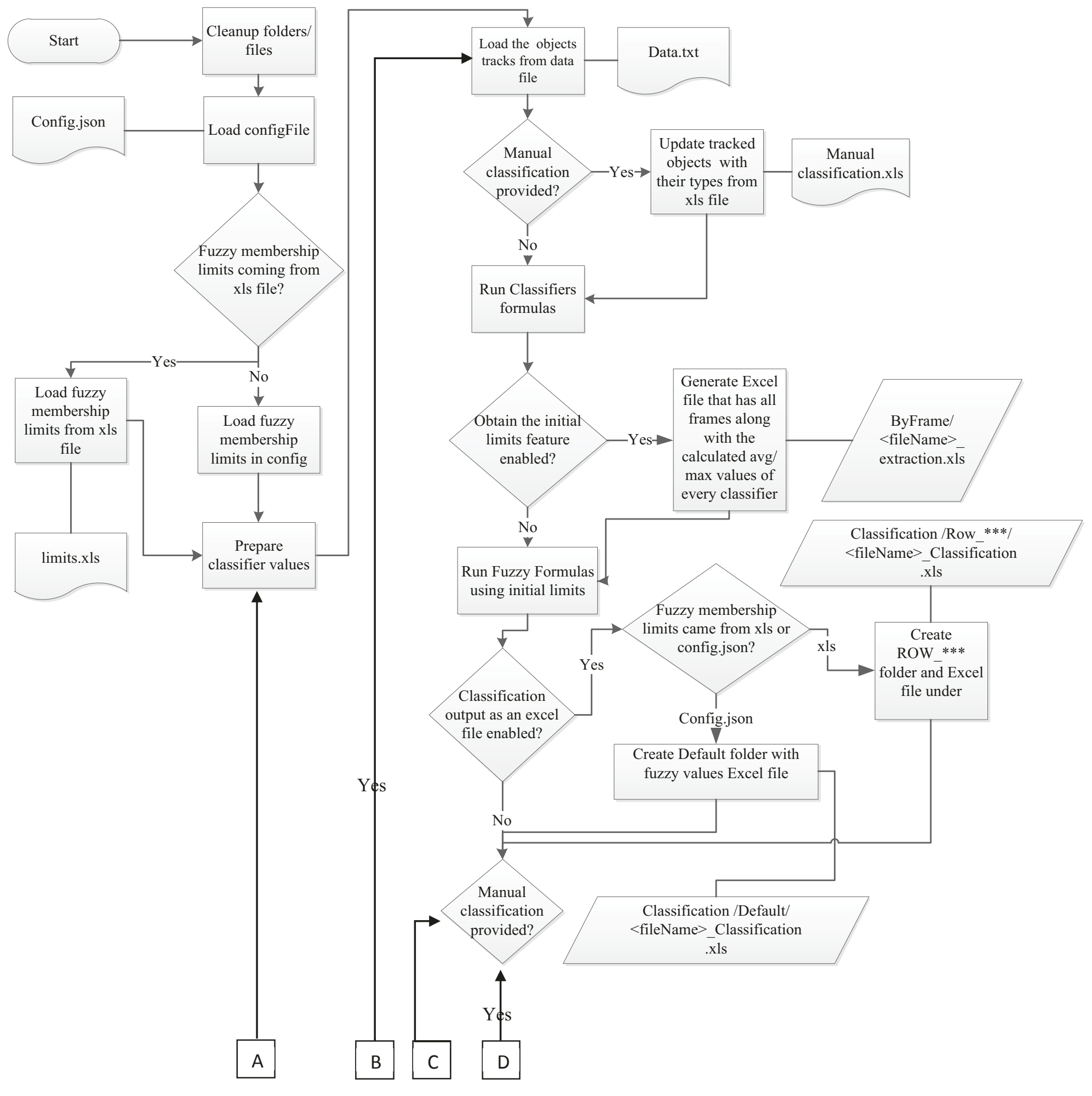

Figure 3. 15 A detailed explanation of the framework mechanism. (part 1) 


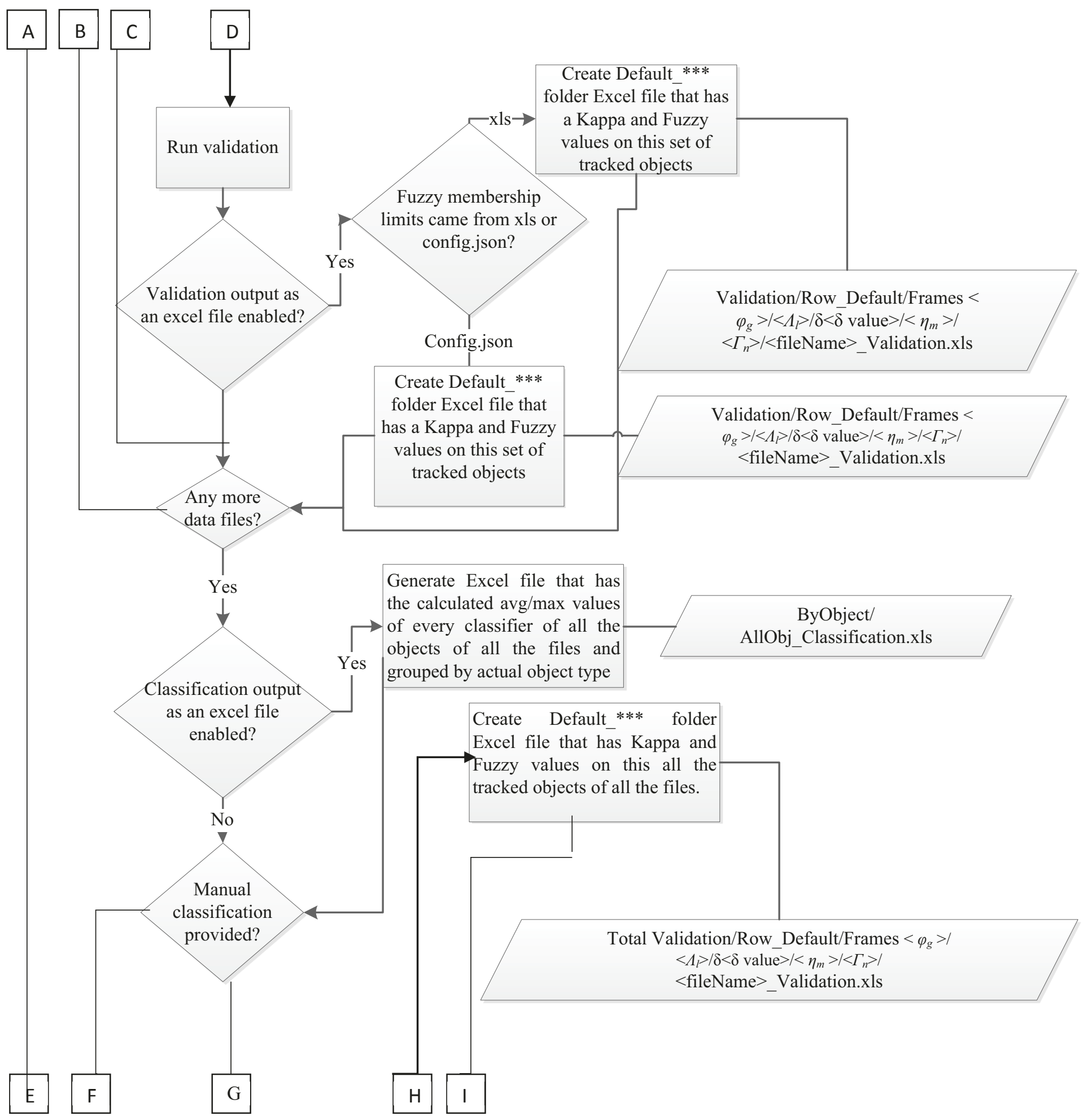

Figure 3. 15 (Part 2) 


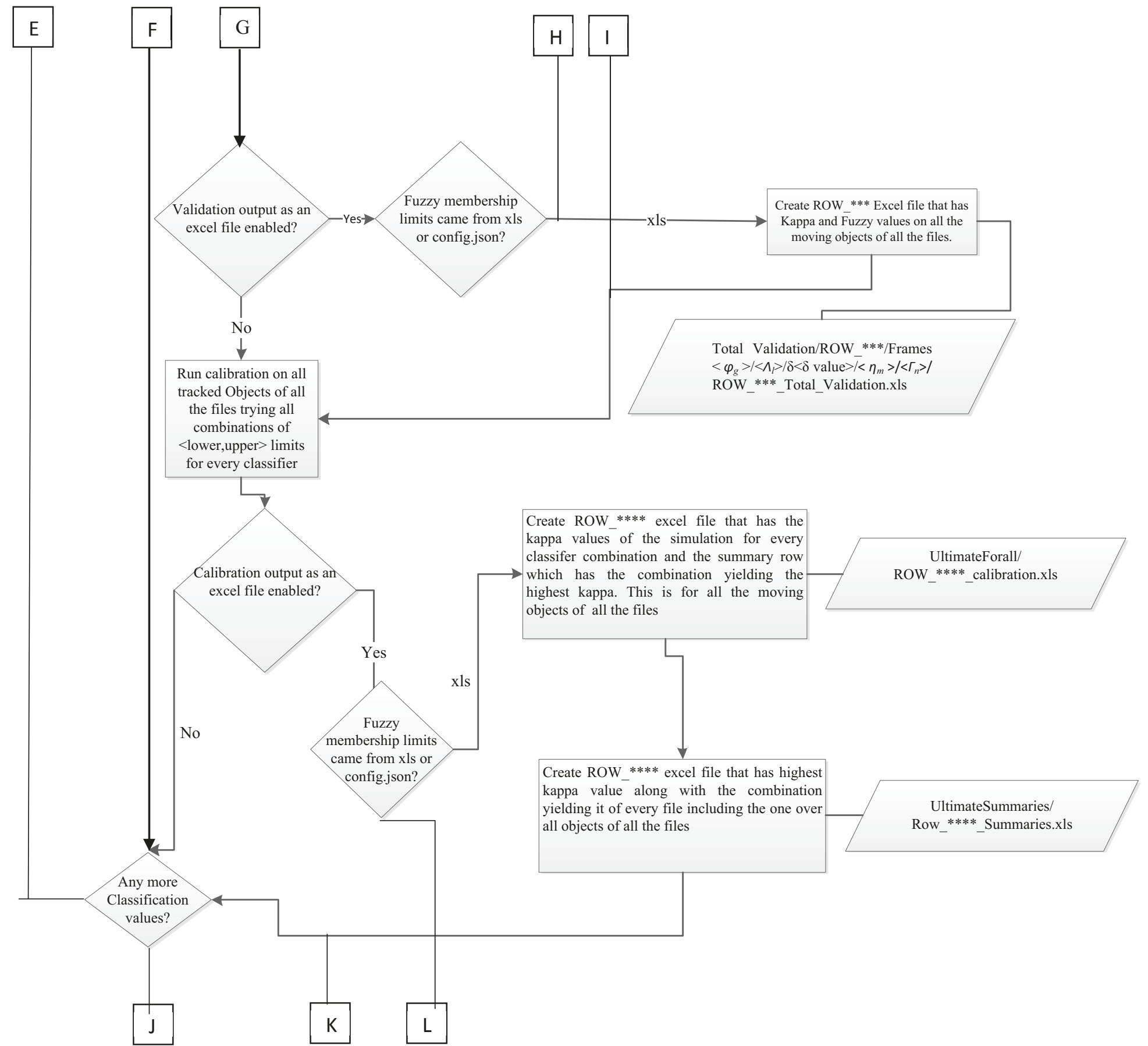

Figure 3. 15 (Part 3) 


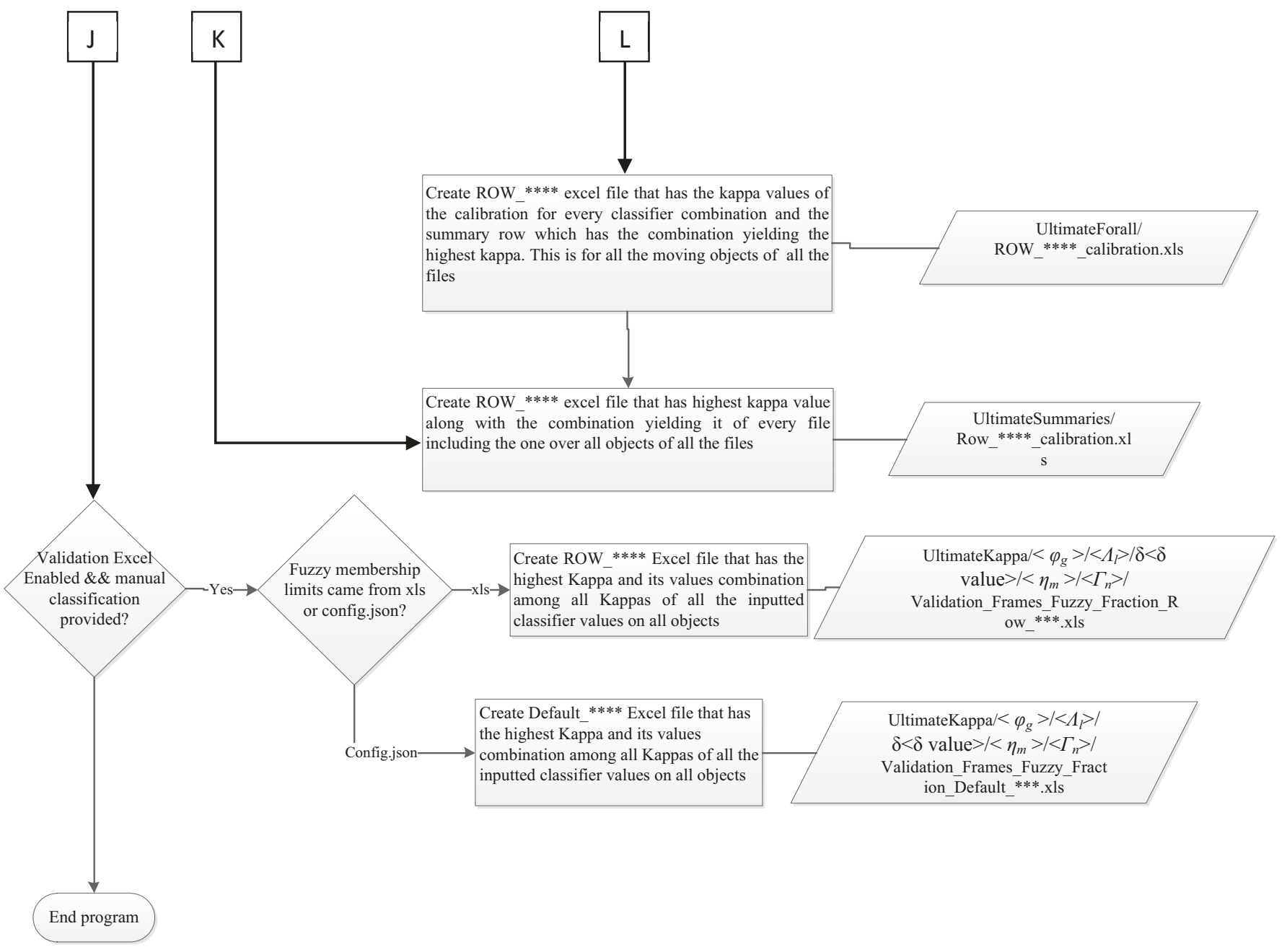

Figure 3. 15 (Part 4) 


\section{Chapter 4: Results and Discussions}

\subsection{Framework Overview}

The proposed framework was designed to classify tracked objects captured in a video sequence using a high-resolution video camera. The classification process utilizes information obtained from the tracked objects tracks, to classify the tracked objects into one of these three categories: vehicles, cyclists, and pedestrians. Four different models have been tested, as is explained in Section 3.1 of this thesis. A large number of configurations were built, calibrated and used to classify the tracked objects. For a configuration to be considered successful, it must meet the following criteria:

1. it should not yield a Kappa coefficient of 0 or less $\left(k_{z, \varphi_{g}} \leq 0\right)$ during the validation. where: $k_{o, \varphi_{g}}$ is the Kappa coefficient for the configuration $o$ for group $g$ of $\varphi_{g}$;

2. It should not get excluded during the null hypothesis test in calibration or any of the three validations.

This chapter illustrates the different models used to classify tracked objects; the effect of the duration of tracked object's appearance; the performance of fuzzy membership functions; and the performance of the classifiers under different configurations.

\subsection{Framework models}

Four classification models were employed in the research methodology. The first model is designed to classify tracked objects into one of the three main categories examined in this study (vehicles, cyclists, or pedestrians). Tracked objects from the three categories mentioned above 
were used to test this model. The second model was designed to classify the tracked objects into vehicles or cyclists only. Therefore, vehicle and cyclists tracks only were used to test this model. The third model was designed to classify tracked objects into vehicles and pedestrians only. Vehicle and pedestrian tracks only were used to test this model. The fourth model was designed to classify tracked objects into cyclists and pedestrians only, and cyclist and pedestrian tracks were used to test this model.

\subsection{Data distribution}

The data used in this thesis was divided into 4 sets; one set is used during the calibration, the other three sets are used for validations. The data were collected collected in previous work (Kassim, 2014) from two different intersections. The first intersection, as previously mentioned, is located at Laurier Avenue West and Lyon Street in Ottawa, Ontario, Canada. Movements for a total of 639 vehicles, 204 cyclists, and 131 pedestrians were observed at this intersection. The corresponding data were divided approximately in half between the calibration and the first validation. The second intersection is located at Laurier Avenue West and Bay Street, also in Ottawa. Here, movements for a total of 259 vehicles, 149 cyclists, and 183 pedestrians were observed. This data were used for the second validation. A third validation combines data from the first and second validations. The data distribution is shown in Table 4.1, below.

Table 4. 1 Data distribution shows the number of tracked objects used in calibration and validations.

\begin{tabular}{|c|c|c|c|c|}
\hline Category & Calibration & $\mathbf{1}^{\text {st }}$ Validation & $\mathbf{2}^{\text {nd }}$ Validation & $\mathbf{3}^{\text {rd }}$ Validation \\
\hline Vehicles & 340 & 299 & 259 & 558 \\
\hline Cyclists & 104 & 100 & 149 & 249 \\
\hline Pedestrians & 68 & 63 & 183 & 246 \\
\hline
\end{tabular}




\subsection{Calibration Results}

Four different models were studied in this thesis. For each model, the calibration was performed using multiple configurations. A sample of the results is illustrated in this chapter. For a list of comprehensive results, please refer to the Appendices at the end of this manuscript (B, $\mathrm{C}, \mathrm{D}$, and E). The Appendices include the calibration and results for all used configuration(s).

\subsubsection{Obtain the initial limits for the fuzzy membership}

As explained in section 3.5.2 of this thesis, in order to find the calibrated limits $\left(a_{j, q}, d_{j, q},\right)$ for the fuzzy membership $\Lambda_{l}$, the calibration process requires initial limits. These are the initial values for lower limit $\left(a i_{j, q}\right)$ and upper limit $\left(d i_{j, q}\right)$ of the fuzzy which were obtained from the same dataset used in calibration. These include 340 vehicles, 104 cyclists, and 68 pedestrians. The way in which this study obtained these initial values was explained in section 3.5.2. Table 4.2, below, illustrates the obtained initial values. Figures 4.1, 4.2 and 4.3 illustrate the observed average speeds for the vehicles, cyclists, and pedestrians ${ }^{3}$.

\footnotetext{
${ }^{3}$ Section A.1 of Appendix A, which includes the histograms of the remaining classifiers for the same data set.
} 
Table 4. 2 The initial values obtained from the tracked objects.

\begin{tabular}{|l|c|c|c|c|c|c|}
\hline \multicolumn{1}{|c|}{ Classifiers } & \multicolumn{2}{c|}{ Vehicles } & \multicolumn{2}{c|}{ Cyclists } & \multicolumn{2}{c|}{ Pedestrians } \\
\hline \multicolumn{1}{|c|}{ Limits } & Min & Max & Min & Max & Min & Max \\
\hline Average speed (m/s) & 0.68 & 17.18 & 0.9 & 8.69 & 0.9 & 3.8 \\
\hline Maximum speed (m/s) & 0.72 & 17.42 & 1.06 & 9.57 & 1.22 & 5.85 \\
\hline Average acceleration (m/s2) & 0 & 10.34 & 0 & 16.07 & 0 & 12.59 \\
\hline Maximum acceleration (m/s2) & 0 & 180.09 & 0 & 50.88 & 0 & 31.03 \\
\hline Average deceleration (m/s2) & 0 & 14.09 & 0 & 18.45 & 0 & 34.23 \\
\hline Maximum deceleration (m/s2) & 0 & 120.58 & 0 & 106.92 & 0 & 47.38 \\
\hline Average direction (degree) & 81.61 & 276.95 & 84 & 249.06 & 2.81 & 344.03 \\
\hline Maximum direction (degree) & 88.08 & 294.03 & 86.16 & 273.3 & 2.81 & 359.84 \\
\hline Average area (m2) & 0.93 & 19.82 & 0.01 & 4.73 & 0.02 & 2.47 \\
\hline Maximum area (m2) & 1.33 & 49.95 & 0.01 & 8.1 & 0.02 & 8 \\
\hline Average length (m) & 0.76 & 5.99 & 0.12 & 1.67 & 0.12 & 2.27 \\
\hline Maximum length (m) & 0.86 & 7.9 & 0.13 & 2.9 & 0.12 & 3.02 \\
\hline Average width (m) & 0.72 & 6.59 & 0.08 & 2.67 & 0.13 & 2.05 \\
\hline Maximum width (m) & 1.32 & 9.35 & 0.08 & 3.83 & 0.13 & 3.17 \\
\hline Peaks in speed per second & 0 & 15.76 & 0.11 & 30 & 0 & 20.36 \\
\hline Effective weighted average frequency (Hz) & 0 & 151.78 & 0 & 53.31 & 0 & 26.29 \\
\hline Effective frequency (Hz) & 0 & 29.33 & 0 & 29.55 & 0 & 27.86 \\
\hline Average of change in direction (degree) & -1.33 & 0.77 & -6.73 & 0.64 & -27 & 3.45 \\
\hline Average of cosine of change in direction (degree) & -0.36 & 0.99 & -0.9 & 0.97 & -0.39 & 1 \\
\hline
\end{tabular}

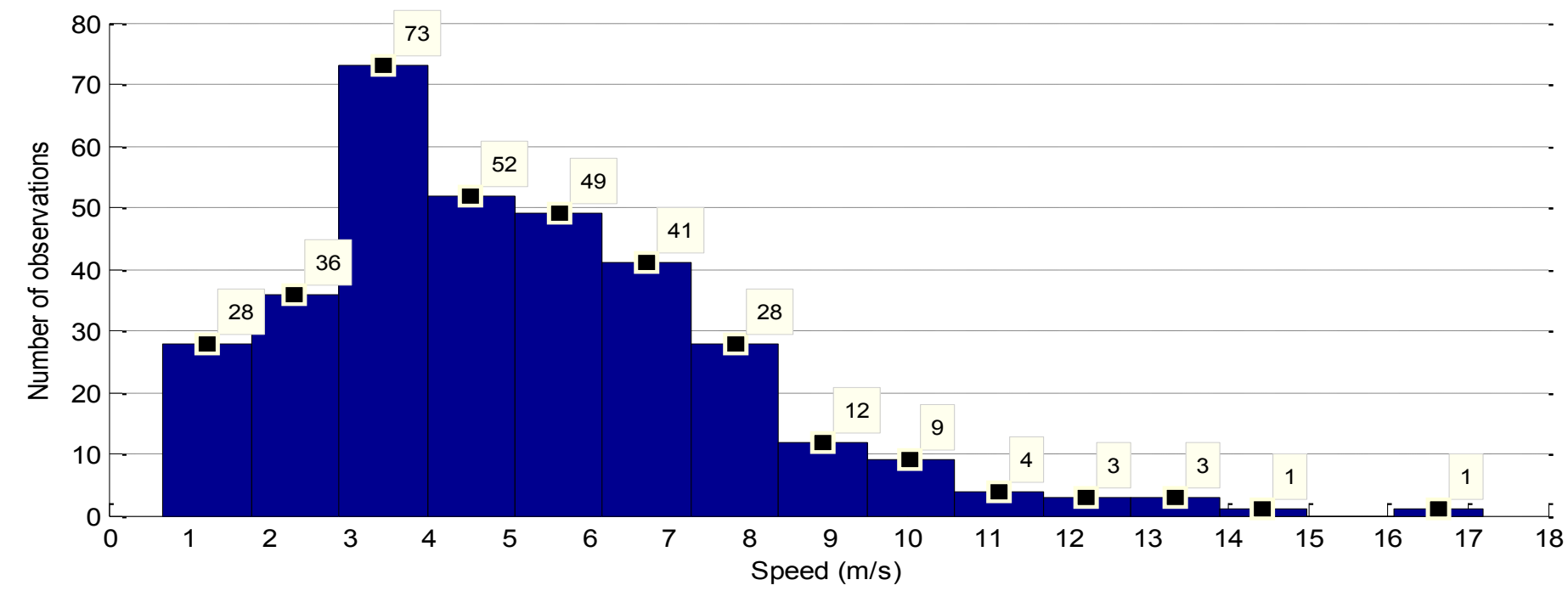

Figure 4. 1 The observed average speed histogram for vehicles 


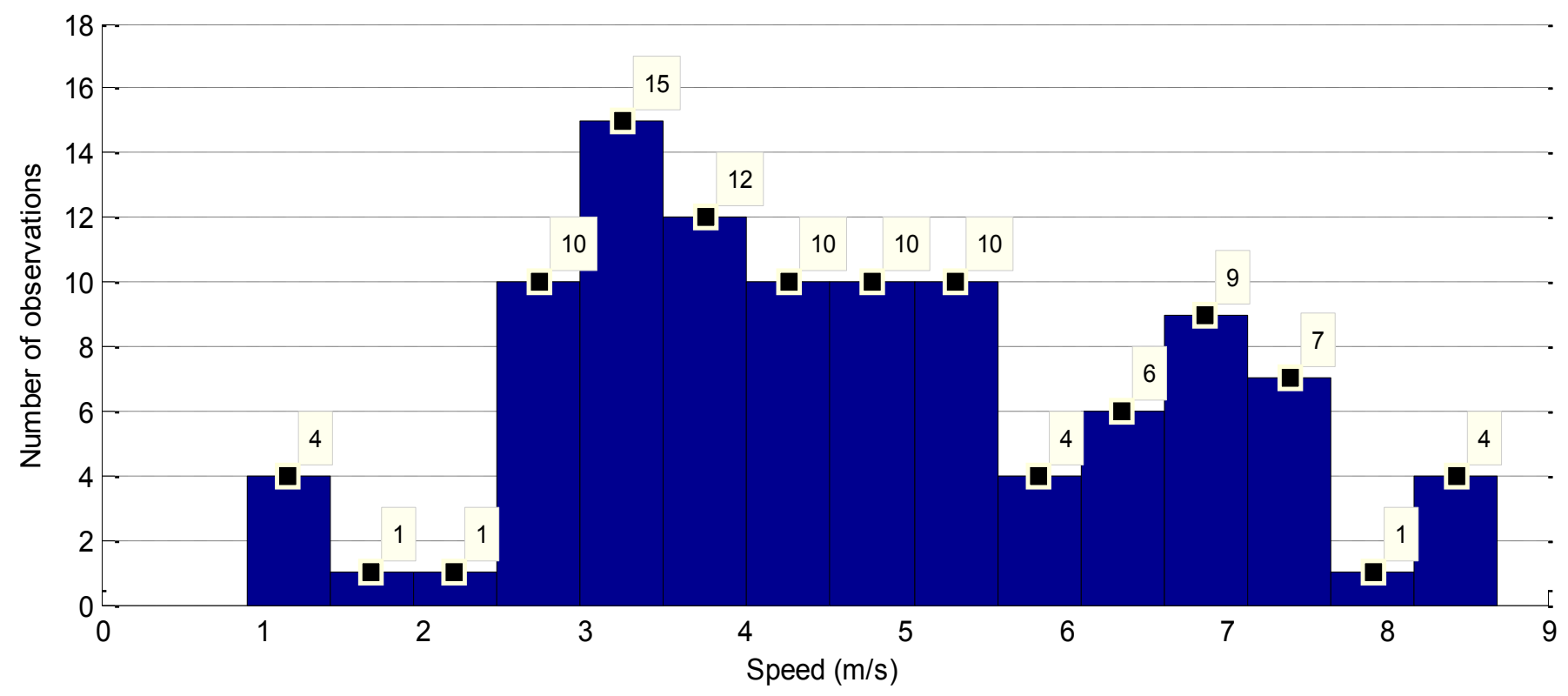

Figure 4. 2 The observed average speed histogram for cyclists

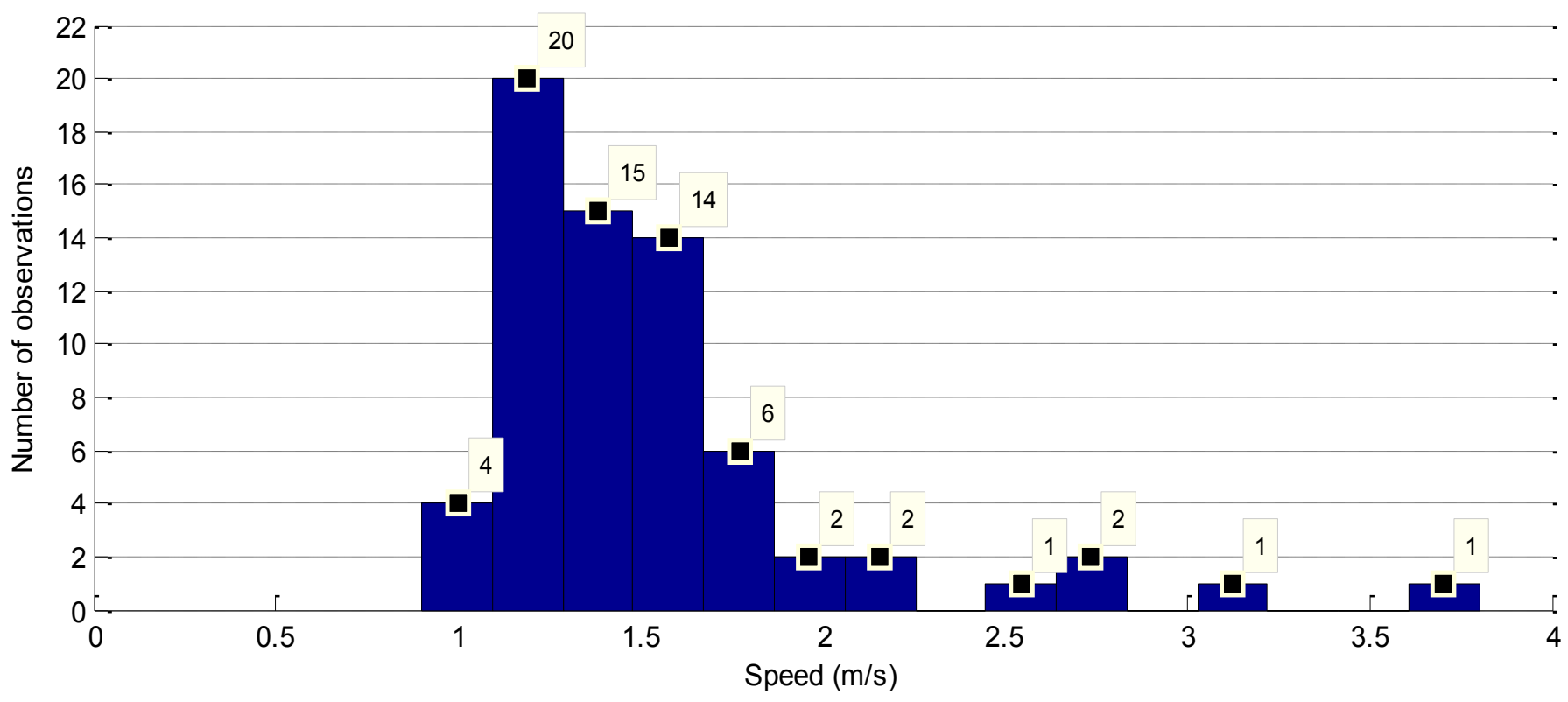

Figure 4. 3 The observed average speed histogram for pedestrians 


\subsubsection{Obtain the calibrated limits for the fuzzy membership}

Obtaining the calibrated limits $\left(a_{j, q}, d_{j, q},\right)$ of the fuzzy membership function in a specific setting offers the ability to understand ways in which different configurations affect the calibration and validations processes. This accounts for the minimum duration of the tracked object's appearance $\varphi_{g}$ as well as the type of fuzzy membership function $\Lambda_{l}$. It also answers questions regarding whether various classifiers improve the results of classification or not.

\subsubsection{Calibration procedure 1 vs procedure 2}

The main differences between calibration procedure 1 and calibration procedure 2 are: [i] calibration procedure 2 can be only used if the calibration is being performed on more than one classifier. [ii] the required results are to keep the effective classifiers only. The effective classifiers are those that significantly improve the classification process. Therefore, if a classifier is terminated when using the calibration procedure 2 , it is not necessarily harmful to the classification overall. Therefore, when a classifier is excluded, it is because this classifier did not improve the classification in a statistically significant way. Figures 4.4 and 4.5 illustrate the two types of calibration used on a triangular fuzzy membership function with $\delta=0.5$. In both figures, the $y$-axis refers to the Kappa coefficient. The $x$-axis refers to the number of calibration trails. The figure shows the improvement of calibration results with each calibration trail. After approximately 95,000 calibration trails, an acceptable results achieved for both type of calibrations under different durations of tracked object's appearance 
(a)

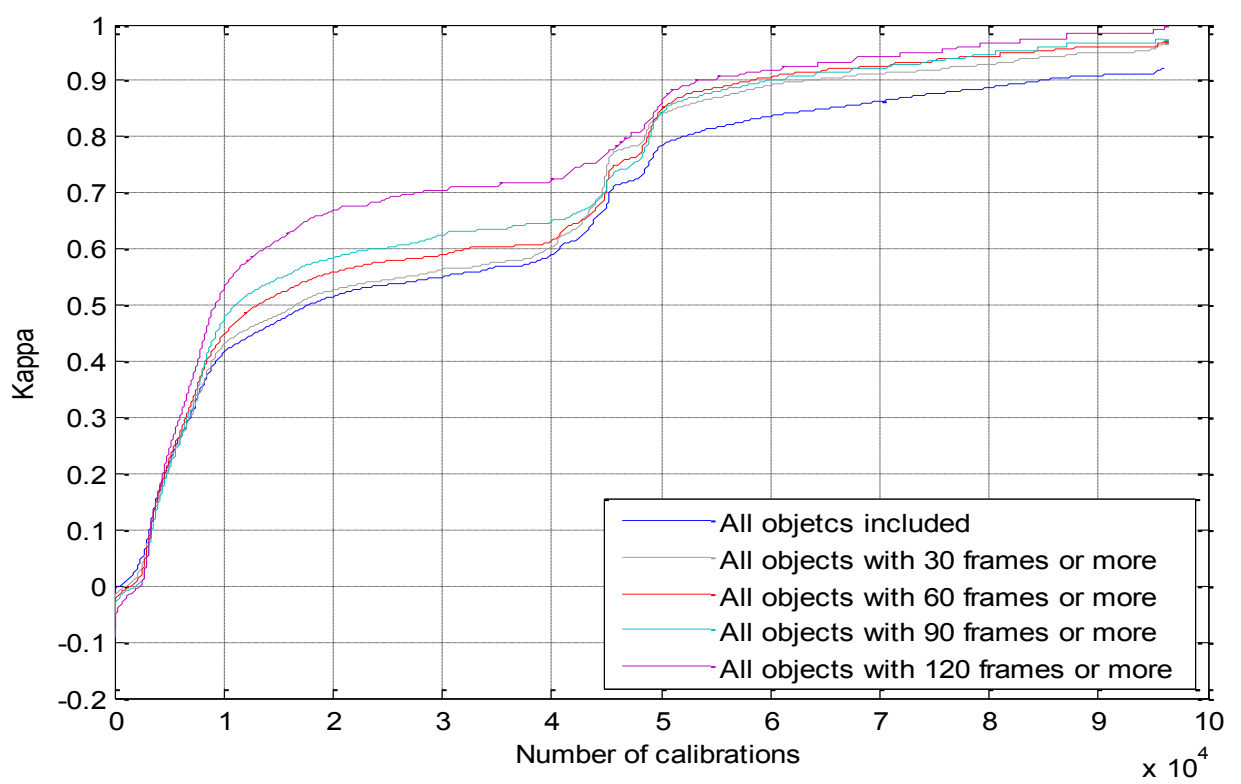

(b)

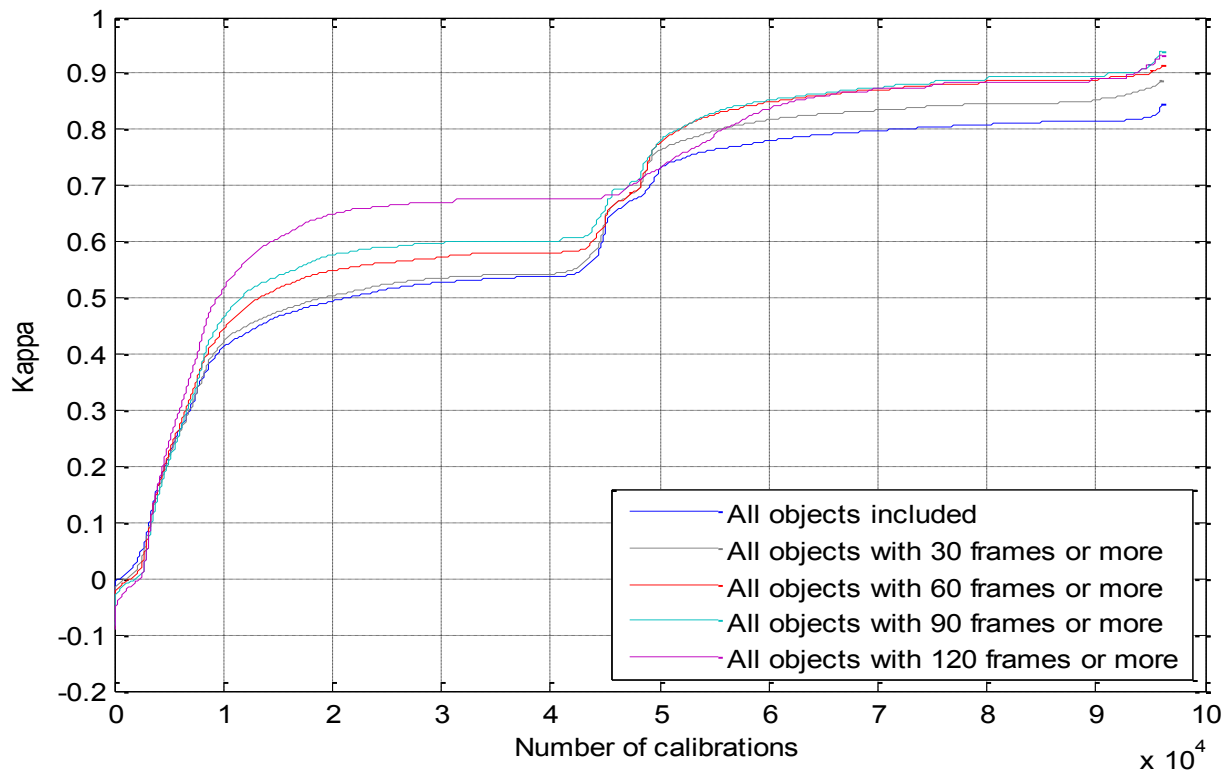

Figure 4. 4 a) Cumulative curves show the improvement of Kappa coefficient using calibration procedure 1 b) Cumulative curves show the improvement of Kappa value using calibration procedure 2 . 
(a)

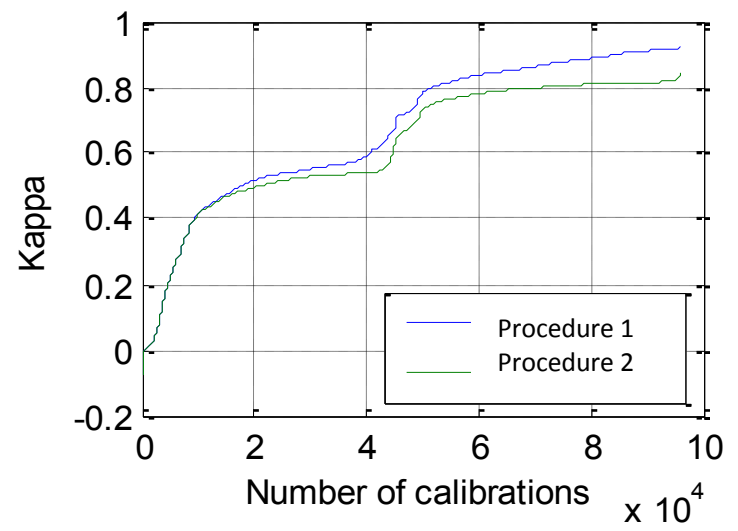

(c)

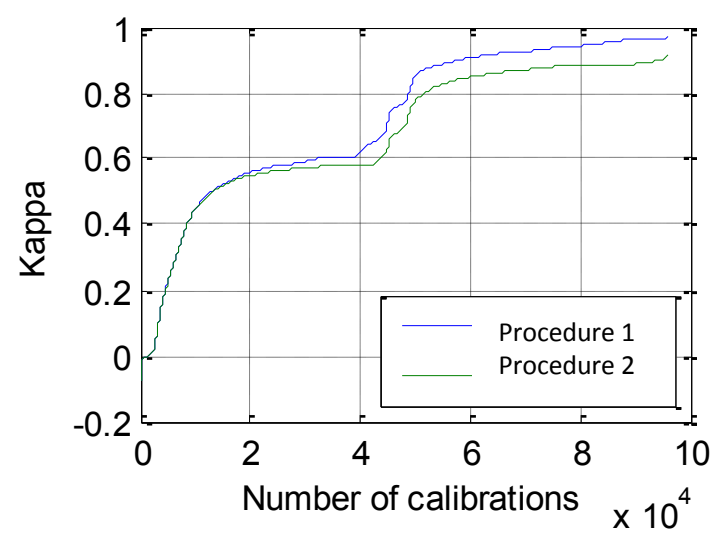

(b)

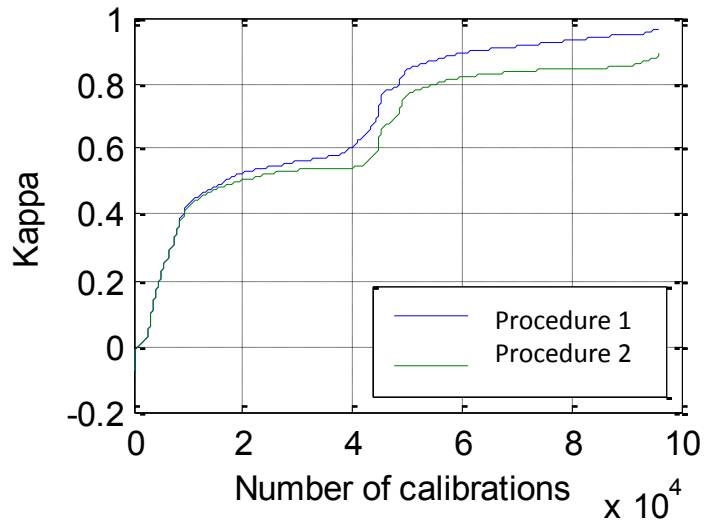

(d)

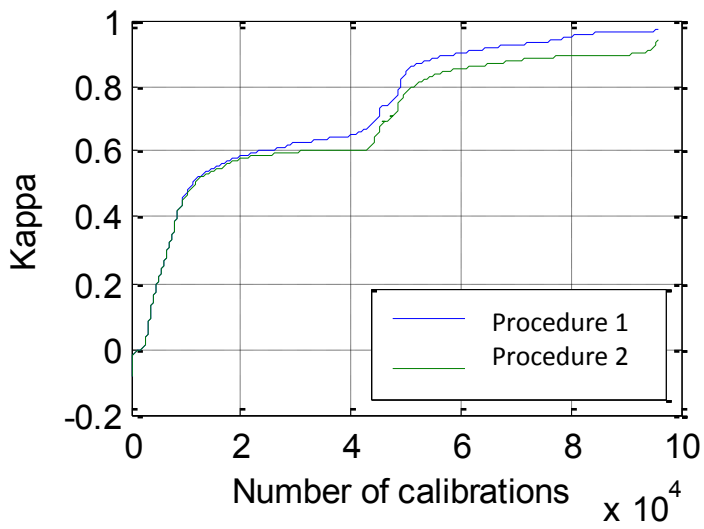

(e)

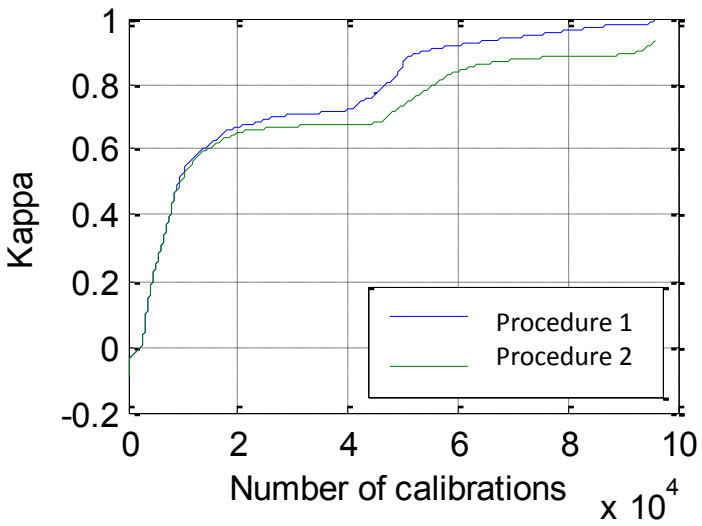

Figure 4. 5 Cumulative curves show the improvement of Kappa coefficient with calibration for calibration procedure 1 and calibration procedure 2 .

where the minimum duration of the tracked objects' appearance in the video sequence is a) 0 frames, b) 30 frames, c) 60 frames, d) 90 frames, e) 120 frames. 
It is noticeable that the results of calibration using procedure 2 yielded lower results comparing to the results from procedure $1^{4}$. The coefficient of determination is calculated to measures agreement between the two types of calibration. Table 4.2 shows the summary results of the relationship between procedure 1and procedure 2 calibrations. The results did show some agreement for the First model. This wasn't the case for the other models.

a)

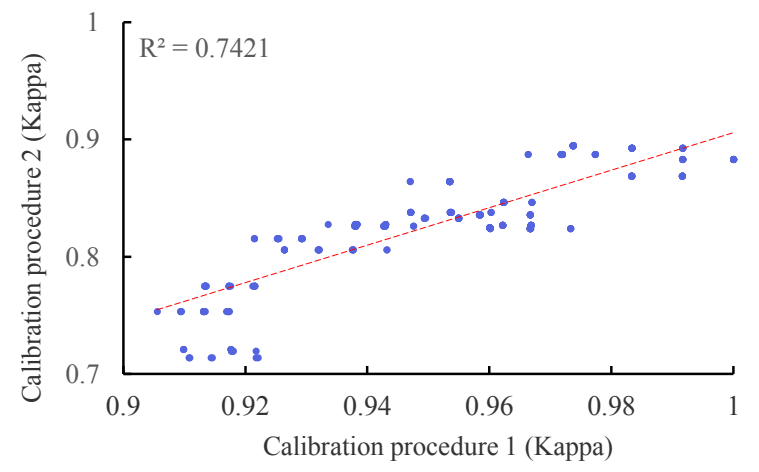

c)

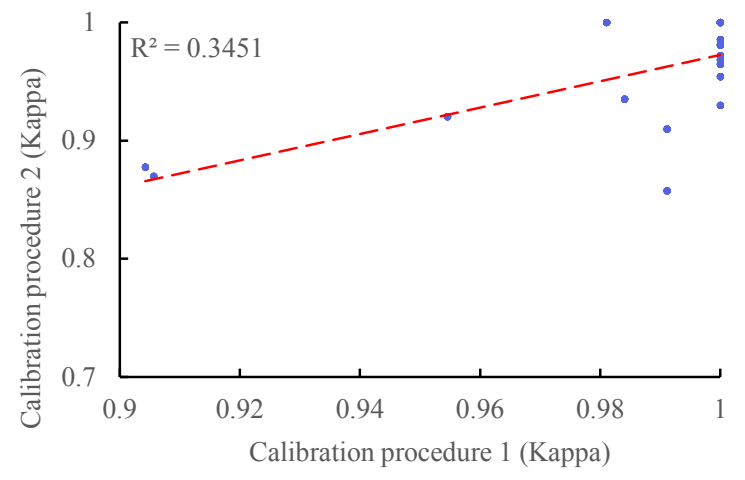

b)

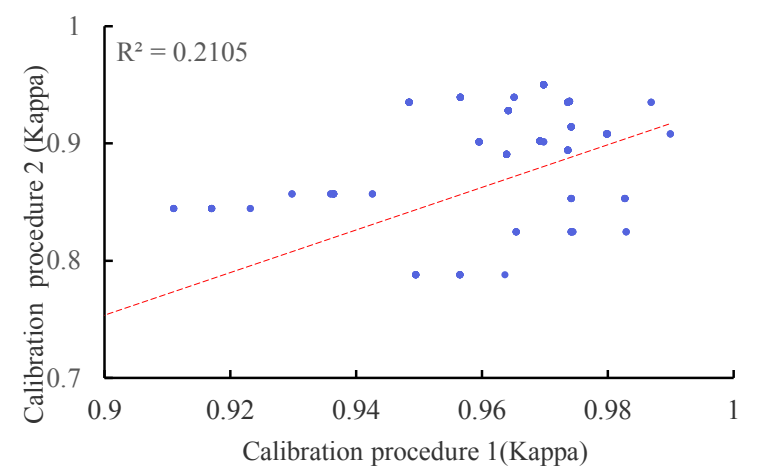

d)

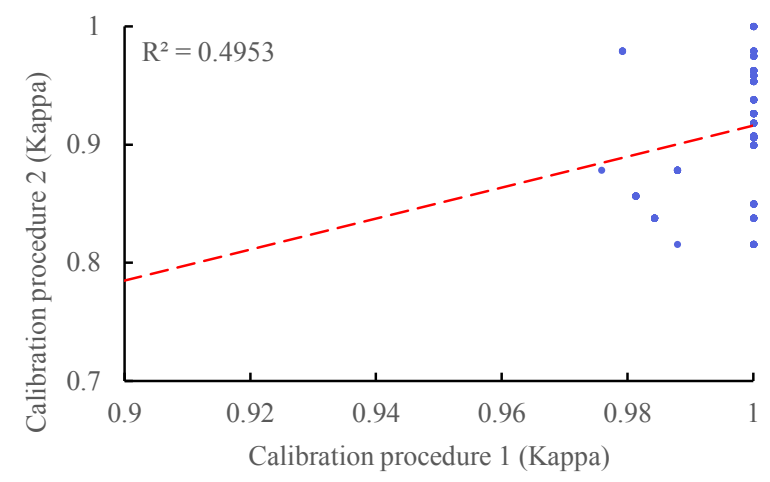

Figure 4. 6 Relationship between calibration procedure 1 and calibration procedure 2. a) for the first model; b) for the second model; c) for the third model; d) for the fourth model.

\footnotetext{
${ }^{4}$ Please refer to Tables B.1.1, B.1.2, C.1.1, C.1.2, D.1.1, D.1.2, E.1.1 and E.1.2 found in each respective Appendix for a comprehensive list of these results.
} 
Table 4. 3 Coefficient of determination for the four models

\begin{tabular}{|c|c|}
\hline Model & $\mathbf{R}^{2}$ \\
\hline 1 & 0.742 \\
\hline 2 & 0.211 \\
\hline 3 & 0.345 \\
\hline 4 & 0.495 \\
\hline
\end{tabular}

The calibration results are presented in Kappa coffeient. The results of calibration procedure 1 are clearly higher than the results of calibration procedure 2 . This applies to all used configurations. However, this thesis takes into account that this discrepancy between calibration procedures might not have remained consistent with validation. Therefore, the calibrated configurations from both types of calibration were validated and will be detailed later in this chapter. From testing different configurations using calibration procedure 2, it was noticed that some classifiers did not improve calibration results which is presented in Kappa coffeient. One of classifiers that did not improve the calibration results is declaration classifier. On the other hand, there were some classifiers that actually did improve the calibration results such as the average area. Table 4.4 is a percentage table that illustrates the effectiveness of all classifiers for the four models using calibration procedure 2 . The first model classify the tracked object into vehicles, cyclists and pedestrians. The second model classify the tracked object into vehicles and cyclists only. The third model classify the tracked objects into vehicle and pedestrians only. The fourth model classify tracked object into cyclists and pedestrians only. A classifier is considered effective if it is included. The results are for the 360 different combinations. $100 \%$ value for a classifier indicates that a classifier was included in all 360 configurations. $0 \%$ value for a classifier indicates that a classifier was excluded in all 360 configurations. 
Table 4. 4 The effectiveness of all the classifiers using calibration procedure 2 for the four different models.

\begin{tabular}{|l|c|c|c|c|}
\hline \multicolumn{1}{|c|}{ Classifiers } & Model 1 & Model 2 & Model 3 & Model 4 \\
\hline Average speed & 100 & 100 & 100 & 100 \\
\hline Maximum speed & 100 & 100 & 55 & 5 \\
\hline Average acceleration & 0 & 30 & 5 & 0 \\
\hline Maximum acceleration & 0 & 20 & 0 & 0 \\
\hline Average deceleration & 0 & 0 & 5 & 10 \\
\hline Maximum deceleration & 0 & 0 & 0 & 0 \\
\hline Average direction & 0 & 10 & 0 & 0 \\
\hline Maximum direction & 10 & 25 & 10 & 0 \\
\hline Average area & 95 & 100 & 100 & 0 \\
\hline Maximum area & 100 & 75 & 35 & 0 \\
\hline Average length & 5 & 30 & 35 & 0 \\
\hline Maximum length & 0 & 5 & 35 & 5 \\
\hline Average width & 0 & 0 & 35 & 10 \\
\hline Maximum width & 0 & 0 & 35 & 15 \\
\hline Peaks in speed per second & 0 & 0 & 35 & 5 \\
\hline Effective weighted average frequency & 0 & 0 & 35 & 5 \\
\hline Effective frequency & 0 & 0 & 35 & 5 \\
\hline Average of change in direction & 0 & 35 & 5 \\
\hline Average of cosine of change in direction & 0 & 35 & 5 \\
\hline
\end{tabular}

\subsubsection{Identify the best calibrated configuration(s)}

In the case of multiple configurations, the calibration process is not limited to finding the calibrated lower and upper values of the fuzzy membership function to the configurations only, but also to find the best possible configuration(s). Using $t$-statistic a null hypothesis test was performed, as is discussed in Section 3.5.2 of this thesis. The aim was to exclude any configuration $k_{o, \varphi_{g}}$ with Kappa coffeient falling outside the $(95 \%)$ confidence interval of the 
configuration $k_{z, \varphi_{g}}$ that yielded the highest Kappa coffeient. This exclusion was enforced only for the configurations within the same group which share the same minimum duration of tracked object's appearance. Mathematically, it was explained in equation (3.23). A significant number of configurations for each duration group were excluded, and Appendices B to E include the results of those configurations that fell within the $95 \%$ confidence interval of the configuration that yielded the highest Kappa.

\subsection{Validation Results}

\subsubsection{Effect of the minimum duration of tracked objects appearance $\varphi_{g}$ on results}

The Results for the four models ${ }^{5}$ show that the minimum duration of tracked object appearance $\varphi_{g}$ has limited effect on the classification process of the tracked object. In other word, a tracked object with 10 frames can be classified with the same accuracy as classifying a tracked object with 100 frames. This finding was noticed in most of the validation results. Figures 4.4 and 4.5 illustrate a sample of these results.

\subsubsection{Effect of the fuzzy membership function $\Lambda_{l}$ on results}

Observing results for the four models found that the performance of different fuzzy membership function $\Lambda_{l}$ was almost the same for successful configurations within the group organized according to duration of appearance $l$. The goal of using different types of fuzzy membership functions was to find the best fuzzy membership, which lead to high classification rates. Figure 4.7, below, contains a sample showing fuzzy function performance. The $y$-axis is

\footnotetext{
${ }^{5}$ The results for these four models are found in Appendices B to E.
} 
the Kappa coefficient. The $x$-axis is the number of calibration trails. The graph clearly shows that

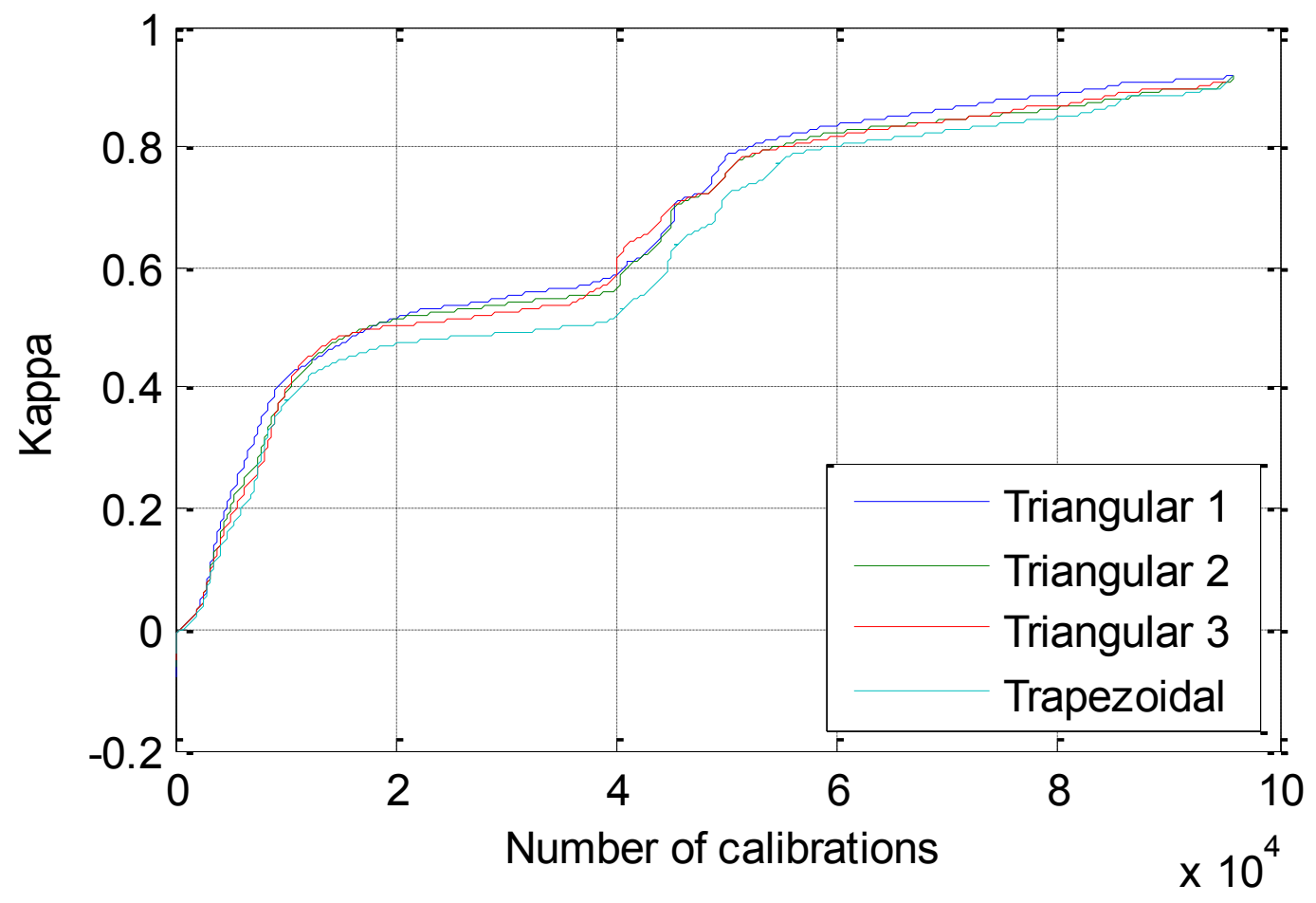

Figure 4. 7 Cumulative curves show the improvement of Kappa value with calibration using different kind of fuzzy membership functions.

Note that triangular 1, 2 and 3 mean triangular fuzzy membership function with $\delta=0.25,0.5$ and 0.75 consecutively.

the different type of fuzzy membership function had no effect on the classification accuracy.

\subsubsection{Effect of the classifiers on results}

The following section outlines results pertaining to the effect of classifiers on the classification process. This discussion is paired alongside Appendices B through E of this thesis, which display detailed results for calibrations and validations. For a more comprehensive listing of results, please consult these Appendices. 


\subsection{3a Validation results: procedure 1 as compared with procedure 2}

This section discusses the validation results obtained using the configuration obtained from calibration procedures 1 and 2. A comparison between the validation results is also detailed in this section. Note that the validation results using configuration obtained from calibration procedures 1 will be called validation type 1 . Similarly, for the validation results using the configuration obtained from calibration procedures 2 will be called validation type 2 .

\section{The first model}

Validation type 1 results presented in Kappa coefficient reveals that a large number of these configurations were eliminated, as opposed to validation type 2 results $^{6}$. The number of configurations in Table B.1.2 is less than Table B.1.1. This is because the effective weight average frequency and the effective frequency were terminated when calibration procedure 2 was used. Comparing the results of the successful configurations based on the minimum duration of the tracked objects appearance $\varphi_{g}$, as well as the type of the fuzzy membership function $\Lambda_{l}$, in both types shows that the results of all validations were almost identical. Importantly, some of the successful configurations used in validation type 2 are failed in validation type 1 . This may indicate that some of the terminated classifiers in validation type 2 would reduce the classification quality if they were included, as in validation type 1 . Overall, the best classification results are obtained from the most successful configurations.

\footnotetext{
${ }^{6}$ Please also see Table B.1.1 and Table B.1.2, found in Appendix B, which outline the results for calibrations and validations for both Types 1 and 2 .
} 


\section{The second model}

The Kappa value for the successful configurations in both validation type 1 and validation type 2 fall within the same Kappa bracket shown in Table 3.8, indicating that there is almost perfect agreement between manual and automated classifications. Also, some successful configurations in validation type 2 were failed in validation type 1 , which supports the earlier observation that not all classifiers are bound to improve the classification process: some may prove irrelevant or detrimental. ${ }^{7}$

\section{The third model}

Comparing successful configurations for both validation type 1 and validation type 2, interestingly, validation type 2 yielded better results than validation type $1 .^{8}$ Overall, the results from Validation type 2 are better than validation type 1. Some classifiers, such as the effective weighted average frequency and the effective frequency, did work in a few successful configurations in validation type 2 . This is notably different than what was observed using the first and second models, in which these classifiers did not produce successful results in both.

\section{The fourth model}

In this case, again, configuration of validation type 2 produced better results than validation type $1 .^{9}$ A few classifiers, as was the case using the third model — again including the effective weighted average frequency and the effective frequency-were successful in

\footnotetext{
${ }^{7}$ Tables C.1.1 and Table C.1.2 in Appendix C include the results for calibrations and validations using both Types 1 and 2 .

${ }^{8}$ Tables D.1.1 and Table D.1.2 in Appendix D include the results for calibrations and validations using both Types 1 and 2 .

${ }^{9}$ Tables E.1.1 and Table E.1.2 in Appendix E include the results for calibrations and validations using both Types 1 and 2 .
} 
configurations of validation type 2 calibrations. However, these configurations failed following the first and second validations.

\section{Summary of the results}

a. For the first and second model, the configuration from calibrations procedure 2 yielded similar results to the configurations from calibration procedure 1.

b. For the third and fourth model, the configuration from calibrations procedure 2 yielded better results to the configurations from calibration procedure 1 .

c. Based on the three validations, $98 \%$ of the successful configuration showed perfect agreement between manual and automated classifications, and 2\% showed substantial agreement between manual observation and automated classification. This ranking is based on Kappa coefficient interpretation as shown in Table 3.8.

d. Classification accuracy for the three categories was measured using the correct percentage value $^{10}$ (see Table 4.5). This includes the correct percentage value for vehicles, cyclists, and pedestrians. The results are based on the three validations.

Table 4. 5 The accuracy rate of classification using the percent correct value. The results are for the successful configurations only in the three validations.

\begin{tabular}{|c|c|c|c|c|c|c|}
\hline \multirow{2}{*}{ Model Number } & \multicolumn{2}{|c|}{ Vehicles } & \multicolumn{2}{c|}{ Cyclists } & \multicolumn{2}{c|}{ Pedestrians } \\
\cline { 2 - 7 } & Min $^{\mathbf{1 1}}$ & Max $^{\mathbf{1 2}}$ & Min & Max & Min & Max \\
\hline Model 1 & 83 & 96 & 73 & 95 & 90 & 100 \\
\hline Model 2 & 73 & 96 & 83 & 100 & - & - \\
\hline Model 3 & 92 & 99 & - & - & 86 & 99 \\
\hline Model 4 & - & - & 82.67 & 100 & 95.24 & 100 \\
\hline
\end{tabular}

\footnotetext{
${ }^{10}$ Please refer to Tables B.2.1, B.2.2, C.2.1, C.2.2, D.2.1, D.2.2, E.2.1 and E.2.2 found in each respective Appendix for a comprehensive list of these results.

${ }^{11}$ Refer to minimum accuracy rate in percentage scale.

${ }^{12}$ Refer to maximum accuracy rate in percentage scale.
} 


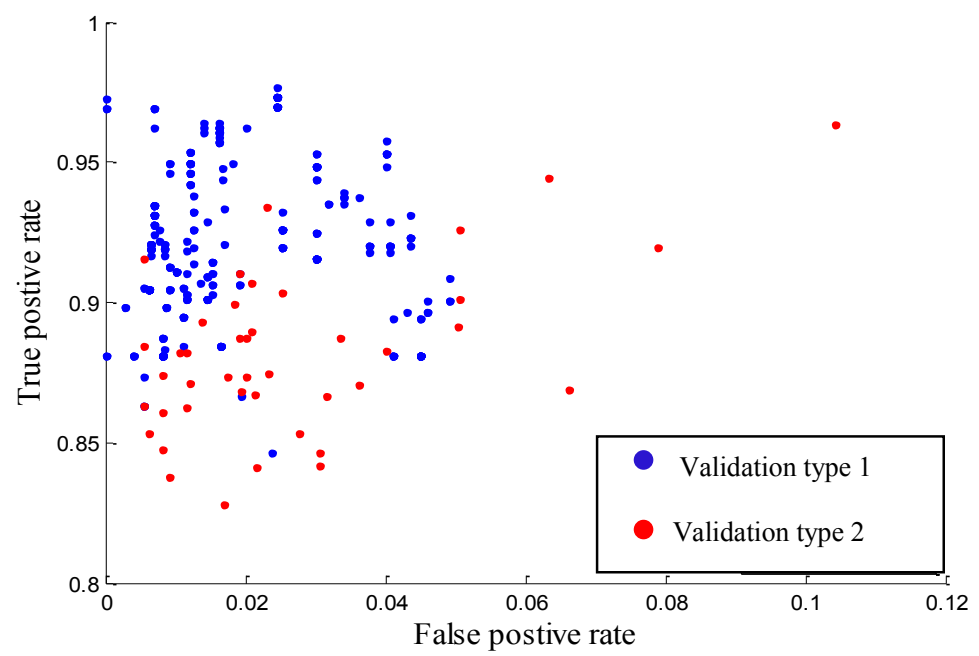

Figure 4. 8 Receiver operating characteristic ${ }^{13}$ for the first model illustrate the validation results for vehicles using validation type 1 and 2 .

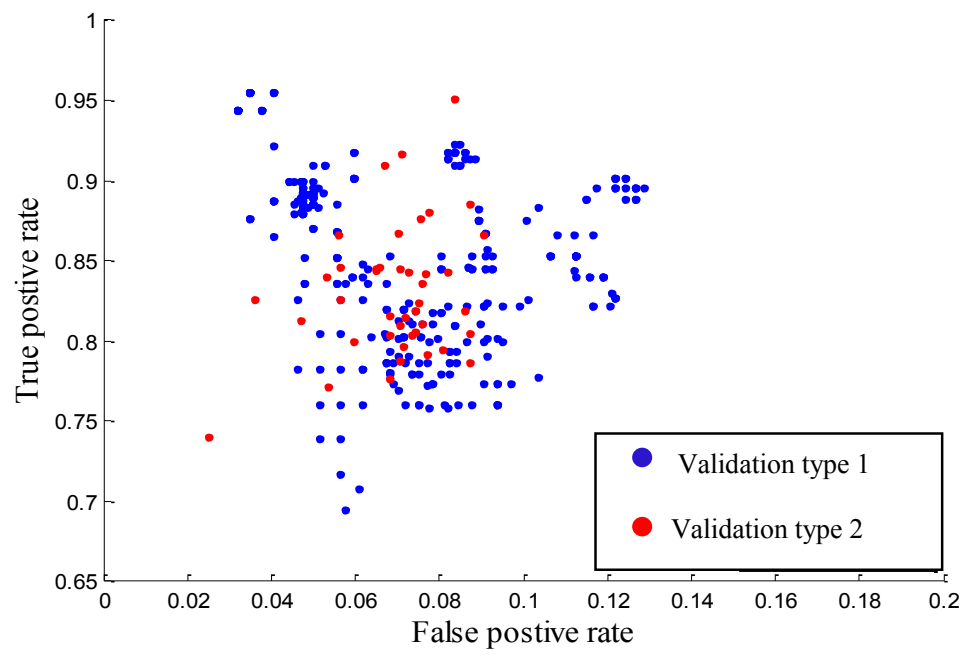

Figure 4. 9 Receiver operating characteristic ${ }^{14}$ for the first model illustrate the validation results for cyclists using validation type 1 and 2 .

\footnotetext{
${ }^{13}$ True positive rate refer to all (vehicles classified as vehicles), false positive rate refer to all (vehicles classified as cyclists and pedestrians).

${ }^{14}$ True positive rate refer to all (cyclists classified as cyclists), false positive rate refer to all (cyclists classified as vehicles and pedestrians).
} 


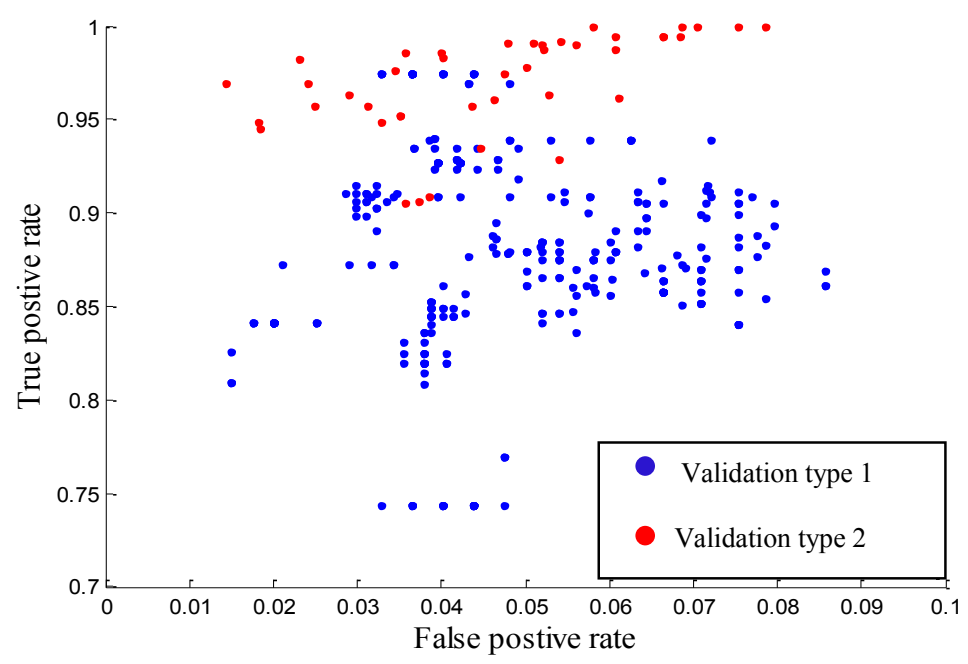

\section{Figure 4. 10 Receiver operating characteristic ${ }^{15}$ for the first model illustrate the validation results for pedestrians using validation type 1 and 2.}

\subsection{3b Tracked object speed}

\section{- Average speed}

From testing the average speed classifier to classify the tracked objects ${ }^{16}$, it was found that this classifier is able to categorize tracked objects with an acceptable accuracy rate in binary classification, if one of the categories being classified is for pedestrians - for example, this works when combining vehicles and pedestrians, or cyclists and pedestrians. The highest classification rate was observed in the fourth model, followed by the third and then the first models. The results from the second model fell below expectations. This may indicate that the algorithm could not provide a sufficiently high classification rate for the vehicles-cyclist classification, a conclusion which is based on the following findings:

\footnotetext{
${ }^{15}$ True positive rate refer to all (pedestrians classified as pedestrians), false positive rate refer to all (pedestrians classified as cyclists and pedestrians).

${ }^{16}$ For the results of using the average speed to classify tracked objects using the four models, see Tables B.1.3, C.1.3, D.1.3, and E.1.3, found in each respective Appendix.
} 
- Higher classification results are for the third and fourth models, in which one of the categories is always the pedestrian category.

- The mutual categories between the first and second models are the vehicle and cyclist categories. As the results of the first and second models are lowest. This supports the conclusion that the algorithm could not accurately provide an appropriate classification rate within this category. This conclusion is acceptable because vehicle and cyclists tracks were captured at signalized intersections where road users are expected to slow down. Table 4.6, below, illustrates the classification accuracy range for the three categories using the percent correct value ${ }^{17}$.

Table 4. 6 The accuracy rate of classification using the percent correct value. The results are for the successful configurations only in the three validations.

\begin{tabular}{|c|c|c|c|c|c|c|}
\hline \multirow{2}{*}{ Model Number } & \multicolumn{2}{|c|}{ Vehicles } & \multicolumn{2}{c|}{ Cyclists } & \multicolumn{2}{c|}{ Pedestrians } \\
\cline { 2 - 7 } & Min & Max & Min & Max & Min & Max \\
\hline Model 1 & 31 & 74 & 26 & 77 & 82 & 98 \\
\hline Model 2 & 18 & 59 & 39 & 88 & - & - \\
\hline Model 3 & 76 & 92 & - & - & 88 & 98 \\
\hline Model 4 & - & - & 83 & 100 & 95 & 100 \\
\hline
\end{tabular}

The configurations for the four models are ranked as follows (the ranking is based on Kappa coefficient interpretation as presented in Table 3.8):

a) In the first model:

- $66 \%$ of successful configurations show moderate agreement between manual and automated classification;

\footnotetext{
${ }^{17}$ For the percentage results of using the average speed to classify tracked objects for the four models, see Tables B.2.3, C.2.3, D.2.3, and E.2.3, found in each respective Appendix.
} 
- $34 \%$ of successful configurations show fair agreement between manual and automated classification.

b) In the second model:

- $100 \%$ of successful configurations show a slight agreement between manual and automated classification.

c) In the third model

- $92 \%$ of successful configurations show a substantial agreement between manual and automated classification;

- $8 \%$ of successful configurations show an almost perfect agreement between manual and automated classification.

d) In the fourth model:

- $100 \%$ of successful configurations show an almost perfect agreement between manual and automated classification.

- Maximum speed

The results of using the maximum speed to classify the track objects for the four models are in Tables B.1.4, C.1.4, D.1.4, and E.1.4. The performance of these classifiers shares the same characteristics as was achieved while using the average speed classifier. This classifier can also categorize tracked objects with an acceptable accuracy rate in binary classification, as long as one of the categories being classified is for pedestrian. The highest classification rate occurred using the fourth model, followed by the third. As opposed to the average speed classifier, the maximum speed classifier did provide a higher level of classification while in the second model. This also supports the conclusion that vehicles and cyclists may travel through an intersection at similar speeds, but at different speeds through the entire trip, especially after crossing the 
intersection. Table 4.7 illustrates the classification accuracy ranges for the three categories, using the percent correct value to show their accuracy.

Table 4. 7 The accuracy for the rate of classification, using a percent correct value. These results are based on the three validations.

\begin{tabular}{|c|c|c|c|c|c|c|}
\hline \multirow{2}{*}{ Model Number } & \multicolumn{2}{|c|}{ Vehicles } & \multicolumn{2}{c|}{ Cyclists } & \multicolumn{2}{c|}{ Pedestrians } \\
\cline { 2 - 7 } & Min & Max & Min & Max & Min & Max \\
\hline Model 1 & 45 & 81 & 63 & 76 & 87 & 97 \\
\hline Model 2 & 45 & 81 & 78 & 98 & - & - \\
\hline Model 3 & 88 & 95 & - & - & 87 & 96 \\
\hline Model 4 & - & - & 76 & 100 & 83 & 96 \\
\hline
\end{tabular}

Using Table 3.8 as a reference, the ranking of the configurations for the four models is as follows:

a) In the first model:

- $43 \%$ of successful configurations show moderate agreement between manual and automated classification;

- $57 \%$ of successful configurations show fair agreement between manual and automated classification.

b) In the second model:

- $27 \%$ of successful configurations show fair agreement between manual and automated classification;

- $73 \%$ of successful configurations show moderate agreement between manual and automated classification.

c) In the third model: 
- $33 \%$ of successful configurations show a substantial agreement between manual and automated classification;

- $67 \%$ of successful configurations show an almost perfect agreement between manual and automated classification.

d) In the fourth model:

- $100 \%$ of successful configurations show an almost perfect agreement between manual and automated classification.

\subsection{3c Acceleration and decelerations}

The results of the average and maximum accelerations for all four models were not satisfying ${ }^{18}$. The same applies to the average and maximum decelerations for the models ${ }^{19}$. The majority of successful configurations show a slight agreement between manual and automated classification based on the interpretation of the Kappa coefficient ${ }^{20}$. For some models, the entire set of configurations for the maximum acceleration and declaration has failed. This failure can be attributed to the acceleration and deceleration being measured at each frame-or, in other words, every 0.033 seconds. Therefore, a new technique is used wherein the moving average filter is included in obtaining the mentioned classifiers. This technique is explained in further detail in chapter 5 .

\footnotetext{
${ }^{18}$ For the results of using the average and maximum acceleration to classify tracked objects using the four models, see Tables B.1.5, B.1.6, C.1.5, C.1.6, D.1.5, D.1.6, E.1.5 and E.1.6, found in each respective Appendix.

${ }^{19}$ For the results of using the average and maximum deceleration to classify tracked objects using the four models, see Tables B.1.7, B.1.8, C.1.7, C.1.8, D.1.7, D.1.8, E.1.7 and E.1.8, found in each respective Appendix.

${ }^{20}$ This interpretation is based on Table 3.8
} 


\subsection{3d Tracked object direction}

The results of the average direction ${ }^{21}$, maximum direction ${ }^{22}$, average of change in direction $^{23}$ and average of cosine of change in direction ${ }^{24}$ classifiers were below expectations. The majority of successful configurations for the four models show only a slight agreement between manual and automated classification ${ }^{20}$. There was only one exception, and this was observed using the maximum direction classifier in the second model; in this case, there was a moderate degree of agreement between manual and automated classification ${ }^{20}$. On the one hand, this may indicate that the maximum direction classifier cannot be used to classify pedestrians, because pedestrians, as opposed to vehicles and cyclists, are able to move in different directions while navigating an intersection. On the other hand, the average direction classifier also did not yield good results for the vehicles-cyclists classification, and therefore, using this classifier to classify tracked objects seems generally risky. For some models, the entire sets of configurations using average direction and average of change in direction failed.

\subsection{3d Tracked object dimensions}

This section discusses results using the average and maximum area, length and width, classifiers. ${ }^{25}$ Using the dimensions of tracked objects to classify them have yielded acceptable

${ }^{21}$ For the results of using the average direction to classify tracked objects using the four models, see Tables B.1.9, C.1.9, D.1.9, and E.1.9, found in each respective Appendix.

${ }^{22}$ For the results of using the maximum direction to classify tracked objects using the four models, see Tables B.1.10, C.1.10, D.1.10, and E.1.10, found in each respective Appendix.

${ }^{23}$ For the results of using the average of change in direction to classify tracked objects using the four models, see Tables B.1.11, C.1.11, D.1.11, and E.1.11, found in each respective Appendix.

${ }^{24}$ For the results of using the average of cosine of change in direction to classify tracked objects using the four models, see Tables B.1.12, C.1.12, D.1.12, and E.1.12, found in each respective Appendix.

${ }^{25}$ For a full listing of results, please refer to Tables B.1.13 to B.1.18, C.1.13 to C.1.18, D.1.13 to D.1.18, and E.1.13 to E.1.18 in Appendices B, C, D, and E. 
results in the first, second and third models ${ }^{26}$. With the exception of the fourth model, the results varied between showing moderate to almost perfect agreement among manual and automated classification. Note that the results obtained from the second and third models were higher than the first model, which indicates that average and maximum dimensions this include the average and maximum area, and length and width cannot properly discriminate between cyclists and pedestrians $^{26}$. This is an acceptable argument: if we include limitations in the tracker system and the change in size of the bounding box, the size for both categories - cyclists and pedestriansare likely identical.

\subsection{3e Tracked object frequency}

\section{- Peaks in speed}

The results were generally below expectation, ${ }^{27}$ with the exception of the third model. The results ranged between fair and moderate agreement between manual and automated classification $^{20}$. Analyzing the successful configurations on a percentage scale reveals that peaks in speed provided the highest classification rate for vehicles for various four models.

\section{- The effective weighted average frequency}

One of the main contributions of this thesis is using the frequency of tracked objects to categorize them into one of the three categories (vehicles, cyclists, or pedestrians). the summary of the results as follow:

\footnotetext{
${ }^{26}$ The first model is designed to classify vehicles, cyclists and pedestrian. The second model is designed for vehicles-cyclists classification. The third model is designed for vehicles-pedestrians classification, the fourth model is designed for cyclists-pedestrians classification.

${ }^{27}$ The results of using peak speed to classify the tracked objects for the four models can be found in Tables B.1.19, C.1.19, D.1.19, and E.1.19 in Appendices B, C, D, and E. to view the results on percentage scale, please consult Tables B.2.19, C.2.19, D.2.19, and E.2.19.
} 
1. Using the effective weighted average frequency to classify tracked objects in the first and second models yielded acceptable results ${ }^{28}$. The interesting findings are as follows:

a) The longer the object appeared in the video sequence the higher the results are.

b) The successful configuration(s) only occurred with the triangular membership function set to $\delta=0.25$. This may indicates that the performance of this fuzzy membership function is better than that of other that is used in this framework.

c) Whether the frequency group was for the highest 5 powers or the highest 30 powers, the performances were the same: each produced Kappa coefficient fell within the 95\% confidence interval of the group of frequencies that yielded the highest Kappa coefficient.

d) The ranking of results based on Kappa coefficient interpretations in Table 3.8 shows that they range between fair and moderate agreement among manual and automated classification.

e) From observing the correct percentage value for each category in the first model, it was found that the highest classification rate was for the pedestrian category, followed by vehicles, and then cyclists. (Please see Table 4.8.)

f) From observing the correct percentage value for each category in the second model, it was found that the highest classification rate was for the cyclist category, followed by vehicles. (Please also see Table 4.8.)

2. Using the effective weighted average frequency to classify tracked objects in the third model yielded the best results ${ }^{29}$ among all models. This interesting finding is elaborated as the following:

\footnotetext{
${ }^{28}$ For the detailed results, please consult Tables B.1.20, C.1.20 in Appendices B, C.

${ }^{29}$ For the detailed results, please consult Tables D.1.20 in Appendices D.
} 
a) The longer the object appeared in the video sequence, the higher the results.

b) Successful configurations came from various fuzzy sets.

c) Whether the frequency group was for the highest 5 powers or the highest 30 powers, the performances were the same: each produced Kappa coefficient fell within the 95\% confidence interval of the group of frequencies that yielded the highest Kappa coefficient.

d) The ranking of results based on Kappa coefficient interpretations in Table 3.8 shows that they range between fair and substantial agreement among manual and automated classification $^{30}$.

e) By observing the correct percentage value for each category in the third model, it was found that the highest classification rate was for the pedestrian category, followed by vehicles. (Please also see Table 4.8)

3. Using the effective weighted average frequency to classify tracked objects in the fourth model yielded the lowest results ${ }^{31}$ among all models. The following points elaborate further:

a) The longer the object appeared in the video sequence, the higher the results.

b) Successful configuration(s) came from different types of fuzzy sets.

c) Whether the frequency group was for the highest 5 powers or the highest 30 powers, the performances were the same: each produced Kappa coefficient fell within the 95\% confidence interval of the group of frequencies that yielded the highest Kappa coefficient.

d) The ranking of results based on Kappa coefficient interpretations in Table 3.8 shows that they range between slight and fair agreement among manual observation and automated classification.

\footnotetext{
${ }^{30}$ For the detailed results, please consult Tables D.1.20 in Appendices D.

${ }^{31}$ For the detailed results, please consult Tables E.1.20 in Appendices E.
} 
e) Through observing the correct percentage value for each category in the fourth model, it was found that the highest classification rate was for the pedestrian category, followed by cyclists, and then vehicles.

Table 4. 8 The accuracy rate of classification using a percent correct value. The results are for the successful configurations only in the three validations.

\begin{tabular}{|c|c|c|c|c|c|c|}
\hline \multirow{2}{*}{ Model Number } & \multicolumn{2}{|c|}{ Vehicles } & \multicolumn{2}{c|}{ Cyclists } & \multicolumn{2}{c|}{ Pedestrians } \\
\cline { 2 - 7 } & Min & Max & Min & Max & Min & Max \\
\hline Model 1 & 34 & 75 & 9 & 30 & 89 & 100 \\
\hline Model 2 & 34 & 75 & 83 & 100 & - & - \\
\hline Model 3 & 41 & 78 & - & - & 89 & 100 \\
\hline Model 4 & - & - & 5 & 37 & 70 & 100 \\
\hline
\end{tabular}

\section{- The effective frequency}

Using the effective frequency to classify tracked objects in the four models produced results below expectation for the four models, when compared with the results for the effective weighted average frequency. ${ }^{32}$ The ranking ${ }^{20}$ of the majority of successful configurations for the four models shows a slight agreement between manual observation and the automated classification. In addition, a fair agreement between manual and the automated classification is observed in binary classification, if one of the categories is vehicles. Arriving at percent correct values for each category reveals that this classifier does categorize vehicles with an acceptable accuracy rate in binary classification (e.g., vehicles-cyclists).

\footnotetext{
${ }^{32}$ For the detailed results, please see Tables B.1.21, C.1.21, D.1.21 and E.1.21 in Appendices B, C, D, and E.
} 
Table 4.9 The accuracy rate of classification using a percent correct value. The results are for the successful configurations only in the three validations.

\begin{tabular}{|c|c|c|c|c|c|c|}
\hline \multirow{2}{*}{ Model Number } & \multicolumn{2}{|c|}{ Vehicles } & \multicolumn{2}{c|}{ Cyclists } & \multicolumn{2}{c|}{ Pedestrians } \\
\cline { 2 - 7 } & Min & Max & Min & Max & Min & Max \\
\hline Model 1 & 0 & 1 & 0 & 6 & 23 & 70 \\
\hline Model 2 & 62 & 96 & 0 & 24 & - & - \\
\hline Model 3 & 69 & 99 & - & - & 5 & 30 \\
\hline Model 4 & - & - & 0 & 93 & 0 & 52 \\
\hline
\end{tabular}




\section{Chapter 5: The Effect of Filtration}

\subsection{Overview}

Some of the extracted information from objects' tracks could not be used to classify tracked objects, due to the noise in the time series, such as the speed. The speed of a tracked object often changes due to multiple factors, like the behaviour of a driver, or the way in which traffic is being enforced through the intersection. Any change in speed of the tracked object may produce noise in the speed signal. As explained in Chapter Three (Development of a Framework for Object Classification) of this thesis, noise can also be caused when the tracking system fails to capture the object for a certain number of frames, this would lead to missing tracks for some frames. The substituted values for the missing frames are obtained using linear interpolation. It is acknowledged that linear interpolation is based on a simple assumption which may not be true. The tracking system has proven to produce an acceptable agreement between the actual speed of the tracked objects, and the video-measured speed (Kassim, 2014). The actual speed of the tracked object is the field measure speed. Therefore, this chapter is not questioning the accuracy of speed measurement. However, There is a concern that measuring the speed at each frame (which is approximately every 0.033 seconds), alongside the noise in the speed signal, may reflect on the results of other classifiers that mainly depend on speed. This would include average and maximum acceleration and declaration, as well as the effective frequency and the effective weighted average frequency. Therefore, this chapter asks: can better results be obtained when using the classifiers mentioned above by filtering the speed signal? 


\subsection{The Moving Average Filter}

Smoothing a noisy signal, such as speed profile in time, can be performed using mathematical filters commonly used in trend analysis and signal processing. Filters are tools that convert a time domain signal to another type of signal, and multiple kinds of filters are available. For the purposes of this research, a filter called the moving average filter was used. The moving average filter is a simple technique, and it works as follows: the value of speed at each frame in the output signal is produced by averaging a pre-assigned number of speed values from the input signal, as is shown below:

$$
y_{i}=\frac{1}{w} \sum_{k=0}^{w-1} x_{i+k}
$$

where: $x$ is the input signal

$y$ is the output signal

$w$ is the windows size

Assigning the number of speed values is called the window size. In this thesis, we are dealing with a number of tracked objects. Some of the tracked objects appeared in the video sequence for 60 frames, others for 300 frames. This variation made it impossible to assign an integer value as a window size without excluding any tracked objects that has the same number of frames as the assigned window size. Therefore, the window size is assigned as a percentage value of the duration of the tracked object's appearance in the video sequence. For example, if a tracked object appeared for ten frames, the window size is $20 \%$. This means that the window size is $20 \%$ of the ten frames, which is equal to 2 . In case the integer has decimals, the value is rounded down to the nearest lowest integer. For the purposes of this chapter, six window sizes were selected: $5 \%, 10 \%, 15 \%, 20 \%, 25 \%$ and $30 \%$. In order to distinguish between the output of 
the moving average filter and its input, all of these values were tested on the speed of the tracked objects. The output speed is called the moving average speed.

a)

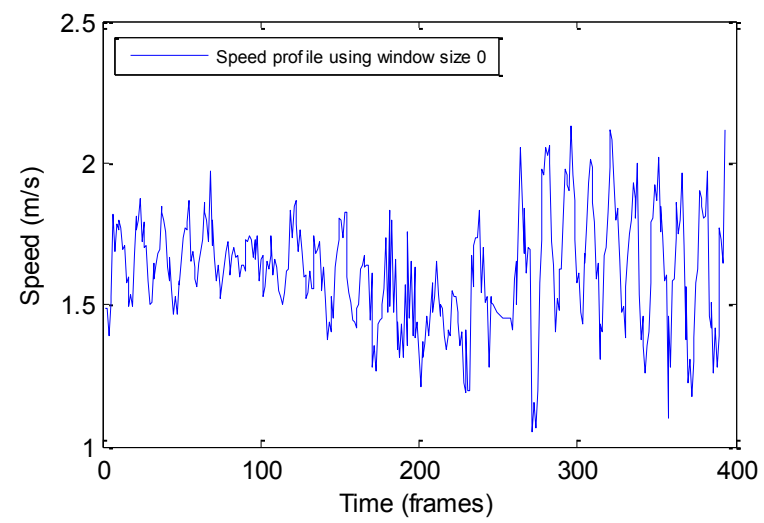

c)

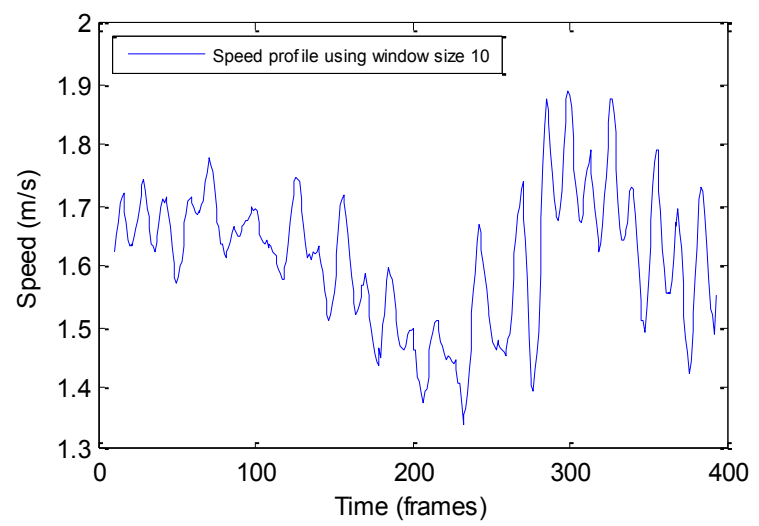

b)

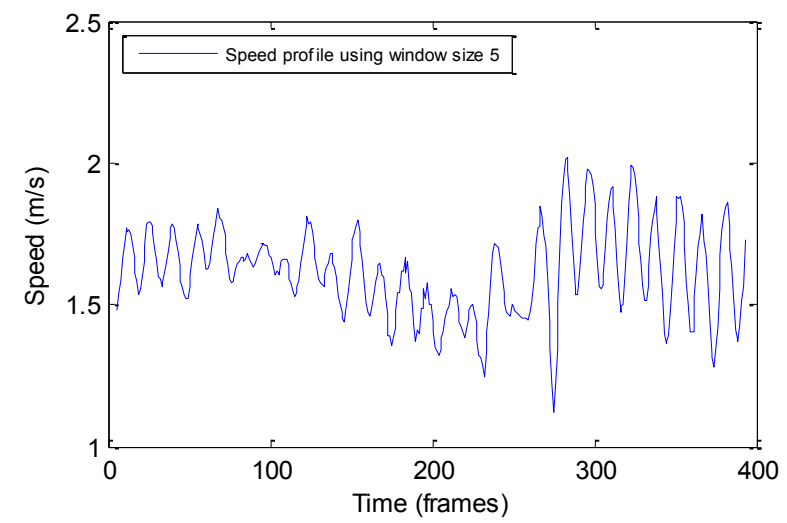

d)

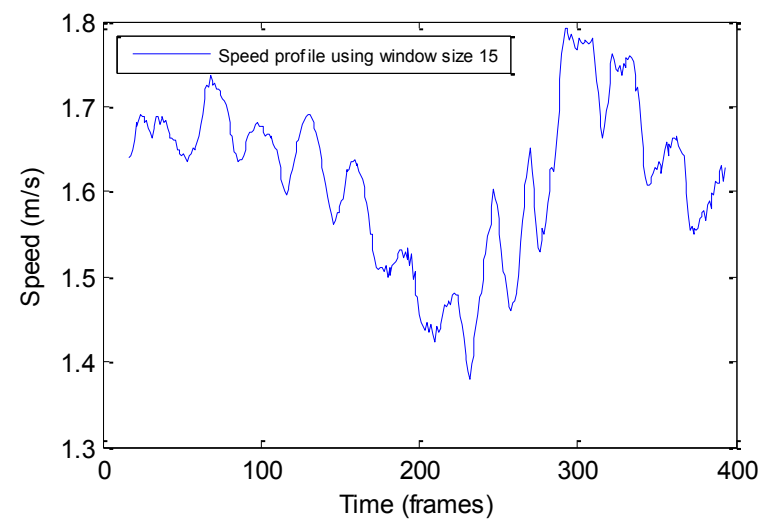

Figure 5. 1 (part 1) A speed profile of a pedestrian under different window sizes. The window sizes are represented as a percentage value. 
e)

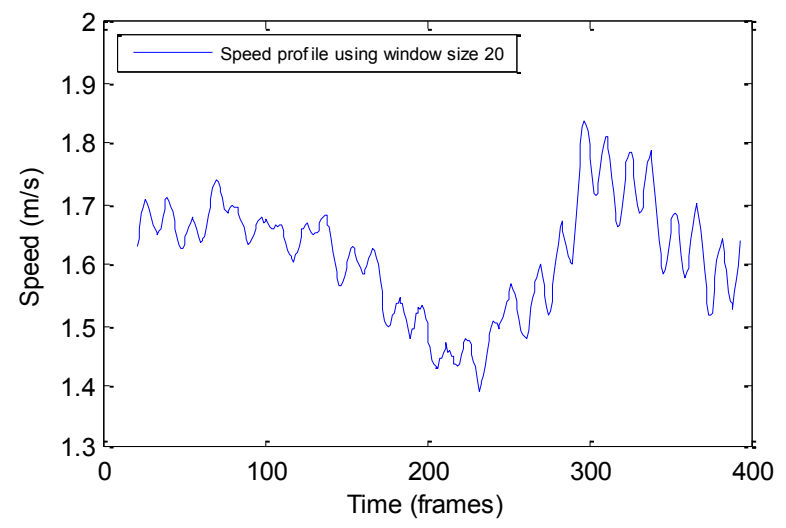

f)

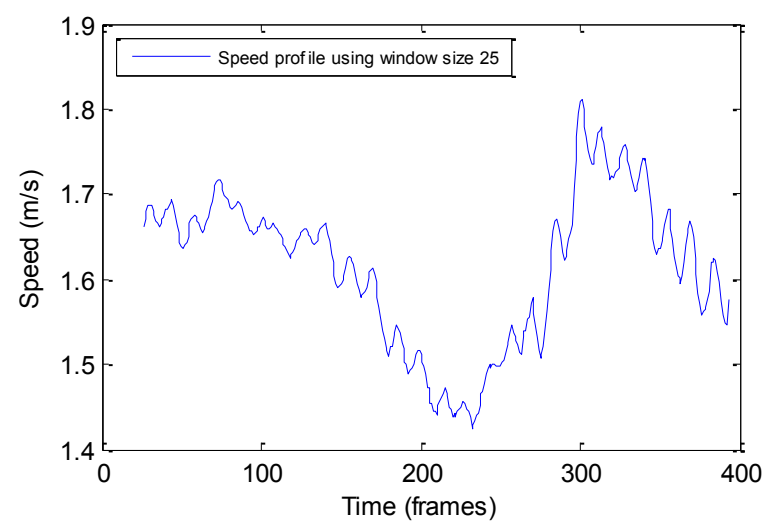

g)

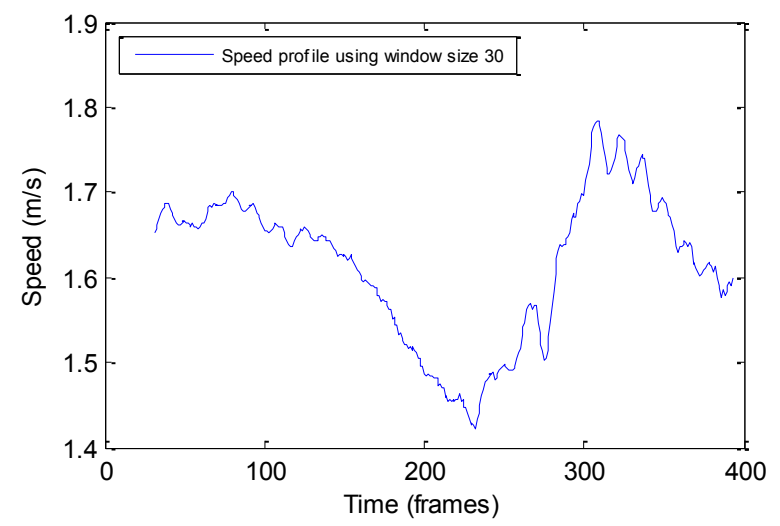

Figure 5.1 (part 2)

\subsection{Tracked Object Acceleration and Deceleration}

As is explained in Section 3.3.2, Acceleration (or conversely deceleration) of a tracked object is calculated by recording the change in speed between two consecutive frames. Using this approach, the acceleration and declaration of the tracked object are calculated by subtracting the final moving average speed from the initial moving average speed between successive frames. The initial speed of the tracked object is the speed at the (frame ${ }_{i}$ ) and the final speed of that object is the speed at the (frame $i+1)$. If the subtracted result is a positive value, i.e., the tracked 
object is going faster, then this means that the tracked object is accelerating. If the subtracted result is a negative value, i.e., the tracked object is slowing down, then this means that the tracked object is decelerating. The result in each frame is multiplied by the video frame rates to convert the units to $\left(\mathrm{m} / \mathrm{s}^{2}\right)$.

$$
A_{i}=\left(M S_{i, f_{j+1}}-M S_{i, f_{j+1}}\right) ; * f_{r} ; f \in\left\{n_{1}, n_{2}\right\}
$$

where: $A$ is the acceleration or deceleration in $\left(\mathrm{m} / \mathrm{s}^{2}\right)$ for object $i ; M S_{i, f, j+1}$ and $M S_{i, f, j}$ are the moving average speeds of object $i$ object in the successive frames.

The average acceleration for a tracked object is the sum of all the acceleration values of this object, divided by the total number of the acceleration for this object. The maximum acceleration of a tracked object is the highest acceleration value for this object within all the frames this object was tracked in.

The average deceleration for a tracked object is the sum of the absolute deceleration values for this object, divided by the total number of decelerations for this object. The maximum deceleration of a tracked object is the absolute highest deceleration value for this object within all the frames this object was tracked in.

\subsection{Tracked Object Frequency}

The new algorithm used to obtain tracked object's frequency is similar to the one explained in Section 3.3.5 of this thesis, albeit with one difference. The new algorithm is as follows:

1- The speed deviation is calculated for each frame by subtracting the speed of the tracked object in each frame from the moving average speed of that object. 
2- Perform a Fast Fourier transform (FFT) on the time series ( in this case it is the speed of the tracked objects). Let the FFT return the coefficient $\Omega$, the amount of $\Omega$ equal to the length of time series. The coefficient $\Omega$ is complex number which represents the amplitude and phase of the sinusoidal waves. a complex number consist of a real number and an imaginary numbers in the following format:

$$
C_{m}=R_{e}+I_{m} i
$$

where $: C_{m}$ represents the complex number, $R_{e}$ represent the real number and $I_{m}$ represents the imaginary number.

3- Calculate the power of a signal using the FFT coefficient $\Omega$ from step 1, as in the following:

$$
P_{w, h}=\left(\frac{\Omega_{h}}{s_{l} / 2}\right)^{2} ; h \in\left\{1, \ldots, s_{l} / 2\right\}
$$

where: $P_{w}$ is the power of a signal in Watt/Hertz $(\mathrm{W} / \mathrm{Hz})$;

$s_{l}$ Represent the length of the time series

4- Calculate the frequency for each power, as in:

$$
F=\frac{h * f_{r}}{s_{l}} ; h \in\left\{1, \ldots, s_{l} / 2\right\}
$$

where: $F$ is the frequency in hertz;

\subsection{Calibration Modifications}

The calibration and validation approaches used here employ the same procedure that was explained in Section 3.5, with some minor changes, as are put forth in what follows here.

1. The minimum duration of tracked object's appearance $\varphi_{g}$ used in the previous chapter was divided into five groups as shown in Table 3.5. For the purpose of this chapter, group 1 was 
excluded, because some of the tracked objects appeared for only few frames, and the tracks of such objects may not be accurate due to limitations of the tracker system.

2. Identifying the different configuration combinations. The process for identifying different configuration combinations was also changed slightly. Three type sets for configurations were built manually.

a) The first set was created to measure acceleration and deceleration, and resulted in ninetysix configurations. It was built using:

- The four groups of minimum duration of tracked object's appearance $\varphi_{g}$;

- The four types of fuzzy membership functions $\Lambda_{l}$;

- The six window sizes $w$.

b) The second configuration set was built to obtain the effective frequency. This set resulted in 288 configurations. It was built using:

- The four groups of minimum duration of tracked object's appearance $\varphi_{g}$;

- The four types of fuzzy membership functions $\Lambda_{l}$;

- The six window sizes $w$;

- The used frequencies $\eta_{m}$.

c) The third configuration set was built to measure the effective weighted frequency. This set resulted in 576 configurations. It was similarly built using:

- The four groups of minimum duration of tracked object's appearance $\varphi_{g}$;

- The four types of fuzzy membership functions $\Lambda_{l}$;

- The six window sizes $w$;

- The group of frequencies based on their power $\Gamma_{n}$. 
3. Obtain the initial limits for the fuzzy membership function. In order to start calibrating the configuration sets, the initial lower and upper limits for the fuzzy membership function under the different window sizes is needed. The same procedure used in Section 3.5.2 of this thesis was also employed here using the six window sizes. The initial values were obtained from the same data set used in calibration, and this set included 305 vehicles, 89 cyclists, and 58 pedestrians. Figures 5.2, 5.3 and 5.4 illustrates some samples of observed average acceleration for the vehicles, cyclists, and pedestrians under window size 30 .

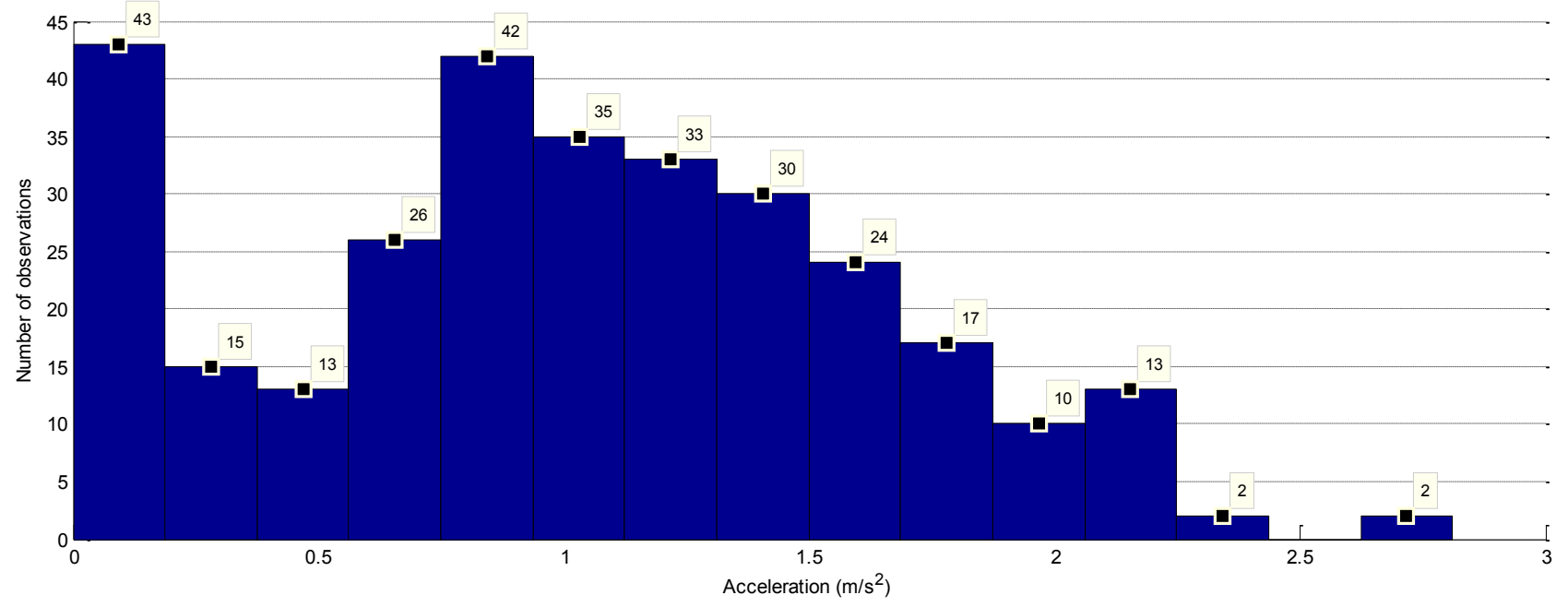

Figure 5. 2 The observed average acceleration histogram for vehicles. 


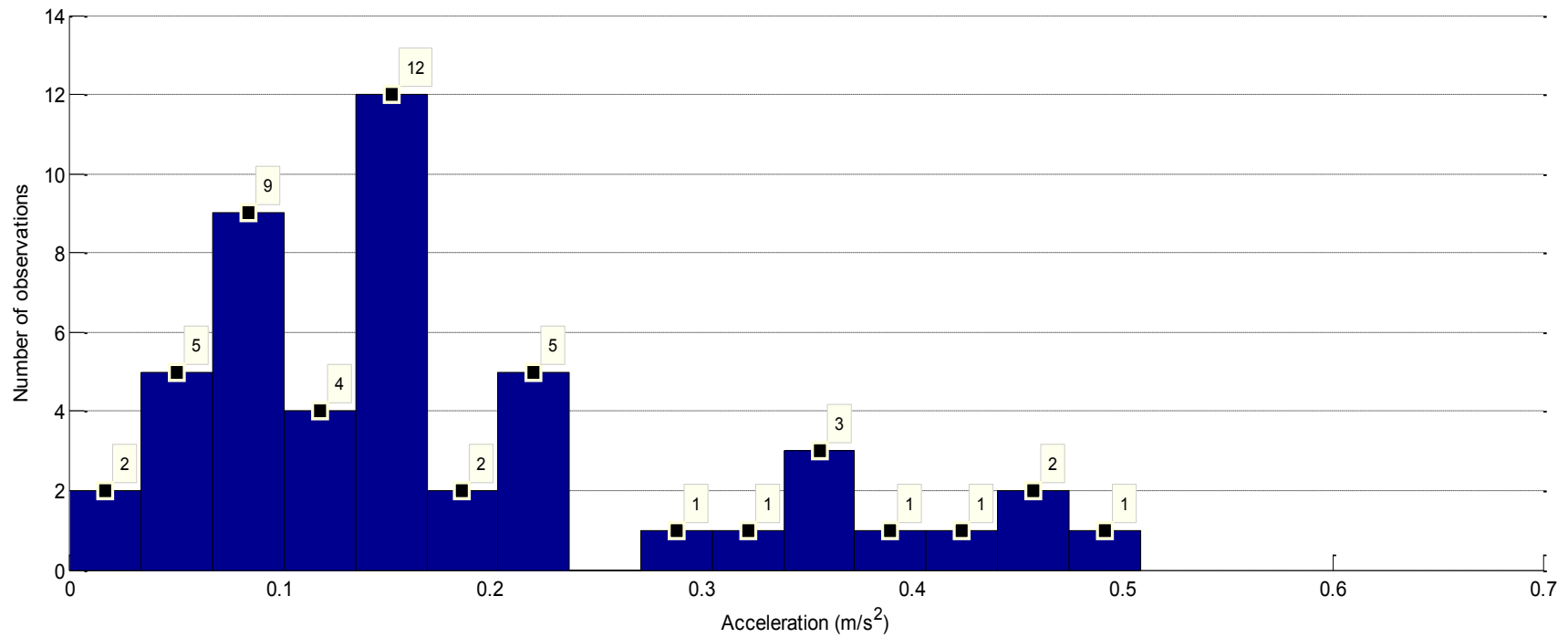

Figure 5. 3 The observed average acceleration histogram for cyclists.

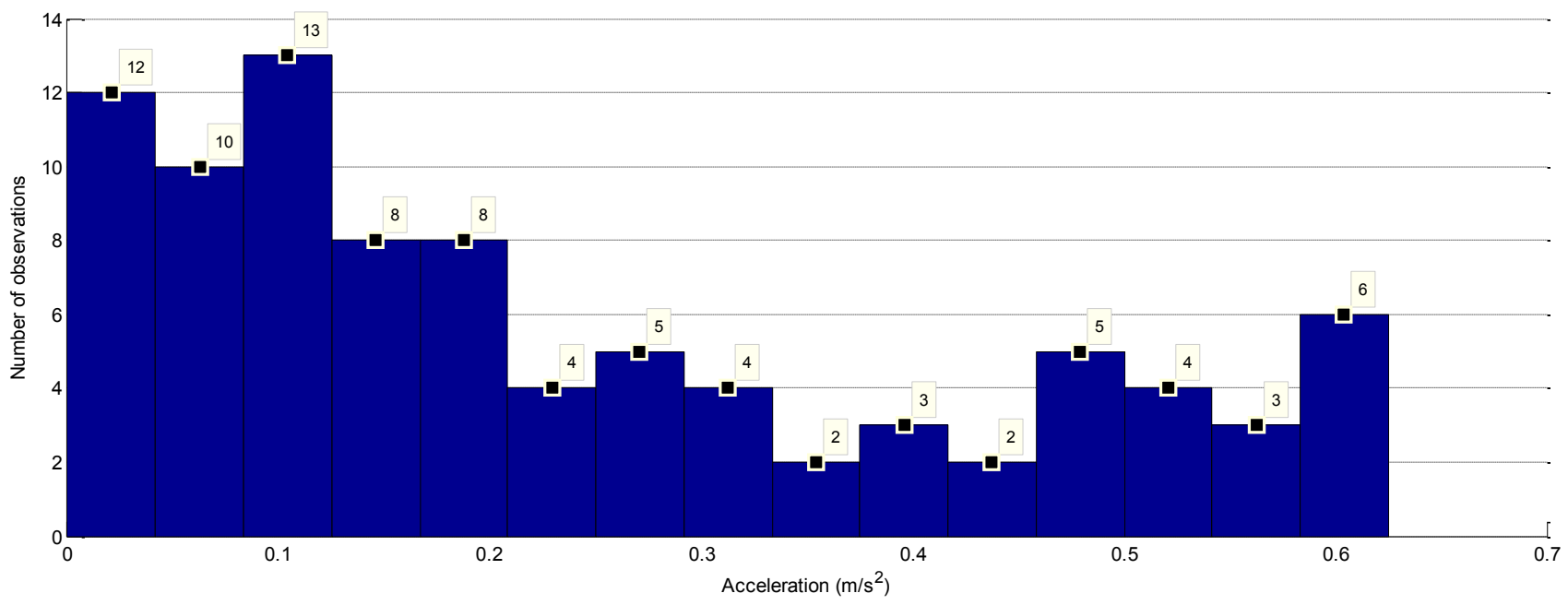

Figure 5. 4 The observed average acceleration histogram for pedestrians. 


\subsection{Results}

\subsubsection{Accelerations and declarations}

Classifying tracked objects based on their average and maximum accelerations did not yield better results as compared to the results found when using the approach described in section 4.4.3c of this thesis. This conclusion is based on the ranking of successful configurations $^{33}$ for the four models, a ranking that is based on Kappa coefficient measured for each successful configuration during validations of the four models. Based on Table 3.8, the results show only slight agreement between manual and automated classification. The same results were obtained using classifiers based on tracked objects' average and maximum decelerations. In this case, the results show only slight agreement between manual observation and automated classification.

\subsubsection{Effective weighted average frequency}

For the first model, the classification results demonstrate a fair agreement between manual observation and automated classification ${ }^{20}$. Classification accuracy increased alongside an increase in the duration of appearance. However, rankings for different groups according to duration of appearance fall within the same category as the Kappa coefficient interpretation illustrates in Table 3.8. There is no evidence suggesting that the type of fuzzy membership function created any improvement in the method of classification. Percentage correct values for the three categories (vehicles, cyclists, and pedestrians) using the first model show the highest classification accuracy for the pedestrian category, as is shown in Table 5.1.

\footnotetext{
${ }^{33}$ The definition of successful configuration is found in section 4.1.
} 
For the second and fourth models, results show only a slight agreement between manual and automated classification ${ }^{20}$. The accuracy of classification did not increase with the increase in the minimum duration of appearance. These results suggest no evidence that the type of fuzzy membership function improved the classification method, as successful configurations were variously created using different fuzzy types. Percentage correct values for the three categories using the second model demonstrate the highest classification accuracy for the cyclist category, as is shown in Table 5.1.

For the third model, results indicate fair agreement between manual and automated classification $^{20}$. The accuracy of classification did not increase alongside an increase in the duration of appearance. The results also fail to prove that the fuzzy membership function improves classification. Percentage correct values show the highest classification accuracy for the pedestrian category, as in Table 5.1

Table 5. 1 The accuracy rate of classification using a percentage correct value. The results are for the successful configurations only in the three validations.

\begin{tabular}{|c|c|c|c|c|c|c|}
\hline \multirow{2}{*}{ Model Number } & \multicolumn{2}{|c|}{ Vehicles } & \multicolumn{2}{c|}{ Cyclists } & \multicolumn{2}{c|}{ Pedestrians } \\
\cline { 2 - 7 } & Min & Max & Min & Max & Min & Max \\
\hline Model 1 & 25 & 61 & 5 & 24 & 62 & 100 \\
\hline Model 2 & 7 & 48 & 48 & 86 & - & - \\
\hline Model 3 & 31 & 64 & - & - & 88 & 100 \\
\hline Model 4 & - & - & 3 & 33 & 43 & 100 \\
\hline
\end{tabular}

\subsubsection{The effective frequency}

Using the effective frequency to classify tracked objects for the four models fell below expectations when compared with results for the effective weighted average frequency. The ranking of most successful configurations for the four models, based on Table 3.8, shows a slight agreement between manual and automated classification. There was, however, a fair agreement 
between manual and automated classification with the third model. Table 5.2 illustrated the percentage correct values of the classification accuracy for the four models.

Table 5. 2 The accuracy rate of classification using a percentage correct value. The results are for the successful configurations only in the three validations.

\begin{tabular}{|c|c|c|c|c|c|c|}
\hline \multirow{2}{*}{ Model Number } & \multicolumn{2}{|c|}{ Vehicles } & \multicolumn{2}{c|}{ Cyclists } & \multicolumn{2}{c|}{ Pedestrians } \\
\cline { 2 - 7 } & Min & Max & Min & Max & Min & Max \\
\hline Model 1 & 0 & 0 & 0 & 6 & 25 & 72 \\
\hline Model 2 & 51 & 100 & 0 & 53 & - & - \\
\hline Model 3 & 70 & 99 & - & - & 3 & 30 \\
\hline Model 4 & - & - & 0 & 5 & 25 & 100 \\
\hline
\end{tabular}




\section{Chapter 6: Summary and Conclusion}

\subsection{Research Summary}

Video monitoring of traffic is common practice in major cities. Cameras are often used to monitor traffic patterns, road use, and even traffic law violations. (Ismail et al., 2011). The data generated by video monitoring has practical uses such as traffic analysis for city planning. However, the usefulness of video monitoring of traffic is limited unless there is also a reliable way to automatically classify road users. Manually analysing traffic data to count road users would take an exorbitant number of man-hours and is inefficient in comparison with automated classification systems. As a result, there are a variety of different automated classification systems that have been designed which work in various ways to accurately construct the features of the various road users on the video sequence and thus to determine what category each road user falls into. This thesis presented a framework which attempted to use road users' motion cues to classify them. Overall, the performance of proposed classification approach found to be satisfactory based on its simplicity of application, transferability, and validation results.

The framework that is designed to classify three categories of tracked objects on the road captured using a high-resolution video camera $(1920 \times 1080$ at approximately 30 frames/second). The tracked objects are vehicles, cyclists and pedestrians. Tracking an object in a video sequence is the process of locating the position of the tracked objects in each video frame and extracting all relevant information of that object (Prakash et al., 2012). This can be done using tracking system such as computer vision techniques. From this sequence of positions, valuable information can be extracted regarding the road users such as speed, acceleration, the direction of movement, and variations thereof. The separate pieces of information are gained 
from these motion cues are hereafter called the Classifiers. There are nineteen classifiers, which included the average and maximum speed; the average and maximum acceleration; the average and maximum deceleration; the average and maximum direction; average of change in direction; the average of cosine of change in direction; the average and maximum area; the average and maximum length; the average and maximum width; Peaks in speed; the effective weighted average frequency; and the effective frequency.

A total of 898 vehicles, 535 cyclists, and 314 pedestrians were observed and used. The provided data were collected from two different intersections in the downtown area of Ottawa, data that was also collected for and employed in a study of road users authored by (Kassim, 2014). The first intersection, as previously mentioned, is located at Laurier Avenue West and Lyon Street in Ottawa, Ontario, Canada. Movements for a total of 639 vehicles, 204 cyclists, and 131 pedestrians were observed at this intersection. The corresponding data were divided approximately in half between the calibration and the first validation. The second intersection is located at Laurier Avenue West and Bay Street, also in Ottawa. Here, movements for a total of 259 vehicles, 149 cyclists, and 183 pedestrians were observed. This data were used for the second validation. A third validation combines data from the first and second validations. The provided data are constituted of objects' tracks, which include the positions of the vertices of the bounding box in world coordinates, as well as the sequence of average displacement of a tracked object in each frame.

After obtaining the classifiers' values for the tracked object, each value was assessed in order to discriminate between tracked objects. The term "classifiers values" refer to value of each classifier. Assessing a classifier was performed using fuzzy set theory. Assessing a classifier is the process of finding the Fuzzy value for the tracked object in each category based 
on that classifier. The higher the assessment value for a classifier in a category, the more likely the tracked object that associated with the assessed classifier truly belongs to that category.

Each category of tracked objects has some unique characterises that distinguish them from the other categories. For example, a tracked object traveling at a speed of $100 \mathrm{~km} / \mathrm{hr}$ is likely to be a vehicle. However, in some circumstances, two or more categories of tracked objects might share similar characterises, e.g., a vehicle is capable of traveling at the same speed as a cyclist or a pedestrian. This means there is an unavoidable level of uncertainty in classifying the tracked objects. Therefore, after obtaining the classifiers' values for the tracked objects, these values need to be assessed in order to utilize them to discriminate between the tracked objects. The term "classifiers values" refer to value of each classifier (e.g., average speed). It was described as $C v_{i, j}$ where it to the value of classifier $j$ for object $i$. The used approach for assessment is the Fuzzy Set theory. Assessing a classifier of a tracked object is the process of finding the Fuzzy value for the tracked object in each category based on that classifier. The higher the assessment value for a classifier in a category, the more likely the tracked object that associated with the assessed classifier truly belongs to that category.

After building a classification algorithm, it was calibrated in order to provide maximum accuracy in classifying different tracked objects. Calibration is the process of finding the calibrated limits of the fuzzy membership function for a specific configuration. In the case of multiple configurations, calibration should be able to identify the best configuration(s). The configuration consists of four elements. The elements divided into: $[i]$ essential elements, and [ii] optional elements. The essential elements are mandatory for any classification. The essential elements consist of: [i] The duration of the tracked objects appearance in the video sequence and [ii] The type of the fuzzy membership function. The optional elements are only required if the 
effective frequency and the effective weighted average frequency are used for classification. The classification system needs to know which frequency or group of frequencies shall use for classification.

Two procedures were created for calibration. The first procedure will be called calibration procedure 1 , it is used to obtain the calibrated limits for the fuzzy membership in two cases: $[i]$ the calibration is being performed on one classifier only; or [ii] the calibration is being performed on more than one classifier, regardless of whether the new classifiers provide a significant improvement to the results or not. The second procedure will be called calibration procedure 2 , it is used to obtain the calibrated limits for the fuzzy membership in two cases: $[i]$ the calibration is being performed on more than one classifier; and [ii] the required results are to keep the effective classifiers only. The effective classifiers are those that significantly improve the classification process. This calibration was performed for four different models; the first model is designed to classify tracked objects into one of the three main categories examined in this study (vehicles, cyclists, or pedestrians). Tracked objects from the three categories mentioned above were used to test this model. The second model was designed to classify the tracked objects into vehicles or cyclists only. Therefore, vehicle and cyclists tracks only were used to test this model. The third model was designed to classify tracked objects into vehicles and pedestrians only. Vehicle and pedestrian tracks only were used to test this model. The fourth model was designed to classify tracked objects into cyclists and pedestrians only, and cyclist and pedestrian tracks were used to test this model. The calibration results from both procedures 1and 2 were validated. The validation was done using three sets of data which resulted in 3 validations. 
The results of the four models show that the minimum duration of tracked object appearance has limited effect on the classification process of the tracked object. The results of the four models also show that the performance of different fuzzy membership functions was almost the same for successful configurations within the group organized according to duration of appearance. The effect of different classifier was notice in the validation results. There were some superior classifiers, such as area, length, width, and average and maximum speeds. Likewise, there were some classifiers that did not improve the results, such as the average and maximum acceleration and deceleration. Different results are obtained depending on the type model or the selected classifiers which all listed in appendices B, C, D and E.

Some results gleaned from objects' tracks could be misleading, because signals in a time domain, such as speed, may contain a lot of trend components. There was a concern that measuring the speed at each frame (which is approximately every 0.033 seconds), alongside the varied nature of trend components, may reflect on the results of other classifiers that mainly depend on speed. This would include average and maximum acceleration and declaration, as well as the effective frequency and the effective weighted average frequency. Therefore, a simple filter called the moving average filter was used. The moving average filter is a simple technique, and it works as follows: the value of speed at each frame in the output signal is produced by averaging a pre-assigned number of speed values from the input signal. Using this approach for tracked objects classification did not provide any improvement to the classification process. 


\subsection{Recommendations}

As mentioned above, the results of this research were overall quite satisfactory, although improvements to the process of classification employed can also be made in future research that aims to classify road users in heavily trafficked urban areas. Based on the finding of this research as well as in which the process detailed throughout this thesis could be improved upon, the following are recommended to future research directions:

1. Improve the tracking system especially when the partial tracks occur, as this framework is highly affected by tracking quality.

2. Include more data from different intersections and follow the same validation approach in order to obtain the best configuration that yields the highest classification accuracy.

3. Improve the tracked object's classification based on their frequency, this include using signal processing and signal filtration technique to extract the frequency of the tracked objects.

4. The effect of harmonic frequencies on the classification process was not considered in this thesis, this could be a possible limitation when classifying road users based on their frequency. Harmonic frequencies are "[component] frequencies that are integer multiples of the fundamental power frequency" (Ingale, 2014). Harmonic frequencies could occur due to limitation in the tracking system, or in case of partial tracks. Therefore, it is recommended to measure the total harmonic distortion (THD). THD measure the amount of the harmonic frequencies compared to the fundamental frequency, it is usually described as percentage values. Once the THD value is obtained, it is recommended to divide the road users into subclasses based on their THD value, classify road users in each subclass, and compare the classification accuracy of different subclasses to the classification accuracy of subclass with 
lowest THD value (preferably to the subclass that has THD value of $0 \%$ ). This gives the ability to identify at which THD value the harmonic frequencies reduce the classification accuracy.

5. Obtain longer tracking durations for each tracked object. Longer tracking duration provide better understanding to the road user behavior. This can be done by: [i] using tracking GPS, or [ii] by installing multiple cameras to cover an entire road, then connect the tracks of the road users captured in all the cameras.

6. Develop an algorithm for binary- classification of tracked objects as vehicles-non vehicles, cyclists-non cyclists and pedestrians-non pedestrians.

7. Develop a technique that capable to categorize road users of the same category (e.g., motorcycles, busses and heavy tracks).

8. Obtain tracks of road users on the road, not from intersections. Road users may slow down at intersections which could affect the classification results. 


\section{References}

1. World Health Organization, 2013. Global status report on road safety. Available at: http://www.who.int/violence injury_prevention/road_safety_status/2013/report/en/

2. World Health Organization, 2009. Global status report on road safety. Available at: http://whqlibdoc.who.int/publications/2009/9789241563840 eng.pdf?ua=1

3. Transport Canada, 2011. Road Safety in Canada. Available at: https://www.tc.gc.ca/eng/motorvehiclesafety/tp-tp15145-1201.htm.

4. National Highway Traffic Safety Administration, 2011. Traffic safety facts 2011. DOT HS 811754

5. K. Ismail, T. Sayed, M. Zaki, and F. Alrukaibi, 2011. Automated detection of spatial traffic violations through use of video sensors. Transportation research record, pp. 8798.

6. K. Ismail, T. Sayed, and N. Saunier, 2010. Automated analysis of pedestrian-vehicle conflicts. Transportation research record, pp. 52-64.

7. N. Saunier, and T. Sayed, 2006. A feature-based tracking algorithm for vehicle in intersections. IEEE, in proceedings of the 3rd Canadian Conference of Computer and Robot Vision, CRV 2006, pp. 59

8. C. Curio, J. Edelbrunner, T. Kalinke, C. Tzomakas, and W. Seelen, 2000. Walking pedestrian recognition. IEEE Transactions on intelligent transportation systems, vol. 1, no. 3 , pp. $155-163$.

9. M. Bertozzi, A. Broggi, and A. Fascioli, 1998. An extension to the inverse perspective mapping to handle non-flat roads. IEEE Intelligent Vehicles Symp, pp. 305-310. 
10. D. P. Huttenlocher, J. J. Noh, and W. J. Rucklidge, 1993. Tracking non-rigid objects in complex scenes. IEEE, in proceedings of the 4th international Conference of Computer Vision. pp. 93-101.

11. R. E. Kalman, 1960. A new approach to linear filtering and prediction problems. Trans. ASME-J. Basic Eng., vol. 82, no. 1, pp. 35-45.

12. Y. ran, R. Chellappa, and Q. Zheng, 2006. Finding gait in space and time. IEEE, in Proceedings - International Conference of Pattern Recognition, vol 4, 2006, pp. 586-589

13. M. Zaki, and T. Sayed, 2014. Using automated walking gait analysis for the identification of pedestrian attributes. Transportation Research Part C 48 (2014). pp. 16-36.

14. C. Pai, H. Tyan, Y. Liang, H. Liao, and S. Chen, 2003. Pedestrian detection and tracking at crossroads. IEEE, in Proceedings - International Conference of image processing, 2003. vol. 3, pp. 14-17.

15. B. Lucas, and T. Kanade, 1981. An Iterative Image Registration Technique with an Application to Stereo Vision. IJCAI, vol .130, pp. 121-130.

16. D. Beymer, P. McLauchlan, B. Coifman, and J. Malik, 1997. A real-time computer vision system for measuring traffic parameters. IEEE, in proceedings of the 1997 Conference of Computer Vision and Pattern Recognition (CVPR. '97), pp. 495-501.

17. Vlachos, M., G. Kollios, and D. Gunopulos, 2005. Elastic translation invariant matching of trajectories. Machine Learning, vol. 58, 2005, pp. 301-334.

18. M. Zaki, and T. Sayed, 2013 A framework for automated road-users classification using movement trajectories. Transportation Research Part C 33 (2013). pp. 50-73. 
19. A. Golyandina, 2005. Automatic extraction and forecast of time series cyclic components within the framework of SSA. IEEE, In Proceedings of the 5th workshop on simulation, pp. $45-50$.

20. M. Zaki, T. Sayed, and Greg Mori, 2013. Classifying road users in urban scenes using movement patterns. The journal of computing in civil engineering, vol. 27, no. 4. pp. $395-406$.

21. G. H. Golub, and C. F. Van Loan, 1996. Matrix computations, Johns Hopkins University Press, Baltimore, MD.

22. S. Messelodi, C. Modena and M. Zanin, 2005. A computer vision system for the detection and classification of vehicles at urban road intersections. Pattern Anal Applic (2005), vol. 8, pp. 17-31.

23. N. Buch J. Orwell S.A. Velastin, 2010. Urban road user detection and classification using 3D wire frame models. IET Computer Vision, vol. 4, no. 2, pp. 105-116.

24. B. Morris, and M. Trivedi, 2006. Robust classification and tracking of vehicles in traffic video streams. IEEE, In Proceedings of the intelligent transportation systems conference (2006), pp. 1078-1083.

25. S. Gupte, O. Masoud, R. Martin, and N. Papanikolopoulos, 2002. Detection and classification of vehicles. IEEE Transactions on intelligent transportation systems, vol. 1, no. 3. pp. 37-47.

26. J. Hsieh, S. Yu, Y. Chen, and W. Hu, 2006. Automatic traffic surveillance system for vehicle tracking and classification. IEEE Transactions on intelligent transportation systems, vol. 7, no. 2. pp. 175-187. 
27. N. Dalal and B. Triggs, 2005. Histograms of oriented gradients for human detection. IEEE, Proceedings - 2005 IEEE Computer Society Conference on Computer Vision and Pattern Recognition, CVPR 2005. vol. 1 article number 1467360, pages 886-893.

28. B. Bilgic, BK. Horn, and I. Masaki, 2010. Efficient integral image computation on the GPU. Intelligent Vehicles Symposium, vol. 1, pp. 528-533.

29. P. Viola, and MJ. Jones, 2001. Rapid object detection using a boosted cascade of simple features. IEEE Computer Society Conference on Computer Vision and Pattern Recognition, vol. 1, pp. 511-518.

30. B. Schölkopf, A.J. Smola, 2002. Learning with Kernels. MIT Press, Cambridge, MA.

31. Y. Xu, L Xu, D. Li, and Y. Wu, 2011. Pedestrian detection using background subtraction assisted Support Vector Machine. IEEE, Intelligent Systems Design and Applications (ISDA), pp. $837-842$.

32. Z. Chen, T. Ellis, 2013. Efficient annotation of video for vehicle type classification. IEEE, Intelligent Transportation Systems - (ITSC), pp. 59-64.

33. K. Yang, E. Du, E. Delp, P. Jiang, F. Jiang, Y. Chen, R. Sherony, and H. Takahashi, 2013. An extreme learning machine-based pedestrian detection method. Intelligent Vehicles Symposium (IV), 2013 IEEE. pp. 1404 - 1409.

34. S. Nigam, K. Deb, A. Khare, 2013. Moment invariants based object recognition for different pose and appearances in real scenes. IEEE, In 2013 International Conference of Informatics, Electronics \& Vision (ICIEV), pp. $1-5$.

35. G. Somasundaram, R. Sivalingam, V. Morellas, N. Papanikolopoulos., 2013. Classification and counting of composite objects in traffic scenes sing global and local 
image analysis. Intelligent Transportation Systems, IEEE Transactions on 2013. vol.14, no.1, pp. 69-81.

36. G. Hong, B. Kim, Y. Hwang, K. Kwon, 2015. Multimedia Tools and Applications.

37. P. Govardhan, U.C. Pati., 2014. NIR image based pedestrian detection in night vision with cascade classification and validation. IEEE, 2014 international conference of advanced Communication Control and Computing Technologies (ICACCCT), pp. 14351438.

38. J. Hariyono, V. Hoang, and K. Jo, 2014. Location classification of detected pedestrian. IEEE, 2014 14th International Conference of Control, Automation and Systems (ICCAS), pp. 599-602.

39. L. Ng, S. Suandi, and S. Teoh, 2014. Vehicle classification using visual background extractor and multi-class Support Vector Machines. Springer, the 8th international conference on robotic, vision, signal processing \& power applications. pp. 221-226.

40. G. Ballesteros, L. Salgado, 2014. Optimized HOG for on-road video based vehicle verification. 2014 Proceedings of the 22nd European Signal Processing Conference (EUSIPCO), pp. 805-809.

41. C. Liang, and C. Juang, 2014. Moving object classification using local shape and HOG features inwavelet-transformed space with hierarchical SVM classifiers. Applied Soft Computing 28 (2015), pp. 483-497.

42. J.R.R. Uijlings, I.C. Duta, N. Rostamzadeh, and N. Sebe. 2014. Realtime Video Classification using Dense HOF/HOG.

43. G. Santoshi, and S.R. Mishra, 2015. Pedestrian with Direction Detection Using the Combination of Decision Tree Learning and SVM. Springer International Publishing 
Switzerland 2015 S.C. Satapathy et al. (eds.), Emerging ICT for Bridging the Future vol 1, Advances in Intelligent Systems and Computing 337.

44. S. Messelodi, C. Modena, and G. Cattoni. 2007. Vision-based bicycle/motorcycle classification. ScienceDirect, Pattern Recognition Letters 28 (2007), pp. 1719-1726.

45. L. Chen, R. Feris, Yun Zhai, L. Brown, 2008. IEEE Fifth International Conference of Advanced Video and Signal Based Surveillance, 2008. AVSS '08. pp. 52 - 59.

46. Z. Zhang, M. Li, K. Huang, T. Tan, 2008. IEEE, In 19th International Conference of Pattern Recognition, 2008. ICPR 2008. pp. 1-4.

47. D. G. Lowe, 2004. Distinctive image features from scale invariant key points. IJCV, 2004.D. G.S.

48. S. Lazebnik, C. Schmid, and J. Ponce, 2005. A sparse texture representation using local affine regions. IEEE Transactions on PAMI, vol. 7, no. 8, pp. 1265-1278.

49. T. Takahashi, K. HyungKwan, S. Kamijo, 2012. Urban road user classification framework using local feature descriptors and HMM. IEEE, 15th International IEEE Conference of Intelligent Transportation Systems (ITSC), 2012. pp. 67-72.

50. S. Kamijo, Y. Matsushita, and K. Ikeuchi, 2000. Occlusion robust tracking utilizing spatio-temporal markov random field model. IEEE, in Proceedings. 15th International Conference of Pattern Recognition, 2000, vol. 1. pp. 140-144

51. Q. Du, 2007. Modified Fisher's Linear Discriminant Analysis for Hyperspectral Imagery . IEEE, Geoscience and Remote Sensing Letters, IEEE, volume.4, no. 4, pp. 503-507.

52. Q. Du, and N.H. Younan, 2008. On the performance improvement for linear discriminant analysis-based hyperspectral image classification. IEEE, Pattern Recognition in Remote Sensing (PRRS 2008), pp. 1-4. 
53. L.E. Baum and T. Petrie, 1966. statistical inference for probabilistic functions of finite state Markov chains. Ann.Math.Stat. vol. 37, pp.1554-1563,

54. A. Møgelmose, A. Prioletti, M. Trivedi, A. Broggi, and T. Moeslund, 2012. Two-stage Part-Based Pedestrian Detection. IEEE, 15th International IEEE Conference of Intelligent Transportation Systems (ITSC), pp. 73-77.

55. W. Cheng, and D. MaoJhan, 2012. A self-constructing cascade classifier with AdaBoost and SVM for pedestrian detection. Engineering Applications of Artificial Intelligence 26 (2013), pp. 1016-1028.

56. O. Prakash, M. Khare, C. Sharma, A. Kushwaha, 2012. Moving object tracking in video sequences based on energy of daubechies complex wavelet transforms. International Journal of Computer Applications (IJCA). pp. 6-10.

57. A. Kassim, 2014. Innovative techniques for analyzing cyclist behaviour and predicting cyclist safety. PhD. thesis, Carleton University.

58. P. Wendykier, 2013. The FFT package used in this thesis. It is available at http://incanter.org/docs/parallelcolt/api/edu/emory/mathcs/jtransforms/fft/packagesummary.html.

59. M. Dhar, 2012. On Geometrical Representation of Fuzzy Numbers. International Journal of Energy, Information and Communications vol. 3, no 2, pp. 29-34.

60. A.Viera, and J. Garrett, 2005. Understanding Interobserver Agreement: The Kappa Statistic. Family Medicine. pp. 360-363.

61. H. T. Chen, H. H. Lin, and T. L. Liu, 2001. Multi-object tracking using dynamical graph matching. IEEE Computer Society Conference on Computer Vision and Pattern Recognition, vol. 2, pp. 210- 217. 
62. Rajesh Ingale, 2014. Harmonic Analysis Using FFT and STFT. International Journal of Signal Processing, Image Processing and Pattern Recognition. vol 7, no.4 pp. 345-362. 


\section{Appendix A}

Figures show the performance of different classifiers for a selected configuration. 
A.1 Histogram of different classifiers for the tracked obje cts sample used in calibrations

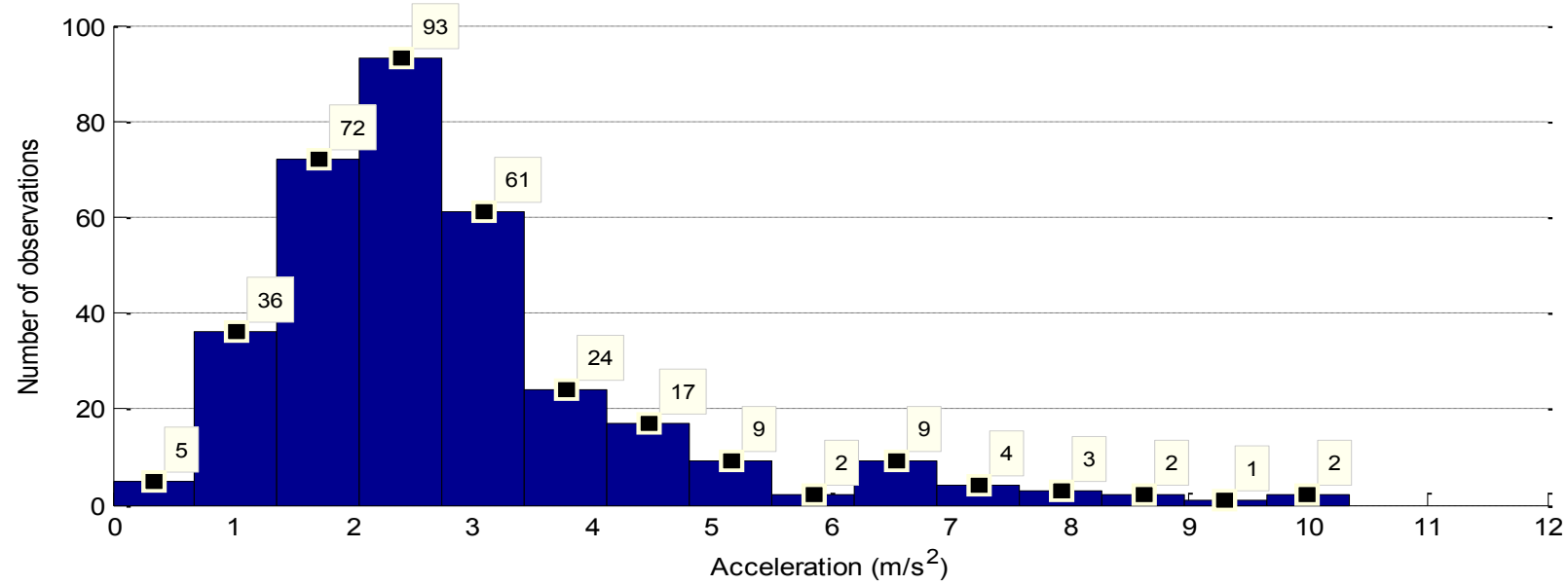

Fig.A.1.1 observed average acceleration histogram for vehicles

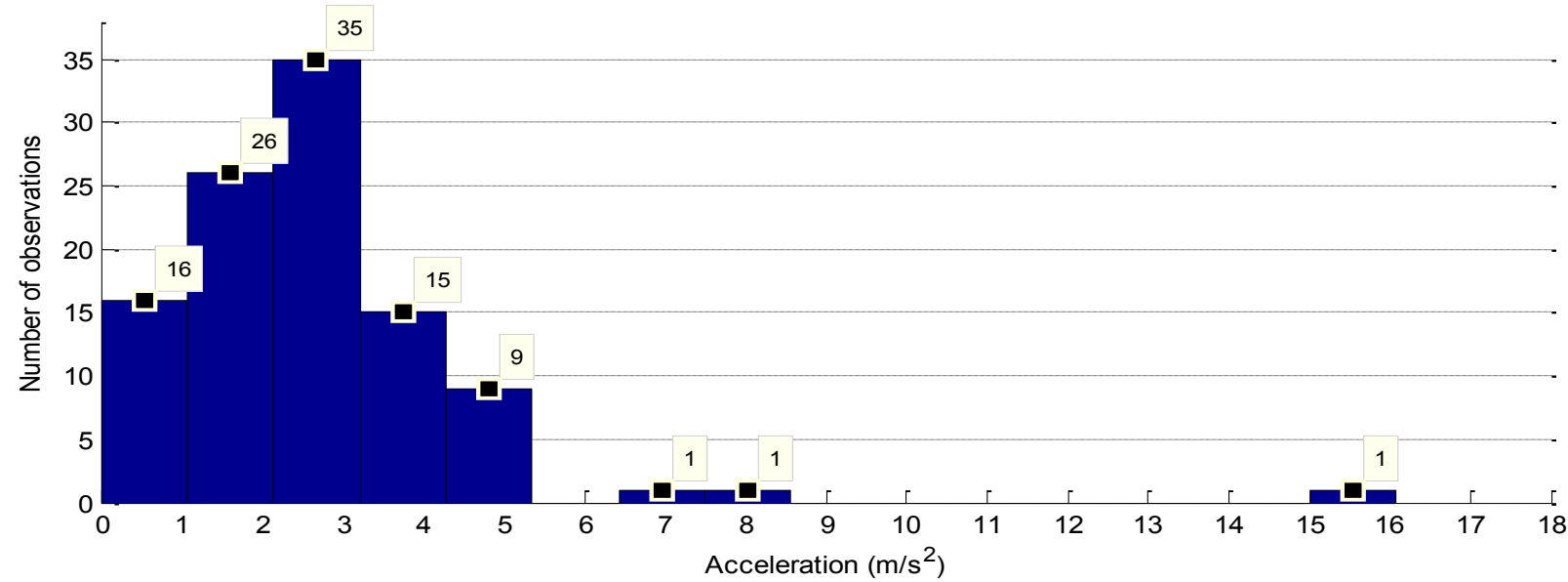

Fig.A.1.2 observed average acceleration histogram for cyclists

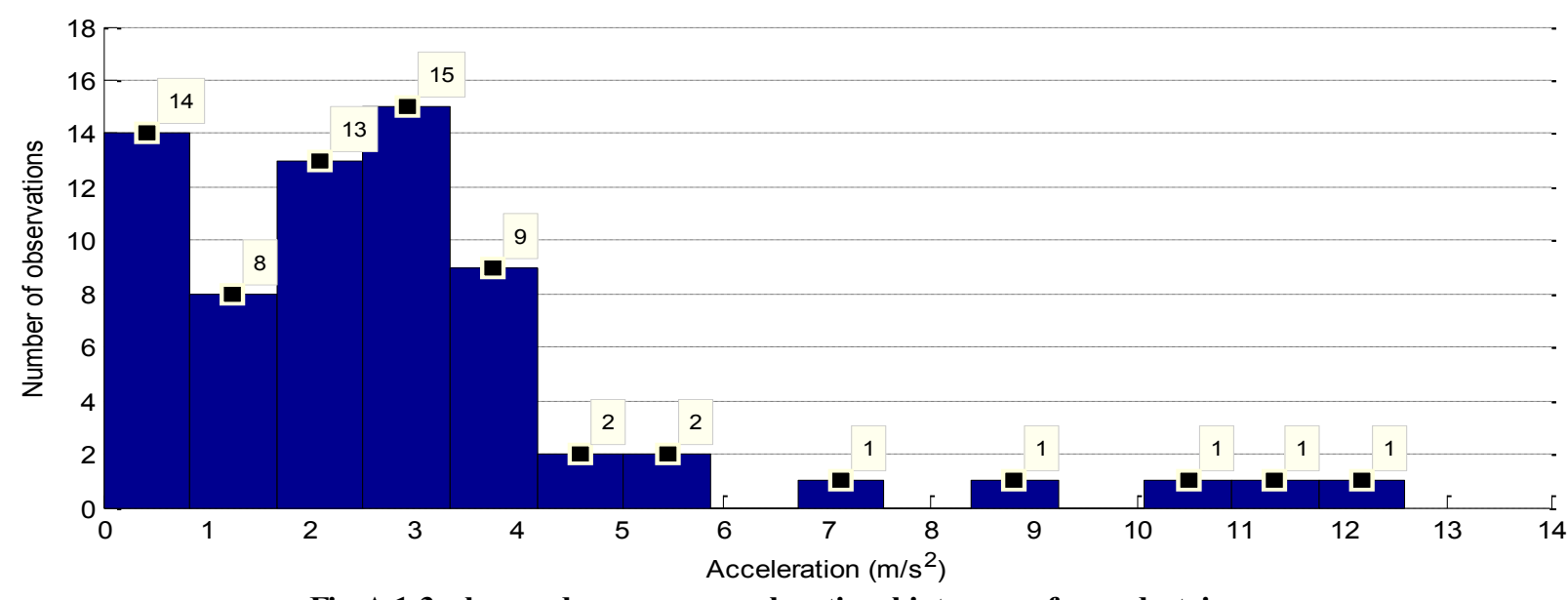

Fig.A.1.3 observed average acceleration histogram for pedestrians 


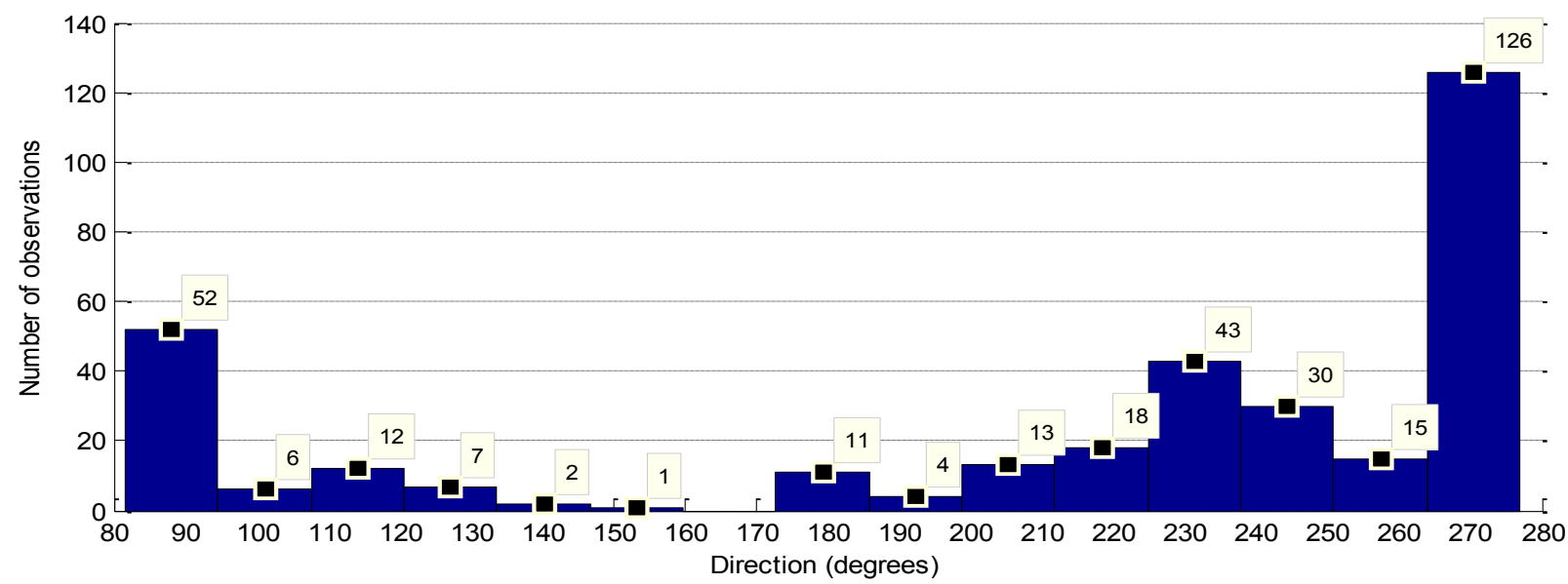

Fig.A.1.4 observed average direction histogram for vehicles

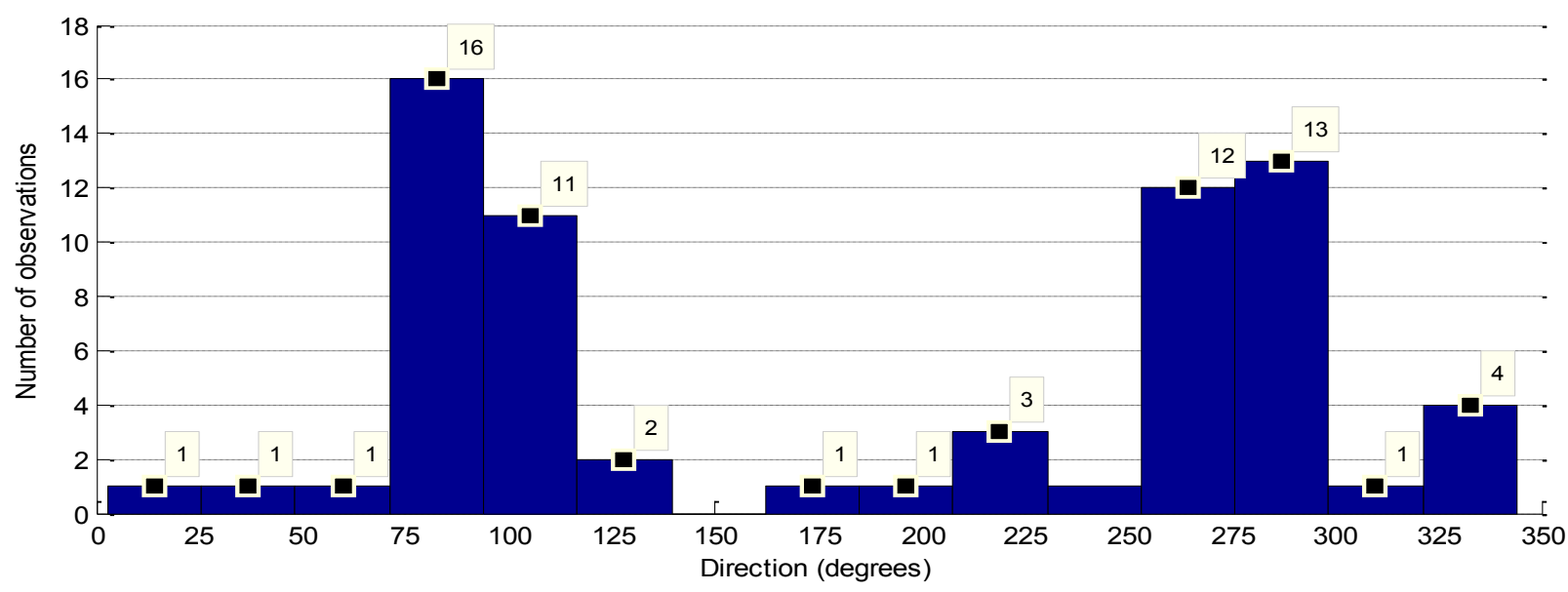

Fig.A.1.5 observed average direction histogram for cyclists

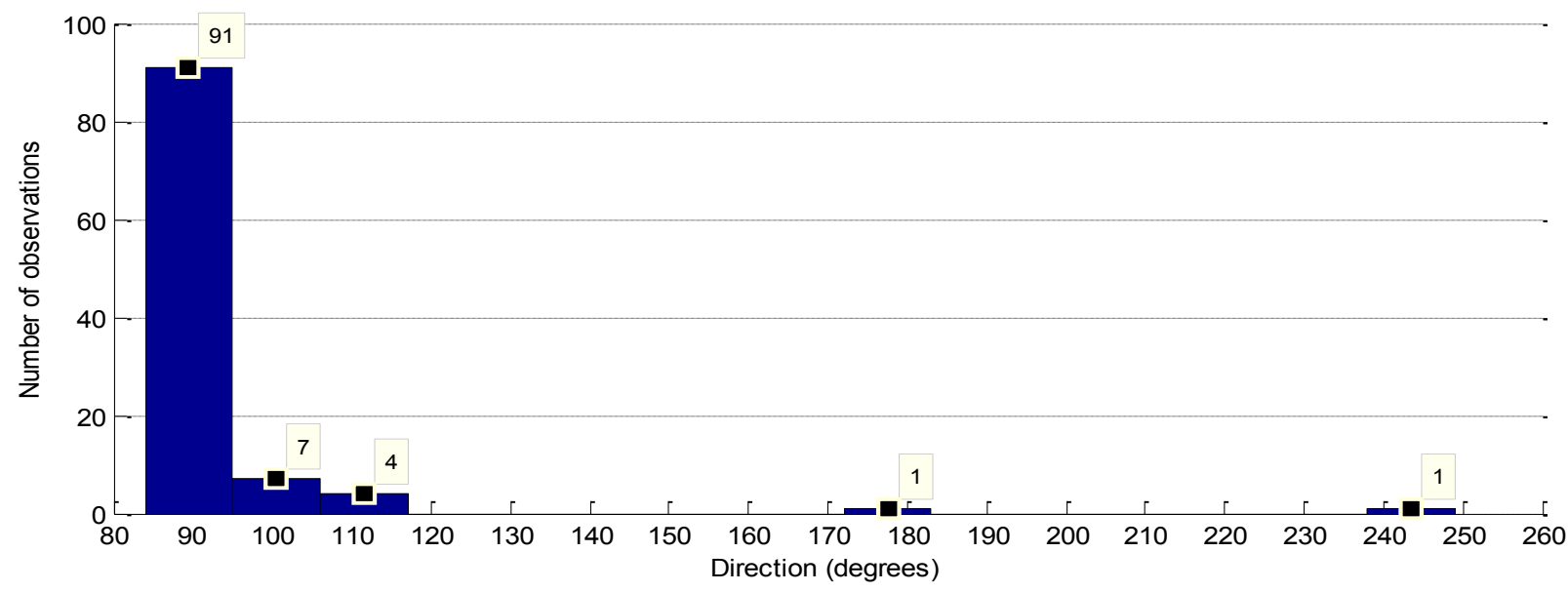

Fig.A.1.6 observed average direction histogram for pedestrians 


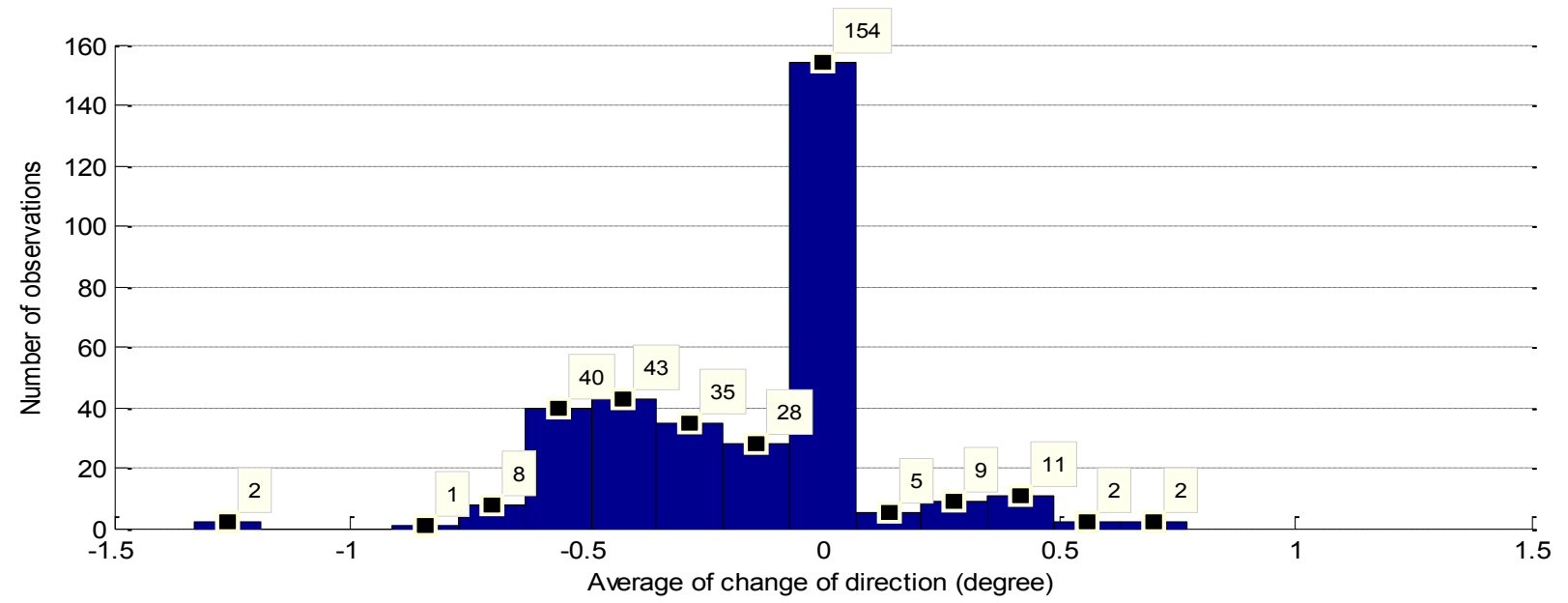

Fig.A.1.7 observed average of change in direction histogram for vehicles

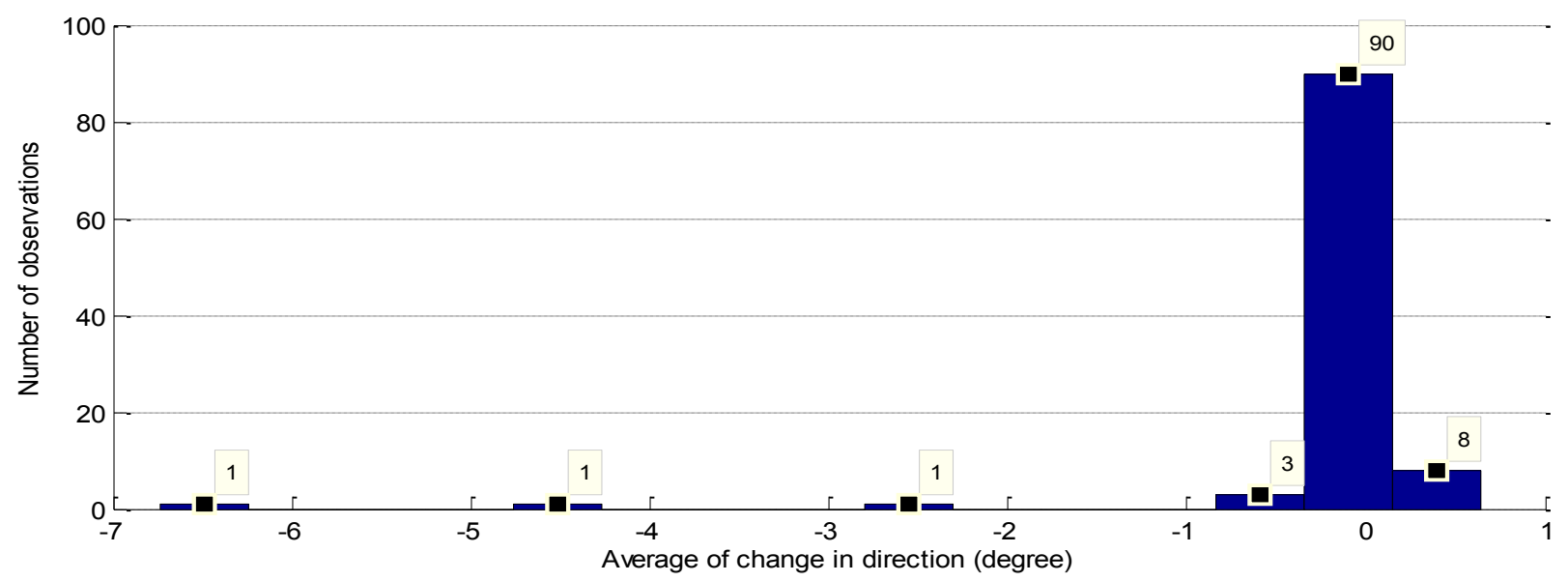

Fig.A.1.8 observed average of change in direction histogram for cyclists

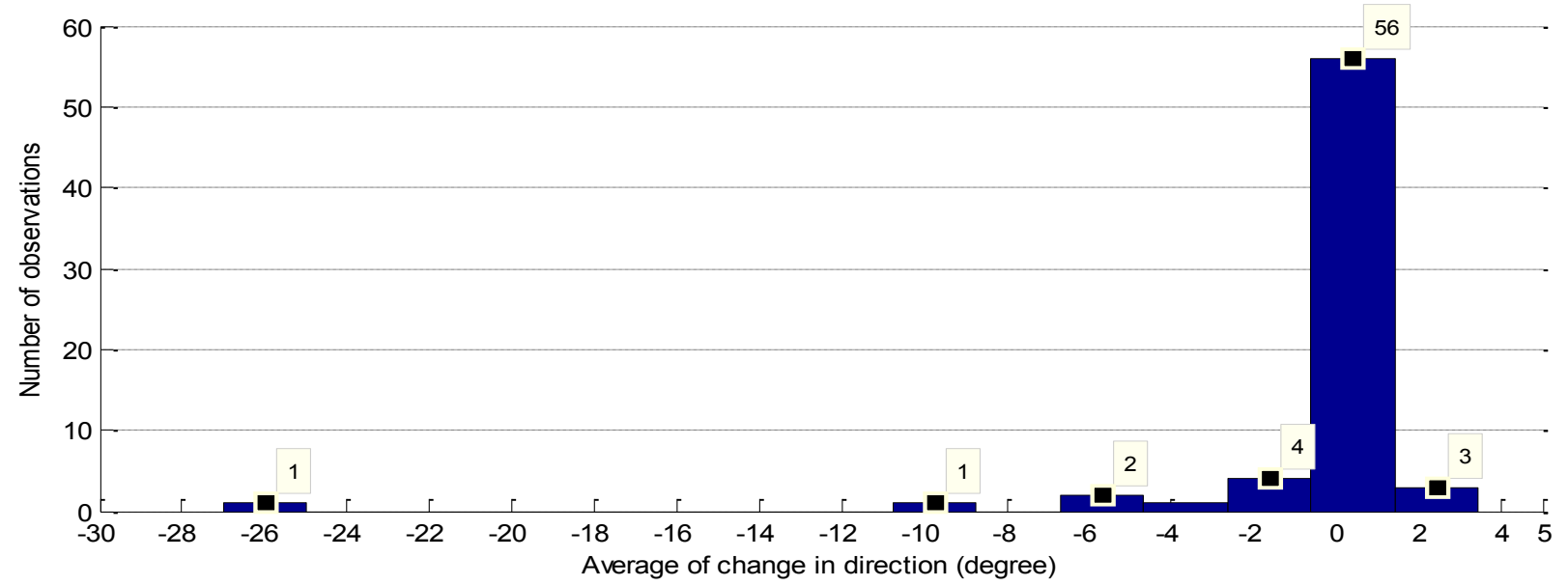

Fig.A.1.9 observed average of change in direction histogram for pedestrians 


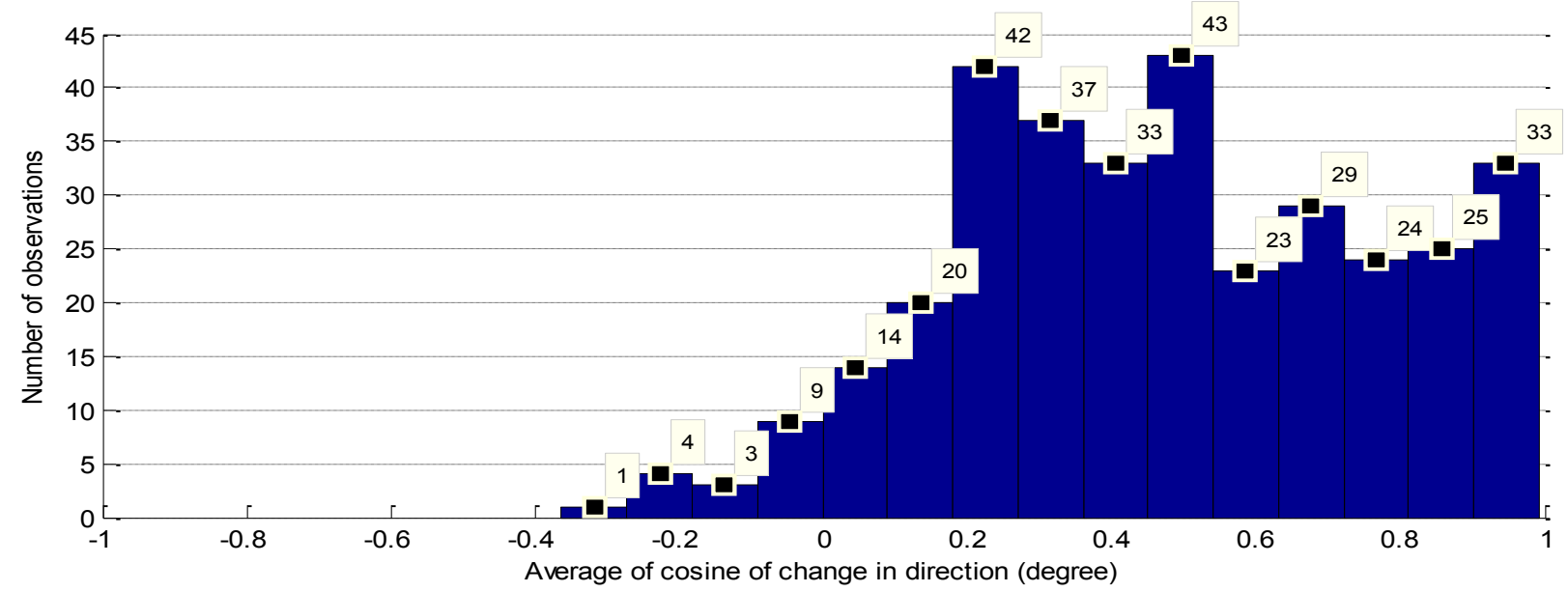

Fig.A.1.10 observed average of cosine of change in direction histogram for vehicles

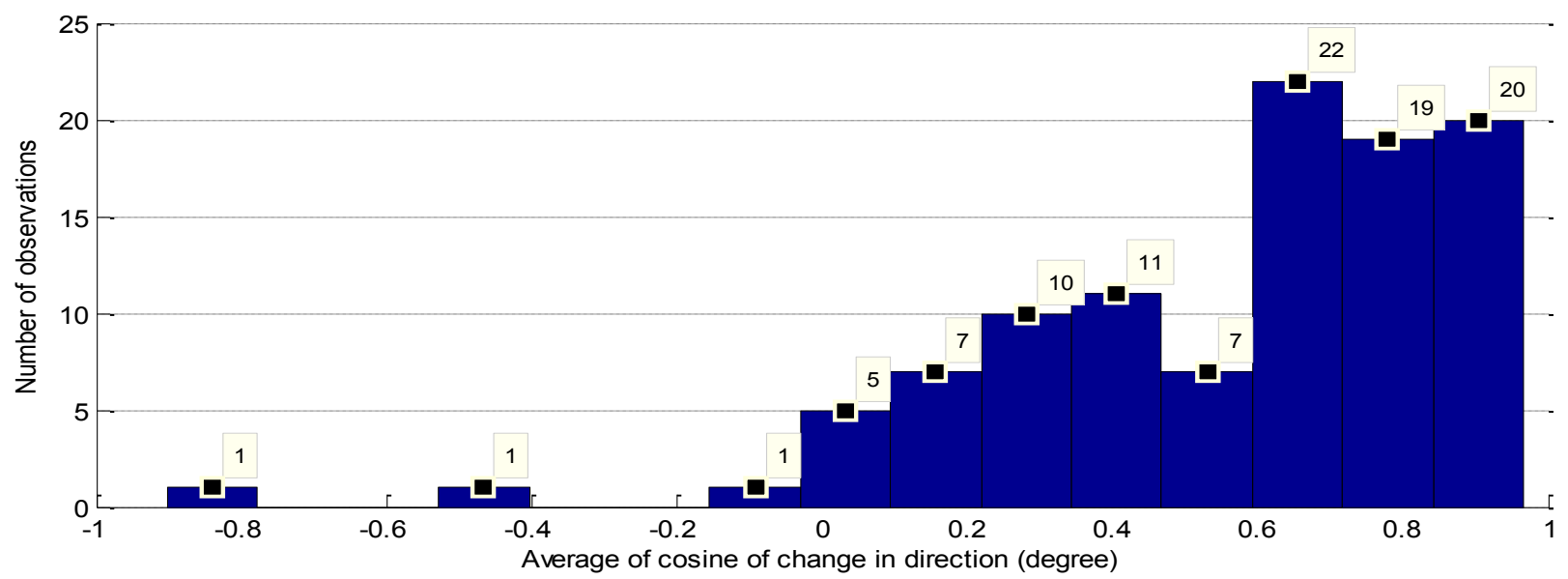

Fig.A.1.11 observed average of cosine of change in direction histogram for cyclists

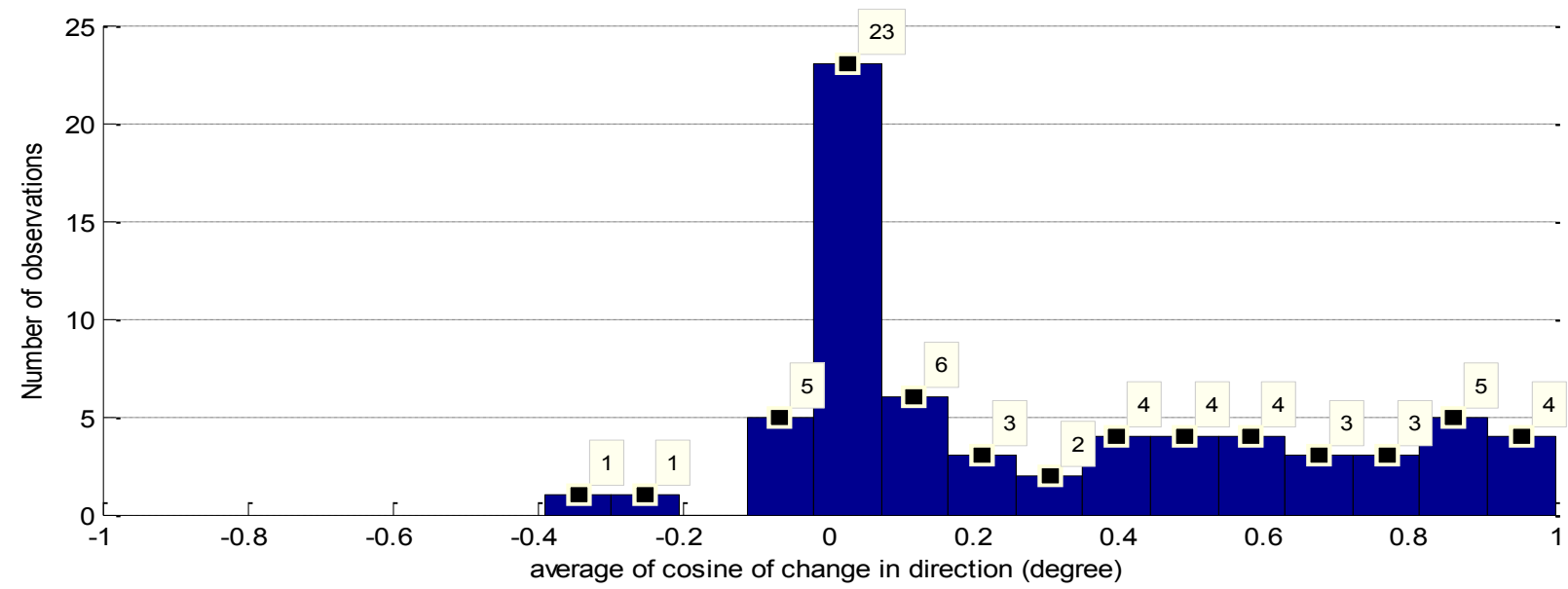

Fig.A.1.12 observed average of cosine of change in direction histogram for pedestrians 


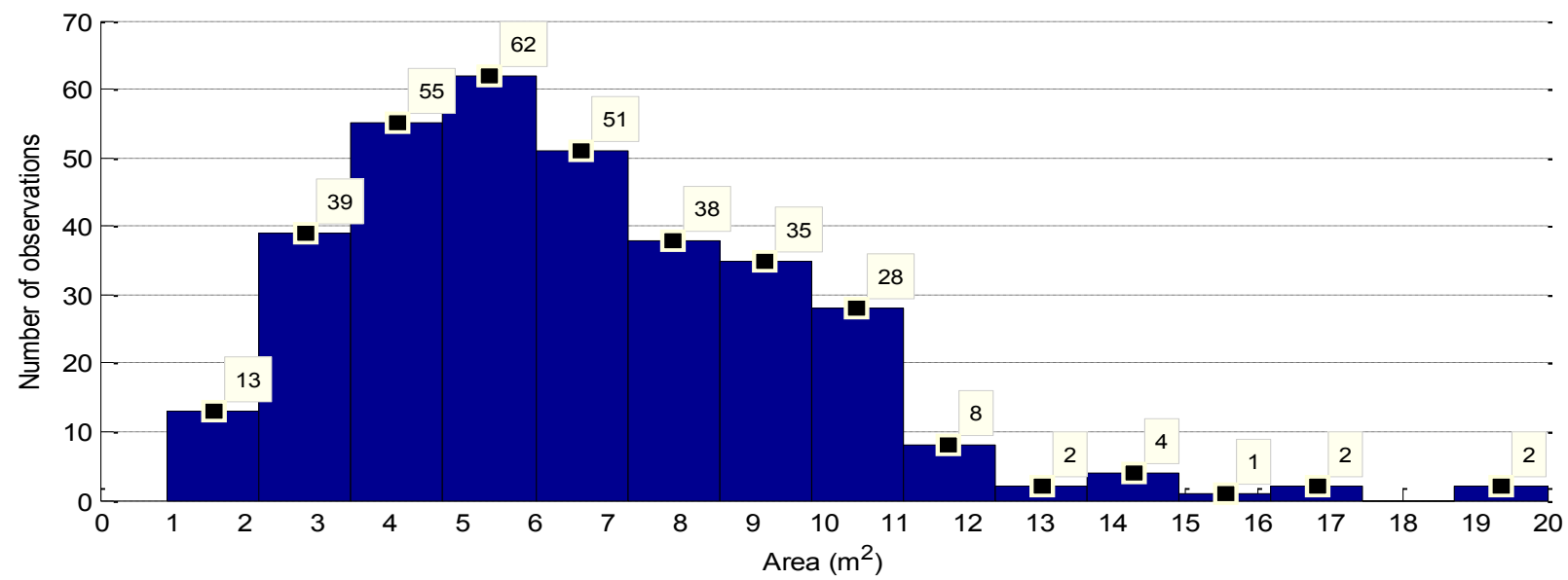

Fig.A.1.13 observed average area histogram for vehicles

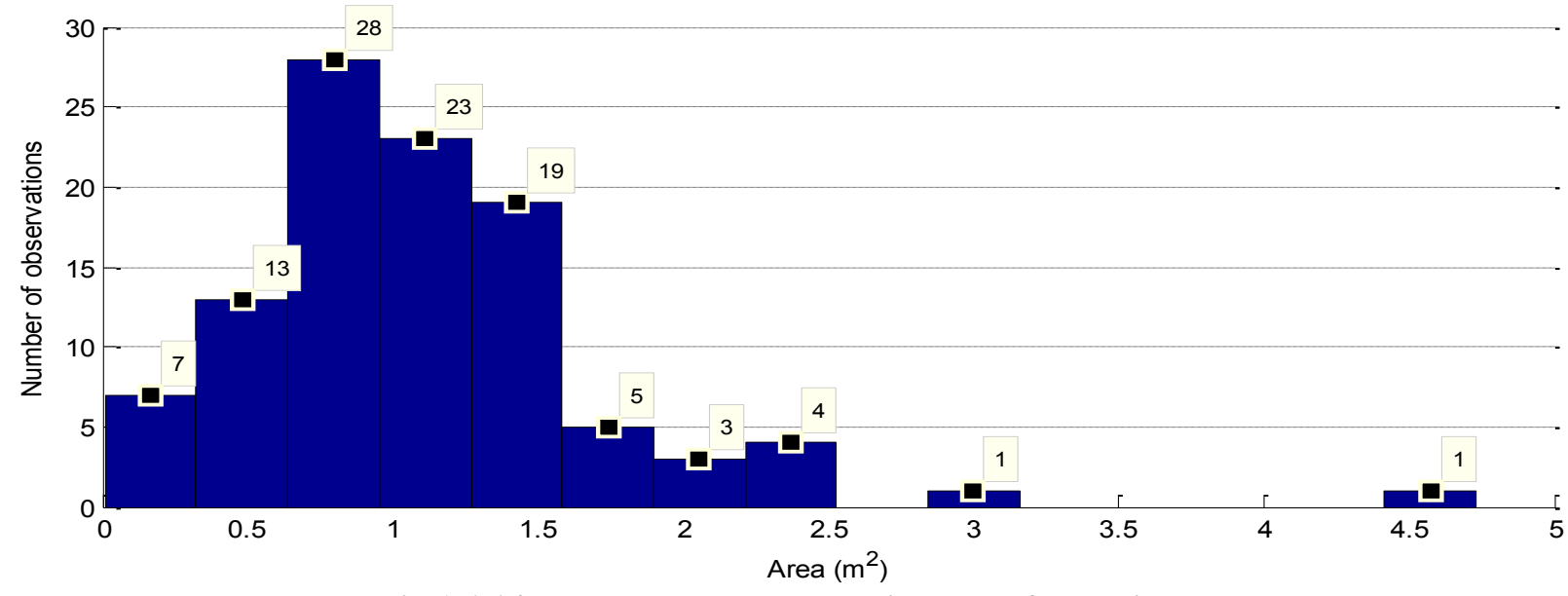

Fig.A.1.14 observed average area histogram for cyclists

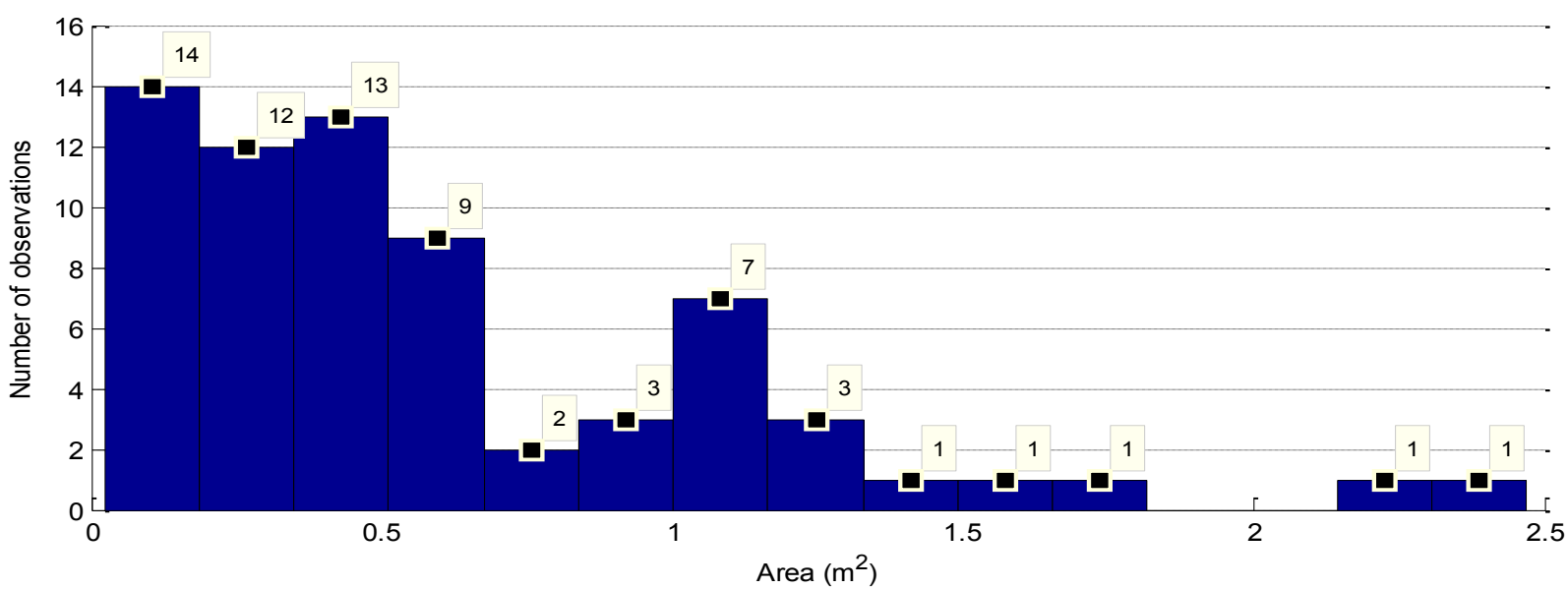

Fig.A.1.15 observed average area histogram for pedestrians 


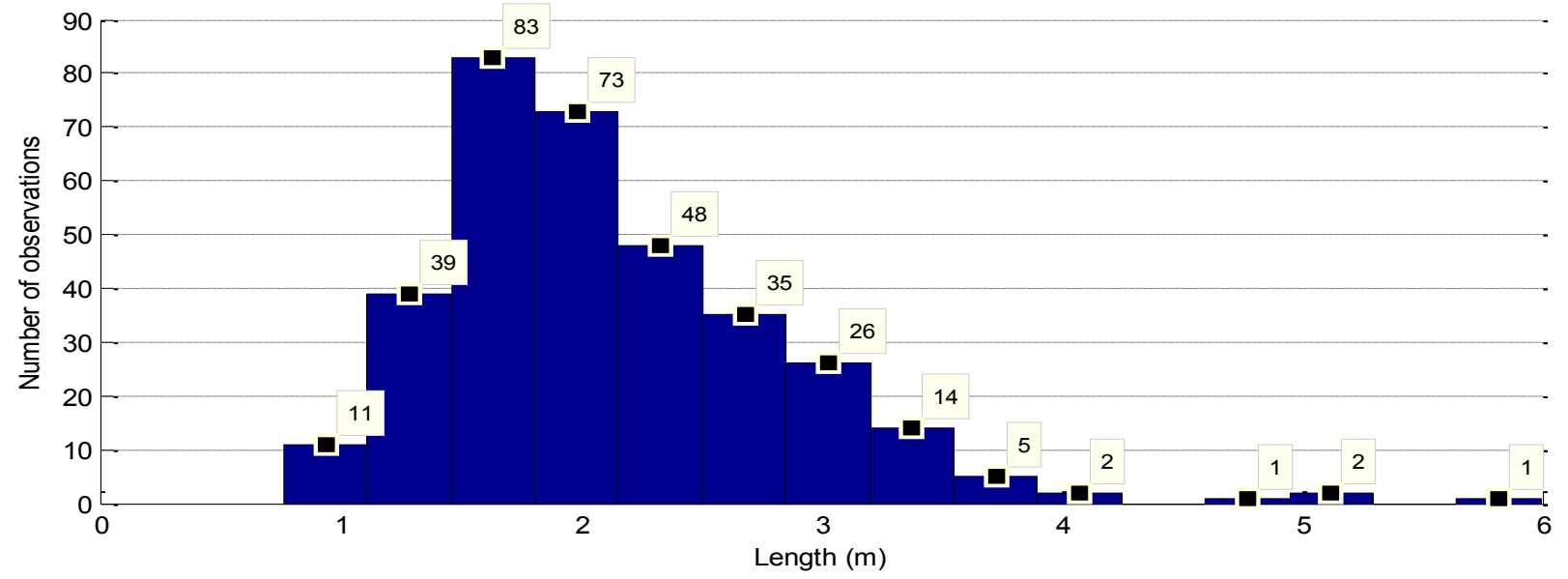

Fig.A.1.16 observed average length histogram for vehicles

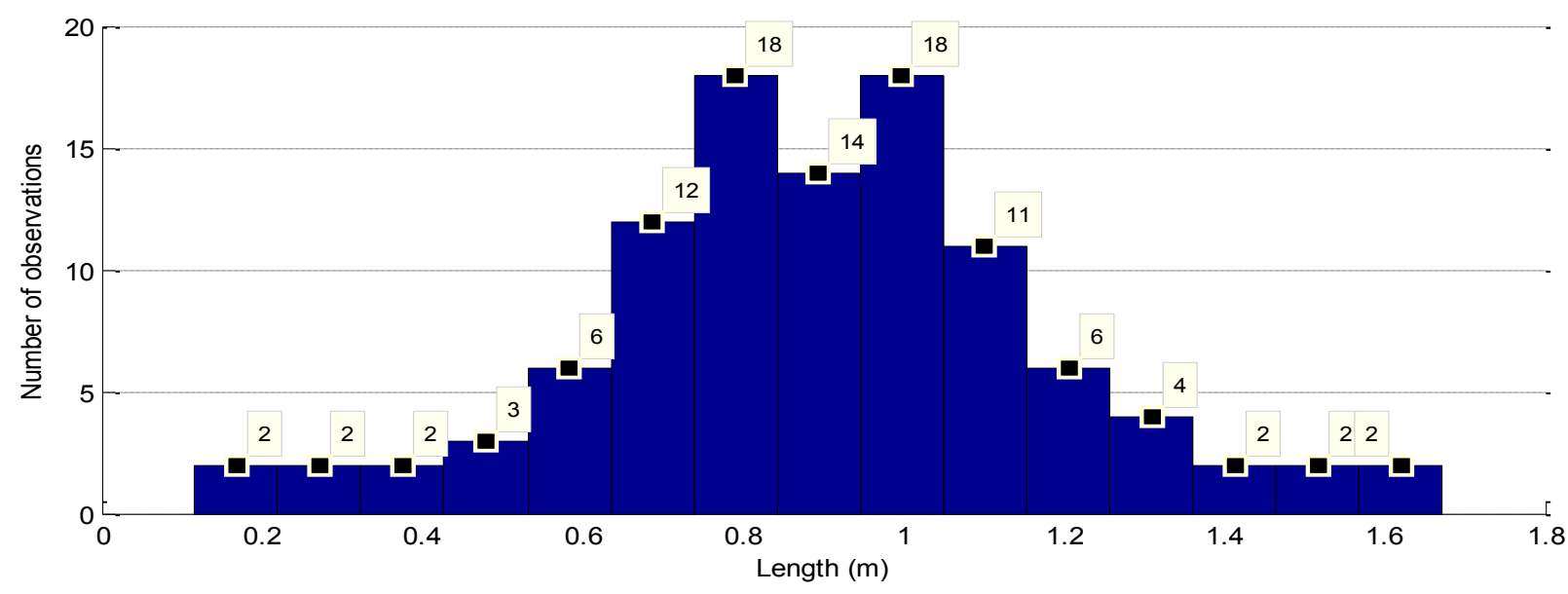

Fig.A.1.17 observed average length histogram for cyclists

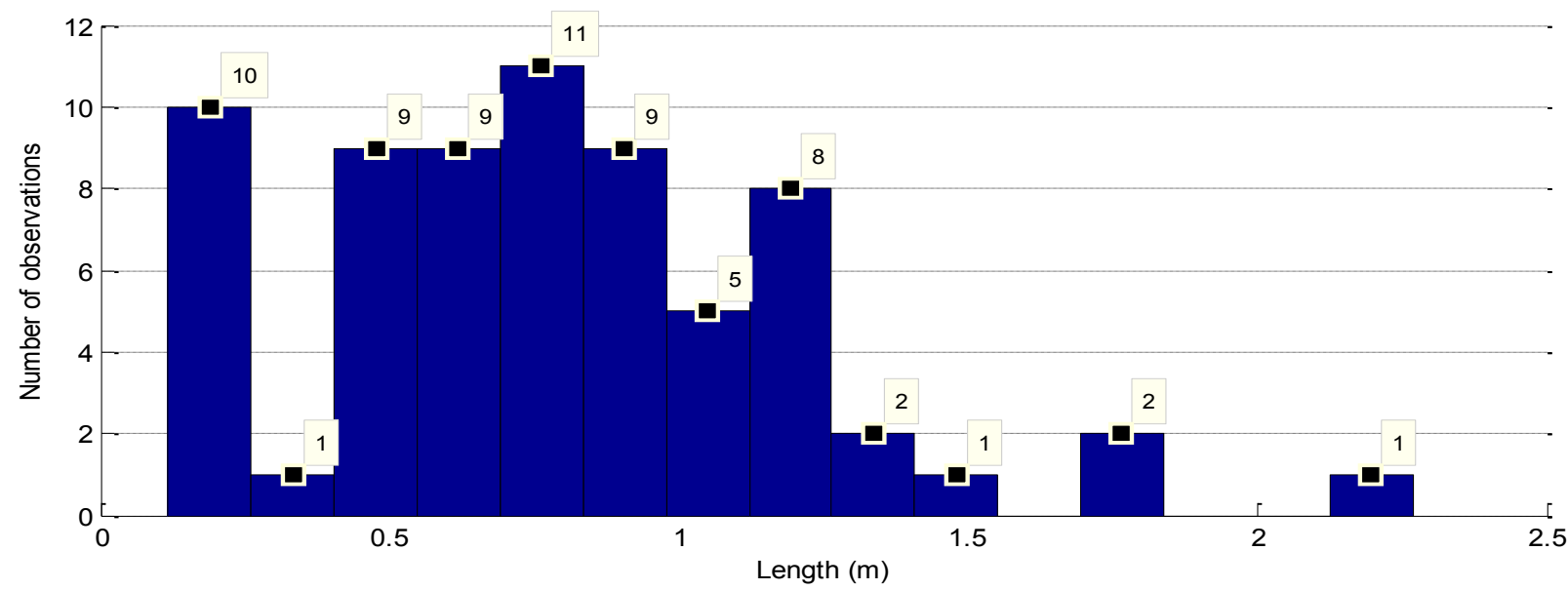

Fig.A.1.18 observed average length histogram for pedestrians 


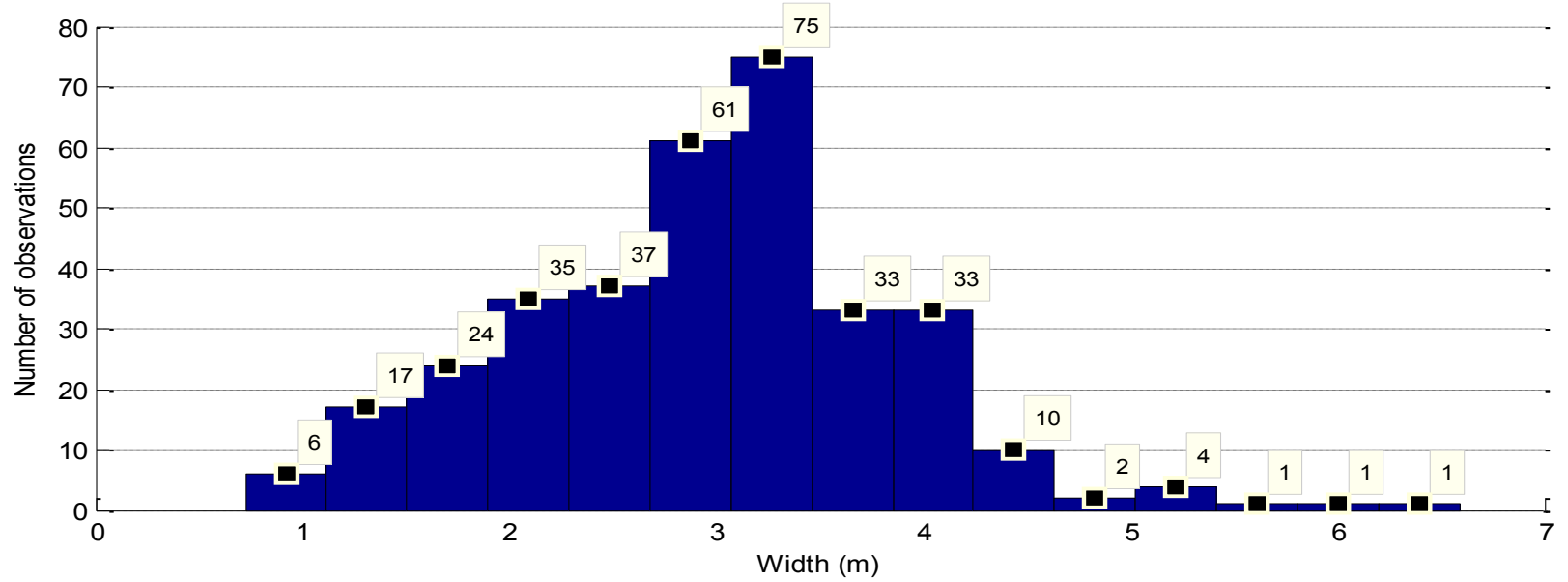

Fig.A.1.19 observed average width histogram for vehicles

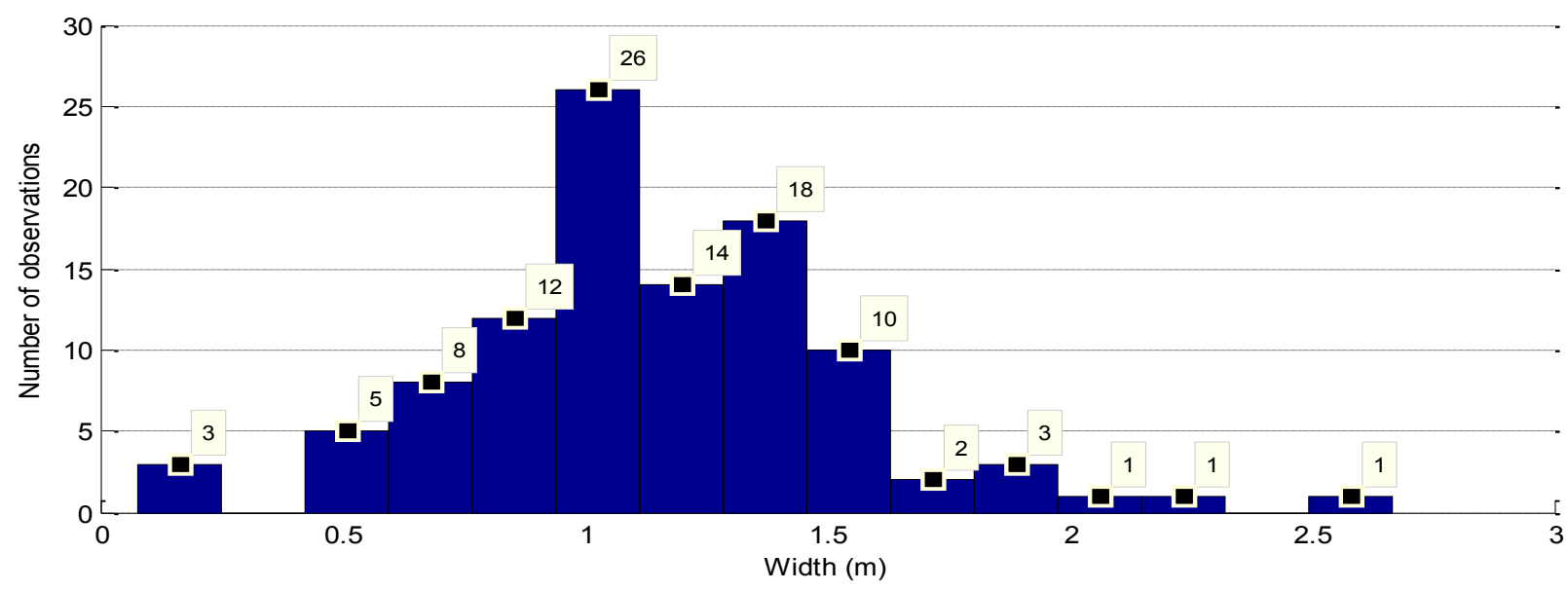

Fig.A.1.20 observed average width histogram for cyclists

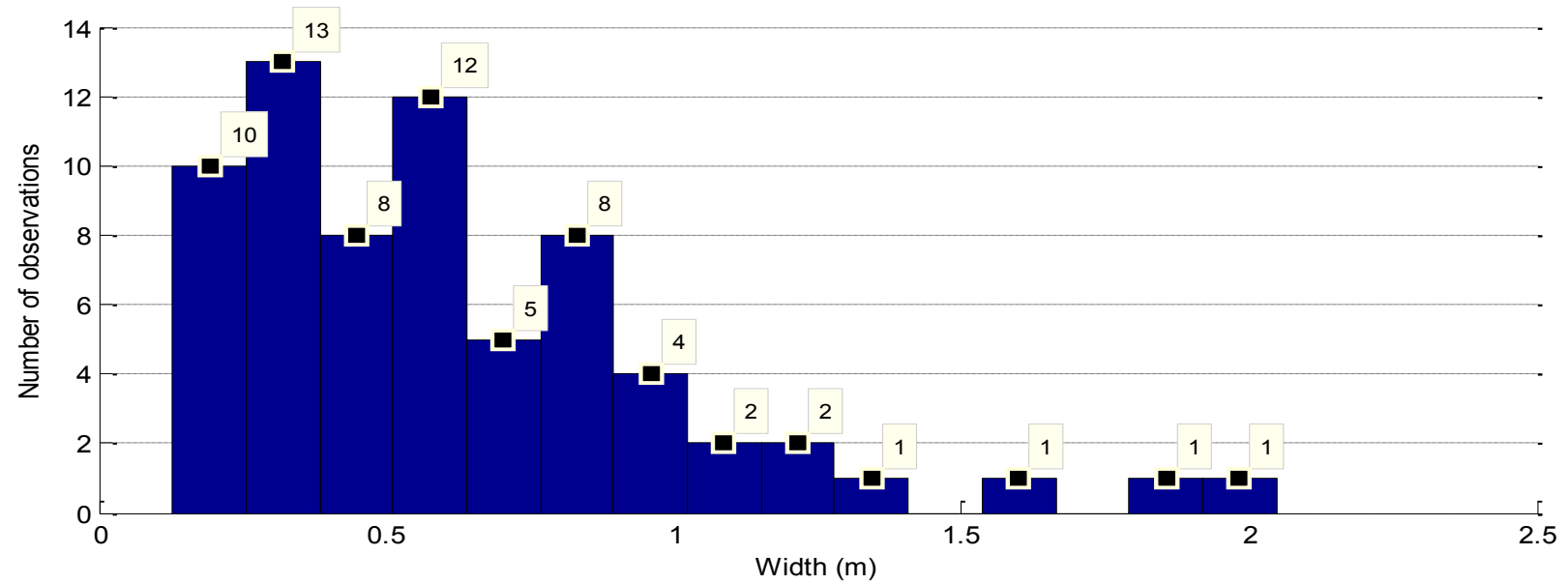

Fig.A.1.21 observed average width histogram for pedestrians 


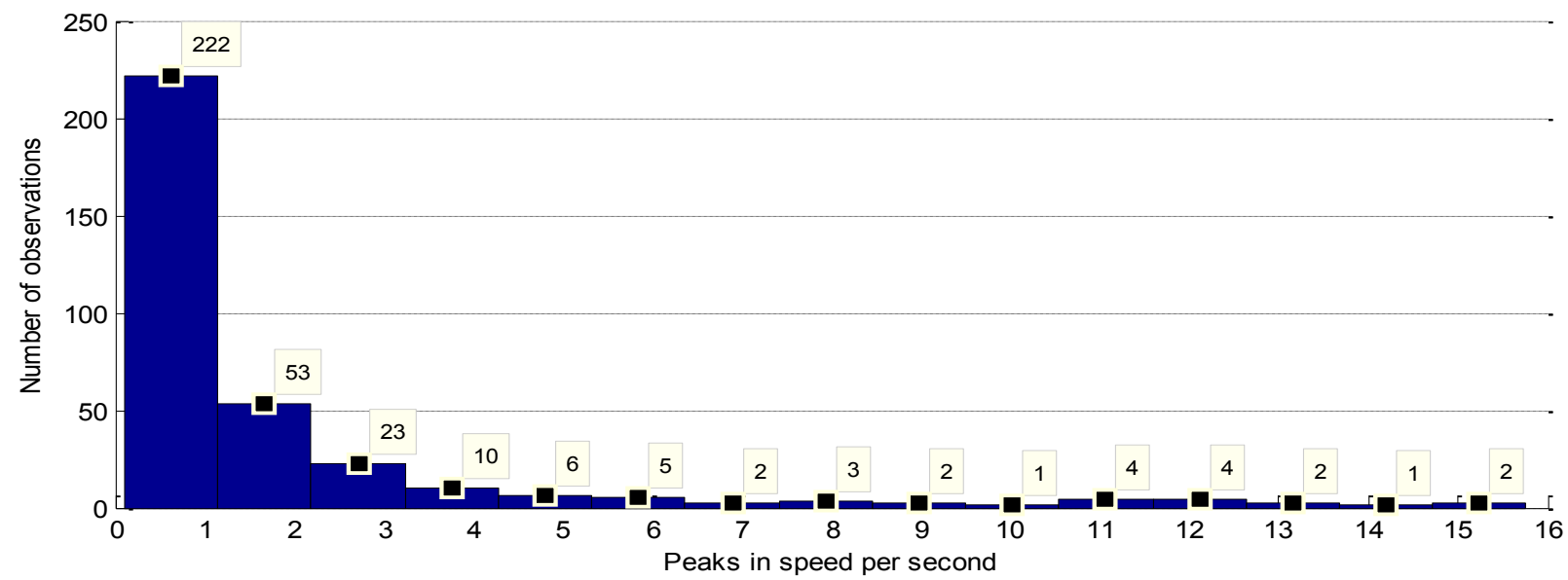

Fig.A.1.22 observed peaks in speed histogram for vehicles

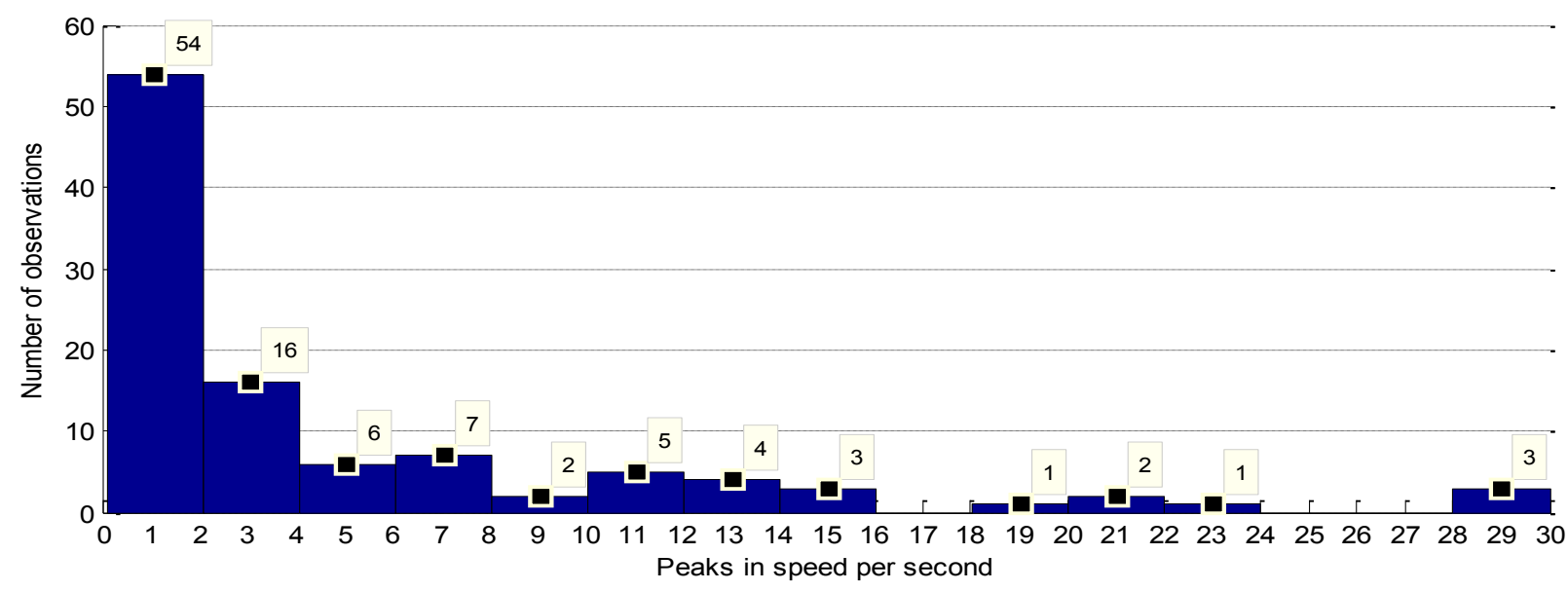

Fig.A.1.23 observed peaks in speed histogram for cyclists

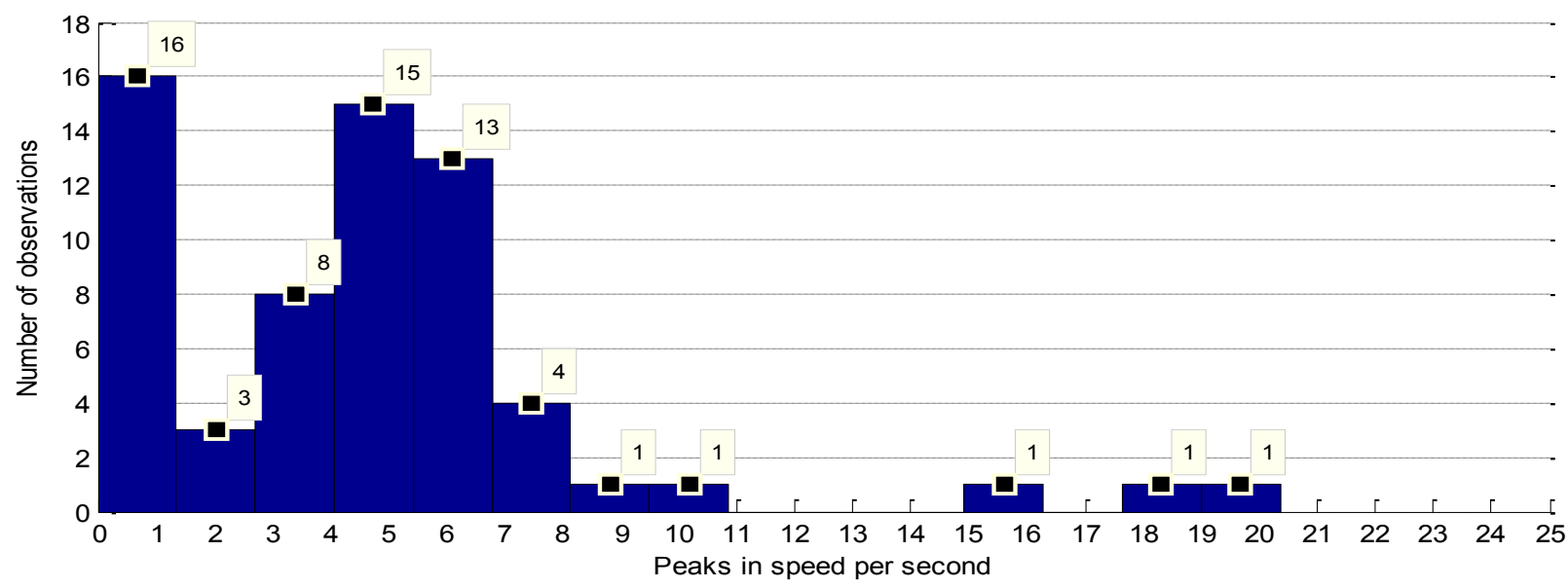

Fig.A.1.24 observed peaks in speed histogram for pedestrians 


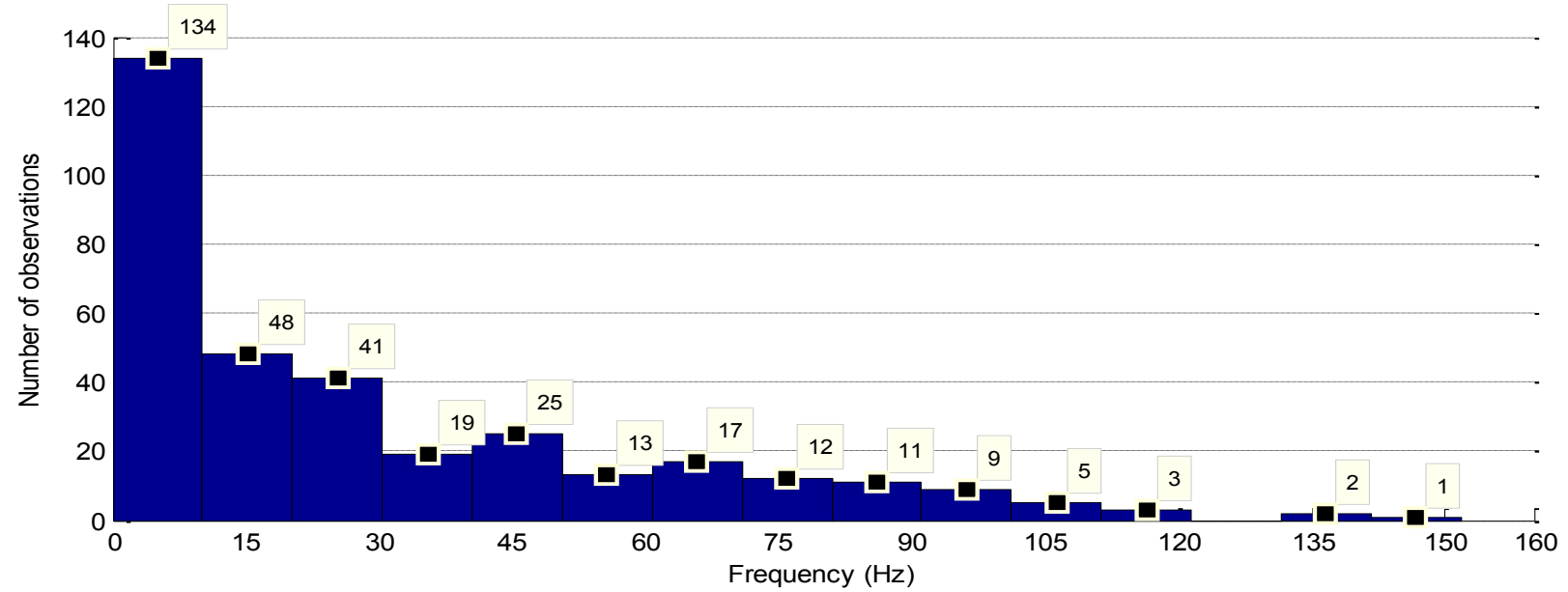

Fig.A.1.25 observed effective weighted average frequency of the highest 5 powers for vehicles

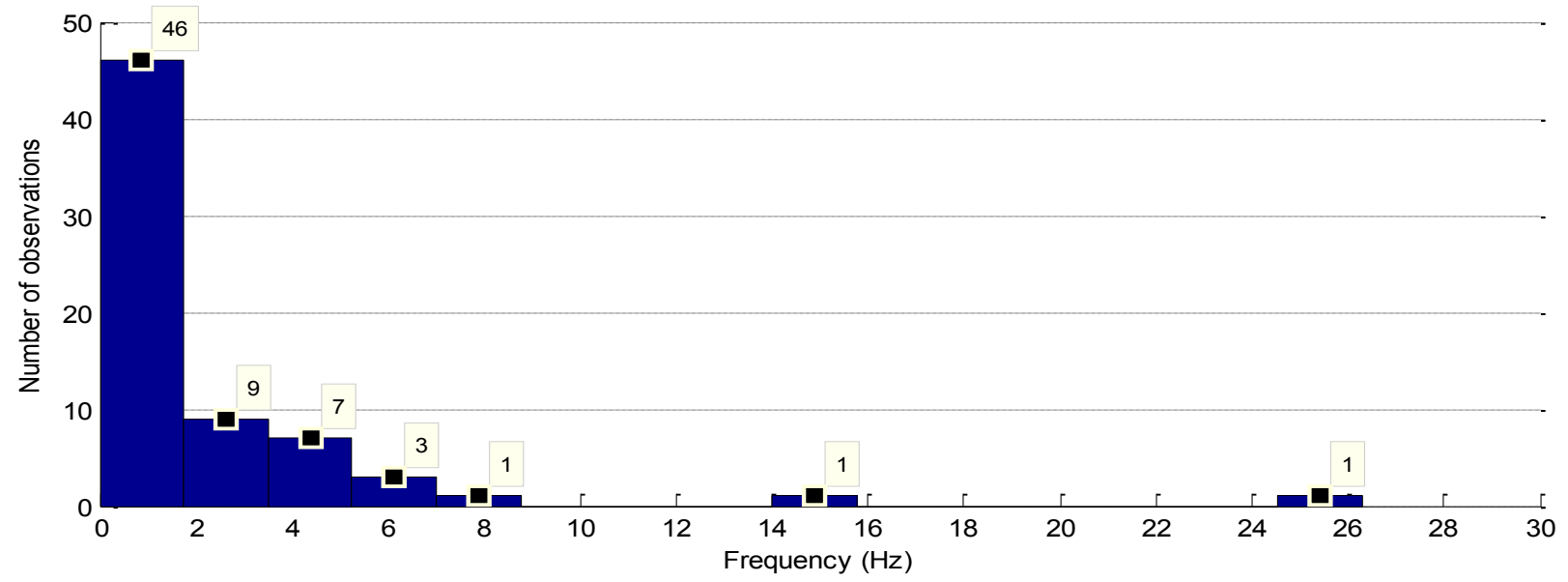

Fig.A.1.26 observed effective weighted average frequency of the highest 5 powers for cyclists

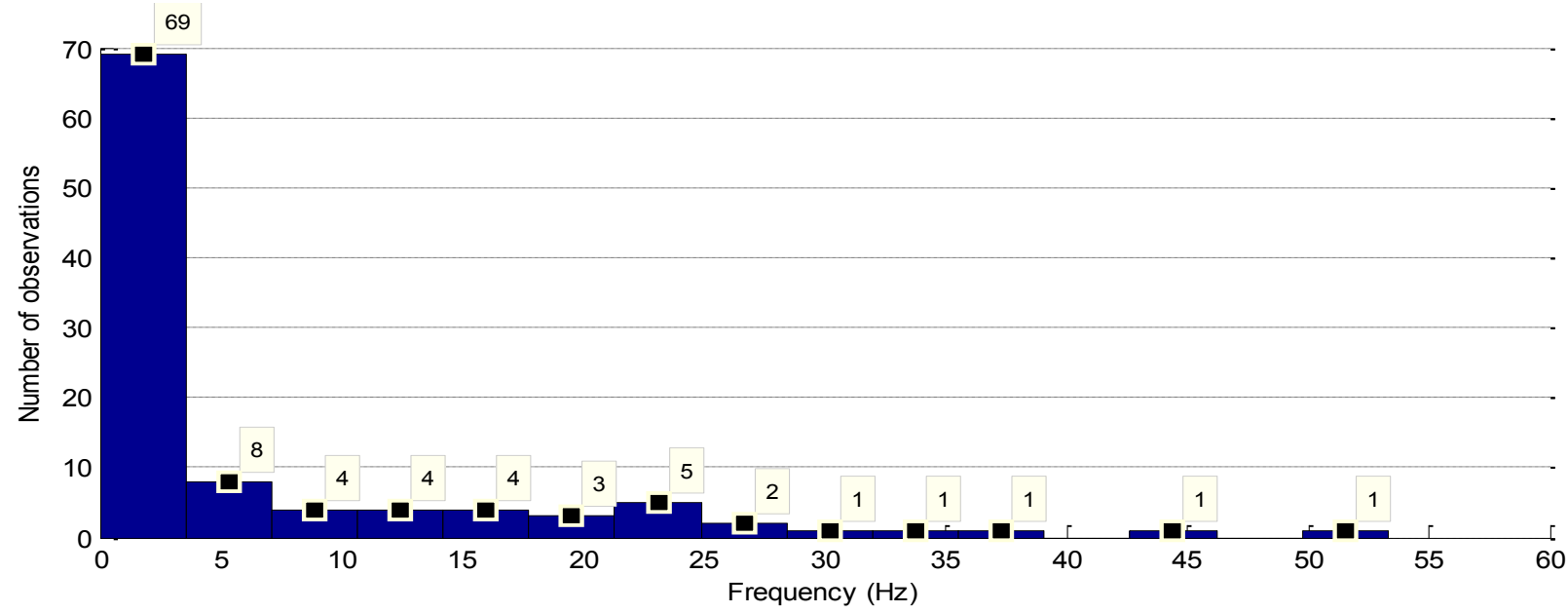

Fig.A.1.27 observed effective weighted average frequency of the highest 5 powers for pedestrians 


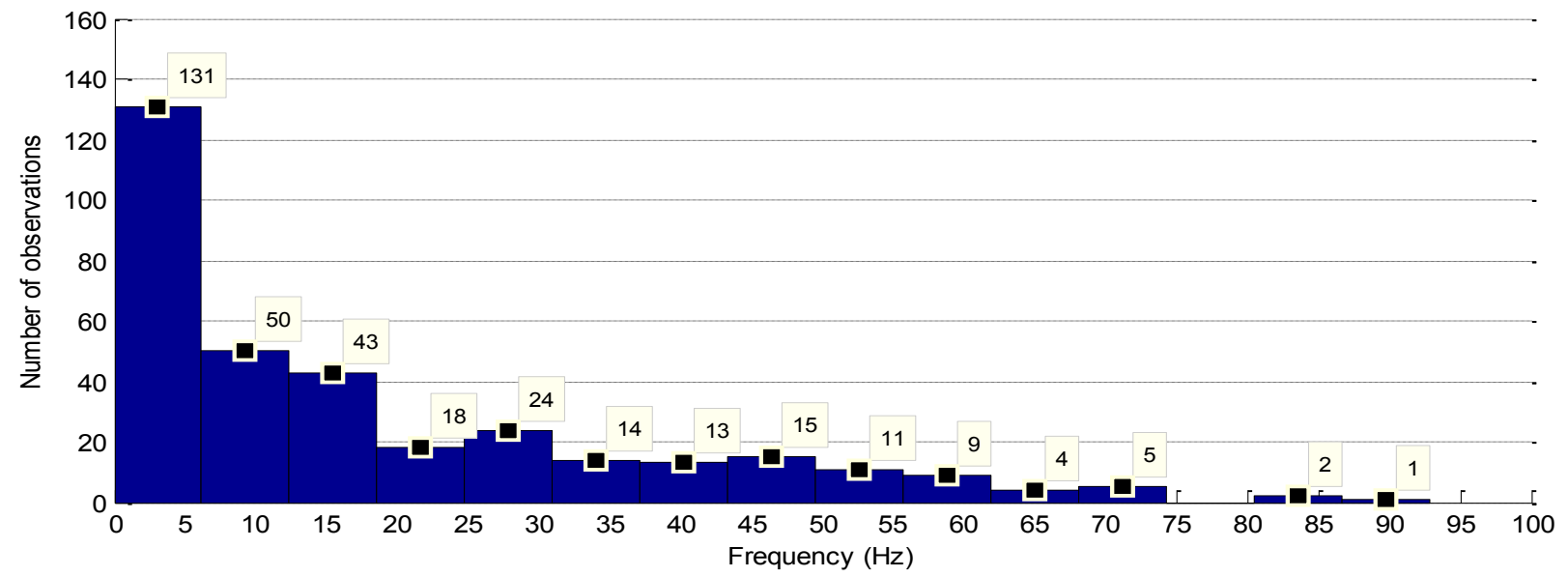

Fig.A.1.28 observed effective weighted average frequency of the highest 10 powers for vehicles

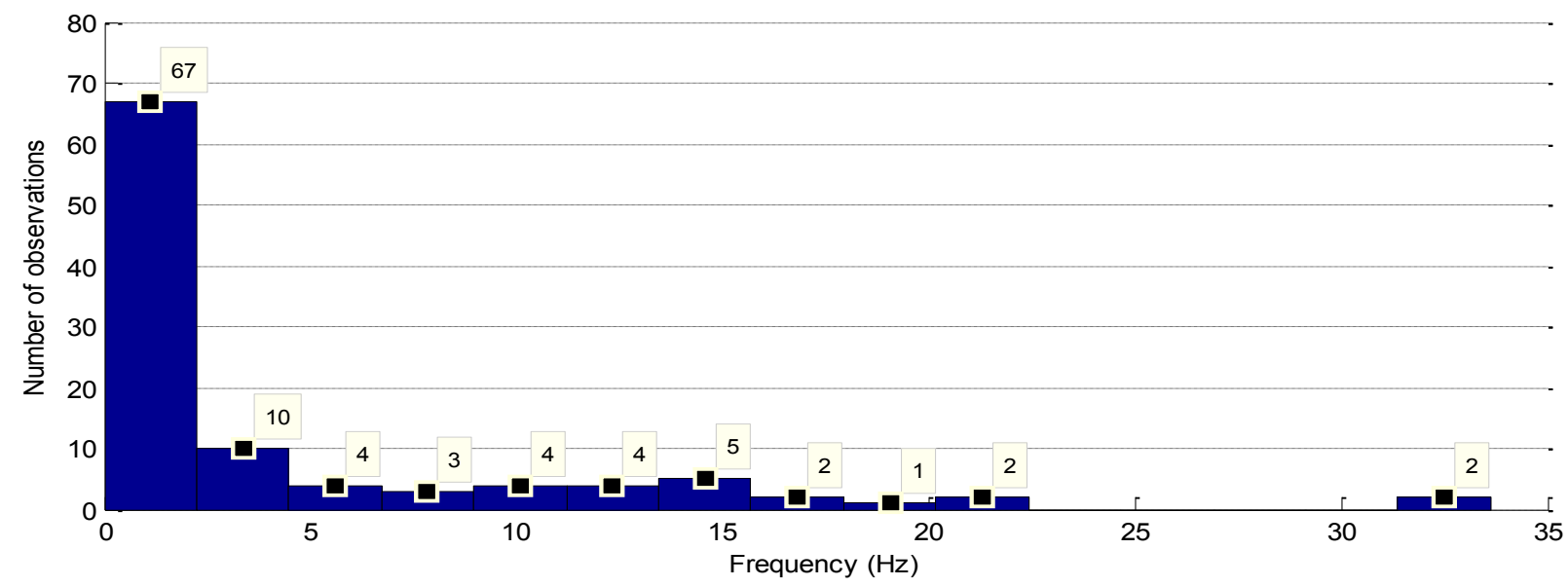

Fig.A.1.29 observed effective weighted average frequency of the highest 10 powers for cyclists

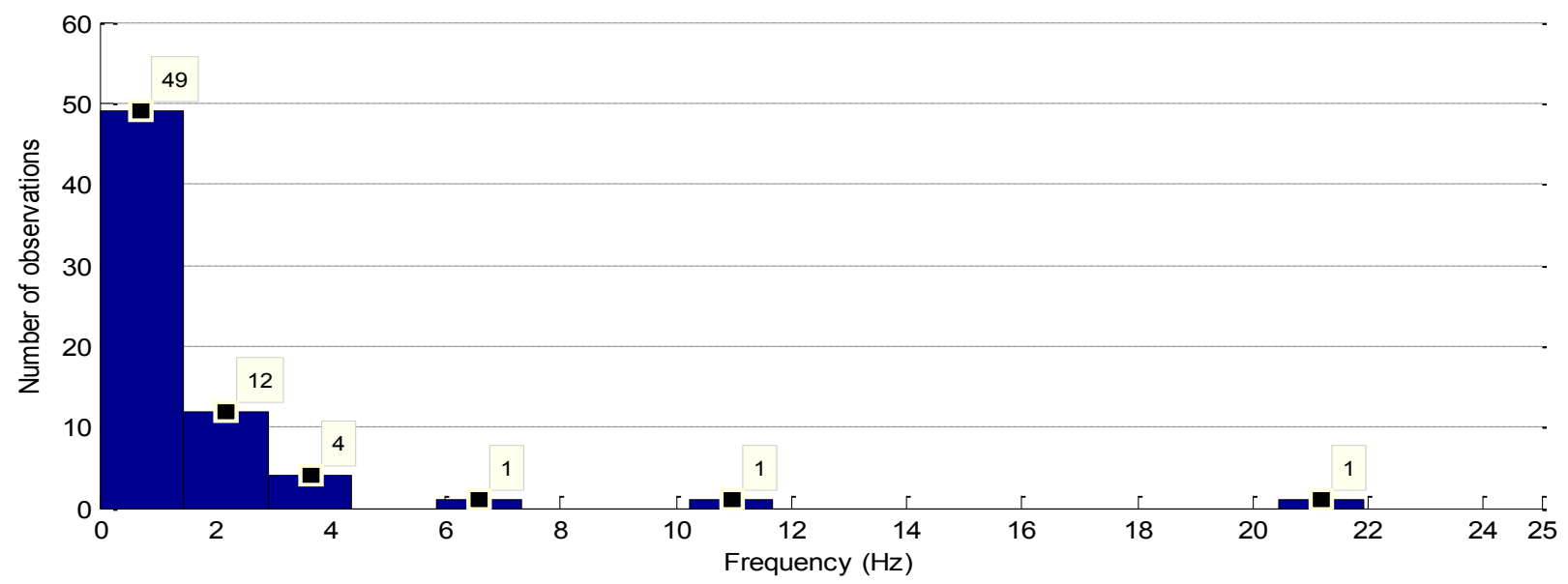

Fig.A.1.30 observed effective weighted average frequency of the highest 10 powers for pedestrians 


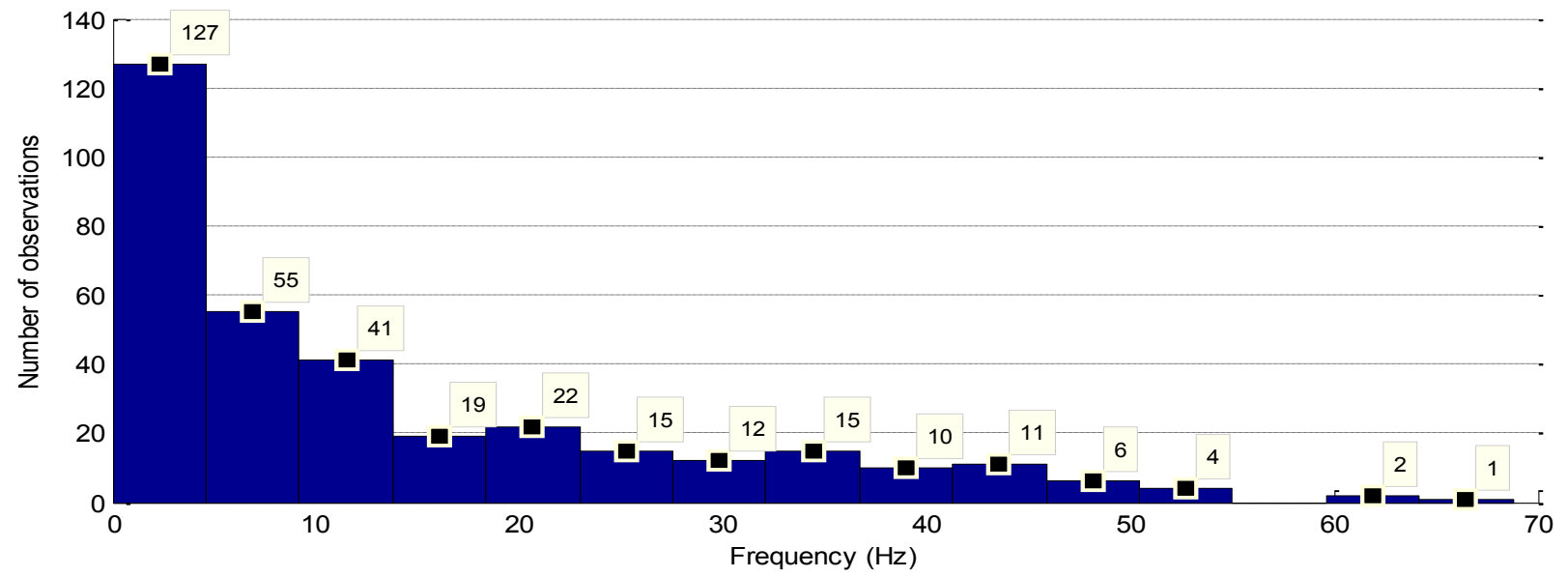

Fig.A.1.31 observed effective weighted average frequency of the highest 15 powers for vehicles

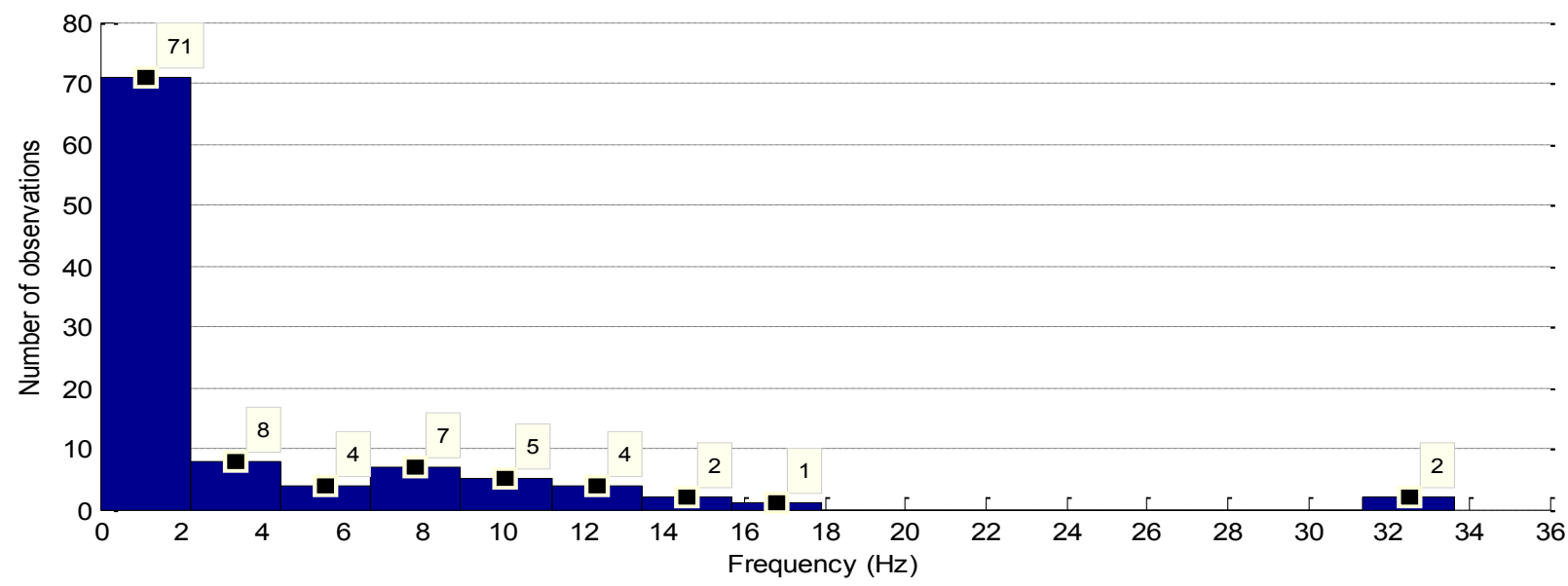

Fig.A.1.32 observed effective weighted average frequency of the highest 15 powers for cyclists

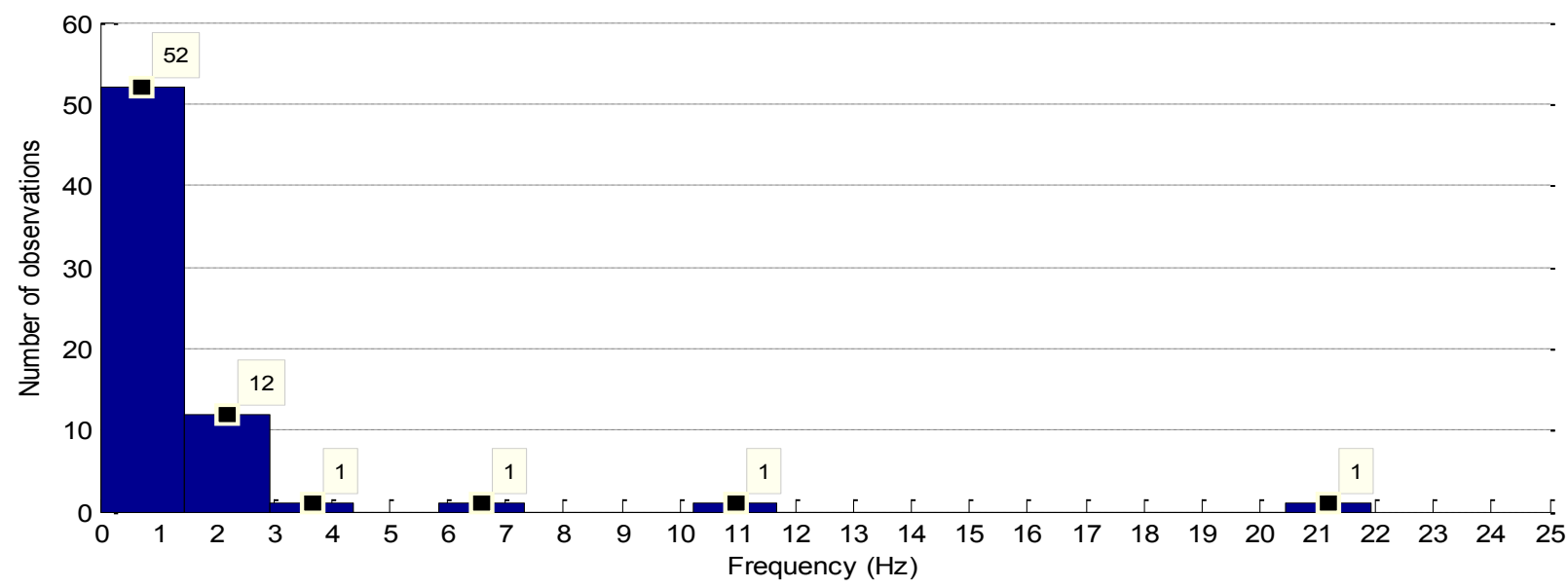

Fig.A.1.33 observed effective weighted average frequency of the highest 15 powers for pedestrians 


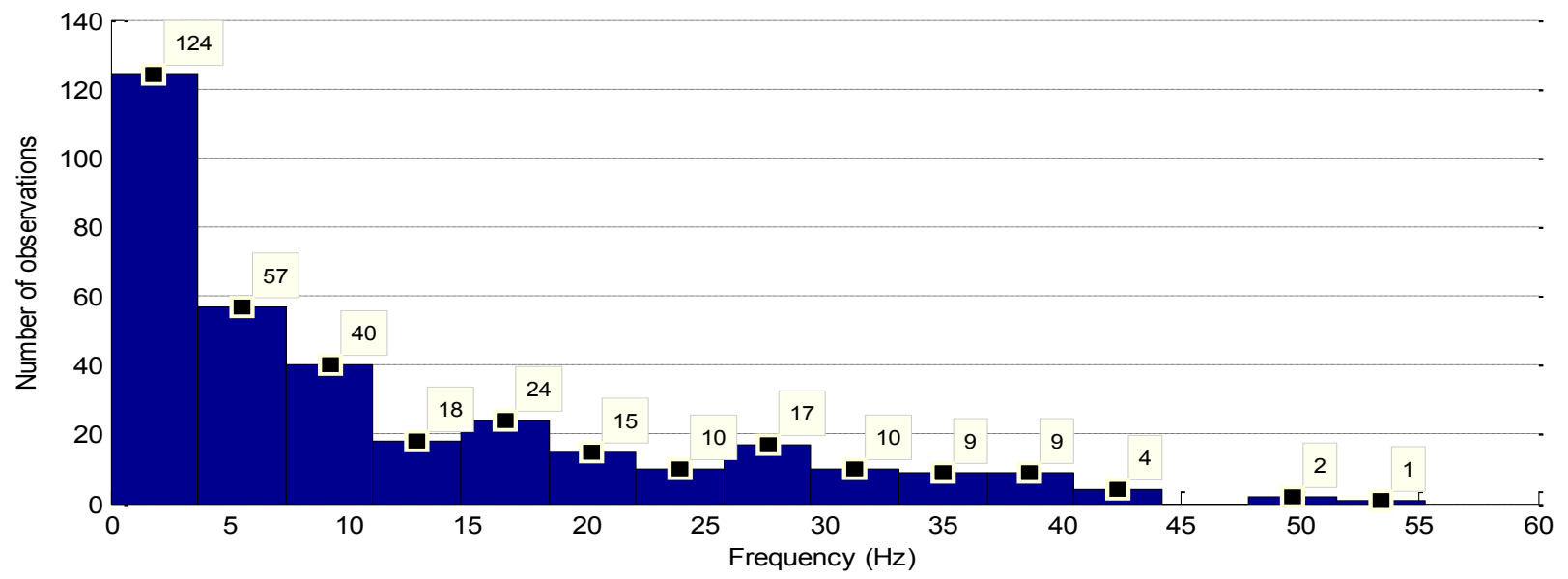

Fig.A.1.34 observed effective weighted average frequency of the highest 20 powers for vehicles

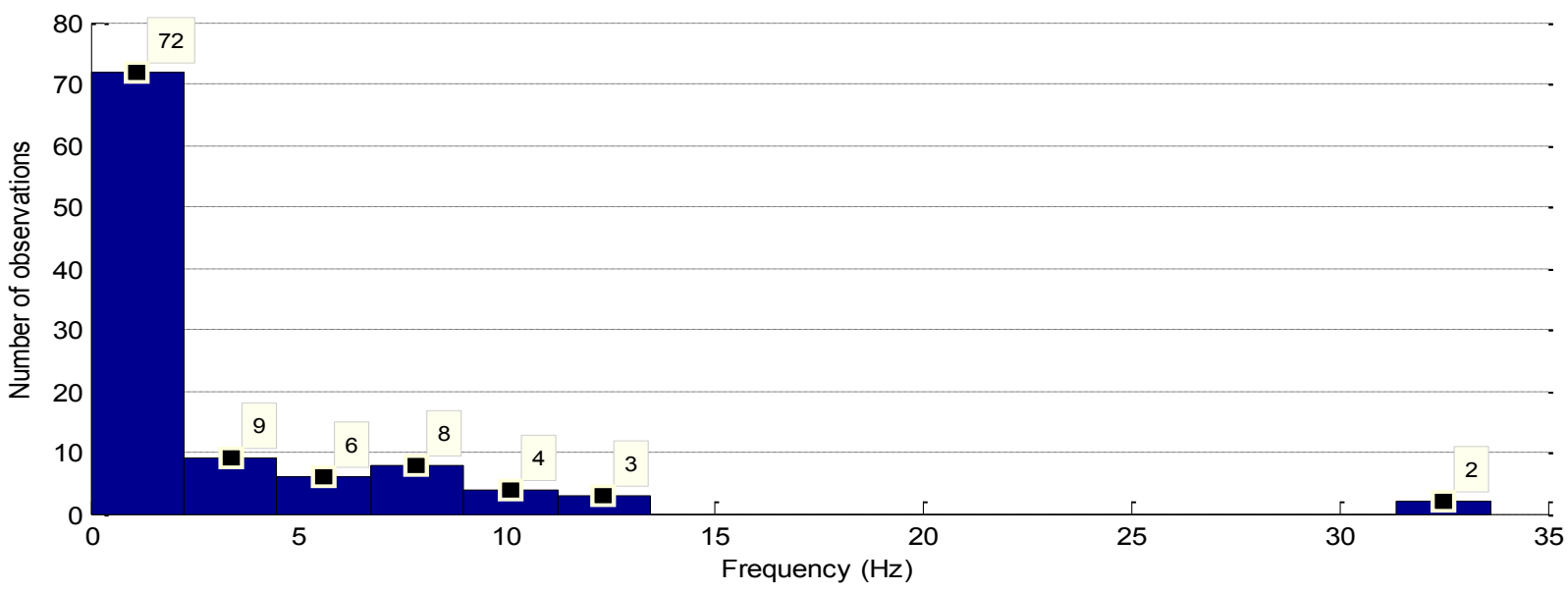

Fig.A.1.35 observed effective weighted average frequency of the highest 20 powers for cyclists

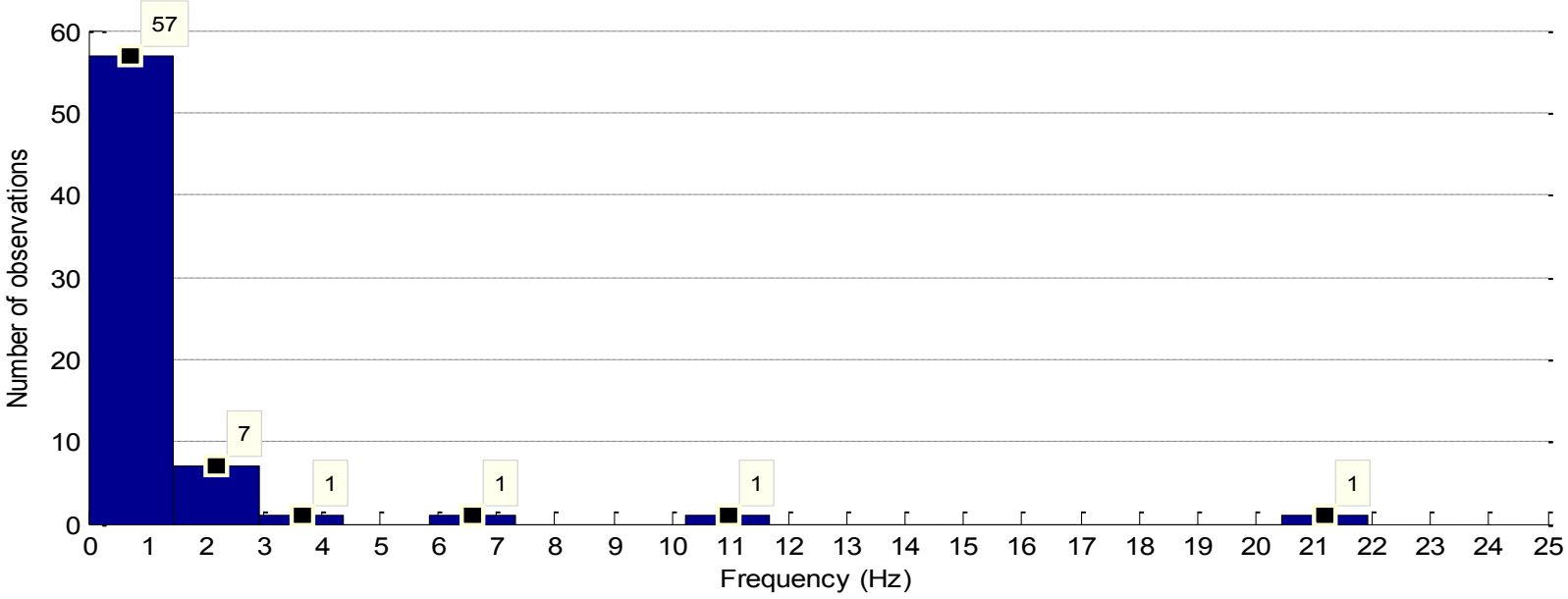

Fig.A.1.36 observed effective weighted average frequency of the highest 20 powers for pedestrians 


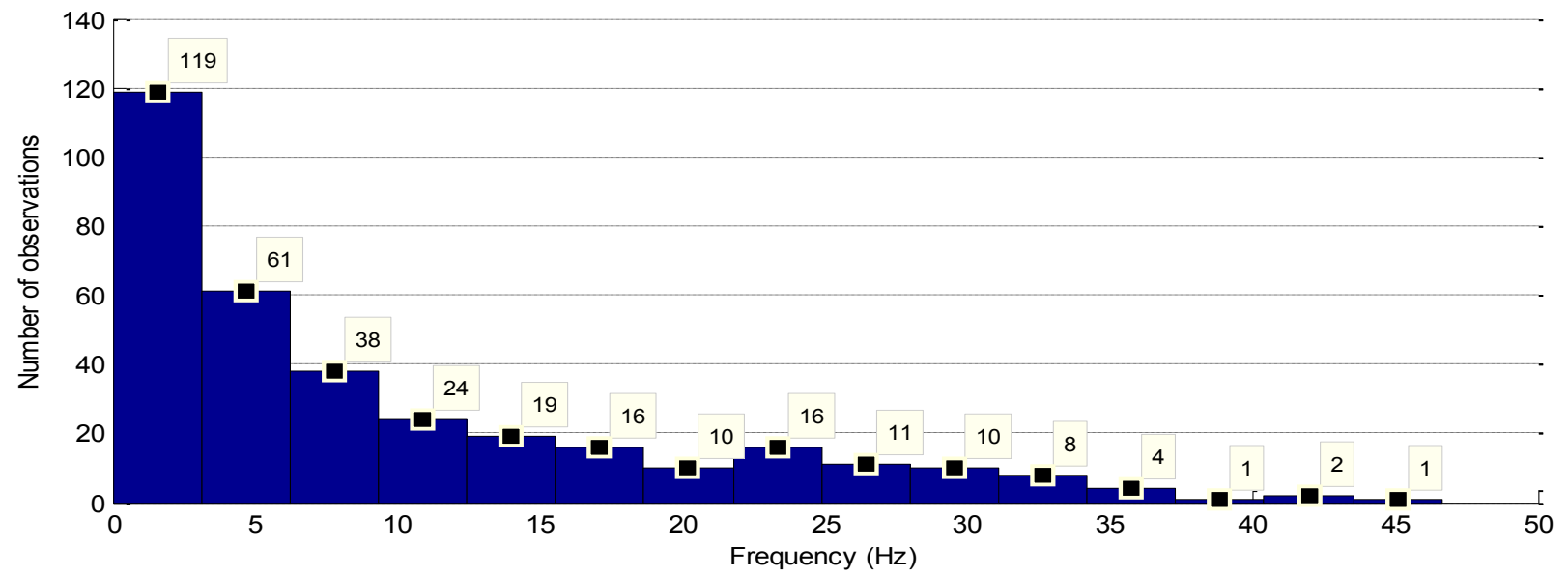

Fig.A.1.37 observed effective weighted average frequency of the highest 25 powers for vehicles

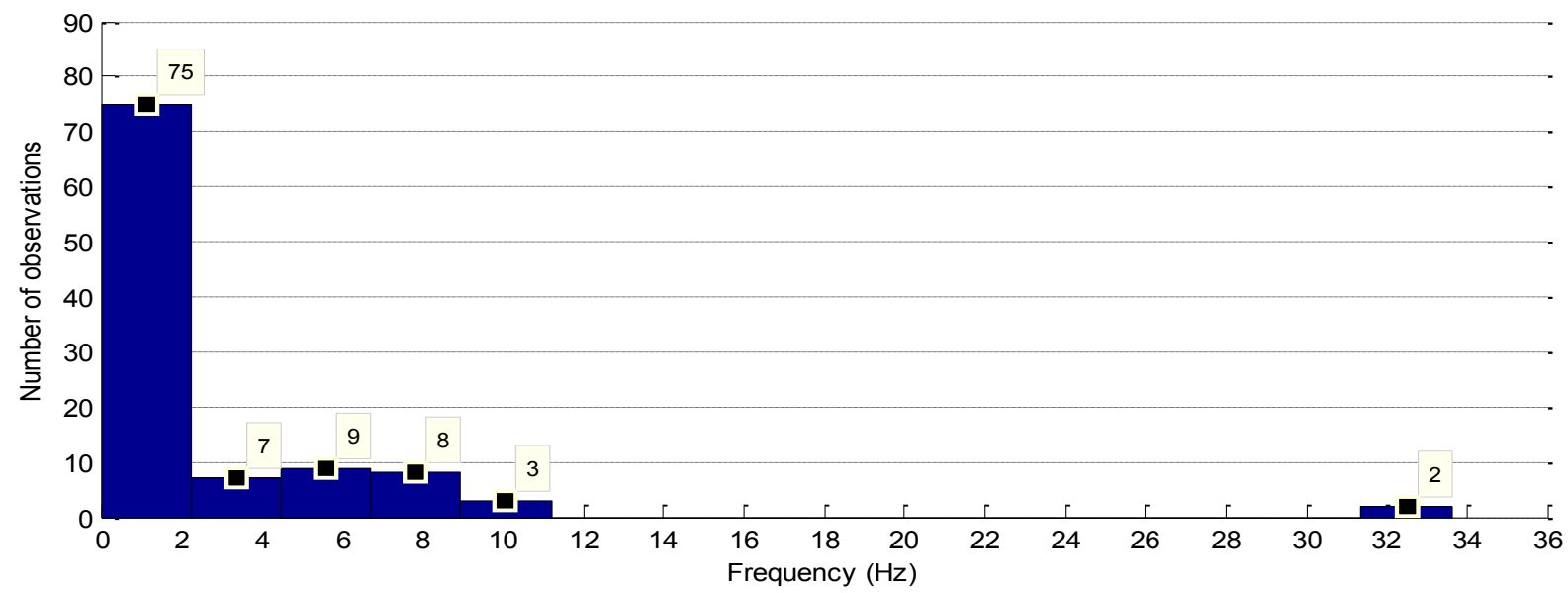

Fig.A.1.38 observed effective weighted average frequency of the highest 25 powers for cyclists

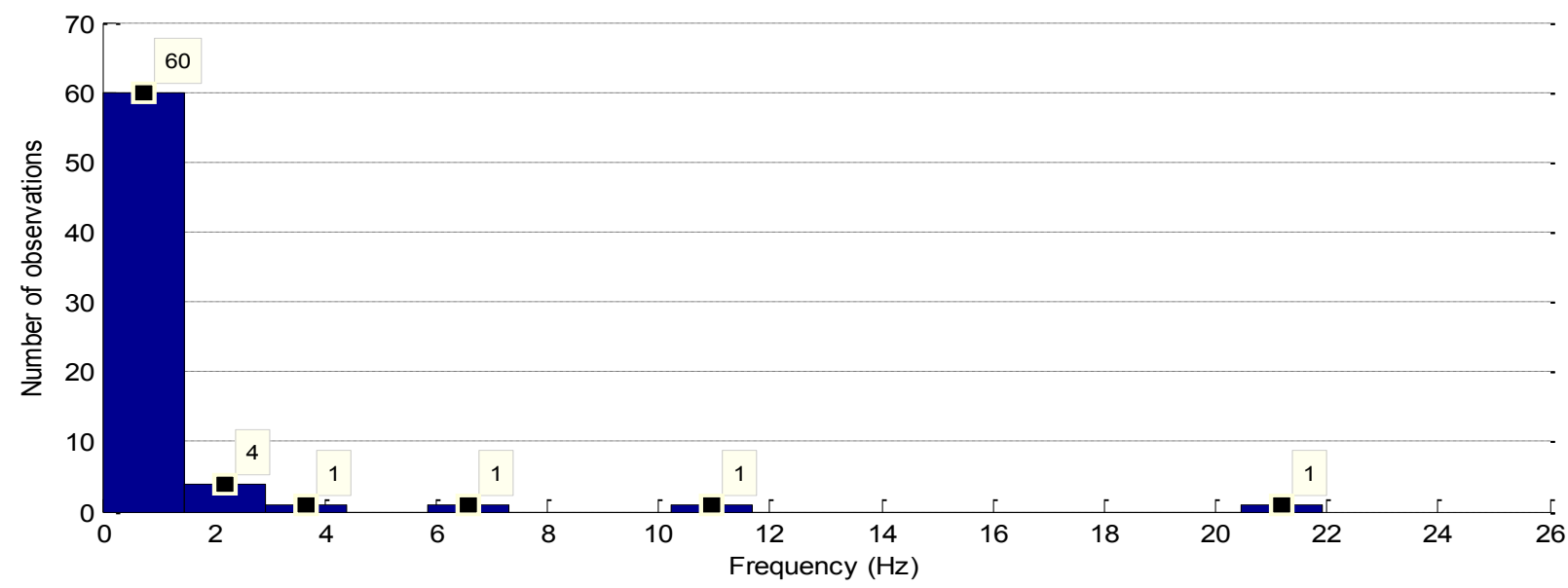

Fig.A.1.63 observed effective weighted average frequency of the highest 25 powers for pedestrians 


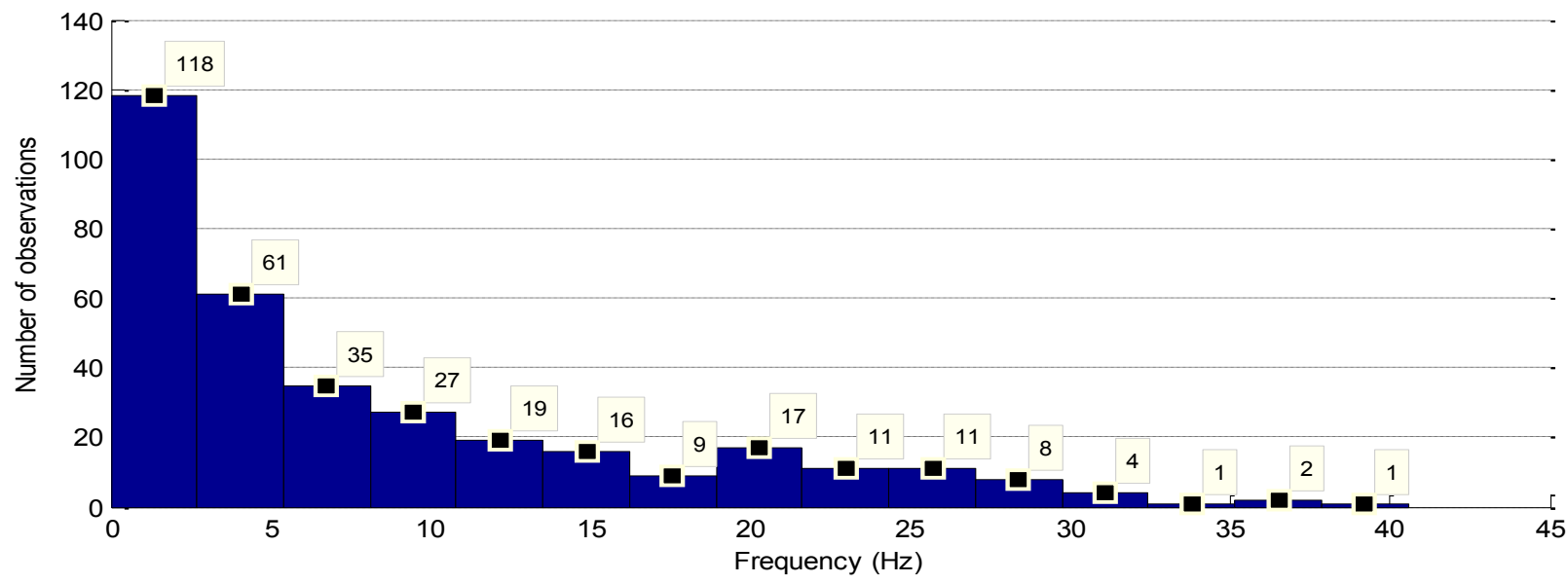

Fig.A.1.39 observed effective weighted average frequency of the highest 30 powers for vehicles

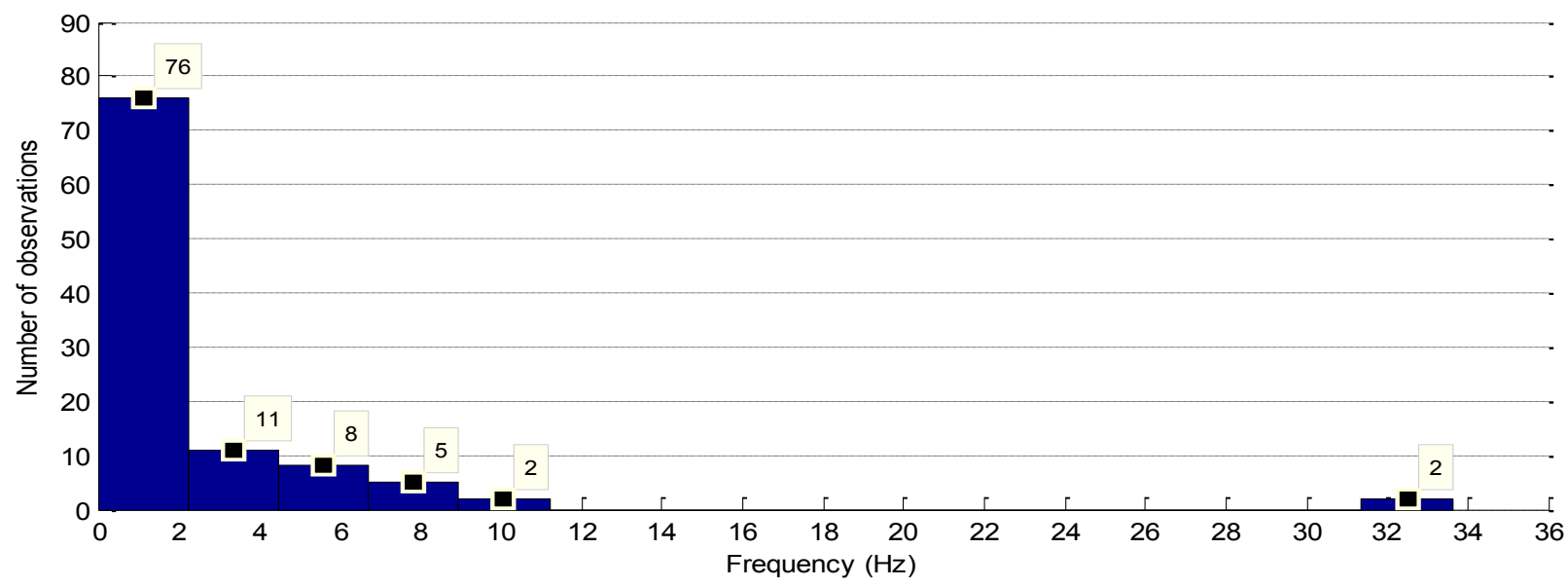

Fig.A.1.40 observed effective weighted average frequency of the highest 30 powers for cyclists

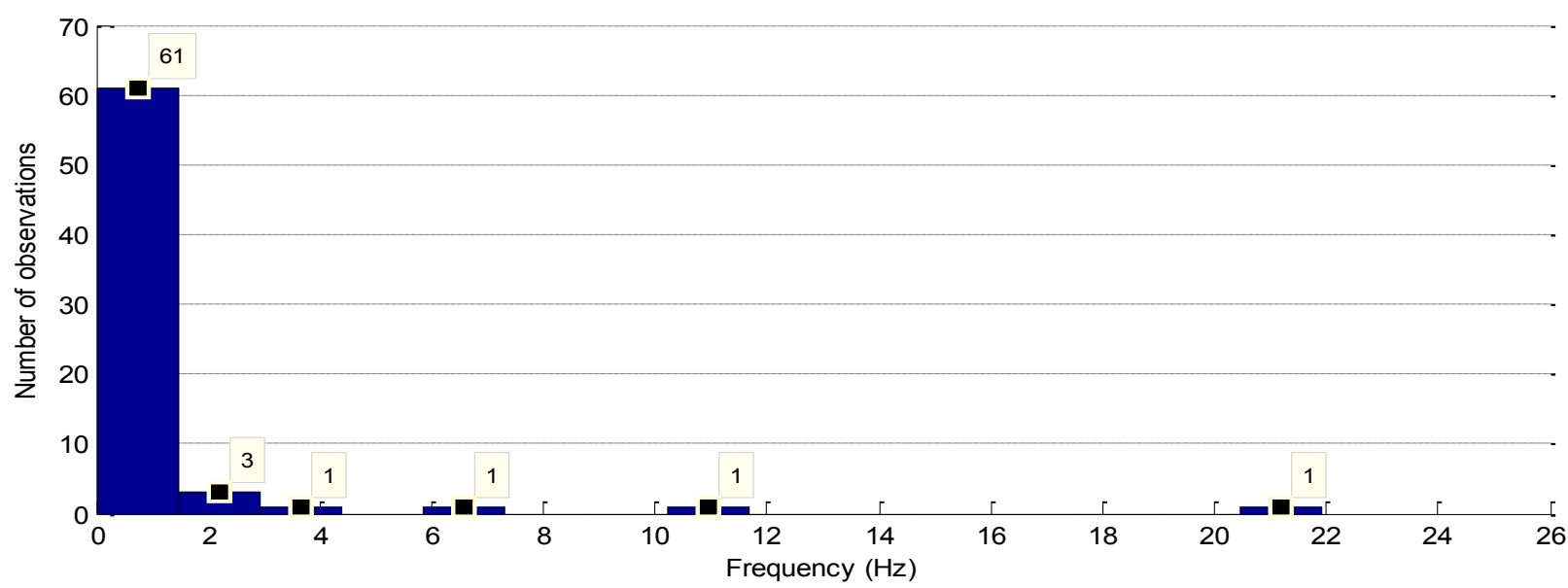

Fig.A.1.41 observed effective weighted average frequency of the highest 30 powers for pedestrians 


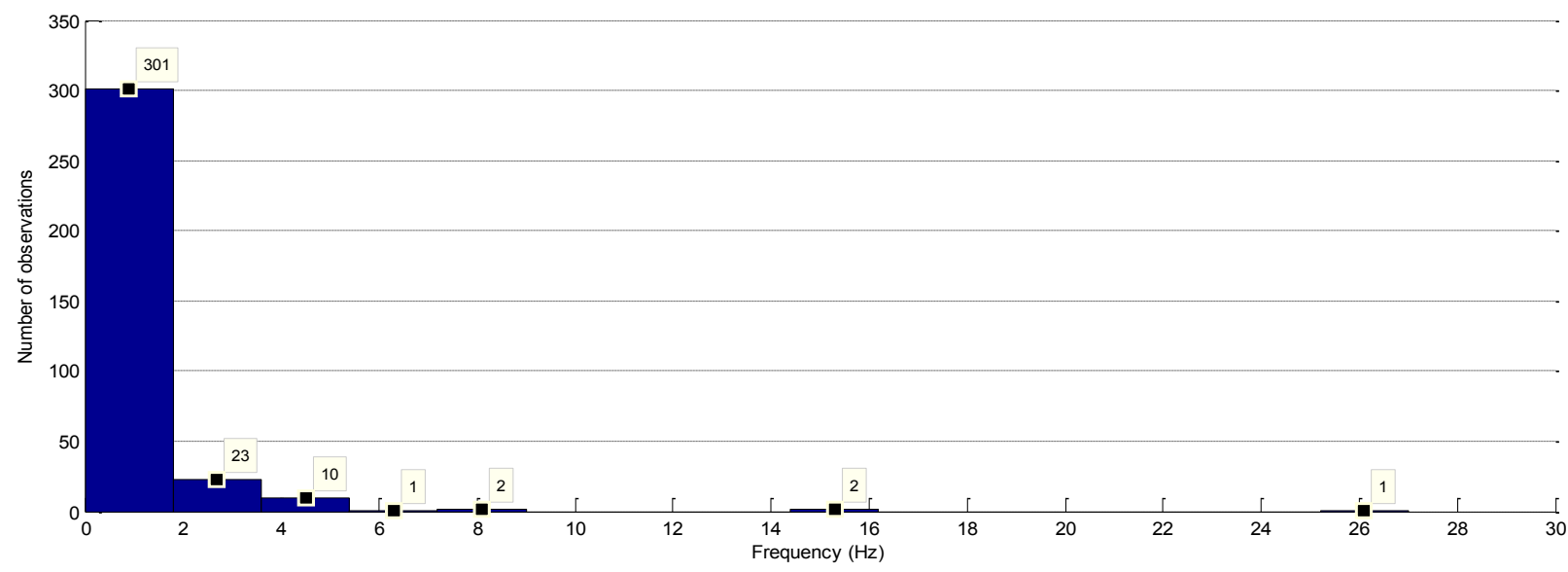

Fig.A.1.42 observed frequency of the highest power for vehicles

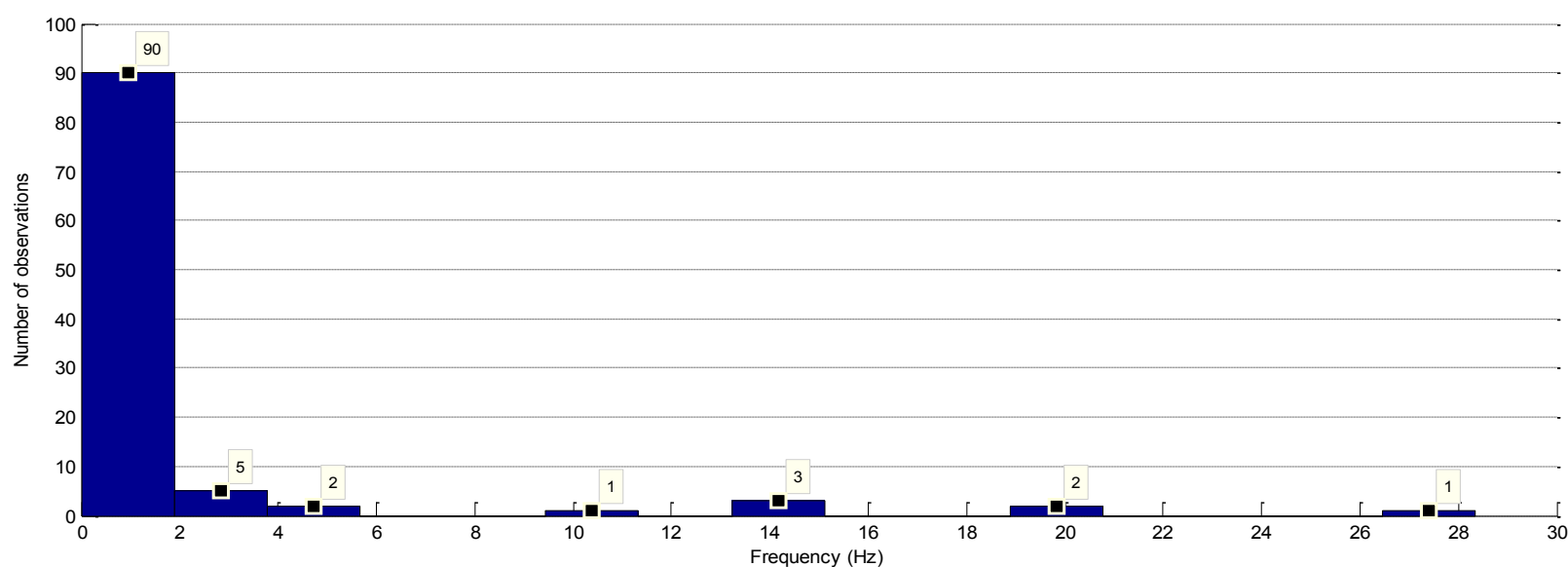

Fig.A.1.43 observed frequency of the highest power for cyclists

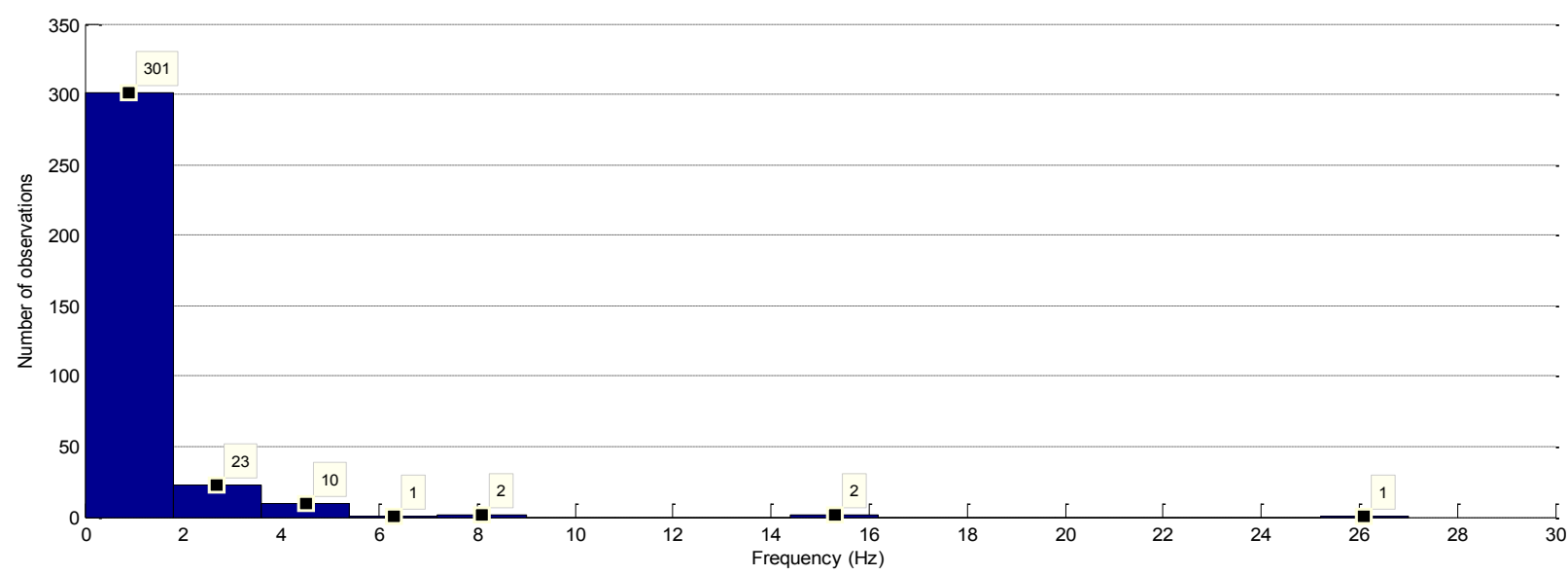

Fig.A.1.44 observed frequency of the highest power for pedestrians 


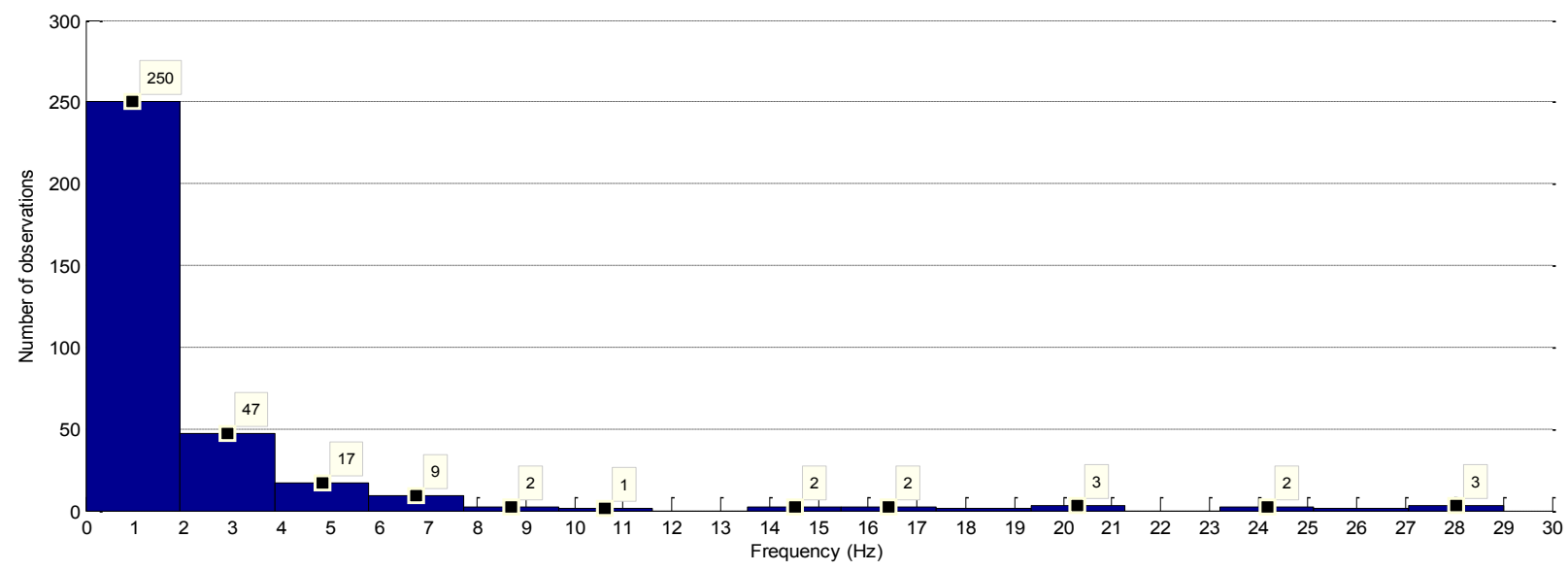

Fig.A.1.45 observed frequency of the $2^{\text {nd }}$ highest power for vehicles

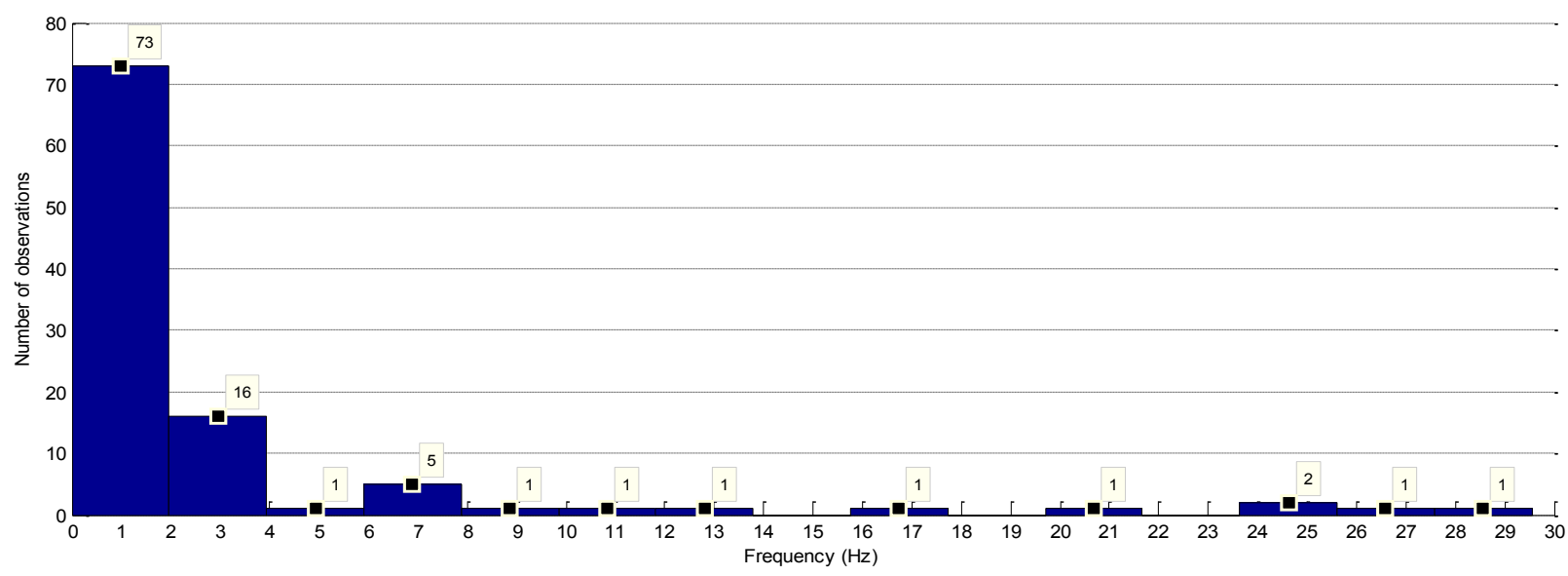

Fig.A.1.46 observed frequency of the $2^{\text {nd }}$ highest power for cyclists

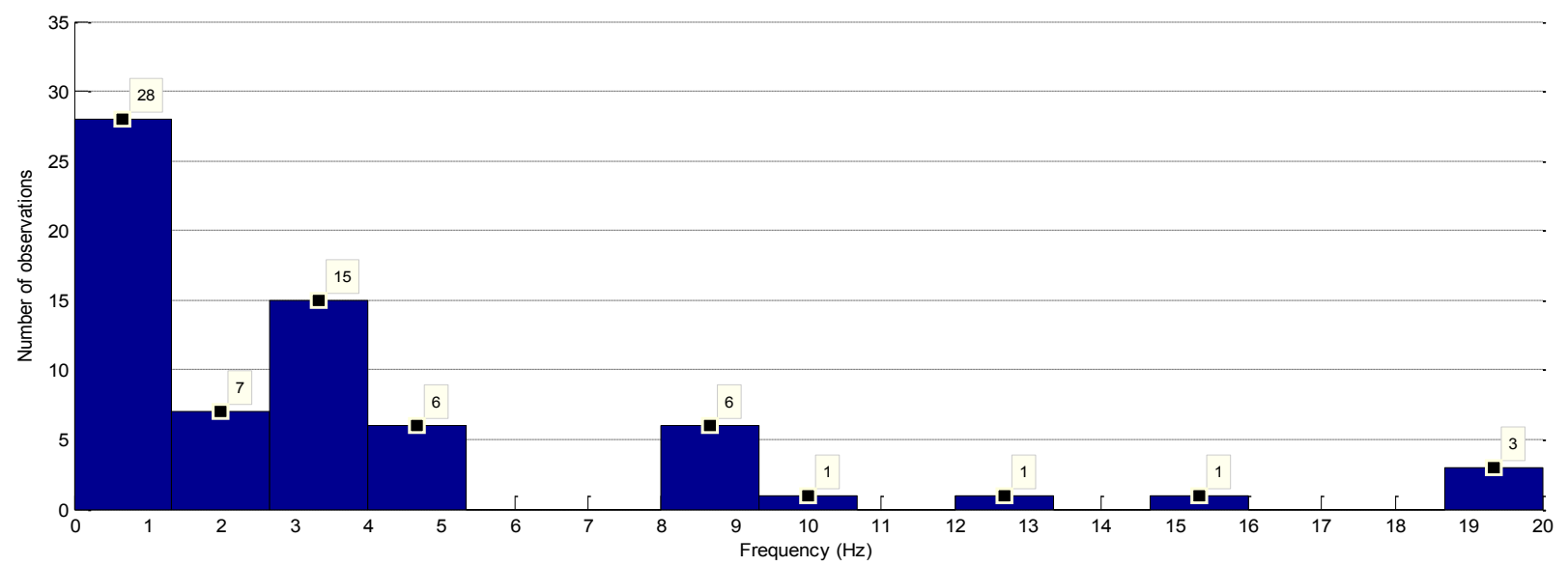

Fig.A.1.47 observed frequency of the $2^{\text {nd }}$ highest power for pedestrians 


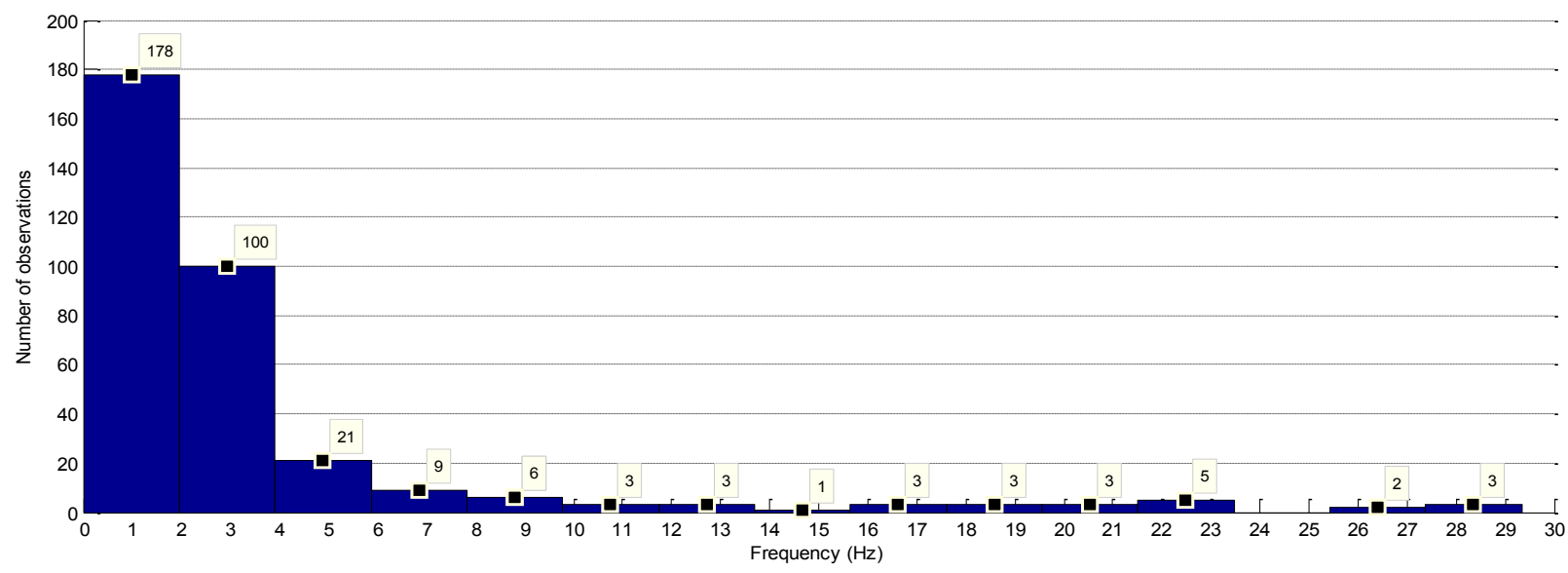

Fig.A.1.48 observed frequency of the $3^{\text {rd }}$ highest power for vehicle

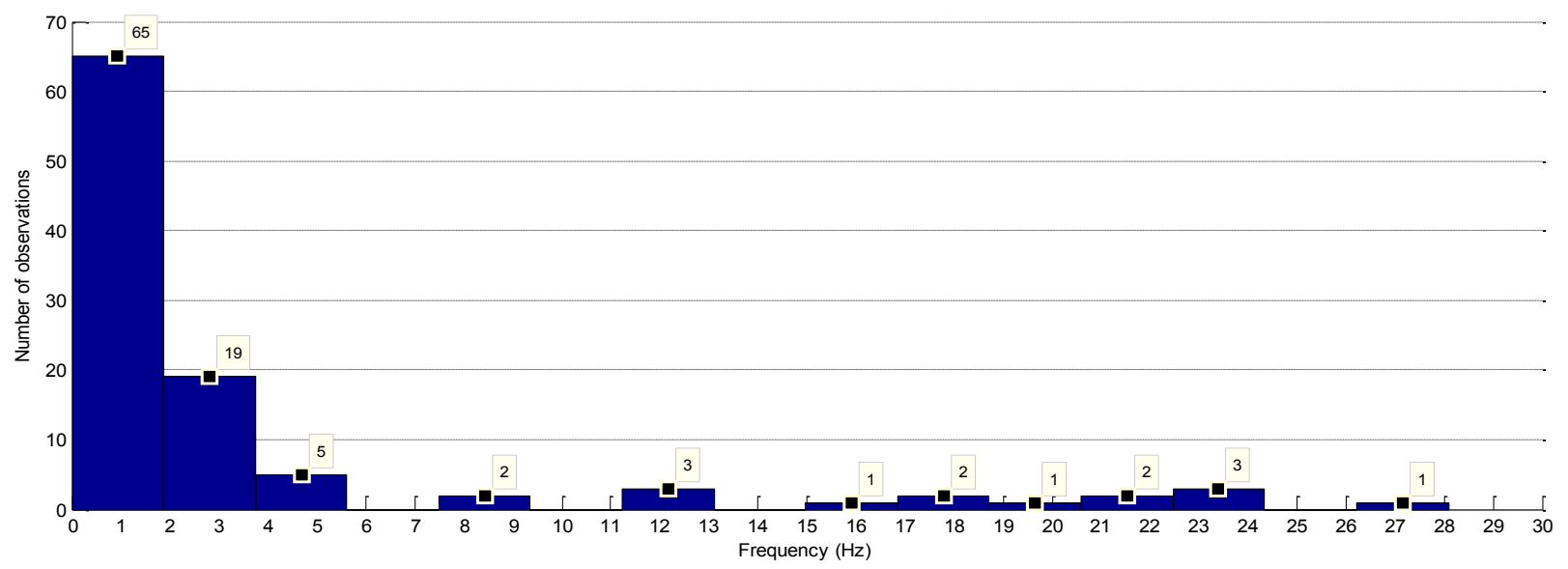

Fig.A.1.49 observed frequency of the $3^{\text {rd }}$ highest power for cyclists

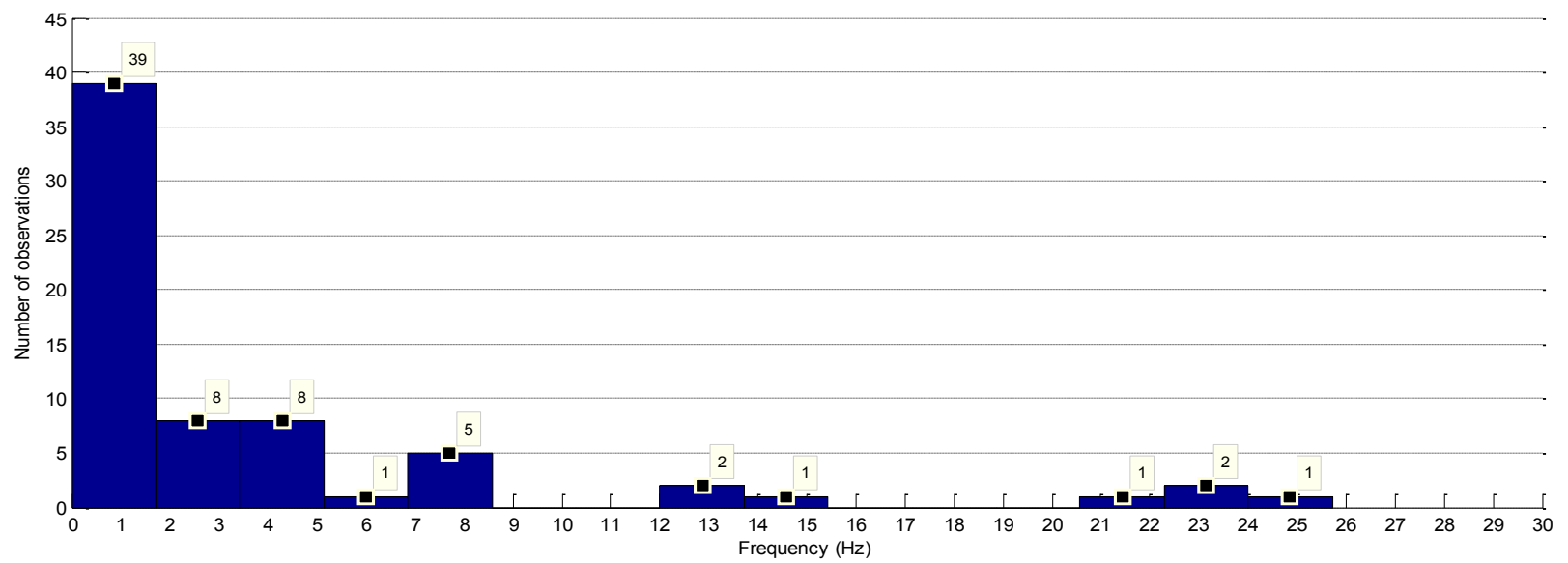

Fig.A.1.50 observed frequency of the $3^{\text {rd }}$ highest power for pedestrians 


\section{A.2 Cumulative curves show the improvement of Kappa over calibration}

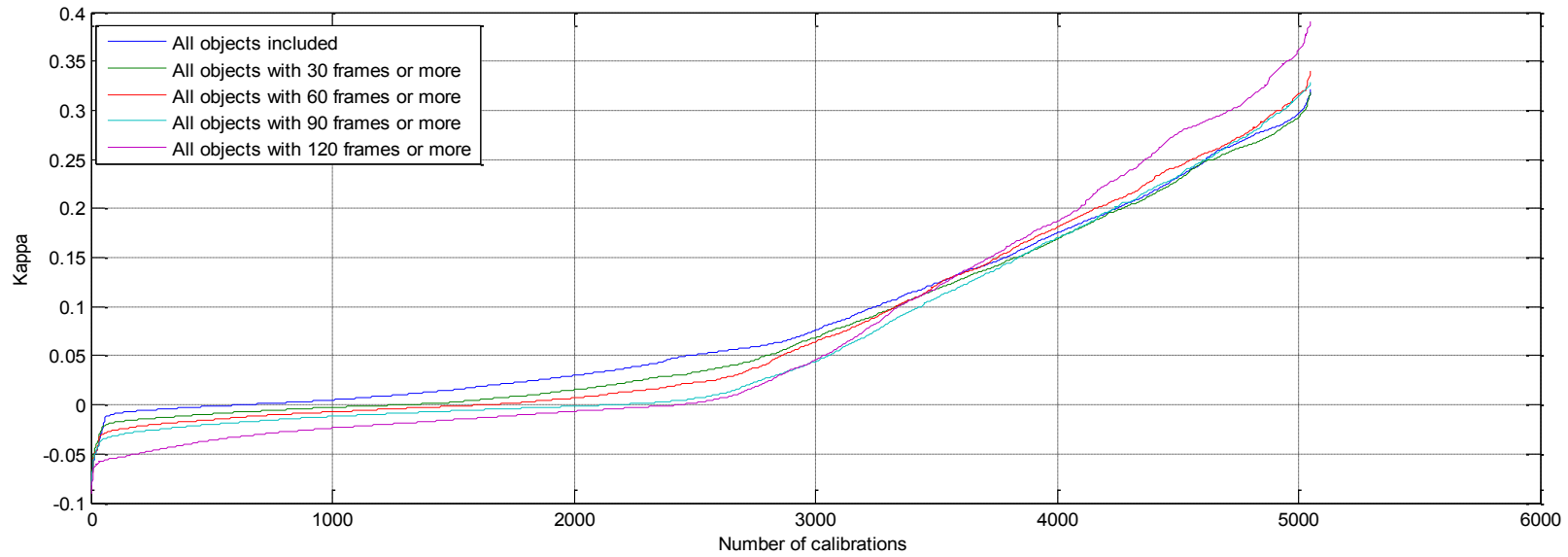

Fig.A.2.1 Cumulative curves show the improvement of Kappa over calibration using the average speed

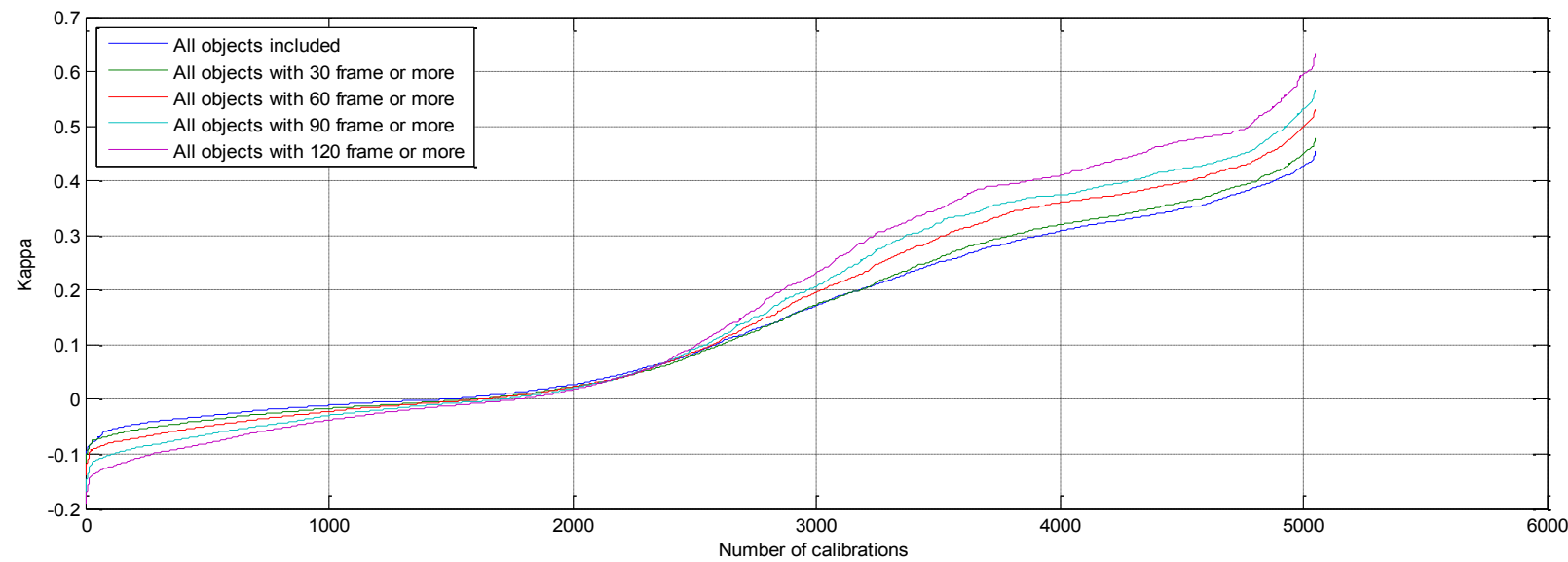

Fig.A.2.2 Cumulative curves show the improvement of Kappa over calibration using the maximum speed

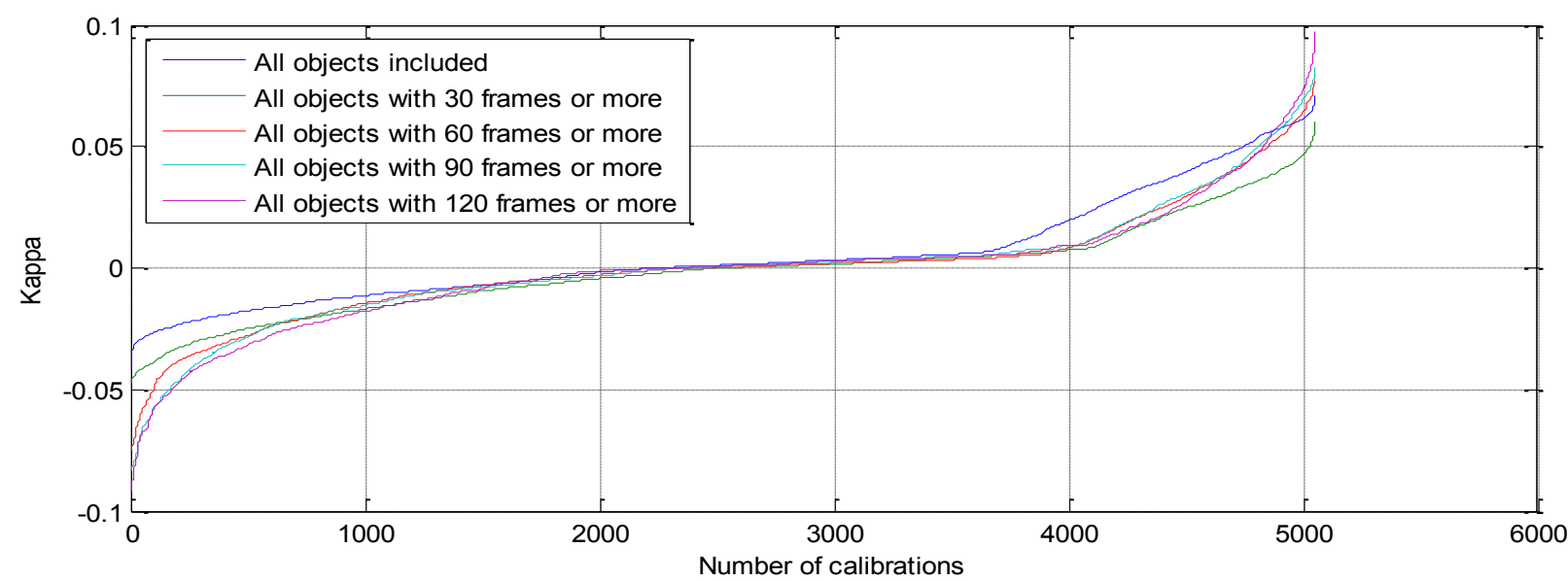

Fig.A.2.3 Cumulative curves show the improvement of Kappa over calibration using the average acceleration 


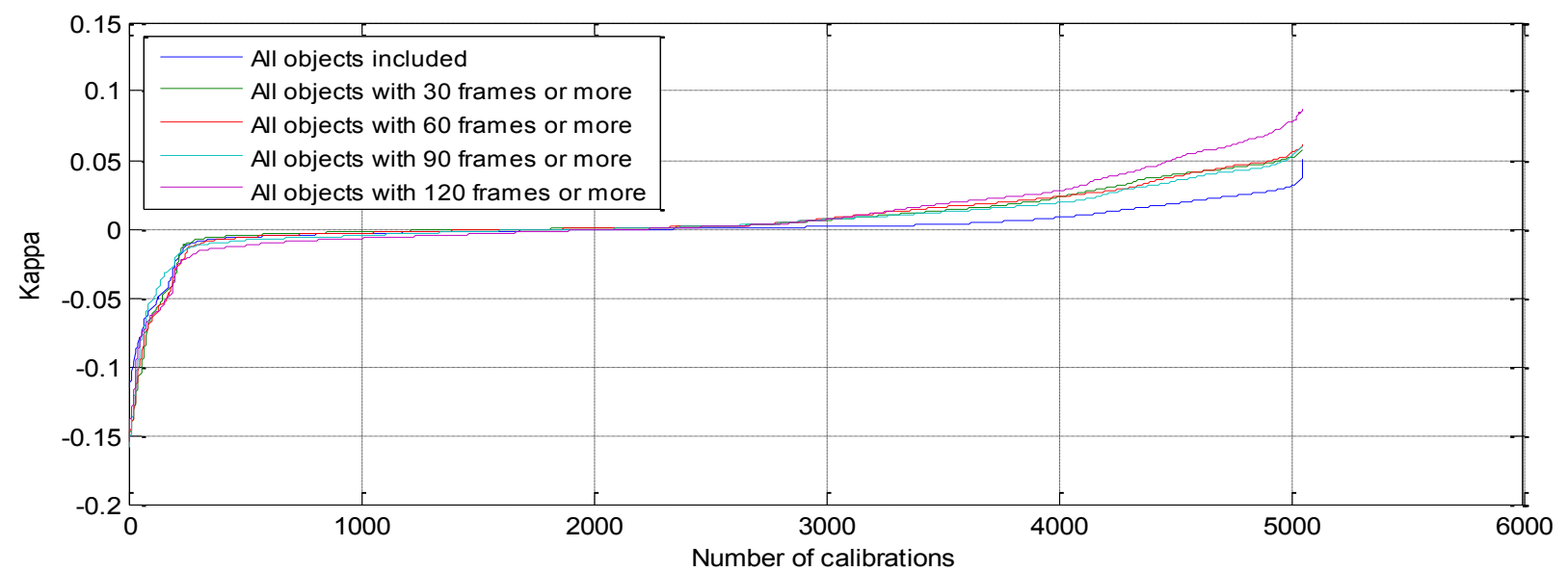

Fig.A.2.4 Cumulative curves show the improvement of Kappa over calibration using the maximum acceleration

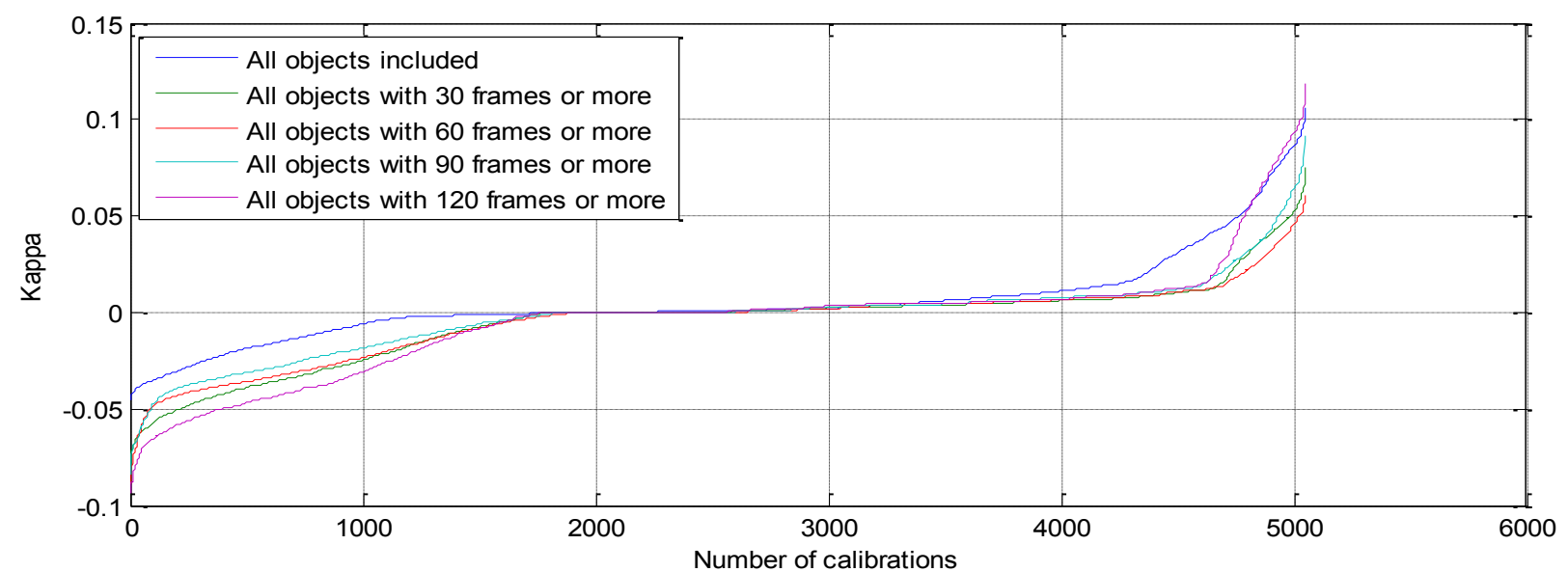

Fig.A.2.5 Cumulative curves show the improvement of Kappa over calibration using the average deceleration

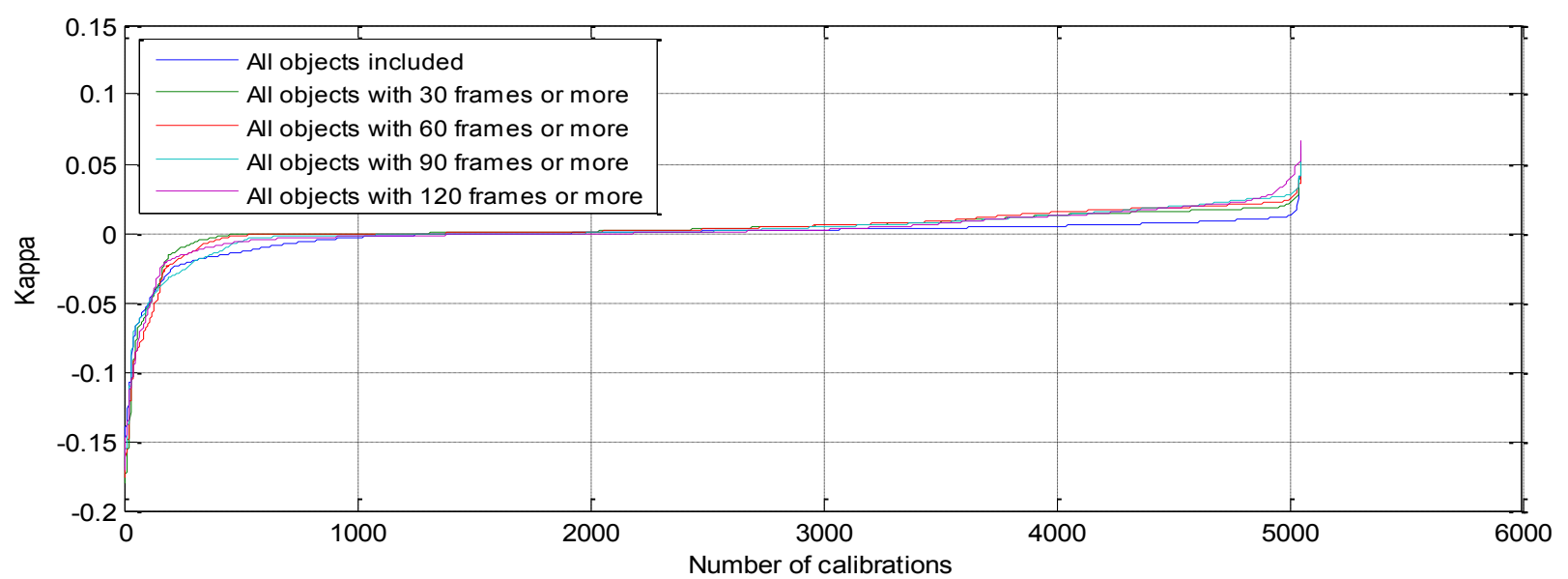

Fig.A.2.6 Cumulative curves show the improvement of Kappa over calibration using the maximum deceleration 


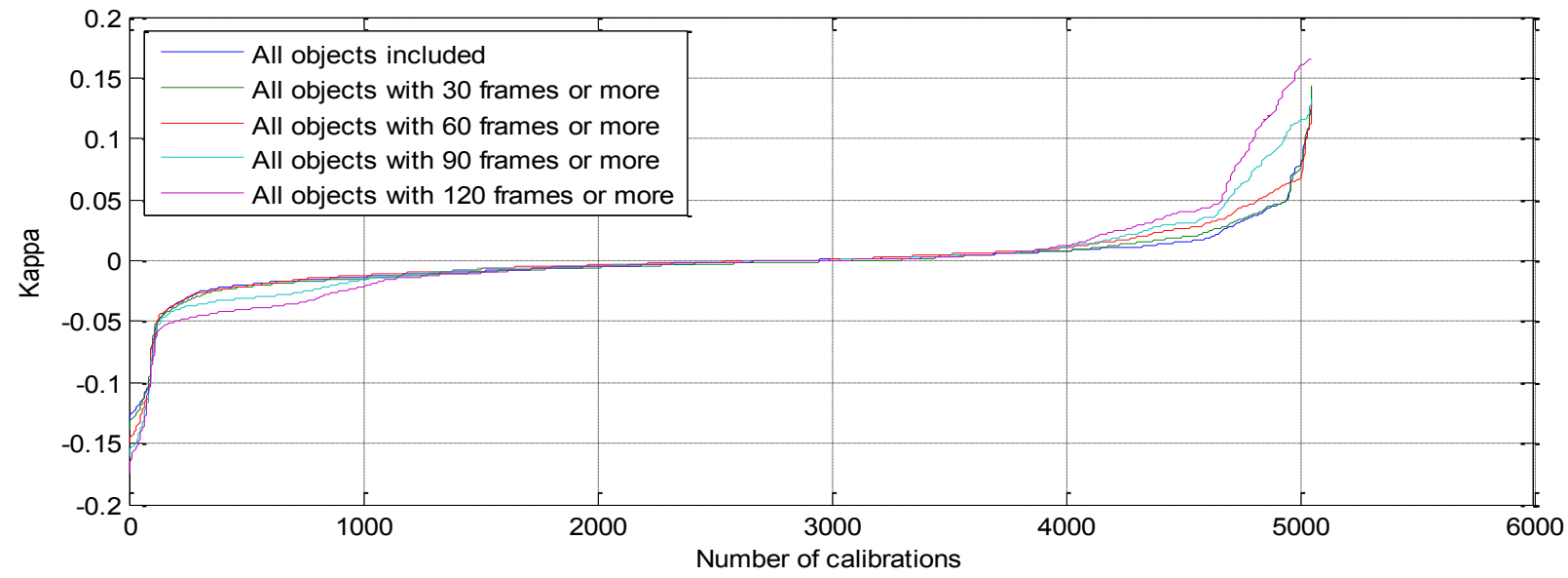

Fig.A.2.7 Cumulative curves show the improvement of Kappa over calibration using the average direction

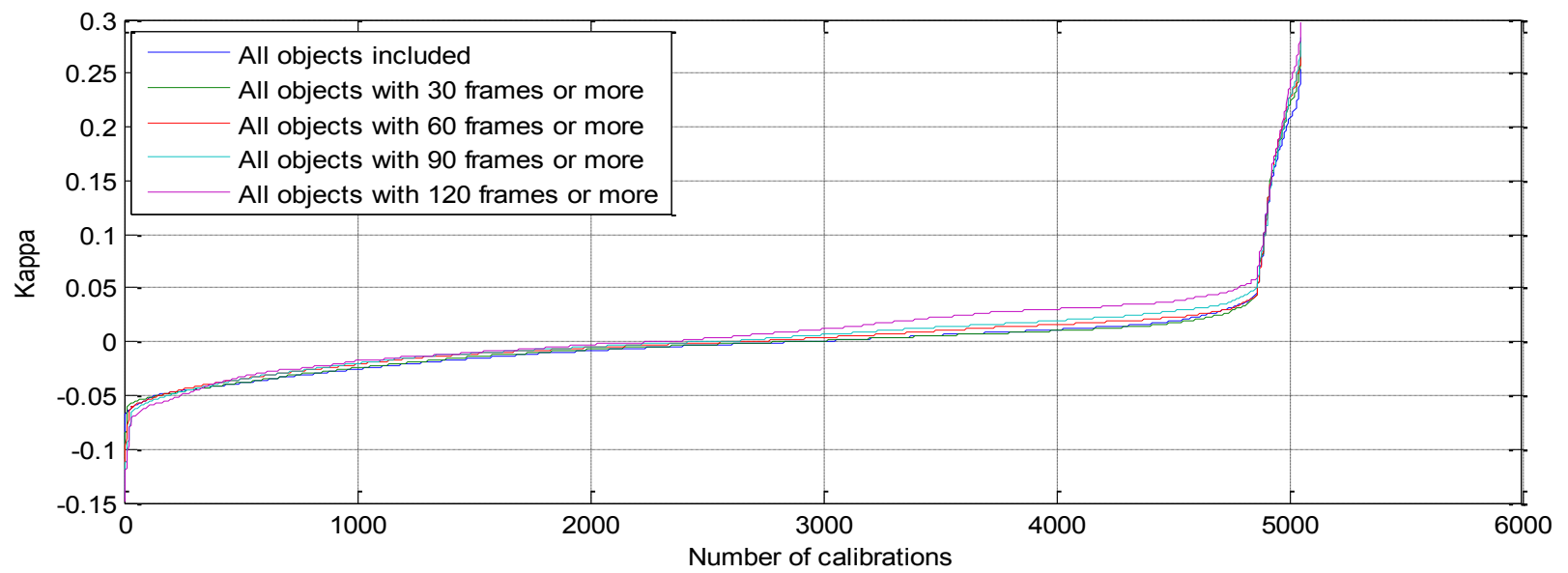

Fig.A.2.8 Cumulative curves show the improvement of Kappa over calibration using the maximum direction

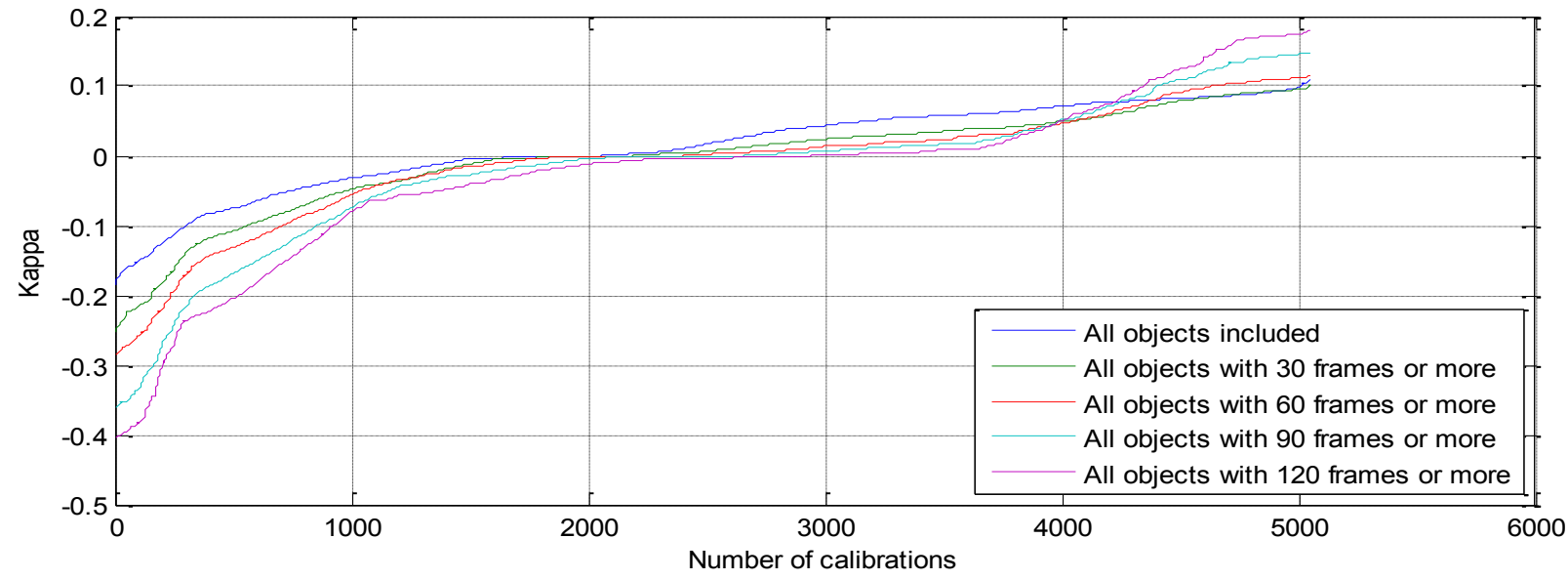

Fig.A.2.9 Cumulative curves show the improvement of Kappa over calibration using the average of change in direction 


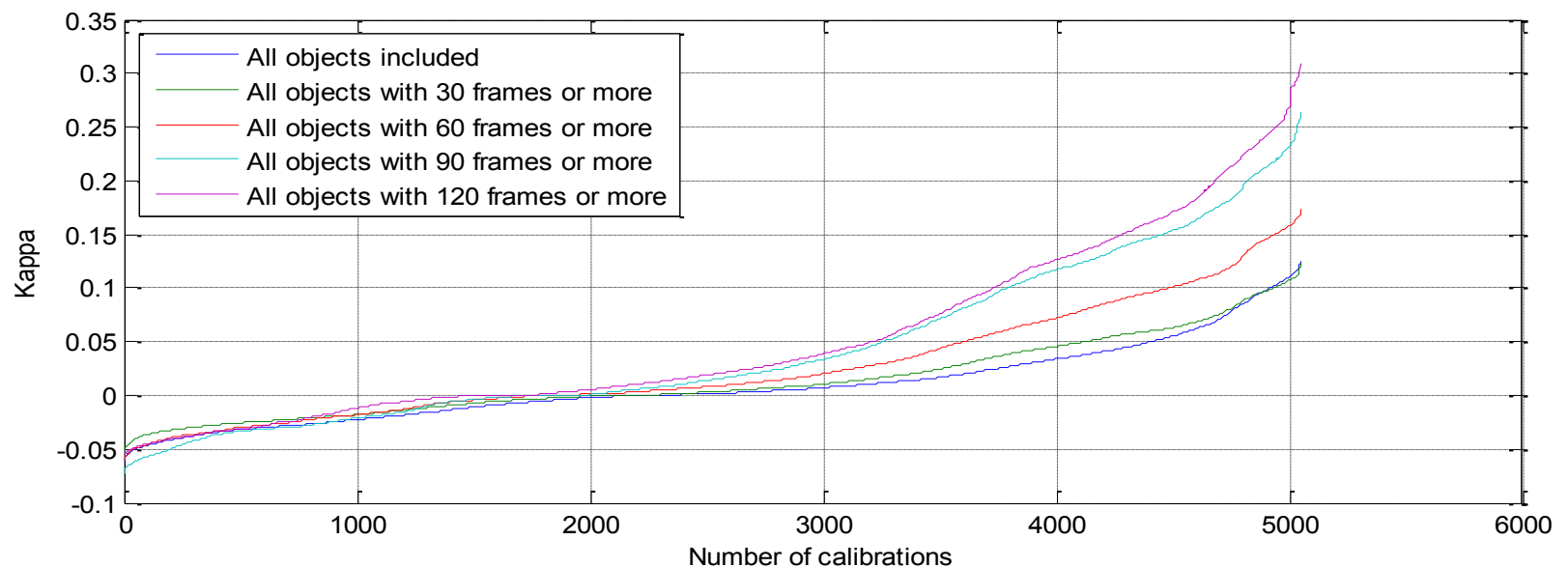

Fig.A.2.10 Cumulative curves show the improvement of Kappa over calibration using the average of cosine of change in direction

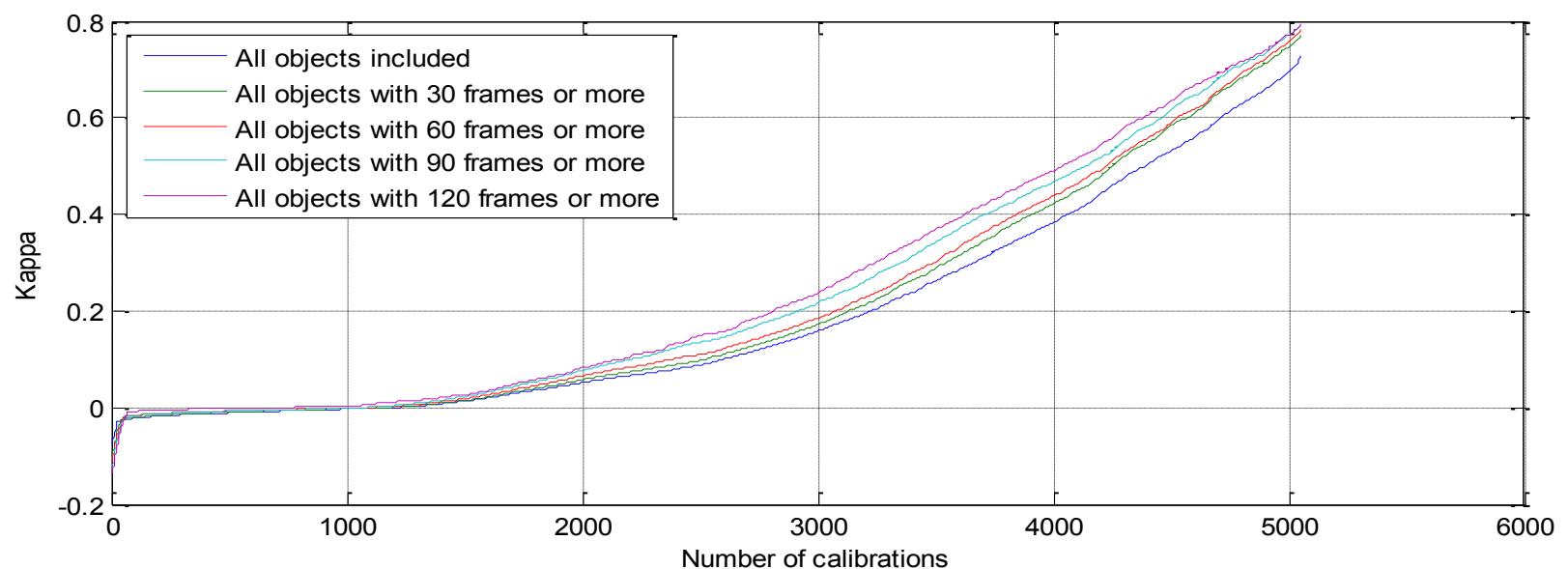

Fig.A.2.11 Cumulative curves show the improvement of Kappa over calibration using the average area

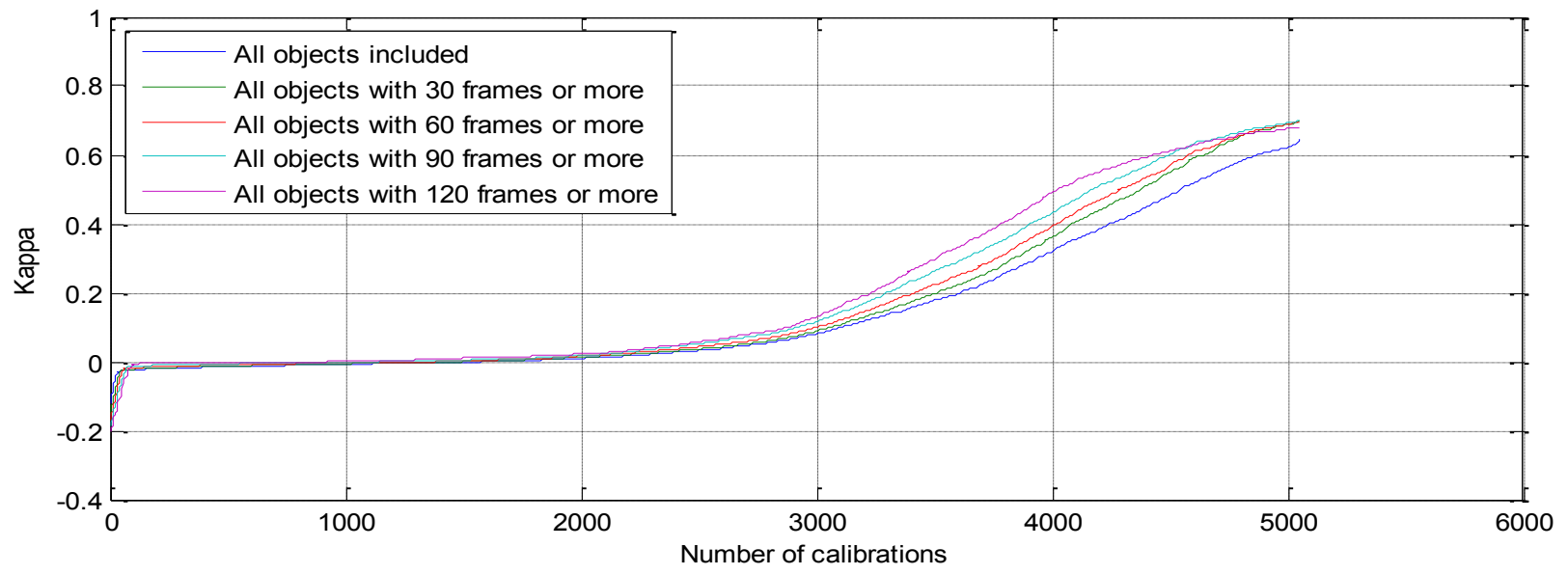

Fig.A.2.12 Cumulative curves show the improvement of Kappa over calibration using the maximum area 


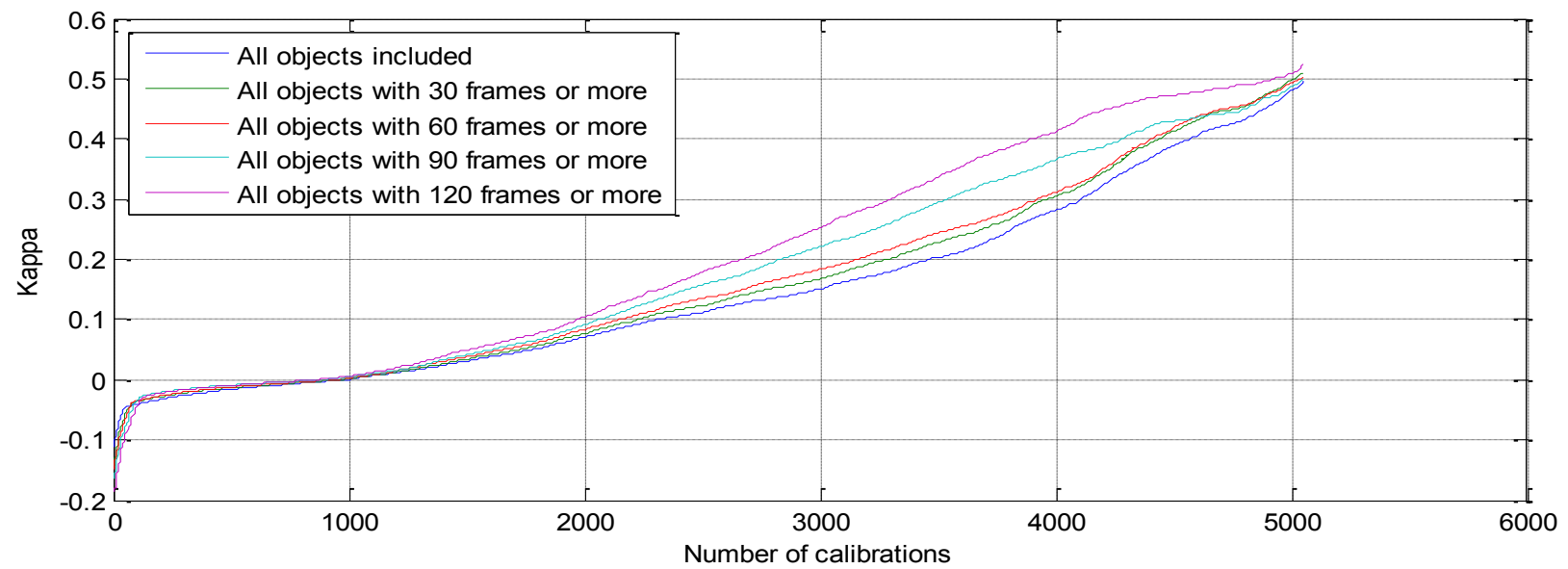

Fig.A.2.13 Cumulative curves show the improvement of Kappa over calibration using the average length

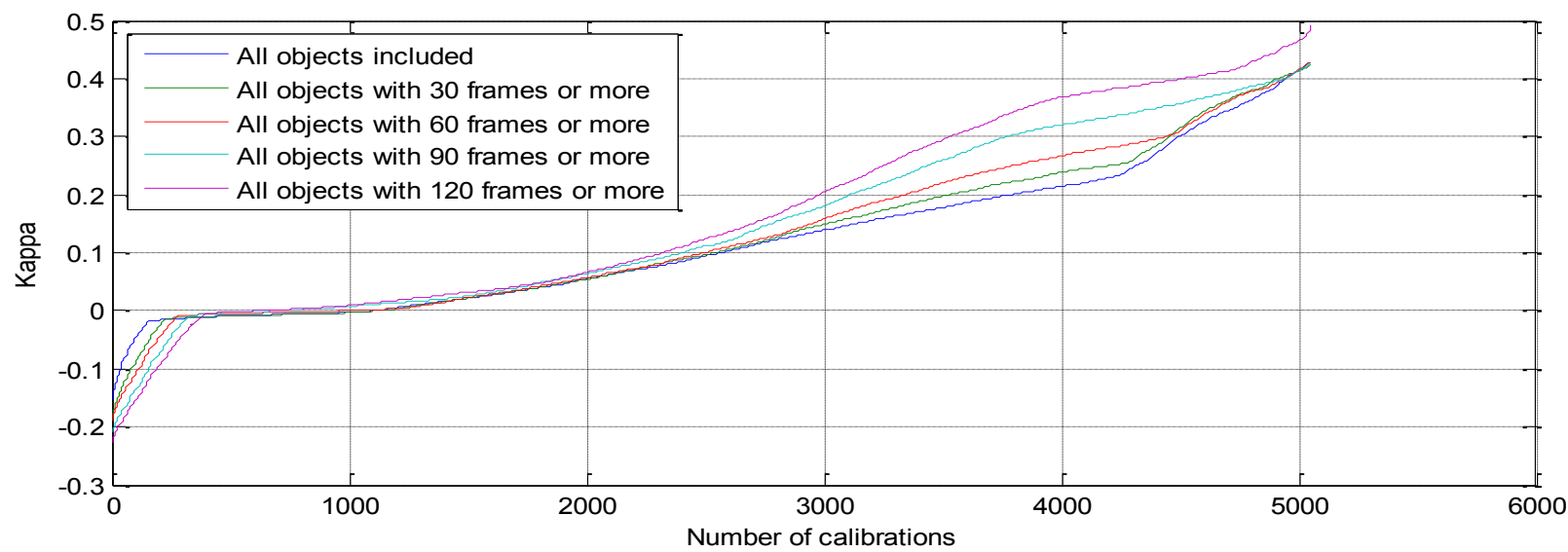

Fig.A.2.14 Cumulative curves show the improvement of Kappa over calibration using the maximum length

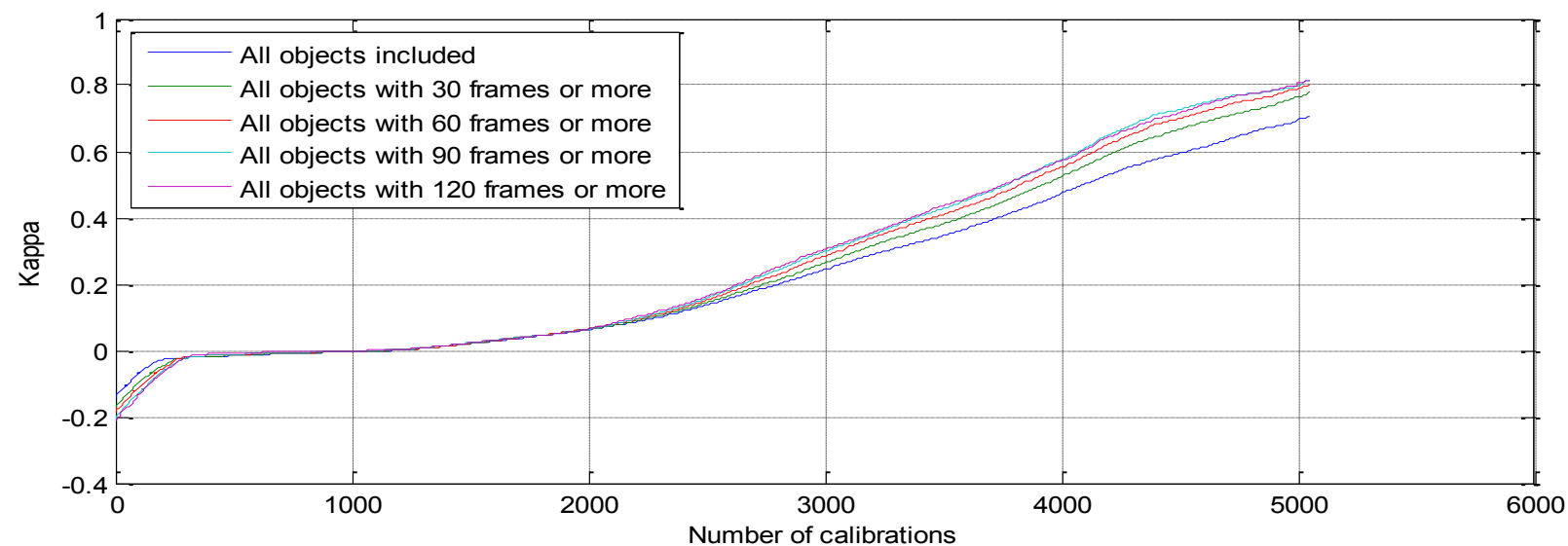

Fig.A.2.15 Cumulative curves show the improvement of Kappa over calibration using the average width 


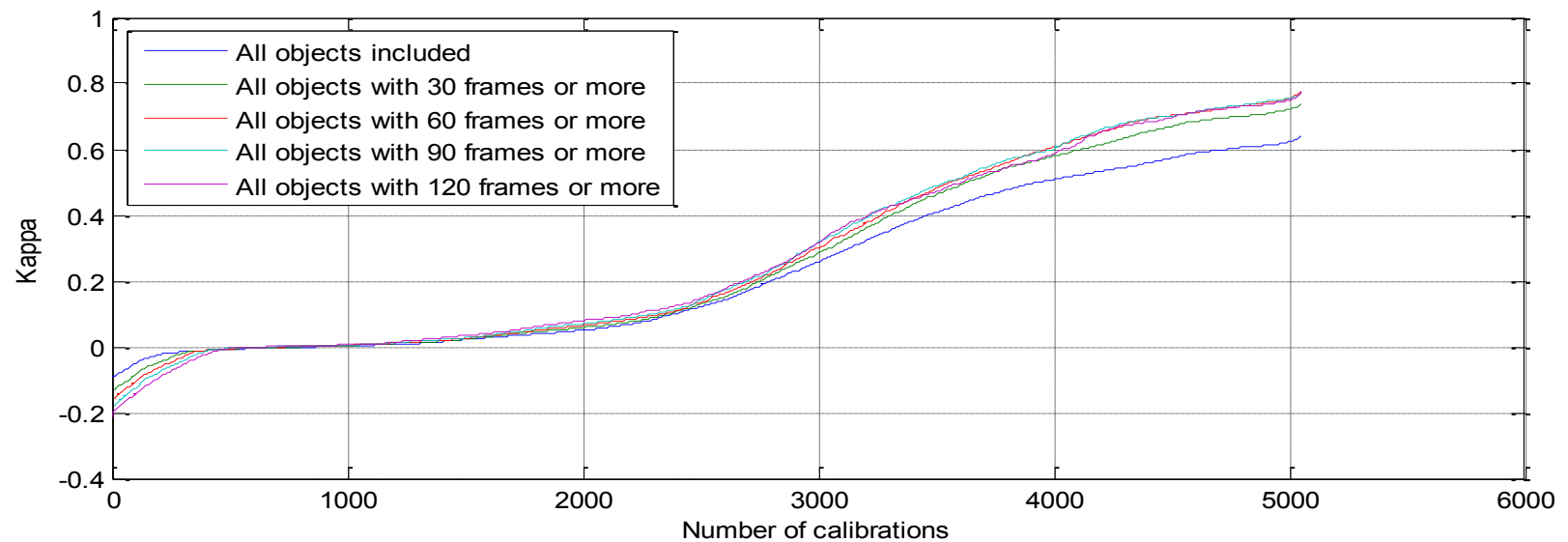

Fig.A.2.16 Cumulative curves show the improvement of Kappa over calibration using the maximum width

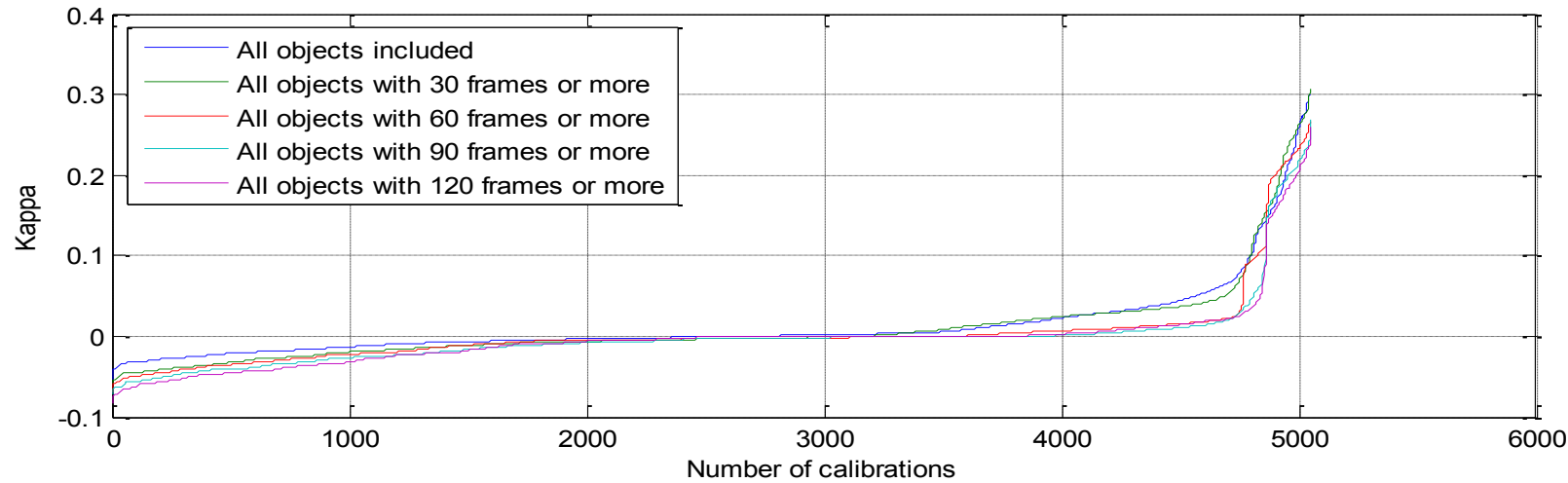

Fig.A.2.17 Cumulative curves show the improvement of Kappa over calibration using the peaks in speed per second

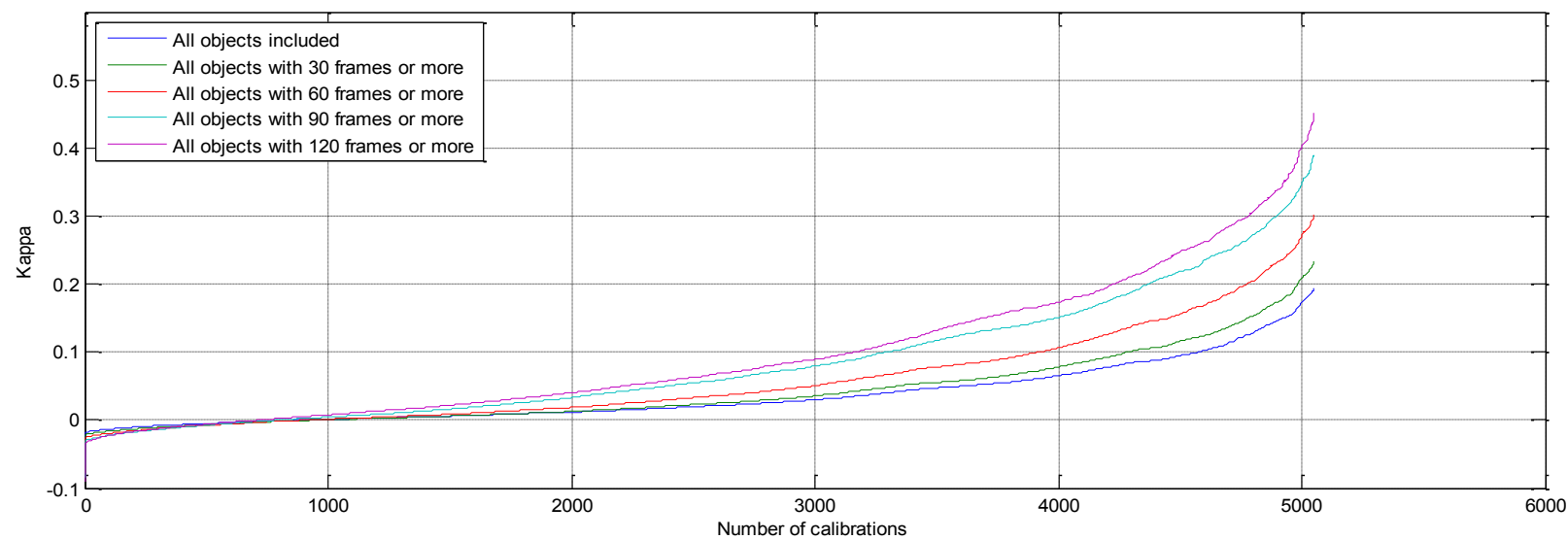

Fig.A.2.18 Cumulative curves show the improvement of Kappa over calibration using the effective weighted average frequency of the highest 5 powers 


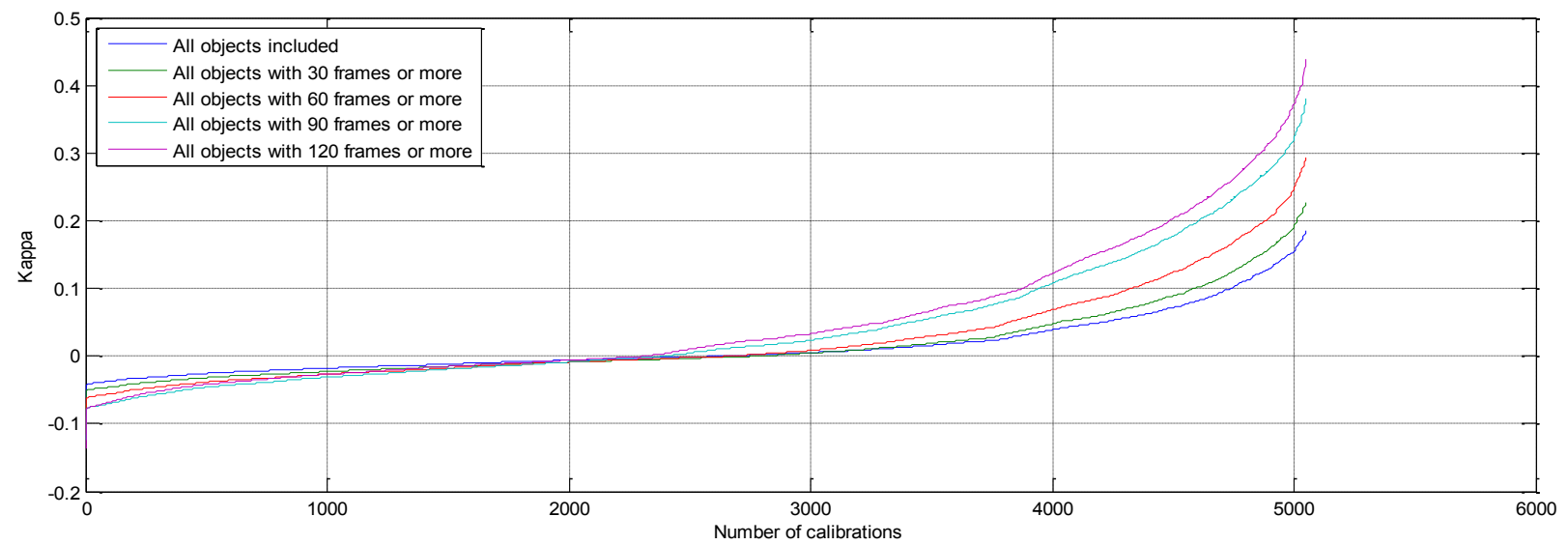

Fig.A.2.19 Cumulative curves show the improve ment of Kappa over calibration using the effective weighted average frequency of the highest 10 powers

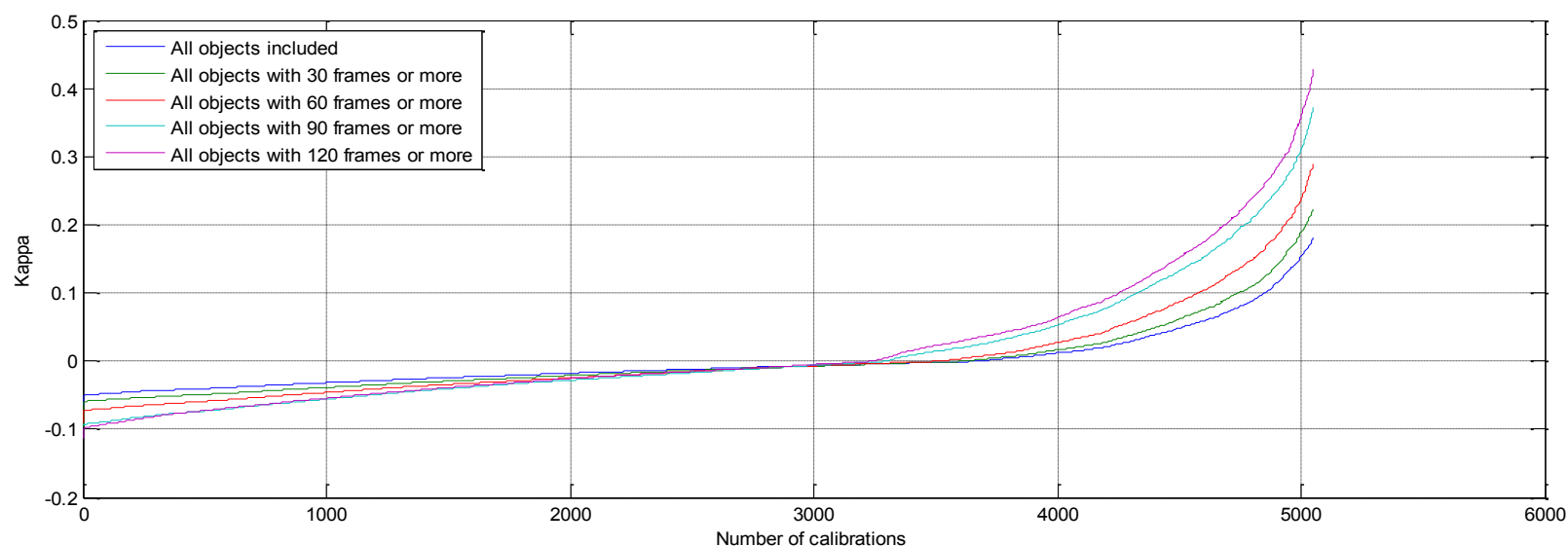

Fig.A.2.20 Cumulative curves show the improve ment of Kappa over calibration using the effective weighted average frequency of the highest 15 powers

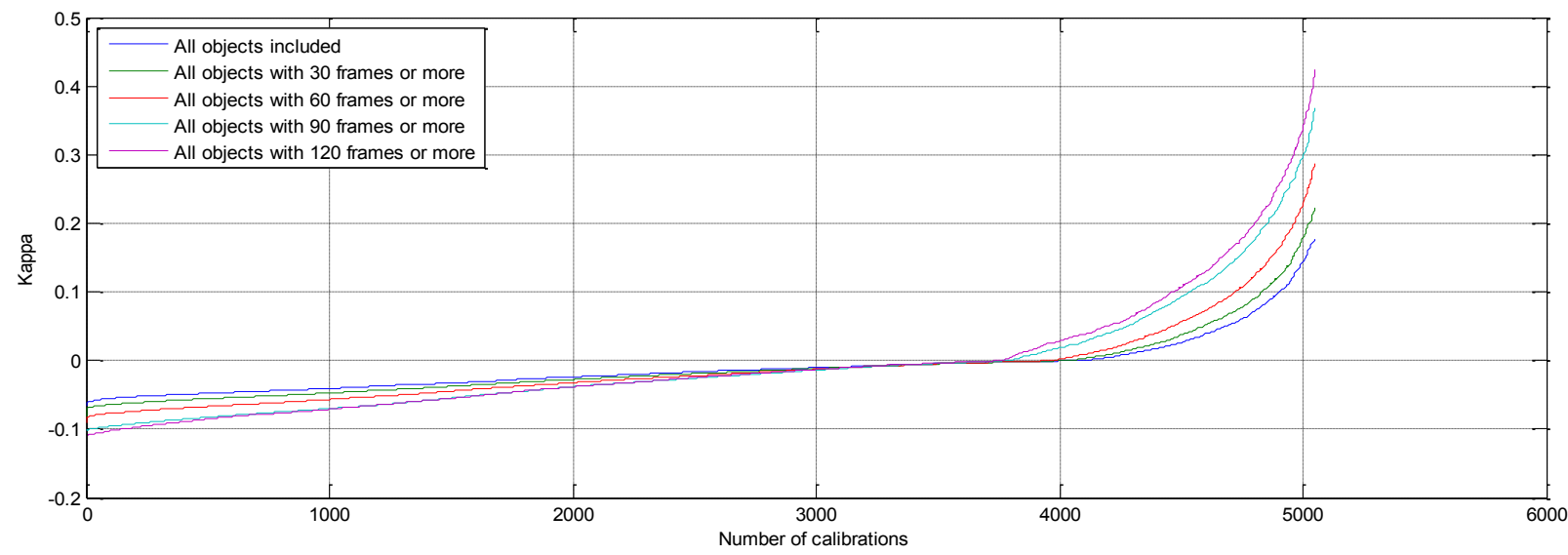

Fig.A.2.21 Cumulative curves show the improvement of Kappa over calibration using the effective weighted average frequency of the highest 20 powers 


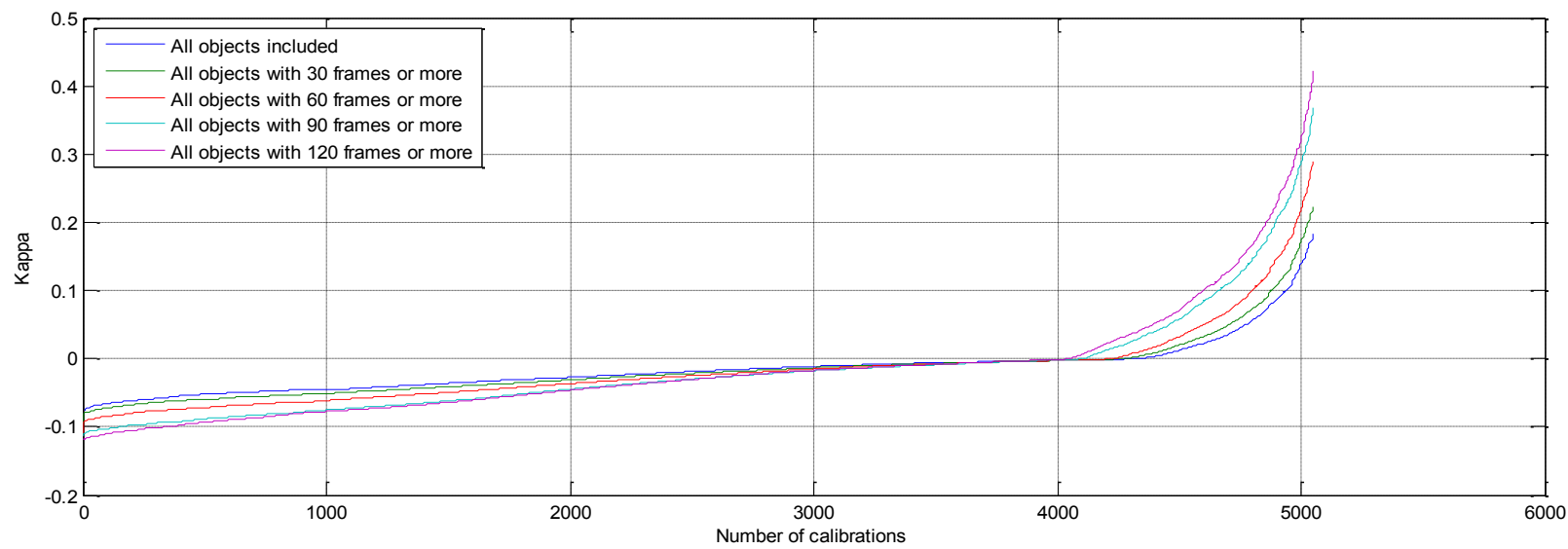

Fig.A.2.22 Cumulative curves show the improve ment of Kappa over calibration using the effective weighted average frequency of the highest 25 powers

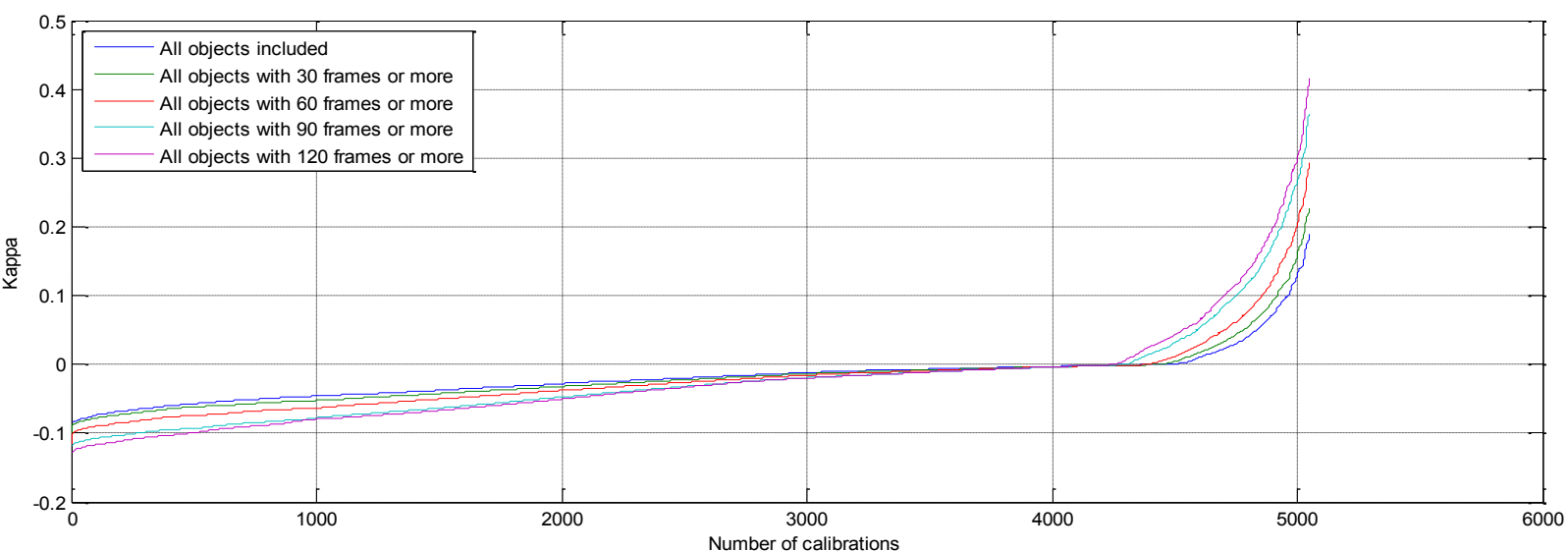

Fig.A.2.23 Cumulative curves show the improve ment of Kappa over calibration using the effective weighted average frequency of the highest 30 powers

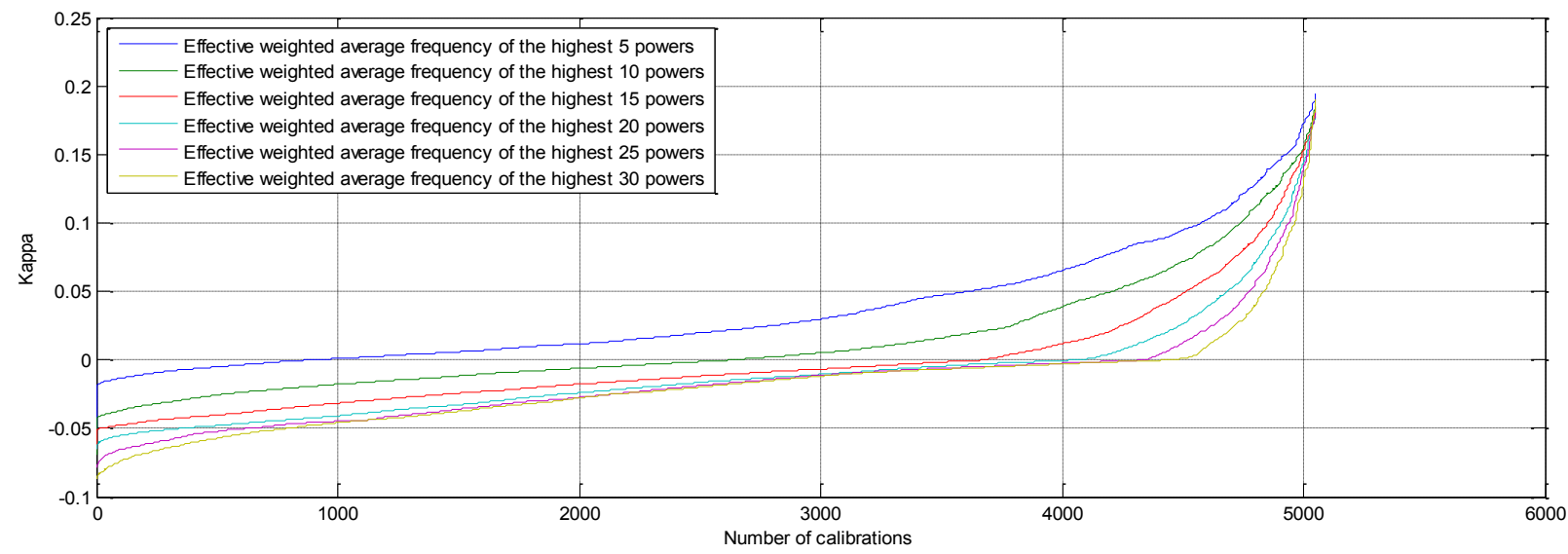

Fig.A.2.24 Cumulative curves show the improvement of Kappa over calibration using the effective weighted average frequency where the minimum length of appearance for tracked object is 0 frames or more. 


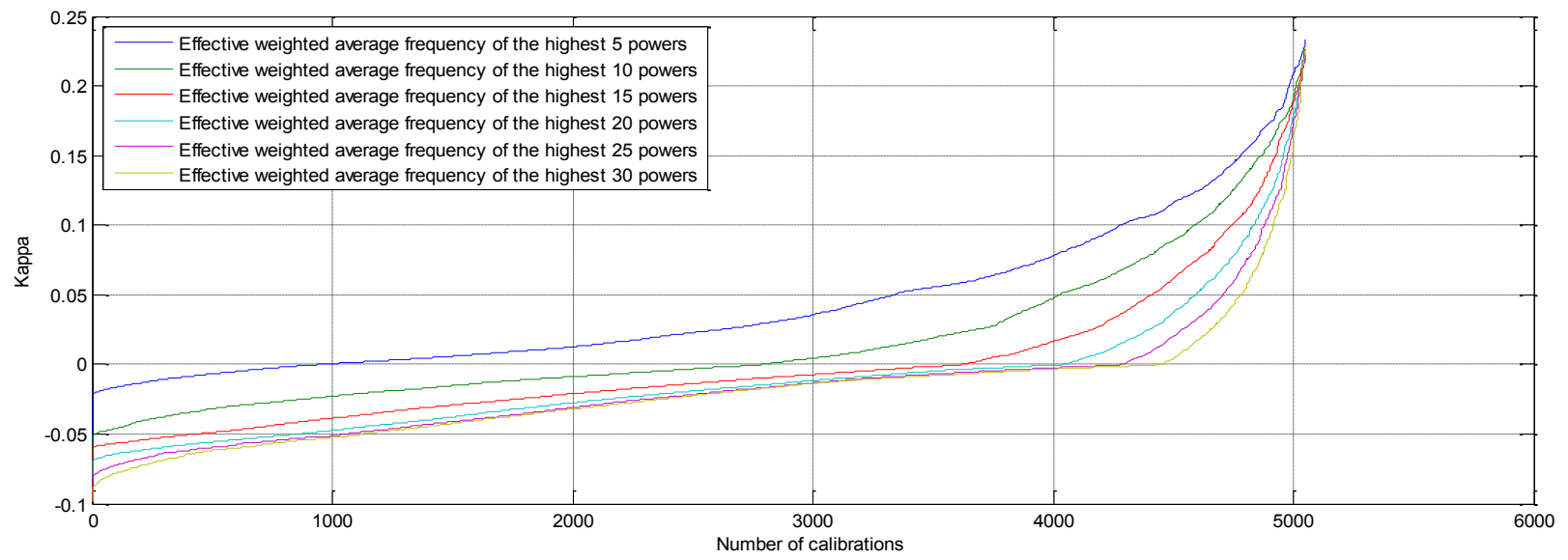

Fig.A.2.25 Cumulative curves show the improvement of Kappa over calibration using the effective weighted average frequency where the minimum length of appearance for tracked object is 30 frames or more.

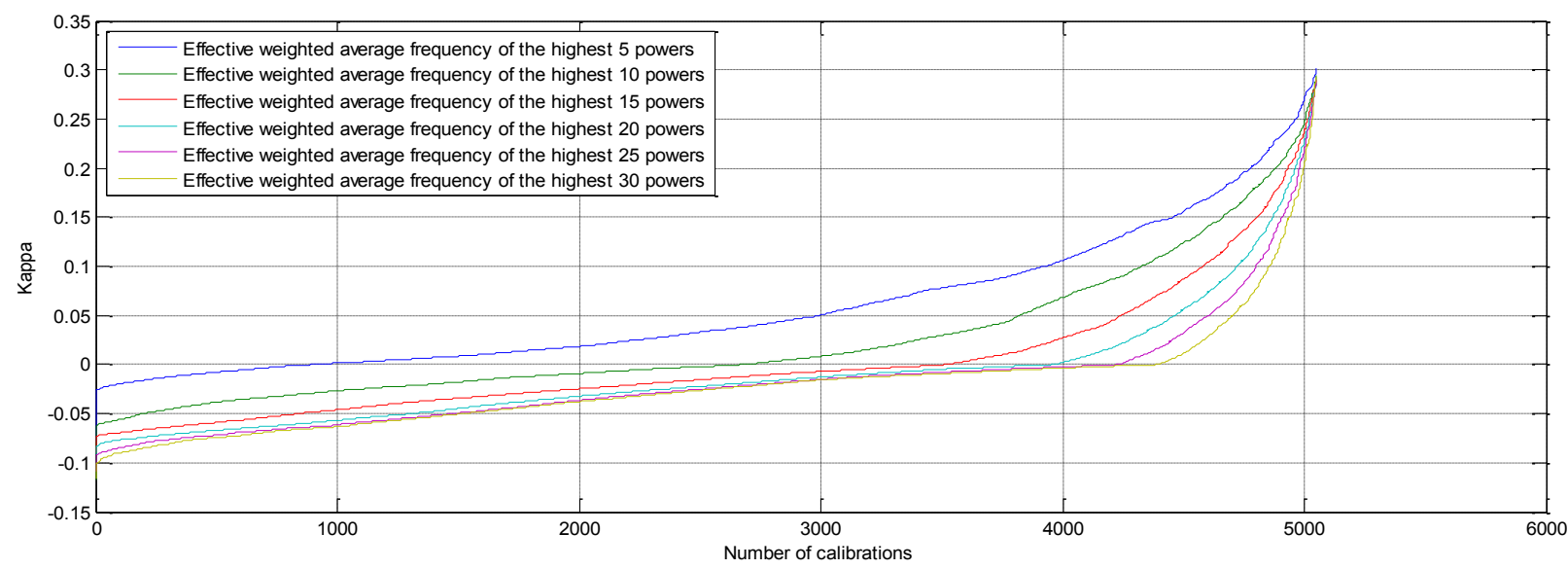

Fig.A.2.26 Cumulative curves show the improvement of Kappa over calibration using the effective weighted average frequency where the minimum length of appearance for tracked object is 60 frames or more.

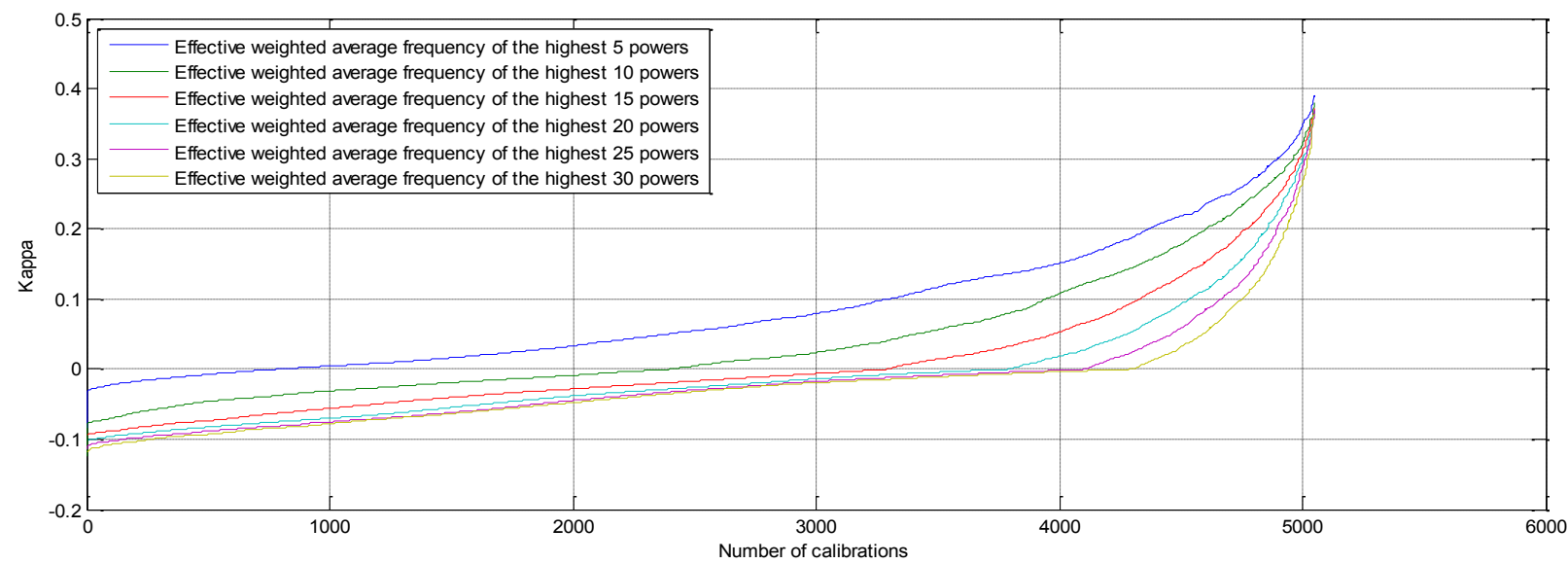

Fig.A.2.27 Cumulative curves show the improvement of Kappa over calibration using the effective weighted average frequency where the minimum length of appearance for tracked object is 90 frames or more. 


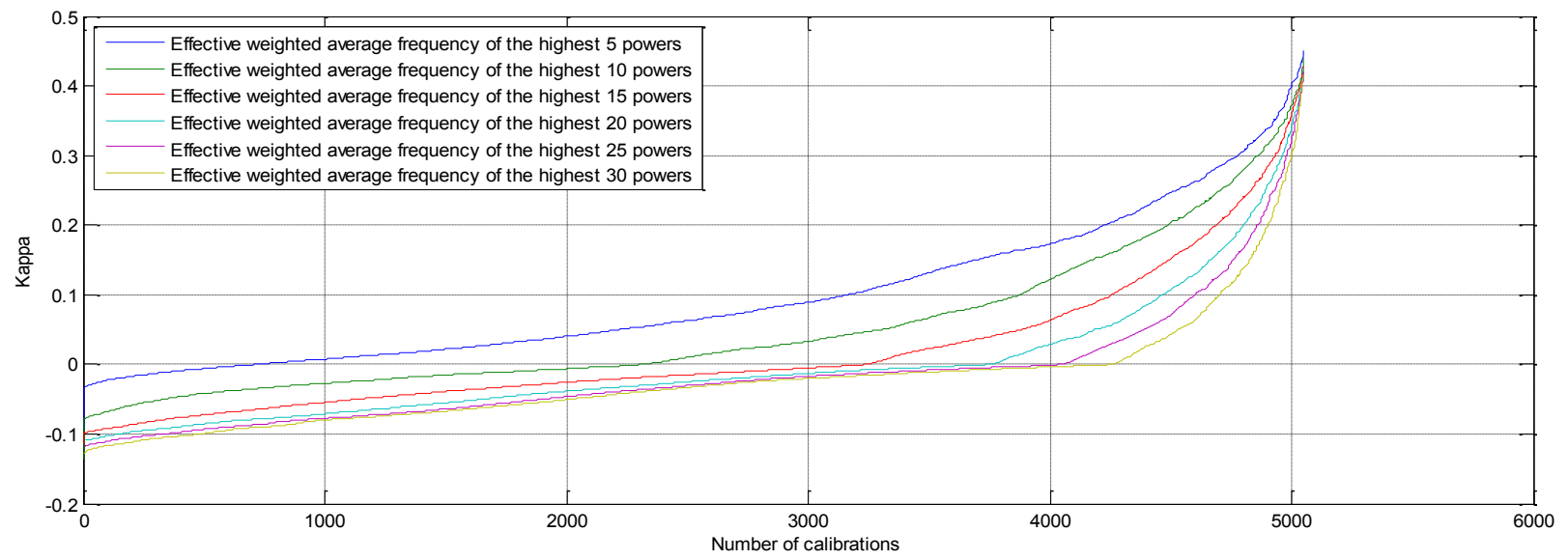

Fig.A.2.28 Cumulative curves show the improvement of Kappa over calibration using the effective weighted average frequency where the minimum length of appearance for tracked object is 120 frames or more.

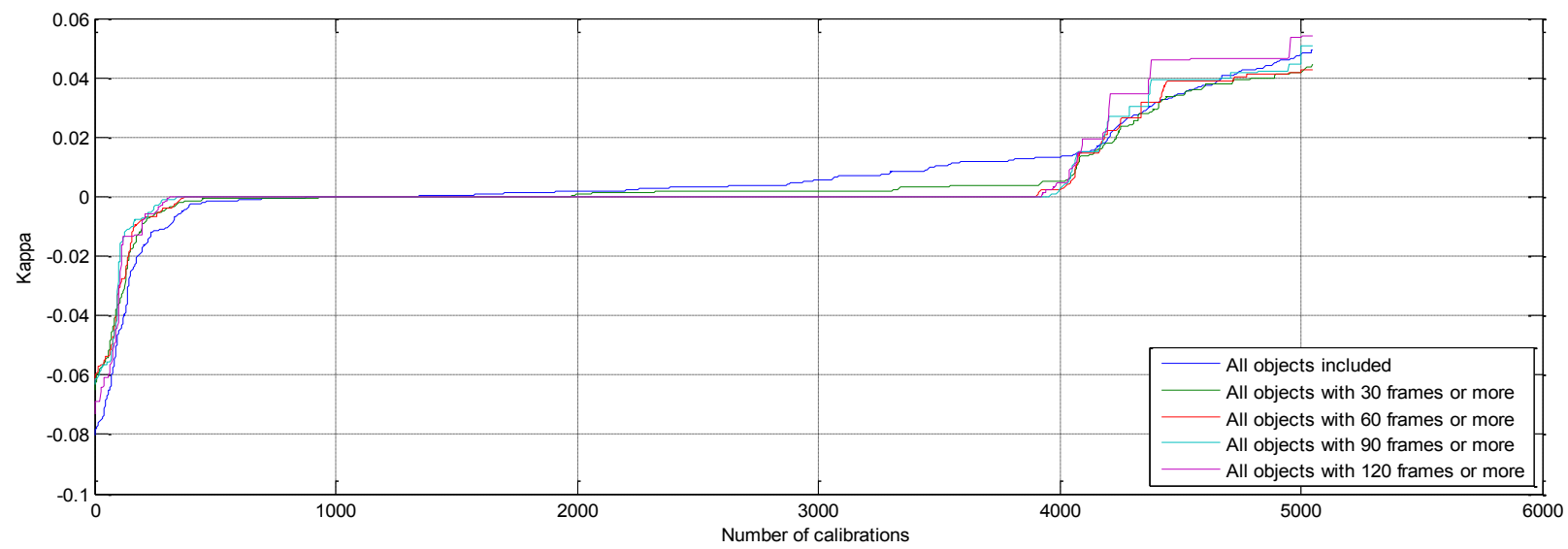

Fig.A.2.29 Cumulative curves show the improvement of Kappa over calibration using the effective frequency of the highest power.

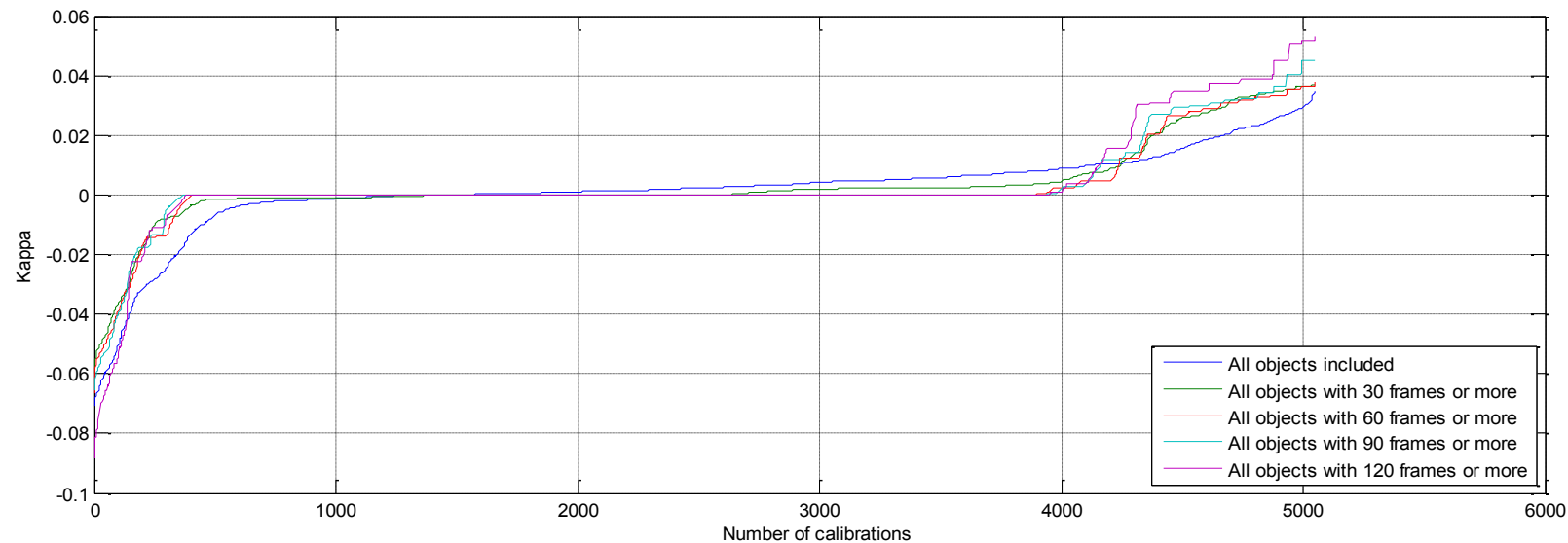

Fig.A.2.30 Cumulative curves show the improvement of Kappa over calibration using the effective frequency of the $2^{\text {nd }}$ highest power. 


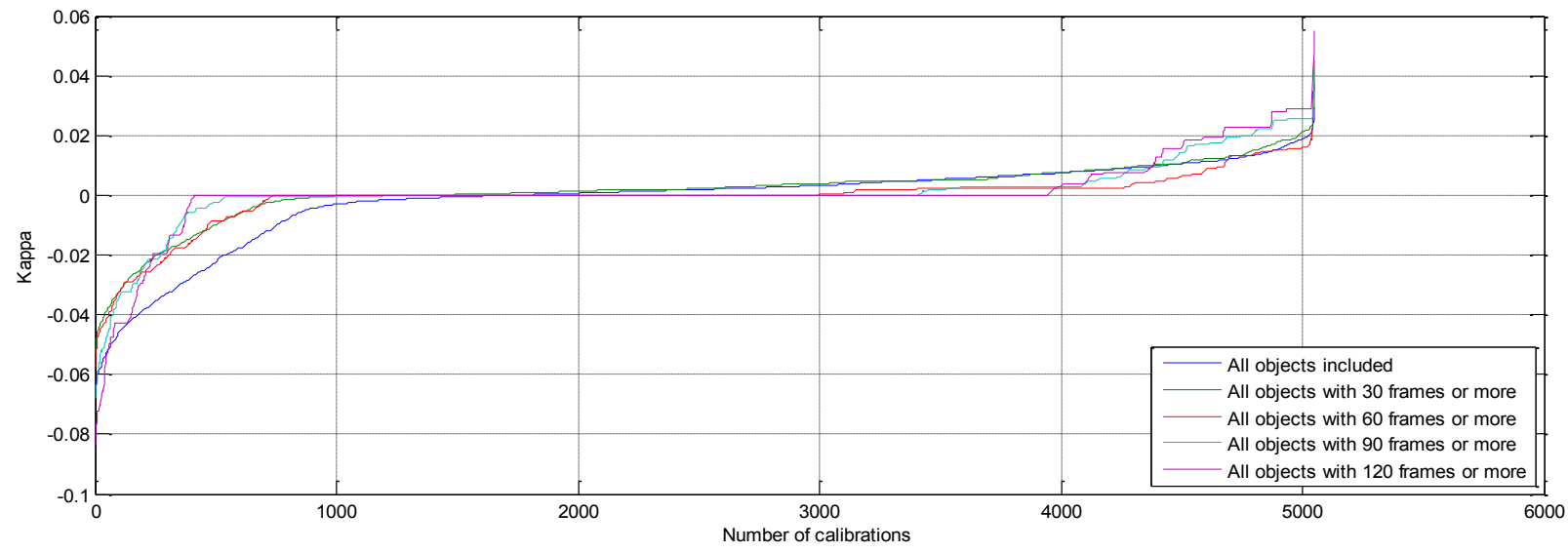

Fig.A.2.31 Cumulative curves show the improvement of Kappa over calibration using the effective frequency of the $3^{\text {rd }}$ highest power.

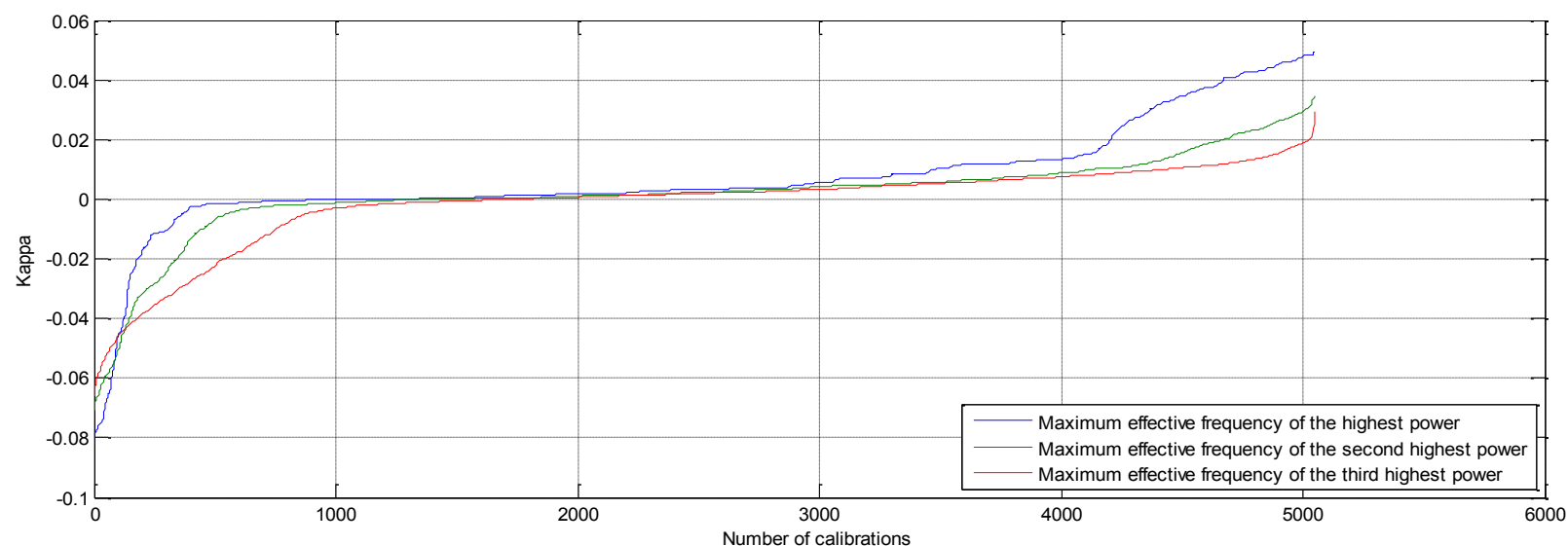

Fig.A.2.32 Cumulative curves show the improve ment of Kappa over calibration using the effective frequency where the minimum length of appearance for tracked object is 0 frames or more.

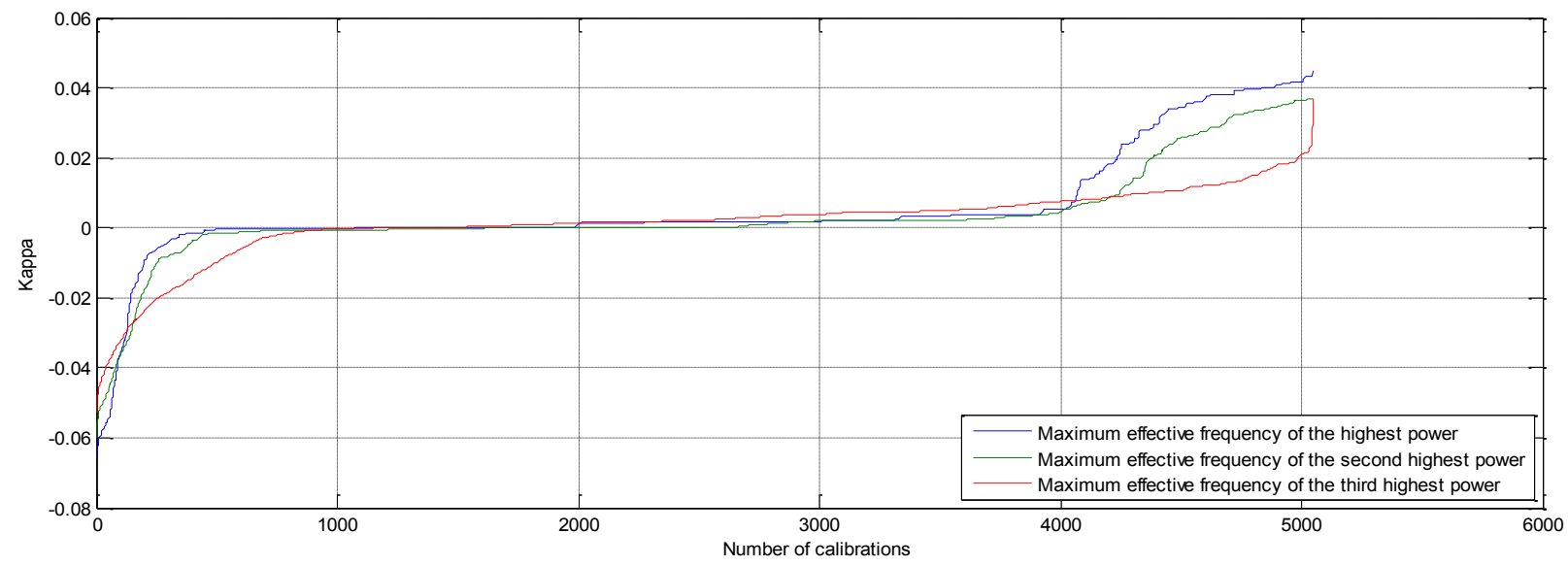

Fig.A.2.33 Cumulative curves show the improvement of Kappa over calibration using the effective frequency where the minimum length of appearance for tracked object is 30 frames or more. 


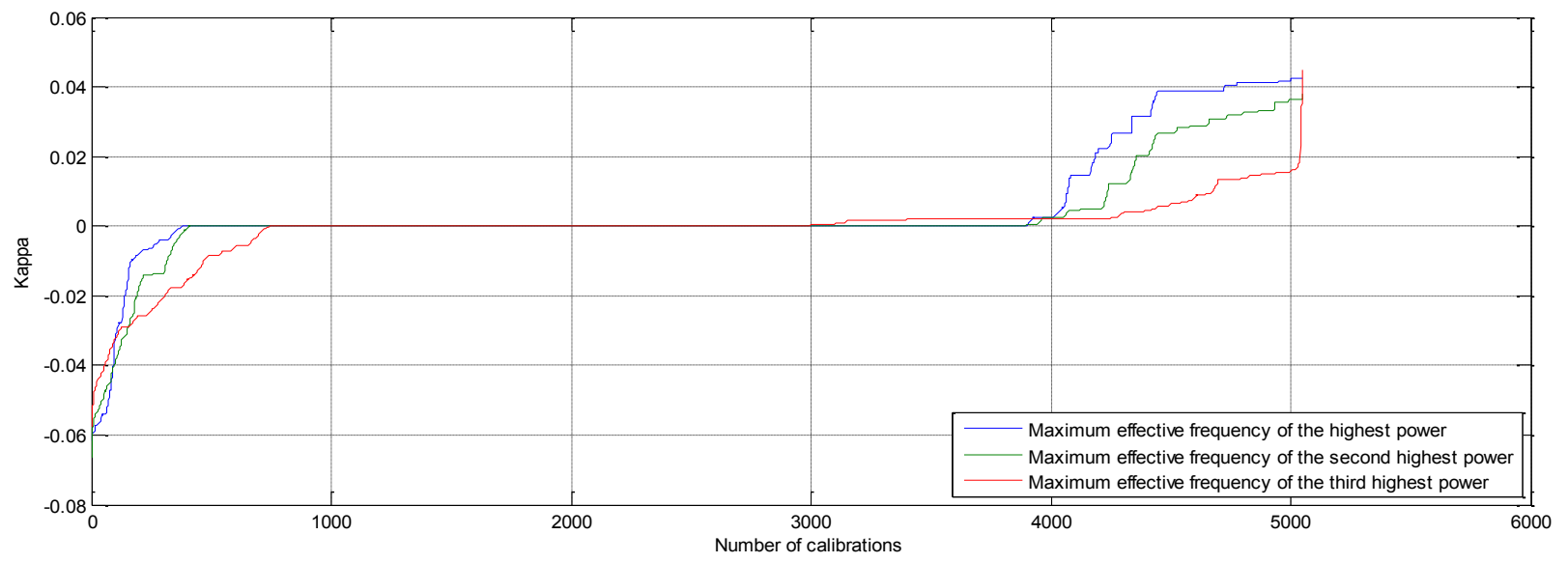

Fig.A.2.34 Cumulative curves show the improvement of Kappa over calibration using the effective frequency where the minimum length of appearance for tracked object is 60 frames or more.

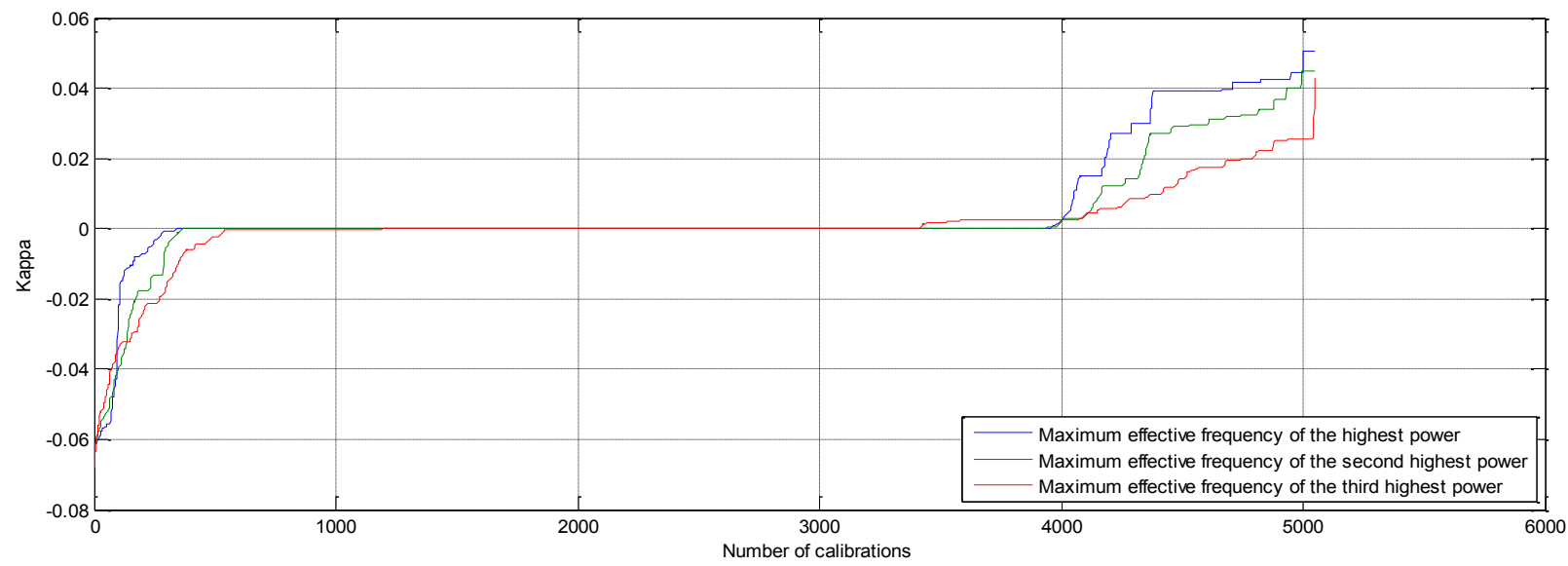

Fig.A.2.35 Cumulative curves show the improve ment of Kappa over calibration using the effective frequency where the minimum length of appearance for tracked object is 90 frames or more.

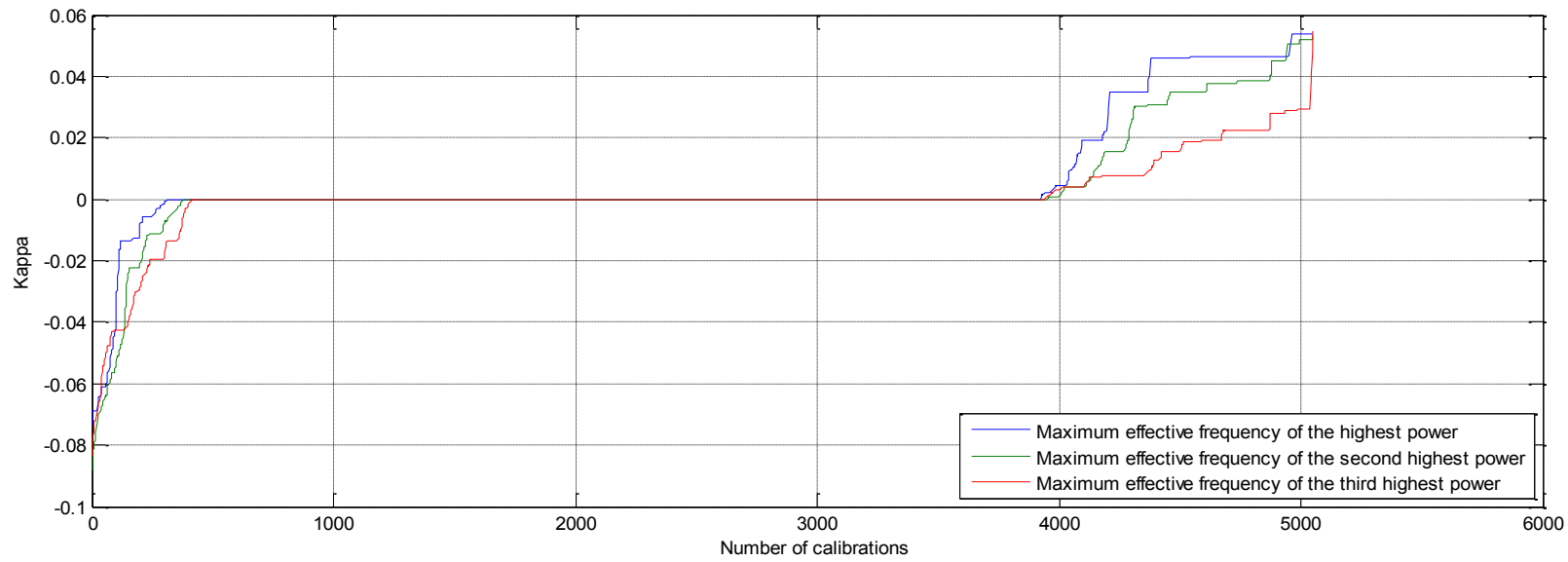

Fig.A.2.36 Cumulative curves show the improve ment of Kappa over calibration using the effective frequency where the minimum length of appearance for tracked object is $\mathbf{1 2 0}$ frames or more. 


\section{Appendix B}

The Results of the Calibrations and Validations for the first model "Vehicles, Cyclists and Pedestrians" 


\section{B.1 The results of calibrations and validations using Kappa statistics}

Table B.1.1 the results of calibration procedure 1 and its validations.

\begin{tabular}{|c|c|c|c|c|c|c|c|}
\hline \multicolumn{4}{|c|}{ Configurations } & \multirow{2}{*}{$\begin{array}{c}\text { Calibration } \\
\text { Kappa } \\
\end{array}$} & \multirow{2}{*}{$\frac{1^{\text {st }} \text { Validation }}{\text { Kappa }}$} & \multirow{2}{*}{$\begin{array}{c}2^{\text {nd }} \text { Validation } \\
\text { Kappa }\end{array}$} & \multirow{2}{*}{$\begin{array}{c}\mathbf{3}^{\text {rd }} \text { Validation } \\
\text { Kappa } \\
\end{array}$} \\
\hline$\varphi_{g}{ }^{29}$ & $\Lambda_{l}^{29}$ & $\Gamma_{n}{ }^{29}$ & $\eta_{m}{ }^{29}$ & & & & \\
\hline 1 & 1 & 1 & 1 & 0.92 & 0.88 & 0.89 & 0.89 \\
\hline 1 & 1 & 1 & 2 & 0.93 & 0.88 & 0.90 & 0.89 \\
\hline 1 & 1 & 1 & 3 & 0.93 & 0.88 & 0.89 & 0.89 \\
\hline 1 & 1 & 2 & 1 & 0.92 & 0.88 & 0.88 & 0.88 \\
\hline 1 & 1 & 2 & 2 & 0.93 & 0.88 & 0.89 & 0.89 \\
\hline 1 & 1 & 2 & 3 & 0.93 & 0.88 & 0.89 & 0.89 \\
\hline 1 & 1 & 3 & 1 & 0.92 & 0.87 & 0.90 & 0.89 \\
\hline 1 & 1 & 3 & 2 & 0.93 & 0.88 & 0.89 & 0.89 \\
\hline 1 & 1 & 3 & 3 & 0.93 & 0.87 & 0.89 & 0.88 \\
\hline 1 & 1 & 4 & 1 & 0.93 & 0.87 & 0.89 & 0.89 \\
\hline 1 & 1 & 4 & 2 & 0.93 & 0.88 & 0.89 & 0.89 \\
\hline 1 & 1 & 4 & 3 & 0.93 & 0.88 & 0.89 & 0.89 \\
\hline 1 & 1 & 1 & 1 & 0.93 & 0.88 & 0.89 & 0.89 \\
\hline 1 & 1 & 1 & 2 & 0.93 & 0.89 & 0.89 & 0.89 \\
\hline 1 & 1 & 1 & 3 & 0.93 & 0.88 & 0.89 & 0.89 \\
\hline 1 & 1 & 6 & 1 & 0.93 & 0.88 & 0.89 & 0.89 \\
\hline 1 & 1 & 6 & 2 & 0.93 & 0.88 & 0.89 & 0.89 \\
\hline 1 & 1 & 6 & 3 & 0.93 & 0.88 & 0.88 & 0.88 \\
\hline 1 & 2 & 1 & 1 & 0.92 & 0.84 & $--^{30}$ & - \\
\hline 1 & 2 & 1 & 2 & 0.91 & 0.84 & - & - \\
\hline 1 & 2 & 1 & 3 & 0.92 & 0.84 & - & - \\
\hline 1 & 2 & 2 & 1 & 0.92 & 0.85 & - & - \\
\hline 1 & 2 & 2 & 2 & 0.91 & 0.84 & - & - \\
\hline 1 & 2 & 2 & 3 & 0.91 & 0.84 & - & - \\
\hline 1 & 2 & 3 & 1 & 0.92 & 0.86 & - & - \\
\hline 1 & 2 & 3 & 2 & 0.91 & 0.85 & - & - \\
\hline 1 & 2 & 3 & 3 & 0.91 & 0.85 & - & - \\
\hline 1 & 2 & 4 & 1 & 0.91 & - & - & - \\
\hline 1 & 2 & 4 & 2 & 0.91 & - & - & - \\
\hline 1 & 2 & 4 & 3 & 0.91 & - & - & - \\
\hline 1 & 2 & 1 & 1 & 0.92 & 0.84 & - & - \\
\hline 1 & 2 & 1 & 2 & 0.91 & 0.84 & - & - \\
\hline 1 & 2 & 1 & 3 & 0.91 & 0.83 & - & - \\
\hline 1 & 2 & 6 & 1 & 0.92 & 0.84 & - & - \\
\hline 1 & 2 & 6 & 2 & 0.91 & 0.83 & - & - \\
\hline 1 & 2 & 6 & 3 & 0.91 & 0.83 & - & - \\
\hline 1 & 3 & 1 & 1 & 0.92 & - & - & - \\
\hline 1 & 3 & 1 & 2 & 0.92 & - & - & - \\
\hline 1 & 3 & 1 & 3 & 0.91 & - & - & - \\
\hline 1 & 3 & 2 & 1 & 0.92 & - & - & - \\
\hline 1 & 3 & 2 & 2 & 0.92 & - & - & - \\
\hline 1 & 3 & 2 & 3 & 0.91 & - & - & - \\
\hline 1 & 3 & 3 & 1 & 0.92 & - & - & - \\
\hline 1 & 3 & 3 & 2 & 0.92 & - & - & - \\
\hline 1 & 3 & 3 & 3 & 0.91 & - & - & - \\
\hline
\end{tabular}

29 The meaning of the symbols is in section 3.5

30 The meaning of (-) are: 1 . Has Kappa coefficient $\leq 0$, or

2. Failed the null hypothes is test as explained in 3.5.2. 
Table B.1.1 the results of calibration procedure 1 and its validations.

\begin{tabular}{|c|c|c|c|c|c|c|c|}
\hline \multicolumn{4}{|c|}{ Configurations } & \multirow{2}{*}{$\begin{array}{c}\text { Calibration } \\
\text { Kappa }\end{array}$} & \multirow{2}{*}{$\begin{array}{c}\mathbf{1}^{\text {st }} \text { Validation } \\
\text { Kappa }\end{array}$} & \multirow{2}{*}{$\begin{array}{c}2^{\text {nd }} \text { Validation } \\
\text { Kappa }\end{array}$} & \multirow{2}{*}{$\begin{array}{c}3^{\text {rd }} \text { Validation } \\
\text { Kappa }\end{array}$} \\
\hline$\varphi_{g}{ }^{29}$ & $\Lambda_{l}^{29}$ & $\Gamma_{n}{ }^{29}$ & $\eta_{m}{ }^{29}$ & & & & \\
\hline 1 & 3 & 4 & 1 & 0.92 & - & - & - \\
\hline 1 & 3 & 4 & 2 & 0.92 & - & - & - \\
\hline 1 & 3 & 4 & 3 & 0.91 & - & - & - \\
\hline 1 & 3 & 1 & 1 & 0.92 & - & - & - \\
\hline 1 & 3 & 1 & 2 & 0.92 & - & - & - \\
\hline 1 & 3 & 1 & 3 & 0.91 & - & - & - \\
\hline 1 & 3 & 6 & 1 & 0.91 & - & - & - \\
\hline 1 & 3 & 6 & 2 & 0.91 & - & - & - \\
\hline 1 & 3 & 6 & 3 & 0.91 & - & - & - \\
\hline 1 & 4 & 1 & 1 & 0.92 & 0.83 & - & - \\
\hline 1 & 4 & 1 & 2 & 0.91 & - & - & - \\
\hline 1 & 4 & 1 & 3 & 0.91 & - & - & - \\
\hline 1 & 4 & 2 & 1 & 0.92 & - & - & - \\
\hline 1 & 4 & 2 & 2 & 0.91 & - & - & - \\
\hline 1 & 4 & 2 & 3 & 0.91 & 0.84 & - & - \\
\hline 1 & 4 & 3 & 1 & 0.92 & 0.83 & - & - \\
\hline 1 & 4 & 3 & 2 & 0.91 & - & - & - \\
\hline 1 & 4 & 3 & 3 & 0.91 & 0.86 & - & - \\
\hline 1 & 4 & 4 & 1 & 0.92 & - & - & - \\
\hline 1 & 4 & 4 & 2 & 0.92 & - & - & - \\
\hline 1 & 4 & 4 & 3 & 0.92 & 0.86 & - & - \\
\hline 1 & 4 & 1 & 1 & 0.92 & - & - & - \\
\hline 1 & 4 & 1 & 2 & 0.92 & - & - & - \\
\hline 1 & 4 & 1 & 3 & 0.91 & 0.84 & - & - \\
\hline 1 & 4 & 6 & 1 & 0.92 & - & - & - \\
\hline 1 & 4 & 6 & 2 & 0.92 & - & - & - \\
\hline 1 & 4 & 6 & 3 & 0.92 & 0.85 & - & - \\
\hline 2 & 1 & 1 & 1 & 0.97 & - & - & - \\
\hline 2 & 1 & 1 & 2 & 0.96 & - & - & - \\
\hline 2 & 1 & 1 & 3 & 0.97 & - & - & - \\
\hline 2 & 1 & 2 & 1 & 0.96 & - & - & - \\
\hline 2 & 1 & 2 & 2 & 0.96 & - & - & - \\
\hline 2 & 1 & 2 & 3 & 0.96 & - & - & - \\
\hline 2 & 1 & 3 & 1 & 0.97 & - & - & - \\
\hline 2 & 1 & 3 & 2 & 0.96 & - & - & - \\
\hline 2 & 1 & 3 & 3 & 0.97 & - & - & - \\
\hline 2 & 1 & 4 & 1 & 0.96 & - & - & - \\
\hline 2 & 1 & 4 & 2 & 0.96 & - & - & - \\
\hline 2 & 1 & 4 & 3 & 0.96 & - & - & - \\
\hline 2 & 1 & 1 & 1 & 0.96 & - & - & - \\
\hline 2 & 1 & 1 & 2 & 0.96 & - & - & - \\
\hline 2 & 1 & 1 & 3 & 0.96 & - & - & - \\
\hline 2 & 1 & 6 & 1 & 0.96 & - & - & - \\
\hline 2 & 1 & 6 & 2 & 0.96 & - & - & - \\
\hline 2 & 1 & 6 & 3 & 0.96 & - & - & - \\
\hline 2 & 2 & 1 & 1 & 0.94 & - & - & - \\
\hline 2 & 2 & 1 & 2 & 0.94 & - & - & - \\
\hline 2 & 2 & 1 & 3 & 0.94 & - & - & - \\
\hline 2 & 2 & 2 & 1 & 0.94 & - & - & - \\
\hline 2 & 2 & 2 & 2 & 0.94 & - & - & - \\
\hline 2 & 2 & 2 & 3 & 0.94 & - & - & - \\
\hline
\end{tabular}


Table B.1.1 the results of calibration procedure 1 and its validations.

\begin{tabular}{|c|c|c|c|c|c|c|c|}
\hline \multicolumn{4}{|c|}{ Configurations } & \multirow{2}{*}{$\begin{array}{c}\text { Calibration } \\
\text { Kappa }\end{array}$} & \multirow{2}{*}{$\begin{array}{c}\mathbf{1}^{\text {st }} \text { Validation } \\
\text { Kappa }\end{array}$} & \multirow{2}{*}{$\begin{array}{c}2^{\text {nd }} \text { Validation } \\
\text { Kappa } \\
\end{array}$} & \multirow{2}{*}{$\begin{array}{c}3^{\text {rd }} \text { Validation } \\
\text { Kappa }\end{array}$} \\
\hline$\varphi_{g}{ }^{29}$ & $\Lambda_{l}^{29}$ & $\Gamma_{n}{ }^{29}$ & $\eta_{m}{ }^{29}$ & & & & \\
\hline 2 & 2 & 3 & 1 & 0.94 & - & - & - \\
\hline 2 & 2 & 3 & 2 & 0.94 & - & - & - \\
\hline 2 & 2 & 3 & 3 & 0.94 & - & - & - \\
\hline 2 & 2 & 4 & 1 & 0.94 & - & - & - \\
\hline 2 & 2 & 4 & 2 & 0.95 & - & - & - \\
\hline 2 & 2 & 4 & 3 & 0.95 & - & - & - \\
\hline 2 & 2 & 1 & 1 & 0.94 & - & - & - \\
\hline 2 & 2 & 1 & 2 & 0.94 & - & - & - \\
\hline 2 & 2 & 1 & 3 & 0.94 & - & - & - \\
\hline 2 & 2 & 6 & 1 & 0.94 & - & - & - \\
\hline 2 & 2 & 6 & 2 & 0.94 & - & - & - \\
\hline 2 & 2 & 6 & 3 & 0.94 & - & - & - \\
\hline 2 & 3 & 1 & 1 & 0.94 & 0.89 & - & - \\
\hline 2 & 3 & 1 & 2 & 0.94 & 0.88 & - & - \\
\hline 2 & 3 & 1 & 3 & 0.94 & 0.90 & 0.79 & 0.84 \\
\hline 2 & 3 & 2 & 1 & 0.93 & 0.87 & - & - \\
\hline 2 & 3 & 2 & 2 & 0.94 & 0.86 & - & - \\
\hline 2 & 3 & 2 & 3 & 0.94 & 0.87 & - & - \\
\hline 2 & 3 & 3 & 1 & 0.94 & 0.87 & 0.80 & 0.84 \\
\hline 2 & 3 & 3 & 2 & 0.94 & 0.86 & - & - \\
\hline 2 & 3 & 3 & 3 & 0.94 & 0.86 & - & - \\
\hline 2 & 3 & 4 & 1 & 0.94 & 0.88 & 0.80 & 0.84 \\
\hline 2 & 3 & 4 & 2 & 0.94 & 0.87 & - & - \\
\hline 2 & 3 & 4 & 3 & 0.94 & 0.89 & 0.81 & 0.84 \\
\hline 2 & 3 & 1 & 1 & 0.94 & 0.87 & 0.81 & 0.84 \\
\hline 2 & 3 & 1 & 2 & 0.94 & 0.87 & - & - \\
\hline 2 & 3 & 1 & 3 & 0.94 & 0.87 & - & - \\
\hline 2 & 3 & 6 & 1 & 0.94 & 0.88 & 0.81 & 0.84 \\
\hline 2 & 3 & 6 & 2 & 0.94 & 0.87 & - & - \\
\hline 2 & 3 & 6 & 3 & 0.94 & 0.89 & 0.81 & 0.84 \\
\hline 2 & 4 & 1 & 1 & 0.97 & 0.87 & 0.81 & 0.84 \\
\hline 2 & 4 & 1 & 2 & 0.97 & 0.87 & 0.81 & 0.84 \\
\hline 2 & 4 & 1 & 3 & 0.97 & 0.87 & 0.81 & 0.84 \\
\hline 2 & 4 & 2 & 1 & 0.97 & 0.88 & 0.81 & 0.84 \\
\hline 2 & 4 & 2 & 2 & 0.97 & 0.88 & 0.81 & 0.84 \\
\hline 2 & 4 & 2 & 3 & 0.97 & 0.88 & 0.81 & 0.84 \\
\hline 2 & 4 & 3 & 1 & 0.97 & 0.88 & 0.82 & 0.84 \\
\hline 2 & 4 & 3 & 2 & 0.97 & 0.88 & 0.82 & 0.84 \\
\hline 2 & 4 & 3 & 3 & 0.97 & 0.88 & 0.81 & 0.84 \\
\hline 2 & 4 & 4 & 1 & 0.96 & 0.88 & 0.81 & 0.84 \\
\hline 2 & 4 & 4 & 2 & 0.96 & 0.87 & 0.81 & 0.84 \\
\hline 2 & 4 & 4 & 3 & 0.96 & 0.88 & 0.81 & 0.84 \\
\hline 2 & 4 & 1 & 1 & 0.96 & 0.88 & 0.82 & 0.84 \\
\hline 2 & 4 & 1 & 2 & 0.96 & 0.87 & 0.82 & 0.84 \\
\hline 2 & 4 & 1 & 3 & 0.96 & 0.88 & 0.81 & 0.84 \\
\hline 2 & 4 & 6 & 1 & 0.96 & 0.87 & 0.82 & 0.84 \\
\hline 2 & 4 & 6 & 2 & 0.96 & 0.86 & 0.82 & 0.84 \\
\hline 2 & 4 & 6 & 3 & 0.96 & 0.87 & 0.82 & 0.84 \\
\hline 3 & 1 & 1 & 1 & 0.97 & 0.77 & - & - \\
\hline 3 & 1 & 1 & 2 & 0.97 & 0.79 & - & - \\
\hline 3 & 1 & 1 & 3 & 0.98 & 0.77 & - & - \\
\hline
\end{tabular}


Table B.1.1 the results of calibration procedure 1 and its validations.

\begin{tabular}{|c|c|c|c|c|c|c|c|}
\hline \multicolumn{4}{|c|}{ Configurations } & \multirow{2}{*}{$\begin{array}{c}\text { Calibration } \\
\text { Kappa }\end{array}$} & \multirow{2}{*}{$\begin{array}{c}\mathbf{1}^{\text {st }} \text { Validation } \\
\text { Kappa }\end{array}$} & \multirow{2}{*}{$\begin{array}{c}2^{\text {nd }} \text { Validation } \\
\text { Kappa }\end{array}$} & \multirow{2}{*}{$\begin{array}{c}3^{\text {rd }} \text { Validation } \\
\text { Kappa }\end{array}$} \\
\hline$\varphi_{g}{ }^{29}$ & $\Lambda_{l}^{29}$ & $\Gamma_{n}{ }^{29}$ & $\eta_{m}{ }^{29}$ & & & & \\
\hline 3 & 1 & 2 & 1 & 0.97 & 0.77 & - & - \\
\hline 3 & 1 & 2 & 2 & 0.97 & 0.79 & - & - \\
\hline 3 & 1 & 2 & 3 & 0.98 & 0.77 & - & - \\
\hline 3 & 1 & 3 & 1 & 0.97 & 0.77 & - & - \\
\hline 3 & 1 & 3 & 2 & 0.97 & 0.78 & - & - \\
\hline 3 & 1 & 3 & 3 & 0.98 & 0.77 & - & - \\
\hline 3 & 1 & 4 & 1 & 0.97 & 0.77 & - & - \\
\hline 3 & 1 & 4 & 2 & 0.97 & 0.79 & - & - \\
\hline 3 & 1 & 4 & 3 & 0.98 & 0.77 & - & - \\
\hline 3 & 1 & 1 & 1 & 0.97 & 0.77 & - & - \\
\hline 3 & 1 & 1 & 2 & 0.97 & 0.78 & - & - \\
\hline 3 & 1 & 1 & 3 & 0.98 & 0.77 & - & - \\
\hline 3 & 1 & 6 & 1 & 0.97 & 0.78 & - & - \\
\hline 3 & 1 & 6 & 2 & 0.97 & 0.77 & - & - \\
\hline 3 & 1 & 6 & 3 & 0.97 & 0.76 & 0.77 & 0.78 \\
\hline 3 & 2 & 1 & 1 & 0.95 & - & - & - \\
\hline 3 & 2 & 1 & 2 & 0.95 & - & - & - \\
\hline 3 & 2 & 1 & 3 & 0.95 & - & - & - \\
\hline 3 & 2 & 2 & 1 & 0.95 & - & - & - \\
\hline 3 & 2 & 2 & 2 & 0.95 & - & - & - \\
\hline 3 & 2 & 2 & 3 & 0.95 & - & - & - \\
\hline 3 & 2 & 3 & 1 & 0.95 & - & - & - \\
\hline 3 & 2 & 3 & 2 & 0.95 & - & - & - \\
\hline 3 & 2 & 3 & 3 & 0.95 & - & - & - \\
\hline 3 & 2 & 4 & 1 & 0.95 & - & - & - \\
\hline 3 & 2 & 4 & 2 & 0.95 & - & - & - \\
\hline 3 & 2 & 4 & 3 & 0.95 & - & - & - \\
\hline 3 & 2 & 1 & 1 & 0.95 & - & - & - \\
\hline 3 & 2 & 1 & 2 & 0.95 & - & - & - \\
\hline 3 & 2 & 1 & 3 & 0.95 & - & - & - \\
\hline 3 & 2 & 6 & 1 & 0.95 & 0.72 & - & - \\
\hline 3 & 2 & 6 & 2 & 0.95 & 0.72 & - & - \\
\hline 3 & 2 & 6 & 3 & 0.95 & - & - & - \\
\hline 3 & 3 & 1 & 1 & - & - & - & - \\
\hline 3 & 3 & 1 & 2 & - & - & - & - \\
\hline 3 & 3 & 1 & 3 & - & - & - & - \\
\hline 3 & 3 & 2 & 1 & - & - & - & - \\
\hline 3 & 3 & 2 & 2 & - & - & - & - \\
\hline 3 & 3 & 2 & 3 & - & - & - & - \\
\hline 3 & 3 & 3 & 1 & - & - & - & - \\
\hline 3 & 3 & 3 & 2 & - & - & - & - \\
\hline 3 & 3 & 3 & 3 & - & - & - & - \\
\hline 3 & 3 & 4 & 1 & - & - & - & - \\
\hline 3 & 3 & 4 & 2 & 0.94 & 0.78 & 0.82 & 0.82 \\
\hline 3 & 3 & 4 & 3 & - & - & - & - \\
\hline 3 & 3 & 1 & 1 & - & - & - & - \\
\hline 3 & 3 & 1 & 2 & 0.94 & 0.77 & 0.82 & 0.81 \\
\hline 3 & 3 & 1 & 3 & - & - & - & - \\
\hline 3 & 3 & 6 & 1 & - & - & - & - \\
\hline 3 & 3 & 6 & 2 & - & - & - & - \\
\hline 3 & 3 & 6 & 3 & - & - & - & - \\
\hline
\end{tabular}


Table B.1.1 the results of calibration procedure 1 and its validations.

\begin{tabular}{|c|c|c|c|c|c|c|c|}
\hline \multicolumn{4}{|c|}{ Configurations } & \multirow{2}{*}{$\begin{array}{c}\text { Calibration } \\
\text { Kappa }\end{array}$} & \multirow{2}{*}{$\begin{array}{c}\mathbf{1}^{\text {st }} \text { Validation } \\
\text { Kappa }\end{array}$} & \multirow{2}{*}{$\begin{array}{c}2^{\text {nd }} \text { Validation } \\
\text { Kappa }\end{array}$} & \multirow{2}{*}{$\begin{array}{c}3^{\text {rd }} \text { Validation } \\
\text { Kappa }\end{array}$} \\
\hline$\varphi_{g}{ }^{29}$ & $\Lambda_{l}^{29}$ & $\Gamma_{n}{ }^{29}$ & $\eta_{m}{ }^{29}$ & & & & \\
\hline 3 & 4 & 1 & 1 & 0.96 & 0.80 & - & - \\
\hline 3 & 4 & 1 & 2 & 0.96 & 0.80 & - & - \\
\hline 3 & 4 & 1 & 3 & 0.96 & 0.79 & - & - \\
\hline 3 & 4 & 2 & 1 & 0.96 & 0.79 & - & - \\
\hline 3 & 4 & 2 & 2 & 0.96 & 0.79 & - & - \\
\hline 3 & 4 & 2 & 3 & 0.96 & 0.79 & - & - \\
\hline 3 & 4 & 3 & 1 & 0.96 & 0.79 & - & - \\
\hline 3 & 4 & 3 & 2 & 0.96 & 0.79 & - & - \\
\hline 3 & 4 & 3 & 3 & 0.96 & 0.79 & - & - \\
\hline 3 & 4 & 4 & 1 & 0.96 & 0.79 & - & - \\
\hline 3 & 4 & 4 & 2 & 0.96 & 0.79 & - & - \\
\hline 3 & 4 & 4 & 3 & 0.96 & 0.79 & - & - \\
\hline 3 & 4 & 1 & 1 & 0.96 & 0.79 & - & - \\
\hline 3 & 4 & 1 & 2 & 0.96 & 0.79 & - & - \\
\hline 3 & 4 & 1 & 3 & 0.96 & 0.79 & - & - \\
\hline 3 & 4 & 6 & 1 & 0.96 & 0.79 & - & - \\
\hline 3 & 4 & 6 & 2 & 0.96 & 0.79 & - & - \\
\hline 3 & 4 & 6 & 3 & 0.96 & 0.79 & - & - \\
\hline 4 & 1 & 1 & 1 & 0.97 & - & - & - \\
\hline 4 & 1 & 1 & 2 & 0.97 & - & - & - \\
\hline 4 & 1 & 1 & 3 & 0.97 & - & - & - \\
\hline 4 & 1 & 2 & 1 & 0.97 & 0.78 & - & - \\
\hline 4 & 1 & 2 & 2 & 0.97 & 0.78 & - & - \\
\hline 4 & 1 & 2 & 3 & 0.97 & 0.78 & - & - \\
\hline 4 & 1 & 3 & 1 & 0.97 & - & - & - \\
\hline 4 & 1 & 3 & 2 & 0.97 & - & - & - \\
\hline 4 & 1 & 3 & 3 & 0.97 & - & - & - \\
\hline 4 & 1 & 4 & 1 & 0.97 & - & - & - \\
\hline 4 & 1 & 4 & 2 & 0.97 & 0.77 & - & - \\
\hline 4 & 1 & 4 & 3 & 0.97 & 0.77 & - & - \\
\hline 4 & 1 & 1 & 1 & 0.97 & - & - & - \\
\hline 4 & 1 & 1 & 2 & 0.97 & 0.77 & - & - \\
\hline 4 & 1 & 1 & 3 & 0.97 & 0.77 & - & - \\
\hline 4 & 1 & 6 & 1 & 0.97 & - & - & - \\
\hline 4 & 1 & 6 & 2 & 0.97 & - & - & - \\
\hline 4 & 1 & 6 & 3 & 0.97 & - & - & - \\
\hline 4 & 2 & 1 & 1 & 0.96 & - & - & - \\
\hline 4 & 2 & 1 & 2 & 0.96 & - & - & - \\
\hline 4 & 2 & 1 & 3 & 0.95 & - & - & - \\
\hline 4 & 2 & 2 & 1 & 0.95 & - & - & - \\
\hline 4 & 2 & 2 & 2 & 0.95 & - & - & - \\
\hline 4 & 2 & 2 & 3 & 0.95 & - & - & - \\
\hline 4 & 2 & 3 & 1 & 0.95 & - & - & - \\
\hline 4 & 2 & 3 & 2 & 0.95 & - & - & - \\
\hline 4 & 2 & 3 & 3 & 0.95 & - & - & - \\
\hline 4 & 2 & 4 & 1 & 0.95 & - & - & - \\
\hline 4 & 2 & 4 & 2 & 0.95 & - & - & - \\
\hline 4 & 2 & 4 & 3 & 0.95 & - & - & - \\
\hline 4 & 2 & 1 & 1 & 0.95 & - & - & - \\
\hline 4 & 2 & 1 & 2 & 0.96 & - & - & - \\
\hline 4 & 2 & 1 & 3 & 0.95 & - & - & - \\
\hline
\end{tabular}


Table B.1.1 the results of calibration procedure 1 and its validations.

\begin{tabular}{|c|c|c|c|c|c|c|c|}
\hline \multicolumn{4}{|c|}{ Configurations } & \multirow{2}{*}{$\begin{array}{c}\text { Calibration } \\
\text { Kappa }\end{array}$} & \multirow{2}{*}{$\begin{array}{c}\mathbf{1}^{\text {st }} \text { Validation } \\
\text { Kappa }\end{array}$} & \multirow{2}{*}{$\begin{array}{c}2^{\text {nd }} \text { Validation } \\
\text { Kappa } \\
\end{array}$} & \multirow{2}{*}{$\begin{array}{c}3^{\text {rd }} \text { Validation } \\
\text { Kappa }\end{array}$} \\
\hline$\varphi_{g}{ }^{29}$ & $\Lambda_{l}^{29}$ & $\Gamma_{n}{ }^{29}$ & $\eta_{m}{ }^{29}$ & & & & \\
\hline 4 & 2 & 6 & 1 & 0.95 & - & - & - \\
\hline 4 & 2 & 6 & 2 & 0.95 & - & - & - \\
\hline 4 & 2 & 6 & 3 & 0.95 & - & - & - \\
\hline 4 & 3 & 1 & 1 & 0.97 & 0.80 & 0.77 & 0.80 \\
\hline 4 & 3 & 1 & 2 & 0.97 & 0.79 & 0.79 & 0.81 \\
\hline 4 & 3 & 1 & 3 & 0.97 & 0.79 & 0.78 & 0.80 \\
\hline 4 & 3 & 2 & 1 & 0.97 & 0.79 & 0.77 & 0.79 \\
\hline 4 & 3 & 2 & 2 & 0.97 & 0.78 & 0.79 & 0.80 \\
\hline 4 & 3 & 2 & 3 & 0.97 & 0.78 & 0.78 & 0.80 \\
\hline 4 & 3 & 3 & 1 & 0.97 & 0.81 & 0.77 & 0.80 \\
\hline 4 & 3 & 3 & 2 & 0.97 & 0.79 & 0.79 & 0.81 \\
\hline 4 & 3 & 3 & 3 & 0.97 & 0.79 & 0.79 & 0.80 \\
\hline 4 & 3 & 4 & 1 & 0.97 & 0.79 & 0.78 & 0.80 \\
\hline 4 & 3 & 4 & 2 & 0.97 & 0.79 & 0.80 & 0.81 \\
\hline 4 & 3 & 4 & 3 & 0.97 & 0.79 & 0.79 & 0.81 \\
\hline 4 & 3 & 1 & 1 & 0.97 & 0.80 & 0.77 & 0.80 \\
\hline 4 & 3 & 1 & 2 & 0.96 & 0.79 & 0.81 & 0.82 \\
\hline 4 & 3 & 1 & 3 & 0.96 & 0.79 & 0.79 & 0.80 \\
\hline 4 & 3 & 6 & 1 & 0.97 & 0.80 & 0.78 & 0.80 \\
\hline 4 & 3 & 6 & 2 & 0.96 & 0.79 & 0.79 & 0.81 \\
\hline 4 & 3 & 6 & 3 & 0.97 & 0.80 & - & - \\
\hline 4 & 4 & 1 & 1 & 0.95 & 0.84 & 0.79 & 0.82 \\
\hline 4 & 4 & 1 & 2 & 0.95 & 0.84 & 0.79 & 0.82 \\
\hline 4 & 4 & 1 & 3 & 0.95 & 0.84 & 0.79 & 0.82 \\
\hline 4 & 4 & 2 & 1 & 0.95 & 0.84 & 0.79 & 0.82 \\
\hline 4 & 4 & 2 & 2 & 0.95 & 0.84 & 0.79 & 0.82 \\
\hline 4 & 4 & 2 & 3 & 0.95 & 0.84 & 0.79 & 0.82 \\
\hline 4 & 4 & 3 & 1 & 0.95 & 0.84 & 0.77 & 0.81 \\
\hline 4 & 4 & 3 & 2 & 0.95 & 0.83 & 0.77 & 0.81 \\
\hline 4 & 4 & 3 & 3 & 0.95 & 0.83 & 0.77 & 0.81 \\
\hline 4 & 4 & 4 & 1 & 0.95 & 0.83 & 0.79 & 0.82 \\
\hline 4 & 4 & 4 & 2 & 0.95 & 0.82 & 0.79 & 0.81 \\
\hline 4 & 4 & 4 & 3 & 0.95 & 0.82 & 0.79 & 0.81 \\
\hline 4 & 4 & 1 & 1 & 0.95 & 0.82 & 0.79 & 0.82 \\
\hline 4 & 4 & 1 & 2 & 0.95 & 0.82 & 0.79 & 0.82 \\
\hline 4 & 4 & 1 & 3 & 0.95 & 0.82 & 0.79 & 0.82 \\
\hline 4 & 4 & 6 & 1 & 0.95 & 0.84 & 0.80 & 0.83 \\
\hline 4 & 4 & 6 & 2 & 0.95 & 0.83 & 0.80 & 0.82 \\
\hline 4 & 4 & 6 & 3 & 0.95 & 0.83 & 0.80 & 0.82 \\
\hline 5 & 1 & 1 & 1 & 1.00 & 0.80 & - & - \\
\hline 5 & 1 & 1 & 2 & 1.00 & 0.79 & - & - \\
\hline 5 & 1 & 1 & 3 & 1.00 & 0.80 & - & - \\
\hline 5 & 1 & 2 & 1 & 0.99 & 0.79 & - & - \\
\hline 5 & 1 & 2 & 2 & 1.00 & 0.77 & - & - \\
\hline 5 & 1 & 2 & 3 & 0.99 & 0.79 & - & - \\
\hline 5 & 1 & 3 & 1 & 1.00 & 0.81 & - & - \\
\hline 5 & 1 & 3 & 2 & 1.00 & 0.80 & - & - \\
\hline 5 & 1 & 3 & 3 & 1.00 & 0.81 & - & - \\
\hline 5 & 1 & 4 & 1 & 0.99 & 0.80 & - & - \\
\hline 5 & 1 & 4 & 2 & 1.00 & 0.79 & - & - \\
\hline 5 & 1 & 4 & 3 & 0.99 & 0.80 & - & - \\
\hline
\end{tabular}


Table B.1.1 the results of calibration procedure 1 and its validations.

\begin{tabular}{|c|c|c|c|c|c|c|c|}
\hline \multicolumn{4}{|c|}{ Configurations } & \multirow{2}{*}{$\begin{array}{c}\text { Calibration } \\
\text { Kappa }\end{array}$} & \multirow{2}{*}{$\begin{array}{c}\mathbf{1}^{\text {st }} \text { Validation } \\
\text { Kappa }\end{array}$} & \multirow{2}{*}{$\begin{array}{c}2^{\text {nd }} \text { Validation } \\
\text { Kappa } \\
\end{array}$} & \multirow{2}{*}{$\begin{array}{c}3^{\text {rd }} \text { Validation } \\
\text { Kappa }\end{array}$} \\
\hline$\varphi_{g}{ }^{29}$ & $\Lambda_{l}^{29}$ & $\Gamma_{n}{ }^{29}$ & $\eta_{m}{ }^{29}$ & & & & \\
\hline 5 & 1 & 1 & 1 & 0.99 & 0.79 & - & - \\
\hline 5 & 1 & 1 & 2 & 0.99 & 0.78 & - & - \\
\hline 5 & 1 & 1 & 3 & 0.99 & 0.79 & - & - \\
\hline 5 & 1 & 6 & 1 & 1.00 & 0.79 & - & - \\
\hline 5 & 1 & 6 & 2 & 1.00 & 0.78 & - & - \\
\hline 5 & 1 & 6 & 3 & 1.00 & 0.79 & - & - \\
\hline 5 & 2 & 1 & 1 & 0.99 & 0.77 & - & - \\
\hline 5 & 2 & 1 & 2 & 0.99 & 0.75 & - & - \\
\hline 5 & 2 & 1 & 3 & 0.99 & 0.75 & - & - \\
\hline 5 & 2 & 2 & 1 & 0.99 & 0.77 & - & - \\
\hline 5 & 2 & 2 & 2 & 0.99 & 0.75 & - & - \\
\hline 5 & 2 & 2 & 3 & 0.99 & 0.75 & - & - \\
\hline 5 & 2 & 3 & 1 & 0.98 & - & - & - \\
\hline 5 & 2 & 3 & 2 & 0.98 & - & - & - \\
\hline 5 & 2 & 3 & 3 & 0.98 & - & - & - \\
\hline 5 & 2 & 4 & 1 & 0.98 & 0.75 & - & - \\
\hline 5 & 2 & 4 & 2 & 0.98 & - & - & - \\
\hline 5 & 2 & 4 & 3 & 0.98 & - & - & - \\
\hline 5 & 2 & 1 & 1 & 0.98 & - & - & - \\
\hline 5 & 2 & 1 & 2 & 0.98 & - & - & - \\
\hline 5 & 2 & 1 & 3 & 0.98 & - & - & - \\
\hline 5 & 2 & 6 & 1 & 0.98 & - & - & - \\
\hline 5 & 2 & 6 & 2 & 0.98 & - & - & - \\
\hline 5 & 2 & 6 & 3 & 0.98 & - & - & - \\
\hline 5 & 3 & 1 & 1 & - & - & - & - \\
\hline 5 & 3 & 1 & 2 & - & - & - & - \\
\hline 5 & 3 & 1 & 3 & - & - & - & - \\
\hline 5 & 3 & 2 & 1 & - & - & - & - \\
\hline 5 & 3 & 2 & 2 & - & - & - & - \\
\hline 5 & 3 & 2 & 3 & - & - & - & - \\
\hline 5 & 3 & 3 & 1 & - & - & - & - \\
\hline 5 & 3 & 3 & 2 & - & - & - & - \\
\hline 5 & 3 & 3 & 3 & - & - & - & - \\
\hline 5 & 3 & 4 & 1 & - & - & - & - \\
\hline 5 & 3 & 4 & 2 & - & - & - & - \\
\hline 5 & 3 & 4 & 3 & - & - & - & - \\
\hline 5 & 3 & 1 & 1 & - & - & - & - \\
\hline 5 & 3 & 1 & 2 & - & - & - & - \\
\hline 5 & 3 & 1 & 3 & - & - & - & - \\
\hline 5 & 3 & 6 & 1 & - & - & - & - \\
\hline 5 & 3 & 6 & 2 & - & - & - & - \\
\hline 5 & 3 & 6 & 3 & - & - & - & - \\
\hline 5 & 4 & 1 & 1 & 0.98 & 0.78 & 0.77 & 0.79 \\
\hline 5 & 4 & 1 & 2 & 0.98 & 0.80 & 0.79 & 0.81 \\
\hline 5 & 4 & 1 & 3 & 0.98 & 0.77 & 0.79 & 0.80 \\
\hline 5 & 4 & 2 & 1 & 0.98 & 0.81 & 0.79 & 0.81 \\
\hline 5 & 4 & 2 & 2 & 0.99 & 0.81 & 0.81 & 0.82 \\
\hline 5 & 4 & 2 & 3 & 0.99 & 0.78 & 0.81 & 0.82 \\
\hline 5 & 4 & 3 & 1 & 0.98 & 0.82 & 0.80 & 0.82 \\
\hline 5 & 4 & 3 & 2 & 0.99 & 0.82 & 0.83 & 0.84 \\
\hline 5 & 4 & 3 & 3 & 0.99 & 0.80 & 0.82 & 0.83 \\
\hline
\end{tabular}


Table B.1.1 the results of calibration procedure 1 and its validations.

\begin{tabular}{|c|c|c|c|c|c|c|c|}
\hline \multicolumn{4}{|c|}{ Configurations } & Calibration & $1^{\text {st }}$ Validation & $2^{\text {nd }}$ Validation & $3^{\text {rd }}$ Validation \\
\hline$\varphi_{g}{ }^{29}$ & $\Lambda_{l}^{29}$ & $\Gamma_{n}{ }^{29}$ & $\eta_{m}{ }^{29}$ & Kappa & Kappa & Kappa & Kappa \\
\hline 5 & 4 & 4 & 1 & 0.98 & 0.83 & 0.79 & 0.82 \\
\hline 5 & 4 & 4 & 2 & 0.99 & 0.83 & 0.82 & 0.84 \\
\hline 5 & 4 & 4 & 3 & 0.99 & 0.81 & 0.83 & 0.83 \\
\hline 5 & 4 & 1 & 1 & 0.98 & 0.80 & 0.77 & 0.80 \\
\hline 5 & 4 & 1 & 2 & 0.98 & 0.81 & 0.77 & 0.80 \\
\hline 5 & 4 & 1 & 3 & 0.98 & 0.79 & 0.77 & 0.80 \\
\hline 5 & 4 & 6 & 1 & 0.98 & 0.82 & 0.79 & 0.82 \\
\hline 5 & 4 & 6 & 2 & 0.99 & 0.82 & 0.81 & 0.83 \\
\hline 5 & 4 & 6 & 3 & 0.99 & 0.81 & 0.81 & 0.83 \\
\hline
\end{tabular}

Table B.1.2 the results of calibration procedure 2 and its validations.

\begin{tabular}{|c|c|c|c|c|c|}
\hline \multicolumn{2}{|c|}{ Configurations } & \multirow{2}{*}{$\begin{array}{c}\text { Calibration } \\
\text { Kappa }\end{array}$} & \multirow{2}{*}{$\begin{array}{c}\mathbf{1}^{\text {st }} \text { Validation } \\
\text { Kappa }\end{array}$} & \multirow{2}{*}{$\begin{array}{cl}2^{\text {nd }} & \text { Validation } \\
\text { Kappa }\end{array}$} & \multirow{2}{*}{$\frac{3^{\text {rd }} \text { Validation }}{\text { Kappa }}$} \\
\hline$\varphi_{g}$ & $\Lambda_{l}$ & & & & \\
\hline 1 & 1 & 0.82 & 0.82 & 0.82 & 0.83 \\
\hline 1 & 2 & 0.75 & - & - & - \\
\hline 1 & 3 & 0.78 & 0.83 & 0.80 & 0.82 \\
\hline 1 & 4 & 0.72 & 0.78 & - & - \\
\hline 2 & 1 & 0.85 & 0.84 & 0.83 & 0.84 \\
\hline 2 & 2 & 0.83 & 0.80 & 0.82 & 0.82 \\
\hline 2 & 3 & 0.83 & 0.81 & - & - \\
\hline 2 & 4 & 0.83 & 0.80 & 0.82 & 0.82 \\
\hline 3 & 1 & 0.89 & 0.83 & 0.82 & 0.83 \\
\hline 3 & 2 & 0.83 & 0.78 & 0.83 & 0.82 \\
\hline 3 & 3 & - & - & - & - \\
\hline 3 & 4 & 0.82 & 0.77 & 0.80 & 0.80 \\
\hline 4 & 1 & 0.89 & 0.80 & 0.85 & 0.84 \\
\hline 4 & 2 & 0.84 & 0.76 & 0.86 & 0.84 \\
\hline 4 & 3 & - & - & - & - \\
\hline 4 & 4 & 0.86 & 0.80 & 0.86 & 0.85 \\
\hline 5 & 1 & 0.88 & 0.83 & 0.84 & 0.85 \\
\hline 5 & 2 & 0.89 & 0.79 & 0.86 & 0.85 \\
\hline 5 & 3 & 0.84 & 0.75 & 0.84 & - \\
\hline 5 & 4 & 0.87 & 0.83 & 0.89 & 0.88 \\
\hline
\end{tabular}


Table B.1.3 the results of calibration and validations based on the average speed

\begin{tabular}{|c|c|c|c|c|c|}
\hline \multicolumn{2}{|c|}{ Configurations } & \multirow{2}{*}{$\begin{array}{c}\text { Calibration } \\
\text { Kappa }\end{array}$} & \multirow{2}{*}{$\frac{1^{\text {st }} \text { Validation }}{\text { Kappa }}$} & \multirow{2}{*}{$\begin{array}{c}2^{\text {nd }} \text { Validation } \\
\text { Kappa }\end{array}$} & \multirow{2}{*}{$\frac{3^{\text {rd }} \text { Validation }}{\text { Kappa }}$} \\
\hline$\varphi_{g}$ & $\Lambda_{l}$ & & & & \\
\hline 1 & 1 & 0.32 & 0.35 & 0.45 & 0.43 \\
\hline 1 & 2 & 0.28 & 0.33 & 0.48 & 0.43 \\
\hline 1 & 3 & 0.26 & 0.33 & 0.46 & 0.42 \\
\hline 1 & 4 & - & - & - & - \\
\hline 2 & 1 & 0.32 & 0.36 & 0.46 & 0.44 \\
\hline 2 & 2 & 0.29 & 0.33 & 0.46 & 0.43 \\
\hline 2 & 3 & 0.25 & 0.30 & 0.48 & 0.42 \\
\hline 2 & 4 & 0.25 & 0.27 & - & - \\
\hline 3 & 1 & 0.34 & 0.39 & 0.48 & 0.47 \\
\hline 3 & 2 & 0.32 & 0.36 & 0.48 & 0.46 \\
\hline 3 & 3 & 0.27 & 0.29 & 0.46 & - \\
\hline 3 & 4 & 0.29 & 0.31 & 0.41 & - \\
\hline 4 & 1 & 0.33 & 0.39 & 0.52 & 0.51 \\
\hline 4 & 2 & 0.33 & 0.36 & 0.52 & 0.50 \\
\hline 4 & 3 & 0.31 & 0.32 & 0.51 & 0.47 \\
\hline 4 & 4 & 0.29 & 0.33 & 0.44 & 0.43 \\
\hline 5 & 1 & 0.39 & 0.43 & 0.53 & 0.53 \\
\hline 5 & 2 & 0.39 & 0.42 & 0.53 & 0.53 \\
\hline 5 & 3 & 0.39 & 0.38 & 0.53 & 0.51 \\
\hline 5 & 4 & 0.35 & 0.37 & 0.47 & 0.47 \\
\hline
\end{tabular}

Table B.1.4 the results of calibration and validations based on the maximum speed

\begin{tabular}{|c|c|c|c|c|c|}
\hline \multicolumn{2}{|c|}{ Configurations } & \multirow{2}{*}{$\begin{array}{c}\text { Calibration } \\
\text { Kappa } \\
\end{array}$} & \multirow{2}{*}{$\begin{array}{c}\mathbf{1}^{\text {st }} \text { Validation } \\
\text { Kappa } \\
\end{array}$} & \multirow{2}{*}{$\begin{array}{cl}2^{\text {nd }} & \text { Validation } \\
\text { Kappa } \\
\end{array}$} & \multirow{2}{*}{$\begin{array}{c}3^{\text {rd }} \text { Validation } \\
\text { Kappa } \\
\end{array}$} \\
\hline$\varphi_{g}$ & $\Lambda_{l}$ & & & & \\
\hline 1 & 1 & - & - & - & - \\
\hline 1 & 2 & 0.54 & 0.58 & 0.57 & 0.59 \\
\hline 1 & 3 & 0.52 & 0.57 & 0.56 & 0.58 \\
\hline 1 & 4 & - & - & - & - \\
\hline 2 & 1 & 0.48 & 0.51 & - & - \\
\hline 2 & 2 & 0.55 & 0.57 & 0.57 & 0.59 \\
\hline 2 & 3 & 0.53 & 0.53 & 0.58 & 0.58 \\
\hline 2 & 4 & - & - & - & - \\
\hline 3 & 1 & 0.53 & 0.57 & - & - \\
\hline 3 & 2 & 0.60 & 0.60 & 0.57 & 0.61 \\
\hline 3 & 3 & 0.58 & 0.56 & 0.59 & 0.60 \\
\hline 3 & 4 & - & - & - & - \\
\hline 4 & 1 & 0.57 & 0.61 & - & - \\
\hline 4 & 2 & 0.62 & 0.61 & 0.60 & 0.63 \\
\hline 4 & 3 & 0.63 & 0.58 & 0.63 & 0.64 \\
\hline 4 & 4 & - & - & - & - \\
\hline 5 & 1 & 0.63 & 0.65 & - & - \\
\hline 5 & 2 & 0.68 & 0.65 & 0.57 & 0.64 \\
\hline 5 & 3 & 0.68 & 0.64 & 0.64 & 0.67 \\
\hline 5 & 4 & 0.58 & - & - & - \\
\hline
\end{tabular}


Table B.1.5 the results of calibration and validations based on the average acceleration

\begin{tabular}{|c|c|c|c|c|c|}
\hline \multicolumn{2}{|c|}{ Configurations } & \multirow{2}{*}{$\begin{array}{c}\text { Calibration } \\
\text { Kappa }\end{array}$} & \multirow{2}{*}{$\frac{1^{\text {st }} \text { Validation }}{\text { Kappa }}$} & \multirow{2}{*}{$\begin{array}{c}2^{\text {nd }} \text { Validation } \\
\text { Kappa }\end{array}$} & \multirow{2}{*}{$\begin{array}{c}3^{\text {rd }} \text { Validation } \\
\text { Kappa }\end{array}$} \\
\hline$\varphi_{g}$ & $\Lambda_{l}$ & & & & \\
\hline 1 & 1 & 0.07 & 0.03 & 0.12 & 0.09 \\
\hline 1 & 2 & 0.08 & 0.02 & 0.13 & 0.09 \\
\hline 1 & 3 & 0.08 & 0.03 & 0.11 & 0.08 \\
\hline 1 & 4 & 0.06 & 0.03 & 0.07 & 0.06 \\
\hline 2 & 1 & 0.06 & 0.06 & 0.08 & 0.06 \\
\hline 2 & 2 & 0.07 & 0.04 & 0.09 & 0.08 \\
\hline 2 & 3 & 0.07 & 0.06 & 0.10 & 0.09 \\
\hline 2 & 4 & 0.05 & 0.04 & 0.07 & 0.05 \\
\hline 3 & 1 & 0.08 & 0.04 & 0.08 & 0.07 \\
\hline 3 & 2 & 0.08 & 0.03 & 0.10 & 0.08 \\
\hline 3 & 3 & 0.09 & 0.06 & 0.10 & 0.09 \\
\hline 3 & 4 & 0.06 & 0.01 & 0.05 & 0.04 \\
\hline 4 & 1 & 0.08 & 0.03 & 0.09 & 0.08 \\
\hline 4 & 2 & 0.09 & 0.02 & 0.10 & 0.08 \\
\hline 4 & 3 & 0.10 & - & - & - \\
\hline 4 & 4 & 0.08 & 0.03 & 0.07 & 0.07 \\
\hline 5 & 1 & 0.10 & 0.01 & 0.10 & 0.09 \\
\hline 5 & 2 & 0.11 & 0.01 & 0.11 & 0.09 \\
\hline 5 & 3 & 0.10 & 0.02 & 0.12 & 0.10 \\
\hline 5 & 4 & 0.07 & 0.04 & 0.05 & 0.06 \\
\hline
\end{tabular}

Table B.1.6 the results of calibration and validations based on the maximum acceleration

\begin{tabular}{|c|c|c|c|c|c|}
\hline \multicolumn{2}{|c|}{ Configurations } & \multirow{2}{*}{$\begin{array}{c}\text { Calibration } \\
\text { Kappa } \\
\end{array}$} & \multirow{2}{*}{$\begin{array}{c}\mathbf{1}^{\text {st }} \text { Validation } \\
\text { Kappa } \\
\end{array}$} & \multirow{2}{*}{$\begin{array}{cl}2^{\text {nd }} & \text { Validation } \\
& \text { Kappa } \\
\end{array}$} & \multirow{2}{*}{$\begin{array}{c}3^{\text {rd }} \text { Validation } \\
\text { Kappa } \\
\end{array}$} \\
\hline$\varphi_{g}$ & $\Lambda_{l}$ & & & & \\
\hline 1 & 1 & 0.05 & 0.02 & 0.07 & 0.05 \\
\hline 1 & 2 & 0.05 & 0.02 & 0.07 & 0.05 \\
\hline 1 & 3 & 0.04 & 0.02 & 0.06 & 0.04 \\
\hline 1 & 4 & 0.05 & 0.02 & 0.07 & 0.04 \\
\hline 2 & 1 & 0.06 & - & - & - \\
\hline 2 & 2 & 0.06 & - & - & - \\
\hline 2 & 3 & 0.05 & - & - & - \\
\hline 2 & 4 & 0.06 & - & - & - \\
\hline 3 & 1 & 0.06 & 0.01 & - & - \\
\hline 3 & 2 & 0.06 & 0.01 & - & - \\
\hline 3 & 3 & 0.06 & 0.02 & - & - \\
\hline 3 & 4 & 0.06 & 0.01 & - & - \\
\hline 4 & 1 & 0.06 & 0.01 & - & - \\
\hline 4 & 2 & 0.06 & 0.01 & - & - \\
\hline 4 & 3 & 0.06 & 0.01 & - & - \\
\hline 4 & 4 & 0.06 & 0.01 & - & - \\
\hline 5 & 1 & 0.09 & 0.01 & - & - \\
\hline 5 & 2 & 0.09 & - & - & - \\
\hline 5 & 3 & 0.08 & - & - & - \\
\hline 5 & 4 & 0.09 & - & - & - \\
\hline
\end{tabular}


Table B.1.7 the results of calibration and validations based on the average deceleration

\begin{tabular}{|c|c|c|c|c|c|}
\hline \multicolumn{2}{|c|}{ Configurations } & \multirow{2}{*}{$\begin{array}{c}\text { Calibration } \\
\text { Kappa }\end{array}$} & \multirow{2}{*}{$\frac{\mathbf{1}^{\text {st }} \text { Validation }}{\text { Kappa }}$} & \multirow{2}{*}{$\begin{array}{c}2^{\text {nd }} \text { Validation } \\
\text { Kappa }\end{array}$} & \multirow{2}{*}{$\frac{3^{\text {rd }} \text { Validation }}{\text { Kappa }}$} \\
\hline$\varphi_{g}$ & $\Lambda_{l}$ & & & & \\
\hline 1 & 1 & 0.11 & 0.08 & 0.14 & 0.11 \\
\hline 1 & 2 & 0.10 & 0.07 & - & - \\
\hline 1 & 3 & 0.10 & 0.05 & 0.20 & 0.13 \\
\hline 1 & 4 & 0.07 & 0.05 & 0.16 & 0.10 \\
\hline 2 & 1 & 0.08 & 0.08 & 0.14 & 0.11 \\
\hline 2 & 2 & 0.07 & 0.06 & 0.19 & 0.13 \\
\hline 2 & 3 & 0.08 & 0.07 & 0.18 & 0.13 \\
\hline 2 & 4 & 0.06 & 0.05 & 0.16 & 0.11 \\
\hline 3 & 1 & 0.06 & 0.08 & 0.16 & 0.12 \\
\hline 3 & 2 & 0.06 & 0.08 & 0.21 & 0.15 \\
\hline 3 & 3 & 0.07 & 0.07 & 0.19 & 0.13 \\
\hline 3 & 4 & 0.05 & 0.05 & 0.17 & 0.11 \\
\hline 4 & 1 & 0.09 & 0.06 & 0.22 & 0.14 \\
\hline 4 & 2 & 0.09 & 0.09 & 0.23 & 0.16 \\
\hline 4 & 3 & 0.09 & 0.06 & 0.18 & 0.13 \\
\hline 4 & 4 & 0.08 & 0.05 & 0.20 & 0.12 \\
\hline 5 & 1 & 0.12 & 0.11 & 0.20 & 0.15 \\
\hline 5 & 2 & 0.11 & 0.07 & - & - \\
\hline 5 & 3 & 0.12 & 0.08 & 0.13 & 0.11 \\
\hline 5 & 4 & 0.11 & 0.07 & 0.21 & 0.14 \\
\hline
\end{tabular}

Table B.1.8 the results of calibration and validations based on the maximum deceleration

\begin{tabular}{|c|c|c|c|c|c|}
\hline \multicolumn{2}{|c|}{ Configurations } & \multirow{2}{*}{$\begin{array}{c}\text { Calibration } \\
\text { Kappa } \\
\end{array}$} & \multirow{2}{*}{$\begin{array}{c}\mathbf{1}^{\text {st }} \text { Validation } \\
\text { Kappa } \\
\end{array}$} & \multirow{2}{*}{$\begin{array}{cl}2^{\text {nd }} & \text { Validation } \\
\text { Kappa } \\
\end{array}$} & \multirow{2}{*}{$\begin{array}{c}3^{\text {rd }} \text { Validation } \\
\text { Kappa } \\
\end{array}$} \\
\hline$\varphi_{g}$ & $\Lambda_{l}$ & & & & \\
\hline 1 & 1 & 0.06 & 0.04 & 0.09 & 0.07 \\
\hline 1 & 2 & 0.03 & 0.02 & 0.05 & 0.04 \\
\hline 1 & 3 & 0.03 & 0.01 & - & - \\
\hline 1 & 4 & 0.03 & 0.02 & - & - \\
\hline 2 & 1 & 0.06 & 0.05 & - & - \\
\hline 2 & 2 & 0.03 & 0.02 & 0.10 & 0.08 \\
\hline 2 & 3 & 0.04 & 0.02 & - & - \\
\hline 2 & 4 & 0.03 & 0.02 & - & - \\
\hline 3 & 1 & 0.06 & 0.07 & - & - \\
\hline 3 & 2 & 0.03 & 0.02 & - & - \\
\hline 3 & 3 & 0.04 & 0.04 & - & - \\
\hline 3 & 4 & 0.03 & 0.02 & - & - \\
\hline 4 & 1 & 0.05 & 0.11 & 0.08 & 0.10 \\
\hline 4 & 2 & 0.04 & 0.05 & 0.05 & 0.06 \\
\hline 4 & 3 & 0.03 & - & - & - \\
\hline 4 & 4 & 0.03 & - & - & - \\
\hline 5 & 1 & 0.07 & 0.12 & 0.10 & 0.12 \\
\hline 5 & 2 & 0.05 & 0.02 & - & - \\
\hline 5 & 3 & 0.06 & 0.02 & - & - \\
\hline 5 & 4 & 0.05 & - & - & - \\
\hline
\end{tabular}


Table B.1.9 the results of calibration and validations based on the average direction

\begin{tabular}{|c|c|c|c|c|c|}
\hline \multicolumn{2}{|c|}{ Configurations } & \multirow{2}{*}{$\begin{array}{c}\text { Calibration } \\
\text { Kappa }\end{array}$} & \multirow{2}{*}{$\frac{\mathbf{1}^{\text {st }} \text { Validation }}{\text { Kappa }}$} & \multirow{2}{*}{$\begin{array}{c}2^{\text {nd }} \text { Validation } \\
\text { Kappa }\end{array}$} & \multirow{2}{*}{$\frac{3^{\text {rd }} \text { Validation }}{\text { Kappa }}$} \\
\hline$\varphi_{g}$ & $\Lambda_{l}$ & & & & \\
\hline 1 & 1 & 0.14 & 0.11 & 0.02 & 0.07 \\
\hline 1 & 2 & 0.13 & 0.11 & 0.02 & 0.07 \\
\hline 1 & 3 & 0.11 & 0.09 & 0.02 & 0.06 \\
\hline 1 & 4 & 0.13 & 0.11 & 0.02 & 0.07 \\
\hline 2 & 1 & 0.14 & 0.11 & 0.02 & 0.07 \\
\hline 2 & 2 & 0.13 & 0.11 & 0.02 & 0.07 \\
\hline 2 & 3 & 0.11 & 0.10 & 0.03 & 0.07 \\
\hline 2 & 4 & 0.13 & 0.11 & 0.02 & 0.07 \\
\hline 3 & 1 & 0.13 & 0.10 & 0.02 & 0.07 \\
\hline 3 & 2 & 0.12 & 0.10 & 0.03 & 0.07 \\
\hline 3 & 3 & 0.09 & 0.08 & 0.03 & 0.07 \\
\hline 3 & 4 & 0.12 & 0.10 & 0.03 & 0.07 \\
\hline 4 & 1 & 0.13 & 0.09 & 0.07 & 0.08 \\
\hline 4 & 2 & 0.13 & 0.06 & 0.07 & 0.07 \\
\hline 4 & 3 & 0.12 & 0.04 & - & - \\
\hline 4 & 4 & 0.12 & 0.04 & 0.04 & - \\
\hline 5 & 1 & 0.16 & 0.05 & 0.03 & 0.04 \\
\hline 5 & 2 & 0.16 & 0.06 & 0.03 & 0.04 \\
\hline 5 & 3 & 0.17 & 0.05 & - & - \\
\hline 5 & 4 & 0.16 & 0.06 & 0.03 & 0.04 \\
\hline
\end{tabular}

Table B.1.10 the results of calibration and validations based on the maximum direction

\begin{tabular}{|c|c|c|c|c|c|}
\hline \multicolumn{2}{|c|}{ Configurations } & \multirow{2}{*}{$\begin{array}{c}\text { Calibration } \\
\text { Kappa } \\
\end{array}$} & \multirow{2}{*}{$\begin{array}{c}\mathbf{1}^{\text {st }} \text { Validation } \\
\text { Kappa } \\
\end{array}$} & \multirow{2}{*}{$\begin{array}{cl}2^{\text {nd }} & \text { Validation } \\
& \text { Kappa } \\
\end{array}$} & \multirow{2}{*}{$\begin{array}{c}3^{\text {rd }} \text { Validation } \\
\text { Kappa } \\
\end{array}$} \\
\hline$\varphi_{g}$ & $\Lambda_{l}$ & & & & \\
\hline 1 & 1 & 0.25 & 0.21 & 0.04 & 0.12 \\
\hline 1 & 2 & 0.24 & 0.20 & 0.04 & 0.12 \\
\hline 1 & 3 & 0.23 & 0.19 & 0.06 & 0.13 \\
\hline 1 & 4 & 0.23 & 0.20 & 0.04 & 0.12 \\
\hline 2 & 1 & 0.27 & 0.21 & 0.04 & 0.12 \\
\hline 2 & 2 & 0.26 & 0.20 & 0.04 & 0.12 \\
\hline 2 & 3 & 0.24 & 0.21 & 0.04 & 0.12 \\
\hline 2 & 4 & 0.26 & 0.20 & 0.04 & 0.12 \\
\hline 3 & 1 & 0.28 & 0.21 & 0.04 & 0.13 \\
\hline 3 & 2 & 0.27 & 0.20 & 0.04 & 0.12 \\
\hline 3 & 3 & 0.25 & 0.21 & 0.04 & 0.12 \\
\hline 3 & 4 & 0.26 & 0.20 & 0.04 & 0.12 \\
\hline 4 & 1 & 0.28 & 0.20 & 0.04 & 0.13 \\
\hline 4 & 2 & 0.27 & 0.18 & 0.04 & 0.13 \\
\hline 4 & 3 & 0.25 & 0.19 & 0.04 & 0.13 \\
\hline 4 & 4 & 0.26 & 0.18 & 0.04 & 0.13 \\
\hline 5 & 1 & 0.30 & 0.21 & 0.03 & 0.14 \\
\hline 5 & 2 & 0.28 & 0.20 & 0.04 & 0.13 \\
\hline 5 & 3 & 0.26 & 0.20 & 0.03 & 0.13 \\
\hline 5 & 4 & 0.27 & 0.20 & 0.04 & 0.13 \\
\hline
\end{tabular}


Table B.1.11 the results of calibration and validations based on the average of change in direction

\begin{tabular}{|c|c|c|c|c|c|}
\hline \multicolumn{2}{|c|}{ Configurations } & \multirow{2}{*}{$\begin{array}{c}\text { Calibration } \\
\text { Kappa }\end{array}$} & \multirow{2}{*}{$\begin{array}{c}\mathbf{1}^{\text {st }} \text { Validation } \\
\text { Kappa } \\
\end{array}$} & \multirow{2}{*}{$\begin{array}{cl}2^{\text {nd }} & \text { Validation } \\
\text { Kappa } \\
\end{array}$} & \multirow{2}{*}{$\begin{array}{c}3^{\text {rd }} \text { Validation } \\
\text { Kappa }\end{array}$} \\
\hline$\varphi_{g}$ & $\Lambda_{l}$ & & & & \\
\hline 1 & 1 & 0.11 & 0.01 & - & - \\
\hline 1 & 2 & 0.11 & 0.01 & - & - \\
\hline 1 & 3 & 0.11 & 0.01 & - & - \\
\hline 1 & 4 & 0.12 & 0.05 & - & - \\
\hline 2 & 1 & 0.10 & - & - & - \\
\hline 2 & 2 & 0.10 & - & - & - \\
\hline 2 & 3 & 0.10 & - & - & - \\
\hline 2 & 4 & 0.10 & - & - & - \\
\hline 3 & 1 & 0.12 & - & - & - \\
\hline 3 & 2 & 0.12 & - & - & - \\
\hline 3 & 3 & 0.12 & - & - & - \\
\hline 3 & 4 & 0.12 & - & - & - \\
\hline 4 & 1 & 0.15 & 0.06 & - & - \\
\hline 4 & 2 & 0.15 & 0.06 & - & - \\
\hline 4 & 3 & 0.15 & 0.06 & - & - \\
\hline 4 & 4 & 0.15 & 0.06 & - & - \\
\hline 5 & 1 & 0.18 & 0.06 & - & - \\
\hline 5 & 2 & 0.18 & 0.06 & - & - \\
\hline 5 & 3 & 0.18 & 0.06 & - & - \\
\hline 5 & 4 & 0.18 & 0.06 & - & - \\
\hline
\end{tabular}

Table B.1.12 the results of calibration and validations based on the average of cosine of change in direction

\begin{tabular}{|c|c|c|c|c|c|}
\hline \multicolumn{2}{|c|}{ Configurations } & Calibration & $1^{\text {st }}$ Validation & $2^{\text {nd }}$ Validation & $3^{\text {rd }}$ Validation \\
\hline$\varphi_{g}$ & $\Lambda_{l}$ & Kappa & Kappa & Kappa & Kappa \\
\hline 1 & 1 & 0.13 & 0.09 & 0.09 & 0.09 \\
\hline 1 & 2 & 0.13 & 0.10 & 0.10 & 0.10 \\
\hline 1 & 3 & 0.08 & 0.08 & 0.06 & 0.07 \\
\hline 1 & 4 & - & - & - & - \\
\hline 2 & 1 & 0.12 & 0.09 & 0.09 & 0.09 \\
\hline 2 & 2 & 0.12 & 0.10 & 0.10 & 0.10 \\
\hline 2 & 3 & 0.07 & 0.09 & 0.06 & 0.07 \\
\hline 2 & 4 & - & - & - & - \\
\hline 3 & 1 & 0.17 & 0.10 & 0.10 & 0.09 \\
\hline 3 & 2 & 0.17 & 0.13 & 0.12 & 0.13 \\
\hline 3 & 3 & 0.09 & 0.09 & 0.07 & - \\
\hline 3 & 4 & - & - & - & - \\
\hline 4 & 1 & 0.26 & 0.12 & 0.10 & 0.11 \\
\hline 4 & 2 & 0.24 & 0.15 & 0.13 & 0.15 \\
\hline 4 & 3 & - & - & - & - \\
\hline 4 & 4 & - & - & - & - \\
\hline 5 & 1 & 0.31 & 0.12 & 0.11 & 0.13 \\
\hline 5 & 2 & 0.27 & 0.16 & 0.15 & 0.17 \\
\hline 5 & 3 & - & - & - & - \\
\hline 5 & 4 & - & - & - & - \\
\hline
\end{tabular}


Table B.1.13 the results of calibration and validations based on the average area

\begin{tabular}{|c|c|c|c|c|c|}
\hline \multicolumn{2}{|c|}{ Configurations } & Calibration & $1^{\text {st }}$ Validation & $2^{\text {nd }}$ Validation & $3^{\text {rd }}$ Validation \\
\hline$\varphi_{g}$ & $\Lambda_{l}$ & Kappa & Kappa & Kappa & Kappa \\
\hline 1 & 1 & 0.73 & 0.66 & 0.54 & 0.61 \\
\hline 1 & 2 & - & - & - & - \\
\hline 1 & 3 & - & - & - & - \\
\hline 1 & 4 & - & - & - & - \\
\hline 2 & 1 & 0.77 & 0.69 & 0.53 & 0.60 \\
\hline 2 & 2 & - & - & - & - \\
\hline 2 & 3 & - & - & - & - \\
\hline 2 & 4 & - & - & - & - \\
\hline 3 & 1 & 0.78 & 0.66 & 0.55 & 0.61 \\
\hline 3 & 2 & - & - & - & - \\
\hline 3 & 3 & - & - & - & - \\
\hline 3 & 4 & - & - & - & - \\
\hline 4 & 1 & 0.79 & 0.67 & 0.61 & 0.67 \\
\hline 4 & 2 & - & - & - & - \\
\hline 4 & 3 & - & - & - & - \\
\hline 4 & 4 & - & - & - & - \\
\hline 5 & 1 & 0.79 & 0.73 & 0.67 & 0.72 \\
\hline 5 & 2 & - & - & - & - \\
\hline 5 & 3 & - & - & - & - \\
\hline 5 & 4 & - & - & - & - \\
\hline
\end{tabular}

Table B.1.14 the results of calibration and validations based on the maximum area

\begin{tabular}{|c|c|c|c|c|c|}
\hline \multicolumn{2}{|c|}{ Configurations } & \multirow{2}{*}{$\begin{array}{c}\text { Calibration } \\
\text { Kappa } \\
\end{array}$} & \multirow{2}{*}{$\begin{array}{c}\mathbf{1}^{\text {st }} \text { Validation } \\
\text { Kappa } \\
\end{array}$} & \multirow{2}{*}{$\begin{array}{cl}2^{\text {nd }} & \text { Validation } \\
\text { Kappa } \\
\end{array}$} & \multirow{2}{*}{$\begin{array}{c}3^{\text {rd }} \text { Validation } \\
\text { Kappa } \\
\end{array}$} \\
\hline$\varphi_{g}$ & $\Lambda_{l}$ & & & & \\
\hline 1 & 1 & 0.64 & 0.62 & 0.46 & 0.53 \\
\hline 1 & 2 & 0.57 & - & - & - \\
\hline 1 & 3 & - & - & - & - \\
\hline 1 & 4 & - & - & - & - \\
\hline 2 & 1 & 0.70 & 0.63 & 0.44 & 0.52 \\
\hline 2 & 2 & - & - & - & - \\
\hline 2 & 3 & - & - & - & - \\
\hline 2 & 4 & - & - & - & - \\
\hline 3 & 1 & 0.70 & 0.63 & 0.38 & 0.48 \\
\hline 3 & 2 & 0.61 & 0.56 & 0.37 & 0.45 \\
\hline 3 & 3 & - & - & - & - \\
\hline 3 & 4 & - & - & - & - \\
\hline 4 & 1 & 0.70 & 0.66 & 0.36 & 0.48 \\
\hline 4 & 2 & 0.63 & 0.60 & 0.32 & 0.44 \\
\hline 4 & 3 & - & - & - & - \\
\hline 4 & 4 & - & - & - & - \\
\hline 5 & 1 & 0.68 & 0.67 & 0.34 & 0.48 \\
\hline 5 & 2 & 0.61 & 0.57 & 0.30 & 0.42 \\
\hline 5 & 3 & - & - & - & - \\
\hline 5 & 4 & - & - & - & - \\
\hline
\end{tabular}


Table B.1.15 the results of calibration and validations based on the average length

\begin{tabular}{|c|c|c|c|c|c|}
\hline \multicolumn{2}{|c|}{ Configurations } & \multirow{2}{*}{$\begin{array}{c}\text { Calibration } \\
\text { Kappa }\end{array}$} & \multirow{2}{*}{$\frac{\mathbf{1}^{\text {st }} \text { Validation }}{\text { Kappa }}$} & \multirow{2}{*}{$\begin{array}{c}2^{\text {nd }} \text { Validation } \\
\text { Kappa }\end{array}$} & \multirow{2}{*}{$\begin{array}{c}3^{\text {rd }} \text { Validation } \\
\text { Kappa }\end{array}$} \\
\hline$\varphi_{g}$ & $\Lambda_{l}$ & & & & \\
\hline 1 & 1 & 0.50 & 0.37 & 0.41 & 0.40 \\
\hline 1 & 2 & 0.47 & 0.37 & 0.46 & 0.43 \\
\hline 1 & 3 & 0.51 & 0.35 & 0.45 & 0.41 \\
\hline 1 & 4 & - & - & - & - \\
\hline 2 & 1 & 0.51 & 0.38 & 0.41 & 0.40 \\
\hline 2 & 2 & 0.50 & 0.34 & 0.44 & 0.40 \\
\hline 2 & 3 & 0.53 & 0.36 & 0.44 & 0.41 \\
\hline 2 & 4 & - & - & - & - \\
\hline 3 & 1 & 0.50 & 0.38 & 0.39 & 0.39 \\
\hline 3 & 2 & 0.50 & 0.32 & 0.40 & 0.37 \\
\hline 3 & 3 & 0.55 & 0.31 & 0.36 & 0.34 \\
\hline 3 & 4 & - & - & - & - \\
\hline 4 & 1 & 0.50 & 0.39 & 0.33 & 0.35 \\
\hline 4 & 2 & 0.50 & - & - & - \\
\hline 4 & 3 & 0.59 & 0.32 & 0.29 & 0.30 \\
\hline 4 & 4 & - & - & - & - \\
\hline 5 & 1 & - & - & - & - \\
\hline 5 & 2 & 0.58 & 0.28 & 0.16 & 0.20 \\
\hline 5 & 3 & 0.66 & 0.33 & 0.20 & 0.25 \\
\hline 5 & 4 & - & - & - & - \\
\hline
\end{tabular}

Table B.1.16 the results of calibration and validations based on the maximum length

\begin{tabular}{|c|c|c|c|c|c|}
\hline \multicolumn{2}{|c|}{ Configurations } & \multirow{2}{*}{$\begin{array}{c}\text { Calibration } \\
\text { Kappa } \\
\end{array}$} & \multirow{2}{*}{$\begin{array}{c}\mathbf{1}^{\text {st }} \text { Validation } \\
\text { Kappa } \\
\end{array}$} & \multirow{2}{*}{$\begin{array}{cl}2^{\text {nd }} & \text { Validation } \\
\text { Kappa } \\
\end{array}$} & \multirow{2}{*}{$\begin{array}{c}3^{\text {rd }} \text { Validation } \\
\text { Kappa } \\
\end{array}$} \\
\hline$\varphi_{g}$ & $\Lambda_{l}$ & & & & \\
\hline 1 & 1 & 0.43 & 0.36 & 0.55 & 0.49 \\
\hline 1 & 2 & 0.44 & 0.35 & 0.52 & 0.46 \\
\hline 1 & 3 & 0.49 & 0.37 & - & - \\
\hline 1 & 4 & - & - & - & - \\
\hline 2 & 1 & 0.42 & 0.34 & 0.53 & 0.48 \\
\hline 2 & 2 & 0.44 & 0.34 & 0.51 & 0.46 \\
\hline 2 & 3 & 0.51 & 0.36 & - & - \\
\hline 2 & 4 & - & - & - & - \\
\hline 3 & 1 & 0.43 & 0.33 & 0.51 & 0.46 \\
\hline 3 & 2 & 0.45 & 0.27 & - & - \\
\hline 3 & 3 & 0.53 & 0.33 & - & - \\
\hline 3 & 4 & 0.32 & 0.25 & - & - \\
\hline 4 & 1 & - & - & - & - \\
\hline 4 & 2 & 0.50 & 0.27 & - & - \\
\hline 4 & 3 & 0.57 & 0.33 & 0.34 & 0.34 \\
\hline 4 & 4 & - & - & - & - \\
\hline 5 & 1 & 0.63 & - & - & - \\
\hline 5 & 2 & 0.57 & 0.32 & - & - \\
\hline 5 & 3 & 0.63 & 0.39 & 0.29 & 0.34 \\
\hline 5 & 4 & 0.57 & - & - & - \\
\hline
\end{tabular}


Table B.1.17 the results of calibration and validations based on the average width

\begin{tabular}{|c|c|c|c|c|c|}
\hline \multicolumn{2}{|c|}{ Configurations } & \multirow{2}{*}{$\begin{array}{c}\text { Calibration } \\
\text { Kappa }\end{array}$} & \multirow{2}{*}{$\frac{1^{\text {st }} \text { Validation }}{\text { Kappa }}$} & \multirow{2}{*}{$\begin{array}{c}\text { Validation } 2 \\
\text { Kappa }\end{array}$} & \multirow{2}{*}{$\frac{3^{\text {rd }} \text { Validation }}{\text { Kappa }}$} \\
\hline$\varphi_{g}$ & $\Lambda_{l}$ & & & & \\
\hline 1 & 1 & 0.71 & 0.64 & 0.52 & 0.58 \\
\hline 1 & 2 & 0.69 & 0.62 & 0.46 & 0.54 \\
\hline 1 & 3 & - & - & - & - \\
\hline 1 & 4 & - & - & - & - \\
\hline 2 & 1 & 0.78 & 0.68 & 0.52 & 0.59 \\
\hline 2 & 2 & 0.76 & 0.64 & 0.46 & 0.54 \\
\hline 2 & 3 & - & - & - & - \\
\hline 2 & 4 & - & - & - & - \\
\hline 3 & 1 & 0.80 & 0.66 & 0.52 & 0.59 \\
\hline 3 & 2 & 0.79 & 0.63 & 0.46 & 0.54 \\
\hline 3 & 3 & - & - & - & - \\
\hline 3 & 4 & - & - & - & - \\
\hline 4 & 1 & 0.81 & 0.71 & 0.55 & 0.63 \\
\hline 4 & 2 & 0.80 & 0.66 & 0.49 & 0.58 \\
\hline 4 & 3 & - & - & - & - \\
\hline 4 & 4 & - & - & - & - \\
\hline 5 & 1 & 0.82 & 0.72 & 0.54 & 0.64 \\
\hline 5 & 2 & 0.80 & 0.68 & 0.50 & 0.60 \\
\hline 5 & 3 & - & - & - & - \\
\hline 5 & 4 & - & - & - & - \\
\hline
\end{tabular}

Table B.1.18 the results of calibration and validations based on the maximum width

\begin{tabular}{|c|c|c|c|c|c|}
\hline \multicolumn{2}{|c|}{ Configurations } & \multirow{2}{*}{$\begin{array}{c}\text { Calibration } \\
\text { Kappa }\end{array}$} & \multirow{2}{*}{$\begin{array}{c}\mathbf{1}^{\text {st }} \text { Validation } \\
\text { Kappa }\end{array}$} & \multirow{2}{*}{$\begin{array}{ll}2^{\text {nd }} & \text { Validation } \\
& \text { Kappa }\end{array}$} & \multirow{2}{*}{$\begin{array}{c}\mathbf{3}^{\text {rd }} \text { Validation } \\
\text { Kappa }\end{array}$} \\
\hline$\varphi_{g}$ & $\Lambda_{l}$ & & & & \\
\hline 1 & 1 & 0.64 & 0.71 & 0.66 & 0.69 \\
\hline 1 & 2 & 0.63 & 0.69 & 0.64 & 0.67 \\
\hline 1 & 3 & 0.56 & 0.65 & 0.61 & - \\
\hline 1 & 4 & - & - & - & - \\
\hline 2 & 1 & 0.74 & 0.73 & 0.67 & 0.70 \\
\hline 2 & 2 & 0.73 & 0.72 & 0.65 & 0.69 \\
\hline 2 & 3 & - & - & - & - \\
\hline 2 & 4 & - & - & - & - \\
\hline 3 & 1 & 0.77 & 0.74 & 0.70 & 0.73 \\
\hline 3 & 2 & 0.76 & 0.73 & 0.67 & 0.71 \\
\hline 3 & 3 & - & - & - & - \\
\hline 3 & 4 & - & - & - & - \\
\hline 4 & 1 & 0.77 & 0.71 & - & - \\
\hline 4 & 2 & 0.75 & 0.76 & 0.69 & 0.74 \\
\hline 4 & 3 & 0.68 & 0.75 & 0.71 & 0.74 \\
\hline 4 & 4 & - & - & - & - \\
\hline 5 & 1 & 0.78 & 0.70 & - & - \\
\hline 5 & 2 & 0.74 & 0.74 & 0.71 & 0.75 \\
\hline 5 & 3 & - & - & - & - \\
\hline 5 & 4 & - & - & - & - \\
\hline
\end{tabular}


Table B.1.19 the results of calibration and validations based on the peaks in speed

\begin{tabular}{|c|c|c|c|c|c|}
\hline \multicolumn{2}{|c|}{ Configurations } & \multirow{2}{*}{$\begin{array}{c}\text { Calibration } \\
\text { Kappa }\end{array}$} & \multirow{2}{*}{$\frac{1^{\text {st }} \text { Validation }}{\text { Kappa }}$} & \multirow{2}{*}{$\begin{array}{c}2^{\text {nd }} \text { Validation } \\
\text { Kappa }\end{array}$} & \multirow{2}{*}{$\frac{3^{\text {rd }} \text { Validation }}{\text { Kappa }}$} \\
\hline$\varphi_{g}$ & $\Lambda_{l}$ & & & & \\
\hline 1 & 1 & 0.30 & 0.29 & 0.21 & 0.24 \\
\hline 1 & 2 & 0.28 & 0.29 & 0.19 & 0.23 \\
\hline 1 & 3 & 0.25 & 0.26 & 0.16 & 0.20 \\
\hline 1 & 4 & 0.20 & 0.24 & 0.20 & 0.22 \\
\hline 2 & 1 & 0.31 & 0.29 & 0.19 & 0.23 \\
\hline 2 & 2 & 0.30 & 0.28 & 0.18 & 0.22 \\
\hline 2 & 3 & 0.27 & 0.26 & 0.15 & 0.19 \\
\hline 2 & 4 & 0.21 & 0.26 & 0.20 & 0.23 \\
\hline 3 & 1 & 0.27 & 0.23 & 0.19 & 0.21 \\
\hline 3 & 2 & 0.26 & 0.23 & 0.17 & 0.20 \\
\hline 3 & 3 & 0.24 & 0.22 & 0.14 & 0.18 \\
\hline 3 & 4 & 0.21 & 0.23 & 0.20 & 0.23 \\
\hline 4 & 1 & 0.27 & 0.29 & 0.24 & 0.27 \\
\hline 4 & 2 & 0.24 & 0.30 & 0.22 & 0.26 \\
\hline 4 & 3 & 0.23 & 0.27 & 0.21 & 0.25 \\
\hline 4 & 4 & 0.21 & 0.22 & 0.18 & 0.22 \\
\hline 5 & 1 & 0.26 & 0.29 & 0.19 & 0.25 \\
\hline 5 & 2 & 0.24 & 0.28 & 0.18 & 0.24 \\
\hline 5 & 3 & 0.23 & 0.25 & 0.19 & 0.24 \\
\hline 5 & 4 & 0.21 & 0.23 & 0.21 & 0.24 \\
\hline
\end{tabular}

Table B.1.20 the results of calibration and validations based on the effective weighted average frequency

\begin{tabular}{|c|c|c|c|c|c|c|}
\hline \multicolumn{3}{|c|}{ Configurations } & Calibration & $1^{\text {st }}$ Validation & $2^{\text {nd }}$ Validation & $3^{\text {rd }}$ Validation \\
\hline$\varphi_{g}$ & $\Lambda_{l}$ & $\Gamma_{n}$ & Kappa & Kappa & Kappa & Kappa \\
\hline 1 & 1 & 1 & 0.19 & 0.26 & 0.26 & 0.28 \\
\hline 1 & 1 & 2 & 0.19 & 0.26 & 0.26 & 0.28 \\
\hline 1 & 1 & 3 & 0.18 & 0.25 & 0.26 & 0.27 \\
\hline 1 & 1 & 4 & 0.18 & 0.25 & 0.23 & 0.26 \\
\hline 1 & 1 & 1 & 0.18 & 0.25 & 0.28 & 0.29 \\
\hline 1 & 1 & 6 & 0.19 & 0.25 & 0.28 & 0.29 \\
\hline 1 & 2 & 1 & 0.16 & 0.21 & - & - \\
\hline 1 & 2 & 2 & 0.16 & 0.20 & - & - \\
\hline 1 & 2 & 3 & 0.15 & 0.20 & - & - \\
\hline 1 & 2 & 4 & 0.15 & 0.20 & - & - \\
\hline 1 & 2 & 1 & 0.15 & 0.21 & - & - \\
\hline 1 & 2 & 6 & 0.16 & 0.20 & - & - \\
\hline 1 & 3 & 1 & 0.14 & - & - & - \\
\hline 1 & 3 & 2 & 0.13 & - & - & - \\
\hline 1 & 3 & 3 & 0.13 & - & - & - \\
\hline 1 & 3 & 4 & 0.13 & - & - & - \\
\hline 1 & 3 & 1 & 0.14 & - & - & - \\
\hline 1 & 3 & 6 & 0.14 & - & - & - \\
\hline 1 & 4 & 1 & 0.17 & 0.20 & 0.22 & - \\
\hline 1 & 4 & 2 & 0.17 & 0.21 & - & - \\
\hline 1 & 4 & 3 & 0.16 & 0.20 & - & - \\
\hline 1 & 4 & 4 & 0.16 & 0.21 & - & - \\
\hline 1 & 4 & 1 & 0.16 & 0.21 & - & - \\
\hline 1 & 4 & 6 & 0.17 & 0.21 & - & - \\
\hline
\end{tabular}


Table B.1.20 the results of calibration and validations based on the effective weighted average frequency

\begin{tabular}{|c|c|c|c|c|c|c|}
\hline \multicolumn{3}{|c|}{ Configurations } & \multirow{2}{*}{$\begin{array}{c}\text { Calibration } \\
\text { Kappa } \\
\end{array}$} & \multirow{2}{*}{$\begin{array}{c}\mathbf{1}^{\text {st }} \text { Validation } \\
\text { Kappa } \\
\end{array}$} & \multirow{2}{*}{$\begin{array}{cl}2^{\text {nd }} & \text { Validation } \\
\text { Kappa } \\
\end{array}$} & \multirow{2}{*}{$\begin{array}{c}3^{\text {rd }} \text { Validation } \\
\text { Kappa } \\
\end{array}$} \\
\hline$\varphi_{g}$ & $\Lambda_{l}$ & $\Gamma_{n}$ & & & & \\
\hline 2 & 1 & 1 & 0.23 & 0.28 & 0.27 & 0.30 \\
\hline 2 & 1 & 2 & 0.23 & 0.28 & 0.28 & 0.30 \\
\hline 2 & 1 & 3 & 0.22 & 0.27 & 0.27 & 0.29 \\
\hline 2 & 1 & 4 & 0.22 & 0.27 & 0.24 & 0.28 \\
\hline 2 & 1 & 1 & 0.22 & 0.28 & 0.26 & 0.29 \\
\hline 2 & 1 & 6 & 0.23 & 0.27 & 0.29 & 0.31 \\
\hline 2 & 2 & 1 & 0.19 & 0.22 & - & - \\
\hline 2 & 2 & 2 & 0.19 & 0.22 & - & - \\
\hline 2 & 2 & 3 & 0.19 & 0.21 & - & - \\
\hline 2 & 2 & 4 & 0.19 & 0.21 & - & - \\
\hline 2 & 2 & 1 & 0.19 & 0.22 & - & - \\
\hline 2 & 2 & 6 & 0.19 & 0.22 & - & - \\
\hline 2 & 3 & 1 & 0.17 & - & - & - \\
\hline 2 & 3 & 2 & 0.17 & - & - & - \\
\hline 2 & 3 & 3 & 0.16 & - & - & - \\
\hline 2 & 3 & 4 & 0.16 & - & - & - \\
\hline 2 & 3 & 1 & 0.17 & - & - & - \\
\hline 2 & 3 & 6 & 0.17 & - & - & - \\
\hline 2 & 4 & 1 & 0.20 & 0.22 & 0.23 & - \\
\hline 2 & 4 & 2 & 0.20 & 0.22 & - & - \\
\hline 2 & 4 & 3 & 0.19 & 0.22 & - & - \\
\hline 2 & 4 & 4 & 0.20 & 0.22 & - & - \\
\hline 2 & 4 & 1 & 0.20 & 0.22 & - & - \\
\hline 2 & 4 & 6 & 0.20 & 0.23 & - & - \\
\hline 3 & 1 & 1 & 0.30 & 0.35 & 0.33 & 0.37 \\
\hline 3 & 1 & 2 & 0.29 & 0.34 & 0.34 & 0.37 \\
\hline 3 & 1 & 3 & 0.29 & 0.33 & 0.33 & 0.36 \\
\hline 3 & 1 & 4 & 0.29 & 0.33 & 0.34 & 0.36 \\
\hline 3 & 1 & 1 & 0.29 & 0.34 & 0.32 & 0.36 \\
\hline 3 & 1 & 6 & 0.29 & 0.34 & 0.35 & 0.38 \\
\hline 3 & 2 & 1 & 0.24 & 0.27 & - & - \\
\hline 3 & 2 & 2 & 0.24 & 0.27 & - & - \\
\hline 3 & 2 & 3 & 0.24 & 0.26 & - & - \\
\hline 3 & 2 & 4 & 0.24 & 0.26 & - & - \\
\hline 3 & 2 & 1 & 0.24 & 0.27 & - & - \\
\hline 3 & 2 & 6 & 0.24 & 0.27 & - & - \\
\hline 3 & 3 & 1 & - & - & - & - \\
\hline 3 & 3 & 2 & - & - & - & - \\
\hline 3 & 3 & 3 & - & - & - & - \\
\hline 3 & 3 & 4 & - & - & - & - \\
\hline 3 & 3 & 1 & - & - & - & - \\
\hline 3 & 3 & 6 & 0.21 & - & - & - \\
\hline 3 & 4 & 1 & 0.25 & 0.26 & 0.28 & - \\
\hline 3 & 4 & 2 & 0.25 & 0.27 & - & - \\
\hline 3 & 4 & 3 & 0.24 & 0.26 & - & - \\
\hline 3 & 4 & 4 & 0.24 & 0.27 & - & - \\
\hline 3 & 4 & 1 & 0.25 & 0.27 & - & - \\
\hline 3 & 4 & 6 & 0.25 & 0.27 & - & - \\
\hline 4 & 1 & 1 & 0.39 & 0.38 & 0.46 & 0.46 \\
\hline 4 & 1 & 2 & 0.38 & 0.39 & 0.45 & 0.46 \\
\hline
\end{tabular}


Table B.1.20 the results of calibration and validations based on the effective weighted average frequency

\begin{tabular}{|c|c|c|c|c|c|c|}
\hline \multicolumn{3}{|c|}{ Configurations } & \multirow{2}{*}{$\begin{array}{c}\text { Calibration } \\
\text { Kappa }\end{array}$} & \multirow{2}{*}{$\begin{array}{c}\mathbf{1}^{\text {st }} \text { Validation } \\
\text { Kappa }\end{array}$} & \multirow{2}{*}{$\begin{array}{cl}2^{\text {nd }} & \text { Validation } \\
\text { Kappa } \\
\end{array}$} & \multirow{2}{*}{$\frac{3^{\text {rd }} \text { Validation }}{\text { Kappa }}$} \\
\hline$\varphi_{g}$ & $\Lambda_{l}$ & $\Gamma_{n}$ & & & & \\
\hline 4 & 1 & 3 & 0.37 & 0.38 & 0.44 & 0.45 \\
\hline 4 & 1 & 4 & 0.37 & 0.39 & 0.41 & 0.44 \\
\hline 4 & 1 & 1 & 0.37 & 0.39 & 0.42 & 0.44 \\
\hline 4 & 1 & 6 & 0.36 & 0.38 & 0.43 & 0.45 \\
\hline 4 & 2 & 1 & 0.31 & 0.30 & - & - \\
\hline 4 & 2 & 2 & 0.31 & 0.30 & - & - \\
\hline 4 & 2 & 3 & 0.31 & 0.30 & - & - \\
\hline 4 & 2 & 4 & 0.31 & 0.30 & - & - \\
\hline 4 & 2 & 1 & 0.31 & 0.31 & - & - \\
\hline 4 & 2 & 6 & 0.32 & 0.31 & - & - \\
\hline 4 & 3 & 1 & - & - & - & - \\
\hline 4 & 3 & 2 & - & - & - & - \\
\hline 4 & 3 & 3 & - & - & - & - \\
\hline 4 & 3 & 4 & - & - & - & - \\
\hline 4 & 3 & 1 & - & - & - & - \\
\hline 4 & 3 & 6 & - & - & - & - \\
\hline 4 & 4 & 1 & 0.30 & 0.29 & - & - \\
\hline 4 & 4 & 2 & 0.30 & 0.30 & - & - \\
\hline 4 & 4 & 3 & 0.31 & 0.30 & - & - \\
\hline 4 & 4 & 4 & 0.31 & 0.30 & - & - \\
\hline 4 & 4 & 1 & 0.31 & 0.30 & - & - \\
\hline 4 & 4 & 6 & 0.32 & 0.31 & - & - \\
\hline 5 & 1 & 1 & 0.45 & 0.45 & 0.56 & 0.55 \\
\hline 5 & 1 & 2 & 0.44 & 0.45 & 0.55 & 0.54 \\
\hline 5 & 1 & 3 & 0.43 & 0.43 & 0.53 & 0.53 \\
\hline 5 & 1 & 4 & 0.42 & 0.44 & 0.49 & 0.51 \\
\hline 5 & 1 & 1 & 0.42 & 0.44 & 0.51 & 0.52 \\
\hline 5 & 1 & 6 & 0.42 & 0.43 & 0.51 & 0.51 \\
\hline 5 & 2 & 1 & 0.37 & 0.37 & - & - \\
\hline 5 & 2 & 2 & 0.36 & 0.36 & - & - \\
\hline 5 & 2 & 3 & 0.37 & 0.37 & - & - \\
\hline 5 & 2 & 4 & 0.37 & 0.36 & - & - \\
\hline 5 & 2 & 1 & 0.36 & 0.37 & - & - \\
\hline 5 & 2 & 6 & 0.36 & 0.37 & - & - \\
\hline 5 & 3 & 1 & - & - & - & - \\
\hline 5 & 3 & 2 & - & - & - & - \\
\hline 5 & 3 & 3 & - & - & - & - \\
\hline 5 & 3 & 4 & - & - & - & - \\
\hline 5 & 3 & 1 & - & - & - & - \\
\hline 5 & 3 & 6 & - & - & - & - \\
\hline 5 & 4 & 1 & 0.35 & 0.35 & - & - \\
\hline 5 & 4 & 2 & 0.35 & 0.36 & - & - \\
\hline 5 & 4 & 3 & 0.37 & 0.36 & - & - \\
\hline 5 & 4 & 4 & 0.36 & 0.37 & - & - \\
\hline 5 & 4 & 1 & 0.36 & 0.36 & - & - \\
\hline 5 & 4 & 6 & 0.37 & 0.37 & - & - \\
\hline
\end{tabular}


Table B.1.21 the results of calibration and validations based on the effective frequency

\begin{tabular}{|c|c|c|c|c|c|c|}
\hline \multicolumn{3}{|c|}{ Configurations } & \multirow{2}{*}{$\begin{array}{c}\text { Calibration } \\
\text { Kappa }\end{array}$} & \multirow{2}{*}{$\frac{\mathbf{1}^{\text {st }} \text { Validation }}{\text { Kappa }}$} & \multirow{2}{*}{$\begin{array}{cl}2^{\text {nd }} & \text { Validation } \\
\text { Kappa }\end{array}$} & \multirow{2}{*}{$\frac{3^{\text {rd }} \text { Validation }}{\text { Kappa }}$} \\
\hline$\varphi_{g}$ & $\Lambda_{l}$ & $\eta_{m}$ & & & & \\
\hline 1 & 1 & 1 & 0.05 & 0.04 & 0.06 & 0.05 \\
\hline 1 & 1 & 2 & 0.03 & 0.06 & - & - \\
\hline 1 & 1 & 3 & 0.03 & - & - & - \\
\hline 1 & 2 & 1 & 0.05 & 0.04 & 0.05 & 0.05 \\
\hline 1 & 2 & 2 & 0.04 & 0.06 & - & - \\
\hline 1 & 2 & 3 & 0.03 & - & - & - \\
\hline 1 & 3 & 1 & 0.05 & 0.04 & 0.05 & 0.05 \\
\hline 1 & 3 & 2 & 0.04 & 0.06 & 0.04 & 0.05 \\
\hline 1 & 3 & 3 & 0.04 & - & - & - \\
\hline 1 & 4 & 1 & 0.04 & 0.04 & 0.05 & 0.05 \\
\hline 1 & 4 & 2 & 0.03 & 0.06 & - & - \\
\hline 1 & 4 & 3 & - & - & - & - \\
\hline 2 & 1 & 1 & 0.04 & 0.05 & 0.05 & 0.05 \\
\hline 2 & 1 & 2 & 0.04 & 0.05 & 0.03 & 0.04 \\
\hline 2 & 1 & 3 & 0.04 & - & - & - \\
\hline 2 & 2 & 1 & 0.05 & 0.05 & 0.05 & 0.05 \\
\hline 2 & 2 & 2 & 0.04 & - & - & - \\
\hline 2 & 2 & 3 & 0.04 & - & - & - \\
\hline 2 & 3 & 1 & 0.05 & 0.04 & 0.05 & 0.05 \\
\hline 2 & 3 & 2 & 0.04 & 0.05 & 0.03 & 0.04 \\
\hline 2 & 3 & 3 & 0.05 & - & - & - \\
\hline 2 & 4 & 1 & 0.04 & 0.04 & 0.05 & 0.05 \\
\hline 2 & 4 & 2 & 0.04 & 0.05 & 0.03 & 0.04 \\
\hline 2 & 4 & 3 & 0.03 & - & - & - \\
\hline 3 & 1 & 1 & 0.04 & 0.05 & 0.07 & 0.07 \\
\hline 3 & 1 & 2 & 0.04 & - & - & - \\
\hline 3 & 1 & 3 & 0.04 & - & - & - \\
\hline 3 & 2 & 1 & 0.04 & 0.05 & 0.07 & 0.07 \\
\hline 3 & 2 & 2 & 0.05 & - & - & - \\
\hline 3 & 2 & 3 & 0.05 & - & - & - \\
\hline 3 & 3 & 1 & 0.04 & 0.05 & 0.07 & 0.07 \\
\hline 3 & 3 & 2 & 0.04 & 0.03 & 0.08 & - \\
\hline 3 & 3 & 3 & 0.06 & - & - & - \\
\hline 3 & 4 & 1 & 0.04 & 0.05 & 0.07 & 0.07 \\
\hline 3 & 4 & 2 & 0.04 & 0.06 & 0.04 & 0.05 \\
\hline 3 & 4 & 3 & 0.03 & - & - & - \\
\hline 4 & 1 & 1 & 0.05 & 0.04 & - & - \\
\hline 4 & 1 & 2 & 0.04 & 0.07 & 0.07 & 0.08 \\
\hline 4 & 1 & 3 & 0.04 & - & - & - \\
\hline 4 & 2 & 1 & 0.05 & 0.04 & 0.08 & 0.07 \\
\hline 4 & 2 & 2 & 0.05 & - & - & - \\
\hline 4 & 2 & 3 & 0.05 & - & - & - \\
\hline 4 & 3 & 1 & 0.05 & 0.04 & 0.08 & 0.07 \\
\hline 4 & 3 & 2 & 0.04 & 0.07 & 0.07 & 0.08 \\
\hline 4 & 3 & 3 & 0.07 & - & - & - \\
\hline 4 & 4 & 1 & 0.05 & 0.04 & 0.07 & 0.07 \\
\hline 4 & 4 & 2 & 0.04 & 0.07 & 0.08 & 0.08 \\
\hline 4 & 4 & 3 & 0.04 & - & - & - \\
\hline 5 & 1 & 1 & 0.05 & 0.06 & 0.10 & 0.09 \\
\hline 5 & 1 & 2 & 0.05 & - & - & - \\
\hline 5 & 1 & 3 & 0.05 & - & - & - \\
\hline
\end{tabular}


Table B.1.21 the results of calibration and validations based on the effective frequency

\begin{tabular}{|c|c|c|c|c|c|c|}
\hline \multicolumn{3}{|c|}{ Configurations } & Calibration & $1^{\text {st }}$ Validation & $2^{\text {nd }}$ Validation & $3^{\text {rd }}$ Validation \\
\hline$\overline{\varphi_{g}}$ & $\Lambda_{l}$ & $\eta_{m}$ & Kappa & Kappa & Kappa & Kappa \\
\hline 5 & 2 & 1 & 0.05 & 0.06 & 0.10 & 0.09 \\
\hline 5 & 2 & 2 & 0.07 & - & - & - \\
\hline 5 & 2 & 3 & 0.06 & 0.04 & - & - \\
\hline 5 & 3 & 1 & 0.05 & 0.05 & 0.10 & 0.09 \\
\hline 5 & 3 & 2 & 0.05 & 0.04 & 0.06 & - \\
\hline 5 & 3 & 3 & 0.08 & 0.04 & 0.05 & - \\
\hline 5 & 4 & 1 & 0.05 & 0.06 & 0.10 & 0.09 \\
\hline 5 & 4 & 2 & 0.05 & 0.08 & 0.07 & 0.08 \\
\hline 5 & 4 & 3 & 0.05 & - & - & - \\
\hline
\end{tabular}




\section{B.2 The results of calibrations and validations in percentage scale}

Table B.2.1 the results of calibration procedure 1 and its validations.

\begin{tabular}{|c|c|c|c|c|c|c|c|c|c|c|c|c|c|c|c|}
\hline \multicolumn{4}{|c|}{ Configurations } & \multicolumn{3}{|c|}{ Calibration } & \multicolumn{3}{|c|}{$1^{\text {st }}$ Validation } & \multicolumn{3}{|c|}{$2^{\text {nd }}$ Validation } & \multicolumn{3}{|c|}{$3^{\text {rd }}$ Validation } \\
\hline$\varphi_{g}$ & $\Lambda_{l}$ & $\Gamma_{n}$ & $\eta_{m}$ & Vehicles & Cyclists & Pedestrians & Vehicles & Cyclists & Pedestrians & Vehicles & Cyclists & Pedestrians & Vehicles & Cyclists & Pedestrians \\
\hline 1 & 1 & 1 & 1 & 98.82 & 89.42 & 92.65 & 96.99 & 89.00 & 84.13 & 94.21 & 89.26 & 93.44 & 95.70 & 89.16 & 91.06 \\
\hline 1 & 1 & 1 & 2 & 99.12 & 89.42 & 92.65 & 97.32 & 89.00 & 84.13 & 94.98 & 89.93 & 93.44 & 96.24 & 89.56 & 91.06 \\
\hline 1 & 1 & 1 & 3 & 98.82 & 90.38 & 92.65 & 96.99 & 89.00 & 84.13 & 94.98 & 89.26 & 92.90 & 96.06 & 89.16 & 90.65 \\
\hline 1 & 1 & 2 & 1 & 98.82 & 89.42 & 92.65 & 96.99 & 89.00 & 84.13 & 94.21 & 87.92 & 93.44 & 95.70 & 88.35 & 91.06 \\
\hline 1 & 1 & 2 & 2 & 99.12 & 89.42 & 92.65 & 97.32 & 89.00 & 84.13 & 95.37 & 88.59 & 93.44 & 96.42 & 88.76 & 91.06 \\
\hline 1 & 1 & 2 & 3 & 98.82 & 90.38 & 92.65 & 96.99 & 89.00 & 84.13 & 95.37 & 87.92 & 92.90 & 96.24 & 88.35 & 90.65 \\
\hline 1 & 1 & 3 & 1 & 98.82 & 88.46 & 94.12 & 96.99 & 87.00 & 84.13 & 95.37 & 89.93 & 93.44 & 96.24 & 88.76 & 91.06 \\
\hline 1 & 1 & 3 & 2 & 99.12 & 89.42 & 94.12 & 97.32 & 90.00 & 84.13 & 94.98 & 88.59 & 92.35 & 96.24 & 89.16 & 90.24 \\
\hline 1 & 1 & 3 & 3 & 98.82 & 90.38 & 92.65 & 96.99 & 87.00 & 84.13 & 94.98 & 89.26 & 92.35 & 96.06 & 88.35 & 90.24 \\
\hline 1 & 1 & 4 & 1 & 98.82 & 90.38 & 92.65 & 96.99 & 88.00 & 84.13 & 94.21 & 89.93 & 93.99 & 95.70 & 89.16 & 91.46 \\
\hline 1 & 1 & 4 & 2 & 99.12 & 90.38 & 92.65 & 97.32 & 89.00 & 84.13 & 94.59 & 89.26 & 93.99 & 96.06 & 89.16 & 91.46 \\
\hline 1 & 1 & 4 & 3 & 98.82 & 91.35 & 92.65 & 96.99 & 89.00 & 84.13 & 94.59 & 89.26 & 93.44 & 95.88 & 89.16 & 91.06 \\
\hline 1 & 1 & 1 & 1 & 98.82 & 90.38 & 92.65 & 97.32 & 90.00 & 84.13 & 94.59 & 89.93 & 92.90 & 96.06 & 89.96 & 90.65 \\
\hline 1 & 1 & 1 & 2 & 99.12 & 90.38 & 92.65 & 97.66 & 90.00 & 84.13 & 94.98 & 89.26 & 92.90 & 96.42 & 89.56 & 90.65 \\
\hline 1 & 1 & 1 & 3 & 98.82 & 91.35 & 92.65 & 97.32 & 90.00 & 84.13 & 94.98 & 88.59 & 92.35 & 96.24 & 89.16 & 90.24 \\
\hline 1 & 1 & 6 & 1 & 98.82 & 90.38 & 94.12 & 97.32 & 91.00 & 82.54 & 94.98 & 88.59 & 92.35 & 96.24 & 89.56 & 89.84 \\
\hline 1 & 1 & 6 & 2 & 98.82 & 90.38 & 94.12 & 97.32 & 91.00 & 80.95 & 94.98 & 87.92 & 92.90 & 96.24 & 89.16 & 89.84 \\
\hline 1 & 1 & 6 & 3 & 98.82 & 91.35 & 92.65 & 97.32 & 91.00 & 80.95 & 94.59 & 88.59 & 91.80 & 96.06 & 89.56 & 89.02 \\
\hline 1 & 2 & 1 & 1 & 99.12 & 84.62 & 97.06 & 96.66 & 77.00 & 92.06 & - & - & - & - & - & - \\
\hline 1 & 2 & 1 & 2 & 99.12 & 83.65 & 97.06 & 96.66 & 77.00 & 92.06 & - & - & - & - & - & - \\
\hline 1 & 2 & 1 & 3 & 99.12 & 84.62 & 97.06 & 96.66 & 77.00 & 92.06 & - & - & - & - & - & - \\
\hline 1 & 2 & 2 & 1 & 99.12 & 84.62 & 97.06 & 96.66 & 78.00 & 92.06 & - & - & - & - & - & - \\
\hline 1 & 2 & 2 & 2 & 99.12 & 82.69 & 97.06 & 96.66 & 78.00 & 90.48 & - & - & - & - & - & - \\
\hline 1 & 2 & 2 & 3 & 99.12 & 83.65 & 97.06 & 96.66 & 78.00 & 90.48 & - & - & - & - & - & - \\
\hline 1 & 2 & 3 & 1 & 99.12 & 84.62 & 97.06 & 96.99 & 80.00 & 92.06 & - & - & - & - & - & - \\
\hline 1 & 2 & 3 & 2 & 99.12 & 82.69 & 97.06 & 96.99 & 80.00 & 90.48 & - & - & - & - & - & - \\
\hline 1 & 2 & 3 & 3 & 99.12 & 83.65 & 97.06 & 96.99 & 80.00 & 90.48 & - & - & - & - & - & - \\
\hline 1 & 2 & 4 & 1 & 98.82 & 85.58 & 95.59 & - & - & - & - & - & - & - & - & - \\
\hline 1 & 2 & 4 & 2 & 98.82 & 83.65 & 95.59 & - & - & - & - & - & - & - & - & - \\
\hline 1 & 2 & 4 & 3 & 98.82 & 83.65 & 97.06 & - & - & - & - & - & - & - & - & - \\
\hline 1 & 2 & 1 & 1 & 99.12 & 85.58 & 95.59 & 96.32 & 79.00 & 90.48 & - & - & - & - & - & - \\
\hline 1 & 2 & 1 & 2 & 99.12 & 83.65 & 97.06 & 96.32 & 79.00 & 90.48 & - & - & - & - & - & - \\
\hline 1 & 2 & 1 & 3 & 99.12 & 83.65 & 97.06 & 96.32 & 78.00 & 88.89 & - & - & - & - & - & - \\
\hline 1 & 2 & 6 & 1 & 98.82 & 85.58 & 97.06 & 95.65 & 79.00 & 92.06 & - & - & - & - & - & - \\
\hline
\end{tabular}


Table B.2.1 the results of calibration procedure 1 and its validations.

\begin{tabular}{|c|c|c|c|c|c|c|c|c|c|c|c|c|c|c|c|}
\hline \multicolumn{4}{|c|}{ Configurations } & \multicolumn{3}{|c|}{ Calibration } & \multicolumn{3}{|c|}{$1^{\text {st }}$ Validation } & \multicolumn{3}{|c|}{$2^{\text {nd }}$ Validation } & \multicolumn{3}{|c|}{$3^{\text {rd }}$ Validation } \\
\hline$\varphi_{g}$ & $\Lambda_{l}$ & $\overline{\Gamma_{n}}$ & $\eta_{m}$ & Vehicles & Cyclists & Pedestrians & Vehicles & Cyclists & Pedestrians & Vehicles & Cyclists & Pedestrians & Vehicles & Cyclists & Pedestrians \\
\hline 1 & 2 & 6 & 2 & 98.82 & 83.65 & 97.06 & 95.65 & 79.00 & 90.48 & - & - & - & - & - & - \\
\hline 1 & 2 & 6 & 3 & 98.82 & 84.62 & 97.06 & 95.65 & 79.00 & 90.48 & - & - & - & - & - & - \\
\hline 1 & 3 & 1 & 1 & 98.53 & 92.31 & 88.24 & - & - & - & - & - & - & - & - & - \\
\hline 1 & 3 & 1 & 2 & 98.53 & 92.31 & 89.71 & - & - & - & - & - & - & - & - & - \\
\hline 1 & 3 & 1 & 3 & 98.53 & 90.38 & 89.71 & - & - & - & - & - & - & - & - & - \\
\hline 1 & 3 & 2 & 1 & 98.53 & 90.38 & 91.18 & - & - & - & - & - & - & - & - & - \\
\hline 1 & 3 & 2 & 2 & 98.53 & 90.38 & 91.18 & - & - & - & - & - & - & - & - & - \\
\hline 1 & 3 & 2 & 3 & 98.53 & 89.42 & 91.18 & - & - & - & - & - & - & - & - & - \\
\hline 1 & 3 & 3 & 1 & 98.53 & 90.38 & 91.18 & - & - & - & - & - & - & - & - & - \\
\hline 1 & 3 & 3 & 2 & 98.53 & 90.38 & 91.18 & - & - & - & - & - & - & - & - & - \\
\hline 1 & 3 & 3 & 3 & 98.53 & 89.42 & 91.18 & - & - & - & - & - & - & - & - & - \\
\hline 1 & 3 & 4 & 1 & 98.53 & 92.31 & 89.71 & - & - & - & - & - & - & - & - & - \\
\hline 1 & 3 & 4 & 2 & 98.53 & 91.35 & 91.18 & - & - & - & - & - & - & - & - & - \\
\hline 1 & 3 & 4 & 3 & 98.53 & 90.38 & 89.71 & - & - & - & - & - & - & - & - & - \\
\hline 1 & 3 & 1 & 1 & 98.24 & 92.31 & 91.18 & - & - & - & - & - & - & - & - & - \\
\hline 1 & 3 & 1 & 2 & 98.24 & 92.31 & 91.18 & - & - & - & - & - & - & - & - & - \\
\hline 1 & 3 & 1 & 3 & 98.24 & 90.38 & 91.18 & - & - & - & - & - & - & - & - & - \\
\hline 1 & 3 & 6 & 1 & 98.53 & 89.42 & 91.18 & - & - & - & - & - & - & - & - & - \\
\hline 1 & 3 & 6 & 2 & 98.53 & 89.42 & 91.18 & - & - & - & - & - & - & - & - & - \\
\hline 1 & 3 & 6 & 3 & 98.53 & 88.46 & 92.65 & - & - & - & - & - & - & - & - & - \\
\hline 1 & 4 & 1 & 1 & 98.24 & 89.42 & 94.12 & 95.99 & 87.00 & 76.19 & - & - & - & - & - & - \\
\hline 1 & 4 & 1 & 2 & 97.94 & 89.42 & 92.65 & - & - & - & - & - & - & - & - & - \\
\hline 1 & 4 & 1 & 3 & 97.35 & 91.35 & 94.12 & - & - & - & - & - & - & - & - & - \\
\hline 1 & 4 & 2 & 1 & 98.24 & 89.42 & 94.12 & - & - & - & - & - & - & - & - & - \\
\hline 1 & 4 & 2 & 2 & 97.94 & 89.42 & 92.65 & - & - & - & - & - & - & - & - & - \\
\hline 1 & 4 & 2 & 3 & 96.76 & 91.35 & 95.59 & 92.64 & 89.00 & 90.48 & - & - & - & - & - & - \\
\hline 1 & 4 & 3 & 1 & 98.24 & 89.42 & 94.12 & 95.99 & 87.00 & 76.19 & - & - & - & - & - & - \\
\hline 1 & 4 & 3 & 2 & 97.94 & 88.46 & 94.12 & - & - & - & - & - & - & - & - & - \\
\hline 1 & 4 & 3 & 3 & 96.76 & 91.35 & 97.06 & 93.98 & 89.00 & 90.48 & - & - & - & - & - & - \\
\hline 1 & 4 & 4 & 1 & 97.94 & 90.38 & 94.12 & - & - & - & - & - & - & - & - & - \\
\hline 1 & 4 & 4 & 2 & 98.53 & 89.42 & 92.65 & - & - & - & - & - & - & - & - & - \\
\hline 1 & 4 & 4 & 3 & 97.94 & 91.35 & 94.12 & 93.65 & 89.00 & 92.06 & - & - & - & - & - & - \\
\hline 1 & 4 & 1 & 1 & 97.94 & 90.38 & 94.12 & - & - & - & - & - & - & - & - & - \\
\hline 1 & 4 & 1 & 2 & 97.94 & 90.38 & 94.12 & - & - & - & - & - & - & - & - & - \\
\hline 1 & 4 & 1 & 3 & 96.76 & 90.38 & 97.06 & 92.31 & 90.00 & 90.48 & - & - & - & - & - & - \\
\hline 1 & 4 & 6 & 1 & 98.24 & 90.38 & 94.12 & - & - & - & - & - & - & - & - & - \\
\hline
\end{tabular}


Table B.2.1 the results of calibration procedure 1 and its validations.

\begin{tabular}{|c|c|c|c|c|c|c|c|c|c|c|c|c|c|c|c|}
\hline \multicolumn{4}{|c|}{ Configurations } & \multicolumn{3}{|c|}{ Calibration } & \multicolumn{3}{|c|}{$1^{\text {st }}$ Validation } & \multicolumn{3}{|c|}{$2^{\text {nd }}$ Validation } & \multicolumn{3}{|c|}{$3^{\text {rd }}$ Validation } \\
\hline$\varphi_{g}$ & $\Lambda_{l}$ & $\overline{\Gamma_{n}}$ & $\eta_{m}$ & Vehicles & Cyclists & Pedestrians & Vehicles & Cyclists & Pedestrians & Vehicles & Cyclists & Pedestrians & Vehicles & Cyclists & Pedestrians \\
\hline 1 & 4 & 6 & 2 & 97.94 & 90.38 & 94.12 & - & - & - & - & - & - & - & - & - \\
\hline 1 & 4 & 6 & 3 & 97.65 & 91.35 & 95.59 & 93.31 & 89.00 & 92.06 & - & - & - & - & - & - \\
\hline 2 & 1 & 1 & 1 & 98.36 & 97.75 & 100.00 & - & - & - & - & - & - & - & - & - \\
\hline 2 & 1 & 1 & 2 & 98.03 & 97.75 & 100.00 & - & - & - & - & - & - & - & - & - \\
\hline 2 & 1 & 1 & 3 & 98.36 & 97.75 & 100.00 & - & - & - & - & - & - & - & - & - \\
\hline 2 & 1 & 2 & 1 & 98.03 & 97.75 & 100.00 & - & - & - & - & - & - & - & - & - \\
\hline 2 & 1 & 2 & 2 & 98.03 & 97.75 & 100.00 & - & - & - & - & - & - & - & - & - \\
\hline 2 & 1 & 2 & 3 & 98.03 & 97.75 & 100.00 & - & - & - & - & - & - & - & - & - \\
\hline 2 & 1 & 3 & 1 & 98.36 & 97.75 & 100.00 & - & - & - & - & - & - & - & - & - \\
\hline 2 & 1 & 3 & 2 & 98.03 & 97.75 & 100.00 & - & - & - & - & - & - & - & - & - \\
\hline 2 & 1 & 3 & 3 & 98.36 & 97.75 & 100.00 & - & - & - & - & - & - & - & - & - \\
\hline 2 & 1 & 4 & 1 & 98.03 & 97.75 & 100.00 & - & - & - & - & - & - & - & - & - \\
\hline 2 & 1 & 4 & 2 & 98.03 & 97.75 & 100.00 & - & - & - & - & - & - & - & - & - \\
\hline 2 & 1 & 4 & 3 & 98.03 & 97.75 & 100.00 & - & - & - & - & - & - & - & - & - \\
\hline 2 & 1 & 1 & 1 & 98.03 & 97.75 & 100.00 & - & - & - & - & - & - & - & - & - \\
\hline 2 & 1 & 1 & 2 & 98.03 & 97.75 & 100.00 & - & - & - & - & - & - & - & - & - \\
\hline 2 & 1 & 1 & 3 & 98.03 & 97.75 & 100.00 & - & - & - & - & - & - & - & - & - \\
\hline 2 & 1 & 6 & 1 & 98.03 & 97.75 & 100.00 & - & - & - & - & - & - & - & - & - \\
\hline 2 & 1 & 6 & 2 & 98.03 & 97.75 & 100.00 & - & - & - & - & - & - & - & - & - \\
\hline 2 & 1 & 6 & 3 & 98.03 & 97.75 & 100.00 & - & - & - & - & - & - & - & - & - \\
\hline 2 & 2 & 1 & 1 & 99.34 & 88.76 & 97.96 & - & - & - & - & - & - & - & - & - \\
\hline 2 & 2 & 1 & 2 & 99.34 & 89.89 & 97.96 & - & - & - & - & - & - & - & - & - \\
\hline 2 & 2 & 1 & 3 & 99.34 & 89.89 & 97.96 & - & - & - & - & - & - & - & - & - \\
\hline 2 & 2 & 2 & 1 & 99.34 & 88.76 & 97.96 & - & - & - & - & - & - & - & - & - \\
\hline 2 & 2 & 2 & 2 & 99.34 & 88.76 & 97.96 & - & - & - & - & - & - & - & - & - \\
\hline 2 & 2 & 2 & 3 & 99.34 & 88.76 & 97.96 & - & - & - & - & - & - & - & - & - \\
\hline 2 & 2 & 3 & 1 & 99.34 & 88.76 & 97.96 & - & - & - & - & - & - & - & - & - \\
\hline 2 & 2 & 3 & 2 & 99.34 & 88.76 & 97.96 & - & - & - & - & - & - & - & - & - \\
\hline 2 & 2 & 3 & 3 & 99.34 & 88.76 & 97.96 & - & - & - & - & - & - & - & - & - \\
\hline 2 & 2 & 4 & 1 & 99.34 & 89.89 & 97.96 & - & - & - & - & - & - & - & - & - \\
\hline 2 & 2 & 4 & 2 & 99.34 & 91.01 & 97.96 & - & - & - & - & - & - & - & - & - \\
\hline 2 & 2 & 4 & 3 & 99.34 & 91.01 & 97.96 & - & - & - & - & - & - & - & - & - \\
\hline 2 & 2 & 1 & 1 & 99.02 & 91.01 & 97.96 & - & - & - & - & - & - & - & - & - \\
\hline 2 & 2 & 1 & 2 & 99.02 & 91.01 & 97.96 & - & - & - & - & - & - & - & - & - \\
\hline 2 & 2 & 1 & 3 & 99.02 & 91.01 & 97.96 & - & - & - & - & - & - & - & - & - \\
\hline 2 & 2 & 6 & 1 & 99.34 & 88.76 & 97.96 & - & - & - & - & - & - & - & - & - \\
\hline
\end{tabular}


Table B.2.1 the results of calibration procedure 1 and its validations.

\begin{tabular}{|c|c|c|c|c|c|c|c|c|c|c|c|c|c|c|c|}
\hline \multicolumn{4}{|c|}{ Configurations } & \multicolumn{3}{|c|}{ Calibration } & \multicolumn{3}{|c|}{$1^{\text {st }}$ Validation } & \multicolumn{3}{|c|}{$2^{\text {nd }}$ Validation } & \multicolumn{3}{|c|}{$3^{\text {rd }}$ Validation } \\
\hline$\varphi_{g}$ & $\Lambda_{l}$ & $\Gamma_{n}$ & $\eta_{m}$ & Vehicles & Cyclists & Pedestrians & Vehicles & Cyclists & Pedestrians & Vehicles & Cyclists & Pedestrians & Vehicles & Cyclists & Pedestrians \\
\hline 2 & 2 & 6 & 2 & 99.34 & 89.89 & 97.96 & - & - & - & - & - & - & - & - & - \\
\hline 2 & 2 & 6 & 3 & 99.34 & 89.89 & 97.96 & - & - & - & - & - & - & - & - & - \\
\hline 2 & 3 & 1 & 1 & 98.36 & 94.38 & 93.88 & 96.55 & 92.13 & 85.45 & - & - & - & - & - & - \\
\hline 2 & 3 & 1 & 2 & 98.36 & 95.51 & 93.88 & 96.55 & 91.01 & 85.45 & - & - & - & - & - & - \\
\hline 2 & 3 & 1 & 3 & 98.69 & 94.38 & 91.84 & 97.24 & 92.13 & 87.27 & 90.08 & 81.12 & 85.79 & 93.91 & 85.34 & 86.13 \\
\hline 2 & 3 & 2 & 1 & 98.36 & 93.26 & 93.88 & 96.55 & 87.64 & 85.45 & - & - & - & - & - & - \\
\hline 2 & 3 & 2 & 2 & 98.36 & 95.51 & 93.88 & 96.55 & 86.52 & 85.45 & - & - & - & - & - & - \\
\hline 2 & 3 & 2 & 3 & 98.36 & 94.38 & 93.88 & 96.55 & 87.64 & 85.45 & - & - & - & - & - & - \\
\hline 2 & 3 & 3 & 1 & 99.02 & 93.26 & 91.84 & 96.90 & 86.52 & 87.27 & 89.68 & 81.82 & 87.98 & 93.54 & 83.62 & 87.82 \\
\hline 2 & 3 & 3 & 2 & 98.36 & 95.51 & 93.88 & 96.55 & 86.52 & 83.64 & - & - & - & - & - & - \\
\hline 2 & 3 & 3 & 3 & 98.36 & 94.38 & 93.88 & 96.55 & 87.64 & 83.64 & - & - & - & - & - & - \\
\hline 2 & 3 & 4 & 1 & 99.02 & 93.26 & 91.84 & 96.90 & 88.76 & 87.27 & 89.68 & 81.82 & 87.98 & 93.54 & 84.48 & 87.82 \\
\hline 2 & 3 & 4 & 2 & 98.36 & 94.38 & 93.88 & 96.90 & 86.52 & 85.45 & - & - & - & - & - & - \\
\hline 2 & 3 & 4 & 3 & 99.02 & 92.13 & 93.88 & 96.90 & 87.64 & 90.91 & 90.08 & 81.82 & 87.98 & 93.73 & 84.05 & 88.66 \\
\hline 2 & 3 & 1 & 1 & 99.02 & 93.26 & 91.84 & 96.21 & 88.76 & 87.27 & 90.87 & 81.12 & 87.98 & 93.73 & 84.05 & 87.82 \\
\hline 2 & 3 & 1 & 2 & 98.36 & 95.51 & 93.88 & 96.55 & 88.76 & 83.64 & - & - & - & - & - & - \\
\hline 2 & 3 & 1 & 3 & 98.36 & 94.38 & 93.88 & 96.55 & 89.89 & 83.64 & - & - & - & - & - & - \\
\hline 2 & 3 & 6 & 1 & 99.02 & 93.26 & 91.84 & 96.90 & 88.76 & 87.27 & 89.68 & 81.12 & 89.07 & 93.54 & 84.05 & 88.66 \\
\hline 2 & 3 & 6 & 2 & 98.36 & 94.38 & 93.88 & 96.90 & 87.64 & 83.64 & - & - & - & - & - & - \\
\hline 2 & 3 & 6 & 3 & 99.02 & 92.13 & 93.88 & 96.90 & 87.64 & 90.91 & 90.08 & 81.12 & 89.07 & 93.73 & 83.62 & 89.50 \\
\hline 2 & 4 & 1 & 1 & 98.69 & 97.75 & 97.96 & 93.10 & 94.38 & 92.73 & 90.48 & 89.51 & 82.51 & 91.88 & 91.38 & 84.87 \\
\hline 2 & 4 & 1 & 2 & 98.69 & 97.75 & 97.96 & 93.10 & 94.38 & 92.73 & 90.48 & 89.51 & 81.97 & 91.88 & 91.38 & 84.45 \\
\hline 2 & 4 & 1 & 3 & 98.69 & 97.75 & 97.96 & 93.10 & 94.38 & 92.73 & 90.48 & 89.51 & 81.42 & 91.88 & 91.38 & 84.03 \\
\hline 2 & 4 & 2 & 1 & 98.69 & 97.75 & 97.96 & 93.45 & 94.38 & 92.73 & 90.48 & 88.81 & 81.97 & 92.07 & 90.95 & 84.45 \\
\hline 2 & 4 & 2 & 2 & 98.69 & 97.75 & 97.96 & 93.45 & 94.38 & 92.73 & 90.48 & 88.81 & 82.51 & 92.07 & 90.95 & 84.87 \\
\hline 2 & 4 & 2 & 3 & 98.69 & 97.75 & 97.96 & 93.45 & 94.38 & 92.73 & 90.48 & 88.81 & 81.97 & 92.07 & 90.95 & 84.45 \\
\hline 2 & 4 & 3 & 1 & 98.69 & 97.75 & 97.96 & 93.45 & 94.38 & 92.73 & 90.48 & 90.21 & 82.51 & 92.07 & 91.81 & 84.87 \\
\hline 2 & 4 & 3 & 2 & 98.69 & 97.75 & 97.96 & 93.45 & 94.38 & 92.73 & 90.48 & 90.21 & 82.51 & 92.07 & 91.81 & 84.87 \\
\hline 2 & 4 & 3 & 3 & 98.69 & 97.75 & 97.96 & 93.45 & 94.38 & 92.73 & 90.48 & 90.21 & 81.97 & 92.07 & 91.81 & 84.45 \\
\hline 2 & 4 & 4 & 1 & 98.36 & 97.75 & 97.96 & 93.10 & 95.51 & 92.73 & 90.48 & 89.51 & 81.97 & 91.88 & 91.81 & 84.45 \\
\hline 2 & 4 & 4 & 2 & 98.36 & 97.75 & 97.96 & 92.76 & 95.51 & 92.73 & 90.48 & 90.21 & 81.97 & 91.70 & 92.24 & 84.45 \\
\hline 2 & 4 & 4 & 3 & 98.36 & 97.75 & 97.96 & 93.10 & 95.51 & 92.73 & 90.48 & 89.51 & 80.87 & 91.88 & 91.81 & 83.61 \\
\hline 2 & 4 & 1 & 1 & 98.36 & 97.75 & 97.96 & 93.10 & 95.51 & 92.73 & 90.48 & 89.51 & 83.06 & 91.88 & 91.81 & 85.29 \\
\hline 2 & 4 & 1 & 2 & 98.36 & 97.75 & 97.96 & 92.76 & 95.51 & 92.73 & 90.48 & 90.21 & 82.51 & 91.70 & 92.24 & 84.87 \\
\hline 2 & 4 & 1 & 3 & 98.36 & 97.75 & 97.96 & 93.10 & 95.51 & 92.73 & 90.48 & 89.51 & 81.97 & 91.88 & 91.81 & 84.45 \\
\hline 2 & 4 & 6 & 1 & 98.36 & 97.75 & 97.96 & 92.76 & 95.51 & 90.91 & 91.27 & 88.81 & 83.61 & 92.07 & 91.38 & 85.29 \\
\hline
\end{tabular}


Table B.2.1 the results of calibration procedure 1 and its validations.

\begin{tabular}{|c|c|c|c|c|c|c|c|c|c|c|c|c|c|c|c|}
\hline \multicolumn{4}{|c|}{ Configurations } & \multicolumn{3}{|c|}{ Calibration } & \multicolumn{3}{|c|}{$1^{\text {st }}$ Validation } & \multicolumn{3}{|c|}{$2^{\text {nd }}$ Validation } & \multicolumn{3}{|c|}{$3^{\text {rd }}$ Validation } \\
\hline$\varphi_{g}$ & $\Lambda_{l}$ & $\Gamma_{n}$ & $\eta_{m}$ & Vehicles & Cyclists & Pedestrians & Vehicles & Cyclists & Pedestrians & Vehicles & Cyclists & Pedestrians & Vehicles & Cyclists & Pedestrians \\
\hline 2 & 4 & 6 & 2 & 98.36 & 97.75 & 97.96 & 92.41 & 95.51 & 90.91 & 91.27 & 89.51 & 83.06 & 91.88 & 91.81 & 84.87 \\
\hline 2 & 4 & 6 & 3 & 98.36 & 97.75 & 97.96 & 92.76 & 95.51 & 90.91 & 91.27 & 88.81 & 83.61 & 92.07 & 91.38 & 85.29 \\
\hline 3 & 1 & 1 & 1 & 98.01 & 100.00 & 100.00 & 87.95 & 86.11 & 91.49 & - & - & - & - & - & - \\
\hline 3 & 1 & 1 & 2 & 98.41 & 98.67 & 100.00 & 89.16 & 86.11 & 91.49 & - & - & - & - & - & - \\
\hline 3 & 1 & 1 & 3 & 98.80 & 98.67 & 100.00 & 89.16 & 83.33 & 89.36 & - & - & - & - & - & - \\
\hline 3 & 1 & 2 & 1 & 98.01 & 100.00 & 100.00 & 87.95 & 86.11 & 91.49 & - & - & - & - & - & - \\
\hline 3 & 1 & 2 & 2 & 98.41 & 98.67 & 100.00 & 89.16 & 86.11 & 91.49 & - & - & - & - & - & - \\
\hline 3 & 1 & 2 & 3 & 98.80 & 98.67 & 100.00 & 89.16 & 83.33 & 89.36 & - & - & - & - & - & - \\
\hline 3 & 1 & 3 & 1 & 98.01 & 100.00 & 100.00 & 87.95 & 84.72 & 91.49 & - & - & - & - & - & - \\
\hline 3 & 1 & 3 & 2 & 98.41 & 98.67 & 100.00 & 89.16 & 84.72 & 91.49 & - & - & - & - & - & - \\
\hline 3 & 1 & 3 & 3 & 98.80 & 98.67 & 100.00 & 89.16 & 81.94 & 89.36 & - & - & - & - & - & - \\
\hline 3 & 1 & 4 & 1 & 98.01 & 100.00 & 100.00 & 87.95 & 86.11 & 91.49 & - & - & - & - & - & - \\
\hline 3 & 1 & 4 & 2 & 98.41 & 98.67 & 100.00 & 89.16 & 86.11 & 91.49 & - & - & - & - & - & - \\
\hline 3 & 1 & 4 & 3 & 98.80 & 98.67 & 100.00 & 89.16 & 83.33 & 89.36 & - & - & - & - & - & - \\
\hline 3 & 1 & 1 & 1 & 98.01 & 100.00 & 100.00 & 87.55 & 86.11 & 91.49 & - & - & - & - & - & - \\
\hline 3 & 1 & 1 & 2 & 98.41 & 98.67 & 100.00 & 88.76 & 86.11 & 91.49 & - & - & - & - & - & - \\
\hline 3 & 1 & 1 & 3 & 98.80 & 98.67 & 100.00 & 89.16 & 83.33 & 89.36 & - & - & - & - & - & - \\
\hline 3 & 1 & 6 & 1 & 98.01 & 100.00 & 100.00 & 88.76 & 84.72 & 91.49 & - & - & - & - & - & - \\
\hline 3 & 1 & 6 & 2 & 98.01 & 98.67 & 100.00 & 88.35 & 84.72 & 91.49 & - & - & - & - & - & - \\
\hline 3 & 1 & 6 & 3 & 98.01 & 98.67 & 100.00 & 88.35 & 81.94 & 91.49 & 84.65 & 83.04 & 86.81 & 86.64 & 82.61 & 87.77 \\
\hline 3 & 2 & 1 & 1 & 98.01 & 96.00 & 97.56 & - & - & - & - & - & - & - & - & - \\
\hline 3 & 2 & 1 & 2 & 98.01 & 96.00 & 100.00 & - & - & - & - & - & - & - & - & - \\
\hline 3 & 2 & 1 & 3 & 98.01 & 96.00 & 100.00 & - & - & - & - & - & - & - & - & - \\
\hline 3 & 2 & 2 & 1 & 98.01 & 96.00 & 97.56 & - & - & - & - & - & - & - & - & - \\
\hline 3 & 2 & 2 & 2 & 98.01 & 96.00 & 100.00 & - & - & - & - & - & - & - & - & - \\
\hline 3 & 2 & 2 & 3 & 98.01 & 96.00 & 100.00 & - & - & - & - & - & - & - & - & - \\
\hline 3 & 2 & 3 & 1 & 98.01 & 96.00 & 97.56 & - & - & - & - & - & - & - & - & - \\
\hline 3 & 2 & 3 & 2 & 98.01 & 96.00 & 100.00 & - & - & - & - & - & - & - & - & - \\
\hline 3 & 2 & 3 & 3 & 98.01 & 96.00 & 100.00 & - & - & - & - & - & - & - & - & - \\
\hline 3 & 2 & 4 & 1 & 98.01 & 96.00 & 97.56 & - & - & - & - & - & - & - & - & - \\
\hline 3 & 2 & 4 & 2 & 98.01 & 96.00 & 100.00 & - & - & - & - & - & - & - & - & - \\
\hline 3 & 2 & 4 & 3 & 98.01 & 96.00 & 100.00 & - & - & - & - & - & - & - & - & - \\
\hline 3 & 2 & 1 & 1 & 98.01 & 96.00 & 97.56 & - & - & - & - & - & - & - & - & - \\
\hline 3 & 2 & 1 & 2 & 98.01 & 96.00 & 100.00 & - & - & - & - & - & - & - & - & - \\
\hline 3 & 2 & 1 & 3 & 98.01 & 96.00 & 100.00 & - & - & - & - & - & - & - & - & - \\
\hline 3 & 2 & 6 & 1 & 98.01 & 96.00 & 97.56 & 86.75 & 83.33 & 80.85 & - & - & - & - & - & - \\
\hline
\end{tabular}


Table B.2.1 the results of calibration procedure 1 and its validations.

\begin{tabular}{|c|c|c|c|c|c|c|c|c|c|c|c|c|c|c|c|}
\hline \multicolumn{4}{|c|}{ Configurations } & \multicolumn{3}{|c|}{ Calibration } & \multicolumn{3}{|c|}{$1^{\text {st }}$ Validation } & \multicolumn{3}{|c|}{$2^{\text {nd }}$ Validation } & \multicolumn{3}{|c|}{$3^{\text {rd }}$ Validation } \\
\hline$\varphi_{g}$ & $\Lambda_{l}$ & $\overline{\Gamma_{n}}$ & $\eta_{m}$ & Vehicles & Cyclists & Pedestrians & Vehicles & Cyclists & Pedestrians & Vehicles & Cyclists & Pedestrians & Vehicles & Cyclists & Pedestrians \\
\hline 3 & 2 & 6 & 2 & 98.01 & 96.00 & 100.00 & 86.75 & 83.33 & 80.85 & - & - & - & - & - & - \\
\hline 3 & 2 & 6 & 3 & 97.61 & 96.00 & 100.00 & - & - & - & - & - & - & - & - & - \\
\hline 3 & 3 & 1 & 1 & - & - & - & - & - & - & - & - & - & - & - & - \\
\hline 3 & 3 & 1 & 2 & - & - & - & - & - & - & - & - & - & - & - & - \\
\hline 3 & 3 & 1 & 3 & - & - & - & - & - & - & - & - & - & - & - & - \\
\hline 3 & 3 & 2 & 1 & - & - & - & - & - & - & - & - & - & - & - & - \\
\hline 3 & 3 & 2 & 2 & - & - & - & - & - & - & - & - & - & - & - & - \\
\hline 3 & 3 & 2 & 3 & - & - & - & - & - & - & - & - & - & - & - & - \\
\hline 3 & 3 & 3 & 1 & - & - & - & - & - & - & - & - & - & - & - & - \\
\hline 3 & 3 & 3 & 2 & - & - & - & - & - & - & - & - & - & - & - & - \\
\hline 3 & 3 & 3 & 3 & - & - & - & - & - & - & - & - & - & - & - & - \\
\hline 3 & 3 & 4 & 1 & - & - & - & - & - & - & - & - & - & - & - & - \\
\hline 3 & 3 & 4 & 2 & 99.20 & 89.33 & 100.00 & 94.78 & 69.44 & 87.23 & 90.70 & 88.39 & 85.71 & 92.89 & 80.98 & 86.03 \\
\hline 3 & 3 & 4 & 3 & - & - & - & - & - & - & - & - & - & - & - & - \\
\hline 3 & 3 & 1 & 1 & - & - & - & - & - & - & - & - & - & - & - & - \\
\hline 3 & 3 & 1 & 2 & 99.20 & 89.33 & 100.00 & 94.38 & 70.83 & 85.11 & 92.09 & 87.50 & 84.62 & 93.32 & 80.98 & 84.72 \\
\hline 3 & 3 & 1 & 3 & - & - & - & - & - & - & - & - & - & - & - & - \\
\hline 3 & 3 & 6 & 1 & - & - & - & - & - & - & - & - & - & - & - & - \\
\hline 3 & 3 & 6 & 2 & - & - & - & - & - & - & - & - & - & - & - & - \\
\hline 3 & 3 & 6 & 3 & - & - & - & - & - & - & - & - & - & - & - & - \\
\hline 3 & 4 & 1 & 1 & 99.20 & 93.33 & 100.00 & 94.38 & 75.00 & 87.23 & - & - & - & - & - & - \\
\hline 3 & 4 & 1 & 2 & 99.20 & 93.33 & 100.00 & 94.38 & 76.39 & 85.11 & - & - & - & - & - & - \\
\hline 3 & 4 & 1 & 3 & 99.20 & 93.33 & 100.00 & 94.38 & 73.61 & 87.23 & - & - & - & - & - & - \\
\hline 3 & 4 & 2 & 1 & 99.20 & 93.33 & 100.00 & 93.98 & 75.00 & 87.23 & - & - & - & - & - & - \\
\hline 3 & 4 & 2 & 2 & 99.20 & 93.33 & 100.00 & 93.98 & 76.39 & 85.11 & - & - & - & - & - & - \\
\hline 3 & 4 & 2 & 3 & 99.20 & 93.33 & 100.00 & 93.98 & 73.61 & 87.23 & - & - & - & - & - & - \\
\hline 3 & 4 & 3 & 1 & 99.20 & 93.33 & 100.00 & 93.98 & 75.00 & 87.23 & - & - & - & - & - & - \\
\hline 3 & 4 & 3 & 2 & 99.20 & 93.33 & 100.00 & 93.98 & 76.39 & 85.11 & - & - & - & - & - & - \\
\hline 3 & 4 & 3 & 3 & 99.20 & 93.33 & 100.00 & 93.98 & 73.61 & 87.23 & - & - & - & - & - & - \\
\hline 3 & 4 & 4 & 1 & 99.20 & 93.33 & 100.00 & 93.98 & 75.00 & 87.23 & - & - & - & - & - & - \\
\hline 3 & 4 & 4 & 2 & 99.20 & 93.33 & 100.00 & 93.98 & 76.39 & 85.11 & - & - & - & - & - & - \\
\hline 3 & 4 & 4 & 3 & 99.20 & 93.33 & 100.00 & 93.98 & 73.61 & 87.23 & - & - & - & - & - & - \\
\hline 3 & 4 & 1 & 1 & 99.20 & 93.33 & 100.00 & 93.98 & 75.00 & 87.23 & - & - & - & - & - & - \\
\hline 3 & 4 & 1 & 2 & 99.20 & 93.33 & 100.00 & 93.98 & 76.39 & 85.11 & - & - & - & - & - & - \\
\hline 3 & 4 & 1 & 3 & 99.20 & 93.33 & 100.00 & 93.98 & 73.61 & 87.23 & - & - & - & - & - & - \\
\hline 3 & 4 & 6 & 1 & 99.20 & 93.33 & 100.00 & 93.98 & 75.00 & 87.23 & - & - & - & - & - & - \\
\hline
\end{tabular}


Table B.2.1 the results of calibration procedure 1 and its validations.

\begin{tabular}{|c|c|c|c|c|c|c|c|c|c|c|c|c|c|c|c|}
\hline \multicolumn{4}{|c|}{ Configurations } & \multicolumn{3}{|c|}{ Calibration } & \multicolumn{3}{|c|}{$1^{\text {st }}$ Validation } & \multicolumn{3}{|c|}{$2^{\text {nd }}$ Validation } & \multicolumn{3}{|c|}{$3^{\text {rd }}$ Validation } \\
\hline$\varphi_{g}$ & $\Lambda_{l}$ & $\overline{\Gamma_{n}}$ & $\eta_{m}$ & Vehicles & Cyclists & Pedestrians & Vehicles & Cyclists & Pedestrians & Vehicles & Cyclists & Pedestrians & Vehicles & Cyclists & Pedestrians \\
\hline 3 & 4 & 6 & 2 & 99.20 & 93.33 & 100.00 & 93.98 & 76.39 & 85.11 & - & - & - & - & - & - \\
\hline 3 & 4 & 6 & 3 & 99.20 & 93.33 & 100.00 & 93.98 & 73.61 & 87.23 & - & - & - & - & - & - \\
\hline 4 & 1 & 1 & 1 & 98.40 & 98.51 & 100.00 & - & - & - & - & - & - & - & - & - \\
\hline 4 & 1 & 1 & 2 & 98.40 & 98.51 & 100.00 & - & - & - & - & - & - & - & - & - \\
\hline 4 & 1 & 1 & 3 & 98.40 & 98.51 & 100.00 & - & - & - & - & - & - & - & - & - \\
\hline 4 & 1 & 2 & 1 & 98.40 & 98.51 & 100.00 & 87.32 & 88.52 & 92.31 & - & - & - & - & - & - \\
\hline 4 & 1 & 2 & 2 & 98.40 & 98.51 & 100.00 & 87.32 & 88.52 & 92.31 & - & - & - & - & - & - \\
\hline 4 & 1 & 2 & 3 & 98.40 & 98.51 & 100.00 & 87.32 & 88.52 & 92.31 & - & - & - & - & - & - \\
\hline 4 & 1 & 3 & 1 & 98.40 & 98.51 & 100.00 & - & - & - & - & - & - & - & - & - \\
\hline 4 & 1 & 3 & 2 & 98.40 & 98.51 & 100.00 & - & - & - & - & - & - & - & - & - \\
\hline 4 & 1 & 3 & 3 & 98.40 & 98.51 & 100.00 & - & - & - & - & - & - & - & - & - \\
\hline 4 & 1 & 4 & 1 & 98.40 & 98.51 & 100.00 & - & - & - & - & - & - & - & - & - \\
\hline 4 & 1 & 4 & 2 & 98.40 & 98.51 & 100.00 & 88.73 & 85.25 & 89.74 & - & - & - & - & - & - \\
\hline 4 & 1 & 4 & 3 & 98.40 & 98.51 & 100.00 & 88.73 & 85.25 & 89.74 & - & - & - & - & - & - \\
\hline 4 & 1 & 1 & 1 & 98.40 & 98.51 & 100.00 & - & - & - & - & - & - & - & - & - \\
\hline 4 & 1 & 1 & 2 & 98.40 & 98.51 & 100.00 & 88.73 & 85.25 & 89.74 & - & - & - & - & - & - \\
\hline 4 & 1 & 1 & 3 & 98.40 & 98.51 & 100.00 & 88.73 & 85.25 & 89.74 & - & - & - & - & - & - \\
\hline 4 & 1 & 6 & 1 & 98.40 & 98.51 & 100.00 & - & - & - & - & - & - & - & - & - \\
\hline 4 & 1 & 6 & 2 & 98.40 & 98.51 & 100.00 & - & - & - & - & - & - & - & - & - \\
\hline 4 & 1 & 6 & 3 & 98.40 & 98.51 & 100.00 & - & - & - & - & - & - & - & - & - \\
\hline 4 & 2 & 1 & 1 & 99.47 & 94.03 & 97.30 & - & - & - & - & - & - & - & - & - \\
\hline 4 & 2 & 1 & 2 & 99.47 & 94.03 & 97.30 & - & - & - & - & - & - & - & - & - \\
\hline 4 & 2 & 1 & 3 & 99.47 & 92.54 & 97.30 & - & - & - & - & - & - & - & - & - \\
\hline 4 & 2 & 2 & 1 & 98.93 & 94.03 & 94.59 & - & - & - & - & - & - & - & - & - \\
\hline 4 & 2 & 2 & 2 & 98.93 & 94.03 & 94.59 & - & - & - & - & - & - & - & - & - \\
\hline 4 & 2 & 2 & 3 & 98.93 & 94.03 & 94.59 & - & - & - & - & - & - & - & - & - \\
\hline 4 & 2 & 3 & 1 & 98.93 & 94.03 & 94.59 & - & - & - & - & - & - & - & - & - \\
\hline 4 & 2 & 3 & 2 & 98.93 & 94.03 & 94.59 & - & - & - & - & - & - & - & - & - \\
\hline 4 & 2 & 3 & 3 & 98.93 & 94.03 & 94.59 & - & - & - & - & - & - & - & - & - \\
\hline 4 & 2 & 4 & 1 & 99.47 & 94.03 & 94.59 & - & - & - & - & - & - & - & - & - \\
\hline 4 & 2 & 4 & 2 & 99.47 & 94.03 & 94.59 & - & - & - & - & - & - & - & - & - \\
\hline 4 & 2 & 4 & 3 & 99.47 & 94.03 & 94.59 & - & - & - & - & - & - & - & - & - \\
\hline 4 & 2 & 1 & 1 & 99.47 & 94.03 & 94.59 & - & - & - & - & - & - & - & - & - \\
\hline 4 & 2 & 1 & 2 & 99.47 & 94.03 & 97.30 & - & - & - & - & - & - & - & - & - \\
\hline 4 & 2 & 1 & 3 & 99.47 & 94.03 & 94.59 & - & - & - & - & - & - & - & - & - \\
\hline 4 & 2 & 6 & 1 & 98.93 & 94.03 & 97.30 & - & - & - & - & - & - & - & - & - \\
\hline
\end{tabular}


Table B.2.1 the results of calibration procedure 1 and its validations.

\begin{tabular}{|c|c|c|c|c|c|c|c|c|c|c|c|c|c|c|c|}
\hline \multicolumn{4}{|c|}{ Configurations } & \multicolumn{3}{|c|}{ Calibration } & \multicolumn{3}{|c|}{$1^{\text {st }}$ Validation } & \multicolumn{3}{|c|}{$2^{\text {nd }}$ Validation } & \multicolumn{3}{|c|}{$3^{\text {rd }}$ Validation } \\
\hline$\varphi_{g}$ & $\Lambda_{l}$ & $\Gamma_{n}$ & $\eta_{m}$ & Vehicles & Cyclists & Pedestrians & Vehicles & Cyclists & Pedestrians & Vehicles & Cyclists & Pedestrians & Vehicles & Cyclists & Pedestrians \\
\hline 4 & 2 & 6 & 2 & 98.93 & 94.03 & 97.30 & - & - & - & - & - & - & - & - & - \\
\hline 4 & 2 & 6 & 3 & 98.93 & 92.54 & 97.30 & - & - & - & - & - & - & - & - & - \\
\hline 4 & 3 & 1 & 1 & 100.00 & 95.52 & 97.30 & 95.31 & 81.97 & 74.36 & 88.08 & 77.33 & 86.39 & 92.31 & 79.41 & 84.13 \\
\hline 4 & 3 & 1 & 2 & 100.00 & 94.03 & 97.30 & 94.84 & 80.33 & 74.36 & 88.08 & 77.33 & 89.94 & 92.03 & 78.68 & 87.02 \\
\hline 4 & 3 & 1 & 3 & 100.00 & 94.03 & 97.30 & 94.84 & 80.33 & 74.36 & 88.08 & 77.33 & 88.17 & 92.03 & 78.68 & 85.58 \\
\hline 4 & 3 & 2 & 1 & 100.00 & 95.52 & 97.30 & 94.84 & 81.97 & 74.36 & 88.08 & 76.00 & 86.98 & 92.03 & 78.68 & 84.62 \\
\hline 4 & 3 & 2 & 2 & 100.00 & 94.03 & 97.30 & 94.37 & 80.33 & 74.36 & 88.08 & 76.00 & 90.53 & 91.76 & 77.94 & 87.50 \\
\hline 4 & 3 & 2 & 3 & 100.00 & 94.03 & 97.30 & 94.37 & 80.33 & 74.36 & 88.08 & 76.00 & 88.76 & 91.76 & 77.94 & 86.06 \\
\hline 4 & 3 & 3 & 1 & 100.00 & 95.52 & 97.30 & 95.31 & 83.61 & 74.36 & 88.08 & 76.00 & 86.98 & 92.31 & 79.41 & 84.62 \\
\hline 4 & 3 & 3 & 2 & 100.00 & 94.03 & 97.30 & 94.84 & 81.97 & 74.36 & 88.08 & 76.00 & 90.53 & 92.03 & 78.68 & 87.50 \\
\hline 4 & 3 & 3 & 3 & 100.00 & 94.03 & 97.30 & 94.84 & 81.97 & 74.36 & 88.08 & 76.00 & 89.35 & 92.03 & 78.68 & 86.54 \\
\hline 4 & 3 & 4 & 1 & 100.00 & 95.52 & 97.30 & 95.31 & 80.33 & 74.36 & 88.08 & 77.33 & 86.98 & 92.31 & 78.68 & 84.62 \\
\hline 4 & 3 & 4 & 2 & 100.00 & 94.03 & 97.30 & 94.84 & 78.69 & 76.92 & 88.08 & 77.33 & 91.12 & 92.03 & 77.94 & 88.46 \\
\hline 4 & 3 & 4 & 3 & 100.00 & 94.03 & 97.30 & 94.84 & 78.69 & 76.92 & 88.08 & 77.33 & 89.94 & 92.03 & 77.94 & 87.50 \\
\hline 4 & 3 & 1 & 1 & 99.47 & 95.52 & 97.30 & 95.77 & 80.33 & 74.36 & 89.40 & 76.00 & 85.80 & 93.13 & 77.94 & 83.65 \\
\hline 4 & 3 & 1 & 2 & 99.47 & 94.03 & 97.30 & 95.31 & 80.33 & 74.36 & 89.40 & 78.67 & 90.53 & 92.86 & 79.41 & 87.50 \\
\hline 4 & 3 & 1 & 3 & 99.47 & 94.03 & 97.30 & 95.31 & 78.69 & 74.36 & 89.40 & 76.00 & 88.17 & 92.86 & 77.21 & 85.58 \\
\hline 4 & 3 & 6 & 1 & 99.47 & 95.52 & 97.30 & 95.31 & 81.97 & 74.36 & 88.08 & 77.33 & 86.98 & 92.31 & 79.41 & 84.62 \\
\hline 4 & 3 & 6 & 2 & 99.47 & 94.03 & 97.30 & 94.84 & 80.33 & 74.36 & 88.08 & 76.00 & 90.53 & 92.03 & 77.94 & 87.50 \\
\hline 4 & 3 & 6 & 3 & 100.00 & 95.52 & 94.59 & 95.31 & 86.89 & 66.67 & - & - & - & - & - & - \\
\hline 4 & 4 & 1 & 1 & 100.00 & 89.55 & 100.00 & 91.08 & 91.80 & 97.44 & 88.08 & 85.33 & 85.80 & 89.84 & 88.24 & 87.98 \\
\hline 4 & 4 & 1 & 2 & 100.00 & 89.55 & 100.00 & 91.08 & 90.16 & 97.44 & 88.08 & 85.33 & 85.80 & 89.84 & 87.50 & 87.98 \\
\hline 4 & 4 & 1 & 3 & 100.00 & 89.55 & 100.00 & 91.08 & 90.16 & 97.44 & 88.08 & 85.33 & 85.80 & 89.84 & 87.50 & 87.98 \\
\hline 4 & 4 & 2 & 1 & 100.00 & 89.55 & 100.00 & 91.08 & 91.80 & 97.44 & 88.08 & 84.00 & 85.80 & 89.84 & 87.50 & 87.98 \\
\hline 4 & 4 & 2 & 2 & 99.47 & 89.55 & 100.00 & 91.08 & 90.16 & 97.44 & 88.08 & 84.00 & 86.39 & 89.84 & 86.76 & 88.46 \\
\hline 4 & 4 & 2 & 3 & 99.47 & 89.55 & 100.00 & 91.08 & 90.16 & 97.44 & 88.08 & 84.00 & 86.39 & 89.84 & 86.76 & 88.46 \\
\hline 4 & 4 & 3 & 1 & 100.00 & 89.55 & 100.00 & 91.55 & 88.52 & 97.44 & 88.08 & 82.67 & 84.02 & 90.11 & 85.29 & 86.54 \\
\hline 4 & 4 & 3 & 2 & 100.00 & 89.55 & 100.00 & 91.55 & 86.89 & 97.44 & 88.08 & 82.67 & 84.02 & 90.11 & 84.56 & 86.54 \\
\hline 4 & 4 & 3 & 3 & 100.00 & 89.55 & 100.00 & 91.55 & 86.89 & 97.44 & 88.08 & 82.67 & 84.02 & 90.11 & 84.56 & 86.54 \\
\hline 4 & 4 & 4 & 1 & 100.00 & 89.55 & 100.00 & 91.55 & 86.89 & 97.44 & 88.08 & 84.00 & 85.21 & 90.11 & 85.29 & 87.50 \\
\hline 4 & 4 & 4 & 2 & 100.00 & 89.55 & 100.00 & 91.55 & 85.25 & 97.44 & 88.08 & 84.00 & 85.21 & 90.11 & 84.56 & 87.50 \\
\hline 4 & 4 & 4 & 3 & 100.00 & 89.55 & 100.00 & 91.55 & 85.25 & 97.44 & 88.08 & 84.00 & 85.21 & 90.11 & 84.56 & 87.50 \\
\hline 4 & 4 & 1 & 1 & 100.00 & 89.55 & 100.00 & 91.55 & 85.25 & 97.44 & 88.08 & 85.33 & 85.80 & 90.11 & 85.29 & 87.98 \\
\hline 4 & 4 & 1 & 2 & 100.00 & 89.55 & 100.00 & 91.55 & 83.61 & 97.44 & 88.08 & 85.33 & 85.80 & 90.11 & 84.56 & 87.98 \\
\hline 4 & 4 & 1 & 3 & 100.00 & 89.55 & 100.00 & 91.55 & 83.61 & 97.44 & 88.08 & 85.33 & 85.80 & 90.11 & 84.56 & 87.98 \\
\hline 4 & 4 & 6 & 1 & 100.00 & 89.55 & 100.00 & 92.49 & 85.25 & 97.44 & 88.74 & 85.33 & 86.39 & 90.93 & 85.29 & 88.46 \\
\hline
\end{tabular}


Table B.2.1 the results of calibration procedure 1 and its validations.

\begin{tabular}{|c|c|c|c|c|c|c|c|c|c|c|c|c|c|c|c|}
\hline \multicolumn{4}{|c|}{ Configurations } & \multicolumn{3}{|c|}{ Calibration } & \multicolumn{3}{|c|}{$1^{\text {st }}$ Validation } & \multicolumn{3}{|c|}{$2^{\text {nd }}$ Validation } & \multicolumn{3}{|c|}{$3^{\text {rd }}$ Validation } \\
\hline$\varphi_{g}$ & $\Lambda_{l}$ & $\overline{\Gamma_{n}}$ & $\eta_{m}$ & Vehicles & Cyclists & Pedestrians & Vehicles & Cyclists & Pedestrians & Vehicles & Cyclists & Pedestrians & Vehicles & Cyclists & Pedestrians \\
\hline 4 & 4 & 6 & 2 & 100.00 & 89.55 & 100.00 & 92.49 & 83.61 & 97.44 & 88.74 & 85.33 & 86.39 & 90.93 & 84.56 & 88.46 \\
\hline 4 & 4 & 6 & 3 & 100.00 & 89.55 & 100.00 & 92.49 & 83.61 & 97.44 & 88.74 & 85.33 & 86.39 & 90.93 & 84.56 & 88.46 \\
\hline 5 & 1 & 1 & 1 & 100.00 & 100.00 & 100.00 & 90.74 & 80.43 & 96.97 & - & - & - & - & - & - \\
\hline 5 & 1 & 1 & 2 & 100.00 & 100.00 & 100.00 & 90.74 & 80.43 & 93.94 & - & - & - & - & - & - \\
\hline 5 & 1 & 1 & 3 & 100.00 & 100.00 & 100.00 & 90.74 & 80.43 & 96.97 & - & - & - & - & - & - \\
\hline 5 & 1 & 2 & 1 & 99.28 & 100.00 & 100.00 & 89.51 & 80.43 & 96.97 & - & - & - & - & - & - \\
\hline 5 & 1 & 2 & 2 & 100.00 & 100.00 & 100.00 & 88.89 & 80.43 & 93.94 & - & - & - & - & - & - \\
\hline 5 & 1 & 2 & 3 & 99.28 & 100.00 & 100.00 & 89.51 & 80.43 & 96.97 & - & - & - & - & - & - \\
\hline 5 & 1 & 3 & 1 & 100.00 & 100.00 & 100.00 & 90.12 & 84.78 & 96.97 & - & - & - & - & - & - \\
\hline 5 & 1 & 3 & 2 & 100.00 & 100.00 & 100.00 & 90.12 & 84.78 & 93.94 & - & - & - & - & - & - \\
\hline 5 & 1 & 3 & 3 & 100.00 & 100.00 & 100.00 & 90.12 & 84.78 & 96.97 & - & - & - & - & - & - \\
\hline 5 & 1 & 4 & 1 & 99.28 & 100.00 & 100.00 & 89.51 & 84.78 & 96.97 & - & - & - & - & - & - \\
\hline 5 & 1 & 4 & 2 & 100.00 & 100.00 & 100.00 & 88.89 & 84.78 & 93.94 & - & - & - & - & - & - \\
\hline 5 & 1 & 4 & 3 & 99.28 & 100.00 & 100.00 & 89.51 & 84.78 & 96.97 & - & - & - & - & - & - \\
\hline 5 & 1 & 1 & 1 & 99.28 & 100.00 & 100.00 & 89.51 & 80.43 & 96.97 & - & - & - & - & - & - \\
\hline 5 & 1 & 1 & 2 & 99.28 & 100.00 & 100.00 & 89.51 & 80.43 & 93.94 & - & - & - & - & - & - \\
\hline 5 & 1 & 1 & 3 & 99.28 & 100.00 & 100.00 & 89.51 & 80.43 & 96.97 & - & - & - & - & - & - \\
\hline 5 & 1 & 6 & 1 & 100.00 & 100.00 & 100.00 & 89.51 & 80.43 & 96.97 & - & - & - & - & - & - \\
\hline 5 & 1 & 6 & 2 & 100.00 & 100.00 & 100.00 & 89.51 & 80.43 & 93.94 & - & - & - & - & - & - \\
\hline 5 & 1 & 6 & 3 & 100.00 & 100.00 & 100.00 & 89.51 & 80.43 & 96.97 & - & - & - & - & - & - \\
\hline 5 & 2 & 1 & 1 & 100.00 & 98.08 & 100.00 & 90.74 & 73.91 & 93.94 & - & - & - & - & - & - \\
\hline 5 & 2 & 1 & 2 & 100.00 & 98.08 & 100.00 & 90.74 & 67.39 & 93.94 & - & - & - & - & - & - \\
\hline 5 & 2 & 1 & 3 & 100.00 & 98.08 & 100.00 & 90.74 & 67.39 & 93.94 & - & - & - & - & - & - \\
\hline 5 & 2 & 2 & 1 & 100.00 & 98.08 & 100.00 & 90.74 & 73.91 & 93.94 & - & - & - & - & - & - \\
\hline 5 & 2 & 2 & 2 & 100.00 & 98.08 & 100.00 & 90.74 & 67.39 & 93.94 & - & - & - & - & - & - \\
\hline 5 & 2 & 2 & 3 & 100.00 & 98.08 & 100.00 & 90.74 & 67.39 & 93.94 & - & - & - & - & - & - \\
\hline 5 & 2 & 3 & 1 & 99.28 & 98.08 & 100.00 & - & - & - & - & - & - & - & - & - \\
\hline 5 & 2 & 3 & 2 & 99.28 & 98.08 & 100.00 & - & - & - & - & - & - & - & - & - \\
\hline 5 & 2 & 3 & 3 & 99.28 & 98.08 & 100.00 & - & - & - & - & - & - & - & - & - \\
\hline 5 & 2 & 4 & 1 & 100.00 & 96.15 & 100.00 & 90.74 & 71.74 & 90.91 & - & - & - & - & - & - \\
\hline 5 & 2 & 4 & 2 & 100.00 & 96.15 & 100.00 & - & - & - & - & - & - & - & - & - \\
\hline 5 & 2 & 4 & 3 & 100.00 & 96.15 & 100.00 & - & - & - & - & - & - & - & - & - \\
\hline 5 & 2 & 1 & 1 & 100.00 & 96.15 & 100.00 & - & - & - & - & - & - & - & - & - \\
\hline 5 & 2 & 1 & 2 & 100.00 & 96.15 & 100.00 & - & - & - & - & - & - & - & - & - \\
\hline 5 & 2 & 1 & 3 & 100.00 & 96.15 & 100.00 & - & - & - & - & - & - & - & - & - \\
\hline 5 & 2 & 6 & 1 & 100.00 & 96.15 & 100.00 & - & - & - & - & - & - & - & - & - \\
\hline
\end{tabular}


Table B.2.1 the results of calibration procedure 1 and its validations.

\begin{tabular}{|c|c|c|c|c|c|c|c|c|c|c|c|c|c|c|c|}
\hline \multicolumn{4}{|c|}{ Configurations } & \multicolumn{3}{|c|}{ Calibration } & \multicolumn{3}{|c|}{$1^{\text {st }}$ Validation } & \multicolumn{3}{|c|}{$2^{\text {nd }}$ Validation } & \multicolumn{3}{|c|}{$3^{\text {rd }}$ Validation } \\
\hline$\varphi_{g}$ & $\Lambda_{l}$ & $\Gamma_{n}$ & $\eta_{m}$ & Vehicles & Cyclists & Pedestrians & Vehicles & Cyclists & Pedestrians & Vehicles & Cyclists & Pedestrians & Vehicles & Cyclists & Pedestrians \\
\hline 5 & 2 & 6 & 2 & 100.00 & 96.15 & 100.00 & - & - & - & - & - & - & - & - & - \\
\hline 5 & 2 & 6 & 3 & 100.00 & 96.15 & 100.00 & - & - & - & - & - & - & - & - & - \\
\hline 5 & 3 & 1 & 1 & - & - & - & - & - & - & - & - & - & - & - & - \\
\hline 5 & 3 & 1 & 2 & - & - & - & - & - & - & - & - & - & - & - & - \\
\hline 5 & 3 & 1 & 3 & - & - & - & - & - & - & - & - & - & - & - & - \\
\hline 5 & 3 & 2 & 1 & - & - & - & - & - & - & - & - & - & - & - & - \\
\hline 5 & 3 & 2 & 2 & - & - & - & - & - & - & - & - & - & - & - & - \\
\hline 5 & 3 & 2 & 3 & - & - & - & - & - & - & - & - & - & - & - & - \\
\hline 5 & 3 & 3 & 1 & - & - & - & - & - & - & - & - & - & - & - & - \\
\hline 5 & 3 & 3 & 2 & - & - & - & - & - & - & - & - & - & - & - & - \\
\hline 5 & 3 & 3 & 3 & - & - & - & - & - & - & - & - & - & - & - & - \\
\hline 5 & 3 & 4 & 1 & - & - & - & - & - & - & - & - & - & - & - & - \\
\hline 5 & 3 & 4 & 2 & - & - & - & - & - & - & - & - & - & - & - & - \\
\hline 5 & 3 & 4 & 3 & - & - & - & - & - & - & - & - & - & - & - & - \\
\hline 5 & 3 & 1 & 1 & - & - & - & - & - & - & - & - & - & - & - & - \\
\hline 5 & 3 & 1 & 2 & - & - & - & - & - & - & - & - & - & - & - & - \\
\hline 5 & 3 & 1 & 3 & - & - & - & - & - & - & - & - & - & - & - & - \\
\hline 5 & 3 & 6 & 1 & - & - & - & - & - & - & - & - & - & - & - & - \\
\hline 5 & 3 & 6 & 2 & - & - & - & - & - & - & - & - & - & - & - & - \\
\hline 5 & 3 & 6 & 3 & - & - & - & - & - & - & - & - & - & - & - & - \\
\hline 5 & 4 & 1 & 1 & 100.00 & 96.15 & 100.00 & 91.98 & 73.91 & 90.91 & 88.42 & 77.78 & 86.86 & 90.66 & 75.82 & 87.65 \\
\hline 5 & 4 & 1 & 2 & 100.00 & 96.15 & 100.00 & 92.59 & 78.26 & 90.91 & 88.42 & 82.22 & 87.59 & 91.05 & 80.22 & 88.24 \\
\hline 5 & 4 & 1 & 3 & 100.00 & 96.15 & 100.00 & 91.98 & 71.74 & 90.91 & 88.42 & 80.00 & 88.32 & 90.66 & 75.82 & 88.82 \\
\hline 5 & 4 & 2 & 1 & 99.28 & 98.08 & 100.00 & 91.36 & 80.43 & 96.97 & 88.42 & 84.44 & 86.13 & 90.27 & 82.42 & 88.24 \\
\hline 5 & 4 & 2 & 2 & 100.00 & 98.08 & 100.00 & 92.59 & 78.26 & 93.94 & 89.47 & 80.00 & 89.78 & 91.44 & 79.12 & 90.59 \\
\hline 5 & 4 & 2 & 3 & 100.00 & 98.08 & 100.00 & 91.98 & 73.91 & 93.94 & 89.47 & 80.00 & 90.51 & 91.05 & 76.92 & 91.18 \\
\hline 5 & 4 & 3 & 1 & 99.28 & 98.08 & 100.00 & 91.98 & 82.61 & 96.97 & 88.42 & 86.67 & 86.86 & 90.66 & 84.62 & 88.82 \\
\hline 5 & 4 & 3 & 2 & 100.00 & 98.08 & 100.00 & 93.21 & 80.43 & 93.94 & 90.53 & 82.22 & 90.51 & 92.22 & 81.32 & 91.18 \\
\hline 5 & 4 & 3 & 3 & 100.00 & 98.08 & 100.00 & 92.59 & 76.09 & 93.94 & 90.53 & 80.00 & 91.24 & 91.83 & 78.02 & 91.76 \\
\hline 5 & 4 & 4 & 1 & 99.28 & 98.08 & 100.00 & 92.59 & 82.61 & 96.97 & 88.42 & 86.67 & 86.13 & 91.05 & 84.62 & 88.24 \\
\hline 5 & 4 & 4 & 2 & 100.00 & 98.08 & 100.00 & 93.83 & 80.43 & 93.94 & 90.53 & 82.22 & 89.78 & 92.61 & 81.32 & 90.59 \\
\hline 5 & 4 & 4 & 3 & 100.00 & 98.08 & 100.00 & 93.21 & 76.09 & 93.94 & 90.53 & 82.22 & 90.51 & 92.22 & 79.12 & 91.18 \\
\hline 5 & 4 & 1 & 1 & 100.00 & 96.15 & 100.00 & 92.59 & 78.26 & 90.91 & 86.32 & 82.22 & 86.13 & 90.27 & 80.22 & 87.06 \\
\hline 5 & 4 & 1 & 2 & 100.00 & 96.15 & 100.00 & 93.21 & 78.26 & 90.91 & 87.37 & 82.22 & 85.40 & 91.05 & 80.22 & 86.47 \\
\hline 5 & 4 & 1 & 3 & 100.00 & 96.15 & 100.00 & 92.59 & 76.09 & 90.91 & 86.32 & 82.22 & 86.13 & 90.27 & 79.12 & 87.06 \\
\hline 5 & 4 & 6 & 1 & 99.28 & 98.08 & 100.00 & 91.98 & 84.78 & 93.94 & 88.42 & 86.67 & 86.13 & 90.66 & 85.71 & 87.65 \\
\hline
\end{tabular}


Table B.2.1 the results of calibration procedure 1 and its validations.

\begin{tabular}{|c|c|c|c|c|c|c|c|c|c|c|c|c|c|c|c|}
\hline \multicolumn{4}{|c|}{ Configurations } & \multicolumn{3}{|c|}{ Calibration } & \multicolumn{3}{|c|}{$1^{\text {st }}$ Validation } & \multicolumn{3}{|c|}{$2^{\text {nd }}$ Validation } & \multicolumn{3}{|c|}{$3^{\text {rd }}$ Validation } \\
\hline$\varphi_{g}$ & $\Lambda_{l}$ & $\Gamma_{n}$ & $\eta_{m}$ & Vehicles & Cyclists & Pedestrians & Vehicles & Cyclists & Pedestrians & Vehicles & Cyclists & Pedestrians & Vehicles & Cyclists & Pedestrians \\
\hline 5 & 4 & 6 & 2 & 100.00 & 98.08 & 100.00 & 92.59 & 82.61 & 93.94 & 89.47 & 82.22 & 89.05 & 91.44 & 82.42 & 90.00 \\
\hline 5 & 4 & 6 & 3 & 100.00 & 98.08 & 100.00 & 92.59 & 78.26 & 93.94 & 89.47 & 82.22 & 89.78 & 91.44 & 80.22 & 90.59 \\
\hline
\end{tabular}

Table B.2.2 the results of calibration procedure 2 and its validations.

\begin{tabular}{|c|c|c|c|c|c|c|c|c|c|c|c|c|c|}
\hline \multicolumn{2}{|c|}{ Configurations } & \multicolumn{3}{|c|}{ Calibration } & \multicolumn{3}{|c|}{$1^{\text {st }}$ Validation } & \multicolumn{3}{|c|}{$2^{\text {nd }}$ Validation } & \multicolumn{3}{|c|}{$3^{\text {rd }}$ Validation } \\
\hline$\varphi_{g}$ & $\Lambda_{l}$ & Vehicles & Cyclists & Pedestrians & Vehicles & Cyclists & Pedestrians & Vehicles & Cyclists & Pedestrians & Vehicles & Cyclists & Pedestrians \\
\hline 1 & 1 & 94 & 80 & 88 & 90 & 88 & 95 & 84 & 82 & 100 & 87 & 84 & 99 \\
\hline 1 & 2 & 92 & 68 & 96 & - & - & - & - & - & - & - & - & - \\
\hline 1 & 3 & 98 & 61 & 91 & 96 & 74 & 95 & 87 & 79 & 93 & 92 & 77 & 93 \\
\hline 1 & 4 & 93 & 64 & 82 & 90 & 79 & 90 & - & - & - & - & - & - \\
\hline 2 & 1 & 95 & 85 & 90 & 91 & 91 & 96 & 85 & 80 & 100 & 88 & 84 & 99 \\
\hline 2 & 2 & 94 & 82 & 92 & 89 & 84 & 96 & 85 & 80 & 99 & 87 & 81 & 99 \\
\hline 2 & 3 & 98 & 71 & 94 & 94 & 73 & 98 & - & - & - & - & - & - \\
\hline 2 & 4 & 94 & 80 & 96 & 89 & 88 & 95 & 84 & 81 & 99 & 87 & 84 & 98 \\
\hline 3 & 1 & 97 & 89 & 90 & 90 & 92 & 96 & 83 & 79 & 100 & 87 & 84 & 99 \\
\hline 3 & 2 & 96 & 81 & 88 & 89 & 82 & 96 & 85 & 80 & 100 & 87 & 81 & 99 \\
\hline 3 & 3 & - & - & - & - & - & - & - & - & - & - & - & - \\
\hline 3 & 4 & 96 & 76 & 93 & 89 & 81 & 96 & 84 & 78 & 96 & 87 & 79 & 96 \\
\hline 4 & 1 & 96 & 91 & 92 & 87 & 95 & 95 & 85 & 80 & 100 & 86 & 87 & 99 \\
\hline 4 & 2 & 95 & 81 & 92 & 88 & 79 & 97 & 86 & 84 & 99 & 87 & 82 & 99 \\
\hline 4 & 3 & - & - & - & - & - & - & - & - & - & - & - & - \\
\hline 4 & 4 & 96 & 82 & 95 & 89 & 89 & 95 & 87 & 81 & 99 & 88 & 85 & 99 \\
\hline 5 & 1 & 96 & 87 & 94 & 94 & 83 & 91 & 92 & 87 & 91 & 93 & 85 & 91 \\
\hline 5 & 2 & 97 & 87 & 94 & 90 & 80 & 97 & 86 & 84 & 98 & 89 & 82 & 98 \\
\hline 5 & 3 & 96 & 75 & 94 & 89 & 74 & 97 & 84 & 78 & 99 & - & - & - \\
\hline 5 & 4 & 96 & 83 & 94 & 93 & 83 & 97 & 88 & 87 & 99 & 91 & 85 & 98 \\
\hline
\end{tabular}


Table B.2.3 the results of calibration and validations based on the average speed

\begin{tabular}{|c|c|c|c|c|c|c|c|c|c|c|c|c|c|}
\hline \multicolumn{2}{|c|}{ Configurations } & \multicolumn{3}{|c|}{ Calibration } & \multicolumn{3}{|c|}{$1^{\text {st }}$ Validation } & \multicolumn{3}{|c|}{$2^{\text {nd }}$ Validation } & \multicolumn{3}{|c|}{$3^{\text {rd }}$ Validation } \\
\hline$\varphi_{g}$ & $\Lambda_{l}$ & Vehicles & Cyclists & Pedestrians & Vehicles & Cyclists & Pedestrians & Vehicles & Cyclists & Pedestrians & Vehicles & Cyclists & Pedestrians \\
\hline 1 & 1 & 73 & 25 & 85 & 74 & 26 & 92 & 61 & 33 & 96 & 68 & 30 & 95 \\
\hline 1 & 2 & 49 & 51 & 91 & 48 & 60 & 98 & 36 & 75 & 97 & 42 & 69 & 97 \\
\hline 1 & 3 & 45 & 53 & 91 & 46 & 63 & 98 & 31 & 77 & 97 & 39 & 71 & 97 \\
\hline 1 & 4 & - & - & - & - & - & - & - & - & - & - & - & - \\
\hline 2 & 1 & 71 & 28 & 96 & 73 & 28 & 96 & 59 & 34 & 96 & 66 & 32 & 96 \\
\hline 2 & 2 & 67 & 28 & 96 & 69 & 28 & 96 & 59 & 35 & 96 & 64 & 32 & 96 \\
\hline 2 & 3 & 43 & 57 & 96 & 45 & 62 & 95 & 34 & 76 & 95 & 40 & 71 & 95 \\
\hline 2 & 4 & 59 & 28 & 92 & 61 & 28 & 85 & - & - & - & - & - & - \\
\hline 3 & 1 & 71 & 32 & 98 & 73 & 35 & 96 & 57 & 37 & 96 & 66 & 36 & 96 \\
\hline 3 & 2 & 69 & 32 & 98 & 69 & 35 & 96 & 56 & 38 & 96 & 63 & 36 & 96 \\
\hline 3 & 3 & 60 & 32 & 98 & 59 & 35 & 96 & 52 & 39 & 95 & - & - & - \\
\hline 3 & 4 & 64 & 32 & 93 & 63 & 35 & 87 & 51 & 37 & 84 & - & - & - \\
\hline 4 & 1 & 65 & 36 & 97 & 73 & 39 & 90 & 54 & 44 & 94 & 65 & 42 & 93 \\
\hline 4 & 2 & 65 & 36 & 97 & 69 & 39 & 90 & 53 & 45 & 94 & 62 & 43 & 93 \\
\hline 4 & 3 & 63 & 36 & 97 & 62 & 39 & 95 & 49 & 47 & 95 & 57 & 43 & 95 \\
\hline 4 & 4 & 61 & 36 & 92 & 64 & 39 & 85 & 48 & 44 & 83 & 58 & 42 & 83 \\
\hline 5 & 1 & 64 & 46 & 97 & 70 & 50 & 88 & 48 & 47 & 93 & 62 & 48 & 92 \\
\hline 5 & 2 & 64 & 46 & 97 & 69 & 50 & 88 & 48 & 49 & 93 & 61 & 49 & 92 \\
\hline 5 & 3 & 63 & 46 & 97 & 62 & 50 & 94 & 45 & 51 & 96 & 56 & 51 & 95 \\
\hline 5 & 4 & 63 & 38 & 91 & 65 & 46 & 85 & 48 & 49 & 82 & 59 & 47 & 83 \\
\hline
\end{tabular}


Table B.2.4 the results of calibration and validations based on the maximum speed

\begin{tabular}{|c|c|c|c|c|c|c|c|c|c|c|c|c|c|}
\hline \multicolumn{2}{|c|}{ Configurations } & \multicolumn{3}{|c|}{ Calibration } & \multicolumn{3}{|c|}{$1^{\text {st }}$ Validation } & \multicolumn{3}{|c|}{$2^{\text {nd }}$ Validation } & \multicolumn{3}{|c|}{$3^{\text {rd }}$ Validation } \\
\hline$\varphi_{g}$ & $\Lambda_{l}$ & Vehicles & Cyclists & Pedestrians & Vehicles & Cyclists & Pedestrians & Vehicles & Cyclists & Pedestrians & Vehicles & Cyclists & Pedestrians \\
\hline 1 & 1 & - & - & - & - & - & - & - & - & - & - & - & - \\
\hline 1 & 2 & 78 & 60 & 88 & 77 & 66 & 89 & 51 & 72 & 97 & 65 & 70 & 95 \\
\hline 1 & 3 & 75 & 60 & 88 & 74 & 72 & 89 & 51 & 72 & 97 & 63 & 72 & 95 \\
\hline 1 & 4 & - & - & - & - & - & - & - & - & - & - & - & - \\
\hline 2 & 1 & 80 & 54 & 67 & 80 & 57 & 64 & - & - & - & - & - & - \\
\hline 2 & 2 & 78 & 65 & 90 & 77 & 65 & 91 & 52 & 72 & 97 & 65 & 69 & 95 \\
\hline 2 & 3 & 75 & 65 & 90 & 74 & 63 & 91 & 53 & 71 & 97 & 64 & 68 & 95 \\
\hline 2 & 4 & - & - & - & - & - & - & - & - & - & - & - & - \\
\hline 3 & 1 & 82 & 57 & 73 & 84 & 63 & 70 & - & - & - & - & - & - \\
\hline 3 & 2 & 79 & 69 & 93 & 80 & 67 & 89 & 49 & 72 & 97 & 66 & 70 & 95 \\
\hline 3 & 3 & 78 & 69 & 93 & 77 & 64 & 89 & 53 & 71 & 97 & 66 & 68 & 95 \\
\hline 3 & 4 & - & - & - & - & - & - & - & - & - & - & - & - \\
\hline 4 & 1 & 81 & 63 & 78 & 85 & 66 & 72 & - & - & - & - & - & - \\
\hline 4 & 2 & 78 & 75 & 92 & 79 & 72 & 87 & 49 & 72 & 96 & 67 & 72 & 95 \\
\hline 4 & 3 & 79 & 73 & 92 & 79 & 66 & 87 & 54 & 72 & 96 & 69 & 69 & 95 \\
\hline 4 & 4 & - & - & - & - & - & - & - & - & - & - & - & - \\
\hline 5 & 1 & 83 & 73 & 75 & 86 & 70 & 79 & - & - & - & - & - & - \\
\hline 5 & 2 & 78 & 83 & 91 & 80 & 76 & 88 & 45 & 73 & 91 & 67 & 75 & 91 \\
\hline 5 & 3 & 80 & 81 & 91 & 81 & 70 & 94 & 52 & 73 & 96 & 70 & 71 & 96 \\
\hline 5 & 4 & 81 & 69 & 59 & - & - & - & - & - & - & - & - & - \\
\hline
\end{tabular}


Table B.2.5 the results of calibration and validations based on the average acceleration

\begin{tabular}{|c|c|c|c|c|c|c|c|c|c|c|c|c|c|}
\hline \multicolumn{2}{|c|}{ Configurations } & \multicolumn{3}{|c|}{ Calibration } & \multicolumn{3}{|c|}{$1^{\text {st }}$ Validation } & \multicolumn{3}{|c|}{$2^{\text {nd }}$ Validation } & \multicolumn{3}{|c|}{$3^{\text {rd }}$ Validation } \\
\hline$\overline{\varphi_{g}}$ & $\Lambda_{l}$ & Vehicles & Cyclists & Pedestrians & Vehicles & Cyclists & Pedestrians & Vehicles & Cyclists & Pedestrians & Vehicles & Cyclists & Pedestrians \\
\hline 1 & 1 & 77 & 11 & 12 & 85 & 5 & 6 & 90 & 7 & 15 & 87 & 6 & 13 \\
\hline 1 & 2 & 74 & 13 & 13 & 82 & 5 & 10 & 90 & 7 & 16 & 85 & 6 & 15 \\
\hline 1 & 3 & 75 & 13 & 13 & 83 & 5 & 10 & 90 & 6 & 16 & 86 & 6 & 14 \\
\hline 1 & 4 & 76 & 0 & 0 & 85 & 0 & 0 & 89 & 1 & 0 & 87 & 0 & 0 \\
\hline 2 & 1 & 33 & 39 & 18 & 35 & 34 & 24 & 45 & 17 & 19 & 40 & 23 & 20 \\
\hline 2 & 2 & 55 & 13 & 24 & 65 & 7 & 16 & 62 & 8 & 17 & 63 & 7 & 17 \\
\hline 2 & 3 & 51 & 16 & 22 & 61 & 10 & 18 & 60 & 8 & 17 & 61 & 9 & 17 \\
\hline 2 & 4 & 29 & 29 & 16 & 30 & 28 & 24 & 37 & 12 & 17 & 33 & 18 & 19 \\
\hline 3 & 1 & 51 & 16 & 27 & 57 & 7 & 23 & 53 & 9 & 18 & 55 & 8 & 19 \\
\hline 3 & 2 & 58 & 12 & 27 & 66 & 6 & 17 & 63 & 9 & 17 & 64 & 8 & 17 \\
\hline 3 & 3 & 54 & 15 & 24 & 62 & 10 & 19 & 61 & 8 & 16 & 62 & 9 & 17 \\
\hline 3 & 4 & 45 & 8 & 7 & 47 & 4 & 0 & 47 & 7 & 2 & 47 & 6 & 2 \\
\hline 4 & 1 & 60 & 12 & 27 & 67 & 5 & 13 & 67 & 4 & 17 & 67 & 4 & 16 \\
\hline 4 & 2 & 60 & 10 & 27 & 67 & 5 & 13 & 67 & 5 & 17 & 67 & 5 & 16 \\
\hline 4 & 3 & 67 & 9 & 16 & - & - & - & - & - & - & - & - & - \\
\hline 4 & 4 & 74 & 1 & 0 & 75 & 0 & 0 & 74 & 0 & 0 & 74 & 0 & 0 \\
\hline 5 & 1 & 62 & 12 & 28 & 69 & 2 & 12 & 67 & 4 & 20 & 68 & 3 & 18 \\
\hline 5 & 2 & 61 & 12 & 28 & 68 & 2 & 12 & 67 & 7 & 20 & 68 & 4 & 19 \\
\hline 5 & 3 & 62 & 12 & 28 & 69 & 2 & 12 & 67 & 7 & 20 & 68 & 4 & 18 \\
\hline 5 & 4 & 75 & 0 & 0 & 75 & 0 & 0 & 72 & 0 & 0 & 74 & 0 & 0 \\
\hline
\end{tabular}


Table B.2.6 the results of calibration and validations based on the maximum acceleration

\begin{tabular}{|c|c|c|c|c|c|c|c|c|c|c|c|c|c|}
\hline \multicolumn{2}{|c|}{ Configurations } & \multicolumn{3}{|c|}{ Calibration } & \multicolumn{3}{|c|}{$1^{\text {st }}$ Validation } & \multicolumn{3}{|c|}{$2^{\text {nd }}$ Validation } & \multicolumn{3}{|c|}{$3^{\text {rd }}$ Validation } \\
\hline$\varphi_{g}$ & $\Lambda_{l}$ & Vehicles & Cyclists & Pedestrians & Vehicles & Cyclists & Pedestrians & Vehicles & Cyclists & Pedestrians & Vehicles & Cyclists & Pedestrians \\
\hline 1 & 1 & 38 & 2 & 0 & 29 & 0 & 2 & 46 & 1 & 0 & 37 & 0 & 0 \\
\hline 1 & 2 & 38 & 0 & 0 & 29 & 0 & 2 & 45 & 1 & 0 & 36 & 1 & 0 \\
\hline 1 & 3 & 37 & 0 & 0 & 29 & 0 & 2 & 42 & 1 & 0 & 35 & 0 & 0 \\
\hline 1 & 4 & 37 & 0 & 0 & 29 & 0 & 0 & 44 & 1 & 0 & 36 & 1 & 0 \\
\hline 2 & 1 & 4 & 58 & 59 & - & - & - & - & - & - & - & - & - \\
\hline 2 & 2 & 5 & 55 & 57 & - & - & - & - & - & - & - & - & - \\
\hline 2 & 3 & 5 & 51 & 57 & - & - & - & - & - & - & - & - & - \\
\hline 2 & 4 & 4 & 55 & 55 & - & - & - & - & - & - & - & - & - \\
\hline 3 & 1 & 4 & 63 & 56 & 0 & 57 & 47 & - & - & - & - & - & - \\
\hline 3 & 2 & 5 & 59 & 56 & 0 & 51 & 49 & - & - & - & - & - & - \\
\hline 3 & 3 & 5 & 53 & 56 & 0 & 47 & 49 & - & - & - & - & - & - \\
\hline 3 & 4 & 5 & 59 & 56 & 0 & 51 & 49 & - & - & - & - & - & - \\
\hline 4 & 1 & 5 & 64 & 51 & 0 & 61 & 44 & - & - & - & - & - & - \\
\hline 4 & 2 & 7 & 60 & 51 & 1 & 54 & 44 & - & - & - & - & - & - \\
\hline 4 & 3 & 7 & 54 & 51 & 1 & 49 & 44 & - & - & - & - & - & - \\
\hline 4 & 4 & 6 & 60 & 51 & 0 & 54 & 44 & - & - & - & - & - & - \\
\hline 5 & 1 & 7 & 71 & 50 & 1 & 65 & 39 & - & - & - & - & - & - \\
\hline 5 & 2 & 10 & 65 & 50 & - & - & - & - & - & - & - & - & - \\
\hline 5 & 3 & 10 & 58 & 50 & - & - & - & - & - & - & - & - & - \\
\hline 5 & 4 & 8 & 65 & 50 & - & - & - & - & - & - & - & - & - \\
\hline
\end{tabular}


Table B.2.7 the results of calibration and validations based on the average deceleration

\begin{tabular}{|c|c|c|c|c|c|c|c|c|c|c|c|c|c|}
\hline \multicolumn{2}{|c|}{ Configurations } & \multicolumn{3}{|c|}{ Calibration } & \multicolumn{3}{|c|}{$1^{\text {st }}$ Validation } & \multicolumn{3}{|c|}{$2^{\text {nd }}$ Validation } & \multicolumn{3}{|c|}{$3^{\text {rd }}$ Validation } \\
\hline$\varphi_{g}$ & $\Lambda_{l}$ & Vehicles & Cyclists & Pedestrians & Vehicles & Cyclists & Pedestrians & Vehicles & Cyclists & Pedestrians & Vehicles & Cyclists & Pedestrians \\
\hline 1 & 1 & 56 & 38 & 15 & 60 & 36 & 8 & 74 & 23 & 20 & 66 & 28 & 17 \\
\hline 1 & 2 & 63 & 30 & 13 & 70 & 26 & 5 & - & - & - & - & - & - \\
\hline 1 & 3 & 59 & 29 & 22 & 61 & 23 & 16 & 81 & 19 & 30 & 70 & 21 & 26 \\
\hline 1 & 4 & 32 & 24 & 25 & 32 & 19 & 19 & 49 & 17 & 32 & 40 & 18 & 29 \\
\hline 2 & 1 & 56 & 39 & 8 & 60 & 38 & 7 & 75 & 22 & 20 & 67 & 28 & 17 \\
\hline 2 & 2 & 54 & 33 & 20 & 56 & 29 & 15 & 72 & 26 & 29 & 63 & 27 & 26 \\
\hline 2 & 3 & 55 & 30 & 20 & 59 & 25 & 20 & 73 & 20 & 30 & 65 & 22 & 28 \\
\hline 2 & 4 & 31 & 28 & 24 & 31 & 24 & 20 & 46 & 20 & 37 & 38 & 22 & 33 \\
\hline 3 & 1 & 55 & 39 & 10 & 59 & 39 & 6 & 77 & 22 & 20 & 67 & 29 & 17 \\
\hline 3 & 2 & 49 & 36 & 22 & 52 & 33 & 19 & 73 & 27 & 30 & 62 & 29 & 28 \\
\hline 3 & 3 & 53 & 31 & 22 & 57 & 26 & 19 & 76 & 19 & 30 & 66 & 22 & 28 \\
\hline 3 & 4 & 31 & 33 & 24 & 30 & 29 & 19 & 49 & 23 & 32 & 39 & 26 & 30 \\
\hline 4 & 1 & 32 & 46 & 30 & 29 & 51 & 15 & 48 & 51 & 34 & 37 & 51 & 31 \\
\hline 4 & 2 & 43 & 37 & 30 & 43 & 43 & 15 & 62 & 36 & 35 & 51 & 39 & 31 \\
\hline 4 & 3 & 50 & 31 & 27 & 54 & 26 & 15 & 70 & 23 & 28 & 60 & 24 & 26 \\
\hline 4 & 4 & 37 & 34 & 27 & 35 & 28 & 15 & 56 & 31 & 31 & 44 & 29 & 28 \\
\hline 5 & 1 & 40 & 50 & 22 & 43 & 50 & 15 & 62 & 42 & 26 & 50 & 46 & 24 \\
\hline 5 & 2 & 59 & 37 & 9 & 64 & 26 & 6 & - & - & - & - & - & - \\
\hline 5 & 3 & 66 & 35 & 13 & 73 & 20 & 9 & 82 & 9 & 20 & 76 & 14 & 18 \\
\hline 5 & 4 & 39 & 40 & 25 & 39 & 28 & 15 & 62 & 36 & 29 & 47 & 32 & 26 \\
\hline
\end{tabular}


Table B.2.8 the results of calibration and validations based on the maximum deceleration

\begin{tabular}{|c|c|c|c|c|c|c|c|c|c|c|c|c|c|}
\hline \multicolumn{2}{|c|}{ Configurations } & \multicolumn{3}{|c|}{ Calibration } & \multicolumn{3}{|c|}{ 1st Validation } & \multicolumn{3}{|c|}{ 2nd Validation } & \multicolumn{3}{|c|}{ 3rd Validation } \\
\hline$\varphi_{g}$ & $\Lambda_{l}$ & Vehicles & Cyclists & Pedestrians & Vehicles & Cyclists & Pedestrians & Vehicles & Cyclists & Pedestrians & Vehicles & Cyclists & Pedestrians \\
\hline 1 & 1 & 44 & 0 & 1 & 43 & 0 & 0 & 56 & 1 & 1 & 49 & 0 & 0 \\
\hline 1 & 2 & 29 & 1 & 1 & 27 & 1 & 0 & 37 & 3 & 1 & 32 & 2 & 0 \\
\hline 1 & 3 & 3 & 40 & 37 & 1 & 39 & 38 & - & - & - & - & - & - \\
\hline 1 & 4 & 21 & 0 & 1 & 20 & 0 & 0 & - & - & - & - & - & - \\
\hline 2 & 1 & 43 & 0 & 2 & 43 & 0 & 0 & - & - & - & - & - & - \\
\hline 2 & 2 & 4 & 44 & 47 & 2 & 40 & 53 & 55 & 0 & 1 & 47 & 0 & 0 \\
\hline 2 & 3 & 2 & 47 & 45 & 1 & 45 & 45 & - & - & - & - & - & - \\
\hline 2 & 4 & 3 & 37 & 47 & 1 & 33 & 53 & - & - & - & - & - & - \\
\hline 3 & 1 & 39 & 0 & 0 & 41 & 0 & 0 & - & - & - & - & - & - \\
\hline 3 & 2 & 2 & 24 & 76 & 1 & 21 & 77 & - & - & - & - & - & - \\
\hline 3 & 3 & 3 & 49 & 44 & 1 & 49 & 57 & - & - & - & - & - & - \\
\hline 3 & 4 & 1 & 21 & 76 & 0 & 17 & 77 & - & - & - & - & - & - \\
\hline 4 & 1 & 53 & 0 & 0 & 63 & 0 & 0 & 66 & 1 & 1 & 64 & 1 & 0 \\
\hline 4 & 2 & 32 & 0 & 0 & 36 & 0 & 3 & 40 & 1 & 1 & 38 & 1 & 1 \\
\hline 4 & 3 & 1 & 3 & 27 & - & - & - & - & - & - & - & - & - \\
\hline 4 & 4 & 2 & 22 & 73 & - & - & - & - & - & - & - & - & - \\
\hline 5 & 1 & 53 & 0 & 0 & 64 & 0 & 0 & 71 & 0 & 0 & 66 & 0 & 0 \\
\hline 5 & 2 & 4 & 29 & 84 & 1 & 30 & 76 & - & - & - & - & - & - \\
\hline 5 & 3 & 6 & 60 & 44 & 1 & 61 & 39 & - & - & - & - & - & - \\
\hline 5 & 4 & 2 & 25 & 78 & - & - & - & - & - & - & - & - & - \\
\hline
\end{tabular}


Table B.2.9 the results of calibration and validations based on the average direction

\begin{tabular}{|c|c|c|c|c|c|c|c|c|c|c|c|c|c|}
\hline \multicolumn{2}{|c|}{ Configurations } & \multicolumn{3}{|c|}{ Calibration } & \multicolumn{3}{|c|}{$1^{\text {st }}$ Validation } & \multicolumn{3}{|c|}{$2^{\text {nd }}$ Validation } & \multicolumn{3}{|c|}{$3^{\text {rd }}$ Validation } \\
\hline$\varphi_{g}$ & $\Lambda_{l}$ & Vehicles & Cyclists & Pedestrians & Vehicles & Cyclists & Pedestrians & Vehicles & Cyclists & Pedestrians & Vehicles & Cyclists & Pedestrians \\
\hline 1 & 1 & 49 & 0 & 31 & 44 & 0 & 16 & 2 & 0 & 15 & 25 & 0 & 15 \\
\hline 1 & 2 & 47 & 0 & 32 & 43 & 0 & 19 & 2 & 0 & 18 & 24 & 0 & 18 \\
\hline 1 & 3 & 44 & 0 & 32 & 40 & 0 & 19 & 2 & 0 & 18 & 22 & 0 & 18 \\
\hline 1 & 4 & 46 & 0 & 29 & 43 & 0 & 19 & 2 & 0 & 18 & 24 & 0 & 18 \\
\hline 2 & 1 & 49 & 0 & 37 & 44 & 0 & 18 & 2 & 0 & 15 & 24 & 0 & 16 \\
\hline 2 & 2 & 47 & 0 & 37 & 43 & 0 & 22 & 2 & 0 & 18 & 24 & 0 & 19 \\
\hline 2 & 3 & 43 & 0 & 33 & 40 & 0 & 20 & 2 & 0 & 18 & 23 & 0 & 18 \\
\hline 2 & 4 & 47 & 0 & 37 & 43 & 0 & 22 & 2 & 0 & 18 & 24 & 0 & 19 \\
\hline 3 & 1 & 47 & 0 & 37 & 41 & 0 & 19 & 2 & 0 & 15 & 23 & 0 & 16 \\
\hline 3 & 2 & 45 & 0 & 37 & 41 & 0 & 21 & 1 & 0 & 18 & 22 & 0 & 19 \\
\hline 3 & 3 & 41 & 0 & 34 & 37 & 0 & 19 & 2 & 0 & 18 & 21 & 0 & 18 \\
\hline 3 & 4 & 45 & 0 & 37 & 41 & 0 & 21 & 1 & 0 & 18 & 22 & 0 & 19 \\
\hline 4 & 1 & 0 & 88 & 0 & 0 & 80 & 0 & 0 & 65 & 1 & 0 & 72 & 1 \\
\hline 4 & 2 & 1 & 87 & 0 & 3 & 70 & 0 & 3 & 63 & 1 & 3 & 66 & 1 \\
\hline 4 & 3 & 37 & 0 & 86 & 22 & 0 & 77 & - & - & - & - & - & - \\
\hline 4 & 4 & 37 & 0 & 43 & 22 & 0 & 26 & 1 & 0 & 24 & - & - & - \\
\hline 5 & 1 & 46 & 0 & 44 & 24 & 0 & 27 & 1 & 0 & 20 & 16 & 0 & 21 \\
\hline 5 & 2 & 46 & 0 & 44 & 25 & 0 & 27 & 1 & 0 & 20 & 16 & 0 & 21 \\
\hline 5 & 3 & 46 & 0 & 84 & 25 & 0 & 73 & - & - & - & - & - & - \\
\hline 5 & 4 & 46 & 0 & 44 & 25 & 0 & 27 & 1 & 0 & 20 & 16 & 0 & 21 \\
\hline
\end{tabular}


Table B.2.10 the results of calibration and validations based on the maximum direction

\begin{tabular}{|c|c|c|c|c|c|c|c|c|c|c|c|c|c|}
\hline \multicolumn{2}{|c|}{ Configurations } & \multicolumn{3}{|c|}{ Calibration } & \multicolumn{3}{|c|}{$1^{\text {st }}$ Validation } & \multicolumn{3}{|c|}{$2^{\text {nd }}$ Validation } & \multicolumn{3}{|c|}{$3^{\text {rd }}$ Validation } \\
\hline$\varphi_{g}$ & $\Lambda_{l}$ & Vehicles & Cyclists & Pedestrians & Vehicles & Cyclists & Pedestrians & Vehicles & Cyclists & Pedestrians & Vehicles & Cyclists & Pedestrians \\
\hline 1 & 1 & 65 & 0 & 43 & 58 & 0 & 29 & 2 & 1 & 25 & 32 & 0 & 26 \\
\hline 1 & 2 & 63 & 0 & 43 & 56 & 0 & 29 & 2 & 1 & 25 & 31 & 0 & 26 \\
\hline 1 & 3 & 60 & 1 & 47 & 55 & 0 & 32 & 2 & 1 & 32 & 30 & 0 & 32 \\
\hline 1 & 4 & 62 & 0 & 40 & 56 & 0 & 29 & 2 & 1 & 25 & 31 & 0 & 26 \\
\hline 2 & 1 & 67 & 0 & 51 & 58 & 0 & 33 & 2 & 1 & 25 & 32 & 0 & 27 \\
\hline 2 & 2 & 65 & 0 & 51 & 56 & 0 & 33 & 2 & 1 & 25 & 31 & 0 & 27 \\
\hline 2 & 3 & 63 & 0 & 51 & 56 & 0 & 36 & 2 & 1 & 26 & 31 & 0 & 28 \\
\hline 2 & 4 & 64 & 0 & 51 & 56 & 0 & 33 & 2 & 1 & 25 & 31 & 0 & 27 \\
\hline 3 & 1 & 69 & 0 & 51 & 58 & 0 & 34 & 2 & 0 & 25 & 32 & 0 & 27 \\
\hline 3 & 2 & 67 & 0 & 51 & 56 & 0 & 34 & 2 & 0 & 25 & 31 & 0 & 27 \\
\hline 3 & 3 & 65 & 0 & 51 & 55 & 0 & 38 & 2 & 0 & 25 & 31 & 0 & 28 \\
\hline 3 & 4 & 66 & 0 & 51 & 55 & 0 & 34 & 2 & 0 & 25 & 31 & 0 & 27 \\
\hline 4 & 1 & 69 & 0 & 49 & 57 & 0 & 28 & 1 & 0 & 24 & 34 & 0 & 25 \\
\hline 4 & 2 & 66 & 0 & 49 & 54 & 0 & 28 & 1 & 0 & 24 & 32 & 0 & 25 \\
\hline 4 & 3 & 64 & 0 & 49 & 54 & 0 & 33 & 1 & 0 & 25 & 32 & 0 & 26 \\
\hline 4 & 4 & 65 & 0 & 49 & 54 & 0 & 28 & 1 & 0 & 24 & 32 & 0 & 25 \\
\hline 5 & 1 & 71 & 0 & 47 & 57 & 0 & 33 & 1 & 0 & 23 & 36 & 0 & 25 \\
\hline 5 & 2 & 68 & 0 & 47 & 54 & 0 & 33 & 1 & 0 & 23 & 34 & 0 & 25 \\
\hline 5 & 3 & 65 & 0 & 47 & 53 & 0 & 36 & 1 & 0 & 23 & 34 & 0 & 25 \\
\hline 5 & 4 & 66 & 0 & 47 & 53 & 0 & 33 & 1 & 0 & 23 & 34 & 0 & 25 \\
\hline
\end{tabular}


Table B.2.11 the results of calibration and validations based on the average of change in direction

\begin{tabular}{|c|c|c|c|c|c|c|c|c|c|c|c|c|c|}
\hline \multicolumn{2}{|c|}{ Configurations } & \multicolumn{3}{|c|}{ Calibration } & \multicolumn{3}{|c|}{$1^{\text {st }}$ Validation } & \multicolumn{3}{|c|}{$2^{\text {nd }}$ Validation } & \multicolumn{3}{|c|}{$3^{\text {rd }}$ Validation } \\
\hline$\varphi_{g}$ & $\Lambda_{l}$ & Vehicles & Cyclists & Pedestrians & Vehicles & Cyclists & Pedestrians & Vehicles & Cyclists & Pedestrians & Vehicles & Cyclists & Pedestrians \\
\hline 1 & 1 & 74 & 2 & 1 & 57 & 1 & 0 & - & - & - & - & - & - \\
\hline 1 & 2 & 74 & 2 & 1 & 57 & 1 & 0 & - & - & - & - & - & - \\
\hline 1 & 3 & 74 & 2 & 1 & 57 & 1 & 0 & - & - & - & - & - & - \\
\hline 1 & 4 & 83 & 1 & 1 & 74 & 1 & 0 & - & - & - & - & - & - \\
\hline 2 & 1 & 77 & 0 & 2 & - & - & - & - & - & - & - & - & - \\
\hline 2 & 2 & 77 & 0 & 2 & - & - & - & - & - & - & - & - & - \\
\hline 2 & 3 & 77 & 0 & 2 & - & - & - & - & - & - & - & - & - \\
\hline 2 & 4 & 77 & 0 & 2 & - & - & - & - & - & - & - & - & - \\
\hline 3 & 1 & 71 & 0 & 0 & - & - & - & - & - & - & - & - & - \\
\hline 3 & 2 & 71 & 0 & 0 & - & - & - & - & - & - & - & - & - \\
\hline 3 & 3 & 71 & 0 & 0 & - & - & - & - & - & - & - & - & - \\
\hline 3 & 4 & 71 & 0 & 0 & - & - & - & - & - & - & - & - & - \\
\hline 4 & 1 & 52 & 0 & 0 & 31 & 0 & 0 & - & - & - & - & - & - \\
\hline 4 & 2 & 52 & 0 & 0 & 31 & 0 & 0 & - & - & - & - & - & - \\
\hline 4 & 3 & 52 & 0 & 0 & 31 & 0 & 0 & - & - & - & - & - & - \\
\hline 4 & 4 & 52 & 0 & 0 & 31 & 0 & 0 & - & - & - & - & - & - \\
\hline 5 & 1 & 63 & 0 & 0 & 38 & 0 & 0 & - & - & - & - & - & - \\
\hline 5 & 2 & 63 & 0 & 0 & 38 & 0 & 0 & - & - & - & - & - & - \\
\hline 5 & 3 & 63 & 0 & 0 & 38 & 0 & 0 & - & - & - & - & - & - \\
\hline 5 & 4 & 63 & 0 & 0 & 38 & 0 & 0 & - & - & - & - & - & - \\
\hline
\end{tabular}


Table B.2.12 the results of calibration and validations based on the average of cosine of change in direction

\begin{tabular}{|c|c|c|c|c|c|c|c|c|c|c|c|c|c|}
\hline \multicolumn{2}{|c|}{ Configurations } & \multicolumn{3}{|c|}{ Calibration } & \multicolumn{3}{|c|}{$1^{\text {st }}$ Validation } & \multicolumn{3}{|c|}{$2^{\text {nd }}$ Validation } & \multicolumn{3}{|c|}{$3^{\text {rd }}$ Validation } \\
\hline$\varphi_{g}$ & $\Lambda_{l}$ & Vehicles & Cyclists & Pedestrians & Vehicles & Cyclists & Pedestrians & Vehicles & Cyclists & Pedestrians & Vehicles & Cyclists & Pedestrians \\
\hline 1 & 1 & 59 & 2 & 35 & 61 & 2 & 24 & 64 & 0 & 20 & 62 & 1 & 21 \\
\hline 1 & 2 & 55 & 2 & 40 & 56 & 2 & 33 & 56 & 0 & 30 & 56 & 1 & 31 \\
\hline 1 & 3 & 23 & 2 & 49 & 21 & 2 & 49 & 17 & 0 & 36 & 19 & 1 & 39 \\
\hline 1 & 4 & - & - & - & - & - & - & - & - & - & - & - & - \\
\hline 2 & 1 & 62 & 1 & 31 & 60 & 2 & 24 & 62 & 0 & 20 & 61 & 1 & 21 \\
\hline 2 & 2 & 58 & 1 & 35 & 56 & 2 & 33 & 55 & 1 & 30 & 55 & 1 & 31 \\
\hline 2 & 3 & 24 & 1 & 47 & 21 & 2 & 53 & 17 & 0 & 36 & 19 & 1 & 39 \\
\hline 2 & 4 & - & - & - & - & - & - & - & - & - & - & - & - \\
\hline 3 & 1 & 76 & 1 & 59 & 75 & 1 & 38 & 80 & 0 & 31 & 77 & 1 & 33 \\
\hline 3 & 2 & 67 & 1 & 39 & 62 & 1 & 36 & 61 & 0 & 28 & 62 & 1 & 30 \\
\hline 3 & 3 & 27 & 1 & 51 & 22 & 1 & 55 & 19 & 0 & 35 & - & - & - \\
\hline 3 & 4 & - & - & - & - & - & - & - & - & - & - & - & - \\
\hline 4 & 1 & 88 & 0 & 62 & 80 & 0 & 38 & 82 & 0 & 30 & 81 & 0 & 32 \\
\hline 4 & 2 & 80 & 0 & 41 & 68 & 0 & 36 & 65 & 0 & 28 & 67 & 0 & 30 \\
\hline 4 & 3 & - & - & - & - & - & - & - & - & - & - & - & - \\
\hline 4 & 4 & - & - & - & - & - & - & - & - & - & - & - & - \\
\hline 5 & 1 & 91 & 0 & 69 & 82 & 0 & 36 & 83 & 0 & 32 & 82 & 0 & 33 \\
\hline 5 & 2 & 83 & 0 & 44 & 69 & 0 & 39 & 67 & 0 & 31 & 68 & 0 & 33 \\
\hline 5 & 3 & - & - & - & - & - & - & - & - & - & - & - & - \\
\hline 5 & 4 & - & - & - & - & - & - & - & - & - & - & - & - \\
\hline
\end{tabular}


Table B.2.13 the results of calibration and validations based on the average area

\begin{tabular}{|c|c|c|c|c|c|c|c|c|c|c|c|c|c|}
\hline \multicolumn{2}{|c|}{ Configurations } & \multicolumn{3}{|c|}{ Calibration } & \multicolumn{3}{|c|}{$1^{\text {st }}$ Validation } & \multicolumn{3}{|c|}{$2^{\text {nd }}$ Validation } & \multicolumn{3}{|c|}{$3^{\text {rd }}$ Validation } \\
\hline$\varphi_{g}$ & $\Lambda_{l}$ & Vehicles & Cyclists & Pedestrians & Vehicles & Cyclists & Pedestrians & Vehicles & Cyclists & Pedestrians & Vehicles & Cyclists & Pedestrians \\
\hline 1 & 1 & 94 & 77 & 62 & 87 & 70 & 75 & 85 & 16 & 94 & 86 & 38 & 89 \\
\hline 1 & 2 & - & - & - & - & - & - & - & - & - & - & - & - \\
\hline 1 & 3 & - & - & - & - & - & - & - & - & - & - & - & - \\
\hline 1 & 4 & - & - & - & - & - & - & - & - & - & - & - & - \\
\hline 2 & 1 & 95 & 81 & 65 & 87 & 75 & 77 & 85 & 15 & 90 & 86 & 37 & 86 \\
\hline 2 & 2 & - & - & - & - & - & - & - & - & - & - & - & - \\
\hline 2 & 3 & - & - & - & - & - & - & - & - & - & - & - & - \\
\hline 2 & 4 & - & - & - & - & - & - & - & - & - & - & - & - \\
\hline 3 & 1 & 96 & 83 & 63 & 85 & 74 & 74 & 84 & 14 & 90 & 84 & 38 & 86 \\
\hline 3 & 2 & - & - & - & - & - & - & - & - & - & - & - & - \\
\hline 3 & 3 & - & - & - & - & - & - & - & - & - & - & - & - \\
\hline 3 & 4 & - & - & - & - & - & - & - & - & - & - & - & - \\
\hline 4 & 1 & 96 & 84 & 62 & 87 & 72 & 75 & 87 & 20 & 90 & 87 & 45 & 88 \\
\hline 4 & 2 & - & - & - & - & - & - & - & - & - & - & - & - \\
\hline 4 & 3 & - & - & - & - & - & - & - & - & - & - & - & - \\
\hline 4 & 4 & - & - & - & - & - & - & - & - & - & - & - & - \\
\hline 5 & 1 & 96 & 87 & 63 & 88 & 76 & 85 & 87 & 31 & 91 & 88 & 54 & 89 \\
\hline 5 & 2 & - & - & - & - & - & - & - & - & - & - & - & - \\
\hline 5 & 3 & - & - & - & - & - & - & - & - & - & - & - & - \\
\hline 5 & 4 & - & - & - & - & - & - & - & - & - & - & - & - \\
\hline
\end{tabular}


Table B.2.14 the results of calibration and validations based on the maximum area

\begin{tabular}{|c|c|c|c|c|c|c|c|c|c|c|c|c|c|}
\hline \multicolumn{2}{|c|}{ Configurations } & \multicolumn{3}{|c|}{ Calibration } & \multicolumn{3}{|c|}{$1^{\text {st }}$ Validation } & \multicolumn{3}{|c|}{$2^{\text {nd }}$ Validation } & \multicolumn{3}{|c|}{$3^{\text {rd }}$ Validation } \\
\hline$\varphi_{g}$ & $\Lambda_{l}$ & Vehicles & Cyclists & Pedestrians & Vehicles & Cyclists & Pedestrians & Vehicles & Cyclists & Pedestrians & Vehicles & Cyclists & Pedestrians \\
\hline 1 & 1 & 96 & 85 & 12 & 95 & 78 & 14 & 95 & 80 & 7 & 95 & 79 & 9 \\
\hline 1 & 2 & 94 & 65 & 22 & - & - & - & - & - & - & - & - & - \\
\hline 1 & 3 & - & - & - & - & - & - & - & - & - & - & - & - \\
\hline 1 & 4 & - & - & - & - & - & - & - & - & - & - & - & - \\
\hline 2 & 1 & 98 & 84 & 16 & 95 & 78 & 16 & 94 & 76 & 7 & 95 & 76 & 9 \\
\hline 2 & 2 & - & - & - & - & - & - & - & - & - & - & - & - \\
\hline 2 & 3 & - & - & - & - & - & - & - & - & - & - & - & - \\
\hline 2 & 4 & - & - & - & - & - & - & - & - & - & - & - & - \\
\hline 3 & 1 & 98 & 81 & 20 & 94 & 75 & 26 & 90 & 55 & 9 & 92 & 63 & 12 \\
\hline 3 & 2 & 97 & 56 & 34 & 94 & 53 & 32 & 95 & 61 & 9 & 95 & 58 & 14 \\
\hline 3 & 3 & - & - & - & - & - & - & - & - & - & - & - & - \\
\hline 3 & 4 & - & - & - & - & - & - & - & - & - & - & - & - \\
\hline 4 & 1 & 99 & 85 & 14 & 96 & 82 & 18 & 97 & 73 & 7 & 96 & 77 & 9 \\
\hline 4 & 2 & 98 & 58 & 35 & 95 & 56 & 38 & 96 & 56 & 9 & 96 & 56 & 15 \\
\hline 4 & 3 & - & - & - & - & - & - & - & - & - & - & - & - \\
\hline 4 & 4 & - & - & - & - & - & - & - & - & - & - & - & - \\
\hline 5 & 1 & 99 & 85 & 16 & 96 & 80 & 24 & 91 & 62 & 12 & 94 & 71 & 14 \\
\hline 5 & 2 & 98 & 63 & 28 & 96 & 52 & 33 & 95 & 60 & 12 & 95 & 56 & 16 \\
\hline 5 & 3 & - & - & - & - & - & - & - & - & - & - & - & - \\
\hline 5 & 4 & - & - & - & - & - & - & - & - & - & - & - & - \\
\hline
\end{tabular}


Table B.2.15 the results of calibration and validations based on the average length

\begin{tabular}{|c|c|c|c|c|c|c|c|c|c|c|c|c|c|}
\hline \multicolumn{2}{|c|}{ Configurations } & \multicolumn{3}{|c|}{ Calibration } & \multicolumn{3}{|c|}{$1^{\text {st }}$ Validation } & \multicolumn{3}{|c|}{$2^{\text {nd }}$ Validation } & \multicolumn{3}{|c|}{$3^{\text {rd }}$ Validation } \\
\hline$\varphi_{g}$ & $\Lambda_{l}$ & Vehicles & Cyclists & Pedestrians & Vehicles & Cyclists & Pedestrians & Vehicles & Cyclists & Pedestrians & Vehicles & Cyclists & Pedestrians \\
\hline 1 & 1 & 94 & 8 & 74 & 83 & 7 & 67 & 90 & 63 & 17 & 86 & 41 & 30 \\
\hline 1 & 2 & 94 & 17 & 53 & 84 & 19 & 51 & 91 & 88 & 7 & 87 & 60 & 18 \\
\hline 1 & 3 & 92 & 45 & 32 & 79 & 40 & 24 & 86 & 97 & 1 & 82 & 74 & 7 \\
\hline 1 & 4 & - & - & - & - & - & - & - & - & - & - & - & - \\
\hline 2 & 1 & 95 & 2 & 88 & 83 & 6 & 73 & 90 & 63 & 17 & 86 & 41 & 30 \\
\hline 2 & 2 & 93 & 21 & 59 & 79 & 20 & 47 & 86 & 93 & 4 & 82 & 65 & 14 \\
\hline 2 & 3 & 93 & 43 & 41 & 79 & 39 & 27 & 86 & 96 & 1 & 82 & 74 & 7 \\
\hline 2 & 4 & - & - & - & - & - & - & - & - & - & - & - & - \\
\hline 3 & 1 & 96 & 1 & 85 & 82 & 1 & 81 & 90 & 66 & 17 & 86 & 41 & 30 \\
\hline 3 & 2 & 94 & 21 & 56 & 77 & 14 & 51 & 86 & 93 & 4 & 81 & 62 & 14 \\
\hline 3 & 3 & 87 & 67 & 29 & 66 & 56 & 21 & 77 & 89 & 1 & 71 & 76 & 5 \\
\hline 3 & 4 & - & - & - & - & - & - & - & - & - & - & - & - \\
\hline 4 & 1 & 97 & 0 & 86 & 83 & 0 & 85 & 91 & 56 & 17 & 87 & 31 & 29 \\
\hline 4 & 2 & 88 & 49 & 35 & - & - & - & - & - & - & - & - & - \\
\hline 4 & 3 & 89 & 76 & 24 & 62 & 67 & 21 & 75 & 83 & 1 & 68 & 76 & 4 \\
\hline 4 & 4 & - & - & - & - & - & - & - & - & - & - & - & - \\
\hline 5 & 1 & - & - & - & - & - & - & - & - & - & - & - & - \\
\hline 5 & 2 & 89 & 71 & 28 & 56 & 65 & 24 & 54 & 96 & 1 & 55 & 80 & 5 \\
\hline 5 & 3 & 93 & 87 & 22 & 62 & 76 & 12 & 66 & 73 & 0 & 64 & 75 & 2 \\
\hline 5 & 4 & - & - & - & - & - & - & - & - & - & - & - & - \\
\hline
\end{tabular}


Table B.2.16 the results of calibration and validations based on the maximum length

\begin{tabular}{|c|c|c|c|c|c|c|c|c|c|c|c|c|c|}
\hline \multicolumn{2}{|c|}{ Configurations } & \multicolumn{3}{|c|}{ Calibration } & \multicolumn{3}{|c|}{$1^{\text {st }}$ Validation } & \multicolumn{3}{|c|}{$2^{\text {nd }}$ Validation } & \multicolumn{3}{|c|}{$3^{\text {rd }}$ Validation } \\
\hline$\varphi_{g}$ & $\Lambda_{l}$ & Vehicles & Cyclists & Pedestrians & Vehicles & Cyclists & Pedestrians & Vehicles & Cyclists & Pedestrians & Vehicles & Cyclists & Pedestrians \\
\hline 1 & 1 & 95 & 5 & 57 & 94 & 3 & 52 & 98 & 58 & 42 & 96 & 36 & 45 \\
\hline 1 & 2 & 88 & 31 & 44 & 84 & 32 & 30 & 94 & 89 & 14 & 89 & 66 & 18 \\
\hline 1 & 3 & 78 & 82 & 24 & 72 & 71 & 16 & - & - & - & - & - & - \\
\hline 1 & 4 & - & - & - & - & - & - & - & - & - & - & - & - \\
\hline 2 & 1 & 94 & 2 & 61 & 88 & 3 & 56 & 94 & 73 & 31 & 91 & 47 & 37 \\
\hline 2 & 2 & 90 & 27 & 41 & 84 & 29 & 29 & 94 & 88 & 14 & 89 & 66 & 18 \\
\hline 2 & 3 & 81 & 82 & 18 & 73 & 69 & 13 & - & - & - & - & - & - \\
\hline 2 & 4 & - & - & - & - & - & - & - & - & - & - & - & - \\
\hline 3 & 1 & 95 & 3 & 61 & 88 & 1 & 55 & 96 & 73 & 30 & 92 & 45 & 35 \\
\hline 3 & 2 & 86 & 41 & 41 & 78 & 28 & 26 & - & - & - & - & - & - \\
\hline 3 & 3 & 82 & 81 & 20 & 74 & 61 & 13 & - & - & - & - & - & - \\
\hline 3 & 4 & 92 & 0 & 12 & 86 & 0 & 13 & - & - & - & - & - & - \\
\hline 4 & 1 & - & - & - & - & - & - & - & - & - & - & - & - \\
\hline 4 & 2 & 79 & 76 & 24 & 65 & 49 & 26 & - & - & - & - & - & - \\
\hline 4 & 3 & 86 & 84 & 19 & 73 & 59 & 15 & 83 & 99 & 3 & 77 & 81 & 5 \\
\hline 4 & 4 & - & - & - & - & - & - & - & - & - & - & - & - \\
\hline 5 & 1 & 92 & 87 & 19 & - & - & - & - & - & - & - & - & - \\
\hline 5 & 2 & 88 & 77 & 25 & 70 & 54 & 27 & - & - & - & - & - & - \\
\hline 5 & 3 & 92 & 87 & 19 & 79 & 65 & 15 & 83 & 98 & 3 & 81 & 81 & 5 \\
\hline 5 & 4 & 88 & 77 & 25 & - & - & - & - & - & - & - & - & - \\
\hline
\end{tabular}


Table B.2.17 the results of calibration and validations based on the average width

\begin{tabular}{|c|c|c|c|c|c|c|c|c|c|c|c|c|c|}
\hline \multicolumn{2}{|c|}{ Configurations } & \multicolumn{3}{|c|}{ Calibration } & \multicolumn{3}{|c|}{$1^{\text {st }}$ Validation } & \multicolumn{3}{|c|}{$2^{\text {nd }}$ Validation } & \multicolumn{3}{|c|}{$3^{\text {rd }}$ Validation } \\
\hline$\varphi_{g}$ & $\Lambda_{l}$ & Vehicles & Cyclists & Pedestrians & Vehicles & Cyclists & Pedestrians & Vehicles & Cyclists & Pedestrians & Vehicles & Cyclists & Pedestrians \\
\hline 1 & 1 & 92 & 81 & 54 & 87 & 75 & 59 & 81 & 49 & 62 & 84 & 59 & 61 \\
\hline 1 & 2 & 91 & 67 & 71 & 87 & 56 & 76 & 82 & 34 & 63 & 85 & 43 & 67 \\
\hline 1 & 3 & - & - & - & - & - & - & - & - & - & - & - & - \\
\hline 1 & 4 & - & - & - & - & - & - & - & - & - & - & - & - \\
\hline 2 & 1 & 94 & 88 & 63 & 88 & 79 & 64 & 82 & 49 & 62 & 85 & 60 & 62 \\
\hline 2 & 2 & 93 & 73 & 84 & 88 & 56 & 82 & 83 & 34 & 63 & 85 & 42 & 68 \\
\hline 2 & 3 & - & - & - & - & - & - & - & - & - & - & - & - \\
\hline 2 & 4 & - & - & - & - & - & - & - & - & - & - & - & - \\
\hline 3 & 1 & 96 & 88 & 61 & 86 & 79 & 66 & 82 & 50 & 62 & 84 & 61 & 62 \\
\hline 3 & 2 & 95 & 76 & 83 & 86 & 58 & 81 & 82 & 32 & 63 & 84 & 42 & 67 \\
\hline 3 & 3 & - & - & - & - & - & - & - & - & - & - & - & - \\
\hline 3 & 4 & - & - & - & - & - & - & - & - & - & - & - & - \\
\hline 4 & 1 & 97 & 88 & 62 & 88 & 84 & 69 & 85 & 53 & 62 & 87 & 67 & 63 \\
\hline 4 & 2 & 96 & 76 & 84 & 88 & 62 & 79 & 87 & 33 & 59 & 88 & 46 & 63 \\
\hline 4 & 3 & - & - & - & - & - & - & - & - & - & - & - & - \\
\hline 4 & 4 & - & - & - & - & - & - & - & - & - & - & - & - \\
\hline 5 & 1 & 96 & 90 & 63 & 88 & 83 & 73 & 87 & 47 & 64 & 88 & 65 & 65 \\
\hline 5 & 2 & 95 & 79 & 81 & 88 & 63 & 82 & 88 & 33 & 61 & 88 & 48 & 65 \\
\hline 5 & 3 & - & - & - & - & - & - & - & - & - & - & - & - \\
\hline 5 & 4 & - & - & - & - & - & - & - & - & - & - & - & - \\
\hline
\end{tabular}


Table B.2.18 the results of calibration and validations based on the maximum width

\begin{tabular}{|c|c|c|c|c|c|c|c|c|c|c|c|c|c|}
\hline \multicolumn{2}{|c|}{ Configurations } & \multicolumn{3}{|c|}{ Calibration } & \multicolumn{3}{|c|}{$1^{\text {st }}$ Validation } & \multicolumn{3}{|c|}{$2^{\text {nd }}$ Validation } & \multicolumn{3}{|c|}{$3^{\text {rd }}$ Validation } \\
\hline$\varphi_{g}$ & $\Lambda_{l}$ & Vehicles & Cyclists & Pedestrians & Vehicles & Cyclists & Pedestrians & Vehicles & Cyclists & Pedestrians & Vehicles & Cyclists & Pedestrians \\
\hline 1 & 1 & 89 & 76 & 49 & 92 & 72 & 67 & 83 & 56 & 89 & 88 & 62 & 83 \\
\hline 1 & 2 & 90 & 60 & 65 & 93 & 56 & 79 & 86 & 38 & 95 & 90 & 45 & 91 \\
\hline 1 & 3 & 91 & 42 & 65 & 94 & 41 & 86 & 87 & 28 & 96 & - & - & - \\
\hline 1 & 4 & - & - & - & - & - & - & - & - & - & - & - & - \\
\hline 2 & 1 & 94 & 82 & 57 & 93 & 72 & 69 & 83 & 57 & 89 & 89 & 63 & 84 \\
\hline 2 & 2 & 95 & 66 & 76 & 94 & 60 & 82 & 86 & 38 & 95 & 90 & 46 & 92 \\
\hline 2 & 3 & - & - & - & - & - & - & - & - & - & - & - & - \\
\hline 2 & 4 & - & - & - & - & - & - & - & - & - & - & - & - \\
\hline 3 & 1 & 97 & 85 & 51 & 93 & 78 & 68 & 82 & 62 & 88 & 88 & 68 & 84 \\
\hline 3 & 2 & 97 & 71 & 71 & 93 & 68 & 79 & 86 & 39 & 95 & 90 & 51 & 92 \\
\hline 3 & 3 & - & - & - & - & - & - & - & - & - & - & - & - \\
\hline 3 & 4 & - & - & - & - & - & - & - & - & - & - & - & - \\
\hline 4 & 1 & 96 & 82 & 57 & 93 & 80 & 49 & - & - & - & - & - & - \\
\hline 4 & 2 & 97 & 72 & 68 & 94 & 74 & 74 & 88 & 45 & 89 & 92 & 58 & 86 \\
\hline 4 & 3 & 98 & 54 & 68 & 96 & 57 & 90 & 89 & 37 & 95 & 93 & 46 & 94 \\
\hline 4 & 4 & - & - & - & - & - & - & - & - & - & - & - & - \\
\hline 5 & 1 & 97 & 85 & 53 & 94 & 80 & 42 & - & - & - & - & - & - \\
\hline 5 & 2 & 97 & 73 & 63 & 94 & 67 & 76 & 86 & 44 & 91 & 91 & 56 & 88 \\
\hline 5 & 3 & - & - & - & - & - & - & - & - & - & - & - & - \\
\hline 5 & 4 & - & - & - & - & - & - & - & - & - & - & - & - \\
\hline
\end{tabular}


Table B.2.19 the results of calibration and validations based on the peaks in speed

\begin{tabular}{|c|c|c|c|c|c|c|c|c|c|c|c|c|c|}
\hline \multicolumn{2}{|c|}{ Configurations } & \multicolumn{3}{|c|}{ Calibration } & \multicolumn{3}{|c|}{$1^{\text {st }}$ Validation } & \multicolumn{3}{|c|}{$2^{\text {nd }}$ Validation } & \multicolumn{3}{|c|}{$3^{\text {rd }}$ Validation } \\
\hline$\varphi_{g}$ & $\Lambda_{l}$ & Vehicles & Cyclists & Pedestrians & Vehicles & Cyclists & Pedestrians & Vehicles & Cyclists & Pedestrians & Vehicles & Cyclists & Pedestrians \\
\hline 1 & 1 & 90 & 24 & 32 & 93 & 25 & 17 & 96 & 23 & 14 & 94 & 24 & 15 \\
\hline 1 & 2 & 91 & 19 & 29 & 94 & 24 & 17 & 96 & 21 & 13 & 95 & 22 & 14 \\
\hline 1 & 3 & 91 & 12 & 26 & 94 & 19 & 14 & 96 & 13 & 9 & 95 & 15 & 11 \\
\hline 1 & 4 & 86 & 13 & 0 & 90 & 14 & 0 & 91 & 16 & 0 & 91 & 15 & 0 \\
\hline 2 & 1 & 96 & 20 & 33 & 95 & 22 & 20 & 96 & 20 & 14 & 95 & 21 & 16 \\
\hline 2 & 2 & 96 & 19 & 33 & 95 & 21 & 20 & 96 & 18 & 13 & 96 & 19 & 15 \\
\hline 2 & 3 & 96 & 13 & 31 & 95 & 16 & 20 & 96 & 12 & 9 & 96 & 13 & 11 \\
\hline 2 & 4 & 90 & 13 & 0 & 91 & 17 & 0 & 92 & 15 & 0 & 91 & 16 & 0 \\
\hline 3 & 1 & 99 & 12 & 29 & 97 & 13 & 21 & 99 & 14 & 14 & 98 & 14 & 15 \\
\hline 3 & 2 & 99 & 12 & 29 & 97 & 13 & 21 & 99 & 13 & 13 & 98 & 13 & 15 \\
\hline 3 & 3 & 99 & 9 & 27 & 97 & 10 & 21 & 99 & 9 & 9 & 98 & 9 & 11 \\
\hline 3 & 4 & 91 & 9 & 0 & 92 & 10 & 0 & 93 & 11 & 0 & 93 & 10 & 0 \\
\hline 4 & 1 & 82 & 39 & 11 & 85 & 33 & 8 & 92 & 35 & 4 & 88 & 34 & 5 \\
\hline 4 & 2 & 82 & 27 & 11 & 86 & 30 & 8 & 92 & 15 & 4 & 89 & 21 & 4 \\
\hline 4 & 3 & 83 & 22 & 11 & 87 & 20 & 5 & 92 & 9 & 3 & 89 & 14 & 3 \\
\hline 4 & 4 & 95 & 9 & 0 & 94 & 8 & 0 & 95 & 8 & 0 & 95 & 8 & 0 \\
\hline 5 & 1 & 82 & 31 & 13 & 81 & 39 & 9 & 86 & 31 & 2 & 83 & 35 & 4 \\
\hline 5 & 2 & 83 & 23 & 13 & 84 & 28 & 9 & 89 & 9 & 2 & 86 & 19 & 4 \\
\hline 5 & 3 & 82 & 17 & 9 & 81 & 24 & 3 & 86 & 13 & 1 & 83 & 19 & 2 \\
\hline 5 & 4 & 83 & 12 & 0 & 82 & 15 & 0 & 86 & 20 & 0 & 84 & 18 & 0 \\
\hline
\end{tabular}


Table B.2.20 the results of calibration and validations based on the effective weighted average frequency

\begin{tabular}{|c|c|c|c|c|c|c|c|c|c|c|c|c|c|c|}
\hline \multicolumn{3}{|c|}{ Configurations } & \multicolumn{3}{|c|}{ Calibration } & \multicolumn{3}{|c|}{$1^{\text {st }}$ Validation } & \multicolumn{3}{|c|}{$2^{\text {nd }}$ Validation } & \multicolumn{3}{|c|}{$3^{\text {rd }}$ Validation } \\
\hline$\overline{\varphi_{g}}$ & $\overline{\Lambda_{l}}$ & $\Gamma_{n}$ & Vehicles & Cyclists & Pedestrians & Vehicles & Cyclists & Pedestrians & Vehicles & Cyclists & Pedestrians & Vehicles & Cyclists & Pedestrians \\
\hline 1 & 1 & 1 & 46 & 14 & 87 & 53 & 13 & 94 & 36 & 12 & 100 & 45 & 12 & 98 \\
\hline 1 & 1 & 2 & 46 & 13 & 85 & 54 & 12 & 92 & 38 & 12 & 98 & 46 & 12 & 97 \\
\hline 1 & 1 & 3 & 46 & 13 & 85 & 53 & 12 & 92 & 39 & 11 & 97 & 46 & 11 & 96 \\
\hline 1 & 1 & 4 & 43 & 15 & 84 & 51 & 15 & 94 & 34 & 9 & 98 & 43 & 12 & 97 \\
\hline 1 & 1 & 1 & 46 & 13 & 84 & 54 & 13 & 89 & 41 & 15 & 95 & 48 & 14 & 93 \\
\hline 1 & 1 & 6 & 47 & 13 & 84 & 54 & 13 & 89 & 41 & 15 & 95 & 48 & 14 & 93 \\
\hline 1 & 2 & 1 & 37 & 14 & 87 & 42 & 13 & 94 & - & - & - & - & - & - \\
\hline 1 & 2 & 2 & 37 & 14 & 85 & 42 & 13 & 94 & - & - & - & - & - & - \\
\hline 1 & 2 & 3 & 37 & 14 & 85 & 42 & 12 & 94 & - & - & - & - & - & - \\
\hline 1 & 2 & 4 & 36 & 17 & 85 & 43 & 11 & 94 & - & - & - & - & - & - \\
\hline 1 & 2 & 1 & 36 & 15 & 85 & 43 & 14 & 94 & - & - & - & - & - & - \\
\hline 1 & 2 & 6 & 36 & 17 & 84 & 43 & 13 & 92 & - & - & - & - & - & - \\
\hline 1 & 3 & 1 & 31 & 14 & 87 & - & - & - & - & - & - & - & - & - \\
\hline 1 & 3 & 2 & 30 & 17 & 87 & - & - & - & - & - & - & - & - & - \\
\hline 1 & 3 & 3 & 29 & 17 & 85 & - & - & - & - & - & - & - & - & - \\
\hline 1 & 3 & 4 & 30 & 16 & 85 & - & - & - & - & - & - & - & - & - \\
\hline 1 & 3 & 1 & 33 & 13 & 85 & - & - & - & - & - & - & - & - & - \\
\hline 1 & 3 & 6 & 31 & 17 & 85 & - & - & - & - & - & - & - & - & - \\
\hline 1 & 4 & 1 & 37 & 12 & 87 & 41 & 10 & 94 & 29 & 5 & 100 & - & - & - \\
\hline 1 & 4 & 2 & 37 & 13 & 85 & 41 & 12 & 92 & - & - & - & - & - & - \\
\hline 1 & 4 & 3 & 36 & 13 & 85 & 41 & 11 & 92 & - & - & - & - & - & - \\
\hline 1 & 4 & 4 & 34 & 16 & 85 & 42 & 10 & 94 & - & - & - & - & - & - \\
\hline 1 & 4 & 1 & 36 & 15 & 84 & 43 & 11 & 92 & - & - & - & - & - & - \\
\hline 1 & 4 & 6 & 36 & 16 & 84 & 43 & 11 & 92 & - & - & - & - & - & - \\
\hline 2 & 1 & 1 & 51 & 17 & 100 & 55 & 15 & 100 & 37 & 13 & 100 & 46 & 13 & 100 \\
\hline 2 & 1 & 2 & 51 & 15 & 100 & 55 & 13 & 100 & 40 & 13 & 98 & 48 & 13 & 99 \\
\hline 2 & 1 & 3 & 51 & 15 & 100 & 55 & 13 & 98 & 40 & 11 & 97 & 48 & 12 & 97 \\
\hline 2 & 1 & 4 & 48 & 18 & 100 & 52 & 17 & 100 & 35 & 10 & 98 & 44 & 13 & 99 \\
\hline 2 & 1 & 1 & 49 & 18 & 100 & 54 & 16 & 98 & 37 & 12 & 98 & 46 & 13 & 98 \\
\hline 2 & 1 & 6 & 52 & 15 & 100 & 56 & 15 & 95 & 42 & 15 & 95 & 49 & 15 & 95 \\
\hline 2 & 2 & 1 & 42 & 17 & 100 & 43 & 15 & 100 & - & - & - & - & - & - \\
\hline 2 & 2 & 2 & 41 & 17 & 100 & 43 & 15 & 100 & - & - & - & - & - & - \\
\hline 2 & 2 & 3 & 41 & 17 & 100 & 43 & 13 & 100 & - & - & - & - & - & - \\
\hline 2 & 2 & 4 & 40 & 20 & 100 & 44 & 12 & 100 & - & - & - & - & - & - \\
\hline 2 & 2 & 1 & 40 & 20 & 100 & 44 & 13 & 100 & - & - & - & - & - & - \\
\hline 2 & 2 & 6 & 40 & 20 & 100 & 45 & 15 & 98 & - & - & - & - & - & - \\
\hline
\end{tabular}


Table B.2.20 the results of calibration and validations based on the effective weighted average frequency

\begin{tabular}{|c|c|c|c|c|c|c|c|c|c|c|c|c|c|c|}
\hline \multicolumn{3}{|c|}{ Configurations } & \multicolumn{3}{|c|}{ Calibration } & \multicolumn{3}{|c|}{$1^{\text {st }}$ Validation } & \multicolumn{3}{|c|}{$2^{\text {nd }}$ Validation } & \multicolumn{3}{|c|}{$3^{\text {rd }}$ Validation } \\
\hline$\varphi_{g}$ & $\Lambda_{l}$ & $\Gamma_{n}$, & Vehicles & Cyclists & Pedestrians & Vehicles & Cyclists & Pedestrians & Vehicles & Cyclists & Pedestrians & Vehicles & Cyclists & Pedestrians \\
\hline 2 & 3 & 1 & 35 & 17 & 100 & - & - & - & - & - & - & - & - & - \\
\hline 2 & 3 & 2 & 33 & 20 & 100 & - & - & - & - & - & - & - & - & - \\
\hline 2 & 3 & 3 & 33 & 20 & 100 & - & - & - & - & - & - & - & - & - \\
\hline 2 & 3 & 4 & 34 & 19 & 100 & - & - & - & - & - & - & - & - & - \\
\hline 2 & 3 & 1 & 34 & 19 & 100 & - & - & - & - & - & - & - & - & - \\
\hline 2 & 3 & 6 & 35 & 20 & 100 & - & - & - & - & - & - & - & - & - \\
\hline 2 & 4 & 1 & 41 & 13 & 100 & 42 & 11 & 100 & 30 & 6 & 100 & - & - & - \\
\hline 2 & 4 & 2 & 41 & 15 & 100 & 43 & 13 & 98 & - & - & - & - & - & - \\
\hline 2 & 4 & 3 & 40 & 15 & 100 & 43 & 12 & 98 & - & - & - & - & - & - \\
\hline 2 & 4 & 4 & 39 & 18 & 100 & 44 & 11 & 100 & - & - & - & - & - & - \\
\hline 2 & 4 & 1 & 40 & 18 & 100 & 44 & 12 & 98 & - & - & - & - & - & - \\
\hline 2 & 4 & 6 & 40 & 19 & 100 & 44 & 12 & 98 & - & - & - & - & - & - \\
\hline 3 & 1 & 1 & 61 & 20 & 100 & 63 & 18 & 100 & 42 & 13 & 100 & 53 & 15 & 100 \\
\hline 3 & 1 & 2 & 61 & 17 & 100 & 63 & 17 & 100 & 46 & 13 & 98 & 55 & 15 & 99 \\
\hline 3 & 1 & 3 & 61 & 17 & 100 & 63 & 17 & 98 & 46 & 13 & 97 & 55 & 14 & 97 \\
\hline 3 & 1 & 4 & 63 & 13 & 100 & 63 & 17 & 94 & 46 & 13 & 97 & 55 & 15 & 96 \\
\hline 3 & 1 & 1 & 58 & 21 & 100 & 63 & 19 & 98 & 42 & 13 & 98 & 53 & 15 & 98 \\
\hline 3 & 1 & 6 & 61 & 17 & 100 & 64 & 18 & 94 & 47 & 17 & 95 & 56 & 17 & 95 \\
\hline 3 & 2 & 1 & 49 & 20 & 100 & 50 & 18 & 100 & - & - & - & - & - & - \\
\hline 3 & 2 & 2 & 49 & 20 & 100 & 50 & 18 & 100 & - & - & - & - & - & - \\
\hline 3 & 2 & 3 & 49 & 20 & 100 & 49 & 17 & 100 & - & - & - & - & - & - \\
\hline 3 & 2 & 4 & 47 & 24 & 100 & 51 & 15 & 100 & - & - & - & - & - & - \\
\hline 3 & 2 & 1 & 47 & 24 & 100 & 51 & 17 & 100 & - & - & - & - & - & - \\
\hline 3 & 2 & 6 & 48 & 24 & 100 & 51 & 18 & 98 & - & - & - & - & - & - \\
\hline 3 & 3 & 1 & - & - & - & - & - & - & - & - & - & - & - & - \\
\hline 3 & 3 & 2 & - & - & - & - & - & - & - & - & - & - & - & - \\
\hline 3 & 3 & 3 & - & - & - & - & - & - & - & - & - & - & - & - \\
\hline 3 & 3 & 4 & - & - & - & - & - & - & - & - & - & - & - & - \\
\hline 3 & 3 & 1 & - & - & - & - & - & - & - & - & - & - & - & - \\
\hline 3 & 3 & 6 & 41 & 24 & 100 & - & - & - & - & - & - & - & - & - \\
\hline 3 & 4 & 1 & 49 & 16 & 100 & 49 & 14 & 100 & 34 & 5 & 100 & - & - & - \\
\hline 3 & 4 & 2 & 49 & 17 & 100 & 49 & 17 & 98 & - & - & - & - & - & - \\
\hline 3 & 4 & 3 & 45 & 21 & 100 & 49 & 13 & 100 & - & - & - & - & - & - \\
\hline 3 & 4 & 4 & 46 & 21 & 100 & 51 & 14 & 100 & - & - & - & - & - & - \\
\hline 3 & 4 & 1 & 47 & 21 & 100 & 51 & 15 & 98 & - & - & - & - & - & - \\
\hline 3 & 4 & 6 & 47 & 23 & 100 & 51 & 15 & 98 & - & - & - & - & - & - \\
\hline
\end{tabular}


Table B.2.20 the results of calibration and validations based on the effective weighted average frequency

\begin{tabular}{|c|c|c|c|c|c|c|c|c|c|c|c|c|c|c|}
\hline \multicolumn{3}{|c|}{ Configurations } & \multicolumn{3}{|c|}{ Calibration } & \multicolumn{3}{|c|}{$1^{\text {st }}$ Validation } & \multicolumn{3}{|c|}{$2^{\text {nd }}$ Validation } & \multicolumn{3}{|c|}{$3^{\text {rd }}$ Validation } \\
\hline$\varphi_{g}$ & $\Lambda_{l}$ & $\Gamma_{n}$, & Vehicles & Cyclists & Pedestrians & Vehicles & Cyclists & Pedestrians & Vehicles & Cyclists & Pedestrians & Vehicles & Cyclists & Pedestrians \\
\hline 4 & 1 & 1 & 72 & 19 & 100 & 69 & 18 & 100 & 56 & 15 & 100 & 64 & 16 & 100 \\
\hline 4 & 1 & 2 & 71 & 19 & 100 & 70 & 18 & 100 & 56 & 15 & 98 & 64 & 16 & 99 \\
\hline 4 & 1 & 3 & 70 & 19 & 100 & 69 & 18 & 97 & 56 & 15 & 97 & 64 & 16 & 97 \\
\hline 4 & 1 & 4 & 67 & 22 & 100 & 69 & 21 & 100 & 51 & 13 & 98 & 61 & 17 & 98 \\
\hline 4 & 1 & 1 & 66 & 24 & 100 & 69 & 21 & 97 & 52 & 15 & 98 & 62 & 18 & 98 \\
\hline 4 & 1 & 6 & 70 & 18 & 100 & 70 & 20 & 92 & 54 & 17 & 96 & 63 & 18 & 95 \\
\hline 4 & 2 & 1 & 54 & 27 & 100 & 56 & 18 & 100 & - & - & - & - & - & - \\
\hline 4 & 2 & 2 & 55 & 25 & 100 & 56 & 18 & 100 & - & - & - & - & - & - \\
\hline 4 & 2 & 3 & 55 & 27 & 100 & 56 & 18 & 100 & - & - & - & - & - & - \\
\hline 4 & 2 & 4 & 55 & 27 & 100 & 56 & 18 & 100 & - & - & - & - & - & - \\
\hline 4 & 2 & 1 & 55 & 27 & 100 & 56 & 20 & 100 & - & - & - & - & - & - \\
\hline 4 & 2 & 6 & 56 & 27 & 100 & 57 & 21 & 97 & - & - & - & - & - & - \\
\hline 4 & 3 & 1 & - & - & - & - & - & - & - & - & - & - & - & - \\
\hline 4 & 3 & 2 & - & - & - & - & - & - & - & - & - & - & - & - \\
\hline 4 & 3 & 3 & - & - & - & - & - & - & - & - & - & - & - & - \\
\hline 4 & 3 & 4 & - & - & - & - & - & - & - & - & - & - & - & - \\
\hline 4 & 3 & 1 & - & - & - & - & - & - & - & - & - & - & - & - \\
\hline 4 & 3 & 6 & - & - & - & - & - & - & - & - & - & - & - & - \\
\hline 4 & 4 & 1 & 55 & 22 & 100 & 55 & 15 & 100 & - & - & - & - & - & - \\
\hline 4 & 4 & 2 & 54 & 22 & 100 & 56 & 15 & 100 & - & - & - & - & - & - \\
\hline 4 & 4 & 3 & 54 & 24 & 100 & 56 & 16 & 100 & - & - & - & - & - & - \\
\hline 4 & 4 & 4 & 53 & 25 & 100 & 56 & 16 & 100 & - & - & - & - & - & - \\
\hline 4 & 4 & 1 & 55 & 24 & 100 & 56 & 18 & 97 & - & - & - & - & - & - \\
\hline 4 & 4 & 6 & 55 & 25 & 100 & 56 & 18 & 97 & - & - & - & - & - & - \\
\hline 5 & 1 & 1 & 76 & 25 & 100 & 75 & 22 & 100 & 63 & 20 & 100 & 70 & 21 & 100 \\
\hline 5 & 1 & 2 & 74 & 25 & 100 & 74 & 22 & 100 & 64 & 20 & 99 & 70 & 21 & 99 \\
\hline 5 & 1 & 3 & 73 & 25 & 100 & 73 & 22 & 97 & 63 & 20 & 97 & 69 & 21 & 97 \\
\hline 5 & 1 & 4 & 68 & 31 & 100 & 70 & 28 & 100 & 56 & 18 & 98 & 65 & 23 & 98 \\
\hline 5 & 1 & 1 & 68 & 31 & 100 & 73 & 24 & 97 & 59 & 20 & 98 & 68 & 22 & 98 \\
\hline 5 & 1 & 6 & 65 & 35 & 100 & 69 & 30 & 97 & 57 & 22 & 98 & 65 & 26 & 98 \\
\hline 5 & 2 & 1 & 57 & 35 & 100 & 62 & 24 & 100 & - & - & - & - & - & - \\
\hline 5 & 2 & 2 & 56 & 33 & 100 & 61 & 24 & 100 & - & - & - & - & - & - \\
\hline 5 & 2 & 3 & 58 & 35 & 100 & 62 & 24 & 100 & - & - & - & - & - & - \\
\hline 5 & 2 & 4 & 57 & 35 & 100 & 61 & 24 & 100 & - & - & - & - & - & - \\
\hline 5 & 2 & 1 & 56 & 35 & 100 & 62 & 26 & 100 & - & - & - & - & - & - \\
\hline 5 & 2 & 6 & 56 & 35 & 100 & 62 & 26 & 97 & - & - & - & - & - & - \\
\hline
\end{tabular}


Table B.2.20 the results of calibration and validations based on the effective weighted average frequency

\begin{tabular}{|c|c|c|c|c|c|c|c|c|c|c|c|c|c|c|}
\hline \multicolumn{3}{|c|}{ Configurations } & \multicolumn{3}{|c|}{ Calibration } & \multicolumn{3}{|c|}{$1^{\text {st }}$ Validation } & \multicolumn{3}{|c|}{$2^{\text {nd }}$ Validation } & \multicolumn{3}{|c|}{$3^{\text {rd }}$ Validation } \\
\hline$\varphi_{g}$ & $\Lambda_{l}$ & $\Gamma_{n}$, & Vehicles & Cyclists & Pedestrians & Vehicles & Cyclists & Pedestrians & Vehicles & Cyclists & Pedestrians & Vehicles & Cyclists & Pedestrians \\
\hline 5 & 3 & 1 & - & - & - & - & - & - & - & - & - & - & - & - \\
\hline 5 & 3 & 2 & - & - & - & - & - & - & - & - & - & - & - & - \\
\hline 5 & 3 & 3 & - & - & - & - & - & - & - & - & - & - & - & - \\
\hline 5 & 3 & 4 & - & - & - & - & - & - & - & - & - & - & - & - \\
\hline 5 & 3 & 1 & - & - & - & - & - & - & - & - & - & - & - & - \\
\hline 5 & 3 & 6 & - & - & - & - & - & - & - & - & - & - & - & - \\
\hline 5 & 4 & 1 & 57 & 27 & 100 & 61 & 17 & 100 & - & - & - & - & - & - \\
\hline 5 & 4 & 2 & 56 & 29 & 100 & 61 & 20 & 100 & - & - & - & - & - & - \\
\hline 5 & 4 & 3 & 57 & 31 & 100 & 62 & 20 & 100 & - & - & - & - & - & - \\
\hline 5 & 4 & 4 & 55 & 33 & 100 & 61 & 22 & 100 & - & - & - & - & - & - \\
\hline 5 & 4 & 1 & 56 & 31 & 100 & 62 & 22 & 97 & - & - & - & - & - & - \\
\hline 5 & 4 & 6 & 56 & 33 & 100 & 62 & 24 & 97 & - & - & - & - & - & - \\
\hline
\end{tabular}

Table B.2.21 the results of calibration and validations based on the effective frequency

\begin{tabular}{|c|c|c|c|c|c|c|c|c|c|c|c|c|c|c|}
\hline \multicolumn{3}{|c|}{ Configurations } & \multicolumn{3}{|c|}{ Calibration } & \multicolumn{3}{|c|}{$1^{\text {st }}$ Validation } & \multicolumn{3}{|c|}{$2^{\text {nd }}$ Validation } & \multicolumn{3}{|c|}{$3^{\text {rd }}$ Validation } \\
\hline$\varphi_{g}$ & $\Lambda_{l}$ & $\eta_{m}$ & Vehicles & Cyclists & Pedestrians & Vehicles & Cyclists & Pedestrians & Vehicles & Cyclists & Pedestrians & Vehicles & Cyclists & Pedestrians \\
\hline 1 & 1 & 1 & 0 & 7 & 41 & 0 & 2 & 38 & 0 & 6 & 25 & 0 & 4 & 28 \\
\hline 1 & 1 & 2 & 1 & 8 & 29 & 1 & 6 & 44 & - & - & - & - & - & - \\
\hline 1 & 1 & 3 & 0 & 59 & 15 & - & - & - & - & - & - & - & - & - \\
\hline 1 & 2 & 1 & 0 & 6 & 53 & 0 & 1 & 48 & 0 & 5 & 31 & 0 & 4 & 35 \\
\hline 1 & 2 & 2 & 0 & 5 & 43 & 0 & 7 & 52 & - & - & - & - & - & - \\
\hline 1 & 2 & 3 & 0 & 23 & 13 & - & - & - & - & - & - & - & - & - \\
\hline 1 & 3 & 1 & 0 & 3 & 46 & 0 & 0 & 38 & 0 & 1 & 25 & 0 & 0 & 28 \\
\hline 1 & 3 & 2 & 0 & 3 & 47 & 0 & 3 & 52 & 1 & 4 & 28 & 0 & 4 & 35 \\
\hline 1 & 3 & 3 & 1 & 19 & 21 & - & - & - & - & - & - & - & - & - \\
\hline 1 & 4 & 1 & 0 & 3 & 40 & 0 & 0 & 38 & 0 & 1 & 25 & 0 & 0 & 28 \\
\hline 1 & 4 & 2 & 0 & 3 & 38 & 0 & 2 & 52 & - & - & - & - & - & - \\
\hline 1 & 4 & 3 & - & - & - & - & - & - & - & - & - & - & - & - \\
\hline 2 & 1 & 1 & 0 & 2 & 49 & 0 & 1 & 47 & 0 & 4 & 31 & 0 & 3 & 34 \\
\hline 2 & 1 & 2 & 0 & 2 & 43 & 0 & 3 & 53 & 0 & 4 & 23 & 0 & 4 & 30 \\
\hline 2 & 1 & 3 & 0 & 69 & 20 & - & - & - & - & - & - & - & - & - \\
\hline 2 & 2 & 1 & 0 & 2 & 51 & 0 & 1 & 47 & 0 & 4 & 31 & 0 & 3 & 34 \\
\hline 2 & 2 & 2 & 0 & 31 & 12 & - & - & - & - & - & - & - & - & - \\
\hline 2 & 2 & 3 & 0 & 38 & 24 & - & - & - & - & - & - & - & - & - \\
\hline
\end{tabular}


Table B.2.21 the results of calibration and validations based on the effective frequency

\begin{tabular}{|c|c|c|c|c|c|c|c|c|c|c|c|c|c|c|}
\hline \multicolumn{3}{|c|}{ Configurations } & \multicolumn{3}{|c|}{ Calibration } & \multicolumn{3}{|c|}{$1^{\text {st }}$ Validation } & \multicolumn{3}{|c|}{$2^{\text {nd }}$ Validation } & \multicolumn{3}{|c|}{$3^{\text {rd }}$ Validation } \\
\hline$\varphi_{g}$ & $\Lambda_{l}$ & $\eta_{m}$ & Vehicles & Cyclists & Pedestrians & Vehicles & Cyclists & Pedestrians & Vehicles & Cyclists & Pedestrians & Vehicles & Cyclists & Pedestrians \\
\hline 2 & 3 & 1 & 0 & 1 & 51 & 0 & 0 & 47 & 0 & 2 & 31 & 0 & 1 & 34 \\
\hline 2 & 3 & 2 & 1 & 2 & 43 & 0 & 1 & 53 & 1 & 3 & 26 & 0 & 3 & 32 \\
\hline 2 & 3 & 3 & 2 & 22 & 29 & - & - & - & - & - & - & - & - & - \\
\hline 2 & 4 & 1 & 0 & 1 & 49 & 0 & 0 & 47 & 0 & 2 & 31 & 0 & 1 & 34 \\
\hline 2 & 4 & 2 & 0 & 2 & 43 & 0 & 1 & 53 & 0 & 3 & 23 & 0 & 3 & 30 \\
\hline 2 & 4 & 3 & 0 & 19 & 22 & - & - & - & - & - & - & - & - & - \\
\hline 3 & 1 & 1 & 0 & 0 & 44 & 0 & 0 & 47 & 0 & 0 & 31 & 0 & 0 & 34 \\
\hline 3 & 1 & 2 & 0 & 40 & 2 & - & - & - & - & - & - & - & - & - \\
\hline 3 & 1 & 3 & 0 & 81 & 24 & - & - & - & - & - & - & - & - & - \\
\hline 3 & 2 & 1 & 0 & 0 & 44 & 0 & 0 & 47 & 0 & 0 & 31 & 0 & 0 & 34 \\
\hline 3 & 2 & 2 & 0 & 37 & 15 & - & - & - & - & - & - & - & - & - \\
\hline 3 & 2 & 3 & 0 & 45 & 29 & - & - & - & - & - & - & - & - & - \\
\hline 3 & 3 & 1 & 0 & 0 & 44 & 0 & 0 & 47 & 0 & 0 & 31 & 0 & 0 & 34 \\
\hline 3 & 3 & 2 & 2 & 27 & 17 & 1 & 25 & 11 & 0 & 0 & 32 & - & - & - \\
\hline 3 & 3 & 3 & 2 & 27 & 34 & - & - & - & - & - & - & - & - & - \\
\hline 3 & 4 & 1 & 0 & 0 & 44 & 0 & 0 & 47 & 0 & 0 & 31 & 0 & 0 & 34 \\
\hline 3 & 4 & 2 & 0 & 0 & 39 & 0 & 0 & 55 & 0 & 0 & 26 & 0 & 0 & 32 \\
\hline 3 & 4 & 3 & 0 & 23 & 27 & - & - & - & - & - & - & - & - & - \\
\hline 4 & 1 & 1 & 0 & 0 & 46 & 0 & 0 & 38 & - & - & - & - & - & - \\
\hline 4 & 1 & 2 & 0 & 0 & 43 & 0 & 0 & 67 & 0 & 1 & 33 & 0 & 1 & 39 \\
\hline 4 & 1 & 3 & 1 & 55 & 19 & - & - & - & - & - & - & - & - & - \\
\hline 4 & 2 & 1 & 0 & 0 & 46 & 0 & 0 & 38 & 0 & 0 & 32 & 0 & 0 & 33 \\
\hline 4 & 2 & 2 & 0 & 42 & 16 & - & - & - & - & - & - & - & - & - \\
\hline 4 & 2 & 3 & 0 & 36 & 24 & - & - & - & - & - & - & - & - & - \\
\hline 4 & 3 & 1 & 0 & 0 & 46 & 0 & 0 & 38 & 0 & 0 & 32 & 0 & 0 & 33 \\
\hline 4 & 3 & 2 & 0 & 0 & 43 & 0 & 0 & 67 & 0 & 1 & 33 & 0 & 1 & 39 \\
\hline 4 & 3 & 3 & 3 & 30 & 38 & - & - & - & - & - & - & - & - & - \\
\hline 4 & 4 & 1 & 0 & 0 & 46 & 0 & 0 & 38 & 0 & 0 & 33 & 0 & 0 & 33 \\
\hline 4 & 4 & 2 & 0 & 0 & 43 & 0 & 0 & 67 & 0 & 0 & 32 & 0 & 0 & 39 \\
\hline 4 & 4 & 3 & 0 & 24 & 30 & - & - & - & - & - & - & - & - & - \\
\hline 5 & 1 & 1 & 0 & 0 & 47 & 0 & 0 & 52 & 0 & 0 & 41 & 0 & 0 & 43 \\
\hline 5 & 1 & 2 & 0 & 58 & 3 & - & - & - & - & - & - & - & - & - \\
\hline 5 & 1 & 3 & 1 & 92 & 31 & - & - & - & - & - & - & - & - & - \\
\hline 5 & 2 & 1 & 0 & 0 & 47 & 0 & 0 & 52 & 0 & 0 & 41 & 0 & 0 & 43 \\
\hline 5 & 2 & 2 & 0 & 54 & 19 & - & - & - & - & - & - & - & - & - \\
\hline 5 & 2 & 3 & 0 & 54 & 16 & 0 & 43 & 18 & - & - & - & - & - & - \\
\hline
\end{tabular}


Table B.2.21 the results of calibration and validations based on the effective frequency

\begin{tabular}{|c|c|c|c|c|c|c|c|c|c|c|c|c|c|c|}
\hline \multicolumn{3}{|c|}{ Configurations } & \multicolumn{3}{|c|}{ Calibration } & \multicolumn{3}{|c|}{$1^{\text {st }}$ Validation } & \multicolumn{3}{|c|}{$2^{\text {nd }}$ Validation } & \multicolumn{3}{|c|}{$3^{\text {rd }}$ Validation } \\
\hline$\varphi_{q}$ & $\Lambda_{l}$ & $\eta_{m}$ & Vehicles & Cyclists & Pedestrians & Vehicles & Cyclists & Pedestrians & Vehicles & Cyclists & Pedestrians & Vehicles & Cyclists & Pedestrians \\
\hline 5 & 3 & 1 & 0 & 0 & 47 & 0 & 0 & 48 & 0 & 0 & 40 & 0 & 0 & 42 \\
\hline 5 & 3 & 2 & 4 & 38 & 22 & 1 & 39 & 15 & 0 & 16 & 23 & - & - & - \\
\hline 5 & 3 & 3 & 4 & 38 & 38 & 1 & 33 & 27 & 0 & 16 & 22 & - & - & - \\
\hline 5 & 4 & 1 & 0 & 0 & 47 & 0 & 0 & 52 & 0 & 0 & 41 & 0 & 0 & 43 \\
\hline 5 & 4 & 2 & 0 & 0 & 44 & 0 & 0 & 70 & 0 & 0 & 31 & 0 & 0 & 39 \\
\hline
\end{tabular}




\section{Appendix $\mathrm{C}$}

\section{The Results of the Calibrations and Validations for the second model "Vehicles and Cyclists"}




\section{C.1 The results of calibrations and validations using Kappa statistics}

Table C.1.1 the results of calibration procedure 1 and its validations.

\begin{tabular}{|c|c|c|c|c|c|c|c|}
\hline \multicolumn{4}{|c|}{ Configurations } & \multirow{2}{*}{$\begin{array}{c}\text { Calibration } \\
\text { Kappa }\end{array}$} & \multirow{2}{*}{$\frac{\mathbf{1}^{\text {st }} \text { Validation }}{\text { Kappa }}$} & \multirow{2}{*}{$\begin{array}{c}2^{\text {nd }} \text { Validation } \\
\text { Kappa }\end{array}$} & \multirow{2}{*}{$\begin{array}{c}3^{\text {rd }} \text { Validation } \\
\text { Kappa }\end{array}$} \\
\hline$\varphi_{g}$ & $\Lambda_{l}$ & $\Gamma_{n}$ & $\eta_{m}$ & & & & \\
\hline 1 & 1 & 1 & 1 & 0.91 & 0.90 & 0.86 & 0.88 \\
\hline 1 & 1 & 1 & 2 & 0.92 & 0.90 & 0.83 & 0.86 \\
\hline 1 & 1 & 1 & 3 & 0.92 & 0.90 & 0.83 & 0.86 \\
\hline 1 & 1 & 2 & 1 & 0.91 & 0.90 & 0.85 & 0.87 \\
\hline 1 & 1 & 2 & 2 & 0.92 & 0.90 & - & - \\
\hline 1 & 1 & 2 & 3 & 0.92 & 0.90 & - & - \\
\hline 1 & 1 & 3 & 1 & 0.91 & 0.90 & 0.86 & 0.88 \\
\hline 1 & 1 & 3 & 2 & 0.92 & 0.90 & - & - \\
\hline 1 & 1 & 3 & 3 & 0.92 & 0.90 & - & - \\
\hline 1 & 1 & 4 & 1 & 0.91 & 0.90 & 0.85 & 0.87 \\
\hline 1 & 1 & 4 & 2 & 0.92 & 0.90 & 0.83 & 0.86 \\
\hline 1 & 1 & 4 & 3 & 0.92 & 0.90 & 0.83 & 0.86 \\
\hline 1 & 1 & 1 & 1 & 0.91 & 0.90 & 0.87 & 0.88 \\
\hline 1 & 1 & 1 & 2 & 0.92 & 0.90 & 0.85 & 0.87 \\
\hline 1 & 1 & 1 & 3 & 0.92 & 0.90 & 0.85 & 0.87 \\
\hline 1 & 1 & 6 & 1 & 0.92 & 0.90 & 0.86 & 0.88 \\
\hline 1 & 1 & 6 & 2 & 0.92 & 0.90 & 0.83 & 0.86 \\
\hline 1 & 1 & 6 & 3 & 0.92 & 0.90 & 0.83 & 0.86 \\
\hline 1 & 2 & 1 & 1 & 0.94 & 0.90 & 0.87 & 0.89 \\
\hline 1 & 2 & 1 & 2 & 0.94 & 0.90 & 0.87 & 0.89 \\
\hline 1 & 2 & 1 & 3 & 0.94 & 0.90 & 0.87 & 0.89 \\
\hline 1 & 2 & 2 & 1 & 0.94 & 0.88 & 0.87 & 0.88 \\
\hline 1 & 2 & 2 & 2 & 0.94 & 0.88 & 0.87 & 0.88 \\
\hline 1 & 2 & 2 & 3 & 0.94 & 0.88 & 0.87 & 0.88 \\
\hline 1 & 2 & 3 & 1 & 0.95 & 0.88 & 0.88 & 0.88 \\
\hline 1 & 2 & 3 & 2 & 0.95 & 0.88 & 0.88 & 0.88 \\
\hline 1 & 2 & 3 & 3 & 0.95 & 0.88 & 0.88 & 0.88 \\
\hline 1 & 2 & 4 & 1 & 0.94 & 0.90 & 0.88 & 0.89 \\
\hline 1 & 2 & 4 & 2 & 0.94 & 0.90 & 0.88 & 0.89 \\
\hline 1 & 2 & 4 & 3 & 0.94 & 0.90 & 0.88 & 0.89 \\
\hline 1 & 2 & 1 & 1 & 0.94 & 0.90 & 0.88 & 0.89 \\
\hline 1 & 2 & 1 & 2 & 0.94 & 0.90 & 0.88 & 0.89 \\
\hline 1 & 2 & 1 & 3 & 0.94 & 0.90 & 0.88 & 0.89 \\
\hline 1 & 2 & 6 & 1 & 0.94 & 0.88 & 0.87 & 0.88 \\
\hline 1 & 2 & 6 & 2 & 0.94 & 0.88 & 0.87 & 0.88 \\
\hline 1 & 2 & 6 & 3 & 0.94 & 0.88 & 0.87 & 0.88 \\
\hline 1 & 3 & 1 & 1 & 0.94 & 0.85 & - & - \\
\hline 1 & 3 & 1 & 2 & 0.94 & 0.85 & - & - \\
\hline 1 & 3 & 1 & 3 & 0.94 & 0.85 & - & - \\
\hline 1 & 3 & 2 & 1 & 0.94 & 0.85 & - & - \\
\hline 1 & 3 & 2 & 2 & 0.94 & 0.85 & - & - \\
\hline 1 & 3 & 2 & 3 & 0.94 & 0.85 & - & - \\
\hline 1 & 3 & 3 & 1 & 0.93 & 0.84 & - & - \\
\hline 1 & 3 & 3 & 2 & 0.93 & 0.84 & - & - \\
\hline 1 & 3 & 3 & 3 & 0.93 & 0.84 & - & - \\
\hline 1 & 3 & 4 & 1 & 0.94 & 0.85 & - & - \\
\hline 1 & 3 & 4 & 2 & 0.94 & 0.85 & - & - \\
\hline 1 & 3 & 4 & 3 & 0.94 & 0.85 & - & - \\
\hline 1 & 3 & 1 & 1 & 0.94 & 0.85 & - & - \\
\hline
\end{tabular}


Table C.1.1 the results of calibration procedure 1 and its validations.

\begin{tabular}{|c|c|c|c|c|c|c|c|}
\hline \multicolumn{4}{|c|}{ Configurations } & \multirow{2}{*}{$\begin{array}{c}\text { Calibration } \\
\text { Kappa }\end{array}$} & \multirow{2}{*}{$\begin{array}{c}\mathbf{1}^{\text {st }} \text { Validation } \\
\text { Kappa }\end{array}$} & \multirow{2}{*}{$\begin{array}{c}2^{\text {nd }} \text { Validation } \\
\text { Kappa }\end{array}$} & \multirow{2}{*}{$\begin{array}{c}3^{\text {rd }} \text { Validation } \\
\text { Kappa }\end{array}$} \\
\hline$\varphi_{g}$ & $\Lambda_{l}$ & $\Gamma_{n}$ & $\eta_{m}$ & & & & \\
\hline 1 & 3 & 1 & 2 & 0.94 & 0.85 & - & - \\
\hline 1 & 3 & 1 & 3 & 0.94 & 0.85 & - & - \\
\hline 1 & 3 & 6 & 1 & 0.94 & 0.85 & - & - \\
\hline 1 & 3 & 6 & 2 & 0.94 & 0.85 & - & - \\
\hline 1 & 3 & 6 & 3 & 0.94 & 0.85 & - & - \\
\hline 1 & 4 & 1 & 1 & - & - & - & - \\
\hline 1 & 4 & 1 & 2 & - & - & - & - \\
\hline 1 & 4 & 1 & 3 & - & - & - & - \\
\hline 1 & 4 & 2 & 1 & - & - & - & - \\
\hline 1 & 4 & 2 & 2 & - & - & - & - \\
\hline 1 & 4 & 2 & 3 & - & - & - & - \\
\hline 1 & 4 & 3 & 1 & - & - & - & - \\
\hline 1 & 4 & 3 & 2 & - & - & - & - \\
\hline 1 & 4 & 3 & 3 & - & - & - & - \\
\hline 1 & 4 & 4 & 1 & - & - & - & - \\
\hline 1 & 4 & 4 & 2 & - & - & - & - \\
\hline 1 & 4 & 4 & 3 & - & - & - & - \\
\hline 1 & 4 & 1 & 1 & - & - & - & - \\
\hline 1 & 4 & 1 & 2 & - & - & - & - \\
\hline 1 & 4 & 1 & 3 & - & - & - & - \\
\hline 1 & 4 & 6 & 1 & - & - & - & - \\
\hline 1 & 4 & 6 & 2 & - & - & - & - \\
\hline 1 & 4 & 6 & 3 & - & - & - & - \\
\hline 2 & 1 & 1 & 1 & 0.96 & 0.84 & 0.83 & 0.84 \\
\hline 2 & 1 & 1 & 2 & 0.96 & 0.84 & 0.83 & 0.84 \\
\hline 2 & 1 & 1 & 3 & 0.96 & 0.84 & 0.83 & 0.84 \\
\hline 2 & 1 & 2 & 1 & 0.96 & 0.84 & 0.83 & 0.84 \\
\hline 2 & 1 & 2 & 2 & 0.96 & 0.84 & 0.83 & 0.84 \\
\hline 2 & 1 & 2 & 3 & 0.96 & 0.84 & 0.83 & 0.84 \\
\hline 2 & 1 & 3 & 1 & 0.96 & 0.84 & 0.84 & 0.84 \\
\hline 2 & 1 & 3 & 2 & 0.96 & 0.84 & 0.84 & 0.84 \\
\hline 2 & 1 & 3 & 3 & 0.96 & 0.84 & 0.84 & 0.84 \\
\hline 2 & 1 & 4 & 1 & 0.96 & 0.83 & 0.85 & 0.84 \\
\hline 2 & 1 & 4 & 2 & 0.96 & 0.83 & 0.85 & 0.84 \\
\hline 2 & 1 & 4 & 3 & 0.96 & 0.83 & 0.85 & 0.84 \\
\hline 2 & 1 & 1 & 1 & 0.96 & 0.84 & 0.83 & 0.84 \\
\hline 2 & 1 & 1 & 2 & 0.96 & 0.84 & 0.83 & 0.84 \\
\hline 2 & 1 & 1 & 3 & 0.96 & 0.84 & 0.83 & 0.84 \\
\hline 2 & 1 & 6 & 1 & 0.96 & 0.84 & 0.84 & 0.85 \\
\hline 2 & 1 & 6 & 2 & 0.96 & 0.84 & 0.84 & 0.85 \\
\hline 2 & 1 & 6 & 3 & 0.96 & 0.84 & 0.84 & 0.85 \\
\hline 2 & 2 & 1 & 1 & 0.96 & 0.87 & - & - \\
\hline 2 & 2 & 1 & 2 & 0.96 & 0.87 & - & - \\
\hline 2 & 2 & 1 & 3 & 0.96 & 0.87 & - & - \\
\hline 2 & 2 & 2 & 1 & 0.96 & 0.87 & - & - \\
\hline 2 & 2 & 2 & 2 & 0.96 & 0.87 & - & - \\
\hline 2 & 2 & 2 & 3 & 0.96 & 0.87 & - & - \\
\hline 2 & 2 & 3 & 1 & 0.96 & 0.88 & - & - \\
\hline 2 & 2 & 3 & 2 & 0.96 & 0.88 & - & - \\
\hline 2 & 2 & 3 & 3 & 0.96 & 0.88 & - & - \\
\hline 2 & 2 & 4 & 1 & 0.96 & 0.86 & - & - \\
\hline
\end{tabular}


Table C.1.1 the results of calibration procedure 1 and its validations.

\begin{tabular}{|c|c|c|c|c|c|c|c|}
\hline \multicolumn{4}{|c|}{ Configurations } & \multirow{2}{*}{$\begin{array}{c}\text { Calibration } \\
\text { Kappa }\end{array}$} & \multirow{2}{*}{$\begin{array}{c}\mathbf{1}^{\text {st }} \text { Validation } \\
\text { Kappa }\end{array}$} & \multirow{2}{*}{$\begin{array}{c}2^{\text {nd }} \text { Validation } \\
\text { Kappa }\end{array}$} & \multirow{2}{*}{$\begin{array}{c}3^{\text {rd }} \text { Validation } \\
\text { Kappa }\end{array}$} \\
\hline$\varphi_{g}$ & $\Lambda_{l}$ & $\Gamma_{n}$ & $\eta_{m}$ & & & & \\
\hline 2 & 2 & 4 & 2 & 0.96 & 0.86 & - & - \\
\hline 2 & 2 & 4 & 3 & 0.96 & 0.86 & - & - \\
\hline 2 & 2 & 1 & 1 & 0.96 & 0.87 & - & - \\
\hline 2 & 2 & 1 & 2 & 0.96 & 0.87 & - & - \\
\hline 2 & 2 & 1 & 3 & 0.96 & 0.87 & - & - \\
\hline 2 & 2 & 6 & 1 & 0.96 & 0.87 & - & - \\
\hline 2 & 2 & 6 & 2 & 0.96 & 0.87 & - & - \\
\hline 2 & 2 & 6 & 3 & 0.96 & 0.87 & - & - \\
\hline 2 & 3 & 1 & 1 & 0.97 & 0.85 & 0.79 & 0.82 \\
\hline 2 & 3 & 1 & 2 & 0.97 & 0.85 & 0.79 & 0.82 \\
\hline 2 & 3 & 1 & 3 & 0.97 & 0.85 & 0.79 & 0.82 \\
\hline 2 & 3 & 2 & 1 & 0.96 & 0.85 & 0.79 & 0.82 \\
\hline 2 & 3 & 2 & 2 & 0.96 & 0.85 & 0.79 & 0.82 \\
\hline 2 & 3 & 2 & 3 & 0.96 & 0.85 & 0.79 & 0.82 \\
\hline 2 & 3 & 3 & 1 & 0.96 & 0.85 & 0.78 & 0.82 \\
\hline 2 & 3 & 3 & 2 & 0.96 & 0.85 & 0.78 & 0.82 \\
\hline 2 & 3 & 3 & 3 & 0.96 & 0.85 & 0.78 & 0.82 \\
\hline 2 & 3 & 4 & 1 & 0.96 & 0.85 & 0.78 & 0.81 \\
\hline 2 & 3 & 4 & 2 & 0.96 & 0.85 & 0.78 & 0.81 \\
\hline 2 & 3 & 4 & 3 & 0.96 & 0.85 & 0.78 & 0.81 \\
\hline 2 & 3 & 1 & 1 & 0.96 & 0.85 & 0.78 & 0.82 \\
\hline 2 & 3 & 1 & 2 & 0.96 & 0.85 & 0.78 & 0.82 \\
\hline 2 & 3 & 1 & 3 & 0.96 & 0.85 & 0.78 & 0.82 \\
\hline 2 & 3 & 6 & 1 & 0.96 & 0.85 & 0.79 & 0.82 \\
\hline 2 & 3 & 6 & 2 & 0.96 & 0.85 & 0.79 & 0.82 \\
\hline 2 & 3 & 6 & 3 & 0.96 & 0.85 & 0.79 & 0.82 \\
\hline 2 & 4 & 1 & 1 & 0.96 & - & - & - \\
\hline 2 & 4 & 1 & 2 & 0.96 & - & - & - \\
\hline 2 & 4 & 1 & 3 & 0.96 & - & - & - \\
\hline 2 & 4 & 2 & 1 & 0.95 & - & - & - \\
\hline 2 & 4 & 2 & 2 & 0.95 & - & - & - \\
\hline 2 & 4 & 2 & 3 & 0.95 & - & - & - \\
\hline 2 & 4 & 3 & 1 & 0.96 & - & - & - \\
\hline 2 & 4 & 3 & 2 & 0.96 & - & - & - \\
\hline 2 & 4 & 3 & 3 & 0.96 & - & - & - \\
\hline 2 & 4 & 4 & 1 & 0.96 & - & - & - \\
\hline 2 & 4 & 4 & 2 & 0.96 & - & - & - \\
\hline 2 & 4 & 4 & 3 & 0.96 & - & - & - \\
\hline 2 & 4 & 1 & 1 & 0.95 & - & - & - \\
\hline 2 & 4 & 1 & 2 & 0.95 & - & - & - \\
\hline 2 & 4 & 1 & 3 & 0.95 & - & - & - \\
\hline 2 & 4 & 6 & 1 & 0.95 & - & - & - \\
\hline 2 & 4 & 6 & 2 & 0.95 & - & - & - \\
\hline 2 & 4 & 6 & 3 & 0.95 & - & - & - \\
\hline 3 & 1 & 1 & 1 & 0.97 & 0.80 & - & - \\
\hline 3 & 1 & 1 & 2 & 0.97 & 0.80 & - & - \\
\hline 3 & 1 & 1 & 3 & 0.97 & 0.80 & - & - \\
\hline 3 & 1 & 2 & 1 & 0.97 & 0.81 & - & - \\
\hline 3 & 1 & 2 & 2 & 0.97 & 0.81 & - & - \\
\hline 3 & 1 & 2 & 3 & 0.97 & 0.81 & - & - \\
\hline 3 & 1 & 3 & 1 & 0.97 & 0.81 & - & - \\
\hline
\end{tabular}


Table C.1.1 the results of calibration procedure 1 and its validations.

\begin{tabular}{|c|c|c|c|c|c|c|c|}
\hline \multicolumn{4}{|c|}{ Configurations } & \multirow{2}{*}{$\begin{array}{c}\text { Calibration } \\
\text { Kappa }\end{array}$} & \multirow{2}{*}{$\begin{array}{c}\mathbf{1}^{\text {st }} \text { Validation } \\
\text { Kappa }\end{array}$} & \multirow{2}{*}{$\begin{array}{c}2^{\text {nd }} \text { Validation } \\
\text { Kappa }\end{array}$} & \multirow{2}{*}{$\begin{array}{c}3^{\text {rd }} \text { Validation } \\
\text { Kappa }\end{array}$} \\
\hline$\varphi_{g}$ & $\Lambda_{l}$ & $\Gamma_{n}$ & $\eta_{m}$ & & & & \\
\hline 3 & 1 & 3 & 2 & 0.97 & 0.81 & - & - \\
\hline 3 & 1 & 3 & 3 & 0.97 & 0.81 & - & - \\
\hline 3 & 1 & 4 & 1 & 0.97 & 0.80 & - & - \\
\hline 3 & 1 & 4 & 2 & 0.97 & 0.80 & - & - \\
\hline 3 & 1 & 4 & 3 & 0.97 & 0.80 & - & - \\
\hline 3 & 1 & 1 & 1 & 0.97 & 0.80 & - & - \\
\hline 3 & 1 & 1 & 2 & 0.97 & 0.80 & - & - \\
\hline 3 & 1 & 1 & 3 & 0.97 & 0.80 & - & - \\
\hline 3 & 1 & 6 & 1 & 0.97 & 0.83 & - & - \\
\hline 3 & 1 & 6 & 2 & 0.97 & 0.83 & - & - \\
\hline 3 & 1 & 6 & 3 & 0.97 & 0.83 & - & - \\
\hline 3 & 2 & 1 & 1 & 0.97 & 0.80 & 0.80 & 0.80 \\
\hline 3 & 2 & 1 & 2 & 0.97 & 0.80 & 0.80 & 0.80 \\
\hline 3 & 2 & 1 & 3 & 0.97 & 0.80 & 0.80 & 0.80 \\
\hline 3 & 2 & 2 & 1 & 0.97 & - & - & - \\
\hline 3 & 2 & 2 & 2 & 0.97 & - & - & - \\
\hline 3 & 2 & 2 & 3 & 0.97 & - & - & - \\
\hline 3 & 2 & 3 & 1 & 0.98 & 0.80 & 0.78 & 0.79 \\
\hline 3 & 2 & 3 & 2 & 0.98 & 0.80 & 0.78 & 0.79 \\
\hline 3 & 2 & 3 & 3 & 0.98 & 0.80 & 0.78 & 0.79 \\
\hline 3 & 2 & 4 & 1 & 0.98 & 0.80 & 0.78 & 0.79 \\
\hline 3 & 2 & 4 & 2 & 0.98 & 0.80 & 0.78 & 0.79 \\
\hline 3 & 2 & 4 & 3 & 0.98 & 0.80 & 0.78 & 0.79 \\
\hline 3 & 2 & 1 & 1 & 0.98 & - & - & - \\
\hline 3 & 2 & 1 & 2 & 0.98 & - & - & - \\
\hline 3 & 2 & 1 & 3 & 0.98 & - & - & - \\
\hline 3 & 2 & 6 & 1 & 0.97 & - & - & - \\
\hline 3 & 2 & 6 & 2 & 0.97 & - & - & - \\
\hline 3 & 2 & 6 & 3 & 0.97 & - & - & - \\
\hline 3 & 3 & 1 & 1 & 0.98 & - & - & - \\
\hline 3 & 3 & 1 & 2 & 0.98 & - & - & - \\
\hline 3 & 3 & 1 & 3 & 0.98 & - & - & - \\
\hline 3 & 3 & 2 & 1 & 0.97 & 0.84 & 0.78 & 0.81 \\
\hline 3 & 3 & 2 & 2 & 0.97 & 0.84 & 0.78 & 0.81 \\
\hline 3 & 3 & 2 & 3 & 0.97 & 0.84 & 0.78 & 0.81 \\
\hline 3 & 3 & 3 & 1 & 0.97 & 0.85 & 0.77 & 0.81 \\
\hline 3 & 3 & 3 & 2 & 0.97 & 0.85 & 0.77 & 0.81 \\
\hline 3 & 3 & 3 & 3 & 0.97 & 0.85 & 0.77 & 0.81 \\
\hline 3 & 3 & 4 & 1 & 0.97 & 0.85 & 0.77 & 0.81 \\
\hline 3 & 3 & 4 & 2 & 0.97 & 0.85 & 0.77 & 0.81 \\
\hline 3 & 3 & 4 & 3 & 0.97 & 0.85 & 0.77 & 0.81 \\
\hline 3 & 3 & 1 & 1 & 0.97 & 0.81 & 0.74 & 0.77 \\
\hline 3 & 3 & 1 & 2 & 0.97 & 0.81 & 0.74 & 0.77 \\
\hline 3 & 3 & 1 & 3 & 0.97 & 0.81 & 0.74 & 0.77 \\
\hline 3 & 3 & 6 & 1 & 0.97 & 0.81 & 0.75 & 0.78 \\
\hline 3 & 3 & 6 & 2 & 0.97 & 0.81 & 0.75 & 0.78 \\
\hline 3 & 3 & 6 & 3 & 0.97 & 0.81 & 0.75 & 0.78 \\
\hline 3 & 4 & 1 & 1 & 0.96 & 0.86 & - & - \\
\hline 3 & 4 & 1 & 2 & 0.96 & 0.86 & - & - \\
\hline 3 & 4 & 1 & 3 & 0.96 & 0.86 & - & - \\
\hline 3 & 4 & 2 & 1 & 0.96 & 0.88 & - & - \\
\hline
\end{tabular}


Table C.1.1 the results of calibration procedure 1 and its validations.

\begin{tabular}{|c|c|c|c|c|c|c|c|}
\hline \multicolumn{4}{|c|}{ Configurations } & \multirow{2}{*}{$\begin{array}{c}\text { Calibration } \\
\text { Kappa }\end{array}$} & \multirow{2}{*}{$\begin{array}{c}\mathbf{1}^{\text {st }} \text { Validation } \\
\text { Kappa }\end{array}$} & \multirow{2}{*}{$\begin{array}{c}2^{\text {nd }} \text { Validation } \\
\text { Kappa }\end{array}$} & \multirow{2}{*}{$\begin{array}{c}3^{\text {rd }} \text { Validation } \\
\text { Kappa }\end{array}$} \\
\hline$\varphi_{g}$ & $\Lambda_{l}$ & $\Gamma_{n}$ & $\eta_{m}$ & & & & \\
\hline 3 & 4 & 2 & 2 & 0.96 & 0.88 & - & - \\
\hline 3 & 4 & 2 & 3 & 0.96 & 0.88 & - & - \\
\hline 3 & 4 & 3 & 1 & 0.96 & 0.85 & - & - \\
\hline 3 & 4 & 3 & 2 & 0.96 & 0.85 & - & - \\
\hline 3 & 4 & 3 & 3 & 0.96 & 0.85 & - & - \\
\hline 3 & 4 & 4 & 1 & 0.96 & 0.86 & - & - \\
\hline 3 & 4 & 4 & 2 & 0.96 & 0.86 & - & - \\
\hline 3 & 4 & 4 & 3 & 0.96 & 0.86 & - & - \\
\hline 3 & 4 & 1 & 1 & 0.97 & 0.87 & - & - \\
\hline 3 & 4 & 1 & 2 & 0.97 & 0.87 & - & - \\
\hline 3 & 4 & 1 & 3 & 0.97 & 0.87 & - & - \\
\hline 3 & 4 & 6 & 1 & 0.97 & 0.87 & - & - \\
\hline 3 & 4 & 6 & 2 & 0.97 & 0.87 & - & - \\
\hline 3 & 4 & 6 & 3 & 0.97 & 0.87 & - & - \\
\hline 4 & 1 & 1 & 1 & 0.97 & 0.82 & 0.94 & 0.89 \\
\hline 4 & 1 & 1 & 2 & 0.97 & 0.82 & 0.94 & 0.89 \\
\hline 4 & 1 & 1 & 3 & 0.97 & 0.82 & 0.94 & 0.89 \\
\hline 4 & 1 & 2 & 1 & 0.97 & 0.82 & 0.95 & 0.89 \\
\hline 4 & 1 & 2 & 2 & 0.97 & 0.82 & 0.95 & 0.89 \\
\hline 4 & 1 & 2 & 3 & 0.97 & 0.82 & 0.95 & 0.89 \\
\hline 4 & 1 & 3 & 1 & 0.97 & 0.83 & 0.94 & 0.89 \\
\hline 4 & 1 & 3 & 2 & 0.97 & 0.83 & 0.94 & 0.89 \\
\hline 4 & 1 & 3 & 3 & 0.97 & 0.83 & 0.94 & 0.89 \\
\hline 4 & 1 & 4 & 1 & 0.97 & 0.83 & 0.94 & 0.89 \\
\hline 4 & 1 & 4 & 2 & 0.97 & 0.83 & 0.94 & 0.89 \\
\hline 4 & 1 & 4 & 3 & 0.97 & 0.83 & 0.94 & 0.89 \\
\hline 4 & 1 & 1 & 1 & 0.97 & 0.84 & 0.94 & 0.90 \\
\hline 4 & 1 & 1 & 2 & 0.97 & 0.83 & 0.94 & 0.89 \\
\hline 4 & 1 & 1 & 3 & 0.97 & 0.83 & 0.94 & 0.89 \\
\hline 4 & 1 & 6 & 1 & 0.97 & 0.84 & 0.94 & 0.90 \\
\hline 4 & 1 & 6 & 2 & 0.97 & 0.84 & 0.94 & 0.90 \\
\hline 4 & 1 & 6 & 3 & 0.97 & 0.84 & 0.94 & 0.90 \\
\hline 4 & 2 & 1 & 1 & 0.97 & 0.78 & - & - \\
\hline 4 & 2 & 1 & 2 & 0.97 & 0.78 & - & - \\
\hline 4 & 2 & 1 & 3 & 0.97 & 0.78 & - & - \\
\hline 4 & 2 & 2 & 1 & 0.96 & 0.79 & - & - \\
\hline 4 & 2 & 2 & 2 & 0.96 & 0.79 & - & - \\
\hline 4 & 2 & 2 & 3 & 0.96 & 0.79 & - & - \\
\hline 4 & 2 & 3 & 1 & 0.96 & 0.79 & - & - \\
\hline 4 & 2 & 3 & 2 & 0.96 & 0.79 & - & - \\
\hline 4 & 2 & 3 & 3 & 0.96 & 0.79 & - & - \\
\hline 4 & 2 & 4 & 1 & 0.96 & 0.79 & - & - \\
\hline 4 & 2 & 4 & 2 & 0.96 & 0.79 & - & - \\
\hline 4 & 2 & 4 & 3 & 0.96 & 0.79 & - & - \\
\hline 4 & 2 & 1 & 1 & 0.96 & 0.79 & - & - \\
\hline 4 & 2 & 1 & 2 & 0.96 & 0.79 & - & - \\
\hline 4 & 2 & 1 & 3 & 0.96 & 0.79 & - & - \\
\hline 4 & 2 & 6 & 1 & 0.96 & 0.79 & - & - \\
\hline 4 & 2 & 6 & 2 & 0.96 & 0.79 & - & - \\
\hline 4 & 2 & 6 & 3 & 0.96 & 0.79 & - & - \\
\hline 4 & 3 & 1 & 1 & 0.99 & - & - & - \\
\hline
\end{tabular}


Table C.1.1 the results of calibration procedure 1 and its validations.

\begin{tabular}{|c|c|c|c|c|c|c|c|}
\hline \multicolumn{4}{|c|}{ Configurations } & \multirow{2}{*}{$\begin{array}{c}\text { Calibration } \\
\text { Kappa }\end{array}$} & \multirow{2}{*}{$\begin{array}{c}\mathbf{1}^{\text {st }} \text { Validation } \\
\text { Kappa }\end{array}$} & \multirow{2}{*}{$\begin{array}{c}2^{\text {nd }} \text { Validation } \\
\text { Kappa }\end{array}$} & \multirow{2}{*}{$\begin{array}{c}3^{\text {rd }} \text { Validation } \\
\text { Kappa }\end{array}$} \\
\hline$\varphi_{g}$ & $\Lambda_{l}$ & $\Gamma_{n}$ & $\eta_{m}$ & & & & \\
\hline 4 & 3 & 1 & 2 & 0.99 & - & - & - \\
\hline 4 & 3 & 1 & 3 & 0.99 & - & - & - \\
\hline 4 & 3 & 2 & 1 & 0.98 & - & - & - \\
\hline 4 & 3 & 2 & 2 & 0.98 & - & - & - \\
\hline 4 & 3 & 2 & 3 & 0.98 & - & - & - \\
\hline 4 & 3 & 3 & 1 & 0.98 & - & - & - \\
\hline 4 & 3 & 3 & 2 & 0.98 & - & - & - \\
\hline 4 & 3 & 3 & 3 & 0.98 & - & - & - \\
\hline 4 & 3 & 4 & 1 & 0.98 & - & - & - \\
\hline 4 & 3 & 4 & 2 & 0.98 & - & - & - \\
\hline 4 & 3 & 4 & 3 & 0.98 & - & - & - \\
\hline 4 & 3 & 1 & 1 & 0.98 & 0.76 & - & - \\
\hline 4 & 3 & 1 & 2 & 0.98 & 0.76 & - & - \\
\hline 4 & 3 & 1 & 3 & 0.98 & 0.76 & - & - \\
\hline 4 & 3 & 6 & 1 & 0.98 & 0.76 & - & - \\
\hline 4 & 3 & 6 & 2 & 0.98 & 0.76 & - & - \\
\hline 4 & 3 & 6 & 3 & 0.98 & 0.76 & - & - \\
\hline 4 & 4 & 1 & 1 & 0.97 & 0.82 & - & - \\
\hline 4 & 4 & 1 & 2 & 0.97 & 0.82 & - & - \\
\hline 4 & 4 & 1 & 3 & 0.97 & 0.82 & - & - \\
\hline 4 & 4 & 2 & 1 & 0.97 & 0.82 & - & - \\
\hline 4 & 4 & 2 & 2 & 0.97 & 0.82 & - & - \\
\hline 4 & 4 & 2 & 3 & 0.97 & 0.82 & - & - \\
\hline 4 & 4 & 3 & 1 & 0.97 & 0.82 & - & - \\
\hline 4 & 4 & 3 & 2 & 0.97 & 0.82 & - & - \\
\hline 4 & 4 & 3 & 3 & 0.97 & 0.82 & - & - \\
\hline 4 & 4 & 4 & 1 & 0.97 & 0.82 & - & - \\
\hline 4 & 4 & 4 & 2 & 0.97 & 0.82 & - & - \\
\hline 4 & 4 & 4 & 3 & 0.97 & 0.82 & - & - \\
\hline 4 & 4 & 1 & 1 & 0.97 & 0.82 & - & - \\
\hline 4 & 4 & 1 & 2 & 0.97 & 0.82 & - & - \\
\hline 4 & 4 & 1 & 3 & 0.97 & 0.82 & - & - \\
\hline 4 & 4 & 6 & 1 & 0.97 & 0.82 & - & - \\
\hline 4 & 4 & 6 & 2 & 0.97 & 0.82 & - & - \\
\hline 4 & 4 & 6 & 3 & 0.97 & 0.82 & - & - \\
\hline 5 & 1 & 1 & 1 & 0.97 & 0.75 & 0.76 & 0.76 \\
\hline 5 & 1 & 1 & 2 & 0.97 & 0.75 & 0.76 & 0.76 \\
\hline 5 & 1 & 1 & 3 & 0.97 & 0.75 & 0.76 & 0.76 \\
\hline 5 & 1 & 2 & 1 & 0.97 & 0.75 & 0.76 & 0.76 \\
\hline 5 & 1 & 2 & 2 & 0.97 & 0.75 & 0.76 & 0.76 \\
\hline 5 & 1 & 2 & 3 & 0.97 & 0.75 & 0.76 & 0.76 \\
\hline 5 & 1 & 3 & 1 & 0.97 & 0.77 & 0.76 & 0.77 \\
\hline 5 & 1 & 3 & 2 & 0.97 & 0.77 & 0.74 & 0.76 \\
\hline 5 & 1 & 3 & 3 & 0.97 & 0.77 & 0.74 & 0.76 \\
\hline 5 & 1 & 4 & 1 & 0.97 & 0.75 & 0.76 & 0.76 \\
\hline 5 & 1 & 4 & 2 & 0.97 & 0.75 & 0.76 & 0.76 \\
\hline 5 & 1 & 4 & 3 & 0.97 & 0.75 & 0.76 & 0.76 \\
\hline 5 & 1 & 1 & 1 & 0.97 & 0.76 & 0.77 & 0.77 \\
\hline 5 & 1 & 1 & 2 & 0.97 & 0.76 & 0.77 & 0.77 \\
\hline 5 & 1 & 1 & 3 & 0.97 & 0.76 & 0.77 & 0.77 \\
\hline 5 & 1 & 6 & 1 & 0.97 & 0.76 & 0.76 & 0.76 \\
\hline
\end{tabular}


Table C.1.1 the results of calibration procedure 1 and its validations.

\begin{tabular}{|c|c|c|c|c|c|c|c|}
\hline \multicolumn{4}{|c|}{ Configurations } & \multirow{2}{*}{$\begin{array}{c}\text { Calibration } \\
\text { Kappa }\end{array}$} & \multirow{2}{*}{$\frac{1^{\text {st }} \text { Validation }}{\text { Kappa }}$} & \multirow{2}{*}{$\begin{array}{c}2^{\text {nd }} \text { Validation } \\
\text { Kappa }\end{array}$} & \multirow{2}{*}{$\frac{3^{\text {rd }} \text { Validation }}{\text { Kappa }}$} \\
\hline$\varphi_{g}$ & $\Lambda_{l}$ & $\overline{\Gamma_{n}}$ & $\eta_{m}$ & & & & \\
\hline 5 & 1 & 6 & 2 & 0.97 & 0.76 & 0.79 & 0.78 \\
\hline 5 & 1 & 6 & 3 & 0.97 & 0.76 & 0.79 & 0.78 \\
\hline 5 & 2 & 1 & 1 & 0.97 & 0.71 & 0.69 & 0.71 \\
\hline 5 & 2 & 1 & 2 & 0.97 & 0.71 & 0.69 & 0.71 \\
\hline 5 & 2 & 1 & 3 & 0.97 & 0.71 & 0.69 & 0.71 \\
\hline 5 & 2 & 2 & 1 & 0.97 & 0.75 & 0.68 & 0.72 \\
\hline 5 & 2 & 2 & 2 & 0.97 & 0.75 & 0.68 & 0.72 \\
\hline 5 & 2 & 2 & 3 & 0.97 & 0.75 & 0.68 & 0.72 \\
\hline 5 & 2 & 3 & 1 & 0.97 & 0.72 & 0.66 & 0.70 \\
\hline 5 & 2 & 3 & 2 & 0.97 & 0.72 & 0.66 & 0.70 \\
\hline 5 & 2 & 3 & 3 & 0.97 & 0.72 & 0.66 & 0.70 \\
\hline 5 & 2 & 4 & 1 & 0.97 & 0.70 & 0.66 & 0.69 \\
\hline 5 & 2 & 4 & 2 & 0.97 & 0.70 & 0.66 & 0.69 \\
\hline 5 & 2 & 4 & 3 & 0.97 & 0.70 & 0.66 & 0.69 \\
\hline 5 & 2 & 1 & 1 & 0.97 & 0.72 & 0.68 & 0.70 \\
\hline 5 & 2 & 1 & 2 & 0.97 & 0.72 & 0.68 & 0.70 \\
\hline 5 & 2 & 1 & 3 & 0.97 & 0.72 & 0.68 & 0.70 \\
\hline 5 & 2 & 6 & 1 & 0.99 & 0.72 & 0.66 & 0.70 \\
\hline 5 & 2 & 6 & 2 & 0.99 & 0.72 & 0.66 & 0.70 \\
\hline 5 & 2 & 6 & 3 & 0.99 & 0.72 & 0.66 & 0.70 \\
\hline 5 & 3 & 1 & 1 & 0.97 & 0.78 & 0.70 & 0.75 \\
\hline 5 & 3 & 1 & 2 & 0.97 & 0.78 & 0.70 & 0.75 \\
\hline 5 & 3 & 1 & 3 & 0.97 & 0.78 & 0.70 & 0.75 \\
\hline 5 & 3 & 2 & 1 & 0.97 & 0.80 & 0.72 & 0.77 \\
\hline 5 & 3 & 2 & 2 & 0.97 & 0.80 & 0.72 & 0.77 \\
\hline 5 & 3 & 2 & 3 & 0.97 & 0.80 & 0.72 & 0.77 \\
\hline 5 & 3 & 3 & 1 & 0.97 & 0.78 & 0.67 & 0.73 \\
\hline 5 & 3 & 3 & 2 & 0.97 & 0.78 & 0.67 & 0.73 \\
\hline 5 & 3 & 3 & 3 & 0.97 & 0.78 & 0.67 & 0.73 \\
\hline 5 & 3 & 4 & 1 & 0.97 & 0.80 & 0.69 & 0.75 \\
\hline 5 & 3 & 4 & 2 & 0.97 & 0.80 & 0.69 & 0.75 \\
\hline 5 & 3 & 4 & 3 & 0.97 & 0.80 & 0.69 & 0.75 \\
\hline 5 & 3 & 1 & 1 & 0.97 & 0.82 & 0.69 & 0.76 \\
\hline 5 & 3 & 1 & 2 & 0.97 & 0.82 & 0.69 & 0.76 \\
\hline 5 & 3 & 1 & 3 & 0.97 & 0.82 & 0.69 & 0.76 \\
\hline 5 & 3 & 6 & 1 & 0.97 & 0.82 & 0.72 & 0.77 \\
\hline 5 & 3 & 6 & 2 & 0.97 & 0.82 & 0.72 & 0.77 \\
\hline 5 & 3 & 6 & 3 & 0.97 & 0.82 & 0.72 & 0.77 \\
\hline 5 & 4 & 1 & 1 & 0.95 & 0.75 & 0.71 & 0.73 \\
\hline 5 & 4 & 1 & 2 & 0.95 & 0.75 & 0.68 & 0.72 \\
\hline 5 & 4 & 1 & 3 & 0.95 & 0.75 & 0.68 & 0.72 \\
\hline 5 & 4 & 2 & 1 & 0.95 & 0.75 & 0.67 & 0.72 \\
\hline 5 & 4 & 2 & 2 & 0.95 & 0.75 & 0.66 & 0.71 \\
\hline 5 & 4 & 2 & 3 & 0.95 & 0.75 & 0.66 & 0.71 \\
\hline 5 & 4 & 3 & 1 & 0.95 & 0.74 & 0.64 & 0.70 \\
\hline 5 & 4 & 3 & 2 & 0.95 & 0.74 & - & - \\
\hline 5 & 4 & 3 & 3 & 0.95 & 0.74 & - & - \\
\hline 5 & 4 & 4 & 1 & 0.95 & 0.75 & 0.69 & 0.73 \\
\hline 5 & 4 & 4 & 2 & 0.95 & 0.75 & 0.67 & 0.72 \\
\hline 5 & 4 & 4 & 3 & 0.95 & 0.75 & 0.67 & 0.72 \\
\hline 5 & 4 & 1 & 1 & 0.95 & 0.75 & 0.68 & 0.72 \\
\hline
\end{tabular}


Table C.1.1 the results of calibration procedure 1 and its validations.

\begin{tabular}{|c|c|c|c|c|c|c|c|}
\hline \multicolumn{4}{|c|}{ Configurations } & Calibration & $1^{\text {st }}$ Validation & $2^{\text {nd }}$ Validation & $3^{\text {rd }}$ Validation \\
\hline$\varphi_{g}$ & $\Lambda_{l}$ & $\Gamma_{n}$ & $\eta_{m}$ & Kappa & Kappa & Kappa & Kappa \\
\hline 5 & 4 & 1 & 2 & 0.95 & 0.75 & 0.65 & 0.71 \\
\hline 5 & 4 & 1 & 3 & 0.95 & 0.75 & 0.65 & 0.71 \\
\hline 5 & 4 & 6 & 1 & 0.95 & 0.75 & 0.68 & 0.72 \\
\hline 5 & 4 & 6 & 2 & 0.95 & 0.75 & 0.65 & 0.71 \\
\hline 5 & 4 & 6 & 3 & 0.95 & 0.75 & 0.65 & 0.71 \\
\hline
\end{tabular}

Table C.1.2 the results of calibration procedure 2 and its validations.

\begin{tabular}{|c|c|c|c|c|c|}
\hline \multicolumn{2}{|c|}{ Configurations } & \multirow{2}{*}{$\begin{array}{c}\text { Calibration } \\
\text { Kappa }\end{array}$} & \multirow{2}{*}{$\begin{array}{c}1^{\text {st }} \text { Validation } \\
\text { Kappa }\end{array}$} & \multirow{2}{*}{$\begin{array}{cl}2^{\text {nd }} & \text { Validation } \\
\text { Kappa }\end{array}$} & \multirow{2}{*}{$\frac{3^{\text {rd }} \text { Validation }}{\text { Kappa }}$} \\
\hline$\varphi_{g}$ & $\Lambda_{l}$ & & & & \\
\hline 1 & 1 & 0.84 & 0.85 & 0.84 & 0.85 \\
\hline 1 & 2 & - & - & - & - \\
\hline 1 & 3 & 0.86 & 0.78 & - & - \\
\hline 1 & 4 & - & - & - & - \\
\hline 2 & 1 & 0.89 & - & - & - \\
\hline 2 & 2 & 0.93 & 0.89 & 0.80 & 0.85 \\
\hline 2 & 3 & - & - & - & - \\
\hline 2 & 4 & - & - & - & - \\
\hline 3 & 1 & 0.91 & 0.81 & 0.82 & 0.82 \\
\hline 3 & 2 & - & - & - & - \\
\hline 3 & 3 & - & - & - & - \\
\hline 3 & 4 & 0.94 & 0.81 & 0.83 & 0.82 \\
\hline 4 & 1 & 0.90 & 0.73 & - & - \\
\hline 4 & 2 & 0.90 & 0.72 & - & - \\
\hline 4 & 3 & 0.91 & 0.78 & - & - \\
\hline 4 & 4 & 0.95 & 0.79 & 0.85 & 0.82 \\
\hline 5 & 1 & 0.94 & 0.76 & 0.65 & 0.72 \\
\hline 5 & 2 & 0.94 & 0.70 & 0.61 & 0.67 \\
\hline 5 & 3 & 0.89 & 0.65 & 0.70 & 0.68 \\
\hline 5 & 4 & 0.94 & 0.74 & 0.70 & 0.73 \\
\hline
\end{tabular}


Table C.1.3 the results of calibration and validations based on the average speed

\begin{tabular}{|c|c|c|c|c|c|}
\hline \multicolumn{2}{|c|}{ Configurations } & \multirow{2}{*}{$\begin{array}{c}\text { Calibration } \\
\text { Kappa }\end{array}$} & \multirow{2}{*}{$\begin{array}{c}\mathbf{1}^{\text {st }} \text { Validation } \\
\text { Kappa }\end{array}$} & \multirow{2}{*}{$\begin{array}{ll}2^{\text {nd }} & \text { Validation } \\
& \text { Kappa }\end{array}$} & \multirow{2}{*}{$\frac{3^{\text {rd }} \text { Validation }}{\text { Kappa }}$} \\
\hline$\varphi_{g}$ & $\Lambda_{l}$ & & & & \\
\hline 1 & 1 & 0.08 & 0.14 & 0.17 & 0.16 \\
\hline 1 & 2 & 0.07 & 0.14 & 0.16 & 0.16 \\
\hline 1 & 3 & 0.07 & 0.09 & - & - \\
\hline 1 & 4 & 0.07 & 0.15 & 0.16 & 0.16 \\
\hline 2 & 1 & 0.08 & 0.11 & 0.19 & 0.17 \\
\hline 2 & 2 & 0.08 & 0.13 & 0.16 & 0.16 \\
\hline 2 & 3 & 0.07 & 0.11 & 0.19 & 0.17 \\
\hline 2 & 4 & 0.08 & 0.13 & 0.17 & 0.16 \\
\hline 3 & 1 & 0.07 & 0.14 & 0.17 & 0.16 \\
\hline 3 & 2 & 0.07 & 0.13 & 0.17 & 0.16 \\
\hline 3 & 3 & 0.07 & 0.13 & 0.17 & 0.16 \\
\hline 3 & 4 & 0.07 & 0.13 & 0.17 & 0.16 \\
\hline 4 & 1 & 0.07 & 0.05 & 0.04 & 0.05 \\
\hline 4 & 2 & 0.07 & 0.05 & 0.04 & 0.05 \\
\hline 4 & 3 & 0.07 & 0.05 & 0.04 & 0.06 \\
\hline 4 & 4 & 0.07 & 0.05 & 0.04 & 0.05 \\
\hline 5 & 1 & 0.14 & 0.11 & 0.03 & 0.08 \\
\hline 5 & 2 & 0.14 & 0.11 & 0.03 & 0.08 \\
\hline 5 & 3 & 0.14 & 0.12 & 0.02 & 0.09 \\
\hline 5 & 4 & 0.14 & 0.11 & 0.03 & 0.08 \\
\hline
\end{tabular}

Table C.1.4 the results of calibration and validations based on the maximum speed

\begin{tabular}{|c|c|c|c|c|c|}
\hline \multicolumn{2}{|c|}{ Configurations } & \multirow{2}{*}{$\begin{array}{c}\text { Calibration } \\
\text { Kappa } \\
\end{array}$} & \multirow{2}{*}{$\begin{array}{c}\mathbf{1}^{\text {st }} \text { Validation } \\
\text { Kappa } \\
\end{array}$} & \multirow{2}{*}{$\begin{array}{cl}2^{\text {nd }} & \text { Validation } \\
\text { Kappa } \\
\end{array}$} & \multirow{2}{*}{$\begin{array}{c}3^{\text {rd }} \text { Validation } \\
\text { Kappa } \\
\end{array}$} \\
\hline$\varphi_{g}$ & $\Lambda_{l}$ & & & & \\
\hline 1 & 1 & 0.38 & 0.49 & 0.40 & 0.46 \\
\hline 1 & 2 & 0.39 & 0.48 & 0.40 & 0.45 \\
\hline 1 & 3 & 0.37 & 0.48 & 0.38 & 0.44 \\
\hline 1 & 4 & 0.34 & 0.48 & 0.36 & 0.43 \\
\hline 2 & 1 & 0.42 & 0.49 & 0.40 & 0.46 \\
\hline 2 & 2 & 0.43 & 0.48 & 0.38 & 0.45 \\
\hline 2 & 3 & 0.41 & 0.46 & 0.38 & 0.44 \\
\hline 2 & 4 & 0.39 & 0.47 & 0.37 & 0.44 \\
\hline 3 & 1 & 0.46 & 0.54 & 0.38 & 0.46 \\
\hline 3 & 2 & 0.48 & 0.53 & 0.37 & 0.46 \\
\hline 3 & 3 & 0.46 & 0.47 & 0.39 & 0.45 \\
\hline 3 & 4 & 0.44 & 0.51 & 0.37 & 0.45 \\
\hline 4 & 1 & 0.50 & 0.56 & 0.39 & 0.49 \\
\hline 4 & 2 & 0.51 & 0.55 & 0.37 & 0.47 \\
\hline 4 & 3 & 0.51 & 0.53 & 0.39 & 0.47 \\
\hline 4 & 4 & 0.50 & 0.53 & 0.36 & 0.46 \\
\hline 5 & 1 & 0.59 & 0.60 & 0.38 & 0.51 \\
\hline 5 & 2 & 0.59 & 0.57 & 0.34 & 0.48 \\
\hline 5 & 3 & 0.59 & 0.52 & 0.40 & 0.48 \\
\hline 5 & 4 & 0.59 & 0.57 & 0.34 & 0.48 \\
\hline
\end{tabular}


Table C.1.5 the results of calibration and validations based on the average acceleration

\begin{tabular}{|c|c|c|c|c|c|}
\hline \multicolumn{2}{|c|}{ Configurations } & \multirow{2}{*}{$\begin{array}{c}\text { Calibration } \\
\text { Kappa }\end{array}$} & \multirow{2}{*}{$\frac{1^{\text {st }} \text { Validation }}{\text { Kappa }}$} & \multirow{2}{*}{$\begin{array}{c}2^{\text {nd }} \text { Validation } \\
\text { Kappa }\end{array}$} & \multirow{2}{*}{$\begin{array}{c}3^{\text {rd }} \text { Validation } \\
\text { Kappa }\end{array}$} \\
\hline$\varphi_{g}$ & $\Lambda_{l}$ & & & & \\
\hline 1 & 1 & 0.08 & 0.09 & 0.12 & 0.10 \\
\hline 1 & 2 & 0.09 & 0.08 & 0.09 & 0.07 \\
\hline 1 & 3 & 0.10 & 0.08 & 0.07 & 0.06 \\
\hline 1 & 4 & 0.09 & 0.11 & 0.11 & 0.11 \\
\hline 2 & 1 & 0.09 & 0.09 & 0.11 & 0.10 \\
\hline 2 & 2 & 0.09 & 0.10 & 0.11 & 0.10 \\
\hline 2 & 3 & 0.09 & 0.10 & 0.09 & 0.09 \\
\hline 2 & 4 & 0.09 & 0.10 & 0.11 & 0.10 \\
\hline 3 & 1 & 0.09 & 0.10 & 0.10 & 0.10 \\
\hline 3 & 2 & 0.09 & 0.11 & 0.10 & 0.10 \\
\hline 3 & 3 & 0.09 & 0.13 & 0.10 & 0.11 \\
\hline 3 & 4 & 0.09 & 0.11 & 0.10 & 0.10 \\
\hline 4 & 1 & 0.09 & 0.09 & 0.09 & 0.08 \\
\hline 4 & 2 & 0.09 & 0.01 & 0.13 & 0.08 \\
\hline 4 & 3 & 0.10 & 0.01 & 0.13 & 0.08 \\
\hline 4 & 4 & 0.09 & - & - & - \\
\hline 5 & 1 & 0.11 & 0.05 & 0.11 & 0.07 \\
\hline 5 & 2 & 0.11 & 0.08 & 0.10 & 0.08 \\
\hline 5 & 3 & 0.11 & 0.07 & 0.13 & 0.09 \\
\hline 5 & 4 & 0.11 & 0.08 & 0.10 & 0.08 \\
\hline
\end{tabular}

Table C.1.6 the results of calibration and validations based on the maximum acceleration

\begin{tabular}{|c|c|c|c|c|c|}
\hline \multicolumn{2}{|c|}{ Configurations } & \multirow{2}{*}{$\begin{array}{c}\text { Calibration } \\
\text { Kappa } \\
\end{array}$} & \multirow{2}{*}{$\begin{array}{c}\mathbf{1}^{\text {st }} \text { Validation } \\
\text { Kappa } \\
\end{array}$} & \multirow{2}{*}{$\begin{array}{cl}2^{\text {nd }} & \text { Validation } \\
& \text { Kappa } \\
\end{array}$} & \multirow{2}{*}{$\begin{array}{c}3^{\text {rd }} \text { Validation } \\
\text { Kappa } \\
\end{array}$} \\
\hline$\varphi_{g}$ & $\Lambda_{l}$ & & & & \\
\hline 1 & 1 & 0.05 & - & - & - \\
\hline 1 & 2 & 0.05 & - & - & - \\
\hline 1 & 3 & 0.05 & - & - & - \\
\hline 1 & 4 & 0.05 & - & - & - \\
\hline 2 & 1 & 0.06 & 0.01 & - & - \\
\hline 2 & 2 & 0.06 & - & - & - \\
\hline 2 & 3 & 0.06 & - & - & - \\
\hline 2 & 4 & 0.06 & - & - & - \\
\hline 3 & 1 & 0.06 & 0.02 & - & - \\
\hline 3 & 2 & 0.06 & 0.01 & - & - \\
\hline 3 & 3 & 0.06 & 0.01 & - & - \\
\hline 3 & 4 & 0.06 & 0.01 & - & - \\
\hline 4 & 1 & 0.08 & 0.02 & - & - \\
\hline 4 & 2 & 0.07 & - & - & - \\
\hline 4 & 3 & 0.07 & - & - & - \\
\hline 4 & 4 & 0.07 & - & - & - \\
\hline 5 & 1 & 0.09 & 0.02 & - & - \\
\hline 5 & 2 & 0.09 & - & - & - \\
\hline 5 & 3 & 0.09 & - & - & - \\
\hline 5 & 4 & 0.09 & - & - & - \\
\hline
\end{tabular}


Table C.1.7 the results of calibration and validations based on the average deceleration

\begin{tabular}{|c|c|c|c|c|c|}
\hline \multicolumn{2}{|c|}{ Configurations } & \multirow{2}{*}{$\begin{array}{c}\text { Calibration } \\
\text { Kappa }\end{array}$} & \multirow{2}{*}{$\frac{1^{\text {st }} \text { Validation }}{\text { Kappa }}$} & \multirow{2}{*}{$\begin{array}{c}2^{\text {nd }} \text { Validation } \\
\text { Kappa }\end{array}$} & \multirow{2}{*}{$\begin{array}{c}3^{\text {rd }} \text { Validation } \\
\text { Kappa }\end{array}$} \\
\hline$\varphi_{g}$ & $\Lambda_{l}$ & & & & \\
\hline 1 & 1 & 0.09 & 0.05 & 0.20 & 0.11 \\
\hline 1 & 2 & 0.09 & 0.07 & 0.16 & 0.10 \\
\hline 1 & 3 & 0.10 & 0.05 & 0.15 & 0.08 \\
\hline 1 & 4 & 0.06 & 0.04 & 0.12 & 0.07 \\
\hline 2 & 1 & 0.08 & 0.06 & 0.17 & 0.10 \\
\hline 2 & 2 & 0.08 & 0.08 & 0.16 & 0.10 \\
\hline 2 & 3 & 0.08 & 0.06 & 0.15 & 0.08 \\
\hline 2 & 4 & 0.06 & 0.05 & 0.12 & 0.07 \\
\hline 3 & 1 & 0.07 & 0.04 & 0.20 & 0.10 \\
\hline 3 & 2 & 0.07 & 0.08 & 0.16 & 0.10 \\
\hline 3 & 3 & 0.06 & 0.04 & - & - \\
\hline 3 & 4 & 0.05 & 0.04 & 0.12 & 0.07 \\
\hline 4 & 1 & 0.08 & 0.11 & 0.14 & 0.11 \\
\hline 4 & 2 & 0.07 & 0.02 & - & - \\
\hline 4 & 3 & 0.08 & 0.03 & - & - \\
\hline 4 & 4 & 0.06 & 0.07 & 0.12 & 0.08 \\
\hline 5 & 1 & 0.12 & 0.17 & 0.29 & 0.20 \\
\hline 5 & 2 & 0.11 & 0.11 & 0.28 & 0.15 \\
\hline 5 & 3 & 0.12 & 0.04 & 0.09 & - \\
\hline 5 & 4 & 0.09 & 0.09 & 0.15 & 0.10 \\
\hline
\end{tabular}

Table C.1.8 the results of calibration and validations based on the maximum deceleration

\begin{tabular}{|c|c|c|c|c|c|}
\hline \multicolumn{2}{|c|}{ Configurations } & \multirow{2}{*}{$\begin{array}{c}\text { Calibration } \\
\text { Kappa }\end{array}$} & \multirow{2}{*}{$\begin{array}{c}1^{\text {st }} \text { Validation } \\
\text { Kappa }\end{array}$} & \multirow{2}{*}{$\begin{array}{cl}2^{\text {nd }} & \text { Validation } \\
\text { Kappa }\end{array}$} & \multirow{2}{*}{$\begin{array}{c}3^{\text {rd }} \text { Validation } \\
\text { Kappa }\end{array}$} \\
\hline$\varphi_{g}$ & $\Lambda_{l}$ & & & & \\
\hline 1 & 1 & 0.06 & 0.03 & 0.03 & 0.02 \\
\hline 1 & 2 & 0.06 & 0.03 & 0.04 & 0.02 \\
\hline 1 & 3 & 0.06 & 0.02 & 0.04 & 0.03 \\
\hline 1 & 4 & 0.06 & 0.03 & 0.05 & 0.03 \\
\hline 2 & 1 & 0.07 & 0.04 & 0.03 & 0.03 \\
\hline 2 & 2 & 0.07 & 0.04 & 0.03 & 0.03 \\
\hline 2 & 3 & 0.07 & 0.04 & 0.04 & 0.03 \\
\hline 2 & 4 & 0.07 & 0.04 & 0.04 & 0.04 \\
\hline 3 & 1 & 0.06 & 0.05 & 0.03 & 0.03 \\
\hline 3 & 2 & 0.06 & 0.05 & 0.04 & 0.03 \\
\hline 3 & 3 & 0.06 & 0.04 & 0.05 & 0.03 \\
\hline 3 & 4 & 0.06 & 0.05 & 0.05 & 0.04 \\
\hline 4 & 1 & 0.08 & 0.05 & 0.02 & 0.03 \\
\hline 4 & 2 & 0.08 & 0.05 & 0.02 & 0.03 \\
\hline 4 & 3 & 0.07 & 0.05 & 0.03 & 0.03 \\
\hline 4 & 4 & 0.07 & 0.05 & 0.03 & 0.03 \\
\hline 5 & 1 & 0.09 & 0.04 & 0.01 & 0.02 \\
\hline 5 & 2 & 0.09 & 0.04 & 0.02 & 0.03 \\
\hline 5 & 3 & 0.09 & 0.06 & 0.03 & 0.04 \\
\hline 5 & 4 & 0.07 & 0.04 & 0.04 & 0.03 \\
\hline
\end{tabular}


Table C.1.9 the results of calibration and validations based on the average direction

\begin{tabular}{|c|c|c|c|c|c|}
\hline \multicolumn{2}{|c|}{ Configurations } & \multirow{2}{*}{$\begin{array}{c}\text { Calibration } \\
\text { Kappa }\end{array}$} & \multirow{2}{*}{$\begin{array}{c}\mathbf{1}^{\text {st }} \text { Validation } \\
\text { Kappa } \\
\end{array}$} & \multirow{2}{*}{$\begin{array}{c}2^{\text {nd }} \text { Validation } \\
\text { Kappa }\end{array}$} & \multirow{2}{*}{$\frac{3^{\text {rd }} \text { Validation }}{\text { Kappa }}$} \\
\hline$\varphi_{g}$ & $\Lambda_{l}$ & & & & \\
\hline 1 & 1 & 0.25 & 0.21 & - & - \\
\hline 1 & 2 & 0.24 & 0.19 & - & - \\
\hline 1 & 3 & 0.18 & 0.16 & - & - \\
\hline 1 & 4 & 0.20 & 0.17 & - & - \\
\hline 2 & 1 & 0.24 & 0.21 & - & - \\
\hline 2 & 2 & 0.23 & 0.18 & - & - \\
\hline 2 & 3 & 0.18 & 0.15 & - & - \\
\hline 2 & 4 & 0.20 & 0.17 & - & - \\
\hline 3 & 1 & 0.25 & 0.20 & - & - \\
\hline 3 & 2 & 0.24 & 0.18 & - & - \\
\hline 3 & 3 & 0.20 & 0.15 & - & - \\
\hline 3 & 4 & 0.22 & 0.17 & - & - \\
\hline 4 & 1 & 0.24 & 0.17 & 0.01 & - \\
\hline 4 & 2 & 0.24 & 0.15 & - & - \\
\hline 4 & 3 & 0.21 & 0.14 & - & - \\
\hline 4 & 4 & 0.23 & 0.16 & - & - \\
\hline 5 & 1 & 0.24 & 0.17 & 0.01 & - \\
\hline 5 & 2 & 0.23 & 0.15 & 0.01 & - \\
\hline 5 & 3 & 0.19 & 0.09 & - & - \\
\hline 5 & 4 & 0.22 & 0.12 & - & - \\
\hline
\end{tabular}

Table C.1.10 the results of calibration and validations based on the maximum direction

\begin{tabular}{|c|c|c|c|c|c|}
\hline \multicolumn{2}{|c|}{ Configurations } & \multirow{2}{*}{$\begin{array}{c}\text { Calibration } \\
\text { Kappa } \\
\end{array}$} & \multirow{2}{*}{$\begin{array}{c}\mathbf{1}^{\text {st }} \text { Validation } \\
\text { Kappa } \\
\end{array}$} & \multirow{2}{*}{$\begin{array}{cl}2^{\text {nd }} & \text { Validation } \\
\text { Kappa } \\
\end{array}$} & \multirow{2}{*}{$\begin{array}{c}3^{\text {rd }} \text { Validation } \\
\text { Kappa } \\
\end{array}$} \\
\hline$\varphi_{g}$ & $\Lambda_{l}$ & & & & \\
\hline 1 & 1 & 0.65 & 0.55 & 0.30 & 0.43 \\
\hline 1 & 2 & - & - & - & - \\
\hline 1 & 3 & - & - & - & - \\
\hline 1 & 4 & - & - & - & - \\
\hline 2 & 1 & 0.65 & 0.54 & 0.30 & 0.42 \\
\hline 2 & 2 & 0.57 & 0.48 & - & - \\
\hline 2 & 3 & - & - & - & - \\
\hline 2 & 4 & - & - & - & - \\
\hline 3 & 1 & 0.69 & 0.51 & 0.35 & 0.44 \\
\hline 3 & 2 & 0.60 & 0.46 & - & - \\
\hline 3 & 3 & - & - & - & - \\
\hline 3 & 4 & - & - & - & - \\
\hline 4 & 1 & 0.72 & 0.50 & - & - \\
\hline 4 & 2 & 0.62 & 0.45 & - & - \\
\hline 4 & 3 & - & - & - & - \\
\hline 4 & 4 & 0.61 & 0.41 & 0.40 & 0.46 \\
\hline 5 & 1 & 0.75 & 0.51 & 0.43 & 0.49 \\
\hline 5 & 2 & - & - & - & - \\
\hline 5 & 3 & - & - & - & - \\
\hline 5 & 4 & - & - & - & - \\
\hline
\end{tabular}


Table C.1.11 the results of calibration and validations based on the average of change in direction

\begin{tabular}{|c|c|c|c|c|c|}
\hline \multicolumn{2}{|c|}{ Configurations } & \multirow{2}{*}{$\begin{array}{c}\text { Calibration } \\
\text { Kappa }\end{array}$} & \multirow{2}{*}{$\begin{array}{c}\mathbf{1}^{\text {st }} \text { Validation } \\
\text { Kappa } \\
\end{array}$} & \multirow{2}{*}{$\begin{array}{cl}2^{\text {nd }} & \text { Validation } \\
\text { Kappa } \\
\end{array}$} & \multirow{2}{*}{$\begin{array}{c}3^{\text {rd }} \text { Validation } \\
\text { Kappa }\end{array}$} \\
\hline$\varphi_{g}$ & $\Lambda_{l}$ & & & & \\
\hline 1 & 1 & 0.09 & 0.06 & - & - \\
\hline 1 & 2 & 0.09 & 0.06 & - & - \\
\hline 1 & 3 & 0.09 & 0.06 & - & - \\
\hline 1 & 4 & 0.09 & 0.01 & - & - \\
\hline 2 & 1 & 0.09 & 0.06 & - & - \\
\hline 2 & 2 & 0.09 & 0.06 & - & - \\
\hline 2 & 3 & 0.09 & 0.06 & - & - \\
\hline 2 & 4 & 0.09 & 0.06 & - & - \\
\hline 3 & 1 & 0.11 & 0.01 & - & - \\
\hline 3 & 2 & 0.11 & 0.01 & - & - \\
\hline 3 & 3 & 0.11 & 0.01 & - & - \\
\hline 3 & 4 & 0.11 & 0.01 & - & - \\
\hline 4 & 1 & 0.14 & 0.06 & - & - \\
\hline 4 & 2 & 0.14 & 0.06 & - & - \\
\hline 4 & 3 & 0.14 & 0.06 & - & - \\
\hline 4 & 4 & 0.14 & 0.06 & - & - \\
\hline 5 & 1 & 0.18 & 0.05 & - & - \\
\hline 5 & 2 & 0.18 & 0.05 & - & - \\
\hline 5 & 3 & 0.18 & 0.05 & - & - \\
\hline 5 & 4 & 0.18 & 0.05 & - & - \\
\hline
\end{tabular}

Table C.1.12 the results of calibration and validations based on the average of cosine of change in direction

\begin{tabular}{|c|c|c|c|c|c|}
\hline \multicolumn{2}{|c|}{ Configurations } & Calibration & $1^{\text {st }}$ Validation & $2^{\text {nd }}$ Validation & $3^{\text {rd }}$ Validation \\
\hline$\varphi_{g}$ & $\Lambda_{l}$ & Kappa & Kappa & Kappa & Kappa \\
\hline 1 & 1 & 0.08 & 0.05 & 0.07 & 0.06 \\
\hline 1 & 2 & 0.07 & 0.05 & 0.06 & 0.06 \\
\hline 1 & 3 & 0.06 & 0.06 & 0.02 & 0.04 \\
\hline 1 & 4 & 0.07 & 0.04 & 0.04 & 0.04 \\
\hline 2 & 1 & 0.08 & 0.06 & 0.06 & 0.06 \\
\hline 2 & 2 & 0.08 & 0.05 & 0.06 & 0.06 \\
\hline 2 & 3 & 0.06 & 0.06 & 0.02 & 0.04 \\
\hline 2 & 4 & 0.07 & 0.03 & 0.04 & 0.04 \\
\hline 3 & 1 & 0.11 & 0.07 & 0.07 & 0.07 \\
\hline 3 & 2 & 0.11 & 0.04 & 0.03 & 0.04 \\
\hline 3 & 3 & 0.10 & 0.08 & 0.03 & 0.05 \\
\hline 3 & 4 & 0.10 & 0.05 & 0.05 & 0.05 \\
\hline 4 & 1 & 0.17 & 0.05 & 0.09 & 0.07 \\
\hline 4 & 2 & 0.17 & 0.05 & 0.09 & 0.07 \\
\hline 4 & 3 & 0.15 & 0.08 & 0.04 & 0.07 \\
\hline 4 & 4 & 0.15 & 0.06 & 0.06 & 0.06 \\
\hline 5 & 1 & 0.17 & 0.06 & 0.10 & 0.08 \\
\hline 5 & 2 & 0.17 & 0.08 & - & - \\
\hline 5 & 3 & 0.16 & 0.07 & - & - \\
\hline 5 & 4 & 0.16 & 0.04 & 0.06 & 0.05 \\
\hline
\end{tabular}


Table C.1.13 the results of calibration and validations based on the average area

\begin{tabular}{|c|c|c|c|c|c|}
\hline \multicolumn{2}{|c|}{ Configurations } & \multirow{2}{*}{$\begin{array}{c}\text { Calibration } \\
\text { Kappa }\end{array}$} & \multirow{2}{*}{$\frac{1^{\text {st }} \text { Validation }}{\text { Kappa }}$} & \multirow{2}{*}{$\begin{array}{c}2^{\text {nd }} \text { Validation } \\
\text { Kappa }\end{array}$} & \multirow{2}{*}{$\frac{3^{\text {rd }} \text { Validation }}{\text { Kappa }}$} \\
\hline$\varphi_{g}$ & $\Lambda_{l}$ & & & & \\
\hline 1 & 1 & 0.84 & 0.74 & 0.78 & 0.77 \\
\hline 1 & 2 & 0.80 & 0.72 & 0.78 & 0.75 \\
\hline 1 & 3 & 0.78 & 0.68 & 0.74 & 0.72 \\
\hline 1 & 4 & 0.78 & 0.70 & 0.75 & 0.73 \\
\hline 2 & 1 & 0.87 & 0.75 & 0.79 & 0.77 \\
\hline 2 & 2 & 0.83 & 0.71 & 0.74 & 0.73 \\
\hline 2 & 3 & 0.81 & 0.68 & - & - \\
\hline 2 & 4 & 0.83 & 0.71 & 0.73 & 0.73 \\
\hline 3 & 1 & 0.89 & 0.71 & 0.79 & 0.76 \\
\hline 3 & 2 & 0.86 & 0.68 & 0.75 & 0.72 \\
\hline 3 & 3 & 0.83 & 0.63 & - & - \\
\hline 3 & 4 & 0.86 & 0.68 & 0.75 & 0.72 \\
\hline 4 & 1 & 0.92 & 0.73 & - & - \\
\hline 4 & 2 & 0.89 & 0.62 & - & - \\
\hline 4 & 3 & 0.89 & - & - & - \\
\hline 4 & 4 & 0.89 & 0.62 & 0.81 & 0.77 \\
\hline 5 & 1 & 0.92 & 0.66 & 0.57 & 0.62 \\
\hline 5 & 2 & 0.91 & 0.57 & 0.61 & 0.59 \\
\hline 5 & 3 & 0.90 & 0.57 & 0.60 & 0.59 \\
\hline 5 & 4 & 0.91 & 0.57 & 0.61 & 0.59 \\
\hline
\end{tabular}

Table C.1.14 the results of calibration and validations based on the maximum area

\begin{tabular}{|c|c|c|c|c|c|}
\hline \multicolumn{2}{|c|}{ Configurations } & \multirow{2}{*}{$\begin{array}{c}\text { Calibration } \\
\text { Kappa } \\
\end{array}$} & \multirow{2}{*}{$\begin{array}{c}\mathbf{1}^{\text {st }} \text { Validation } \\
\text { Kappa } \\
\end{array}$} & \multirow{2}{*}{$\begin{array}{cl}2^{\text {nd }} & \text { Validation } \\
& \text { Kappa } \\
\end{array}$} & \multirow{2}{*}{$\begin{array}{c}3^{\text {rd }} \text { Validation } \\
\text { Kappa } \\
\end{array}$} \\
\hline$\varphi_{g}$ & $\Lambda_{l}$ & & & & \\
\hline 1 & 1 & 0.86 & 0.86 & 0.91 & 0.89 \\
\hline 1 & 2 & 0.86 & 0.86 & - & - \\
\hline 1 & 3 & 0.86 & 0.86 & - & - \\
\hline 1 & 4 & 0.83 & 0.83 & - & - \\
\hline 2 & 1 & 0.91 & 0.87 & 0.80 & 0.83 \\
\hline 2 & 2 & 0.91 & 0.87 & 0.80 & 0.83 \\
\hline 2 & 3 & 0.91 & 0.87 & 0.79 & 0.83 \\
\hline 2 & 4 & 0.91 & 0.87 & 0.79 & 0.83 \\
\hline 3 & 1 & 0.91 & 0.80 & 0.70 & 0.75 \\
\hline 3 & 2 & 0.91 & 0.80 & 0.70 & 0.75 \\
\hline 3 & 3 & 0.91 & 0.80 & 0.70 & 0.75 \\
\hline 3 & 4 & 0.91 & 0.80 & 0.70 & 0.75 \\
\hline 4 & 1 & 0.93 & 0.83 & 0.74 & 0.79 \\
\hline 4 & 2 & 0.93 & 0.83 & 0.76 & 0.80 \\
\hline 4 & 3 & 0.93 & 0.83 & 0.75 & 0.79 \\
\hline 4 & 4 & 0.93 & 0.83 & 0.76 & 0.80 \\
\hline 5 & 1 & 0.93 & 0.86 & 0.68 & 0.78 \\
\hline 5 & 2 & 0.93 & 0.86 & 0.70 & 0.79 \\
\hline 5 & 3 & 0.93 & 0.86 & 0.68 & 0.78 \\
\hline 5 & 4 & 0.93 & 0.86 & 0.70 & 0.79 \\
\hline
\end{tabular}


Table C.1.15 the results of calibration and validations based on the average length

\begin{tabular}{|c|c|c|c|c|c|}
\hline \multicolumn{2}{|c|}{ Configurations } & \multirow{2}{*}{$\begin{array}{c}\text { Calibration } \\
\text { Kappa }\end{array}$} & \multirow{2}{*}{$\begin{array}{c}\mathbf{1}^{\text {st }} \text { Validation } \\
\text { Kappa }\end{array}$} & \multirow{2}{*}{$\begin{array}{ll}2^{\text {nd }} & \text { Validation } \\
& \text { Kappa }\end{array}$} & \multirow{2}{*}{$\frac{3^{\text {rd }} \text { Validation }}{\text { Kappa }}$} \\
\hline$\varphi_{g}$ & $\Lambda_{l}$ & & & & \\
\hline 1 & 1 & 0.84 & 0.65 & 0.87 & 0.77 \\
\hline 1 & 2 & 0.83 & 0.65 & 0.87 & 0.77 \\
\hline 1 & 3 & 0.82 & 0.64 & 0.86 & 0.76 \\
\hline 1 & 4 & 0.79 & 0.63 & 0.85 & 0.75 \\
\hline 2 & 1 & 0.85 & 0.62 & 0.84 & 0.75 \\
\hline 2 & 2 & 0.83 & 0.62 & 0.84 & 0.75 \\
\hline 2 & 3 & 0.84 & 0.61 & 0.83 & 0.73 \\
\hline 2 & 4 & 0.83 & 0.62 & 0.83 & 0.74 \\
\hline 3 & 1 & 0.87 & 0.58 & 0.86 & 0.73 \\
\hline 3 & 2 & 0.85 & 0.57 & 0.81 & 0.70 \\
\hline 3 & 3 & 0.86 & 0.57 & 0.85 & 0.72 \\
\hline 3 & 4 & 0.85 & 0.57 & 0.81 & 0.70 \\
\hline 4 & 1 & 0.89 & 0.59 & 0.88 & 0.73 \\
\hline 4 & 2 & 0.87 & 0.58 & 0.85 & 0.72 \\
\hline 4 & 3 & 0.88 & 0.58 & 0.88 & 0.73 \\
\hline 4 & 4 & 0.87 & 0.58 & 0.85 & 0.72 \\
\hline 5 & 1 & 0.95 & 0.70 & 0.87 & 0.78 \\
\hline 5 & 2 & 0.93 & 0.69 & 0.83 & 0.76 \\
\hline 5 & 3 & 0.93 & 0.69 & 0.83 & 0.76 \\
\hline 5 & 4 & 0.93 & 0.69 & 0.83 & 0.76 \\
\hline
\end{tabular}

Table C.1.16 the results of calibration and validations based on the maximum length

\begin{tabular}{|c|c|c|c|c|c|}
\hline \multicolumn{2}{|c|}{ Configurations } & \multirow{2}{*}{$\begin{array}{c}\text { Calibration } \\
\text { Kappa } \\
\end{array}$} & \multirow{2}{*}{$\begin{array}{c}\mathbf{1}^{\text {st }} \text { Validation } \\
\text { Kappa }\end{array}$} & \multirow{2}{*}{$\begin{array}{ll}2^{\text {nd }} & \text { Validation } \\
& \text { Kappa } \\
\end{array}$} & \multirow{2}{*}{$\begin{array}{c}\mathbf{3}^{\text {rd }} \text { Validation } \\
\text { Kappa }\end{array}$} \\
\hline$\varphi_{g}$ & $\Lambda_{l}$ & & & & \\
\hline 1 & 1 & 0.81 & 0.68 & 0.96 & 0.84 \\
\hline 1 & 2 & 0.75 & 0.61 & 0.93 & - \\
\hline 1 & 3 & - & - & - & - \\
\hline 1 & 4 & 0.73 & 0.59 & - & - \\
\hline 2 & 1 & 0.83 & 0.59 & 0.92 & 0.78 \\
\hline 2 & 2 & 0.78 & - & - & - \\
\hline 2 & 3 & - & - & - & - \\
\hline 2 & 4 & 0.78 & 0.63 & 0.91 & 0.79 \\
\hline 3 & 1 & 0.86 & 0.60 & 0.95 & 0.81 \\
\hline 3 & 2 & 0.80 & 0.56 & 0.94 & 0.78 \\
\hline 3 & 3 & - & - & - & - \\
\hline 3 & 4 & 0.80 & 0.56 & 0.94 & 0.78 \\
\hline 4 & 1 & 0.89 & 0.61 & 0.95 & 0.79 \\
\hline 4 & 2 & 0.83 & 0.53 & 0.93 & 0.73 \\
\hline 4 & 3 & - & - & - & - \\
\hline 4 & 4 & 0.83 & 0.53 & 0.93 & 0.73 \\
\hline 5 & 1 & 0.91 & - & - & - \\
\hline 5 & 2 & 0.87 & - & - & - \\
\hline 5 & 3 & 0.84 & 0.59 & 0.91 & 0.78 \\
\hline 5 & 4 & 0.87 & 0.74 & 0.95 & 0.84 \\
\hline
\end{tabular}


Table C.1.17 the results of calibration and validations based on the average width

\begin{tabular}{|c|c|c|c|c|c|}
\hline \multicolumn{2}{|c|}{ Configurations } & \multirow{2}{*}{$\begin{array}{c}\text { Calibration } \\
\text { Kappa }\end{array}$} & \multirow{2}{*}{$\frac{1^{\text {st }} \text { Validation }}{\text { Kappa }}$} & \multirow{2}{*}{$\begin{array}{c}\text { Validation } 2 \\
\text { Kappa }\end{array}$} & \multirow{2}{*}{$\frac{3^{\text {rd }} \text { Validation }}{\text { Kappa }}$} \\
\hline$\varphi_{g}$ & $\Lambda_{l}$ & & & & \\
\hline 1 & 1 & 0.78 & 0.75 & 0.73 & 0.74 \\
\hline 1 & 2 & 0.78 & 0.76 & 0.76 & 0.76 \\
\hline 1 & 3 & 0.76 & 0.72 & 0.74 & 0.73 \\
\hline 1 & 4 & 0.75 & 0.74 & 0.74 & 0.74 \\
\hline 2 & 1 & 0.83 & 0.76 & 0.74 & 0.75 \\
\hline 2 & 2 & 0.82 & 0.73 & 0.70 & 0.72 \\
\hline 2 & 3 & 0.79 & 0.71 & 0.68 & 0.70 \\
\hline 2 & 4 & 0.82 & 0.73 & 0.69 & 0.71 \\
\hline 3 & 1 & 0.87 & 0.72 & 0.74 & 0.74 \\
\hline 3 & 2 & 0.86 & 0.70 & 0.69 & 0.70 \\
\hline 3 & 3 & 0.84 & 0.68 & 0.67 & 0.68 \\
\hline 3 & 4 & 0.86 & 0.70 & 0.69 & 0.70 \\
\hline 4 & 1 & 0.89 & 0.75 & 0.76 & 0.76 \\
\hline 4 & 2 & 0.88 & 0.74 & 0.76 & 0.76 \\
\hline 4 & 3 & 0.87 & 0.72 & 0.74 & 0.73 \\
\hline 4 & 4 & 0.88 & 0.74 & 0.76 & 0.76 \\
\hline 5 & 1 & 0.90 & 0.75 & 0.76 & 0.75 \\
\hline 5 & 2 & 0.88 & 0.75 & 0.76 & 0.75 \\
\hline 5 & 3 & 0.87 & 0.65 & 0.64 & - \\
\hline 5 & 4 & 0.88 & 0.75 & 0.76 & 0.75 \\
\hline
\end{tabular}

Table C.1.18 the results of calibration and validations based on the maximum width

\begin{tabular}{|c|c|c|c|c|c|}
\hline \multicolumn{2}{|c|}{ Configurations } & \multirow{2}{*}{$\begin{array}{c}\text { Calibration } \\
\text { Kappa } \\
\end{array}$} & \multirow{2}{*}{$\begin{array}{c}\mathbf{1}^{\text {st }} \text { Validation } \\
\text { Kappa }\end{array}$} & \multirow{2}{*}{$\begin{array}{ll}2^{\text {nd }} & \text { Validation } \\
& \text { Kappa } \\
\end{array}$} & \multirow{2}{*}{$\begin{array}{c}\mathbf{3}^{\text {rd }} \text { Validation } \\
\text { Kappa }\end{array}$} \\
\hline$\varphi_{g}$ & $\Lambda_{l}$ & & & & \\
\hline 1 & 1 & 0.74 & 0.82 & 0.71 & 0.76 \\
\hline 1 & 2 & 0.75 & 0.86 & 0.76 & 0.81 \\
\hline 1 & 3 & 0.75 & 0.82 & 0.70 & 0.76 \\
\hline 1 & 4 & 0.73 & 0.84 & 0.74 & 0.79 \\
\hline 2 & 1 & 0.83 & 0.83 & - & - \\
\hline 2 & 2 & 0.83 & 0.88 & 0.75 & 0.81 \\
\hline 2 & 3 & 0.83 & 0.83 & 0.68 & - \\
\hline 2 & 4 & 0.83 & 0.88 & 0.74 & 0.81 \\
\hline 3 & 1 & 0.89 & 0.83 & 0.66 & 0.74 \\
\hline 3 & 2 & 0.88 & 0.80 & 0.64 & 0.72 \\
\hline 3 & 3 & 0.88 & 0.81 & 0.65 & 0.73 \\
\hline 3 & 4 & 0.88 & 0.80 & 0.64 & 0.72 \\
\hline 4 & 1 & 0.90 & 0.85 & 0.70 & 0.78 \\
\hline 4 & 2 & 0.90 & 0.80 & 0.67 & 0.74 \\
\hline 4 & 3 & 0.89 & 0.83 & 0.68 & 0.76 \\
\hline 4 & 4 & 0.90 & 0.80 & 0.67 & 0.74 \\
\hline 5 & 1 & 0.94 & 0.86 & 0.67 & 0.77 \\
\hline 5 & 2 & 0.94 & 0.86 & 0.68 & 0.78 \\
\hline 5 & 3 & 0.92 & 0.86 & 0.67 & 0.77 \\
\hline 5 & 4 & 0.94 & 0.86 & 0.68 & 0.78 \\
\hline
\end{tabular}


Table C.1.19 the results of calibration and validations based on the peaks in speed

\begin{tabular}{|c|c|c|c|c|c|}
\hline \multicolumn{2}{|c|}{ Configurations } & \multirow{2}{*}{$\begin{array}{c}\text { Calibration } \\
\text { Kappa }\end{array}$} & \multirow{2}{*}{$\frac{1^{\text {st }} \text { Validation }}{\text { Kappa }}$} & \multirow{2}{*}{$\begin{array}{ll}2^{\text {nd }} & \text { Validation } \\
& \text { Kappa }\end{array}$} & \multirow{2}{*}{$\frac{3^{\text {rd }} \text { Validation }}{\text { Kappa }}$} \\
\hline$\varphi_{g}$ & $\Lambda_{l}$ & & & & \\
\hline 1 & 1 & 0.22 & 0.31 & 0.25 & 0.28 \\
\hline 1 & 2 & 0.20 & 0.31 & 0.25 & 0.27 \\
\hline 1 & 3 & 0.20 & 0.26 & 0.21 & 0.23 \\
\hline 1 & 4 & - & 0.28 & 0.23 & 0.25 \\
\hline 2 & 1 & 0.25 & 0.34 & 0.37 & 0.36 \\
\hline 2 & 2 & 0.22 & 0.27 & 0.30 & 0.29 \\
\hline 2 & 3 & 0.24 & 0.23 & 0.25 & - \\
\hline 2 & 4 & 0.24 & 0.28 & - & - \\
\hline 3 & 1 & 0.27 & 0.30 & 0.36 & 0.34 \\
\hline 3 & 2 & 0.26 & 0.25 & 0.34 & 0.31 \\
\hline 3 & 3 & 0.25 & 0.24 & 0.31 & 0.29 \\
\hline 3 & 4 & 0.29 & 0.22 & 0.33 & 0.29 \\
\hline 4 & 1 & 0.29 & 0.29 & 0.35 & 0.33 \\
\hline 4 & 2 & 0.28 & 0.26 & 0.30 & 0.29 \\
\hline 4 & 3 & 0.25 & 0.25 & 0.27 & 0.26 \\
\hline 4 & 4 & 0.33 & 0.20 & 0.30 & 0.26 \\
\hline 5 & 1 & 0.27 & 0.33 & 0.38 & 0.36 \\
\hline 5 & 2 & 0.25 & 0.30 & 0.32 & 0.32 \\
\hline 5 & 3 & 0.23 & 0.26 & 0.23 & 0.25 \\
\hline 5 & 4 & 0.35 & 0.17 & 0.33 & 0.26 \\
\hline
\end{tabular}

Table C.1.20 the results of calibration and validations based on the effective weighted average frequency

\begin{tabular}{|c|c|c|c|c|c|c|}
\hline \multicolumn{3}{|c|}{ Configurations } & Calibration & $1^{\text {st }}$ Validation & $2^{\text {nd }}$ Validation & $3^{\text {rd }}$ Validation \\
\hline$\varphi_{g}$ & $\Lambda_{l}$ & $\Gamma_{n}$ & Kappa & Kappa & Kappa & Kappa \\
\hline 1 & 1 & 1 & 0.21 & 0.33 & 0.28 & 0.32 \\
\hline 1 & 1 & 2 & 0.20 & 0.31 & 0.25 & 0.29 \\
\hline 1 & 1 & 3 & 0.20 & 0.30 & 0.25 & 0.29 \\
\hline 1 & 1 & 4 & 0.20 & 0.31 & 0.25 & 0.29 \\
\hline 1 & 1 & 1 & 0.20 & 0.32 & 0.26 & 0.30 \\
\hline 1 & 1 & 6 & 0.21 & 0.32 & 0.31 & 0.33 \\
\hline 1 & 2 & 1 & 0.18 & 0.25 & 0.23 & - \\
\hline 1 & 2 & 2 & 0.18 & 0.26 & 0.22 & - \\
\hline 1 & 2 & 3 & 0.18 & 0.25 & 0.23 & - \\
\hline 1 & 2 & 4 & 0.18 & 0.26 & 0.22 & - \\
\hline 1 & 2 & 1 & 0.18 & 0.27 & 0.22 & - \\
\hline 1 & 2 & 6 & 0.18 & 0.27 & 0.21 & - \\
\hline 1 & 3 & 1 & 0.15 & - & - & - \\
\hline 1 & 3 & 2 & 0.15 & - & - & - \\
\hline 1 & 3 & 3 & 0.15 & - & - & - \\
\hline 1 & 3 & 4 & 0.15 & 0.24 & - & - \\
\hline 1 & 3 & 1 & 0.16 & 0.24 & 0.21 & - \\
\hline 1 & 3 & 6 & 0.16 & 0.23 & - & - \\
\hline 1 & 4 & 1 & 0.17 & 0.26 & 0.24 & - \\
\hline 1 & 4 & 2 & 0.17 & 0.26 & 0.23 & - \\
\hline 1 & 4 & 3 & 0.17 & 0.25 & 0.23 & - \\
\hline 1 & 4 & 4 & 0.17 & 0.26 & 0.22 & - \\
\hline 1 & 4 & 1 & 0.18 & 0.26 & 0.23 & - \\
\hline 1 & 4 & 6 & 0.18 & 0.26 & 0.22 & - \\
\hline
\end{tabular}


Table C.1.20 the results of calibration and validations based on the effective weighted average frequency

\begin{tabular}{|c|c|c|c|c|c|c|}
\hline \multicolumn{3}{|c|}{ Configurations } & \multirow{2}{*}{$\begin{array}{c}\text { Calibration } \\
\text { Kappa } \\
\end{array}$} & \multirow{2}{*}{$\begin{array}{c}\mathbf{1}^{\text {st }} \text { Validation } \\
\text { Kappa } \\
\end{array}$} & \multirow{2}{*}{$\begin{array}{cl}2^{\text {nd }} & \text { Validation } \\
\text { Kappa } \\
\end{array}$} & \multirow{2}{*}{$\begin{array}{c}3^{\text {rd }} \text { Validation } \\
\text { Kappa } \\
\end{array}$} \\
\hline$\varphi_{g}$ & $\Lambda_{l}$ & $\Gamma_{n}$ & & & & \\
\hline 2 & 1 & 1 & 0.26 & 0.34 & 0.28 & 0.33 \\
\hline 2 & 1 & 2 & 0.25 & 0.33 & 0.30 & 0.33 \\
\hline 2 & 1 & 3 & 0.25 & 0.31 & 0.26 & 0.30 \\
\hline 2 & 1 & 4 & 0.25 & 0.32 & 0.25 & 0.30 \\
\hline 2 & 1 & 1 & 0.25 & 0.33 & 0.27 & 0.31 \\
\hline 2 & 1 & 6 & 0.24 & 0.33 & 0.32 & 0.34 \\
\hline 2 & 2 & 1 & 0.22 & 0.26 & 0.24 & - \\
\hline 2 & 2 & 2 & 0.21 & 0.27 & 0.23 & - \\
\hline 2 & 2 & 3 & 0.21 & 0.26 & 0.23 & - \\
\hline 2 & 2 & 4 & 0.22 & 0.27 & 0.22 & - \\
\hline 2 & 2 & 1 & 0.21 & 0.27 & - & - \\
\hline 2 & 2 & 6 & 0.22 & 0.28 & - & - \\
\hline 2 & 3 & 1 & 0.19 & - & - & - \\
\hline 2 & 3 & 2 & 0.19 & - & - & - \\
\hline 2 & 3 & 3 & 0.19 & - & - & - \\
\hline 2 & 3 & 4 & 0.19 & - & - & - \\
\hline 2 & 3 & 1 & 0.19 & 0.24 & - & - \\
\hline 2 & 3 & 6 & 0.19 & 0.24 & - & - \\
\hline 2 & 4 & 1 & 0.21 & 0.26 & 0.24 & - \\
\hline 2 & 4 & 2 & 0.21 & 0.27 & 0.23 & - \\
\hline 2 & 4 & 3 & 0.21 & 0.26 & 0.23 & - \\
\hline 2 & 4 & 4 & 0.21 & 0.27 & 0.22 & - \\
\hline 2 & 4 & 1 & 0.21 & 0.27 & 0.23 & - \\
\hline 2 & 4 & 6 & 0.22 & 0.27 & - & - \\
\hline 3 & 1 & 1 & 0.34 & 0.40 & 0.32 & 0.37 \\
\hline 3 & 1 & 2 & 0.33 & 0.39 & 0.34 & 0.38 \\
\hline 3 & 1 & 3 & 0.32 & 0.39 & 0.32 & 0.37 \\
\hline 3 & 1 & 4 & 0.32 & 0.38 & 0.29 & 0.35 \\
\hline 3 & 1 & 1 & 0.32 & 0.39 & 0.31 & 0.36 \\
\hline 3 & 1 & 6 & 0.32 & 0.40 & 0.34 & 0.38 \\
\hline 3 & 2 & 1 & 0.28 & 0.30 & 0.26 & - \\
\hline 3 & 2 & 2 & 0.27 & 0.31 & 0.26 & - \\
\hline 3 & 2 & 3 & 0.27 & 0.30 & 0.26 & - \\
\hline 3 & 2 & 4 & 0.28 & 0.31 & 0.25 & - \\
\hline 3 & 2 & 1 & 0.27 & 0.32 & 0.24 & - \\
\hline 3 & 2 & 6 & 0.28 & 0.32 & 0.24 & - \\
\hline 3 & 3 & 1 & 0.23 & - & - & - \\
\hline 3 & 3 & 2 & 0.24 & - & - & - \\
\hline 3 & 3 & 3 & 0.24 & - & - & - \\
\hline 3 & 3 & 4 & 0.24 & - & - & - \\
\hline 3 & 3 & 1 & 0.24 & - & - & - \\
\hline 3 & 3 & 6 & 0.25 & - & - & - \\
\hline 3 & 4 & 1 & 0.27 & 0.30 & 0.26 & - \\
\hline 3 & 4 & 2 & 0.27 & 0.31 & 0.26 & - \\
\hline 3 & 4 & 3 & 0.26 & 0.30 & 0.26 & - \\
\hline 3 & 4 & 4 & 0.26 & 0.31 & 0.24 & - \\
\hline 3 & 4 & 1 & 0.27 & 0.31 & 0.25 & - \\
\hline 3 & 4 & 6 & 0.27 & 0.32 & 0.24 & - \\
\hline 4 & 1 & 1 & 0.45 & 0.44 & 0.43 & 0.44 \\
\hline 4 & 1 & 2 & 0.44 & 0.45 & 0.42 & 0.45 \\
\hline
\end{tabular}


Table C.1.20 the results of calibration and validations based on the effective weighted average frequency

\begin{tabular}{|c|c|c|c|c|c|c|}
\hline \multicolumn{3}{|c|}{ Configurations } & Calibration & $1^{\text {st }}$ Validation & $2^{\text {nd }}$ Validation & $3^{\text {rd }}$ Validation \\
\hline$\overline{\varphi_{g}}$ & $\overline{\Lambda_{l}}$ & $\overline{\Gamma_{n}}$ & Kappa & Kappa & Kappa & Kappa \\
\hline 4 & 1 & 3 & 0.43 & 0.45 & 0.43 & 0.45 \\
\hline 4 & 1 & 4 & 0.43 & 0.45 & 0.38 & 0.43 \\
\hline 4 & 1 & 1 & 0.42 & 0.45 & 0.39 & 0.43 \\
\hline 4 & 1 & 6 & 0.41 & 0.46 & 0.39 & 0.44 \\
\hline 4 & 2 & 1 & 0.37 & 0.35 & - & - \\
\hline 4 & 2 & 2 & 0.36 & 0.35 & 0.30 & - \\
\hline 4 & 2 & 3 & 0.37 & 0.35 & 0.32 & - \\
\hline 4 & 2 & 4 & 0.37 & 0.36 & 0.31 & - \\
\hline 4 & 2 & 1 & 0.37 & 0.36 & 0.31 & - \\
\hline 4 & 2 & 6 & 0.38 & 0.37 & 0.31 & - \\
\hline 4 & 3 & 1 & 0.34 & - & - & - \\
\hline 4 & 3 & 2 & 0.35 & - & - & - \\
\hline 4 & 3 & 3 & 0.35 & - & - & - \\
\hline 4 & 3 & 4 & 0.34 & - & - & - \\
\hline 4 & 3 & 1 & 0.35 & - & - & - \\
\hline 4 & 3 & 6 & 0.35 & - & - & - \\
\hline 4 & 4 & 1 & 0.37 & 0.35 & - & - \\
\hline 4 & 4 & 2 & 0.36 & 0.36 & - & - \\
\hline 4 & 4 & 3 & 0.36 & 0.35 & 0.31 & - \\
\hline 4 & 4 & 4 & 0.36 & 0.35 & 0.31 & - \\
\hline 4 & 4 & 1 & 0.36 & 0.36 & 0.31 & - \\
\hline 4 & 4 & 6 & 0.37 & 0.36 & 0.32 & - \\
\hline 5 & 1 & 1 & 0.48 & 0.47 & 0.48 & 0.49 \\
\hline 5 & 1 & 2 & 0.47 & 0.48 & 0.49 & 0.49 \\
\hline 5 & 1 & 3 & 0.45 & 0.46 & 0.40 & 0.44 \\
\hline 5 & 1 & 4 & 0.45 & 0.47 & 0.40 & 0.45 \\
\hline 5 & 1 & 1 & 0.44 & 0.48 & 0.43 & 0.47 \\
\hline 5 & 1 & 6 & 0.43 & 0.47 & 0.43 & 0.46 \\
\hline 5 & 2 & 1 & 0.41 & 0.41 & 0.34 & 0.39 \\
\hline 5 & 2 & 2 & 0.39 & 0.42 & 0.37 & 0.41 \\
\hline 5 & 2 & 3 & 0.41 & 0.41 & 0.35 & 0.39 \\
\hline 5 & 2 & 4 & 0.40 & 0.40 & 0.36 & 0.40 \\
\hline 5 & 2 & 1 & 0.40 & 0.39 & - & - \\
\hline 5 & 2 & 6 & 0.40 & 0.40 & - & - \\
\hline 5 & 3 & 1 & 0.37 & 0.36 & - & - \\
\hline 5 & 3 & 2 & 0.38 & 0.36 & - & - \\
\hline 5 & 3 & 3 & 0.39 & 0.35 & - & - \\
\hline 5 & 3 & 4 & 0.38 & 0.37 & - & - \\
\hline 5 & 3 & 1 & 0.38 & 0.37 & - & - \\
\hline 5 & 3 & 6 & 0.38 & 0.38 & - & - \\
\hline 5 & 4 & 1 & 0.41 & 0.41 & 0.34 & 0.39 \\
\hline 5 & 4 & 2 & 0.39 & 0.41 & 0.35 & 0.40 \\
\hline 5 & 4 & 3 & 0.40 & 0.40 & 0.36 & 0.39 \\
\hline 5 & 4 & 4 & 0.39 & 0.40 & 0.36 & 0.39 \\
\hline 5 & 4 & 1 & 0.39 & 0.41 & 0.35 & 0.40 \\
\hline 5 & 4 & 6 & 0.39 & 0.42 & 0.35 & 0.40 \\
\hline
\end{tabular}


Table C.1.21 the results of calibration and validations based on the effective frequency

\begin{tabular}{|c|c|c|c|c|c|c|}
\hline \multicolumn{3}{|c|}{ Configurations } & \multirow{2}{*}{$\begin{array}{c}\text { Calibration } \\
\text { Kappa }\end{array}$} & \multirow{2}{*}{$\frac{\mathbf{1}^{\text {st }} \text { Validation }}{\text { Kappa }}$} & \multirow{2}{*}{$\begin{array}{ll}2^{\text {nd }} & \text { Validation } \\
\text { Kappa }\end{array}$} & \multirow{2}{*}{$\frac{3^{\text {rd }} \text { Validation }}{\text { Kappa }}$} \\
\hline$\varphi_{g}$ & $\Lambda_{l}$ & $\eta_{m}$ & & & & \\
\hline 1 & 1 & 1 & 0.14 & 0.04 & 0.10 & 0.06 \\
\hline 1 & 1 & 2 & 0.13 & 0.10 & 0.10 & 0.09 \\
\hline 1 & 1 & 3 & 0.14 & 0.13 & 0.09 & 0.10 \\
\hline 1 & 2 & 1 & 0.14 & 0.03 & 0.08 & 0.05 \\
\hline 1 & 2 & 2 & 0.12 & 0.09 & 0.09 & 0.08 \\
\hline 1 & 2 & 3 & 0.13 & 0.10 & 0.08 & 0.08 \\
\hline 1 & 3 & 1 & 0.12 & 0.02 & 0.06 & 0.03 \\
\hline 1 & 3 & 2 & 0.12 & 0.09 & 0.07 & 0.07 \\
\hline 1 & 3 & 3 & 0.13 & 0.09 & 0.07 & 0.07 \\
\hline 1 & 4 & 1 & 0.12 & 0.04 & 0.06 & 0.04 \\
\hline 1 & 4 & 2 & 0.11 & 0.07 & 0.07 & 0.07 \\
\hline 1 & 4 & 3 & 0.12 & 0.10 & 0.07 & 0.08 \\
\hline 2 & 1 & 1 & 0.11 & 0.04 & 0.07 & 0.05 \\
\hline 2 & 1 & 2 & 0.13 & 0.06 & 0.19 & 0.12 \\
\hline 2 & 1 & 3 & 0.15 & 0.10 & 0.09 & 0.09 \\
\hline 2 & 2 & 1 & 0.11 & 0.04 & 0.07 & 0.05 \\
\hline 2 & 2 & 2 & 0.12 & 0.05 & 0.16 & 0.10 \\
\hline 2 & 2 & 3 & 0.13 & 0.07 & 0.09 & 0.08 \\
\hline 2 & 3 & 1 & 0.11 & 0.03 & - & - \\
\hline 2 & 3 & 2 & 0.11 & 0.06 & 0.14 & 0.10 \\
\hline 2 & 3 & 3 & 0.12 & 0.07 & 0.07 & 0.07 \\
\hline 2 & 4 & 1 & 0.11 & 0.04 & 0.06 & 0.04 \\
\hline 2 & 4 & 2 & 0.10 & 0.06 & - & - \\
\hline 2 & 4 & 3 & 0.12 & 0.08 & 0.07 & 0.07 \\
\hline 3 & 1 & 1 & 0.13 & 0.07 & 0.07 & 0.06 \\
\hline 3 & 1 & 2 & 0.19 & 0.10 & 0.19 & 0.14 \\
\hline 3 & 1 & 3 & 0.13 & 0.10 & 0.20 & 0.15 \\
\hline 3 & 2 & 1 & 0.13 & 0.06 & 0.06 & 0.05 \\
\hline 3 & 2 & 2 & 0.19 & 0.10 & 0.16 & 0.12 \\
\hline 3 & 2 & 3 & 0.12 & 0.10 & 0.19 & 0.14 \\
\hline 3 & 3 & 1 & 0.12 & 0.06 & 0.06 & 0.05 \\
\hline 3 & 3 & 2 & 0.17 & 0.07 & 0.14 & 0.10 \\
\hline 3 & 3 & 3 & 0.12 & 0.09 & 0.16 & 0.12 \\
\hline 3 & 4 & 1 & 0.11 & 0.06 & 0.06 & 0.05 \\
\hline 3 & 4 & 2 & 0.14 & 0.06 & 0.12 & 0.09 \\
\hline 3 & 4 & 3 & 0.12 & 0.08 & 0.13 & 0.10 \\
\hline 4 & 1 & 1 & 0.14 & 0.05 & 0.07 & 0.05 \\
\hline 4 & 1 & 2 & 0.17 & 0.08 & 0.21 & 0.13 \\
\hline 4 & 1 & 3 & 0.12 & 0.09 & 0.13 & 0.10 \\
\hline 4 & 2 & 1 & 0.12 & 0.05 & 0.07 & 0.05 \\
\hline 4 & 2 & 2 & 0.15 & 0.08 & 0.11 & 0.08 \\
\hline 4 & 2 & 3 & 0.11 & 0.15 & 0.14 & 0.14 \\
\hline 4 & 3 & 1 & 0.12 & 0.05 & 0.07 & 0.05 \\
\hline 4 & 3 & 2 & 0.14 & 0.05 & 0.14 & 0.09 \\
\hline 4 & 3 & 3 & 0.11 & 0.08 & 0.11 & 0.08 \\
\hline 4 & 4 & 1 & 0.12 & 0.05 & 0.07 & 0.05 \\
\hline 4 & 4 & 2 & 0.14 & 0.06 & 0.13 & 0.08 \\
\hline 4 & 4 & 3 & 0.11 & 0.08 & 0.10 & 0.08 \\
\hline 5 & 1 & 1 & 0.14 & 0.08 & 0.12 & 0.09 \\
\hline 5 & 1 & 2 & 0.17 & 0.05 & 0.22 & 0.11 \\
\hline 5 & 1 & 3 & 0.20 & 0.13 & 0.04 & 0.10 \\
\hline
\end{tabular}


Table C.1.21 the results of calibration and validations based on the effective frequency

\begin{tabular}{|c|c|c|c|c|c|c|}
\hline \multicolumn{3}{|c|}{ Configurations } & Calibration & $1^{\text {st }}$ Validation & $2^{\text {nd }}$ Validation & $3^{\text {rd }}$ Validation \\
\hline$\varphi_{g}$ & $\Lambda_{l}$ & $\eta_{m}$ & Kappa & Kappa & Kappa & Kappa \\
\hline 5 & 2 & 1 & 0.13 & 0.07 & 0.11 & 0.08 \\
\hline 5 & 2 & 2 & 0.15 & 0.05 & 0.17 & 0.09 \\
\hline 5 & 2 & 3 & 0.19 & 0.12 & - & - \\
\hline 5 & 3 & 1 & 0.13 & 0.06 & 0.09 & 0.06 \\
\hline 5 & 3 & 2 & 0.14 & 0.04 & 0.14 & 0.07 \\
\hline 5 & 3 & 3 & 0.15 & 0.11 & 0.11 & 0.12 \\
\hline 5 & 4 & 1 & 0.12 & 0.06 & 0.07 & 0.05 \\
\hline 5 & 4 & 2 & 0.15 & 0.05 & 0.17 & 0.09 \\
\hline 5 & 4 & 3 & 0.14 & 0.15 & 0.09 & 0.12 \\
\hline
\end{tabular}




\section{C.2 The results of calibrations and validations in percentage scale}

Table C.2.1 the results of calibration procedure 1 and its validations.

\begin{tabular}{|c|c|c|c|c|c|c|c|c|c|c|c|}
\hline \multicolumn{4}{|c|}{ Configurations } & \multicolumn{2}{|c|}{ Calibration } & \multicolumn{2}{|c|}{$1^{\text {st }}$ Validation } & \multicolumn{2}{|c|}{$2^{\text {nd }}$ Validation } & \multicolumn{2}{|c|}{$3^{\text {rd }}$ Validation } \\
\hline$\varphi_{g}$ & $\overline{\Lambda_{l}}$ & $\Gamma_{n}$ & $\eta_{m}$ & Vehicles & Cyclists & Vehicles & Cyclists & Vehicles & Cyclists & Vehicles & Cyclists \\
\hline 1 & 1 & 1 & 1 & 99 & 91 & 96 & 95 & 91 & 98 & 94 & 97 \\
\hline 1 & 1 & 1 & 2 & 99 & 91 & 96 & 95 & 88 & 97 & 93 & 96 \\
\hline 1 & 1 & 1 & 3 & 99 & 91 & 96 & 95 & 88 & 97 & 93 & 96 \\
\hline 1 & 1 & 2 & 1 & 99 & 91 & 96 & 95 & 90 & 98 & 93 & 97 \\
\hline 1 & 1 & 2 & 2 & 99 & 91 & 96 & 95 & - & - & - & - \\
\hline 1 & 1 & 2 & 3 & 99 & 91 & 96 & 95 & - & - & - & - \\
\hline 1 & 1 & 3 & 1 & 99 & 91 & 96 & 95 & 91 & 97 & 94 & 96 \\
\hline 1 & 1 & 3 & 2 & 99 & 91 & 96 & 95 & - & - & - & - \\
\hline 1 & 1 & 3 & 3 & 99 & 91 & 96 & 95 & - & - & - & - \\
\hline 1 & 1 & 4 & 1 & 99 & 91 & 96 & 95 & 90 & 97 & 93 & 96 \\
\hline 1 & 1 & 4 & 2 & 99 & 91 & 96 & 95 & 89 & 97 & 93 & 96 \\
\hline 1 & 1 & 4 & 3 & 99 & 91 & 96 & 95 & 89 & 97 & 93 & 96 \\
\hline 1 & 1 & 1 & 1 & 99 & 91 & 96 & 95 & 92 & 98 & 94 & 97 \\
\hline 1 & 1 & 1 & 2 & 99 & 91 & 96 & 95 & 90 & 97 & 93 & 96 \\
\hline 1 & 1 & 1 & 3 & 99 & 91 & 96 & 95 & 90 & 97 & 93 & 96 \\
\hline 1 & 1 & 6 & 1 & 99 & 91 & 96 & 95 & 91 & 97 & 94 & 96 \\
\hline 1 & 1 & 6 & 2 & 99 & 91 & 96 & 95 & 89 & 97 & 93 & 96 \\
\hline 1 & 1 & 6 & 3 & 99 & 91 & 96 & 95 & 89 & 97 & 93 & 96 \\
\hline 1 & 2 & 1 & 1 & 99 & 95 & 96 & 96 & 92 & 97 & 94 & 96 \\
\hline 1 & 2 & 1 & 2 & 99 & 95 & 96 & 96 & 92 & 97 & 94 & 96 \\
\hline 1 & 2 & 1 & 3 & 99 & 95 & 96 & 96 & 92 & 97 & 94 & 96 \\
\hline 1 & 2 & 2 & 1 & 99 & 95 & 95 & 96 & 92 & 97 & 94 & 96 \\
\hline 1 & 2 & 2 & 2 & 99 & 95 & 95 & 96 & 92 & 97 & 94 & 96 \\
\hline 1 & 2 & 2 & 3 & 99 & 95 & 95 & 96 & 92 & 97 & 94 & 96 \\
\hline 1 & 2 & 3 & 1 & 99 & 95 & 95 & 96 & 93 & 97 & 94 & 96 \\
\hline 1 & 2 & 3 & 2 & 99 & 95 & 95 & 96 & 93 & 97 & 94 & 96 \\
\hline 1 & 2 & 3 & 3 & 99 & 95 & 95 & 96 & 93 & 97 & 94 & 96 \\
\hline 1 & 2 & 4 & 1 & 99 & 95 & 96 & 96 & 93 & 97 & 94 & 97 \\
\hline 1 & 2 & 4 & 2 & 99 & 95 & 96 & 96 & 93 & 97 & 94 & 97 \\
\hline 1 & 2 & 4 & 3 & 99 & 95 & 96 & 96 & 93 & 97 & 94 & 97 \\
\hline 1 & 2 & 1 & 1 & 99 & 95 & 96 & 96 & 93 & 97 & 94 & 96 \\
\hline 1 & 2 & 1 & 2 & 99 & 95 & 96 & 96 & 93 & 97 & 94 & 96 \\
\hline 1 & 2 & 1 & 3 & 99 & 95 & 96 & 96 & 93 & 97 & 94 & 96 \\
\hline 1 & 2 & 6 & 1 & 99 & 95 & 95 & 96 & 92 & 97 & 94 & 96 \\
\hline
\end{tabular}


Table C.2.1 the results of calibration procedure 1 and its validations.

\begin{tabular}{|c|c|c|c|c|c|c|c|c|c|c|c|}
\hline \multicolumn{4}{|c|}{ Configurations } & \multicolumn{2}{|c|}{ Calibration } & \multicolumn{2}{|c|}{$1^{\text {st }}$ Validation } & \multicolumn{2}{|c|}{$2^{\text {nd }}$ Validation } & \multicolumn{2}{|c|}{$3^{\text {rd }}$ Validation } \\
\hline$\varphi_{g}$ & $\Lambda_{l}$ & $\overline{\Gamma_{n}}$ & $\overline{\eta_{m}}$ & Vehicles & Cyclists & Vehicles & Cyclists & Vehicles & Cyclists & Vehicles & Cyclists \\
\hline 1 & 2 & 6 & 2 & 99 & 95 & 95 & 96 & 92 & 97 & 94 & 96 \\
\hline 1 & 2 & 6 & 3 & 99 & 95 & 95 & 96 & 92 & 97 & 94 & 96 \\
\hline 1 & 3 & 1 & 1 & 99 & 92 & 96 & 90 & - & - & - & - \\
\hline 1 & 3 & 1 & 2 & 99 & 92 & 96 & 90 & - & - & - & - \\
\hline 1 & 3 & 1 & 3 & 99 & 92 & 96 & 90 & - & - & - & - \\
\hline 1 & 3 & 2 & 1 & 99 & 93 & 96 & 90 & - & - & - & - \\
\hline 1 & 3 & 2 & 2 & 99 & 93 & 96 & 90 & - & - & - & - \\
\hline 1 & 3 & 2 & 3 & 99 & 93 & 96 & 90 & - & - & - & - \\
\hline 1 & 3 & 3 & 1 & 99 & 92 & 95 & 90 & - & - & - & - \\
\hline 1 & 3 & 3 & 2 & 99 & 92 & 95 & 90 & - & - & - & - \\
\hline 1 & 3 & 3 & 3 & 99 & 92 & 95 & 90 & - & - & - & - \\
\hline 1 & 3 & 4 & 1 & 99 & 93 & 96 & 90 & - & - & - & - \\
\hline 1 & 3 & 4 & 2 & 99 & 93 & 96 & 90 & - & - & - & - \\
\hline 1 & 3 & 4 & 3 & 99 & 93 & 96 & 90 & - & - & - & - \\
\hline 1 & 3 & 1 & 1 & 99 & 93 & 96 & 90 & - & - & - & - \\
\hline 1 & 3 & 1 & 2 & 99 & 93 & 96 & 90 & - & - & - & - \\
\hline 1 & 3 & 1 & 3 & 99 & 93 & 96 & 90 & - & - & - & - \\
\hline 1 & 3 & 6 & 1 & 99 & 93 & 96 & 90 & - & - & - & - \\
\hline 1 & 3 & 6 & 2 & 99 & 93 & 96 & 90 & - & - & - & - \\
\hline 1 & 3 & 6 & 3 & 99 & 93 & 96 & 90 & - & - & - & - \\
\hline 1 & 4 & 1 & 1 & - & - & - & - & - & - & - & - \\
\hline 1 & 4 & 1 & 2 & - & - & - & - & - & - & - & - \\
\hline 1 & 4 & 1 & 3 & - & - & - & - & - & - & - & - \\
\hline 1 & 4 & 2 & 1 & - & - & - & - & - & - & - & - \\
\hline 1 & 4 & 2 & 2 & - & - & - & - & - & - & - & - \\
\hline 1 & 4 & 2 & 3 & - & - & - & - & - & - & - & - \\
\hline 1 & 4 & 3 & 1 & - & - & - & - & - & - & - & - \\
\hline 1 & 4 & 3 & 2 & - & - & - & - & - & - & - & - \\
\hline 1 & 4 & 3 & 3 & - & - & - & - & - & - & - & - \\
\hline 1 & 4 & 4 & 1 & - & - & - & - & - & - & - & - \\
\hline 1 & 4 & 4 & 2 & - & - & - & - & - & - & - & - \\
\hline 1 & 4 & 4 & 3 & - & - & - & - & - & - & - & - \\
\hline 1 & 4 & 1 & 1 & - & - & - & - & - & - & - & - \\
\hline 1 & 4 & 1 & 2 & - & - & - & - & - & - & - & - \\
\hline 1 & 4 & 1 & 3 & - & - & - & - & - & - & - & - \\
\hline 1 & 4 & 6 & 1 & - & - & - & - & - & - & - & - \\
\hline
\end{tabular}


Table C.2.1 the results of calibration procedure 1 and its validations.

\begin{tabular}{|c|c|c|c|c|c|c|c|c|c|c|c|}
\hline \multicolumn{4}{|c|}{ Configurations } & \multicolumn{2}{|c|}{ Calibration } & \multicolumn{2}{|c|}{$1^{\text {st }}$ Validation } & \multicolumn{2}{|c|}{$2^{\text {nd }}$ Validation } & \multicolumn{2}{|c|}{$3^{\text {rd }}$ Validation } \\
\hline$\overline{\varphi_{g}}$ & $\Lambda_{l}$ & $\overline{\Gamma_{n}}$ & $\overline{\eta_{m}}$ & Vehicles & Cyclists & Vehicles & Cyclists & Vehicles & Cyclists & Vehicles & Cyclists \\
\hline 1 & 4 & 6 & 2 & - & - & - & - & - & - & - & - \\
\hline 1 & 4 & 6 & 3 & - & - & - & - & - & - & - & - \\
\hline 2 & 1 & 1 & 1 & \begin{tabular}{|c|}
99 \\
\end{tabular} & 98 & 94 & 93 & 89 & 97 & 92 & 96 \\
\hline 2 & 1 & 1 & 2 & 99 & 98 & 94 & 93 & 89 & 97 & 92 & 96 \\
\hline 2 & 1 & 1 & 3 & 99 & 98 & 94 & 93 & 89 & 97 & 92 & 96 \\
\hline 2 & 1 & 2 & 1 & 99 & 98 & 94 & 93 & 89 & 97 & 92 & 96 \\
\hline 2 & 1 & 2 & 2 & 99 & 98 & 94 & 93 & 89 & 97 & 92 & 96 \\
\hline 2 & 1 & 2 & 3 & 99 & 98 & 94 & 93 & 89 & 97 & 92 & 96 \\
\hline 2 & 1 & 3 & 1 & 99 & 98 & 94 & 93 & 89 & 97 & 92 & 96 \\
\hline 2 & 1 & 3 & 2 & 99 & 98 & 94 & 93 & 89 & 97 & 92 & 96 \\
\hline 2 & 1 & 3 & 3 & 99 & 98 & 94 & 93 & 89 & 97 & 92 & 96 \\
\hline 2 & 1 & 4 & 1 & 99 & 98 & 94 & 91 & 90 & 97 & 92 & 95 \\
\hline 2 & 1 & 4 & 2 & 99 & 98 & 94 & 91 & 90 & 97 & 92 & 95 \\
\hline 2 & 1 & 4 & 3 & 99 & 98 & 94 & 91 & 90 & 97 & 92 & 95 \\
\hline 2 & 1 & 1 & 1 & 99 & 98 & 94 & 92 & 90 & 96 & 92 & 94 \\
\hline 2 & 1 & 1 & 2 & 99 & 98 & 94 & 92 & 90 & 96 & 92 & 94 \\
\hline 2 & 1 & 1 & 3 & 99 & 98 & 94 & 92 & 90 & 96 & 92 & 94 \\
\hline 2 & 1 & 6 & 1 & 99 & 98 & 94 & 93 & 90 & 97 & 92 & 96 \\
\hline 2 & 1 & 6 & 2 & 99 & 98 & 94 & 93 & 90 & 97 & 92 & 96 \\
\hline 2 & 1 & 6 & 3 & 99 & 98 & 94 & 93 & 90 & 97 & 92 & 96 \\
\hline 2 & 2 & 1 & 1 & 99 & 99 & 95 & 96 & - & - & - & - \\
\hline 2 & 2 & 1 & 2 & 99 & 99 & 95 & 96 & - & - & - & - \\
\hline 2 & 2 & 1 & 3 & 99 & 99 & 95 & 96 & - & - & - & - \\
\hline 2 & 2 & 2 & 1 & 99 & 99 & 95 & 96 & - & - & - & - \\
\hline 2 & 2 & 2 & 2 & 99 & 99 & 95 & 96 & - & - & - & - \\
\hline 2 & 2 & 2 & 3 & 99 & 99 & 95 & 96 & - & - & - & - \\
\hline 2 & 2 & 3 & 1 & 99 & 99 & 94 & 99 & - & - & - & - \\
\hline 2 & 2 & 3 & 2 & 99 & 99 & 94 & 99 & - & - & - & - \\
\hline 2 & 2 & 3 & 3 & 99 & 99 & 94 & 99 & - & - & - & - \\
\hline 2 & 2 & 4 & 1 & 99 & 99 & 94 & 97 & - & - & - & - \\
\hline 2 & 2 & 4 & 2 & 99 & 99 & 94 & 97 & - & - & - & - \\
\hline 2 & 2 & 4 & 3 & 99 & 99 & 94 & 97 & - & - & - & - \\
\hline 2 & 2 & 1 & 1 & 99 & 99 & 94 & 97 & - & - & - & - \\
\hline 2 & 2 & 1 & 2 & 99 & 99 & 94 & 97 & - & - & - & - \\
\hline 2 & 2 & 1 & 3 & 99 & 99 & 94 & 97 & - & - & - & - \\
\hline 2 & 2 & 6 & 1 & 99 & 99 & 95 & 97 & - & - & - & - \\
\hline
\end{tabular}


Table C.2.1 the results of calibration procedure 1 and its validations.

\begin{tabular}{|c|c|c|c|c|c|c|c|c|c|c|c|}
\hline \multicolumn{4}{|c|}{ Configurations } & \multicolumn{2}{|c|}{ Calibration } & \multicolumn{2}{|c|}{$1^{\text {st }}$ Validation } & \multicolumn{2}{|c|}{$2^{\text {nd }}$ Validation } & \multicolumn{2}{|c|}{$3^{\text {rd }}$ Validation } \\
\hline$\overline{\varphi_{g}}$ & $\Lambda_{l}$ & $\overline{\Gamma_{n}}$ & $\overline{\eta_{m}}$ & Vehicles & Cyclists & Vehicles & Cyclists & Vehicles & Cyclists & Vehicles & Cyclists \\
\hline 2 & 2 & 6 & 2 & 99 & 99 & 95 & 97 & - & - & - & - \\
\hline 2 & 2 & 6 & 3 & 99 & 99 & 95 & 97 & - & - & - & - \\
\hline 2 & 3 & 1 & 1 & 99 & 98 & 94 & 94 & 88 & 94 & 91 & 94 \\
\hline 2 & 3 & 1 & 2 & 99 & 98 & 94 & 94 & 88 & 94 & 91 & 94 \\
\hline 2 & 3 & 1 & 3 & 99 & 98 & 94 & 94 & 88 & 94 & 91 & 94 \\
\hline 2 & 3 & 2 & 1 & 99 & 98 & 94 & 94 & 87 & 94 & 91 & 94 \\
\hline 2 & 3 & 2 & 2 & 99 & 98 & 94 & 94 & 87 & 94 & 91 & 94 \\
\hline 2 & 3 & 2 & 3 & 99 & 98 & 94 & 94 & 87 & 94 & 91 & 94 \\
\hline 2 & 3 & 3 & 1 & 99 & 98 & 94 & 94 & 87 & 94 & 91 & 94 \\
\hline 2 & 3 & 3 & 2 & 99 & 98 & 94 & 94 & 87 & 94 & 91 & 94 \\
\hline 2 & 3 & 3 & 3 & 99 & 98 & 94 & 94 & 87 & 94 & 91 & 94 \\
\hline 2 & 3 & 4 & 1 & 99 & 98 & 94 & 94 & 87 & 94 & 91 & 94 \\
\hline 2 & 3 & 4 & 2 & 99 & 98 & 94 & 94 & 87 & 94 & 91 & 94 \\
\hline 2 & 3 & 4 & 3 & 99 & 98 & 94 & 94 & 87 & 94 & 91 & 94 \\
\hline 2 & 3 & 1 & 1 & 99 & 98 & 94 & 94 & 87 & 94 & 91 & 94 \\
\hline 2 & 3 & 1 & 2 & 99 & 98 & 94 & 94 & 87 & 94 & 91 & 94 \\
\hline 2 & 3 & 1 & 3 & 99 & 98 & 94 & 94 & 87 & 94 & 91 & 94 \\
\hline 2 & 3 & 6 & 1 & 99 & 98 & 94 & 94 & 87 & 94 & 91 & 94 \\
\hline 2 & 3 & 6 & 2 & 99 & 98 & 94 & 94 & 87 & 94 & 91 & 94 \\
\hline 2 & 3 & 6 & 3 & 99 & 98 & 94 & 94 & 87 & 94 & 91 & 94 \\
\hline 2 & 4 & 1 & 1 & 99 & 97 & - & - & - & - & - & - \\
\hline 2 & 4 & 1 & 2 & 99 & 97 & - & - & - & - & - & - \\
\hline 2 & 4 & 1 & 3 & 99 & 97 & - & - & - & - & - & - \\
\hline 2 & 4 & 2 & 1 & 99 & 97 & - & - & - & - & - & - \\
\hline 2 & 4 & 2 & 2 & 99 & 97 & - & - & - & - & - & - \\
\hline 2 & 4 & 2 & 3 & 99 & 97 & - & - & - & - & - & - \\
\hline 2 & 4 & 3 & 1 & 99 & 97 & - & - & - & - & - & - \\
\hline 2 & 4 & 3 & 2 & 99 & 97 & - & - & - & - & - & - \\
\hline 2 & 4 & 3 & 3 & 99 & 97 & - & - & - & - & - & - \\
\hline 2 & 4 & 4 & 1 & 99 & 97 & - & - & - & - & - & - \\
\hline 2 & 4 & 4 & 2 & 99 & 97 & - & - & - & - & - & - \\
\hline 2 & 4 & 4 & 3 & 99 & 97 & - & - & - & - & - & - \\
\hline 2 & 4 & 1 & 1 & 99 & 97 & - & - & - & - & - & - \\
\hline 2 & 4 & 1 & 2 & 99 & 97 & - & - & - & - & - & - \\
\hline 2 & 4 & 1 & 3 & 99 & 97 & - & - & - & - & - & - \\
\hline 2 & 4 & 6 & 1 & 99 & 97 & - & - & - & - & - & - \\
\hline
\end{tabular}


Table C.2.1 the results of calibration procedure 1 and its validations.

\begin{tabular}{|c|c|c|c|c|c|c|c|c|c|c|c|}
\hline \multicolumn{4}{|c|}{ Configurations } & \multicolumn{2}{|c|}{ Calibration } & \multicolumn{2}{|c|}{$1^{\text {st }}$ Validation } & \multicolumn{2}{|c|}{$2^{\text {nd }}$ Validation } & \multicolumn{2}{|c|}{$3^{\text {rd }}$ Validation } \\
\hline$\overline{\varphi_{g}}$ & $\Lambda_{l}$ & $\overline{\Gamma_{n}}$ & $\overline{\eta_{m}}$ & Vehicles & Cyclists & Vehicles & Cyclists & Vehicles & Cyclists & Vehicles & Cyclists \\
\hline 2 & 4 & 6 & 2 & 99 & 97 & - & - & - & - & - & - \\
\hline 2 & 4 & 6 & 3 & 99 & 97 & - & - & - & - & - & - \\
\hline 3 & 1 & 1 & 1 & \begin{tabular}{|l|}
99 \\
\end{tabular} & $\begin{array}{l}99 \\
\end{array}$ & 92 & 94 & - & - & - & - \\
\hline 3 & 1 & 1 & 2 & 99 & 99 & 92 & 94 & - & - & - & - \\
\hline 3 & 1 & 1 & 3 & 99 & 99 & 92 & 94 & - & - & - & - \\
\hline 3 & 1 & 2 & 1 & 99 & 99 & 92 & 94 & - & - & - & - \\
\hline 3 & 1 & 2 & 2 & 99 & 99 & 92 & 94 & - & - & - & - \\
\hline 3 & 1 & 2 & 3 & 99 & 99 & 92 & 94 & - & - & - & - \\
\hline 3 & 1 & 3 & 1 & 99 & 99 & 92 & 96 & - & - & - & - \\
\hline 3 & 1 & 3 & 2 & 99 & 99 & 92 & 96 & - & - & - & - \\
\hline 3 & 1 & 3 & 3 & 99 & 99 & 92 & 96 & - & - & - & - \\
\hline 3 & 1 & 4 & 1 & 99 & 99 & 92 & 94 & - & - & - & - \\
\hline 3 & 1 & 4 & 2 & 99 & 99 & 92 & 94 & - & - & - & - \\
\hline 3 & 1 & 4 & 3 & 99 & 99 & 92 & 94 & - & - & - & - \\
\hline 3 & 1 & 1 & 1 & 99 & 99 & 92 & 94 & - & - & - & - \\
\hline 3 & 1 & 1 & 2 & 99 & 99 & 92 & 94 & - & - & - & - \\
\hline 3 & 1 & 1 & 3 & 99 & 99 & 92 & 94 & - & - & - & - \\
\hline 3 & 1 & 6 & 1 & 99 & 99 & 92 & 97 & - & - & - & - \\
\hline 3 & 1 & 6 & 2 & 99 & 99 & 92 & 97 & - & - & - & - \\
\hline 3 & 1 & 6 & 3 & 99 & 99 & 92 & 97 & - & - & - & - \\
\hline 3 & 2 & 1 & 1 & 99 & 99 & 91 & 96 & 90 & 92 & 91 & 93 \\
\hline 3 & 2 & 1 & 2 & 99 & 99 & 91 & 96 & 90 & 92 & 91 & 93 \\
\hline 3 & 2 & 1 & 3 & 99 & 99 & 91 & 96 & 90 & 92 & 91 & 93 \\
\hline 3 & 2 & 2 & 1 & 99 & 99 & - & - & - & - & - & - \\
\hline 3 & 2 & 2 & 2 & 99 & 99 & - & - & - & - & - & - \\
\hline 3 & 2 & 2 & 3 & 99 & 99 & - & - & - & - & - & - \\
\hline 3 & 2 & 3 & 1 & 100 & 99 & 92 & 96 & 89 & 92 & 90 & 93 \\
\hline 3 & 2 & 3 & 2 & 100 & 99 & 92 & 96 & 89 & 92 & 90 & 93 \\
\hline 3 & 2 & 3 & 3 & 100 & 99 & 92 & 96 & 89 & 92 & 90 & 93 \\
\hline 3 & 2 & 4 & 1 & 100 & 99 & 92 & 96 & 89 & 92 & 90 & 93 \\
\hline 3 & 2 & 4 & 2 & 100 & 99 & 92 & 96 & 89 & 92 & 90 & 93 \\
\hline 3 & 2 & 4 & 3 & 100 & 99 & 92 & 96 & 89 & 92 & 90 & 93 \\
\hline 3 & 2 & 1 & 1 & 100 & 99 & - & - & - & - & - & - \\
\hline 3 & 2 & 1 & 2 & 100 & 99 & - & - & - & - & - & - \\
\hline 3 & 2 & 1 & 3 & 100 & 99 & - & - & - & - & - & - \\
\hline 3 & 2 & 6 & 1 & 99 & 99 & - & - & - & - & - & - \\
\hline
\end{tabular}


Table C.2.1 the results of calibration procedure 1 and its validations.

\begin{tabular}{|c|c|c|c|c|c|c|c|c|c|c|c|}
\hline \multicolumn{4}{|c|}{ Configurations } & \multicolumn{2}{|c|}{ Calibration } & \multicolumn{2}{|c|}{$1^{\text {st }}$ Validation } & \multicolumn{2}{|c|}{$2^{\text {nd }}$ Validation } & \multicolumn{2}{|c|}{$3^{\text {rd }}$ Validation } \\
\hline$\overline{\varphi_{g}}$ & $\Lambda_{l}$ & $\overline{\Gamma_{n}}$ & $\overline{\eta_{m}}$ & Vehicles & Cyclists & Vehicles & Cyclists & Vehicles & Cyclists & Vehicles & Cyclists \\
\hline 3 & 2 & 6 & 2 & 99 & 99 & - & - & - & - & - & - \\
\hline 3 & 2 & 6 & 3 & 99 & 99 & - & - & - & - & - & - \\
\hline 3 & 3 & 1 & 1 & 99 & 100 & - & - & - & - & - & - \\
\hline 3 & 3 & 1 & 2 & 99 & 100 & - & - & - & - & - & - \\
\hline 3 & 3 & 1 & 3 & 99 & 100 & - & - & - & - & - & - \\
\hline 3 & 3 & 2 & 1 & 99 & 100 & 92 & 99 & 87 & 96 & 90 & 97 \\
\hline 3 & 3 & 2 & 2 & 99 & 100 & 92 & 99 & 87 & 96 & 90 & 97 \\
\hline 3 & 3 & 2 & 3 & 99 & 100 & 92 & 99 & 87 & 96 & 90 & 97 \\
\hline 3 & 3 & 3 & 1 & 99 & 97 & 95 & 94 & 88 & 91 & 92 & 92 \\
\hline 3 & 3 & 3 & 2 & 99 & 97 & 95 & 94 & 88 & 91 & 92 & 92 \\
\hline 3 & 3 & 3 & 3 & 99 & 97 & 95 & 94 & 88 & 91 & 92 & 92 \\
\hline 3 & 3 & 4 & 1 & 99 & 97 & 95 & 94 & 88 & 91 & 92 & 92 \\
\hline 3 & 3 & 4 & 2 & 99 & 97 & 95 & 94 & 88 & 91 & 92 & 92 \\
\hline 3 & 3 & 4 & 3 & 99 & 97 & 95 & 94 & 88 & 91 & 92 & 92 \\
\hline 3 & 3 & 1 & 1 & 99 & 99 & 91 & 99 & 84 & 94 & 88 & 96 \\
\hline 3 & 3 & 1 & 2 & 99 & 99 & 91 & 99 & 84 & 94 & 88 & 96 \\
\hline 3 & 3 & 1 & 3 & 99 & 99 & 91 & 99 & 84 & 94 & 88 & 96 \\
\hline 3 & 3 & 6 & 1 & 99 & 99 & 91 & 99 & 85 & 94 & 88 & 96 \\
\hline 3 & 3 & 6 & 2 & 99 & 99 & 91 & 99 & 85 & 94 & 88 & 96 \\
\hline 3 & 3 & 6 & 3 & 99 & 99 & 91 & 99 & 85 & 94 & 88 & 96 \\
\hline 3 & 4 & 1 & 1 & 99 & 96 & 96 & 92 & - & - & - & - \\
\hline 3 & 4 & 1 & 2 & 99 & 96 & 96 & 92 & - & - & - & - \\
\hline 3 & 4 & 1 & 3 & 99 & 96 & 96 & 92 & - & - & - & - \\
\hline 3 & 4 & 2 & 1 & 99 & 96 & 96 & 94 & - & - & - & - \\
\hline 3 & 4 & 2 & 2 & 99 & 96 & 96 & 94 & - & - & - & - \\
\hline 3 & 4 & 2 & 3 & 99 & 96 & 96 & 94 & - & - & - & - \\
\hline 3 & 4 & 3 & 1 & 99 & 96 & 96 & 92 & - & - & - & - \\
\hline 3 & 4 & 3 & 2 & 99 & 96 & 96 & 92 & - & - & - & - \\
\hline 3 & 4 & 3 & 3 & 99 & 96 & 96 & 92 & - & - & - & - \\
\hline 3 & 4 & 4 & 1 & 99 & 96 & 96 & 92 & - & - & - & - \\
\hline 3 & 4 & 4 & 2 & 99 & 96 & 96 & 92 & - & - & - & - \\
\hline 3 & 4 & 4 & 3 & 99 & 96 & 96 & 92 & - & - & - & - \\
\hline 3 & 4 & 1 & 1 & 100 & 96 & 96 & 92 & - & - & - & - \\
\hline 3 & 4 & 1 & 2 & 100 & 96 & 96 & 92 & - & - & - & - \\
\hline 3 & 4 & 1 & 3 & 100 & 96 & 96 & 92 & - & - & - & - \\
\hline 3 & 4 & 6 & 1 & 100 & 96 & 96 & 92 & - & - & - & - \\
\hline
\end{tabular}


Table C.2.1 the results of calibration procedure 1 and its validations.

\begin{tabular}{|c|c|c|c|c|c|c|c|c|c|c|c|}
\hline \multicolumn{4}{|c|}{ Configurations } & \multicolumn{2}{|c|}{ Calibration } & \multicolumn{2}{|c|}{$1^{\text {st }}$ Validation } & \multicolumn{2}{|c|}{$2^{\text {nd }}$ Validation } & \multicolumn{2}{|c|}{$3^{\text {rd }}$ Validation } \\
\hline$\overline{\varphi_{g}}$ & $\Lambda_{l}$ & $\overline{\Gamma_{n}}$ & $\overline{\eta_{m}}$ & Vehicles & Cyclists & Vehicles & Cyclists & Vehicles & Cyclists & Vehicles & Cyclists \\
\hline 3 & 4 & 6 & 2 & 100 & 96 & 96 & 92 & - & - & - & - \\
\hline 3 & 4 & 6 & 3 & 100 & 96 & 96 & 92 & - & - & - & - \\
\hline 4 & 1 & 1 & 1 & 100 & 96 & 96 & 87 & 96 & 100 & 96 & 94 \\
\hline 4 & 1 & 1 & 2 & 100 & 96 & 96 & 87 & 96 & 100 & 96 & 94 \\
\hline 4 & 1 & 1 & 3 & 100 & 96 & 96 & 87 & 96 & 100 & 96 & 94 \\
\hline 4 & 1 & 2 & 1 & 100 & 96 & 96 & 87 & 97 & 100 & 96 & 94 \\
\hline 4 & 1 & 2 & 2 & 100 & 96 & 96 & 87 & 97 & 100 & 96 & 94 \\
\hline 4 & 1 & 2 & 3 & 100 & 96 & 96 & 87 & 97 & 100 & 96 & 94 \\
\hline 4 & 1 & 3 & 1 & 100 & 96 & 96 & 89 & 96 & 100 & 96 & 95 \\
\hline 4 & 1 & 3 & 2 & 100 & 96 & 96 & 89 & 96 & 100 & 96 & 95 \\
\hline 4 & 1 & 3 & 3 & 100 & 96 & 96 & 89 & 96 & 100 & 96 & 95 \\
\hline 4 & 1 & 4 & 1 & 100 & 96 & 96 & 89 & 96 & 100 & 96 & 95 \\
\hline 4 & 1 & 4 & 2 & 100 & 96 & 96 & 89 & 96 & 100 & 96 & 95 \\
\hline 4 & 1 & 4 & 3 & 100 & 96 & 96 & 89 & 96 & 100 & 96 & 95 \\
\hline 4 & 1 & 1 & 1 & 100 & 96 & 96 & 90 & 96 & 100 & 96 & 96 \\
\hline 4 & 1 & 1 & 2 & 100 & 96 & 96 & 89 & 96 & 100 & 96 & 95 \\
\hline 4 & 1 & 1 & 3 & 100 & 96 & 96 & 89 & 96 & 100 & 96 & 95 \\
\hline 4 & 1 & 6 & 1 & 100 & 96 & 96 & 89 & 96 & 100 & 96 & 95 \\
\hline 4 & 1 & 6 & 2 & 100 & 96 & 96 & 89 & 96 & 100 & 96 & 95 \\
\hline 4 & 1 & 6 & 3 & 100 & 96 & 96 & 89 & 96 & 100 & 96 & 95 \\
\hline 4 & 2 & 1 & 1 & 99 & 99 & 92 & 92 & - & - & - & - \\
\hline 4 & 2 & 1 & 2 & 99 & 99 & 92 & 92 & - & - & - & - \\
\hline 4 & 2 & 1 & 3 & 99 & 99 & 92 & 92 & - & - & - & - \\
\hline 4 & 2 & 2 & 1 & 99 & 97 & 92 & 93 & - & - & - & - \\
\hline 4 & 2 & 2 & 2 & 99 & 97 & 92 & 93 & - & - & - & - \\
\hline 4 & 2 & 2 & 3 & 99 & 97 & 92 & 93 & - & - & - & - \\
\hline 4 & 2 & 3 & 1 & 99 & 97 & 92 & 93 & - & - & - & - \\
\hline 4 & 2 & 3 & 2 & 99 & 97 & 92 & 93 & - & - & - & - \\
\hline 4 & 2 & 3 & 3 & 99 & 97 & 92 & 93 & - & - & - & - \\
\hline 4 & 2 & 4 & 1 & 99 & 97 & 92 & 93 & - & - & - & - \\
\hline 4 & 2 & 4 & 2 & 99 & 97 & 92 & 93 & - & - & - & - \\
\hline 4 & 2 & 4 & 3 & 99 & 97 & 92 & 93 & - & - & - & - \\
\hline 4 & 2 & 1 & 1 & 99 & 97 & 92 & 93 & - & - & - & - \\
\hline 4 & 2 & 1 & 2 & 99 & 97 & 92 & 93 & - & - & - & - \\
\hline 4 & 2 & 1 & 3 & 99 & 97 & 92 & 93 & - & - & - & - \\
\hline 4 & 2 & 6 & 1 & 99 & 97 & 92 & 93 & - & - & - & - \\
\hline
\end{tabular}


Table C.2.1 the results of calibration procedure 1 and its validations.

\begin{tabular}{|c|c|c|c|c|c|c|c|c|c|c|c|}
\hline \multicolumn{4}{|c|}{ Configurations } & \multicolumn{2}{|c|}{ Calibration } & \multicolumn{2}{|c|}{$1^{\text {st }}$ Validation } & \multicolumn{2}{|c|}{$2^{\text {nd }}$ Validation } & \multicolumn{2}{|c|}{$3^{\text {rd }}$ Validation } \\
\hline$\varphi_{g}$ & $\Lambda_{l}$ & $\overline{\Gamma_{n}}$ & $\eta_{m}$ & Vehicles & Cyclists & Vehicles & Cyclists & Vehicles & Cyclists & Vehicles & Cyclists \\
\hline 4 & 2 & 6 & 2 & 99 & 97 & 92 & 93 & - & - & - & - \\
\hline 4 & 2 & 6 & 3 & 99 & 97 & 92 & 93 & - & - & - & - \\
\hline 4 & 3 & 1 & 1 & 99 & 100 & - & - & - & - & - & - \\
\hline 4 & 3 & 1 & 2 & 99 & 100 & - & - & - & - & - & - \\
\hline 4 & 3 & 1 & 3 & 99 & 100 & - & - & - & - & - & - \\
\hline 4 & 3 & 2 & 1 & 99 & 99 & - & - & - & - & - & - \\
\hline 4 & 3 & 2 & 2 & 99 & 99 & - & - & - & - & - & - \\
\hline 4 & 3 & 2 & 3 & 99 & 99 & - & - & - & - & - & - \\
\hline 4 & 3 & 3 & 1 & 99 & 99 & - & - & - & - & - & - \\
\hline 4 & 3 & 3 & 2 & 99 & 99 & - & - & - & - & - & - \\
\hline 4 & 3 & 3 & 3 & 99 & 99 & - & - & - & - & - & - \\
\hline 4 & 3 & 4 & 1 & 99 & 99 & - & - & - & - & - & - \\
\hline 4 & 3 & 4 & 2 & 99 & 99 & - & - & - & - & - & - \\
\hline 4 & 3 & 4 & 3 & 99 & 99 & - & - & - & - & - & - \\
\hline 4 & 3 & 1 & 1 & 99 & 100 & 90 & 93 & - & - & - & - \\
\hline 4 & 3 & 1 & 2 & 99 & 100 & 90 & 93 & - & - & - & - \\
\hline 4 & 3 & 1 & 3 & 99 & 100 & 90 & 93 & - & - & - & - \\
\hline 4 & 3 & 6 & 1 & 99 & 100 & 90 & 93 & - & - & - & - \\
\hline 4 & 3 & 6 & 2 & 99 & 100 & 90 & 93 & - & - & - & - \\
\hline 4 & 3 & 6 & 3 & 99 & 100 & 90 & 93 & - & - & - & - \\
\hline 4 & 4 & 1 & 1 & 99 & 99 & 91 & 100 & - & - & - & - \\
\hline 4 & 4 & 1 & 2 & 99 & 99 & 91 & 100 & - & - & - & - \\
\hline 4 & 4 & 1 & 3 & 99 & 99 & 91 & 100 & - & - & - & - \\
\hline 4 & 4 & 2 & 1 & 99 & 99 & 91 & 100 & - & - & - & - \\
\hline 4 & 4 & 2 & 2 & 99 & 99 & 91 & 100 & - & - & - & - \\
\hline 4 & 4 & 2 & 3 & 99 & 99 & 91 & 100 & - & - & - & - \\
\hline 4 & 4 & 3 & 1 & 99 & 99 & 91 & 100 & - & - & - & - \\
\hline 4 & 4 & 3 & 2 & 99 & 99 & 91 & 100 & - & - & - & - \\
\hline 4 & 4 & 3 & 3 & 99 & 99 & 91 & 100 & - & - & - & - \\
\hline 4 & 4 & 4 & 1 & 99 & 99 & 91 & 100 & - & - & - & - \\
\hline 4 & 4 & 4 & 2 & 99 & 99 & 91 & 100 & - & - & - & - \\
\hline 4 & 4 & 4 & 3 & 99 & 99 & 91 & 100 & - & - & - & - \\
\hline 4 & 4 & 1 & 1 & 99 & 99 & 91 & 100 & - & - & - & - \\
\hline 4 & 4 & 1 & 2 & 99 & 99 & 91 & 100 & - & - & - & - \\
\hline 4 & 4 & 1 & 3 & 99 & 99 & 91 & 100 & - & - & - & - \\
\hline 4 & 4 & 6 & 1 & 99 & 99 & 91 & 100 & - & - & - & - \\
\hline
\end{tabular}


Table C.2.1 the results of calibration procedure 1 and its validations.

\begin{tabular}{|c|c|c|c|c|c|c|c|c|c|c|c|}
\hline \multicolumn{4}{|c|}{ Configurations } & \multicolumn{2}{|c|}{ Calibration } & \multicolumn{2}{|c|}{$1^{\text {st }}$ Validation } & \multicolumn{2}{|c|}{$2^{\text {nd }}$ Validation } & \multicolumn{2}{|c|}{$3^{\text {rd }}$ Validation } \\
\hline$\overline{\varphi_{g}}$ & $\Lambda_{l}$ & $\overline{\Gamma_{n}}$ & $\overline{\eta_{m}}$ & Vehicles & Cyclists & Vehicles & Cyclists & Vehicles & Cyclists & Vehicles & Cyclists \\
\hline 4 & 4 & 6 & 2 & 99 & 99 & 91 & 100 & - & - & - & - \\
\hline 4 & 4 & 6 & 3 & 99 & 99 & 91 & 100 & - & - & - & - \\
\hline 5 & 1 & 1 & 1 & 99 & 100 & 89 & 96 & 84 & 98 & 87 & 97 \\
\hline 5 & 1 & 1 & 2 & 99 & 100 & 89 & 96 & 84 & 98 & 87 & 97 \\
\hline 5 & 1 & 1 & 3 & 99 & 100 & 89 & 96 & 84 & 98 & 87 & 97 \\
\hline 5 & 1 & 2 & 1 & 99 & 100 & 89 & 96 & 84 & 98 & 87 & 97 \\
\hline 5 & 1 & 2 & 2 & 99 & 100 & 89 & 96 & 84 & 98 & 87 & 97 \\
\hline 5 & 1 & 2 & 3 & 99 & 100 & 89 & 96 & 84 & 98 & 87 & 97 \\
\hline 5 & 1 & 3 & 1 & 99 & 100 & 89 & 98 & 83 & 100 & 87 & 99 \\
\hline 5 & 1 & 3 & 2 & 99 & 100 & 89 & 98 & 83 & 98 & 87 & 98 \\
\hline 5 & 1 & 3 & 3 & 99 & 100 & 89 & 98 & 83 & 98 & 87 & 98 \\
\hline 5 & 1 & 4 & 1 & 99 & 100 & 90 & 93 & 84 & 98 & 88 & 96 \\
\hline 5 & 1 & 4 & 2 & 99 & 100 & 90 & 93 & 84 & 98 & 88 & 96 \\
\hline 5 & 1 & 4 & 3 & 99 & 100 & 90 & 93 & 84 & 98 & 88 & 96 \\
\hline 5 & 1 & 1 & 1 & 99 & 100 & 90 & 96 & 84 & 100 & 88 & 98 \\
\hline 5 & 1 & 1 & 2 & 99 & 100 & 90 & 96 & 84 & 100 & 88 & 98 \\
\hline 5 & 1 & 1 & 3 & 99 & 100 & 90 & 96 & 84 & 100 & 88 & 98 \\
\hline 5 & 1 & 6 & 1 & 99 & 100 & 90 & 96 & 84 & 98 & 88 & 97 \\
\hline 5 & 1 & 6 & 2 & 99 & 100 & 90 & 96 & 86 & 98 & 88 & 97 \\
\hline 5 & 1 & 6 & 3 & 99 & 100 & 90 & 96 & 86 & 98 & 88 & 97 \\
\hline 5 & 2 & 1 & 1 & 99 & 98 & 89 & 89 & 83 & 91 & 87 & 90 \\
\hline 5 & 2 & 1 & 2 & 99 & 98 & 89 & 89 & 83 & 91 & 87 & 90 \\
\hline 5 & 2 & 1 & 3 & 99 & 98 & 89 & 89 & 83 & 91 & 87 & 90 \\
\hline 5 & 2 & 2 & 1 & 99 & 98 & 90 & 93 & 83 & 89 & 87 & 91 \\
\hline 5 & 2 & 2 & 2 & 99 & 98 & 90 & 93 & 83 & 89 & 87 & 91 \\
\hline 5 & 2 & 2 & 3 & 99 & 98 & 90 & 93 & 83 & 89 & 87 & 91 \\
\hline 5 & 2 & 3 & 1 & 99 & 98 & 90 & 89 & 83 & 87 & 87 & 88 \\
\hline 5 & 2 & 3 & 2 & 99 & 98 & 90 & 89 & 83 & 87 & 87 & 88 \\
\hline 5 & 2 & 3 & 3 & 99 & 98 & 90 & 89 & 83 & 87 & 87 & 88 \\
\hline 5 & 2 & 4 & 1 & 99 & 98 & 90 & 87 & 83 & 87 & 87 & 87 \\
\hline 5 & 2 & 4 & 2 & 99 & 98 & 90 & 87 & 83 & 87 & 87 & 87 \\
\hline 5 & 2 & 4 & 3 & 99 & 98 & 90 & 87 & 83 & 87 & 87 & 87 \\
\hline 5 & 2 & 1 & 1 & 99 & 98 & 90 & 89 & 83 & 89 & 87 & 89 \\
\hline 5 & 2 & 1 & 2 & 99 & 98 & 90 & 89 & 83 & 89 & 87 & 89 \\
\hline 5 & 2 & 1 & 3 & 99 & 98 & 90 & 89 & 83 & 89 & 87 & 89 \\
\hline 5 & 2 & 6 & 1 & 99 & 100 & 87 & 96 & 83 & 87 & 86 & 91 \\
\hline
\end{tabular}


Table C.2.1 the results of calibration procedure 1 and its validations.

\begin{tabular}{|c|c|c|c|c|c|c|c|c|c|c|c|}
\hline \multicolumn{4}{|c|}{ Configurations } & \multicolumn{2}{|c|}{ Calibration } & \multicolumn{2}{|c|}{$1^{\text {st }}$ Validation } & \multicolumn{2}{|c|}{$2^{\text {nd }}$ Validation } & \multicolumn{2}{|c|}{$3^{\text {rd }}$ Validation } \\
\hline$\varphi_{g}$ & $\Lambda_{l}$ & $\overline{\Gamma_{n}}$ & $\eta_{m}$ & Vehicles & Cyclists & Vehicles & Cyclists & Vehicles & Cyclists & Vehicles & Cyclists \\
\hline 5 & 2 & 6 & 2 & 99 & 100 & 87 & 96 & 83 & 87 & 86 & 91 \\
\hline 5 & 2 & 6 & 3 & 99 & 100 & 87 & 96 & 83 & 87 & 86 & 91 \\
\hline 5 & 3 & 1 & 1 & 99 & 98 & 92 & 91 & 85 & 89 & 89 & 90 \\
\hline 5 & 3 & 1 & 2 & 99 & 98 & 92 & 91 & 85 & 89 & 89 & 90 \\
\hline 5 & 3 & 1 & 3 & 99 & 98 & 92 & 91 & 85 & 89 & 89 & 90 \\
\hline 5 & 3 & 2 & 1 & 99 & 98 & 93 & 93 & 85 & 91 & 90 & 92 \\
\hline 5 & 3 & 2 & 2 & 99 & 98 & 93 & 93 & 85 & 91 & 90 & 92 \\
\hline 5 & 3 & 2 & 3 & 99 & 98 & 93 & 93 & 85 & 91 & 90 & 92 \\
\hline 5 & 3 & 3 & 1 & 99 & 98 & 92 & 91 & 84 & 87 & 89 & 89 \\
\hline 5 & 3 & 3 & 2 & 99 & 98 & 92 & 91 & 84 & 87 & 89 & 89 \\
\hline 5 & 3 & 3 & 3 & 99 & 98 & 92 & 91 & 84 & 87 & 89 & 89 \\
\hline 5 & 3 & 4 & 1 & 99 & 98 & 93 & 93 & 85 & 87 & 90 & 90 \\
\hline 5 & 3 & 4 & 2 & 99 & 98 & 93 & 93 & 85 & 87 & 90 & 90 \\
\hline 5 & 3 & 4 & 3 & 99 & 98 & 93 & 93 & 85 & 87 & 90 & 90 \\
\hline 5 & 3 & 1 & 1 & 99 & 98 & 93 & 96 & 85 & 87 & 90 & 91 \\
\hline 5 & 3 & 1 & 2 & 99 & 98 & 93 & 96 & 85 & 87 & 90 & 91 \\
\hline 5 & 3 & 1 & 3 & 99 & 98 & 93 & 96 & 85 & 87 & 90 & 91 \\
\hline 5 & 3 & 6 & 1 & 99 & 98 & 93 & 93 & 87 & 87 & 91 & 90 \\
\hline 5 & 3 & 6 & 2 & 99 & 98 & 93 & 93 & 87 & 87 & 91 & 90 \\
\hline 5 & 3 & 6 & 3 & 99 & 98 & 93 & 93 & 87 & 87 & 91 & 90 \\
\hline 5 & 4 & 1 & 1 & 97 & 100 & 90 & 93 & 84 & 91 & 88 & 92 \\
\hline 5 & 4 & 1 & 2 & 97 & 100 & 90 & 93 & 82 & 91 & 87 & 92 \\
\hline 5 & 4 & 1 & 3 & 97 & 100 & 90 & 93 & 82 & 91 & 87 & 92 \\
\hline 5 & 4 & 2 & 1 & 97 & 100 & 90 & 93 & 84 & 87 & 88 & 90 \\
\hline 5 & 4 & 2 & 2 & 97 & 100 & 90 & 93 & 82 & 89 & 87 & 91 \\
\hline 5 & 4 & 2 & 3 & 97 & 100 & 90 & 93 & 82 & 89 & 87 & 91 \\
\hline 5 & 4 & 3 & 1 & 97 & 100 & 89 & 93 & 83 & 84 & 87 & 89 \\
\hline 5 & 4 & 3 & 2 & 97 & 100 & 89 & 93 & - & - & - & - \\
\hline 5 & 4 & 3 & 3 & 97 & 100 & 89 & 93 & - & - & - & - \\
\hline 5 & 4 & 4 & 1 & 97 & 100 & 90 & 93 & 83 & 91 & 87 & 92 \\
\hline 5 & 4 & 4 & 2 & 97 & 100 & 90 & 93 & 81 & 91 & 86 & 92 \\
\hline 5 & 4 & 4 & 3 & 97 & 100 & 90 & 93 & 81 & 91 & 86 & 92 \\
\hline 5 & 4 & 1 & 1 & 97 & 100 & 90 & 93 & 83 & 89 & 87 & 91 \\
\hline 5 & 4 & 1 & 2 & 97 & 100 & 90 & 93 & 81 & 89 & 86 & 91 \\
\hline 5 & 4 & 1 & 3 & 97 & 100 & 90 & 93 & 81 & 89 & 86 & 91 \\
\hline 5 & 4 & 6 & 1 & 97 & 100 & 90 & 93 & 82 & 91 & 87 & 92 \\
\hline
\end{tabular}


Table C.2.1 the results of calibration procedure 1 and its validations.

\begin{tabular}{|c|c|c|c|c|c|c|c|c|c|c|c|}
\hline \multicolumn{4}{|c|}{ Configurations } & \multicolumn{2}{|c|}{ Calibration } & \multicolumn{2}{|c|}{$1^{\text {st }}$ Validation } & \multicolumn{2}{|c|}{$2^{\text {nd }}$ Validation } & \multicolumn{2}{|c|}{$3^{\text {rd }}$ Validation } \\
\hline$\varphi_{g}$ & $\Lambda_{l}$ & $\Gamma_{n}$ & $\eta_{m}$ & Vehicles & Cyclists & Vehicles & Cyclists & Vehicles & Cyclists & Vehicles & Cyclists \\
\hline 5 & 4 & 6 & 2 & 97 & 100 & 90 & 93 & 80 & 91 & 86 & 92 \\
\hline 5 & 4 & 6 & 3 & 97 & 100 & 90 & 93 & 80 & 91 & 86 & 92 \\
\hline
\end{tabular}

Table C.2.2 the results of calibration procedure 2 and its validations.

\begin{tabular}{|c|c|c|c|c|c|c|c|c|c|}
\hline \multicolumn{2}{|c|}{ Configurations } & \multicolumn{2}{|c|}{ Calibration } & \multicolumn{2}{|c|}{$1^{\text {st }}$ Validation } & \multicolumn{2}{|c|}{$2^{\text {nd }}$ Validation } & \multicolumn{2}{|c|}{$3^{\text {rd }}$ Validation } \\
\hline$\varphi_{g}$ & $\Lambda_{l}$ & Vehicles & Cyclists & Vehicles & Cyclists & Vehicles & Cyclists & Vehicles & Cyclists \\
\hline 1 & 1 & 96 & 89 & 93 & 96 & 89 & 99 & 91 & 98 \\
\hline 1 & 2 & - & - & - & - & - & - & - & - \\
\hline 1 & 3 & 96 & 90 & 90 & 94 & - & - & - & - \\
\hline 1 & 4 & - & - & - & - & - & - & - & - \\
\hline 2 & 1 & 98 & 91 & - & - & - & - & - & - \\
\hline 2 & 2 & 98 & 96 & 96 & 97 & 86 & 99 & 91 & 98 \\
\hline 2 & 3 & - & - & - & - & - & - & - & - \\
\hline 2 & 4 & - & - & - & - & - & - & - & - \\
\hline 3 & 1 & 98 & 95 & 92 & 97 & 88 & 97 & 90 & 97 \\
\hline 3 & 2 & - & - & - & - & - & - & - & - \\
\hline 3 & 3 & - & - & - & - & - & - & - & - \\
\hline 3 & 4 & 98 & 96 & 91 & 99 & 88 & 98 & 90 & 98 \\
\hline 4 & 1 & 95 & 99 & 86 & 100 & - & - & - & - \\
\hline 4 & 2 & 96 & 97 & 88 & 93 & - & - & - & - \\
\hline 4 & 3 & 98 & 93 & 93 & 87 & - & - & - & - \\
\hline 4 & 4 & 98 & 99 & 90 & 100 & 89 & 100 & 90 & 100 \\
\hline 5 & 1 & 96 & 100 & 88 & 100 & 80 & 91 & 85 & 96 \\
\hline 5 & 2 & 97 & 98 & 86 & 93 & 73 & 98 & 81 & 96 \\
\hline 5 & 3 & 97 & 92 & 88 & 83 & 80 & 98 & 85 & 90 \\
\hline 5 & 4 & 97 & 98 & 90 & 91 & 79 & 98 & 86 & 95 \\
\hline
\end{tabular}


Table C.2.3 the results of calibration and validations based on the average speed

\begin{tabular}{|c|c|c|c|c|c|c|c|c|c|}
\hline \multicolumn{2}{|c|}{ Configurations } & \multicolumn{2}{|c|}{ Calibration } & \multicolumn{2}{|c|}{$1^{\text {st }}$ Validation } & \multicolumn{2}{|c|}{$2^{\text {nd }}$ Validation } & \multicolumn{2}{|c|}{$3^{\text {rd }}$ Validation } \\
\hline$\varphi_{g}$ & $\Lambda_{l}$ & Vehicles & Cyclists & Vehicles & Cyclists & Vehicles & Cyclists & Vehicles & Cyclists \\
\hline 1 & 1 & 38 & 64 & 34 & 79 & 21 & 85 & 28 & 82 \\
\hline 1 & 2 & 36 & 64 & 34 & 79 & 20 & 85 & 28 & 83 \\
\hline 1 & 3 & 21 & 82 & 18 & 88 & - & - & - & - \\
\hline 1 & 4 & 36 & 63 & 34 & 79 & 20 & 85 & 27 & 83 \\
\hline 2 & 1 & 40 & 63 & 42 & 65 & 29 & 80 & 36 & 75 \\
\hline 2 & 2 & 33 & 70 & 33 & 80 & 20 & 85 & 27 & 83 \\
\hline 2 & 3 & 38 & 62 & 41 & 65 & 29 & 80 & 35 & 74 \\
\hline 2 & 4 & 33 & 70 & 33 & 80 & 20 & 85 & 27 & 83 \\
\hline 3 & 1 & 40 & 61 & 44 & 69 & 30 & 75 & 38 & 73 \\
\hline 3 & 2 & 39 & 61 & 43 & 69 & 30 & 75 & 37 & 73 \\
\hline 3 & 3 & 39 & 61 & 42 & 69 & 30 & 75 & 37 & 73 \\
\hline 3 & 4 & 39 & 61 & 43 & 69 & 30 & 75 & 37 & 73 \\
\hline 4 & 1 & 59 & 36 & 54 & 39 & 46 & 41 & 51 & 40 \\
\hline 4 & 2 & 59 & 36 & 54 & 39 & 46 & 44 & 51 & 42 \\
\hline 4 & 3 & 59 & 36 & 54 & 39 & 46 & 48 & 51 & 44 \\
\hline 4 & 4 & 59 & 36 & 54 & 39 & 46 & 44 & 51 & 42 \\
\hline 5 & 1 & 62 & 46 & 56 & 50 & 44 & 44 & 51 & 47 \\
\hline 5 & 2 & 62 & 46 & 56 & 50 & 44 & 47 & 51 & 48 \\
\hline 5 & 3 & 62 & 48 & 59 & 50 & 44 & 53 & 53 & 52 \\
\hline 5 & 4 & 62 & 46 & 56 & 50 & 44 & 47 & 51 & 48 \\
\hline
\end{tabular}


Table C.2.4 the results of calibration and validations based on the maximum speed

\begin{tabular}{|c|c|c|c|c|c|c|c|c|c|}
\hline \multicolumn{2}{|c|}{ Configurations } & \multicolumn{2}{|c|}{ Calibration } & \multicolumn{2}{|c|}{$1^{\text {st }}$ Validation } & \multicolumn{2}{|c|}{$2^{\text {nd }}$ Validation } & \multicolumn{2}{|c|}{$3^{\text {rd }}$ Validation } \\
\hline$\varphi_{g}$ & $\Lambda_{l}$ & Vehicles & Cyclists & Vehicles & Cyclists & Vehicles & Cyclists & Vehicles & Cyclists \\
\hline 1 & 1 & 75 & 65 & 75 & 81 & 49 & 93 & 63 & 88 \\
\hline 1 & 2 & 78 & 66 & 77 & 78 & 51 & 95 & 65 & 88 \\
\hline 1 & 3 & 74 & 68 & 73 & 84 & 48 & 97 & 61 & 92 \\
\hline 1 & 4 & 71 & 64 & 73 & 82 & 45 & 93 & 60 & 88 \\
\hline 2 & 1 & 76 & 71 & 75 & 82 & 50 & 93 & 63 & 89 \\
\hline 2 & 2 & 77 & 74 & 75 & 84 & 48 & 97 & 63 & 92 \\
\hline 2 & 3 & 74 & 74 & 73 & 84 & 48 & 97 & 62 & 92 \\
\hline 2 & 4 & 73 & 72 & 73 & 83 & 46 & 93 & 61 & 89 \\
\hline 3 & 1 & 78 & 75 & 78 & 86 & 49 & 93 & 65 & 90 \\
\hline 3 & 2 & 79 & 76 & 80 & 82 & 49 & 96 & 66 & 90 \\
\hline 3 & 3 & 78 & 76 & 77 & 79 & 53 & 95 & 66 & 89 \\
\hline 3 & 4 & 76 & 73 & 78 & 81 & 49 & 92 & 65 & 88 \\
\hline 4 & 1 & 77 & 79 & 80 & 87 & 52 & 93 & 68 & 90 \\
\hline 4 & 2 & 78 & 79 & 79 & 87 & 49 & 97 & 67 & 93 \\
\hline 4 & 3 & 78 & 79 & 77 & 87 & 51 & 97 & 66 & 93 \\
\hline 4 & 4 & 77 & 79 & 78 & 87 & 49 & 93 & 66 & 90 \\
\hline 5 & 1 & 78 & 88 & 81 & 91 & 49 & 98 & 69 & 95 \\
\hline 5 & 2 & 78 & 88 & 78 & 91 & 45 & 98 & 66 & 95 \\
\hline 5 & 3 & 80 & 87 & 78 & 85 & 52 & 98 & 68 & 91 \\
\hline 5 & 4 & 78 & 88 & 78 & 91 & 45 & 98 & 66 & 95 \\
\hline
\end{tabular}


Table C.2.5 the results of calibration and validations based on the average acceleration

\begin{tabular}{|c|c|c|c|c|c|c|c|c|c|}
\hline \multicolumn{2}{|c|}{ Configurations } & \multicolumn{2}{|c|}{ Calibration } & \multicolumn{2}{|c|}{$1^{\text {st }}$ Validation } & \multicolumn{2}{|c|}{$2^{\text {nd }}$ Validation } & \multicolumn{2}{|c|}{$3^{\text {rd }}$ Validation } \\
\hline$\varphi_{g}$ & $\Lambda_{l}$ & Vehicles & Cyclists & Vehicles & Cyclists & Vehicles & Cyclists & Vehicles & Cyclists \\
\hline 1 & 1 & 51 & 38 & 60 & 32 & 59 & 19 & 60 & 24 \\
\hline 1 & 2 & 63 & 38 & 73 & 32 & 82 & 19 & 77 & 24 \\
\hline 1 & 3 & 64 & 38 & 74 & 30 & 83 & 17 & 78 & 22 \\
\hline 1 & 4 & 54 & 38 & 67 & 32 & 70 & 17 & 68 & 23 \\
\hline 2 & 1 & 50 & 42 & 58 & 36 & 59 & 20 & 59 & 26 \\
\hline 2 & 2 & 51 & 39 & 60 & 34 & 60 & 17 & 60 & 23 \\
\hline 2 & 3 & 59 & 39 & 72 & 33 & 80 & 17 & 76 & 23 \\
\hline 2 & 4 & 51 & 39 & 60 & 34 & 60 & 17 & 60 & 23 \\
\hline 3 & 1 & 53 & 41 & 59 & 39 & 60 & 18 & 60 & 26 \\
\hline 3 & 2 & 53 & 39 & 61 & 38 & 61 & 15 & 61 & 24 \\
\hline 3 & 3 & 57 & 37 & 69 & 36 & 74 & 14 & 72 & 23 \\
\hline 3 & 4 & 53 & 39 & 61 & 38 & 61 & 15 & 61 & 24 \\
\hline 4 & 1 & 55 & 39 & 62 & 36 & 65 & 11 & 63 & 22 \\
\hline 4 & 2 & 74 & 18 & 75 & 11 & 74 & 7 & 74 & 9 \\
\hline 4 & 3 & 74 & 18 & 75 & 11 & 74 & 7 & 74 & 9 \\
\hline 4 & 4 & 67 & 18 & - & - & - & - & - & - \\
\hline 5 & 1 & 56 & 40 & 64 & 28 & 65 & 13 & 64 & 21 \\
\hline 5 & 2 & 55 & 38 & 64 & 30 & 64 & 13 & 64 & 22 \\
\hline 5 & 3 & 60 & 38 & 69 & 28 & 82 & 13 & 74 & 21 \\
\hline 5 & 4 & 55 & 38 & 64 & 30 & 64 & 13 & 64 & 22 \\
\hline
\end{tabular}


Table C.2.6 the results of calibration and validations based on the maximum acceleration

\begin{tabular}{|c|c|c|c|c|c|c|c|c|c|}
\hline \multicolumn{2}{|c|}{ Configurations } & \multicolumn{2}{|c|}{ Calibration } & \multicolumn{2}{|c|}{$1^{\text {st }}$ Validation } & \multicolumn{2}{|c|}{$2^{\text {nd }}$ Validation } & \multicolumn{2}{|c|}{$3^{\text {rd }}$ Validation } \\
\hline$\varphi_{g}$ & $\Lambda_{l}$ & Vehicles & Cyclists & Vehicles & Cyclists & Vehicles & Cyclists & Vehicles & Cyclists \\
\hline 1 & 1 & 4 & 81 & - & - & - & - & - & - \\
\hline 1 & 2 & 4 & 81 & - & - & - & - & - & - \\
\hline 1 & 3 & 4 & 81 & - & - & - & - & - & - \\
\hline 1 & 4 & 4 & 81 & - & - & - & - & - & - \\
\hline 2 & 1 & 5 & 89 & 0 & 79 & - & - & - & - \\
\hline 2 & 2 & 4 & 89 & - & - & - & - & - & - \\
\hline 2 & 3 & 4 & 89 & - & - & - & - & - & - \\
\hline 2 & 4 & 4 & 89 & - & - & - & - & - & - \\
\hline 3 & 1 & 6 & 92 & 0 & 88 & - & - & - & - \\
\hline 3 & 2 & 5 & 92 & 0 & 85 & - & - & - & - \\
\hline 3 & 3 & 5 & 92 & 0 & 85 & - & - & - & - \\
\hline 3 & 4 & 5 & 92 & 0 & 85 & - & - & - & - \\
\hline 4 & 1 & 6 & 96 & 0 & 90 & - & - & - & - \\
\hline 4 & 2 & 6 & 96 & - & - & - & - & - & - \\
\hline 4 & 3 & 6 & 96 & - & - & - & - & - & - \\
\hline 4 & 4 & 6 & 96 & - & - & - & - & - & - \\
\hline 5 & 1 & 9 & 98 & 1 & 93 & - & - & - & - \\
\hline 5 & 2 & 8 & 98 & - & - & - & - & - & - \\
\hline 5 & 3 & 8 & 98 & - & - & - & - & - & - \\
\hline 5 & 4 & 8 & 98 & - & - & - & - & - & - \\
\hline
\end{tabular}


Table C.2.7 the results of calibration and validations based on the average deceleration

\begin{tabular}{|c|c|c|c|c|c|c|c|c|c|}
\hline \multicolumn{2}{|c|}{ Configurations } & \multicolumn{2}{|c|}{ Calibration } & \multicolumn{2}{|c|}{$1^{\text {st }}$ Validation } & \multicolumn{2}{|c|}{$2^{\text {nd }}$ Validation } & \multicolumn{2}{|c|}{$3^{\text {rd }}$ Validation } \\
\hline$\varphi_{g}$ & $\Lambda_{l}$ & Vehicles & Cyclists & Vehicles & Cyclists & Vehicles & Cyclists & Vehicles & Cyclists \\
\hline 1 & 1 & 59 & 49 & 61 & 44 & 80 & 35 & 70 & 39 \\
\hline 1 & 2 & 53 & 43 & 55 & 44 & 71 & 35 & 63 & 39 \\
\hline 1 & 3 & 59 & 38 & 62 & 36 & 83 & 23 & 72 & 28 \\
\hline 1 & 4 & 30 & 38 & 28 & 35 & 47 & 23 & 37 & 28 \\
\hline 2 & 1 & 28 & 75 & 26 & 80 & 43 & 67 & 34 & 72 \\
\hline 2 & 2 & 54 & 45 & 56 & 47 & 72 & 34 & 63 & 39 \\
\hline 2 & 3 & 59 & 39 & 61 & 38 & 83 & 22 & 72 & 28 \\
\hline 2 & 4 & 30 & 39 & 29 & 38 & 47 & 22 & 37 & 28 \\
\hline 3 & 1 & 26 & 75 & 24 & 78 & 46 & 71 & 34 & 73 \\
\hline 3 & 2 & 49 & 47 & 52 & 51 & 73 & 33 & 62 & 40 \\
\hline 3 & 3 & 61 & 33 & 69 & 28 & - & - & - & - \\
\hline 3 & 4 & 24 & 41 & 24 & 47 & 39 & 40 & 31 & 43 \\
\hline 4 & 1 & 38 & 52 & 38 & 66 & 58 & 43 & 46 & 53 \\
\hline 4 & 2 & 57 & 34 & 62 & 26 & - & - & - & - \\
\hline 4 & 3 & 61 & 30 & 65 & 23 & - & - & - & - \\
\hline 4 & 4 & 32 & 37 & 29 & 44 & 48 & 35 & 37 & 39 \\
\hline 5 & 1 & 47 & 65 & 51 & 72 & 74 & 53 & 60 & 63 \\
\hline 5 & 2 & 40 & 65 & 40 & 67 & 72 & 53 & 52 & 60 \\
\hline 5 & 3 & 68 & 37 & 74 & 26 & 92 & 13 & - & - \\
\hline 5 & 4 & 40 & 44 & 39 & 33 & 62 & 27 & 47 & 30 \\
\hline
\end{tabular}


Table C.2.8 the results of calibration and validations based on the maximum deceleration

\begin{tabular}{|c|c|c|c|c|c|c|c|c|c|}
\hline \multicolumn{2}{|c|}{ Configurations } & \multicolumn{2}{|c|}{ Calibration } & \multicolumn{2}{|c|}{$1^{\text {st }}$ Validation } & \multicolumn{2}{|c|}{$2^{\text {nd }}$ Validation } & \multicolumn{2}{|c|}{$3^{\text {rd }}$ Validation } \\
\hline$\varphi_{g}$ & $\Lambda_{l}$ & Vehicles & Cyclists & Vehicles & Cyclists & Vehicles & Cyclists & Vehicles & Cyclists \\
\hline 1 & 1 & 2 & 81 & 0 & 75 & 2 & 56 & 1 & 64 \\
\hline 1 & 2 & 3 & 79 & 1 & 73 & 2 & 54 & 1 & 62 \\
\hline 1 & 3 & 3 & 78 & 1 & 72 & 2 & 54 & 1 & 61 \\
\hline 1 & 4 & 1 & 81 & 0 & 75 & 0 & 57 & 0 & 64 \\
\hline 2 & 1 & 2 & 90 & 0 & 83 & 2 & 57 & 1 & 67 \\
\hline 2 & 2 & 4 & 88 & 1 & 82 & 2 & 55 & 1 & 65 \\
\hline 2 & 3 & 3 & 87 & 1 & 81 & 2 & 54 & 1 & 64 \\
\hline 2 & 4 & 1 & 90 & 0 & 83 & 0 & 57 & 0 & 67 \\
\hline 3 & 1 & 4 & 88 & 1 & 89 & 3 & 60 & 2 & 71 \\
\hline 3 & 2 & 4 & 88 & 1 & 89 & 2 & 60 & 2 & 71 \\
\hline 3 & 3 & 4 & 87 & 1 & 88 & 2 & 59 & 2 & 70 \\
\hline 3 & 4 & 1 & 91 & 0 & 90 & 0 & 63 & 0 & 73 \\
\hline 4 & 1 & 6 & 91 & 1 & 90 & 5 & 59 & 3 & 73 \\
\hline 4 & 2 & 6 & 91 & 1 & 90 & 3 & 59 & 2 & 73 \\
\hline 4 & 3 & 4 & 91 & 1 & 90 & 2 & 59 & 2 & 73 \\
\hline 4 & 4 & 0 & 91 & 0 & 90 & 1 & 59 & 0 & 73 \\
\hline 5 & 1 & 8 & 94 & 1 & 89 & 3 & 64 & 2 & 77 \\
\hline 5 & 2 & 8 & 94 & 1 & 89 & 2 & 64 & 2 & 77 \\
\hline 5 & 3 & 6 & 85 & 1 & 87 & 0 & 56 & 1 & 71 \\
\hline 5 & 4 & 0 & 94 & 0 & 89 & 0 & 64 & 0 & 77 \\
\hline
\end{tabular}


Table C.2.9 the results of calibration and validations based on the average direction

\begin{tabular}{|c|c|c|c|c|c|c|c|c|c|}
\hline \multicolumn{2}{|c|}{ Configurations } & \multicolumn{2}{|c|}{ Calibration } & \multicolumn{2}{|c|}{$1^{\text {st }}$ Validation } & \multicolumn{2}{|c|}{$2^{\text {nd }}$ Validation } & \multicolumn{2}{|c|}{$3^{\text {rd }}$ Validation } \\
\hline$\varphi_{g}$ & $\Lambda_{l}$ & Vehicles & Cyclists & Vehicles & Cyclists & Vehicles & Cyclists & Vehicles & Cyclists \\
\hline 1 & 1 & 77 & 6 & 69 & 9 & - & - & - & - \\
\hline 1 & 2 & 76 & 6 & 66 & 9 & - & - & - & - \\
\hline 1 & 3 & 66 & 7 & 61 & 9 & - & - & - & - \\
\hline 1 & 4 & 68 & 6 & 61 & 8 & - & - & - & - \\
\hline 2 & 1 & 77 & 4 & 68 & 9 & - & - & - & - \\
\hline 2 & 2 & 76 & 4 & 65 & 8 & - & - & - & - \\
\hline 2 & 3 & 69 & 6 & 61 & 8 & - & - & - & - \\
\hline 2 & 4 & 70 & 4 & 61 & 8 & - & - & - & - \\
\hline 3 & 1 & 78 & 5 & 67 & 10 & - & - & - & - \\
\hline 3 & 2 & 76 & 5 & 64 & 8 & - & - & - & - \\
\hline 3 & 3 & 71 & 5 & 60 & 8 & - & - & - & - \\
\hline 3 & 4 & 73 & 5 & 61 & 8 & 3 & 6 & - & - \\
\hline 4 & 1 & 75 & 0 & 63 & 0 & - & - & - & - \\
\hline 4 & 2 & 75 & 0 & 63 & 0 & - & - & - & - \\
\hline 4 & 3 & 72 & 4 & 60 & 8 & - & - & - & - \\
\hline 4 & 4 & 73 & 4 & 60 & 8 & - & - & - & - \\
\hline 5 & 1 & 74 & 0 & 62 & 0 & 3 & 0 & - & - \\
\hline 5 & 2 & 74 & 0 & 62 & 0 & 3 & 0 & - & - \\
\hline 5 & 3 & 70 & 0 & 52 & 0 & - & - & - & - \\
\hline 5 & 4 & 71 & 0 & 54 & 0 & - & - & - & - \\
\hline
\end{tabular}


Table C.2.10 the results of calibration and validations based on the maximum direction

\begin{tabular}{|c|c|c|c|c|c|c|c|c|c|}
\hline \multicolumn{2}{|c|}{ Configurations } & \multicolumn{2}{|c|}{ Calibration } & \multicolumn{2}{|c|}{$1^{\text {st }}$ Validation } & \multicolumn{2}{|c|}{$2^{\text {nd }}$ Validation } & \multicolumn{2}{|c|}{$3^{\text {rd }}$ Validation } \\
\hline$\varphi_{g}$ & $\Lambda_{l}$ & Vehicles & Cyclists & Vehicles & Cyclists & Vehicles & Cyclists & Vehicles & Cyclists \\
\hline 1 & 1 & 82 & 94 & 77 & 88 & 44 & 91 & 62 & 90 \\
\hline 1 & 2 & - & - & - & - & - & - & - & - \\
\hline 1 & 3 & - & - & - & - & - & - & - & - \\
\hline 1 & 4 & - & - & - & - & - & - & - & - \\
\hline 2 & 1 & 83 & 96 & 77 & 89 & 44 & 91 & 62 & 90 \\
\hline 2 & 2 & 75 & 99 & 66 & 100 & - & - & - & - \\
\hline 2 & 3 & - & - & - & - & - & - & - & - \\
\hline 2 & 4 & - & - & - & - & - & - & - & - \\
\hline 3 & 1 & 85 & 96 & 77 & 86 & 49 & 94 & 64 & 91 \\
\hline 3 & 2 & 77 & 99 & 65 & 100 & - & - & - & - \\
\hline 3 & 3 & - & - & - & - & - & - & - & - \\
\hline 3 & 4 & - & - & - & - & - & - & - & - \\
\hline 4 & 1 & 85 & 96 & 77 & 84 & - & - & - & - \\
\hline 4 & 2 & 76 & 99 & 64 & 100 & - & - & - & - \\
\hline 4 & 3 & - & - & - & - & - & - & - & - \\
\hline 4 & 4 & 75 & 97 & 62 & 89 & 57 & 91 & 69 & 88 \\
\hline 5 & 1 & 86 & 98 & 78 & 85 & 62 & 89 & 72 & 87 \\
\hline 5 & 2 & - & - & - & - & - & - & - & - \\
\hline 5 & 3 & - & - & - & - & - & - & - & - \\
\hline 5 & 4 & - & - & - & - & - & - & - & - \\
\hline
\end{tabular}


Table C.2.11 the results of calibration and validations based on the average of change in direction

\begin{tabular}{|c|c|c|c|c|c|c|c|c|c|}
\hline \multicolumn{2}{|c|}{ Configurations } & \multicolumn{2}{|c|}{ Calibration } & \multicolumn{2}{|c|}{$1^{\text {st }}$ Validation } & \multicolumn{2}{|c|}{$2^{\text {nd }}$ Validation } & \multicolumn{2}{|c|}{$3^{\text {rd }}$ Validation } \\
\hline$\varphi_{g}$ & $\Lambda_{l}$ & Vehicles & Cyclists & Vehicles & Cyclists & Vehicles & Cyclists & Vehicles & Cyclists \\
\hline 1 & 1 & 38 & 2 & 29 & 1 & - & - & - & - \\
\hline 1 & 2 & 38 & 2 & 29 & 1 & - & - & - & - \\
\hline 1 & 3 & 38 & 2 & 29 & 1 & - & - & - & - \\
\hline 1 & 4 & 74 & 1 & 58 & 1 & - & - & - & - \\
\hline 2 & 1 & 42 & 0 & 30 & 0 & - & - & - & - \\
\hline 2 & 2 & 42 & 0 & 30 & 0 & - & - & - & - \\
\hline 2 & 3 & 42 & 0 & 30 & 0 & - & - & - & - \\
\hline 2 & 4 & 42 & 0 & 30 & 0 & - & - & - & - \\
\hline 3 & 1 & 71 & 0 & 42 & 0 & - & - & - & - \\
\hline 3 & 2 & 71 & 0 & 42 & 0 & - & - & - & - \\
\hline 3 & 3 & 71 & 0 & 42 & 0 & - & - & - & - \\
\hline 3 & 4 & 71 & 0 & 42 & 0 & - & - & - & - \\
\hline 4 & 1 & 53 & 0 & 32 & 0 & - & - & - & - \\
\hline 4 & 2 & 53 & 0 & 32 & 0 & - & - & - & - \\
\hline 4 & 3 & 53 & 0 & 32 & 0 & - & - & - & - \\
\hline 4 & 4 & 53 & 0 & 32 & 0 & - & - & - & - \\
\hline 5 & 1 & 63 & 0 & 38 & 0 & - & - & - & - \\
\hline 5 & 2 & 63 & 0 & 38 & 0 & - & - & - & - \\
\hline 5 & 3 & 63 & 0 & 38 & 0 & - & - & - & - \\
\hline 5 & 4 & 63 & 0 & 38 & 0 & - & - & - & - \\
\hline
\end{tabular}


Table C.2.12 the results of calibration and validations based on the average of cosine of change in direction

\begin{tabular}{|c|c|c|c|c|c|c|c|c|c|}
\hline \multicolumn{2}{|c|}{ Configurations } & \multicolumn{2}{|c|}{ Calibration } & \multicolumn{2}{|c|}{$1^{\text {st }}$ Validation } & \multicolumn{2}{|c|}{$2^{\text {nd }}$ Validation } & \multicolumn{2}{|c|}{$3^{\text {rd }}$ Validation } \\
\hline$\varphi_{g}$ & $\Lambda_{l}$ & Vehicles & Cyclists & Vehicles & Cyclists & Vehicles & Cyclists & Vehicles & Cyclists \\
\hline 1 & 1 & 65 & 1 & 64 & 0 & 64 & 0 & 64 & 0 \\
\hline 1 & 2 & 62 & 2 & 63 & 2 & 63 & 0 & 63 & 1 \\
\hline 1 & 3 & 51 & 13 & 54 & 13 & 51 & 7 & 53 & 10 \\
\hline 1 & 4 & 55 & 2 & 56 & 2 & 56 & 0 & 56 & 1 \\
\hline 2 & 1 & 68 & 1 & 64 & 2 & 63 & 0 & 64 & 1 \\
\hline 2 & 2 & 66 & 1 & 63 & 2 & 63 & 0 & 63 & 1 \\
\hline 2 & 3 & 55 & 11 & 54 & 13 & 52 & 7 & 53 & 9 \\
\hline 2 & 4 & 58 & 1 & 56 & 2 & 56 & 0 & 56 & 1 \\
\hline 3 & 1 & 74 & 1 & 69 & 1 & 67 & 0 & 68 & 1 \\
\hline 3 & 2 & 66 & 11 & 61 & 7 & 58 & 4 & 59 & 5 \\
\hline 3 & 3 & 63 & 13 & 59 & 14 & 55 & 6 & 58 & 9 \\
\hline 3 & 4 & 66 & 1 & 61 & 1 & 58 & 0 & 59 & 1 \\
\hline 4 & 1 & 82 & 4 & 71 & 0 & 71 & 0 & 71 & 0 \\
\hline 4 & 2 & 81 & 4 & 71 & 0 & 71 & 0 & 71 & 0 \\
\hline 4 & 3 & 75 & 10 & 65 & 11 & 58 & 7 & 62 & 9 \\
\hline 4 & 4 & 75 & 4 & 65 & 0 & 58 & 0 & 62 & 0 \\
\hline 5 & 1 & 87 & 0 & 77 & 0 & 75 & 0 & 76 & 0 \\
\hline 5 & 2 & 78 & 10 & 67 & 13 & - & - & - & - \\
\hline 5 & 3 & 78 & 10 & 65 & 13 & - & - & - & - \\
\hline 5 & 4 & 81 & 0 & 69 & 0 & 65 & 0 & 67 & 0 \\
\hline
\end{tabular}


Table C.2.13 the results of calibration and validations based on the average area

\begin{tabular}{|c|c|c|c|c|c|c|c|c|c|}
\hline \multicolumn{2}{|c|}{ Configurations } & \multicolumn{2}{|c|}{ Calibration } & \multicolumn{2}{|c|}{$1^{\text {st }}$ Validation } & \multicolumn{2}{|c|}{$2^{\text {nd }}$ Validation } & \multicolumn{2}{|c|}{$3^{\text {rd }}$ Validation } \\
\hline$\varphi_{g}$ & $\Lambda_{l}$ & Vehicles & Cyclists & Vehicles & Cyclists & Vehicles & Cyclists & Vehicles & Cyclists \\
\hline 1 & 1 & 93 & 96 & 86 & 99 & 83 & 99 & 85 & 99 \\
\hline 1 & 2 & 91 & 96 & 84 & 99 & 83 & 99 & 83 & 99 \\
\hline 1 & 3 & 89 & 97 & 81 & 100 & 80 & 99 & 80 & 100 \\
\hline 1 & 4 & 91 & 92 & 83 & 96 & 81 & 99 & 82 & 98 \\
\hline 2 & 1 & 94 & 98 & 87 & 99 & 84 & 99 & 85 & 99 \\
\hline 2 & 2 & 92 & 98 & 84 & 99 & 82 & 96 & 83 & 97 \\
\hline 2 & 3 & 91 & 98 & 81 & 100 & - & - & - & - \\
\hline 2 & 4 & 92 & 98 & 84 & 99 & 81 & 96 & 83 & 97 \\
\hline 3 & 1 & 96 & 97 & 85 & 99 & 85 & 100 & 85 & 99 \\
\hline 3 & 2 & 94 & 97 & 83 & 99 & 82 & 98 & 82 & 98 \\
\hline 3 & 3 & 92 & 97 & 79 & 100 & - & - & - & - \\
\hline 3 & 4 & 94 & 97 & 83 & 99 & 82 & 98 & 82 & 98 \\
\hline 4 & 1 & 97 & 97 & 86 & 100 & - & - & - & - \\
\hline 4 & 2 & 95 & 99 & 78 & 100 & - & - & - & - \\
\hline 4 & 3 & 94 & 99 & - & - & - & - & - & - \\
\hline 4 & 4 & 95 & 99 & 78 & 100 & 86 & 100 & 86 & 100 \\
\hline 5 & 1 & 96 & 100 & 81 & 100 & 79 & 76 & 81 & 88 \\
\hline 5 & 2 & 95 & 100 & 75 & 100 & 72 & 98 & 74 & 99 \\
\hline 5 & 3 & 94 & 100 & 75 & 100 & 69 & 100 & 73 & 100 \\
\hline 5 & 4 & 95 & 100 & 75 & 100 & 72 & 98 & 74 & 99 \\
\hline
\end{tabular}


Table C.2.14 the results of calibration and validations based on the maximum area

\begin{tabular}{|c|c|c|c|c|c|c|c|c|c|}
\hline \multicolumn{2}{|c|}{ Configurations } & \multicolumn{2}{|c|}{ Calibration } & \multicolumn{2}{|c|}{$1^{\text {st }}$ Validation } & \multicolumn{2}{|c|}{$2^{\text {nd }}$ Validation } & \multicolumn{2}{|c|}{$3^{\text {rd }}$ Validation } \\
\hline$\varphi_{g}$ & $\Lambda_{l}$ & Vehicles & Cyclists & Vehicles & Cyclists & Vehicles & Cyclists & Vehicles & Cyclists \\
\hline 1 & 1 & 96 & 92 & 95 & 94 & 95 & 96 & 95 & 95 \\
\hline 1 & 2 & 94 & 95 & 93 & 97 & - & - & - & - \\
\hline 1 & 3 & 94 & 95 & 93 & 97 & - & - & - & - \\
\hline 1 & 4 & 94 & 91 & 93 & 94 & - & - & - & - \\
\hline 2 & 1 & 97 & 94 & 94 & 97 & 90 & 90 & 93 & 93 \\
\hline 2 & 2 & 97 & 94 & 94 & 97 & 90 & 90 & 93 & 93 \\
\hline 2 & 3 & 97 & 94 & 94 & 97 & 90 & 90 & 92 & 93 \\
\hline 2 & 4 & 97 & 94 & 94 & 97 & 90 & 90 & 92 & 93 \\
\hline 3 & 1 & 98 & 95 & 91 & 97 & 87 & 82 & 89 & 88 \\
\hline 3 & 2 & 98 & 95 & 91 & 97 & 87 & 82 & 89 & 88 \\
\hline 3 & 3 & 98 & 95 & 91 & 97 & 87 & 82 & 89 & 88 \\
\hline 3 & 4 & 98 & 95 & 91 & 97 & 87 & 82 & 89 & 88 \\
\hline 4 & 1 & 98 & 96 & 92 & 98 & 88 & 87 & 90 & 92 \\
\hline 4 & 2 & 98 & 96 & 92 & 98 & 89 & 87 & 91 & 92 \\
\hline 4 & 3 & 98 & 96 & 92 & 98 & 89 & 87 & 91 & 92 \\
\hline 4 & 4 & 98 & 96 & 92 & 98 & 89 & 87 & 91 & 92 \\
\hline 5 & 1 & 98 & 96 & 93 & 100 & 86 & 82 & 91 & 91 \\
\hline 5 & 2 & 98 & 96 & 93 & 100 & 87 & 82 & 91 & 91 \\
\hline 5 & 3 & 98 & 96 & 93 & 100 & 86 & 82 & 91 & 91 \\
\hline 5 & 4 & 98 & 96 & 93 & 100 & 87 & 82 & 91 & 91 \\
\hline
\end{tabular}


Table C.2.15 the results of calibration and validations based on the average length

\begin{tabular}{|c|c|c|c|c|c|c|c|c|c|}
\hline \multicolumn{2}{|c|}{ Configurations } & \multicolumn{2}{|c|}{ Calibration } & \multicolumn{2}{|c|}{$1^{\text {st }}$ Validation } & \multicolumn{2}{|c|}{$2^{\text {nd }}$ Validation } & \multicolumn{2}{|c|}{$3^{\text {rd }}$ Validation } \\
\hline$\varphi_{g}$ & $\Lambda_{l}$ & Vehicles & Cyclists & Vehicles & Cyclists & Vehicles & Cyclists & Vehicles & Cyclists \\
\hline 1 & 1 & 96 & 88 & 84 & 88 & 91 & 99 & 87 & 95 \\
\hline 1 & 2 & 95 & 88 & 84 & 88 & 91 & 99 & 87 & 95 \\
\hline 1 & 3 & 94 & 89 & 83 & 89 & 90 & 99 & 86 & 95 \\
\hline 1 & 4 & 95 & 85 & 84 & 85 & 89 & 99 & 86 & 94 \\
\hline 2 & 1 & 96 & 90 & 83 & 88 & 90 & 97 & 86 & 94 \\
\hline 2 & 2 & 96 & 88 & 84 & 85 & 90 & 96 & 87 & 92 \\
\hline 2 & 3 & 95 & 90 & 83 & 87 & 90 & 96 & 86 & 92 \\
\hline 2 & 4 & 96 & 88 & 84 & 85 & 90 & 96 & 87 & 92 \\
\hline 3 & 1 & 97 & 91 & 82 & 85 & 90 & 100 & 86 & 94 \\
\hline 3 & 2 & 96 & 91 & 81 & 86 & 88 & 96 & 84 & 92 \\
\hline 3 & 3 & 96 & 91 & 82 & 85 & 90 & 98 & 85 & 93 \\
\hline 3 & 4 & 96 & 91 & 81 & 86 & 88 & 96 & 84 & 92 \\
\hline 4 & 1 & 97 & 91 & 84 & 84 & 91 & 100 & 87 & 93 \\
\hline 4 & 2 & 96 & 91 & 83 & 85 & 90 & 99 & 86 & 93 \\
\hline 4 & 3 & 97 & 91 & 83 & 84 & 91 & 100 & 87 & 93 \\
\hline 4 & 4 & 96 & 91 & 83 & 85 & 90 & 99 & 86 & 93 \\
\hline 5 & 1 & 99 & 96 & 88 & 89 & 92 & 100 & 89 & 95 \\
\hline 5 & 2 & 98 & 96 & 87 & 91 & 89 & 98 & 88 & 95 \\
\hline 5 & 3 & 98 & 96 & 87 & 91 & 89 & 98 & 88 & 95 \\
\hline 5 & 4 & 98 & 96 & 87 & 91 & 89 & 98 & 88 & 95 \\
\hline
\end{tabular}


Table C.2.16 the results of calibration and validations based on the maximum length

\begin{tabular}{|c|c|c|c|c|c|c|c|c|c|}
\hline \multicolumn{2}{|c|}{ Configurations } & \multicolumn{2}{|c|}{ Calibration } & \multicolumn{2}{|c|}{$1^{\text {st }}$ Validation } & \multicolumn{2}{|c|}{$2^{\text {nd }}$ Validation } & \multicolumn{2}{|c|}{$3^{\text {rd }}$ Validation } \\
\hline$\varphi_{g}$ & $\Lambda_{l}$ & Vehicles & Cyclists & Vehicles & Cyclists & Vehicles & Cyclists & Vehicles & Cyclists \\
\hline 1 & 1 & 96 & 85 & 94 & 73 & 98 & 99 & 96 & 88 \\
\hline 1 & 2 & 90 & 91 & 86 & 79 & 95 & 99 & - & - \\
\hline 1 & 3 & - & - & - & - & - & - & - & - \\
\hline 1 & 4 & 90 & 88 & 86 & 76 & - & - & - & - \\
\hline 2 & 1 & 95 & 91 & 87 & 76 & 95 & 99 & 90 & 91 \\
\hline 2 & 2 & 92 & 91 & - & - & - & - & - & - \\
\hline 2 & 3 & - & - & - & - & - & - & - & - \\
\hline 2 & 4 & 92 & 91 & 89 & 76 & 94 & 98 & 92 & 90 \\
\hline 3 & 1 & 97 & 88 & 92 & 68 & 97 & 99 & 94 & 87 \\
\hline 3 & 2 & 93 & 91 & 88 & 71 & 96 & 100 & 91 & 89 \\
\hline 3 & 3 & - & - & - & - & - & - & - & - \\
\hline 3 & 4 & 93 & 91 & 88 & 71 & 96 & 100 & 91 & 89 \\
\hline 4 & 1 & 97 & 91 & 92 & 67 & 97 & 100 & 94 & 85 \\
\hline 4 & 2 & 93 & 93 & 84 & 75 & 95 & 100 & 88 & 89 \\
\hline 4 & 3 & - & - & - & - & - & - & - & - \\
\hline 4 & 4 & 93 & 93 & 84 & 75 & 95 & 100 & 88 & 89 \\
\hline 5 & 1 & 98 & 92 & - & - & - & - & - & - \\
\hline 5 & 2 & 95 & 94 & - & - & - & - & - & - \\
\hline 5 & 3 & 93 & 94 & 87 & 76 & 94 & 99 & 90 & 91 \\
\hline 5 & 4 & 95 & 94 & 96 & 74 & 97 & 100 & 96 & 87 \\
\hline
\end{tabular}


Table C.2.17 the results of calibration and validations based on the average width

\begin{tabular}{|c|c|c|c|c|c|c|c|c|c|}
\hline \multicolumn{2}{|c|}{ Configurations } & \multicolumn{2}{|c|}{ Calibration } & \multicolumn{2}{|c|}{$1^{\text {st }}$ Validation } & \multicolumn{2}{|c|}{$2^{\text {nd }}$ Validation } & \multicolumn{2}{|c|}{$3^{\text {rd }}$ Validation } \\
\hline$\varphi_{g}$ & $\Lambda_{l}$ & Vehicles & Cyclists & Vehicles & Cyclists & Vehicles & Cyclists & Vehicles & Cyclists \\
\hline 1 & 1 & 92 & 90 & 87 & 97 & 81 & 96 & 84 & 96 \\
\hline 1 & 2 & 91 & 91 & 87 & 99 & 82 & 98 & 85 & 98 \\
\hline 1 & 3 & 90 & 92 & 84 & 99 & 80 & 98 & 82 & 98 \\
\hline 1 & 4 & 91 & 88 & 87 & 96 & 80 & 98 & 84 & 97 \\
\hline 2 & 1 & 94 & 93 & 88 & 99 & 82 & 96 & 85 & 97 \\
\hline 2 & 2 & 93 & 94 & 86 & 99 & 78 & 97 & 82 & 98 \\
\hline 2 & 3 & 91 & 94 & 84 & 99 & 77 & 95 & 81 & 97 \\
\hline 2 & 4 & 93 & 94 & 86 & 99 & 77 & 97 & 82 & 98 \\
\hline 3 & 1 & 96 & 93 & 86 & 99 & 82 & 98 & 84 & 98 \\
\hline 3 & 2 & 95 & 93 & 84 & 99 & 77 & 99 & 81 & 99 \\
\hline 3 & 3 & 94 & 93 & 83 & 99 & 76 & 97 & 80 & 98 \\
\hline 3 & 4 & 95 & 93 & 84 & 99 & 77 & 99 & 81 & 99 \\
\hline 4 & 1 & 97 & 93 & 88 & 98 & 84 & 97 & 86 & 98 \\
\hline 4 & 2 & 96 & 93 & 87 & 98 & 83 & 99 & 86 & 99 \\
\hline 4 & 3 & 96 & 93 & 85 & 98 & 83 & 97 & 84 & 98 \\
\hline 4 & 4 & 96 & 93 & 87 & 98 & 83 & 99 & 86 & 99 \\
\hline 5 & 1 & 96 & 94 & 88 & 98 & 85 & 96 & 87 & 97 \\
\hline 5 & 2 & 96 & 94 & 88 & 98 & 84 & 98 & 86 & 98 \\
\hline 5 & 3 & 95 & 94 & 83 & 91 & 84 & 78 & - & - \\
\hline 5 & 4 & 96 & 94 & 88 & 98 & 84 & 98 & 86 & 98 \\
\hline
\end{tabular}


Table C.2.18 the results of calibration and validations based on the maximum width

\begin{tabular}{|c|c|c|c|c|c|c|c|c|c|}
\hline \multicolumn{2}{|c|}{ Configurations } & \multicolumn{2}{|c|}{ Calibration } & \multicolumn{2}{|c|}{$1^{\text {st }}$ Validation } & \multicolumn{2}{|c|}{$2^{\text {nd }}$ Validation } & \multicolumn{2}{|c|}{$3^{\text {rd }}$ Validation } \\
\hline$\varphi_{g}$ & $\Lambda_{l}$ & Vehicles & Cyclists & Vehicles & Cyclists & Vehicles & Cyclists & Vehicles & Cyclists \\
\hline 1 & 1 & 89 & 92 & 91 & 97 & 81 & 92 & 87 & 94 \\
\hline 1 & 2 & 90 & 90 & 93 & 99 & 86 & 93 & 90 & 95 \\
\hline 1 & 3 & 88 & 95 & 90 & 100 & 78 & 96 & 85 & 98 \\
\hline 1 & 4 & 90 & 87 & 93 & 96 & 84 & 93 & 89 & 94 \\
\hline 2 & 1 & 93 & 96 & 92 & 99 & - & - & - & - \\
\hline 2 & 2 & 95 & 90 & 94 & 99 & 86 & 92 & 90 & 94 \\
\hline 2 & 3 & 93 & 96 & 92 & 99 & 79 & 92 & - & - \\
\hline 2 & 4 & 95 & 90 & 94 & 99 & 85 & 92 & 90 & 94 \\
\hline 3 & 1 & 96 & 95 & 92 & 100 & 78 & 94 & 85 & 96 \\
\hline 3 & 2 & 96 & 95 & 91 & 97 & 78 & 90 & 85 & 93 \\
\hline 3 & 3 & 96 & 95 & 91 & 99 & 78 & 92 & 85 & 95 \\
\hline 3 & 4 & 96 & 95 & 91 & 97 & 78 & 90 & 85 & 93 \\
\hline 4 & 1 & 96 & 96 & 93 & 100 & 81 & 93 & 88 & 96 \\
\hline 4 & 2 & 95 & 99 & 90 & 100 & 79 & 93 & 86 & 96 \\
\hline 4 & 3 & 96 & 96 & 92 & 97 & 81 & 91 & 88 & 93 \\
\hline 4 & 4 & 95 & 99 & 90 & 100 & 79 & 93 & 86 & 96 \\
\hline 5 & 1 & 96 & 100 & 94 & 98 & 82 & 89 & 89 & 93 \\
\hline 5 & 2 & 96 & 100 & 93 & 100 & 82 & 91 & 89 & 96 \\
\hline 5 & 3 & 96 & 100 & 94 & 98 & 82 & 89 & 89 & 93 \\
\hline 5 & 4 & 96 & 100 & 93 & 100 & 82 & 91 & 89 & 96 \\
\hline
\end{tabular}


Table C.2.19 the results of calibration and validations based on the peaks in speed

\begin{tabular}{|c|c|c|c|c|c|c|c|c|c|}
\hline \multicolumn{2}{|c|}{ Configurations } & \multicolumn{2}{|c|}{ Calibration } & \multicolumn{2}{|c|}{$1^{\text {st }}$ Validation } & \multicolumn{2}{|c|}{$2^{\text {nd }}$ Validation } & \multicolumn{2}{|c|}{$3^{\text {rd }}$ Validation } \\
\hline$\varphi_{g}$ & $\Lambda_{l}$ & Vehicles & Cyclists & Vehicles & Cyclists & Vehicles & Cyclists & Vehicles & Cyclists \\
\hline 1 & 1 & 93 & 26 & 96 & 29 & 97 & 24 & 96 & 26 \\
\hline 1 & 2 & 93 & 22 & 96 & 28 & 97 & 23 & 96 & 25 \\
\hline 1 & 3 & 93 & 18 & 96 & 22 & 98 & 17 & 97 & 19 \\
\hline 1 & 4 & 90 & 20 & 93 & 23 & 94 & 21 & 93 & 22 \\
\hline 2 & 1 & 93 & 31 & 93 & 36 & 95 & 37 & 94 & 37 \\
\hline 2 & 2 & 94 & 21 & 94 & 26 & 95 & 27 & 94 & 26 \\
\hline 2 & 3 & 94 & 17 & 94 & 18 & 95 & 20 & - & - \\
\hline 2 & 4 & 94 & 19 & 94 & 25 & - & - & - & - \\
\hline 3 & 1 & 91 & 37 & 92 & 31 & 93 & 35 & 93 & 33 \\
\hline 3 & 2 & 90 & 28 & 92 & 22 & 93 & 30 & 93 & 27 \\
\hline 3 & 3 & 90 & 27 & 92 & 19 & 93 & 26 & 93 & 23 \\
\hline 3 & 4 & 90 & 20 & 92 & 15 & 93 & 27 & 93 & 22 \\
\hline 4 & 1 & 95 & 34 & 94 & 30 & 95 & 33 & 95 & 32 \\
\hline 4 & 2 & 94 & 25 & 94 & 23 & 95 & 27 & 95 & 25 \\
\hline 4 & 3 & 94 & 24 & 94 & 21 & 95 & 21 & 95 & 21 \\
\hline 4 & 4 & 94 & 18 & 94 & 13 & 95 & 25 & 95 & 20 \\
\hline 5 & 1 & 91 & 37 & 95 & 30 & 95 & 36 & 95 & 33 \\
\hline 5 & 2 & 91 & 29 & 95 & 26 & 95 & 27 & 95 & 26 \\
\hline 5 & 3 & 91 & 25 & 95 & 20 & 95 & 11 & 95 & 15 \\
\hline 5 & 4 & 96 & 17 & 96 & 11 & 96 & 27 & 96 & 19 \\
\hline
\end{tabular}


Table C.2.20 the results of calibration and validations based on the effective weighted average frequency

\begin{tabular}{|c|c|c|c|c|c|c|c|c|c|c|}
\hline \multicolumn{3}{|c|}{ Configurations } & \multicolumn{2}{|c|}{ Calibration } & \multicolumn{2}{|c|}{$1^{\text {st }}$ Validation } & \multicolumn{2}{|c|}{$2^{\text {nd }}$ Validation } & \multicolumn{2}{|c|}{$3^{\text {rd }}$ Validation } \\
\hline$\varphi_{g}$ & $\Lambda_{l}$ & $\Gamma_{n}$ & Vehicles & Cyclists & Vehicles & Cyclists & Vehicles & Cyclists & Vehicles & Cyclists \\
\hline 1 & 1 & 1 & 46 & 14 & 53 & 13 & 36 & 12 & 45 & 12 \\
\hline 1 & 1 & 2 & 46 & 13 & 54 & 12 & 38 & 12 & 46 & 12 \\
\hline 1 & 1 & 3 & 46 & 13 & 53 & 12 & 39 & 11 & 46 & 11 \\
\hline 1 & 1 & 4 & 43 & 15 & 51 & 15 & 34 & 9 & 43 & 12 \\
\hline 1 & 1 & 1 & 46 & 13 & 54 & 13 & 41 & 15 & 48 & 14 \\
\hline 1 & 1 & 6 & 47 & 13 & 54 & 13 & 41 & 15 & 48 & 14 \\
\hline 1 & 2 & 1 & 37 & 14 & 42 & 13 & - & - & - & - \\
\hline 1 & 2 & 2 & 37 & 14 & 42 & 13 & - & - & - & - \\
\hline 1 & 2 & 3 & 37 & 14 & 42 & 12 & - & - & - & - \\
\hline 1 & 2 & 4 & 36 & 17 & 43 & 11 & - & - & - & - \\
\hline 1 & 2 & 1 & 36 & 15 & 43 & 14 & - & - & - & - \\
\hline 1 & 2 & 6 & 36 & 17 & 43 & 13 & - & - & - & - \\
\hline 1 & 3 & 1 & 31 & 14 & - & - & - & - & - & - \\
\hline 1 & 3 & 2 & 30 & 17 & - & - & - & - & - & - \\
\hline 1 & 3 & 3 & 29 & 17 & - & - & - & - & - & - \\
\hline 1 & 3 & 4 & 30 & 16 & - & - & - & - & - & - \\
\hline 1 & 3 & 1 & 33 & 13 & - & - & - & - & - & - \\
\hline 1 & 3 & 6 & 31 & 17 & - & - & - & - & - & - \\
\hline 1 & 4 & 1 & 37 & 12 & 41 & 10 & 29 & 5 & - & - \\
\hline 1 & 4 & 2 & 37 & 13 & 41 & 12 & - & - & - & - \\
\hline 1 & 4 & 3 & 36 & 13 & 41 & 11 & - & - & - & - \\
\hline 1 & 4 & 4 & 34 & 16 & 42 & 10 & - & - & - & - \\
\hline 1 & 4 & 1 & 36 & 15 & 43 & 11 & - & - & - & - \\
\hline 1 & 4 & 6 & 36 & 16 & 43 & 11 & - & - & - & - \\
\hline 2 & 1 & 1 & 51 & 17 & 55 & 15 & 37 & 13 & 46 & 13 \\
\hline 2 & 1 & 2 & 51 & 15 & 55 & 13 & 40 & 13 & 48 & 13 \\
\hline 2 & 1 & 3 & 51 & 15 & 55 & 13 & 40 & 11 & 48 & 12 \\
\hline 2 & 1 & 4 & 48 & 18 & 52 & 17 & 35 & 10 & 44 & 13 \\
\hline 2 & 1 & 1 & 49 & 18 & 54 & 16 & 37 & 12 & 46 & 13 \\
\hline 2 & 1 & 6 & 52 & 15 & 56 & 15 & 42 & 15 & 49 & 15 \\
\hline 2 & 2 & 1 & 42 & 17 & 43 & 15 & - & - & - & - \\
\hline 2 & 2 & 2 & 41 & 17 & 43 & 15 & - & - & - & - \\
\hline 2 & 2 & 3 & 41 & 17 & 43 & 13 & - & - & - & - \\
\hline 2 & 2 & 4 & 40 & 20 & 44 & 12 & - & - & - & - \\
\hline 2 & 2 & 1 & 40 & 20 & 44 & 13 & - & - & - & - \\
\hline 2 & 2 & 6 & 40 & 20 & 45 & 15 & - & - & - & - \\
\hline
\end{tabular}


Table C.2.20 the results of calibration and validations based on the effective weighted average frequency

\begin{tabular}{|c|c|c|c|c|c|c|c|c|c|c|}
\hline \multicolumn{3}{|c|}{ Configurations } & \multicolumn{2}{|c|}{ Calibration } & \multicolumn{2}{|c|}{$1^{\text {st }}$ Validation } & \multicolumn{2}{|c|}{$2^{\text {nd }}$ Validation } & \multicolumn{2}{|c|}{$3^{\text {rd }}$ Validation } \\
\hline$\varphi_{g}$ & $\Lambda_{l}$ & $\Gamma_{n}$ & Vehicles & Cyclists & Vehicles & Cyclists & Vehicles & Cyclists & Vehicles & Cyclists \\
\hline 2 & 3 & 1 & 35 & 17 & - & - & - & - & - & - \\
\hline 2 & 3 & 2 & 33 & 20 & - & - & - & - & - & - \\
\hline 2 & 3 & 3 & 33 & 20 & - & - & - & - & - & - \\
\hline 2 & 3 & 4 & 34 & 19 & - & - & - & - & - & - \\
\hline 2 & 3 & 1 & 34 & 19 & - & - & - & - & - & - \\
\hline 2 & 3 & 6 & 35 & 20 & - & - & - & - & - & - \\
\hline 2 & 4 & 1 & 41 & 13 & 42 & 11 & 30 & 6 & - & - \\
\hline 2 & 4 & 2 & 41 & 15 & 43 & 13 & - & - & - & - \\
\hline 2 & 4 & 3 & 40 & 15 & 43 & 12 & - & - & - & - \\
\hline 2 & 4 & 4 & 39 & 18 & 44 & 11 & - & - & - & - \\
\hline 2 & 4 & 1 & 40 & 18 & 44 & 12 & - & - & - & - \\
\hline 2 & 4 & 6 & 40 & 19 & 44 & 12 & - & - & - & - \\
\hline 3 & 1 & 1 & 61 & 20 & 63 & 18 & 42 & 13 & 53 & 15 \\
\hline 3 & 1 & 2 & 61 & 17 & 63 & 17 & 46 & 13 & 55 & 15 \\
\hline 3 & 1 & 3 & 61 & 17 & 63 & 17 & 46 & 13 & 55 & 14 \\
\hline 3 & 1 & 4 & 63 & 13 & 63 & 17 & 46 & 13 & 55 & 15 \\
\hline 3 & 1 & 1 & 58 & 21 & 63 & 19 & 42 & 13 & 53 & 15 \\
\hline 3 & 1 & 6 & 61 & 17 & 64 & 18 & 47 & 17 & 56 & 17 \\
\hline 3 & 2 & 1 & 49 & 20 & 50 & 18 & - & - & - & - \\
\hline 3 & 2 & 2 & 49 & 20 & 50 & 18 & - & - & - & - \\
\hline 3 & 2 & 3 & 49 & 20 & 49 & 17 & - & - & - & - \\
\hline 3 & 2 & 4 & 47 & 24 & 51 & 15 & - & - & - & - \\
\hline 3 & 2 & 1 & 47 & 24 & 51 & 17 & - & - & - & - \\
\hline 3 & 2 & 6 & 48 & 24 & 51 & 18 & - & - & - & - \\
\hline 3 & 3 & 1 & - & - & - & - & - & - & - & - \\
\hline 3 & 3 & 2 & - & - & - & - & - & - & - & - \\
\hline 3 & 3 & 3 & - & - & - & - & - & - & - & - \\
\hline 3 & 3 & 4 & - & - & - & - & - & - & - & - \\
\hline 3 & 3 & 1 & - & - & - & - & - & - & - & - \\
\hline 3 & 3 & 6 & 41 & 24 & - & - & - & - & - & - \\
\hline 3 & 4 & 1 & 49 & 16 & 49 & 14 & 34 & 5 & - & - \\
\hline 3 & 4 & 2 & 49 & 17 & 49 & 17 & - & - & - & - \\
\hline 3 & 4 & 3 & 45 & 21 & 49 & 13 & - & - & - & - \\
\hline 3 & 4 & 4 & 46 & 21 & 51 & 14 & - & - & - & - \\
\hline 3 & 4 & 1 & 47 & 21 & 51 & 15 & - & - & - & - \\
\hline 3 & 4 & 6 & 47 & 23 & 51 & 15 & - & - & - & - \\
\hline
\end{tabular}


Table C.2.20 the results of calibration and validations based on the effective weighted average frequency

\begin{tabular}{|c|c|c|c|c|c|c|c|c|c|c|}
\hline \multicolumn{3}{|c|}{ Configurations } & \multicolumn{2}{|c|}{ Calibration } & \multicolumn{2}{|c|}{$1^{\text {st }}$ Validation } & \multicolumn{2}{|c|}{$2^{\text {nd }}$ Validation } & \multicolumn{2}{|c|}{$3^{\text {rd }}$ Validation } \\
\hline$\varphi_{g}$ & $\Lambda_{l}$ & $\Gamma_{n}$ & Vehicles & Cyclists & Vehicles & Cyclists & Vehicles & Cyclists & Vehicles & Cyclists \\
\hline 4 & 1 & 1 & 72 & 19 & 69 & 18 & 56 & 15 & 64 & 16 \\
\hline 4 & 1 & 2 & 71 & 19 & 70 & 18 & 56 & 15 & 64 & 16 \\
\hline 4 & 1 & 3 & 70 & 19 & 69 & 18 & 56 & 15 & 64 & 16 \\
\hline 4 & 1 & 4 & 67 & 22 & 69 & 21 & 51 & 13 & 61 & 17 \\
\hline 4 & 1 & 1 & 66 & 24 & 69 & 21 & 52 & 15 & 62 & 18 \\
\hline 4 & 1 & 6 & 70 & 18 & 70 & 20 & 54 & 17 & 63 & 18 \\
\hline 4 & 2 & 1 & 54 & 27 & 56 & 18 & - & - & - & - \\
\hline 4 & 2 & 2 & 55 & 25 & 56 & 18 & - & - & - & - \\
\hline 4 & 2 & 3 & 55 & 27 & 56 & 18 & - & - & - & - \\
\hline 4 & 2 & 4 & 55 & 27 & 56 & 18 & - & - & - & - \\
\hline 4 & 2 & 1 & 55 & 27 & 56 & 20 & - & - & - & - \\
\hline 4 & 2 & 6 & 56 & 27 & 57 & 21 & - & - & - & - \\
\hline 4 & 3 & 1 & - & - & - & - & - & - & - & - \\
\hline 4 & 3 & 2 & - & - & - & - & - & - & - & - \\
\hline 4 & 3 & 3 & - & - & - & - & - & - & - & - \\
\hline 4 & 3 & 4 & - & - & - & - & - & - & - & - \\
\hline 4 & 3 & 1 & - & - & - & - & - & - & - & - \\
\hline 4 & 3 & 6 & - & - & - & - & - & - & - & - \\
\hline 4 & 4 & 1 & 55 & 22 & 55 & 15 & - & - & - & - \\
\hline 4 & 4 & 2 & 54 & 22 & 56 & 15 & - & - & - & - \\
\hline 4 & 4 & 3 & 54 & 24 & 56 & 16 & - & - & - & - \\
\hline 4 & 4 & 4 & 53 & 25 & 56 & 16 & - & - & - & - \\
\hline 4 & 4 & 1 & 55 & 24 & 56 & 18 & - & - & - & - \\
\hline 4 & 4 & 6 & 55 & 25 & 56 & 18 & - & - & - & - \\
\hline 5 & 1 & 1 & 76 & 25 & 75 & 22 & 63 & 20 & 70 & 21 \\
\hline 5 & 1 & 2 & 74 & 25 & 74 & 22 & 64 & 20 & 70 & 21 \\
\hline 5 & 1 & 3 & 73 & 25 & 73 & 22 & 63 & 20 & 69 & 21 \\
\hline 5 & 1 & 4 & 68 & 31 & 70 & 28 & 56 & 18 & 65 & 23 \\
\hline 5 & 1 & 1 & 68 & 31 & 73 & 24 & 59 & 20 & 68 & 22 \\
\hline 5 & 1 & 6 & 65 & 35 & 69 & 30 & 57 & 22 & 65 & 26 \\
\hline 5 & 2 & 1 & 57 & 35 & 62 & 24 & - & - & - & - \\
\hline 5 & 2 & 2 & 56 & 33 & 61 & 24 & - & - & - & - \\
\hline 5 & 2 & 3 & 58 & 35 & 62 & 24 & - & - & - & - \\
\hline 5 & 2 & 4 & 57 & 35 & 61 & 24 & - & - & - & - \\
\hline 5 & 2 & 1 & 56 & 35 & 62 & 26 & - & - & - & - \\
\hline 5 & 2 & 6 & 56 & 35 & 62 & 26 & - & - & - & - \\
\hline
\end{tabular}


Table C.2.20 the results of calibration and validations based on the effective weighted average frequency

\begin{tabular}{|c|c|c|c|c|c|c|c|c|c|c|}
\hline \multicolumn{3}{|c|}{ Configurations } & \multicolumn{2}{|c|}{ Calibration } & \multicolumn{2}{|c|}{$1^{\text {st }}$ Validation } & \multicolumn{2}{|c|}{$2^{\text {nd }}$ Validation } & \multicolumn{2}{|c|}{$3^{\text {rd }}$ Validation } \\
\hline$\varphi_{g}$ & $\overline{\Lambda_{l}}$ & $\Gamma_{n}$ & Vehicles & Cyclists & Vehicles & Cyclists & Vehicles & Cyclists & Vehicles & Cyclists \\
\hline 5 & 3 & 1 & - & - & - & - & - & - & - & - \\
\hline 5 & 3 & 2 & - & - & - & - & - & - & - & - \\
\hline 5 & 3 & 3 & - & - & - & - & - & - & - & - \\
\hline 5 & 3 & 4 & - & - & - & - & - & - & - & - \\
\hline 5 & 3 & 1 & - & - & - & - & - & - & - & - \\
\hline 5 & 3 & 6 & - & - & - & - & - & - & - & - \\
\hline 5 & 4 & 1 & 57 & 27 & 61 & 17 & - & - & - & - \\
\hline 5 & 4 & 2 & 56 & 29 & 61 & 20 & - & - & - & - \\
\hline 5 & 4 & 3 & 57 & 31 & 62 & 20 & - & - & - & - \\
\hline 5 & 4 & 4 & 55 & 33 & 61 & 22 & - & - & - & - \\
\hline 5 & 4 & 1 & 56 & 31 & 62 & 22 & - & - & - & - \\
\hline 5 & 4 & 6 & 56 & 33 & 62 & 24 & - & - & - & - \\
\hline
\end{tabular}

Table C.2.21 the results of calibration and validations based on the effective frequency

\begin{tabular}{|c|c|c|c|c|c|c|c|c|c|c|}
\hline \multicolumn{3}{|c|}{ Configurations } & \multicolumn{2}{|c|}{ Calibration } & \multicolumn{2}{|c|}{$1^{\text {st }}$ Validation } & \multicolumn{2}{|c|}{$2^{\text {nd }}$ Validation } & \multicolumn{2}{|c|}{$3^{\text {rd }}$ Validation } \\
\hline$\varphi_{g}$ & $\Lambda_{l}$ & $\eta_{m}$ & Vehicles & Cyclists & Vehicles & Cyclists & Vehicles & Cyclists & Vehicles & Cyclists \\
\hline 1 & 1 & 1 & 87 & 7 & 81 & 2 & 95 & 7 & 88 & 5 \\
\hline 1 & 1 & 2 & 80 & 11 & 80 & 10 & 94 & 8 & 86 & 9 \\
\hline 1 & 1 & 3 & 78 & 14 & 78 & 14 & 90 & 9 & 84 & 11 \\
\hline 1 & 2 & 1 & 87 & 6 & 81 & 1 & 95 & 5 & 88 & 4 \\
\hline 1 & 2 & 2 & 79 & 7 & 79 & 5 & 93 & 4 & 86 & 4 \\
\hline 1 & 2 & 3 & 80 & 9 & 79 & 7 & 92 & 3 & 85 & 5 \\
\hline 1 & 3 & 1 & 87 & 3 & 81 & 0 & 95 & 1 & 88 & 0 \\
\hline 1 & 3 & 2 & 79 & 5 & 79 & 4 & 93 & 1 & 86 & 2 \\
\hline 1 & 3 & 3 & 83 & 7 & 80 & 5 & 93 & 3 & 86 & 4 \\
\hline 1 & 4 & 1 & 85 & 3 & 81 & 0 & 93 & 1 & 87 & 0 \\
\hline 1 & 4 & 2 & 82 & 3 & 80 & 4 & 92 & 4 & 86 & 4 \\
\hline 1 & 4 & 3 & 78 & 5 & 78 & 5 & 89 & 4 & 83 & 4 \\
\hline 2 & 1 & 1 & 86 & 2 & 82 & 1 & 95 & 4 & 88 & 3 \\
\hline 2 & 1 & 2 & 74 & 16 & 70 & 15 & 84 & 24 & 77 & 21 \\
\hline 2 & 1 & 3 & 84 & 9 & 80 & 8 & 91 & 8 & 85 & 8 \\
\hline 2 & 2 & 1 & 86 & 2 & 82 & 1 & 95 & 4 & 88 & 3 \\
\hline 2 & 2 & 2 & 74 & 13 & 70 & 11 & 84 & 18 & 77 & 16 \\
\hline 2 & 2 & 3 & 84 & 3 & 80 & 2 & 91 & 5 & 85 & 4 \\
\hline
\end{tabular}


Table C.2.21 the results of calibration and validations based on the effective frequency

\begin{tabular}{|c|c|c|c|c|c|c|c|c|c|c|}
\hline \multicolumn{3}{|c|}{ Configurations } & \multicolumn{2}{|c|}{ Calibration } & \multicolumn{2}{|c|}{$1^{\text {st }}$ Validation } & \multicolumn{2}{|c|}{$2^{\text {nd }}$ Validation } & \multicolumn{2}{|c|}{$3^{\text {rd }}$ Validation } \\
\hline$\varphi_{g}$ & $\Lambda_{l}$ & $\eta_{m}$ & Vehicles & Cyclists & Vehicles & Cyclists & Vehicles & Cyclists & Vehicles & Cyclists \\
\hline 2 & 3 & 1 & 86 & 1 & 82 & 0 & - & - & - & - \\
\hline 2 & 3 & 2 & 74 & 8 & 70 & 9 & 84 & 9 & 77 & 9 \\
\hline 2 & 3 & 3 & 84 & 2 & 80 & 1 & 91 & 2 & 85 & 2 \\
\hline 2 & 4 & 1 & 86 & 1 & 82 & 0 & 94 & 2 & 88 & 1 \\
\hline 2 & 4 & 2 & 84 & 2 & 81 & 2 & - & - & - & - \\
\hline 2 & 4 & 3 & 84 & 3 & 80 & 3 & 90 & 2 & 85 & 3 \\
\hline 3 & 1 & 1 & 83 & 4 & 78 & 4 & 94 & 3 & 86 & 3 \\
\hline 3 & 1 & 2 & 83 & 11 & 76 & 10 & 92 & 16 & 84 & 14 \\
\hline 3 & 1 & 3 & 82 & 4 & 78 & 8 & 92 & 13 & 84 & 11 \\
\hline 3 & 2 & 1 & 83 & 4 & 78 & 1 & 94 & 0 & 86 & 1 \\
\hline 3 & 2 & 2 & 83 & 11 & 78 & 8 & 93 & 9 & 85 & 9 \\
\hline 3 & 2 & 3 & 82 & 3 & 78 & 7 & 92 & 13 & 84 & 10 \\
\hline 3 & 3 & 1 & 83 & 3 & 78 & 1 & 94 & 0 & 86 & 1 \\
\hline 3 & 3 & 2 & 83 & 7 & 78 & 4 & 93 & 6 & 85 & 5 \\
\hline 3 & 3 & 3 & 82 & 3 & 78 & 4 & 92 & 7 & 84 & 6 \\
\hline 3 & 4 & 1 & 83 & 0 & 78 & 1 & 94 & 0 & 86 & 1 \\
\hline 3 & 4 & 2 & 83 & 0 & 76 & 0 & 92 & 2 & 84 & 1 \\
\hline 3 & 4 & 3 & 82 & 1 & 78 & 1 & 92 & 0 & 84 & 1 \\
\hline 4 & 1 & 1 & 78 & 6 & 74 & 2 & 91 & 1 & 81 & 1 \\
\hline 4 & 1 & 2 & 78 & 10 & 73 & 8 & 92 & 15 & 81 & 12 \\
\hline 4 & 1 & 3 & 76 & 4 & 75 & 7 & 91 & 5 & 82 & 6 \\
\hline 4 & 2 & 1 & 78 & 3 & 74 & 2 & 91 & 1 & 81 & 1 \\
\hline 4 & 2 & 2 & 78 & 7 & 74 & 7 & 92 & 4 & 82 & 5 \\
\hline 4 & 2 & 3 & 76 & 3 & 74 & 15 & 90 & 7 & 81 & 10 \\
\hline 4 & 3 & 1 & 78 & 3 & 74 & 0 & 91 & 1 & 81 & 1 \\
\hline 4 & 3 & 2 & 78 & 3 & 73 & 2 & 92 & 3 & 81 & 2 \\
\hline 4 & 3 & 3 & 76 & 3 & 75 & 3 & 91 & 3 & 82 & 3 \\
\hline 4 & 4 & 1 & 78 & 1 & 74 & 0 & 91 & 0 & 81 & 0 \\
\hline 4 & 4 & 2 & 77 & 3 & 72 & 2 & 91 & 0 & 80 & 1 \\
\hline 4 & 4 & 3 & 76 & 1 & 75 & 2 & 91 & 0 & 82 & 1 \\
\hline 5 & 1 & 1 & 69 & 8 & 67 & 4 & 84 & 9 & 73 & 7 \\
\hline 5 & 1 & 2 & 69 & 12 & 63 & 7 & 84 & 20 & 71 & 13 \\
\hline 5 & 1 & 3 & 93 & 17 & 92 & 11 & 83 & 16 & 89 & 13 \\
\hline 5 & 2 & 1 & 69 & 6 & 67 & 2 & 84 & 7 & 73 & 4 \\
\hline 5 & 2 & 2 & 68 & 6 & 62 & 4 & 84 & 9 & 70 & 7 \\
\hline 5 & 2 & 3 & 93 & 15 & 92 & 9 & - & - & - & - \\
\hline
\end{tabular}


Table C.2.21 the results of calibration and validations based on the effective frequency

\begin{tabular}{|c|c|c|c|c|c|c|c|c|c|c|}
\hline \multicolumn{3}{|c|}{ Configurations } & \multicolumn{2}{|c|}{ Calibration } & \multicolumn{2}{|c|}{$1^{\text {st }}$ Validation } & \multicolumn{2}{|c|}{$2^{\text {nd }}$ Validation } & \multicolumn{2}{|c|}{$3^{\text {rd }}$ Validation } \\
\hline$\varphi_{g}$ & $\Lambda_{l}$ & $\eta_{m}$ & Vehicles & Cyclists & Vehicles & Cyclists & Vehicles & Cyclists & Vehicles & Cyclists \\
\hline 5 & 3 & 1 & 71 & 4 & 67 & 0 & 86 & 2 & 74 & 1 \\
\hline 5 & 3 & 2 & 68 & 4 & 62 & 2 & 84 & 0 & 70 & 1 \\
\hline 5 & 3 & 3 & 95 & 10 & 96 & 2 & 95 & 7 & 96 & 4 \\
\hline 5 & 4 & 1 & 71 & 2 & 67 & 0 & 84 & 0 & 73 & 0 \\
\hline 5 & 4 & 2 & 69 & 4 & 63 & 2 & 84 & 7 & 71 & 4 \\
\hline
\end{tabular}




\section{Appendix D}

\section{The Results of the Calibrations and Validations for the third model "Vehicles and Pedestrians"}




\section{D.1 The results of calibrations and validations using Kappa statistics}

Table D.1.1 the results of calibration procedure 1 and its validations.

\begin{tabular}{|c|c|c|c|c|c|c|c|}
\hline \multicolumn{4}{|c|}{ Configurations } & \multirow{2}{*}{$\begin{array}{c}\text { Calibration } \\
\text { Kappa } \\
\end{array}$} & \multirow{2}{*}{$\frac{\mathbf{1}^{\text {st }} \text { Validation }}{\text { Kappa }}$} & \multirow{2}{*}{$\begin{array}{c}2^{\text {nd }} \text { Validation } \\
\text { Kappa } \\
\end{array}$} & \multirow{2}{*}{$\frac{3^{\text {rd }} \text { Validation }}{\text { Kappa }}$} \\
\hline$\varphi_{g}$ & $\Lambda_{l}$ & $\Gamma_{n}$ & $\eta_{m}$ & & & & \\
\hline 1 & 1 & 1 & 1 & - & - & - & - \\
\hline 1 & 1 & 1 & 2 & - & - & - & - \\
\hline 1 & 1 & 1 & 3 & - & - & - & - \\
\hline 1 & 1 & 2 & 1 & - & - & - & - \\
\hline 1 & 1 & 2 & 2 & - & - & - & - \\
\hline 1 & 1 & 2 & 3 & - & - & - & - \\
\hline 1 & 1 & 3 & 1 & - & - & - & - \\
\hline 1 & 1 & 3 & 2 & - & - & - & - \\
\hline 1 & 1 & 3 & 3 & - & - & - & - \\
\hline 1 & 1 & 4 & 1 & - & - & - & - \\
\hline 1 & 1 & 4 & 2 & - & - & - & - \\
\hline 1 & 1 & 4 & 3 & - & - & - & - \\
\hline 1 & 1 & 1 & 1 & - & - & - & - \\
\hline 1 & 1 & 1 & 2 & - & - & - & - \\
\hline 1 & 1 & 1 & 3 & - & - & - & - \\
\hline 1 & 1 & 6 & 1 & - & - & - & - \\
\hline 1 & 1 & 6 & 2 & - & - & - & - \\
\hline 1 & 1 & 6 & 3 & - & - & - & - \\
\hline 1 & 2 & 1 & 1 & 0.99 & 0.96 & 0.95 & 0.96 \\
\hline 1 & 2 & 1 & 2 & 0.99 & 0.96 & 0.95 & 0.96 \\
\hline 1 & 2 & 1 & 3 & 0.99 & 0.96 & 0.95 & 0.96 \\
\hline 1 & 2 & 2 & 1 & 0.99 & 0.95 & 0.95 & 0.96 \\
\hline 1 & 2 & 2 & 2 & 0.99 & 0.95 & 0.95 & 0.96 \\
\hline 1 & 2 & 2 & 3 & 0.99 & 0.95 & 0.95 & 0.96 \\
\hline 1 & 2 & 3 & 1 & 0.99 & 0.97 & 0.95 & 0.96 \\
\hline 1 & 2 & 3 & 2 & 0.99 & 0.97 & 0.95 & 0.96 \\
\hline 1 & 2 & 3 & 3 & 0.99 & 0.97 & 0.95 & 0.96 \\
\hline 1 & 2 & 4 & 1 & 0.99 & 0.96 & 0.96 & 0.96 \\
\hline 1 & 2 & 4 & 2 & 0.99 & 0.96 & 0.95 & 0.96 \\
\hline 1 & 2 & 4 & 3 & 0.99 & 0.96 & 0.95 & 0.96 \\
\hline 1 & 2 & 1 & 1 & 0.99 & 0.98 & 0.95 & 0.96 \\
\hline 1 & 2 & 1 & 2 & 0.99 & 0.98 & 0.95 & 0.96 \\
\hline 1 & 2 & 1 & 3 & 0.99 & 0.98 & 0.95 & 0.96 \\
\hline 1 & 2 & 6 & 1 & 0.99 & 0.97 & 0.96 & 0.96 \\
\hline 1 & 2 & 6 & 2 & 0.99 & 0.97 & 0.96 & 0.96 \\
\hline 1 & 2 & 6 & 3 & 0.99 & 0.97 & 0.96 & 0.96 \\
\hline 1 & 3 & 1 & 1 & 0.99 & - & - & - \\
\hline 1 & 3 & 1 & 2 & 0.99 & - & - & - \\
\hline 1 & 3 & 1 & 3 & 0.99 & - & - & - \\
\hline 1 & 3 & 2 & 1 & 0.99 & - & - & - \\
\hline 1 & 3 & 2 & 2 & 0.99 & - & - & - \\
\hline 1 & 3 & 2 & 3 & 0.99 & - & - & - \\
\hline 1 & 3 & 3 & 1 & 0.99 & - & - & - \\
\hline 1 & 3 & 3 & 2 & 0.99 & - & - & - \\
\hline 1 & 3 & 3 & 3 & 0.99 & - & - & - \\
\hline 1 & 3 & 4 & 1 & 0.99 & - & - & - \\
\hline 1 & 3 & 4 & 2 & 0.99 & - & - & - \\
\hline 1 & 3 & 4 & 3 & 0.99 & - & - & - \\
\hline 1 & 3 & 1 & 1 & 0.99 & - & - & - \\
\hline
\end{tabular}


Table D.1.1 the results of calibration procedure 1 and its validations.

\begin{tabular}{|c|c|c|c|c|c|c|c|}
\hline \multicolumn{4}{|c|}{ Configurations } & \multirow{2}{*}{$\begin{array}{c}\text { Calibration } \\
\text { Kappa } \\
\end{array}$} & \multirow{2}{*}{$\begin{array}{c}\mathbf{1}^{\text {st }} \text { Validation } \\
\text { Kappa } \\
\end{array}$} & \multirow{2}{*}{$\begin{array}{c}2^{\text {nd }} \text { Validation } \\
\text { Kappa } \\
\end{array}$} & \multirow{2}{*}{$\begin{array}{c}3^{\text {rd }} \text { Validation } \\
\text { Kappa } \\
\end{array}$} \\
\hline$\varphi_{g}$ & $\Lambda_{l}$ & $\Gamma_{n}$ & $\eta_{m}$ & & & & \\
\hline 1 & 3 & 1 & 2 & 0.99 & - & - & - \\
\hline 1 & 3 & 1 & 3 & 0.99 & - & - & - \\
\hline 1 & 3 & 6 & 1 & 0.99 & - & - & - \\
\hline 1 & 3 & 6 & 2 & 0.99 & - & - & - \\
\hline 1 & 3 & 6 & 3 & 0.99 & - & - & - \\
\hline 1 & 4 & 1 & 1 & - & - & - & - \\
\hline 1 & 4 & 1 & 2 & - & - & - & - \\
\hline 1 & 4 & 1 & 3 & - & - & - & - \\
\hline 1 & 4 & 2 & 1 & - & - & - & - \\
\hline 1 & 4 & 2 & 2 & - & - & - & - \\
\hline 1 & 4 & 2 & 3 & - & - & - & - \\
\hline 1 & 4 & 3 & 1 & - & - & - & - \\
\hline 1 & 4 & 3 & 2 & - & - & - & - \\
\hline 1 & 4 & 3 & 3 & - & - & - & - \\
\hline 1 & 4 & 4 & 1 & - & - & - & - \\
\hline 1 & 4 & 4 & 2 & - & - & - & - \\
\hline 1 & 4 & 4 & 3 & - & - & - & - \\
\hline 1 & 4 & 1 & 1 & - & - & - & - \\
\hline 1 & 4 & 1 & 2 & - & - & - & - \\
\hline 1 & 4 & 1 & 3 & - & - & - & - \\
\hline 1 & 4 & 6 & 1 & - & - & - & - \\
\hline 1 & 4 & 6 & 2 & - & - & - & - \\
\hline 1 & 4 & 6 & 3 & - & - & - & - \\
\hline 2 & 1 & 1 & 1 & 1.00 & 0.78 & 0.80 & 0.81 \\
\hline 2 & 1 & 1 & 2 & 1.00 & 0.78 & 0.80 & 0.81 \\
\hline 2 & 1 & 1 & 3 & 1.00 & 0.78 & 0.80 & 0.81 \\
\hline 2 & 1 & 2 & 1 & 1.00 & 0.80 & 0.82 & 0.82 \\
\hline 2 & 1 & 2 & 2 & 1.00 & 0.80 & 0.82 & 0.82 \\
\hline 2 & 1 & 2 & 3 & 1.00 & 0.80 & 0.82 & 0.82 \\
\hline 2 & 1 & 3 & 1 & 1.00 & 0.80 & 0.82 & 0.82 \\
\hline 2 & 1 & 3 & 2 & 1.00 & 0.80 & 0.82 & 0.82 \\
\hline 2 & 1 & 3 & 3 & 1.00 & 0.80 & 0.82 & 0.82 \\
\hline 2 & 1 & 4 & 1 & 1.00 & 0.78 & 0.82 & 0.82 \\
\hline 2 & 1 & 4 & 2 & 1.00 & 0.78 & 0.82 & 0.82 \\
\hline 2 & 1 & 4 & 3 & 1.00 & 0.78 & 0.82 & 0.82 \\
\hline 2 & 1 & 1 & 1 & 1.00 & 0.82 & 0.83 & 0.84 \\
\hline 2 & 1 & 1 & 2 & 1.00 & 0.82 & 0.83 & 0.84 \\
\hline 2 & 1 & 1 & 3 & 1.00 & 0.82 & 0.83 & 0.84 \\
\hline 2 & 1 & 6 & 1 & 1.00 & 0.78 & 0.82 & 0.82 \\
\hline 2 & 1 & 6 & 2 & 1.00 & 0.78 & 0.82 & 0.82 \\
\hline 2 & 1 & 6 & 3 & 1.00 & 0.78 & 0.82 & 0.82 \\
\hline 2 & 2 & 1 & 1 & 1.00 & 0.80 & 0.83 & 0.84 \\
\hline 2 & 2 & 1 & 2 & 1.00 & 0.80 & 0.84 & 0.84 \\
\hline 2 & 2 & 1 & 3 & 1.00 & 0.82 & 0.84 & 0.85 \\
\hline 2 & 2 & 2 & 1 & 1.00 & 0.80 & 0.83 & 0.84 \\
\hline 2 & 2 & 2 & 2 & 1.00 & 0.80 & 0.83 & 0.84 \\
\hline 2 & 2 & 2 & 3 & 1.00 & 0.82 & 0.84 & 0.85 \\
\hline 2 & 2 & 3 & 1 & 1.00 & 0.80 & 0.83 & 0.84 \\
\hline 2 & 2 & 3 & 2 & 1.00 & 0.80 & 0.84 & 0.84 \\
\hline 2 & 2 & 3 & 3 & 1.00 & 0.82 & 0.84 & 0.85 \\
\hline 2 & 2 & 4 & 1 & 1.00 & 0.82 & 0.84 & 0.84 \\
\hline
\end{tabular}


Table D.1.1 the results of calibration procedure 1 and its validations.

\begin{tabular}{|c|c|c|c|c|c|c|c|}
\hline \multicolumn{4}{|c|}{ Configurations } & \multirow{2}{*}{$\begin{array}{c}\text { Calibration } \\
\text { Kappa } \\
\end{array}$} & \multirow{2}{*}{$\begin{array}{c}\mathbf{1}^{\text {st }} \text { Validation } \\
\text { Kappa } \\
\end{array}$} & \multirow{2}{*}{$\begin{array}{c}2^{\text {nd }} \text { Validation } \\
\text { Kappa } \\
\end{array}$} & \multirow{2}{*}{$\begin{array}{c}3^{\text {rd }} \text { Validation } \\
\text { Kappa } \\
\end{array}$} \\
\hline$\varphi_{g}$ & $\Lambda_{l}$ & $\Gamma_{n}$ & $\eta_{m}$ & & & & \\
\hline 2 & 2 & 4 & 2 & 1.00 & 0.82 & 0.84 & 0.84 \\
\hline 2 & 2 & 4 & 3 & 1.00 & 0.83 & 0.84 & 0.85 \\
\hline 2 & 2 & 1 & 1 & 1.00 & 0.81 & 0.84 & 0.84 \\
\hline 2 & 2 & 1 & 2 & 1.00 & 0.81 & 0.84 & 0.84 \\
\hline 2 & 2 & 1 & 3 & 1.00 & 0.83 & 0.84 & 0.85 \\
\hline 2 & 2 & 6 & 1 & 1.00 & 0.80 & 0.83 & 0.83 \\
\hline 2 & 2 & 6 & 2 & 1.00 & 0.80 & 0.83 & 0.84 \\
\hline 2 & 2 & 6 & 3 & 1.00 & 0.81 & 0.83 & 0.84 \\
\hline 2 & 3 & 1 & 1 & 1.00 & 0.83 & - & - \\
\hline 2 & 3 & 1 & 2 & 1.00 & 0.82 & - & - \\
\hline 2 & 3 & 1 & 3 & 1.00 & 0.82 & - & - \\
\hline 2 & 3 & 2 & 1 & 1.00 & 0.83 & - & - \\
\hline 2 & 3 & 2 & 2 & 1.00 & 0.82 & - & - \\
\hline 2 & 3 & 2 & 3 & 1.00 & 0.82 & - & - \\
\hline 2 & 3 & 3 & 1 & 1.00 & 0.82 & - & - \\
\hline 2 & 3 & 3 & 2 & 1.00 & 0.81 & - & - \\
\hline 2 & 3 & 3 & 3 & 1.00 & 0.81 & - & - \\
\hline 2 & 3 & 4 & 1 & 1.00 & 0.83 & - & - \\
\hline 2 & 3 & 4 & 2 & 1.00 & 0.82 & - & - \\
\hline 2 & 3 & 4 & 3 & 1.00 & 0.82 & - & - \\
\hline 2 & 3 & 1 & 1 & 1.00 & 0.80 & - & - \\
\hline 2 & 3 & 1 & 2 & 1.00 & 0.79 & - & - \\
\hline 2 & 3 & 1 & 3 & 1.00 & 0.79 & - & - \\
\hline 2 & 3 & 6 & 1 & 1.00 & 0.83 & - & - \\
\hline 2 & 3 & 6 & 2 & 1.00 & 0.82 & - & - \\
\hline 2 & 3 & 6 & 3 & 1.00 & 0.82 & - & - \\
\hline 2 & 4 & 1 & 1 & 1.00 & 0.83 & - & - \\
\hline 2 & 4 & 1 & 2 & 1.00 & 0.83 & - & - \\
\hline 2 & 4 & 1 & 3 & 1.00 & 0.83 & - & - \\
\hline 2 & 4 & 2 & 1 & 1.00 & 0.84 & - & - \\
\hline 2 & 4 & 2 & 2 & 1.00 & 0.84 & - & - \\
\hline 2 & 4 & 2 & 3 & 1.00 & 0.84 & - & - \\
\hline 2 & 4 & 3 & 1 & 1.00 & 0.84 & - & - \\
\hline 2 & 4 & 3 & 2 & 1.00 & 0.84 & - & - \\
\hline 2 & 4 & 3 & 3 & 1.00 & 0.84 & - & - \\
\hline 2 & 4 & 4 & 1 & 1.00 & 0.83 & - & - \\
\hline 2 & 4 & 4 & 2 & 1.00 & 0.83 & - & - \\
\hline 2 & 4 & 4 & 3 & 1.00 & 0.83 & - & - \\
\hline 2 & 4 & 1 & 1 & 1.00 & 0.83 & - & - \\
\hline 2 & 4 & 1 & 2 & 1.00 & 0.83 & - & - \\
\hline 2 & 4 & 1 & 3 & 1.00 & 0.83 & - & - \\
\hline 2 & 4 & 6 & 1 & 1.00 & 0.82 & - & - \\
\hline 2 & 4 & 6 & 2 & 1.00 & 0.82 & 0.78 & 0.81 \\
\hline 2 & 4 & 6 & 3 & 1.00 & 0.82 & 0.78 & 0.81 \\
\hline 3 & 1 & 1 & 1 & 1.00 & 0.79 & 0.80 & 0.81 \\
\hline 3 & 1 & 1 & 2 & 1.00 & 0.79 & 0.80 & 0.81 \\
\hline 3 & 1 & 1 & 3 & 1.00 & 0.79 & 0.80 & 0.81 \\
\hline 3 & 1 & 2 & 1 & 1.00 & 0.79 & 0.81 & 0.82 \\
\hline 3 & 1 & 2 & 2 & 1.00 & 0.79 & 0.81 & 0.82 \\
\hline 3 & 1 & 2 & 3 & 1.00 & 0.79 & 0.81 & 0.82 \\
\hline 3 & 1 & 3 & 1 & 1.00 & 0.80 & 0.81 & 0.83 \\
\hline
\end{tabular}


Table D.1.1 the results of calibration procedure 1 and its validations.

\begin{tabular}{|c|c|c|c|c|c|c|c|}
\hline \multicolumn{4}{|c|}{ Configurations } & \multirow{2}{*}{$\begin{array}{c}\text { Calibration } \\
\text { Kappa } \\
\end{array}$} & \multirow{2}{*}{$\begin{array}{c}\mathbf{1}^{\text {st }} \text { Validation } \\
\text { Kappa } \\
\end{array}$} & \multirow{2}{*}{$\begin{array}{c}2^{\text {nd }} \text { Validation } \\
\text { Kappa } \\
\end{array}$} & \multirow{2}{*}{$\begin{array}{c}3^{\text {rd }} \text { Validation } \\
\text { Kappa } \\
\end{array}$} \\
\hline$\varphi_{g}$ & $\Lambda_{l}$ & $\Gamma_{n}$ & $\eta_{m}$ & & & & \\
\hline 3 & 1 & 3 & 2 & 1.00 & 0.80 & 0.81 & 0.83 \\
\hline 3 & 1 & 3 & 3 & 1.00 & 0.80 & 0.81 & 0.83 \\
\hline 3 & 1 & 4 & 1 & 1.00 & 0.78 & 0.81 & 0.82 \\
\hline 3 & 1 & 4 & 2 & 1.00 & 0.78 & 0.81 & 0.82 \\
\hline 3 & 1 & 4 & 3 & 1.00 & 0.78 & 0.81 & 0.82 \\
\hline 3 & 1 & 1 & 1 & 1.00 & 0.79 & 0.82 & 0.83 \\
\hline 3 & 1 & 1 & 2 & 1.00 & 0.79 & 0.82 & 0.83 \\
\hline 3 & 1 & 1 & 3 & 1.00 & 0.79 & 0.82 & 0.83 \\
\hline 3 & 1 & 6 & 1 & 1.00 & 0.79 & 0.81 & 0.82 \\
\hline 3 & 1 & 6 & 2 & 1.00 & 0.79 & 0.81 & 0.82 \\
\hline 3 & 1 & 6 & 3 & 1.00 & 0.79 & 0.81 & 0.82 \\
\hline 3 & 2 & 1 & 1 & 1.00 & 0.82 & 0.83 & 0.84 \\
\hline 3 & 2 & 1 & 2 & 1.00 & 0.82 & 0.83 & 0.84 \\
\hline 3 & 2 & 1 & 3 & 1.00 & 0.82 & 0.83 & 0.84 \\
\hline 3 & 2 & 2 & 1 & 1.00 & 0.82 & 0.84 & 0.85 \\
\hline 3 & 2 & 2 & 2 & 1.00 & 0.82 & 0.84 & 0.85 \\
\hline 3 & 2 & 2 & 3 & 1.00 & 0.82 & 0.83 & 0.84 \\
\hline 3 & 2 & 3 & 1 & 1.00 & 0.83 & 0.82 & 0.84 \\
\hline 3 & 2 & 3 & 2 & 1.00 & 0.83 & 0.82 & 0.84 \\
\hline 3 & 2 & 3 & 3 & 1.00 & 0.83 & 0.82 & 0.84 \\
\hline 3 & 2 & 4 & 1 & 1.00 & 0.83 & 0.84 & 0.85 \\
\hline 3 & 2 & 4 & 2 & 1.00 & 0.83 & 0.83 & 0.85 \\
\hline 3 & 2 & 4 & 3 & 1.00 & 0.83 & 0.83 & 0.85 \\
\hline 3 & 2 & 1 & 1 & 1.00 & 0.83 & 0.83 & 0.85 \\
\hline 3 & 2 & 1 & 2 & 1.00 & 0.83 & 0.83 & 0.84 \\
\hline 3 & 2 & 1 & 3 & 1.00 & 0.83 & 0.83 & 0.84 \\
\hline 3 & 2 & 6 & 1 & 1.00 & 0.83 & 0.85 & 0.86 \\
\hline 3 & 2 & 6 & 2 & 1.00 & 0.83 & 0.84 & 0.85 \\
\hline 3 & 2 & 6 & 3 & 1.00 & 0.83 & 0.84 & 0.85 \\
\hline 3 & 3 & 1 & 1 & 1.00 & 0.74 & 0.81 & - \\
\hline 3 & 3 & 1 & 2 & 1.00 & 0.74 & 0.82 & 0.82 \\
\hline 3 & 3 & 1 & 3 & 1.00 & 0.74 & 0.80 & - \\
\hline 3 & 3 & 2 & 1 & 1.00 & 0.77 & 0.81 & 0.82 \\
\hline 3 & 3 & 2 & 2 & 1.00 & 0.77 & 0.81 & 0.82 \\
\hline 3 & 3 & 2 & 3 & 1.00 & 0.77 & 0.80 & - \\
\hline 3 & 3 & 3 & 1 & 1.00 & 0.76 & 0.79 & - \\
\hline 3 & 3 & 3 & 2 & 1.00 & - & - & - \\
\hline 3 & 3 & 3 & 3 & 1.00 & - & - & - \\
\hline 3 & 3 & 4 & 1 & 1.00 & 0.76 & - & - \\
\hline 3 & 3 & 4 & 2 & 1.00 & 0.76 & 0.80 & - \\
\hline 3 & 3 & 4 & 3 & 1.00 & 0.76 & - & - \\
\hline 3 & 3 & 1 & 1 & 1.00 & 0.77 & 0.81 & 0.82 \\
\hline 3 & 3 & 1 & 2 & 1.00 & 0.77 & 0.82 & 0.82 \\
\hline 3 & 3 & 1 & 3 & 1.00 & 0.77 & 0.80 & 0.81 \\
\hline 3 & 3 & 6 & 1 & 1.00 & 0.77 & 0.82 & 0.82 \\
\hline 3 & 3 & 6 & 2 & 1.00 & 0.77 & 0.82 & 0.83 \\
\hline 3 & 3 & 6 & 3 & 1.00 & 0.77 & 0.81 & 0.82 \\
\hline 3 & 4 & 1 & 1 & 1.00 & 0.81 & 0.82 & 0.84 \\
\hline 3 & 4 & 1 & 2 & 1.00 & 0.81 & 0.83 & 0.84 \\
\hline 3 & 4 & 1 & 3 & 1.00 & 0.81 & 0.83 & 0.84 \\
\hline 3 & 4 & 2 & 1 & 1.00 & 0.83 & 0.85 & 0.86 \\
\hline
\end{tabular}


Table D.1.1 the results of calibration procedure 1 and its validations.

\begin{tabular}{|c|c|c|c|c|c|c|c|}
\hline \multicolumn{4}{|c|}{ Configurations } & \multirow{2}{*}{$\begin{array}{c}\text { Calibration } \\
\text { Kappa } \\
\end{array}$} & \multirow{2}{*}{$\begin{array}{c}\mathbf{1}^{\text {st }} \text { Validation } \\
\text { Kappa } \\
\end{array}$} & \multirow{2}{*}{$\begin{array}{c}2^{\text {nd }} \text { Validation } \\
\text { Kappa } \\
\end{array}$} & \multirow{2}{*}{$\begin{array}{c}\mathbf{3}^{\text {rd }} \text { Validation } \\
\text { Kappa }\end{array}$} \\
\hline$\varphi_{g}$ & $\Lambda_{l}$ & $\Gamma_{n}$ & $\eta_{m}$ & & & & \\
\hline 3 & 4 & 2 & 2 & 1.00 & 0.83 & 0.86 & 0.86 \\
\hline 3 & 4 & 2 & 3 & 1.00 & 0.83 & 0.86 & 0.86 \\
\hline 3 & 4 & 3 & 1 & 1.00 & 0.83 & 0.83 & 0.84 \\
\hline 3 & 4 & 3 & 2 & 1.00 & 0.83 & 0.84 & 0.85 \\
\hline 3 & 4 & 3 & 3 & 1.00 & 0.83 & 0.84 & 0.85 \\
\hline 3 & 4 & 4 & 1 & 1.00 & 0.85 & 0.83 & 0.85 \\
\hline 3 & 4 & 4 & 2 & 1.00 & 0.85 & 0.84 & 0.86 \\
\hline 3 & 4 & 4 & 3 & 1.00 & 0.85 & 0.84 & 0.86 \\
\hline 3 & 4 & 1 & 1 & 1.00 & 0.84 & 0.83 & 0.85 \\
\hline 3 & 4 & 1 & 2 & 1.00 & 0.84 & 0.84 & 0.85 \\
\hline 3 & 4 & 1 & 3 & 1.00 & 0.84 & 0.84 & 0.85 \\
\hline 3 & 4 & 6 & 1 & 1.00 & 0.84 & 0.82 & 0.84 \\
\hline 3 & 4 & 6 & 2 & 1.00 & 0.84 & 0.83 & 0.85 \\
\hline 3 & 4 & 6 & 3 & 1.00 & 0.84 & 0.83 & 0.85 \\
\hline 4 & 1 & 1 & 1 & 1.00 & 0.72 & - & - \\
\hline 4 & 1 & 1 & 2 & 1.00 & 0.72 & - & - \\
\hline 4 & 1 & 1 & 3 & 1.00 & 0.72 & - & - \\
\hline 4 & 1 & 2 & 1 & 1.00 & - & - & - \\
\hline 4 & 1 & 2 & 2 & 1.00 & - & - & - \\
\hline 4 & 1 & 2 & 3 & 1.00 & - & - & - \\
\hline 4 & 1 & 3 & 1 & 1.00 & 0.77 & - & - \\
\hline 4 & 1 & 3 & 2 & 1.00 & 0.77 & - & - \\
\hline 4 & 1 & 3 & 3 & 1.00 & 0.77 & - & - \\
\hline 4 & 1 & 4 & 1 & 1.00 & 0.73 & - & - \\
\hline 4 & 1 & 4 & 2 & 1.00 & 0.73 & - & - \\
\hline 4 & 1 & 4 & 3 & 1.00 & 0.73 & - & - \\
\hline 4 & 1 & 1 & 1 & 1.00 & - & - & - \\
\hline 4 & 1 & 1 & 2 & 1.00 & - & - & - \\
\hline 4 & 1 & 1 & 3 & 1.00 & - & - & - \\
\hline 4 & 1 & 6 & 1 & 1.00 & 0.73 & - & - \\
\hline 4 & 1 & 6 & 2 & 1.00 & 0.73 & - & - \\
\hline 4 & 1 & 6 & 3 & 1.00 & 0.73 & - & - \\
\hline 4 & 2 & 1 & 1 & 1.00 & 0.79 & 0.92 & 0.89 \\
\hline 4 & 2 & 1 & 2 & 1.00 & 0.79 & 0.91 & 0.89 \\
\hline 4 & 2 & 1 & 3 & 1.00 & 0.79 & 0.91 & 0.89 \\
\hline 4 & 2 & 2 & 1 & 1.00 & 0.84 & 0.89 & 0.89 \\
\hline 4 & 2 & 2 & 2 & 1.00 & 0.84 & 0.89 & 0.89 \\
\hline 4 & 2 & 2 & 3 & 1.00 & 0.84 & 0.88 & 0.88 \\
\hline 4 & 2 & 3 & 1 & 1.00 & 0.84 & 0.89 & 0.89 \\
\hline 4 & 2 & 3 & 2 & 1.00 & 0.84 & 0.89 & 0.89 \\
\hline 4 & 2 & 3 & 3 & 1.00 & 0.84 & 0.88 & 0.88 \\
\hline 4 & 2 & 4 & 1 & 1.00 & 0.84 & 0.90 & 0.89 \\
\hline 4 & 2 & 4 & 2 & 1.00 & 0.84 & 0.89 & 0.89 \\
\hline 4 & 2 & 4 & 3 & 1.00 & 0.82 & 0.89 & 0.88 \\
\hline 4 & 2 & 1 & 1 & 1.00 & 0.84 & 0.90 & 0.89 \\
\hline 4 & 2 & 1 & 2 & 1.00 & 0.84 & 0.89 & 0.89 \\
\hline 4 & 2 & 1 & 3 & 1.00 & 0.84 & 0.89 & 0.89 \\
\hline 4 & 2 & 6 & 1 & 1.00 & 0.84 & 0.89 & 0.89 \\
\hline 4 & 2 & 6 & 2 & 1.00 & 0.84 & 0.89 & 0.89 \\
\hline 4 & 2 & 6 & 3 & 1.00 & 0.84 & 0.89 & 0.89 \\
\hline 4 & 3 & 1 & 1 & 1.00 & - & - & - \\
\hline
\end{tabular}


Table D.1.1 the results of calibration procedure 1 and its validations.

\begin{tabular}{|c|c|c|c|c|c|c|c|}
\hline \multicolumn{4}{|c|}{ Configurations } & \multirow{2}{*}{$\begin{array}{c}\text { Calibration } \\
\text { Kappa } \\
\end{array}$} & \multirow{2}{*}{$\begin{array}{c}\mathbf{1}^{\text {st }} \text { Validation } \\
\text { Kappa } \\
\end{array}$} & \multirow{2}{*}{$\begin{array}{c}2^{\text {nd }} \text { Validation } \\
\text { Kappa } \\
\end{array}$} & \multirow{2}{*}{$\begin{array}{c}\mathbf{3}^{\text {rd }} \text { Validation } \\
\text { Kappa }\end{array}$} \\
\hline$\varphi_{g}$ & $\Lambda_{l}$ & $\Gamma_{n}$ & $\eta_{m}$ & & & & \\
\hline 4 & 3 & 1 & 2 & 1.00 & - & - & - \\
\hline 4 & 3 & 1 & 3 & 1.00 & - & - & - \\
\hline 4 & 3 & 2 & 1 & 1.00 & - & - & - \\
\hline 4 & 3 & 2 & 2 & 1.00 & - & - & - \\
\hline 4 & 3 & 2 & 3 & 1.00 & - & - & - \\
\hline 4 & 3 & 3 & 1 & 1.00 & - & - & - \\
\hline 4 & 3 & 3 & 2 & 1.00 & - & - & - \\
\hline 4 & 3 & 3 & 3 & 1.00 & - & - & - \\
\hline 4 & 3 & 4 & 1 & 1.00 & - & - & - \\
\hline 4 & 3 & 4 & 2 & 1.00 & - & - & - \\
\hline 4 & 3 & 4 & 3 & 1.00 & - & - & - \\
\hline 4 & 3 & 1 & 1 & 1.00 & - & - & - \\
\hline 4 & 3 & 1 & 2 & 1.00 & - & - & - \\
\hline 4 & 3 & 1 & 3 & 1.00 & - & - & - \\
\hline 4 & 3 & 6 & 1 & 1.00 & - & - & - \\
\hline 4 & 3 & 6 & 2 & 1.00 & 0.73 & - & - \\
\hline 4 & 3 & 6 & 3 & 1.00 & - & - & - \\
\hline 4 & 4 & 1 & 1 & 0.98 & 0.72 & 0.87 & 0.85 \\
\hline 4 & 4 & 1 & 2 & 0.98 & - & - & - \\
\hline 4 & 4 & 1 & 3 & 0.98 & - & - & - \\
\hline 4 & 4 & 2 & 1 & 0.98 & 0.73 & - & - \\
\hline 4 & 4 & 2 & 2 & 0.98 & 0.72 & - & - \\
\hline 4 & 4 & 2 & 3 & 0.98 & 0.72 & - & - \\
\hline 4 & 4 & 3 & 1 & 0.98 & 0.73 & 0.87 & 0.85 \\
\hline 4 & 4 & 3 & 2 & 0.98 & 0.72 & 0.87 & - \\
\hline 4 & 4 & 3 & 3 & 0.98 & 0.72 & 0.87 & - \\
\hline 4 & 4 & 4 & 1 & 0.98 & 0.73 & - & - \\
\hline 4 & 4 & 4 & 2 & 0.98 & - & - & - \\
\hline 4 & 4 & 4 & 3 & 0.98 & - & - & - \\
\hline 4 & 4 & 1 & 1 & 0.98 & 0.74 & 0.86 & 0.85 \\
\hline 4 & 4 & 1 & 2 & 0.98 & 0.73 & 0.86 & - \\
\hline 4 & 4 & 1 & 3 & 0.98 & 0.73 & 0.86 & - \\
\hline 4 & 4 & 6 & 1 & 0.98 & 0.75 & 0.86 & 0.85 \\
\hline 4 & 4 & 6 & 2 & 0.98 & 0.74 & 0.86 & - \\
\hline 4 & 4 & 6 & 3 & 0.98 & 0.74 & 0.86 & 0.85 \\
\hline 5 & 1 & 1 & 1 & 1.00 & 0.75 & 0.85 & 0.85 \\
\hline 5 & 1 & 1 & 2 & 1.00 & 0.75 & 0.84 & 0.85 \\
\hline 5 & 1 & 1 & 3 & 1.00 & 0.75 & 0.84 & 0.85 \\
\hline 5 & 1 & 2 & 1 & 1.00 & 0.73 & 0.82 & 0.83 \\
\hline 5 & 1 & 2 & 2 & 1.00 & 0.73 & 0.82 & 0.83 \\
\hline 5 & 1 & 2 & 3 & 1.00 & 0.73 & 0.82 & 0.83 \\
\hline 5 & 1 & 3 & 1 & 1.00 & 0.75 & 0.85 & 0.85 \\
\hline 5 & 1 & 3 & 2 & 1.00 & 0.77 & 0.83 & 0.85 \\
\hline 5 & 1 & 3 & 3 & 1.00 & 0.75 & 0.83 & 0.84 \\
\hline 5 & 1 & 4 & 1 & 1.00 & 0.77 & 0.85 & 0.85 \\
\hline 5 & 1 & 4 & 2 & 1.00 & 0.79 & 0.83 & 0.85 \\
\hline 5 & 1 & 4 & 3 & 1.00 & 0.77 & 0.83 & 0.85 \\
\hline 5 & 1 & 1 & 1 & 1.00 & 0.74 & 0.83 & 0.84 \\
\hline 5 & 1 & 1 & 2 & 1.00 & 0.74 & 0.83 & 0.84 \\
\hline 5 & 1 & 1 & 3 & 1.00 & 0.74 & 0.83 & 0.84 \\
\hline 5 & 1 & 6 & 1 & 1.00 & 0.75 & 0.86 & 0.85 \\
\hline
\end{tabular}


Table D.1.1 the results of calibration procedure 1 and its validations.

\begin{tabular}{|c|c|c|c|c|c|c|c|}
\hline \multicolumn{4}{|c|}{ Configurations } & \multirow{2}{*}{$\begin{array}{c}\text { Calibration } \\
\text { Kappa } \\
\end{array}$} & \multirow{2}{*}{$\begin{array}{c}\mathbf{1}^{\text {st }} \text { Validation } \\
\text { Kappa } \\
\end{array}$} & \multirow{2}{*}{$\begin{array}{c}2^{\text {nd }} \text { Validation } \\
\text { Kappa } \\
\end{array}$} & \multirow{2}{*}{$\begin{array}{c}3^{\text {rd }} \text { Validation } \\
\text { Kappa } \\
\end{array}$} \\
\hline$\varphi_{g}$ & $\Lambda_{l}$ & $\Gamma_{n}$ & $\eta_{m}$ & & & & \\
\hline 5 & 1 & 6 & 2 & 1.00 & 0.77 & 0.84 & 0.85 \\
\hline 5 & 1 & 6 & 3 & 1.00 & 0.75 & 0.84 & 0.85 \\
\hline 5 & 2 & 1 & 1 & 1.00 & 0.65 & 0.87 & 0.82 \\
\hline 5 & 2 & 1 & 2 & 1.00 & 0.67 & 0.86 & 0.82 \\
\hline 5 & 2 & 1 & 3 & 1.00 & 0.64 & 0.85 & 0.81 \\
\hline 5 & 2 & 2 & 1 & 1.00 & - & - & - \\
\hline 5 & 2 & 2 & 2 & 1.00 & 0.65 & 0.86 & 0.82 \\
\hline 5 & 2 & 2 & 3 & 1.00 & - & - & - \\
\hline 5 & 2 & 3 & 1 & 1.00 & - & - & - \\
\hline 5 & 2 & 3 & 2 & 1.00 & 0.65 & 0.86 & 0.82 \\
\hline 5 & 2 & 3 & 3 & 1.00 & - & - & - \\
\hline 5 & 2 & 4 & 1 & 1.00 & - & - & - \\
\hline 5 & 2 & 4 & 2 & 1.00 & 0.65 & 0.87 & 0.82 \\
\hline 5 & 2 & 4 & 3 & 1.00 & - & - & - \\
\hline 5 & 2 & 1 & 1 & 1.00 & - & - & - \\
\hline 5 & 2 & 1 & 2 & 1.00 & 0.65 & 0.87 & 0.82 \\
\hline 5 & 2 & 1 & 3 & 1.00 & - & - & - \\
\hline 5 & 2 & 6 & 1 & 1.00 & - & - & - \\
\hline 5 & 2 & 6 & 2 & 1.00 & 0.65 & 0.87 & 0.82 \\
\hline 5 & 2 & 6 & 3 & 1.00 & - & - & - \\
\hline 5 & 3 & 1 & 1 & 1.00 & 0.66 & - & - \\
\hline 5 & 3 & 1 & 2 & 1.00 & 0.65 & - & - \\
\hline 5 & 3 & 1 & 3 & 1.00 & 0.69 & - & - \\
\hline 5 & 3 & 2 & 1 & 1.00 & 0.68 & - & - \\
\hline 5 & 3 & 2 & 2 & 1.00 & 0.70 & - & - \\
\hline 5 & 3 & 2 & 3 & 1.00 & 0.70 & - & - \\
\hline 5 & 3 & 3 & 1 & 1.00 & 0.67 & - & - \\
\hline 5 & 3 & 3 & 2 & 1.00 & 0.64 & - & - \\
\hline 5 & 3 & 3 & 3 & 1.00 & 0.68 & - & - \\
\hline 5 & 3 & 4 & 1 & 1.00 & 0.64 & - & - \\
\hline 5 & 3 & 4 & 2 & 1.00 & 0.64 & - & - \\
\hline 5 & 3 & 4 & 3 & 1.00 & 0.68 & - & - \\
\hline 5 & 3 & 1 & 1 & 1.00 & 0.67 & - & - \\
\hline 5 & 3 & 1 & 2 & 1.00 & 0.64 & - & - \\
\hline 5 & 3 & 1 & 3 & 1.00 & 0.68 & - & - \\
\hline 5 & 3 & 6 & 1 & 1.00 & 0.67 & - & - \\
\hline 5 & 3 & 6 & 2 & 1.00 & 0.64 & - & - \\
\hline 5 & 3 & 6 & 3 & 1.00 & 0.68 & - & - \\
\hline 5 & 4 & 1 & 1 & 0.98 & 0.64 & 0.89 & 0.83 \\
\hline 5 & 4 & 1 & 2 & 0.98 & - & - & - \\
\hline 5 & 4 & 1 & 3 & 0.98 & - & - & - \\
\hline 5 & 4 & 2 & 1 & 0.98 & 0.66 & 0.86 & 0.82 \\
\hline 5 & 4 & 2 & 2 & 0.98 & 0.65 & 0.88 & 0.83 \\
\hline 5 & 4 & 2 & 3 & 0.98 & 0.65 & 0.86 & 0.82 \\
\hline 5 & 4 & 3 & 1 & 0.98 & 0.68 & 0.87 & 0.83 \\
\hline 5 & 4 & 3 & 2 & 0.98 & 0.66 & 0.88 & 0.84 \\
\hline 5 & 4 & 3 & 3 & 0.98 & 0.66 & 0.87 & 0.83 \\
\hline 5 & 4 & 4 & 1 & 0.98 & 0.66 & 0.87 & 0.83 \\
\hline 5 & 4 & 4 & 2 & 0.98 & 0.65 & 0.89 & 0.83 \\
\hline 5 & 4 & 4 & 3 & 0.98 & 0.65 & 0.87 & 0.82 \\
\hline 5 & 4 & 1 & 1 & 0.98 & 0.68 & 0.86 & 0.83 \\
\hline
\end{tabular}


Table D.1.1 the results of calibration procedure 1 and its validations.

\begin{tabular}{|c|c|c|c|c|c|c|c|}
\hline \multicolumn{4}{|c|}{ Configurations } & Calibration & $\mathbf{1}^{\text {st }}$ Validation & $2^{\text {nd }}$ Validation & $3^{\text {rd }}$ Validation \\
\hline $\boldsymbol{\varphi}_{\boldsymbol{g}}$ & $\boldsymbol{\Lambda}_{\boldsymbol{l}}$ & $\boldsymbol{\Gamma}_{\boldsymbol{n}}$ & $\boldsymbol{\eta}_{\boldsymbol{m}}$ & Kappa & Kappa & Kappa & Kappa \\
\hline 5 & 4 & 1 & 2 & 0.98 & 0.66 & 0.88 & 0.83 \\
\hline 5 & 4 & 1 & 3 & 0.98 & 0.66 & 0.86 & 0.82 \\
\hline 5 & 4 & 6 & 1 & 0.98 & 0.68 & 0.86 & 0.83 \\
\hline 5 & 4 & 6 & 2 & 0.98 & 0.66 & 0.88 & 0.83 \\
\hline 5 & 4 & 6 & 3 & 0.98 & 0.66 & 0.86 & 0.82 \\
\hline
\end{tabular}

Table D.1.2 the results of calibration procedure 2 and its validations.

\begin{tabular}{|c|c|c|c|c|c|c|c|}
\hline \multicolumn{4}{|c|}{ Configurations } & \multirow{2}{*}{$\begin{array}{c}\text { Calibration } \\
\text { Kappa } \\
\end{array}$} & \multirow{2}{*}{$\begin{array}{c}\mathbf{1}^{\text {st }} \text { Validation } \\
\text { Kappa }\end{array}$} & \multirow{2}{*}{$\begin{array}{c}2^{\text {nd }} \text { Validation } \\
\text { Kappa }\end{array}$} & \multirow{2}{*}{$\begin{array}{c}\mathbf{3}^{\text {rd }} \text { Validation } \\
\text { Kappa }\end{array}$} \\
\hline$\varphi_{g}$ & $\Lambda_{l}$ & $\Gamma_{n}$ & $\eta_{m}$ & & & & \\
\hline 1 & 1 & 1 & 1 & 0.88 & 0.90 & 0.83 & 0.86 \\
\hline 1 & 1 & 1 & 2 & 0.88 & 0.90 & 0.83 & 0.86 \\
\hline 1 & 1 & 1 & 3 & 0.87 & 0.89 & 0.83 & 0.86 \\
\hline 1 & 1 & 2 & 1 & 0.88 & 0.90 & 0.83 & 0.86 \\
\hline 1 & 1 & 2 & 2 & 0.88 & 0.90 & 0.83 & 0.86 \\
\hline 1 & 1 & 2 & 3 & 0.87 & 0.89 & 0.83 & 0.86 \\
\hline 1 & 1 & 3 & 1 & 0.88 & 0.90 & 0.83 & 0.86 \\
\hline 1 & 1 & 3 & 2 & 0.88 & 0.90 & 0.83 & 0.86 \\
\hline 1 & 1 & 3 & 3 & 0.87 & 0.89 & 0.83 & 0.86 \\
\hline 1 & 1 & 4 & 1 & 0.88 & 0.90 & 0.83 & 0.86 \\
\hline 1 & 1 & 4 & 2 & 0.88 & 0.90 & 0.83 & 0.86 \\
\hline 1 & 1 & 4 & 3 & 0.87 & 0.89 & 0.83 & 0.86 \\
\hline 1 & 1 & 1 & 1 & 0.88 & 0.90 & 0.83 & 0.86 \\
\hline 1 & 1 & 1 & 2 & 0.88 & 0.90 & 0.83 & 0.86 \\
\hline 1 & 1 & 1 & 3 & 0.87 & 0.89 & 0.83 & 0.86 \\
\hline 1 & 1 & 6 & 1 & 0.88 & 0.90 & 0.83 & 0.86 \\
\hline 1 & 1 & 6 & 2 & 0.88 & 0.90 & 0.83 & 0.86 \\
\hline 1 & 1 & 6 & 3 & 0.87 & 0.89 & 0.83 & 0.86 \\
\hline 1 & 2 & 1 & 1 & 0.92 & - & - & - \\
\hline 1 & 2 & 1 & 2 & 0.92 & - & - & - \\
\hline 1 & 2 & 1 & 3 & 0.92 & - & - & - \\
\hline 1 & 2 & 2 & 1 & 0.92 & - & - & - \\
\hline 1 & 2 & 2 & 2 & 0.92 & - & - & - \\
\hline 1 & 2 & 2 & 3 & 0.92 & - & - & - \\
\hline 1 & 2 & 3 & 1 & 0.92 & - & - & - \\
\hline 1 & 2 & 3 & 2 & 0.92 & - & - & - \\
\hline 1 & 2 & 3 & 3 & 0.92 & - & - & - \\
\hline 1 & 2 & 4 & 1 & 0.92 & - & - & - \\
\hline 1 & 2 & 4 & 2 & 0.92 & - & - & - \\
\hline 1 & 2 & 4 & 3 & 0.92 & - & - & - \\
\hline 1 & 2 & 1 & 1 & 0.92 & - & - & - \\
\hline 1 & 2 & 1 & 2 & 0.92 & - & - & - \\
\hline 1 & 2 & 1 & 3 & 0.92 & - & - & - \\
\hline 1 & 2 & 6 & 1 & 0.92 & - & - & - \\
\hline 1 & 2 & 6 & 2 & 0.92 & - & - & - \\
\hline 1 & 2 & 6 & 3 & 0.92 & - & - & - \\
\hline 1 & 3 & 1 & 1 & 0.91 & 0.90 & 0.85 & 0.88 \\
\hline 1 & 3 & 1 & 2 & 0.91 & 0.90 & 0.85 & 0.88 \\
\hline 1 & 3 & 1 & 3 & 0.91 & 0.90 & 0.85 & 0.88 \\
\hline 1 & 3 & 2 & 1 & 0.91 & 0.90 & 0.85 & 0.88 \\
\hline 1 & 3 & 2 & 2 & 0.91 & 0.90 & 0.85 & 0.88 \\
\hline
\end{tabular}


Table D.1.2 the results of calibration procedure 2 and its validations.

\begin{tabular}{|c|c|c|c|c|c|c|c|}
\hline \multicolumn{4}{|c|}{ Configurations } & \multirow{2}{*}{$\begin{array}{c}\text { Calibration } \\
\text { Kappa } \\
\end{array}$} & \multirow{2}{*}{$\begin{array}{c}\mathbf{1}^{\text {st }} \text { Validation } \\
\text { Kappa } \\
\end{array}$} & \multirow{2}{*}{$\begin{array}{c}2^{\text {nd }} \text { Validation } \\
\text { Kappa } \\
\end{array}$} & \multirow{2}{*}{$\begin{array}{c}\mathbf{3}^{\text {rd }} \text { Validatior } \\
\text { Kappa } \\
\end{array}$} \\
\hline$\varphi_{g}$ & $\Lambda_{l}$ & $\Gamma_{n}$ & $\eta_{m}$ & & & & \\
\hline 1 & 3 & 2 & 3 & 0.91 & 0.90 & 0.85 & 0.88 \\
\hline 1 & 3 & 3 & 1 & 0.91 & 0.90 & 0.85 & 0.88 \\
\hline 1 & 3 & 3 & 2 & 0.91 & 0.90 & 0.85 & 0.88 \\
\hline 1 & 3 & 3 & 3 & 0.91 & 0.90 & 0.85 & 0.88 \\
\hline 1 & 3 & 4 & 1 & 0.91 & 0.90 & 0.85 & 0.88 \\
\hline 1 & 3 & 4 & 2 & 0.91 & 0.90 & 0.85 & 0.88 \\
\hline 1 & 3 & 4 & 3 & 0.91 & 0.90 & 0.85 & 0.88 \\
\hline 1 & 3 & 1 & 1 & 0.91 & 0.90 & 0.85 & 0.88 \\
\hline 1 & 3 & 1 & 2 & 0.91 & 0.90 & 0.85 & 0.88 \\
\hline 1 & 3 & 1 & 3 & 0.91 & 0.90 & 0.85 & 0.88 \\
\hline 1 & 3 & 6 & 1 & 0.91 & 0.90 & 0.85 & 0.88 \\
\hline 1 & 3 & 6 & 2 & 0.91 & 0.90 & 0.85 & 0.88 \\
\hline 1 & 3 & 6 & 3 & 0.91 & 0.90 & 0.85 & 0.88 \\
\hline 1 & 4 & 1 & 1 & 0.86 & 0.84 & 0.88 & 0.87 \\
\hline 1 & 4 & 1 & 2 & 0.86 & 0.84 & 0.88 & 0.87 \\
\hline 1 & 4 & 1 & 3 & 0.86 & 0.84 & 0.88 & 0.87 \\
\hline 1 & 4 & 2 & 1 & 0.86 & 0.84 & 0.88 & 0.87 \\
\hline 1 & 4 & 2 & 2 & 0.86 & 0.84 & 0.88 & 0.87 \\
\hline 1 & 4 & 2 & 3 & 0.86 & 0.84 & 0.88 & 0.87 \\
\hline 1 & 4 & 3 & 1 & 0.86 & 0.84 & 0.88 & 0.87 \\
\hline 1 & 4 & 3 & 2 & 0.86 & 0.84 & 0.88 & 0.87 \\
\hline 1 & 4 & 3 & 3 & 0.86 & 0.84 & 0.88 & 0.87 \\
\hline 1 & 4 & 4 & 1 & 0.86 & 0.84 & 0.88 & 0.87 \\
\hline 1 & 4 & 4 & 2 & 0.86 & 0.84 & 0.88 & 0.87 \\
\hline 1 & 4 & 4 & 3 & 0.86 & 0.84 & 0.88 & 0.87 \\
\hline 1 & 4 & 1 & 1 & 0.86 & 0.84 & 0.88 & 0.87 \\
\hline 1 & 4 & 1 & 2 & 0.86 & 0.84 & 0.88 & 0.87 \\
\hline 1 & 4 & 1 & 3 & 0.86 & 0.84 & 0.88 & 0.87 \\
\hline 1 & 4 & 6 & 1 & 0.86 & 0.84 & 0.88 & 0.87 \\
\hline 1 & 4 & 6 & 2 & 0.86 & 0.84 & 0.88 & 0.87 \\
\hline 1 & 4 & 6 & 3 & 0.86 & 0.84 & 0.88 & 0.87 \\
\hline 2 & 1 & 1 & 1 & - & - & - & - \\
\hline 2 & 1 & 1 & 2 & - & - & - & - \\
\hline 2 & 1 & 1 & 3 & - & - & - & - \\
\hline 2 & 1 & 2 & 1 & - & - & - & - \\
\hline 2 & 1 & 2 & 2 & - & - & - & - \\
\hline 2 & 1 & 2 & 3 & - & - & - & - \\
\hline 2 & 1 & 3 & 1 & - & - & - & - \\
\hline 2 & 1 & 3 & 2 & - & - & - & - \\
\hline 2 & 1 & 3 & 3 & - & - & - & - \\
\hline 2 & 1 & 4 & 1 & - & - & - & - \\
\hline 2 & 1 & 4 & 2 & - & - & - & - \\
\hline 2 & 1 & 4 & 3 & - & - & - & - \\
\hline 2 & 1 & 1 & 1 & - & - & - & - \\
\hline 2 & 1 & 1 & 2 & - & - & - & - \\
\hline 2 & 1 & 1 & 3 & - & - & - & - \\
\hline 2 & 1 & 6 & 1 & - & - & - & - \\
\hline 2 & 1 & 6 & 2 & - & - & - & - \\
\hline 2 & 1 & 6 & 3 & - & - & - & - \\
\hline 2 & 2 & 1 & 1 & - & - & - & - \\
\hline 2 & 2 & 1 & 2 & - & - & - & - \\
\hline
\end{tabular}


Table D.1.2 the results of calibration procedure 2 and its validations.

\begin{tabular}{|c|c|c|c|c|c|c|c|}
\hline \multicolumn{4}{|c|}{ Configurations } & \multirow{2}{*}{$\begin{array}{c}\text { Calibration } \\
\text { Kappa } \\
\end{array}$} & \multirow{2}{*}{$\begin{array}{c}\mathbf{1}^{\text {st }} \text { Validation } \\
\text { Kappa } \\
\end{array}$} & \multirow{2}{*}{$\begin{array}{c}2^{\text {nd }} \text { Validation } \\
\text { Kappa } \\
\end{array}$} & \multirow{2}{*}{$\begin{array}{c}3^{\text {rd }} \text { Validation } \\
\text { Kappa } \\
\end{array}$} \\
\hline$\varphi_{g}$ & $\Lambda_{l}$ & $\Gamma_{n}$ & $\eta_{m}$ & & & & \\
\hline 2 & 2 & 1 & 3 & - & - & - & - \\
\hline 2 & 2 & 2 & 1 & - & - & - & - \\
\hline 2 & 2 & 2 & 2 & - & - & - & - \\
\hline 2 & 2 & 2 & 3 & - & - & - & - \\
\hline 2 & 2 & 3 & 1 & - & - & - & - \\
\hline 2 & 2 & 3 & 2 & - & - & - & - \\
\hline 2 & 2 & 3 & 3 & - & - & - & - \\
\hline 2 & 2 & 4 & 1 & - & - & - & - \\
\hline 2 & 2 & 4 & 2 & - & - & - & - \\
\hline 2 & 2 & 4 & 3 & - & - & - & - \\
\hline 2 & 2 & 1 & 1 & - & - & - & - \\
\hline 2 & 2 & 1 & 2 & - & - & - & - \\
\hline 2 & 2 & 1 & 3 & - & - & - & - \\
\hline 2 & 2 & 6 & 1 & - & - & - & - \\
\hline 2 & 2 & 6 & 2 & - & - & - & - \\
\hline 2 & 2 & 6 & 3 & - & - & - & - \\
\hline 2 & 3 & 1 & 1 & 1.00 & 0.88 & 0.84 & 0.87 \\
\hline 2 & 3 & 1 & 2 & 1.00 & 0.88 & 0.85 & 0.87 \\
\hline 2 & 3 & 1 & 3 & 1.00 & 0.88 & 0.85 & 0.87 \\
\hline 2 & 3 & 2 & 1 & 1.00 & 0.89 & 0.85 & 0.87 \\
\hline 2 & 3 & 2 & 2 & 1.00 & 0.89 & 0.86 & 0.88 \\
\hline 2 & 3 & 2 & 3 & 1.00 & 0.89 & 0.86 & 0.88 \\
\hline 2 & 3 & 3 & 1 & 1.00 & 0.88 & 0.84 & 0.86 \\
\hline 2 & 3 & 3 & 2 & 1.00 & 0.88 & 0.85 & 0.87 \\
\hline 2 & 3 & 3 & 3 & 1.00 & 0.88 & 0.85 & 0.87 \\
\hline 2 & 3 & 4 & 1 & 1.00 & 0.89 & 0.84 & 0.86 \\
\hline 2 & 3 & 4 & 2 & 1.00 & 0.89 & 0.85 & 0.87 \\
\hline 2 & 3 & 4 & 3 & 1.00 & 0.89 & 0.85 & 0.87 \\
\hline 2 & 3 & 1 & 1 & 1.00 & 0.90 & 0.85 & 0.87 \\
\hline 2 & 3 & 1 & 2 & 1.00 & 0.90 & 0.85 & 0.88 \\
\hline 2 & 3 & 1 & 3 & 1.00 & 0.90 & 0.85 & 0.88 \\
\hline 2 & 3 & 6 & 1 & 1.00 & 0.87 & 0.84 & 0.86 \\
\hline 2 & 3 & 6 & 2 & 1.00 & 0.87 & 0.85 & 0.87 \\
\hline 2 & 3 & 6 & 3 & 1.00 & 0.87 & 0.85 & 0.87 \\
\hline 2 & 4 & 1 & 1 & 1.00 & - & - & - \\
\hline 2 & 4 & 1 & 2 & 1.00 & - & - & - \\
\hline 2 & 4 & 1 & 3 & 1.00 & - & - & - \\
\hline 2 & 4 & 2 & 1 & 1.00 & - & - & - \\
\hline 2 & 4 & 2 & 2 & 1.00 & - & - & - \\
\hline 2 & 4 & 2 & 3 & 1.00 & - & - & - \\
\hline 2 & 4 & 3 & 1 & 1.00 & - & - & - \\
\hline 2 & 4 & 3 & 2 & 1.00 & - & - & - \\
\hline 2 & 4 & 3 & 3 & 1.00 & - & - & - \\
\hline 2 & 4 & 4 & 1 & 1.00 & 0.83 & 0.84 & 0.85 \\
\hline 2 & 4 & 4 & 2 & 1.00 & 0.83 & 0.84 & 0.84 \\
\hline 2 & 4 & 4 & 3 & 1.00 & 0.83 & 0.84 & 0.85 \\
\hline 2 & 4 & 1 & 1 & 1.00 & - & - & - \\
\hline 2 & 4 & 1 & 2 & 1.00 & - & - & - \\
\hline 2 & 4 & 1 & 3 & 1.00 & - & - & - \\
\hline 2 & 4 & 6 & 1 & 1.00 & - & - & - \\
\hline 2 & 4 & 6 & 2 & 1.00 & - & - & - \\
\hline
\end{tabular}


Table D.1.2 the results of calibration procedure 2 and its validations.

\begin{tabular}{|c|c|c|c|c|c|c|c|}
\hline \multicolumn{4}{|c|}{ Configurations } & \multirow{2}{*}{$\begin{array}{c}\text { Calibration } \\
\text { Kappa } \\
\end{array}$} & \multirow{2}{*}{$\begin{array}{c}\mathbf{1}^{\text {st }} \text { Validation } \\
\text { Kappa } \\
\end{array}$} & \multirow{2}{*}{$\begin{array}{c}2^{\text {nd }} \text { Validation } \\
\text { Kappa } \\
\end{array}$} & \multirow{2}{*}{$\begin{array}{c}\mathbf{3}^{\text {rd }} \text { Validatior } \\
\text { Kappa } \\
\end{array}$} \\
\hline$\varphi_{g}$ & $\Lambda_{l}$ & $\Gamma_{n}$ & $\eta_{m}$ & & & & \\
\hline 2 & 4 & 6 & 3 & 1.00 & - & - & - \\
\hline 3 & 1 & 1 & 1 & 0.97 & 0.90 & 0.94 & 0.94 \\
\hline 3 & 1 & 1 & 2 & 0.97 & 0.90 & 0.94 & 0.94 \\
\hline 3 & 1 & 1 & 3 & 0.97 & 0.90 & 0.94 & 0.94 \\
\hline 3 & 1 & 2 & 1 & 0.97 & 0.90 & 0.94 & 0.94 \\
\hline 3 & 1 & 2 & 2 & 0.97 & 0.90 & 0.94 & 0.94 \\
\hline 3 & 1 & 2 & 3 & 0.97 & 0.90 & 0.94 & 0.94 \\
\hline 3 & 1 & 3 & 1 & 0.97 & 0.90 & 0.94 & 0.94 \\
\hline 3 & 1 & 3 & 2 & 0.97 & 0.90 & 0.94 & 0.94 \\
\hline 3 & 1 & 3 & 3 & 0.97 & 0.90 & 0.94 & 0.94 \\
\hline 3 & 1 & 4 & 1 & 0.97 & 0.90 & 0.94 & 0.94 \\
\hline 3 & 1 & 4 & 2 & 0.97 & 0.90 & 0.94 & 0.94 \\
\hline 3 & 1 & 4 & 3 & 0.97 & 0.90 & 0.94 & 0.94 \\
\hline 3 & 1 & 1 & 1 & 0.97 & 0.90 & 0.94 & 0.94 \\
\hline 3 & 1 & 1 & 2 & 0.97 & 0.90 & 0.94 & 0.94 \\
\hline 3 & 1 & 1 & 3 & 0.97 & 0.90 & 0.94 & 0.94 \\
\hline 3 & 1 & 6 & 1 & 0.97 & 0.90 & 0.94 & 0.94 \\
\hline 3 & 1 & 6 & 2 & 0.97 & 0.90 & 0.94 & 0.94 \\
\hline 3 & 1 & 6 & 3 & 0.97 & 0.90 & 0.94 & 0.94 \\
\hline 3 & 2 & 1 & 1 & - & - & - & - \\
\hline 3 & 2 & 1 & 2 & - & - & - & - \\
\hline 3 & 2 & 1 & 3 & - & - & - & - \\
\hline 3 & 2 & 2 & 1 & - & - & - & - \\
\hline 3 & 2 & 2 & 2 & - & - & - & - \\
\hline 3 & 2 & 2 & 3 & - & - & - & - \\
\hline 3 & 2 & 3 & 1 & - & - & - & - \\
\hline 3 & 2 & 3 & 2 & - & - & - & - \\
\hline 3 & 2 & 3 & 3 & - & - & - & - \\
\hline 3 & 2 & 4 & 1 & - & - & - & - \\
\hline 3 & 2 & 4 & 2 & - & - & - & - \\
\hline 3 & 2 & 4 & 3 & - & - & - & - \\
\hline 3 & 2 & 1 & 1 & - & - & - & - \\
\hline 3 & 2 & 1 & 2 & - & - & - & - \\
\hline 3 & 2 & 1 & 3 & - & - & - & - \\
\hline 3 & 2 & 6 & 1 & - & - & - & - \\
\hline 3 & 2 & 6 & 2 & - & - & - & - \\
\hline 3 & 2 & 6 & 3 & - & - & - & - \\
\hline 3 & 3 & 1 & 1 & 1.00 & 0.90 & - & - \\
\hline 3 & 3 & 1 & 2 & 1.00 & 0.86 & - & - \\
\hline 3 & 3 & 1 & 3 & 1.00 & 0.86 & - & - \\
\hline 3 & 3 & 2 & 1 & 1.00 & 0.86 & - & - \\
\hline 3 & 3 & 2 & 2 & 1.00 & 0.84 & - & - \\
\hline 3 & 3 & 2 & 3 & 1.00 & 0.84 & - & - \\
\hline 3 & 3 & 3 & 1 & 1.00 & 0.89 & - & - \\
\hline 3 & 3 & 3 & 2 & 1.00 & 0.86 & 0.90 & - \\
\hline 3 & 3 & 3 & 3 & 1.00 & 0.86 & - & - \\
\hline 3 & 3 & 4 & 1 & 1.00 & 0.89 & - & - \\
\hline 3 & 3 & 4 & 2 & 1.00 & 0.86 & 0.90 & - \\
\hline 3 & 3 & 4 & 3 & 1.00 & 0.86 & 0.90 & - \\
\hline 3 & 3 & 1 & 1 & 1.00 & 0.89 & - & - \\
\hline 3 & 3 & 1 & 2 & 1.00 & 0.86 & - & - \\
\hline
\end{tabular}


Table D.1.2 the results of calibration procedure 2 and its validations.

\begin{tabular}{|c|c|c|c|c|c|c|c|}
\hline \multicolumn{4}{|c|}{ Configurations } & \multirow{2}{*}{$\begin{array}{c}\text { Calibration } \\
\text { Kappa } \\
\end{array}$} & \multirow{2}{*}{$\begin{array}{c}\mathbf{1}^{\text {st }} \text { Validation } \\
\text { Kappa } \\
\end{array}$} & \multirow{2}{*}{$\begin{array}{c}2^{\text {nd }} \text { Validation } \\
\text { Kappa } \\
\end{array}$} & \multirow{2}{*}{$\begin{array}{c}\mathbf{3}^{\text {rd }} \text { Validation } \\
\text { Kappa } \\
\end{array}$} \\
\hline$\varphi_{g}$ & $\Lambda_{l}$ & $\Gamma_{n}$ & $\eta_{m}$ & & & & \\
\hline 3 & 3 & 1 & 3 & 1.00 & 0.86 & - & - \\
\hline 3 & 3 & 6 & 1 & 1.00 & 0.89 & - & - \\
\hline 3 & 3 & 6 & 2 & 1.00 & 0.86 & 0.90 & - \\
\hline 3 & 3 & 6 & 3 & 1.00 & 0.86 & - & - \\
\hline 3 & 4 & 1 & 1 & 0.99 & - & - & - \\
\hline 3 & 4 & 1 & 2 & 0.99 & - & - & - \\
\hline 3 & 4 & 1 & 3 & 0.99 & - & - & - \\
\hline 3 & 4 & 2 & 1 & 0.99 & - & - & - \\
\hline 3 & 4 & 2 & 2 & 0.99 & - & - & - \\
\hline 3 & 4 & 2 & 3 & 0.99 & - & - & - \\
\hline 3 & 4 & 3 & 1 & 0.99 & - & - & - \\
\hline 3 & 4 & 3 & 2 & 0.99 & - & - & - \\
\hline 3 & 4 & 3 & 3 & 0.99 & - & - & - \\
\hline 3 & 4 & 4 & 1 & 0.99 & - & - & - \\
\hline 3 & 4 & 4 & 2 & 0.99 & - & - & - \\
\hline 3 & 4 & 4 & 3 & 0.99 & - & - & - \\
\hline 3 & 4 & 1 & 1 & 0.99 & - & - & - \\
\hline 3 & 4 & 1 & 2 & 0.99 & - & - & - \\
\hline 3 & 4 & 1 & 3 & 0.99 & - & - & - \\
\hline 3 & 4 & 6 & 1 & 0.99 & - & - & - \\
\hline 3 & 4 & 6 & 2 & 0.99 & - & - & - \\
\hline 3 & 4 & 6 & 3 & 0.99 & - & - & - \\
\hline 4 & 1 & 1 & 1 & - & - & - & - \\
\hline 4 & 1 & 1 & 2 & - & - & - & - \\
\hline 4 & 1 & 1 & 3 & - & - & - & - \\
\hline 4 & 1 & 2 & 1 & - & - & - & - \\
\hline 4 & 1 & 2 & 2 & - & - & - & - \\
\hline 4 & 1 & 2 & 3 & - & - & - & - \\
\hline 4 & 1 & 3 & 1 & - & - & - & - \\
\hline 4 & 1 & 3 & 2 & - & - & - & - \\
\hline 4 & 1 & 3 & 3 & - & - & - & - \\
\hline 4 & 1 & 4 & 1 & - & - & - & - \\
\hline 4 & 1 & 4 & 2 & - & - & - & - \\
\hline 4 & 1 & 4 & 3 & - & - & - & - \\
\hline 4 & 1 & 1 & 1 & - & - & - & - \\
\hline 4 & 1 & 1 & 2 & - & - & - & - \\
\hline 4 & 1 & 1 & 3 & - & - & - & - \\
\hline 4 & 1 & 6 & 1 & - & - & - & - \\
\hline 4 & 1 & 6 & 2 & - & - & - & - \\
\hline 4 & 1 & 6 & 3 & - & - & - & - \\
\hline 4 & 2 & 1 & 1 & 1.00 & 0.87 & 0.89 & 0.90 \\
\hline 4 & 2 & 1 & 2 & 1.00 & 0.87 & 0.91 & 0.91 \\
\hline 4 & 2 & 1 & 3 & 1.00 & 0.87 & 0.91 & 0.91 \\
\hline 4 & 2 & 2 & 1 & 1.00 & 0.87 & 0.92 & 0.92 \\
\hline 4 & 2 & 2 & 2 & 1.00 & 0.87 & 0.93 & 0.92 \\
\hline 4 & 2 & 2 & 3 & 1.00 & 0.87 & 0.93 & 0.92 \\
\hline 4 & 2 & 3 & 1 & 1.00 & 0.86 & 0.90 & 0.90 \\
\hline 4 & 2 & 3 & 2 & 1.00 & 0.86 & 0.91 & 0.91 \\
\hline 4 & 2 & 3 & 3 & 1.00 & 0.86 & 0.91 & 0.91 \\
\hline 4 & 2 & 4 & 1 & 1.00 & 0.89 & 0.92 & 0.92 \\
\hline 4 & 2 & 4 & 2 & 1.00 & 0.87 & 0.91 & 0.91 \\
\hline
\end{tabular}


Table D.1.2 the results of calibration procedure 2 and its validations.

\begin{tabular}{|c|c|c|c|c|c|c|c|}
\hline \multicolumn{4}{|c|}{ Configurations } & \multirow{2}{*}{$\begin{array}{c}\text { Calibration } \\
\text { Kappa } \\
\end{array}$} & \multirow{2}{*}{$\begin{array}{c}\mathbf{1}^{\text {st }} \text { Validation } \\
\text { Kappa } \\
\end{array}$} & \multirow{2}{*}{$\begin{array}{c}2^{\text {nd }} \text { Validation } \\
\text { Kappa } \\
\end{array}$} & \multirow{2}{*}{$\begin{array}{c}3^{\text {rd }} \text { Validation } \\
\text { Kappa } \\
\end{array}$} \\
\hline$\varphi_{g}$ & $\Lambda_{l}$ & $\Gamma_{n}$ & $\eta_{m}$ & & & & \\
\hline 4 & 2 & 4 & 3 & 1.00 & 0.87 & 0.91 & 0.91 \\
\hline 4 & 2 & 1 & 1 & 1.00 & 0.86 & 0.92 & 0.91 \\
\hline 4 & 2 & 1 & 2 & 1.00 & 0.86 & 0.92 & 0.91 \\
\hline 4 & 2 & 1 & 3 & 1.00 & 0.86 & 0.92 & 0.91 \\
\hline 4 & 2 & 6 & 1 & 1.00 & 0.89 & 0.92 & 0.92 \\
\hline 4 & 2 & 6 & 2 & 1.00 & 0.87 & 0.91 & 0.91 \\
\hline 4 & 2 & 6 & 3 & 1.00 & 0.87 & 0.91 & 0.91 \\
\hline 4 & 3 & 1 & 1 & 0.97 & 0.87 & 0.92 & 0.92 \\
\hline 4 & 3 & 1 & 2 & 0.97 & 0.87 & 0.92 & 0.92 \\
\hline 4 & 3 & 1 & 3 & 0.97 & 0.87 & 0.92 & 0.92 \\
\hline 4 & 3 & 2 & 1 & 0.97 & 0.87 & 0.92 & 0.92 \\
\hline 4 & 3 & 2 & 2 & 0.97 & 0.87 & 0.92 & 0.92 \\
\hline 4 & 3 & 2 & 3 & 0.97 & 0.87 & 0.92 & 0.92 \\
\hline 4 & 3 & 3 & 1 & 0.97 & 0.87 & 0.92 & 0.92 \\
\hline 4 & 3 & 3 & 2 & 0.97 & 0.87 & 0.92 & 0.92 \\
\hline 4 & 3 & 3 & 3 & 0.97 & 0.87 & 0.92 & 0.92 \\
\hline 4 & 3 & 4 & 1 & 0.97 & 0.87 & 0.92 & 0.92 \\
\hline 4 & 3 & 4 & 2 & 0.97 & 0.87 & 0.92 & 0.92 \\
\hline 4 & 3 & 4 & 3 & 0.97 & 0.87 & 0.92 & 0.92 \\
\hline 4 & 3 & 1 & 1 & 0.97 & 0.87 & 0.92 & 0.92 \\
\hline 4 & 3 & 1 & 2 & 0.97 & 0.87 & 0.92 & 0.92 \\
\hline 4 & 3 & 1 & 3 & 0.97 & 0.87 & 0.92 & 0.92 \\
\hline 4 & 3 & 6 & 1 & 0.97 & 0.87 & 0.92 & 0.92 \\
\hline 4 & 3 & 6 & 2 & 0.97 & 0.87 & 0.92 & 0.92 \\
\hline 4 & 3 & 6 & 3 & 0.97 & 0.87 & 0.92 & 0.92 \\
\hline 4 & 4 & 1 & 1 & 1.00 & 0.88 & 0.92 & 0.92 \\
\hline 4 & 4 & 1 & 2 & 1.00 & 0.88 & 0.92 & 0.92 \\
\hline 4 & 4 & 1 & 3 & 1.00 & 0.88 & 0.92 & 0.92 \\
\hline 4 & 4 & 2 & 1 & 1.00 & 0.88 & 0.92 & 0.92 \\
\hline 4 & 4 & 2 & 2 & 1.00 & 0.88 & 0.92 & 0.92 \\
\hline 4 & 4 & 2 & 3 & 1.00 & 0.88 & 0.92 & 0.92 \\
\hline 4 & 4 & 3 & 1 & 1.00 & 0.90 & 0.92 & 0.92 \\
\hline 4 & 4 & 3 & 2 & 1.00 & 0.90 & 0.92 & 0.93 \\
\hline 4 & 4 & 3 & 3 & 1.00 & 0.90 & 0.92 & 0.93 \\
\hline 4 & 4 & 4 & 1 & 1.00 & 0.90 & 0.91 & 0.92 \\
\hline 4 & 4 & 4 & 2 & 1.00 & 0.90 & 0.91 & 0.92 \\
\hline 4 & 4 & 4 & 3 & 1.00 & 0.90 & 0.91 & 0.92 \\
\hline 4 & 4 & 1 & 1 & 1.00 & 0.90 & 0.92 & 0.93 \\
\hline 4 & 4 & 1 & 2 & 1.00 & 0.90 & 0.93 & 0.93 \\
\hline 4 & 4 & 1 & 3 & 1.00 & 0.90 & 0.93 & 0.93 \\
\hline 4 & 4 & 6 & 1 & 1.00 & 0.88 & 0.91 & 0.92 \\
\hline 4 & 4 & 6 & 2 & 1.00 & 0.88 & 0.92 & 0.92 \\
\hline 4 & 4 & 6 & 3 & 1.00 & 0.88 & 0.92 & 0.92 \\
\hline 5 & 1 & 1 & 1 & 1.00 & 0.91 & 0.93 & 0.94 \\
\hline 5 & 1 & 1 & 2 & 1.00 & 0.91 & 0.93 & 0.94 \\
\hline 5 & 1 & 1 & 3 & 1.00 & 0.91 & 0.93 & 0.94 \\
\hline 5 & 1 & 2 & 1 & 1.00 & 0.91 & 0.93 & 0.94 \\
\hline 5 & 1 & 2 & 2 & 1.00 & 0.91 & 0.93 & 0.94 \\
\hline 5 & 1 & 2 & 3 & 1.00 & 0.91 & 0.93 & 0.94 \\
\hline 5 & 1 & 3 & 1 & 1.00 & 0.91 & 0.92 & 0.93 \\
\hline 5 & 1 & 3 & 2 & 1.00 & 0.91 & 0.92 & 0.93 \\
\hline
\end{tabular}


Table D.1.2 the results of calibration procedure 2 and its validations.

\begin{tabular}{|c|c|c|c|c|c|c|c|}
\hline \multicolumn{4}{|c|}{ Configurations } & \multirow{2}{*}{$\begin{array}{c}\text { Calibration } \\
\text { Kappa } \\
\end{array}$} & \multirow{2}{*}{$\begin{array}{c}\mathbf{1}^{\text {st }} \text { Validation } \\
\text { Kappa } \\
\end{array}$} & \multirow{2}{*}{$\begin{array}{c}2^{\text {nd }} \text { Validation } \\
\text { Kappa } \\
\end{array}$} & \multirow{2}{*}{$\begin{array}{c}3^{\text {rd }} \text { Validation } \\
\text { Kappa } \\
\end{array}$} \\
\hline$\varphi_{g}$ & $\Lambda_{l}$ & $\Gamma_{n}$ & $\eta_{m}$ & & & & \\
\hline 5 & 1 & 3 & 3 & 1.00 & 0.91 & 0.92 & 0.93 \\
\hline 5 & 1 & 4 & 1 & 1.00 & 0.91 & 0.92 & 0.93 \\
\hline 5 & 1 & 4 & 2 & 1.00 & 0.91 & 0.93 & 0.94 \\
\hline 5 & 1 & 4 & 3 & 1.00 & 0.91 & 0.93 & 0.94 \\
\hline 5 & 1 & 1 & 1 & 1.00 & 0.91 & 0.91 & 0.93 \\
\hline 5 & 1 & 1 & 2 & 1.00 & 0.91 & 0.91 & 0.93 \\
\hline 5 & 1 & 1 & 3 & 1.00 & 0.91 & 0.91 & 0.93 \\
\hline 5 & 1 & 6 & 1 & 1.00 & 0.91 & 0.90 & 0.92 \\
\hline 5 & 1 & 6 & 2 & 1.00 & 0.91 & 0.91 & 0.93 \\
\hline 5 & 1 & 6 & 3 & 1.00 & 0.91 & 0.91 & 0.93 \\
\hline 5 & 2 & 1 & 1 & 0.98 & 0.85 & 0.94 & 0.92 \\
\hline 5 & 2 & 1 & 2 & 0.98 & 0.85 & 0.94 & 0.92 \\
\hline 5 & 2 & 1 & 3 & 0.98 & 0.85 & 0.94 & 0.92 \\
\hline 5 & 2 & 2 & 1 & 0.98 & 0.85 & 0.94 & 0.92 \\
\hline 5 & 2 & 2 & 2 & 0.98 & 0.85 & 0.94 & 0.92 \\
\hline 5 & 2 & 2 & 3 & 0.98 & 0.85 & 0.94 & 0.92 \\
\hline 5 & 2 & 3 & 1 & 0.98 & 0.85 & 0.94 & 0.92 \\
\hline 5 & 2 & 3 & 2 & 0.98 & 0.85 & 0.94 & 0.92 \\
\hline 5 & 2 & 3 & 3 & 0.98 & 0.85 & 0.94 & 0.92 \\
\hline 5 & 2 & 4 & 1 & 0.98 & 0.85 & 0.94 & 0.92 \\
\hline 5 & 2 & 4 & 2 & 0.98 & 0.85 & 0.94 & 0.92 \\
\hline 5 & 2 & 4 & 3 & 0.98 & 0.85 & 0.94 & 0.92 \\
\hline 5 & 2 & 1 & 1 & 0.98 & 0.85 & 0.94 & 0.92 \\
\hline 5 & 2 & 1 & 2 & 0.98 & 0.85 & 0.94 & 0.92 \\
\hline 5 & 2 & 1 & 3 & 0.98 & 0.85 & 0.94 & 0.92 \\
\hline 5 & 2 & 6 & 1 & 0.98 & 0.85 & 0.94 & 0.92 \\
\hline 5 & 2 & 6 & 2 & 0.98 & 0.85 & 0.94 & 0.92 \\
\hline 5 & 2 & 6 & 3 & 0.98 & 0.85 & 0.94 & 0.92 \\
\hline 5 & 3 & 1 & 1 & 0.98 & 0.90 & 0.95 & 0.94 \\
\hline 5 & 3 & 1 & 2 & 0.98 & 0.90 & 0.95 & 0.94 \\
\hline 5 & 3 & 1 & 3 & 0.98 & 0.90 & 0.95 & 0.94 \\
\hline 5 & 3 & 2 & 1 & 0.98 & 0.90 & 0.95 & 0.94 \\
\hline 5 & 3 & 2 & 2 & 0.98 & 0.90 & 0.95 & 0.94 \\
\hline 5 & 3 & 2 & 3 & 0.98 & 0.90 & 0.95 & 0.94 \\
\hline 5 & 3 & 3 & 1 & 0.98 & 0.90 & 0.95 & 0.94 \\
\hline 5 & 3 & 3 & 2 & 0.98 & 0.90 & 0.95 & 0.94 \\
\hline 5 & 3 & 3 & 3 & 0.98 & 0.90 & 0.95 & 0.94 \\
\hline 5 & 3 & 4 & 1 & 0.98 & 0.90 & 0.95 & 0.94 \\
\hline 5 & 3 & 4 & 2 & 0.98 & 0.90 & 0.95 & 0.94 \\
\hline 5 & 3 & 4 & 3 & 0.98 & 0.90 & 0.95 & 0.94 \\
\hline 5 & 3 & 1 & 1 & 0.98 & 0.90 & 0.95 & 0.94 \\
\hline 5 & 3 & 1 & 2 & 0.98 & 0.90 & 0.95 & 0.94 \\
\hline 5 & 3 & 1 & 3 & 0.98 & 0.90 & 0.95 & 0.94 \\
\hline 5 & 3 & 6 & 1 & 0.98 & 0.90 & 0.95 & 0.94 \\
\hline 5 & 3 & 6 & 2 & 0.98 & 0.90 & 0.95 & 0.94 \\
\hline 5 & 3 & 6 & 3 & 0.98 & 0.90 & 0.95 & 0.94 \\
\hline 5 & 4 & 1 & 1 & 1.00 & 0.86 & 0.89 & 0.90 \\
\hline 5 & 4 & 1 & 2 & 1.00 & 0.86 & 0.92 & 0.92 \\
\hline 5 & 4 & 1 & 3 & 1.00 & 0.86 & 0.92 & 0.92 \\
\hline 5 & 4 & 2 & 1 & 1.00 & 0.88 & 0.91 & 0.92 \\
\hline 5 & 4 & 2 & 2 & 1.00 & 0.88 & 0.93 & 0.93 \\
\hline
\end{tabular}


Table D.1.2 the results of calibration procedure 2 and its validations.

\begin{tabular}{|c|c|c|c|c|c|c|c|}
\hline \multicolumn{4}{|c|}{ Configurations } & \multirow{2}{*}{$\begin{array}{c}\text { Calibration } \\
\text { Kappa } \\
\end{array}$} & \multirow{2}{*}{$\begin{array}{c}\mathbf{1}^{\text {st }} \text { Validation } \\
\text { Kappa }\end{array}$} & \multirow{2}{*}{$\begin{array}{c}2^{\text {nd }} \text { Validation } \\
\text { Kappa } \\
\end{array}$} & \multirow{2}{*}{$\begin{array}{c}3^{\text {rd }} \text { Validation } \\
\text { Kappa }\end{array}$} \\
\hline$\varphi_{g}$ & $\Lambda_{l}$ & $\Gamma_{n}$ & $\eta_{m}$ & & & & \\
\hline 5 & 4 & 2 & 3 & 1.00 & 0.88 & 0.93 & 0.93 \\
\hline 5 & 4 & 3 & 1 & 1.00 & 0.89 & - & - \\
\hline 5 & 4 & 3 & 2 & 1.00 & 0.89 & 0.90 & 0.92 \\
\hline 5 & 4 & 3 & 3 & 1.00 & 0.89 & 0.90 & 0.92 \\
\hline 5 & 4 & 4 & 1 & 1.00 & 0.88 & - & - \\
\hline 5 & 4 & 4 & 2 & 1.00 & 0.88 & - & - \\
\hline 5 & 4 & 4 & 3 & 1.00 & 0.88 & - & - \\
\hline 5 & 4 & 1 & 1 & 1.00 & 0.89 & 0.89 & 0.91 \\
\hline 5 & 4 & 1 & 2 & 1.00 & 0.89 & 0.91 & 0.92 \\
\hline 5 & 4 & 1 & 3 & 1.00 & 0.89 & 0.91 & 0.92 \\
\hline 5 & 4 & 6 & 1 & 1.00 & 0.84 & 0.91 & 0.91 \\
\hline 5 & 4 & 6 & 2 & 1.00 & 0.84 & 0.92 & 0.91 \\
\hline 5 & 4 & 6 & 3 & 1.00 & 0.84 & 0.92 & 0.91 \\
\hline
\end{tabular}

\section{Table D.1.3 the results of calibration and validations based on the average speed}

\begin{tabular}{|c|c|c|c|c|c|}
\hline \multicolumn{2}{|c|}{ Configurations } & \multirow{2}{*}{$\begin{array}{c}\text { Calibration } \\
\text { Kappa } \\
\end{array}$} & \multirow{2}{*}{$\begin{array}{c}1^{\text {st }} \text { Validation } \\
\text { Kappa }\end{array}$} & \multirow{2}{*}{$\begin{array}{c}2^{\text {nd }} \text { Validation } \\
\text { Kappa }\end{array}$} & \multirow{2}{*}{$\begin{array}{c}\mathbf{3}^{\text {rd }} \text { Validation } \\
\text { Kappa } \\
\end{array}$} \\
\hline$\varphi_{g}$ & $\Lambda_{l}$ & & & & \\
\hline 1 & 1 & 0.68 & 0.68 & 0.75 & 0.74 \\
\hline 1 & 2 & 0.61 & 0.63 & 0.74 & 0.72 \\
\hline 1 & 3 & 0.57 & 0.65 & 0.70 & 0.70 \\
\hline 1 & 4 & 0.58 & 0.63 & 0.73 & 0.71 \\
\hline 2 & 1 & 0.69 & 0.68 & 0.75 & 0.75 \\
\hline 2 & 2 & 0.62 & 0.64 & 0.74 & 0.73 \\
\hline 2 & 3 & 0.61 & 0.61 & 0.74 & 0.71 \\
\hline 2 & 4 & 0.62 & 0.64 & 0.73 & 0.72 \\
\hline 3 & 1 & 0.76 & 0.73 & 0.75 & 0.77 \\
\hline 3 & 2 & 0.72 & 0.66 & 0.76 & 0.75 \\
\hline 3 & 3 & 0.71 & 0.66 & 0.74 & 0.74 \\
\hline 3 & 4 & 0.72 & 0.66 & 0.76 & 0.75 \\
\hline 4 & 1 & 0.82 & 0.76 & 0.81 & 0.82 \\
\hline 4 & 2 & 0.82 & 0.68 & 0.80 & 0.79 \\
\hline 4 & 3 & 0.80 & 0.65 & 0.78 & 0.77 \\
\hline 4 & 4 & 0.82 & 0.68 & 0.80 & 0.79 \\
\hline 5 & 1 & 0.86 & 0.69 & 0.79 & 0.79 \\
\hline 5 & 2 & 0.86 & 0.65 & 0.79 & 0.78 \\
\hline 5 & 3 & 0.86 & 0.67 & 0.80 & 0.79 \\
\hline 5 & 4 & 0.86 & 0.65 & 0.79 & 0.78 \\
\hline
\end{tabular}


Table D.1.4 the results of calibration and validations based on the maximum speed

\begin{tabular}{|c|c|c|c|c|c|}
\hline \multicolumn{2}{|c|}{ Configurations } & \multirow{2}{*}{$\begin{array}{c}\text { Calibration } \\
\text { Kappa }\end{array}$} & \multirow{2}{*}{$\begin{array}{c}\mathbf{1}^{\text {st }} \text { Validation } \\
\text { Kappa }\end{array}$} & \multirow{2}{*}{$\begin{array}{c}2^{\text {nd }} \text { Validation } \\
\text { Kappa }\end{array}$} & \multirow{2}{*}{$\frac{3^{\text {rd }} \text { Validation }}{\text { Kappa }}$} \\
\hline$\varphi_{g}$ & $\Lambda_{l}$ & & & & \\
\hline 1 & 1 & 0.79 & 0.70 & 0.84 & 0.81 \\
\hline 1 & 2 & - & - & - & - \\
\hline 1 & 3 & - & - & - & - \\
\hline 1 & 4 & - & - & - & - \\
\hline 2 & 1 & 0.79 & 0.70 & 0.85 & 0.81 \\
\hline 2 & 2 & - & - & - & - \\
\hline 2 & 3 & - & - & - & - \\
\hline 2 & 4 & - & - & - & - \\
\hline 3 & 1 & 0.86 & 0.74 & 0.86 & 0.84 \\
\hline 3 & 2 & 0.77 & 0.66 & 0.84 & 0.80 \\
\hline 3 & 3 & - & - & - & - \\
\hline 3 & 4 & 0.77 & 0.66 & 0.84 & 0.80 \\
\hline 4 & 1 & 0.89 & 0.77 & 0.88 & 0.86 \\
\hline 4 & 2 & 0.82 & - & - & - \\
\hline 4 & 3 & 0.78 & - & - & - \\
\hline 4 & 4 & 0.82 & - & - & - \\
\hline 5 & 1 & 0.89 & 0.79 & 0.88 & 0.87 \\
\hline 5 & 2 & 0.85 & - & - & - \\
\hline 5 & 3 & 0.83 & - & - & - \\
\hline 5 & 4 & 0.85 & - & - & - \\
\hline
\end{tabular}

Table D.1.5 the results of calibration and validations based on the average acceleration

\begin{tabular}{|c|c|c|c|c|c|}
\hline \multicolumn{2}{|c|}{ Configurations } & \multirow{2}{*}{$\begin{array}{c}\text { Calibration } \\
\text { Kappa }\end{array}$} & \multirow{2}{*}{$\begin{array}{c}\mathbf{1}^{\text {st }} \text { Validation } \\
\text { Kappa }\end{array}$} & \multirow{2}{*}{$\begin{array}{c}2^{\text {nd }} \text { Validation } \\
\text { Kappa }\end{array}$} & \multirow{2}{*}{$\begin{array}{c}3^{\text {rd }} \text { Validation } \\
\text { Kappa }\end{array}$} \\
\hline$\varphi_{g}$ & $\Lambda_{l}$ & & & & \\
\hline 1 & 1 & 0.10 & 0.07 & 0.26 & - \\
\hline 1 & 2 & 0.12 & 0.08 & - & - \\
\hline 1 & 3 & 0.17 & 0.09 & - & - \\
\hline 1 & 4 & 0.09 & 0.06 & - & 0.20 \\
\hline 2 & 1 & 0.04 & 0.02 & 0.09 & 0.06 \\
\hline 2 & 2 & 0.04 & 0.06 & 0.17 & 0.12 \\
\hline 2 & 3 & 0.06 & 0.05 & - & - \\
\hline 2 & 4 & 0.03 & 0.03 & 0.12 & 0.08 \\
\hline 3 & 1 & 0.06 & 0.01 & 0.15 & 0.12 \\
\hline 3 & 2 & 0.08 & 0.10 & 0.08 & 0.10 \\
\hline 3 & 3 & 0.10 & 0.10 & - & - \\
\hline 3 & 4 & 0.06 & 0.01 & - & - \\
\hline 4 & 1 & 0.10 & 0.05 & 0.18 & 0.16 \\
\hline 4 & 2 & 0.12 & 0.07 & 0.20 & 0.18 \\
\hline 4 & 3 & 0.13 & 0.08 & - & - \\
\hline 4 & 4 & 0.09 & 0.03 & - & - \\
\hline 5 & 1 & 0.09 & 0.05 & 0.26 & 0.21 \\
\hline 5 & 2 & 0.11 & 0.06 & 0.24 & 0.20 \\
\hline 5 & 3 & 0.14 & 0.10 & - & - \\
\hline 5 & 4 & 0.08 & 0.04 & - & - \\
\hline
\end{tabular}


Table D.1.6 the results of calibration and validations based on the maximum acceleration

\begin{tabular}{|c|c|c|c|c|c|}
\hline \multicolumn{2}{|c|}{ Configurations } & \multirow{2}{*}{$\begin{array}{c}\text { Calibration } \\
\text { Kappa }\end{array}$} & \multirow{2}{*}{$\begin{array}{c}\mathbf{1}^{\text {st }} \text { Validation } \\
\text { Kappa }\end{array}$} & \multirow{2}{*}{$\begin{array}{c}2^{\text {nd }} \text { Validation } \\
\text { Kappa }\end{array}$} & \multirow{2}{*}{$\frac{3^{\text {rd }} \text { Validation }}{\text { Kappa }}$} \\
\hline$\varphi_{g}$ & $\Lambda_{l}$ & & & & \\
\hline 1 & 1 & 0.05 & 0.04 & 0.14 & 0.12 \\
\hline 1 & 2 & 0.04 & 0.04 & 0.12 & 0.10 \\
\hline 1 & 3 & 0.03 & 0.05 & 0.12 & 0.10 \\
\hline 1 & 4 & 0.04 & 0.04 & 0.11 & 0.10 \\
\hline 2 & 1 & 0.03 & 0.01 & 0.02 & 0.01 \\
\hline 2 & 2 & 0.03 & 0.01 & 0.02 & 0.01 \\
\hline 2 & 3 & 0.03 & 0.01 & 0.02 & 0.01 \\
\hline 2 & 4 & 0.03 & 0.01 & 0.02 & 0.01 \\
\hline 3 & 1 & 0.04 & 0.01 & - & - \\
\hline 3 & 2 & 0.04 & 0.02 & - & - \\
\hline 3 & 3 & 0.04 & 0.02 & - & - \\
\hline 3 & 4 & 0.04 & 0.02 & - & - \\
\hline 4 & 1 & 0.05 & - & - & - \\
\hline 4 & 2 & 0.05 & - & - & - \\
\hline 4 & 3 & 0.05 & - & - & - \\
\hline 4 & 4 & 0.05 & - & - & - \\
\hline 5 & 1 & 0.06 & - & - & - \\
\hline 5 & 2 & 0.06 & - & - & - \\
\hline 5 & 3 & 0.06 & - & - & - \\
\hline 5 & 4 & 0.06 & - & - & - \\
\hline
\end{tabular}

Table D.1.7 the results of calibration and validations based on the average deceleration

\begin{tabular}{|c|c|c|c|c|c|}
\hline \multicolumn{2}{|c|}{ Configurations } & \multirow{2}{*}{$\begin{array}{c}\text { Calibration } \\
\text { Kappa } \\
\end{array}$} & \multirow{2}{*}{$\begin{array}{c}\mathbf{1}^{\text {st }} \text { Validation } \\
\text { Kappa } \\
\end{array}$} & \multirow{2}{*}{$\begin{array}{cl}2^{\text {nd }} & \text { Validation } \\
\text { Kappa } \\
\end{array}$} & \multirow{2}{*}{$\begin{array}{c}3^{\text {rd }} \text { Validation } \\
\text { Kappa }\end{array}$} \\
\hline$\varphi_{g}$ & $\Lambda_{l}$ & & & & \\
\hline 1 & 1 & 0.15 & 0.13 & 0.01 & 0.04 \\
\hline 1 & 2 & 0.16 & 0.13 & - & - \\
\hline 1 & 3 & 0.15 & 0.13 & - & - \\
\hline 1 & 4 & 0.15 & 0.11 & - & - \\
\hline 2 & 1 & 0.06 & 0.08 & - & - \\
\hline 2 & 2 & 0.09 & 0.08 & - & - \\
\hline 2 & 3 & 0.09 & 0.08 & - & - \\
\hline 2 & 4 & 0.09 & 0.08 & - & - \\
\hline 3 & 1 & 0.06 & 0.11 & 0.01 & 0.04 \\
\hline 3 & 2 & 0.09 & 0.11 & - & - \\
\hline 3 & 3 & 0.09 & 0.11 & - & - \\
\hline 3 & 4 & 0.09 & 0.11 & - & - \\
\hline 4 & 1 & 0.11 & 0.04 & - & - \\
\hline 4 & 2 & 0.13 & 0.10 & - & - \\
\hline 4 & 3 & 0.13 & 0.10 & - & - \\
\hline 4 & 4 & 0.13 & 0.10 & - & - \\
\hline 5 & 1 & 0.11 & 0.05 & 0.25 & 0.18 \\
\hline 5 & 2 & 0.13 & 0.08 & - & - \\
\hline 5 & 3 & 0.13 & 0.08 & - & - \\
\hline 5 & 4 & 0.13 & 0.08 & - & - \\
\hline
\end{tabular}


Table D.1.8 the results of calibration and validations based on the maximum deceleration

\begin{tabular}{|c|c|c|c|c|c|}
\hline \multicolumn{2}{|c|}{ Configurations } & \multirow{2}{*}{$\begin{array}{c}\text { Calibration } \\
\text { Kappa }\end{array}$} & \multirow{2}{*}{$\begin{array}{c}\mathbf{1}^{\text {st }} \text { Validation } \\
\text { Kappa }\end{array}$} & \multirow{2}{*}{$\frac{2^{\text {nd }} \text { Validation }}{\text { Kappa }}$} & \multirow{2}{*}{$\frac{3^{\text {rd }} \text { Validation }}{\text { Kappa }}$} \\
\hline$\varphi_{g}$ & $\Lambda_{l}$ & & & & \\
\hline 1 & 1 & 0.04 & 0.04 & 0.13 & 0.11 \\
\hline 1 & 2 & 0.03 & 0.02 & 0.11 & 0.07 \\
\hline 1 & 3 & 0.02 & 0.02 & 0.09 & 0.06 \\
\hline 1 & 4 & 0.03 & 0.02 & 0.10 & 0.07 \\
\hline 2 & 1 & 0.03 & 0.04 & 0.10 & 0.10 \\
\hline 2 & 2 & 0.03 & - & - & - \\
\hline 2 & 3 & 0.03 & - & - & - \\
\hline 2 & 4 & 0.03 & - & - & - \\
\hline 3 & 1 & 0.04 & 0.05 & 0.10 & 0.10 \\
\hline 3 & 2 & 0.03 & 0.01 & 0.09 & 0.05 \\
\hline 3 & 3 & 0.03 & 0.01 & 0.09 & 0.05 \\
\hline 3 & 4 & 0.03 & 0.01 & 0.09 & 0.05 \\
\hline 4 & 1 & 0.04 & 0.06 & 0.10 & 0.11 \\
\hline 4 & 2 & 0.04 & 0.01 & 0.10 & 0.06 \\
\hline 4 & 3 & 0.04 & 0.01 & 0.10 & 0.06 \\
\hline 4 & 4 & 0.04 & 0.01 & 0.10 & 0.06 \\
\hline 5 & 1 & 0.04 & 0.01 & 0.11 & 0.06 \\
\hline 5 & 2 & 0.04 & 0.01 & 0.11 & 0.06 \\
\hline 5 & 3 & 0.04 & 0.01 & 0.11 & 0.06 \\
\hline 5 & 4 & 0.04 & 0.01 & 0.11 & 0.06 \\
\hline
\end{tabular}

Table D.1.9 the results of calibration and validations based on the average direction

\begin{tabular}{|c|c|c|c|c|c|}
\hline \multicolumn{2}{|c|}{ Configurations } & \multirow{2}{*}{$\begin{array}{c}\text { Calibration } \\
\text { Kappa } \\
\end{array}$} & \multirow{2}{*}{$\begin{array}{c}\mathbf{1}^{\text {st }} \text { Validation } \\
\text { Kappa } \\
\end{array}$} & \multirow{2}{*}{$\begin{array}{cl}2^{\text {nd }} & \text { Validation } \\
\text { Kappa } \\
\end{array}$} & \multirow{2}{*}{$\begin{array}{c}\mathbf{3}^{\text {rd }} \text { Validation } \\
\text { Kappa } \\
\end{array}$} \\
\hline$\varphi_{g}$ & $\Lambda_{l}$ & & & & \\
\hline 1 & 1 & 0.16 & 0.11 & 0.01 & 0.08 \\
\hline 1 & 2 & 0.18 & 0.14 & 0.02 & 0.09 \\
\hline 1 & 3 & 0.20 & 0.15 & 0.01 & 0.09 \\
\hline 1 & 4 & 0.17 & 0.14 & 0.02 & 0.09 \\
\hline 2 & 1 & 0.16 & 0.12 & 0.01 & 0.08 \\
\hline 2 & 2 & 0.18 & 0.14 & 0.02 & 0.10 \\
\hline 2 & 3 & 0.21 & 0.16 & 0.02 & 0.10 \\
\hline 2 & 4 & 0.18 & 0.14 & 0.02 & 0.10 \\
\hline 3 & 1 & 0.16 & 0.11 & 0.01 & 0.08 \\
\hline 3 & 2 & 0.18 & 0.13 & 0.02 & 0.09 \\
\hline 3 & 3 & 0.21 & 0.15 & 0.02 & 0.10 \\
\hline 3 & 4 & 0.18 & 0.13 & 0.02 & 0.09 \\
\hline 4 & 1 & 0.15 & 0.10 & 0.01 & 0.08 \\
\hline 4 & 2 & 0.18 & 0.13 & 0.02 & 0.10 \\
\hline 4 & 3 & 0.23 & 0.14 & 0.01 & 0.10 \\
\hline 4 & 4 & 0.18 & 0.13 & 0.02 & 0.10 \\
\hline 5 & 1 & 0.18 & 0.05 & 0.02 & 0.03 \\
\hline 5 & 2 & 0.20 & 0.06 & - & - \\
\hline 5 & 3 & 0.25 & 0.09 & - & - \\
\hline 5 & 4 & 0.20 & 0.06 & - & - \\
\hline
\end{tabular}


Table D.1.10 the results of calibration and validations based on the maximum direction

\begin{tabular}{|c|c|c|c|c|c|}
\hline \multicolumn{2}{|c|}{ Configurations } & \multirow{2}{*}{$\begin{array}{c}\text { Calibration } \\
\text { Kappa }\end{array}$} & \multirow{2}{*}{$\begin{array}{c}\mathbf{1}^{\text {st }} \text { Validation } \\
\text { Kappa }\end{array}$} & \multirow{2}{*}{$\frac{2^{\text {nd }} \text { Validation }}{\text { Kappa }}$} & \multirow{2}{*}{$\frac{3^{\text {rd }} \text { Validation }}{\text { Kappa }}$} \\
\hline$\varphi_{g}$ & $\Lambda_{l}$ & & & & \\
\hline 1 & 1 & 0.25 & 0.21 & 0.03 & 0.13 \\
\hline 1 & 2 & 0.26 & 0.22 & 0.06 & 0.15 \\
\hline 1 & 3 & 0.28 & 0.23 & 0.05 & 0.15 \\
\hline 1 & 4 & 0.25 & 0.22 & 0.06 & 0.15 \\
\hline 2 & 1 & 0.28 & 0.22 & 0.03 & 0.13 \\
\hline 2 & 2 & 0.28 & 0.21 & 0.01 & 0.11 \\
\hline 2 & 3 & 0.30 & 0.23 & 0.03 & 0.13 \\
\hline 2 & 4 & 0.28 & 0.21 & 0.01 & 0.11 \\
\hline 3 & 1 & 0.29 & 0.22 & 0.03 & 0.13 \\
\hline 3 & 2 & 0.29 & 0.22 & 0.01 & 0.12 \\
\hline 3 & 3 & 0.32 & 0.23 & 0.03 & 0.14 \\
\hline 3 & 4 & 0.29 & 0.22 & 0.01 & 0.12 \\
\hline 4 & 1 & 0.31 & 0.20 & 0.03 & 0.14 \\
\hline 4 & 2 & 0.32 & 0.20 & 0.01 & 0.12 \\
\hline 4 & 3 & 0.33 & 0.20 & 0.02 & 0.14 \\
\hline 4 & 4 & 0.32 & 0.20 & 0.01 & 0.12 \\
\hline 5 & 1 & 0.33 & 0.23 & 0.02 & 0.16 \\
\hline 5 & 2 & 0.33 & 0.23 & 0.01 & 0.14 \\
\hline 5 & 3 & 0.34 & 0.23 & - & - \\
\hline 5 & 4 & 0.33 & 0.23 & 0.01 & 0.14 \\
\hline
\end{tabular}

Table D.1.12 the results of calibration and validations based on the average of cosine of change in direction

\begin{tabular}{|c|c|c|c|c|c|}
\hline \multicolumn{2}{|c|}{ Configurations } & \multirow{2}{*}{$\begin{array}{c}\text { Calibration } \\
\text { Kappa }\end{array}$} & \multirow{2}{*}{$\begin{array}{c}\mathbf{1}^{\text {st }} \text { Validation } \\
\text { Kappa }\end{array}$} & \multirow{2}{*}{$\begin{array}{c}2^{\text {nd }} \text { Validation } \\
\text { Kappa }\end{array}$} & \multirow{2}{*}{$\begin{array}{c}3^{\text {rd }} \text { Validation } \\
\text { Kappa }\end{array}$} \\
\hline$\varphi_{g}$ & $\Lambda_{l}$ & & & & \\
\hline 1 & 1 & 0.19 & 0.21 & 0.16 & 0.19 \\
\hline 1 & 2 & 0.20 & 0.23 & 0.17 & 0.20 \\
\hline 1 & 3 & - & - & - & - \\
\hline 1 & 4 & - & - & - & - \\
\hline 2 & 1 & 0.21 & 0.22 & 0.16 & 0.19 \\
\hline 2 & 2 & 0.20 & 0.24 & 0.17 & 0.20 \\
\hline 2 & 3 & - & - & - & - \\
\hline 2 & 4 & - & - & - & - \\
\hline 3 & 1 & 0.29 & 0.30 & 0.21 & 0.25 \\
\hline 3 & 2 & 0.26 & 0.30 & 0.19 & 0.24 \\
\hline 3 & 3 & - & - & - & - \\
\hline 3 & 4 & - & - & - & - \\
\hline 4 & 1 & 0.48 & 0.32 & 0.19 & 0.24 \\
\hline 4 & 2 & 0.39 & 0.32 & 0.19 & 0.26 \\
\hline 4 & 3 & - & - & - & - \\
\hline 4 & 4 & - & - & - & - \\
\hline 5 & 1 & 0.63 & 0.35 & 0.23 & 0.30 \\
\hline 5 & 2 & 0.49 & 0.35 & 0.20 & 0.28 \\
\hline 5 & 3 & - & - & - & - \\
\hline 5 & 4 & - & - & - & - \\
\hline
\end{tabular}


Table D.1.13 the results of calibration and validations based on the average area

\begin{tabular}{|c|c|c|c|c|c|}
\hline \multicolumn{2}{|c|}{ Configurations } & \multirow{2}{*}{$\begin{array}{c}\text { Calibration } \\
\text { Kappa } \\
\end{array}$} & \multirow{2}{*}{$\frac{1^{\text {st }} \text { Validation }}{\text { Kappa }}$} & \multirow{2}{*}{$\begin{array}{c}2^{\text {nd }} \text { Validation } \\
\text { Kappa }\end{array}$} & \multirow{2}{*}{$\frac{3^{\text {rd }} \text { Validation }}{\text { Kappa }}$} \\
\hline$\varphi_{g}$ & $\Lambda_{l}$ & & & & \\
\hline 1 & 1 & 0.80 & - & - & - \\
\hline 1 & 2 & 0.87 & 0.77 & 0.90 & 0.86 \\
\hline 1 & 3 & 0.85 & 0.69 & - & - \\
\hline 1 & 4 & 0.85 & 0.69 & - & - \\
\hline 2 & 1 & 0.89 & 0.72 & 0.77 & 0.77 \\
\hline 2 & 2 & 0.90 & 0.73 & 0.78 & 0.78 \\
\hline 2 & 3 & 0.89 & 0.72 & 0.78 & 0.78 \\
\hline 2 & 4 & 0.89 & 0.72 & 0.77 & 0.77 \\
\hline 3 & 1 & 0.92 & 0.67 & 0.78 & 0.76 \\
\hline 3 & 2 & 0.92 & 0.70 & 0.78 & 0.78 \\
\hline 3 & 3 & 0.92 & 0.67 & 0.78 & 0.76 \\
\hline 3 & 4 & 0.91 & 0.69 & 0.78 & 0.77 \\
\hline 4 & 1 & 0.94 & 0.75 & 0.83 & 0.83 \\
\hline 4 & 2 & 0.94 & 0.77 & 0.86 & 0.85 \\
\hline 4 & 3 & 0.94 & 0.75 & 0.83 & 0.83 \\
\hline 4 & 4 & 0.94 & 0.75 & 0.82 & 0.82 \\
\hline 5 & 1 & 0.93 & 0.78 & 0.84 & 0.85 \\
\hline 5 & 2 & 0.93 & 0.79 & 0.87 & 0.86 \\
\hline 5 & 3 & 0.93 & 0.78 & 0.84 & 0.85 \\
\hline 5 & 4 & 0.93 & 0.78 & 0.81 & 0.83 \\
\hline
\end{tabular}

Table D.1.14 the results of calibration and validations based on the maximum area

\begin{tabular}{|c|c|c|c|c|c|}
\hline \multicolumn{2}{|c|}{ Configurations } & \multirow{2}{*}{$\begin{array}{c}\text { Calibration } \\
\text { Kappa } \\
\end{array}$} & \multirow{2}{*}{$\begin{array}{c}\mathbf{1}^{\text {st }} \text { Validation } \\
\text { Kappa }\end{array}$} & \multirow{2}{*}{$\begin{array}{c}2^{\text {nd }} \text { Validation } \\
\text { Kappa }\end{array}$} & \multirow{2}{*}{$\begin{array}{c}3^{\text {rd }} \text { Validation } \\
\text { Kappa } \\
\end{array}$} \\
\hline$\varphi_{g}$ & $\Lambda_{l}$ & & & & \\
\hline 1 & 1 & 0.83 & 0.83 & 0.94 & 0.91 \\
\hline 1 & 2 & 0.82 & 0.77 & - & - \\
\hline 1 & 3 & 0.82 & 0.76 & - & - \\
\hline 1 & 4 & 0.77 & - & - & - \\
\hline 2 & 1 & 0.88 & 0.74 & 0.61 & 0.67 \\
\hline 2 & 2 & 0.88 & 0.73 & 0.61 & 0.67 \\
\hline 2 & 3 & 0.88 & 0.72 & 0.61 & 0.67 \\
\hline 2 & 4 & 0.88 & 0.73 & 0.61 & 0.66 \\
\hline 3 & 1 & 0.89 & 0.71 & 0.61 & 0.66 \\
\hline 3 & 2 & 0.89 & 0.69 & 0.60 & 0.65 \\
\hline 3 & 3 & 0.89 & 0.68 & 0.60 & 0.65 \\
\hline 3 & 4 & 0.89 & 0.69 & 0.60 & 0.65 \\
\hline 4 & 1 & 0.91 & 0.75 & 0.62 & 0.69 \\
\hline 4 & 2 & 0.91 & 0.73 & 0.62 & 0.68 \\
\hline 4 & 3 & 0.91 & 0.72 & 0.62 & 0.68 \\
\hline 4 & 4 & 0.91 & 0.73 & 0.62 & 0.68 \\
\hline 5 & 1 & 0.91 & 0.78 & 0.62 & 0.71 \\
\hline 5 & 2 & 0.91 & 0.76 & 0.61 & 0.70 \\
\hline 5 & 3 & 0.91 & 0.74 & 0.61 & 0.70 \\
\hline 5 & 4 & 0.91 & 0.76 & 0.61 & 0.70 \\
\hline
\end{tabular}


Table D.1.15 the results of calibration and validations based on the average length

\begin{tabular}{|c|c|c|c|c|c|}
\hline \multicolumn{2}{|c|}{ Configurations } & \multirow{2}{*}{$\begin{array}{c}\text { Calibration } \\
\text { Kappa } \\
\end{array}$} & \multirow{2}{*}{$\begin{array}{c}\mathbf{1}^{\text {st }} \text { Validation } \\
\text { Kappa }\end{array}$} & \multirow{2}{*}{$\begin{array}{c}2^{\text {nd }} \text { Validation } \\
\text { Kappa }\end{array}$} & \multirow{2}{*}{$\frac{3^{\text {rd }} \text { Validation }}{\text { Kappa }}$} \\
\hline$\varphi_{g}$ & $\Lambda_{l}$ & & & & \\
\hline 1 & 1 & 0.79 & 0.58 & 0.88 & 0.78 \\
\hline 1 & 2 & 0.78 & 0.57 & 0.88 & 0.77 \\
\hline 1 & 3 & 0.76 & 0.52 & 0.84 & 0.73 \\
\hline 1 & 4 & 0.71 & 0.54 & 0.86 & 0.75 \\
\hline 2 & 1 & 0.80 & 0.55 & 0.85 & 0.75 \\
\hline 2 & 2 & 0.78 & 0.56 & 0.88 & 0.78 \\
\hline 2 & 3 & 0.76 & 0.51 & 0.83 & 0.73 \\
\hline 2 & 4 & 0.78 & 0.56 & 0.87 & 0.77 \\
\hline 3 & 1 & 0.80 & 0.54 & - & - \\
\hline 3 & 2 & 0.79 & 0.58 & 0.88 & 0.79 \\
\hline 3 & 3 & 0.77 & 0.52 & 0.83 & 0.74 \\
\hline 3 & 4 & 0.79 & 0.58 & 0.88 & 0.79 \\
\hline 4 & 1 & 0.85 & 0.56 & - & - \\
\hline 4 & 2 & 0.83 & 0.60 & 0.91 & 0.82 \\
\hline 4 & 3 & 0.81 & 0.52 & 0.86 & - \\
\hline 4 & 4 & 0.83 & 0.60 & 0.91 & 0.82 \\
\hline 5 & 1 & 0.89 & 0.59 & 0.86 & 0.79 \\
\hline 5 & 2 & 0.87 & 0.57 & 0.83 & 0.76 \\
\hline 5 & 3 & 0.86 & 0.56 & - & - \\
\hline 5 & 4 & 0.87 & 0.57 & 0.83 & 0.76 \\
\hline
\end{tabular}

Table D.1.16 the results of calibration and validations based on the maximum length

\begin{tabular}{|c|c|c|c|c|c|}
\hline \multicolumn{2}{|c|}{ Configurations } & \multirow{2}{*}{$\begin{array}{c}\text { Calibration } \\
\text { Kappa }\end{array}$} & \multirow{2}{*}{$\frac{1^{\text {st }} \text { Validation }}{\text { Kappa }}$} & \multirow{2}{*}{$\begin{array}{c}2^{\text {nd }} \text { Validation } \\
\text { Kappa }\end{array}$} & \multirow{2}{*}{$\frac{3^{\text {rd }} \text { Validation }}{\text { Kappa }}$} \\
\hline$\varphi_{g}$ & $\Lambda_{l}$ & & & & \\
\hline 1 & 1 & 0.64 & 0.58 & 0.95 & 0.85 \\
\hline 1 & 2 & 0.56 & 0.46 & 0.93 & - \\
\hline 1 & 3 & - & - & - & - \\
\hline 1 & 4 & 0.52 & - & - & - \\
\hline 2 & 1 & 0.63 & 0.50 & 0.93 & 0.80 \\
\hline 2 & 2 & 0.55 & 0.44 & 0.93 & 0.77 \\
\hline 2 & 3 & - & - & - & - \\
\hline 2 & 4 & 0.55 & 0.44 & 0.92 & 0.77 \\
\hline 3 & 1 & 0.65 & 0.53 & 0.95 & 0.84 \\
\hline 3 & 2 & 0.58 & 0.47 & 0.95 & 0.81 \\
\hline 3 & 3 & - & - & - & - \\
\hline 3 & 4 & 0.58 & 0.47 & 0.95 & 0.81 \\
\hline 4 & 1 & 0.70 & 0.54 & 0.96 & 0.86 \\
\hline 4 & 2 & 0.62 & 0.48 & 0.96 & 0.83 \\
\hline 4 & 3 & - & - & - & - \\
\hline 4 & 4 & 0.62 & 0.48 & 0.96 & 0.83 \\
\hline 5 & 1 & 0.71 & 0.40 & 0.81 & 0.71 \\
\hline 5 & 2 & 0.68 & 0.40 & 0.85 & 0.73 \\
\hline 5 & 3 & 0.64 & 0.37 & 0.85 & 0.71 \\
\hline 5 & 4 & 0.68 & 0.40 & 0.85 & 0.73 \\
\hline
\end{tabular}


Table D.1.17 the results of calibration and validations based on the average width

\begin{tabular}{|c|c|c|c|c|c|}
\hline \multicolumn{2}{|c|}{ Configurations } & \multirow{2}{*}{$\begin{array}{c}\text { Calibration } \\
\text { Kappa } \\
\end{array}$} & \multirow{2}{*}{$\begin{array}{c}\mathbf{1}^{\text {st }} \text { Validation } \\
\text { Kappa } \\
\end{array}$} & \multirow{2}{*}{$\begin{array}{c}\text { Validation } 2 \\
\text { Kappa } \\
\end{array}$} & \multirow{2}{*}{$\begin{array}{c}3^{\text {rd }} \text { Validation } \\
\text { Kappa }\end{array}$} \\
\hline$\varphi_{g}$ & $\Lambda_{l}$ & & & & \\
\hline 1 & 1 & 0.81 & 0.79 & 0.85 & 0.84 \\
\hline 1 & 2 & - & - & - & - \\
\hline 1 & 3 & 0.85 & 0.85 & 0.89 & 0.88 \\
\hline 1 & 4 & 0.78 & - & - & - \\
\hline 2 & 1 & 0.90 & 0.82 & 0.83 & 0.85 \\
\hline 2 & 2 & 0.89 & 0.78 & 0.85 & 0.85 \\
\hline 2 & 3 & 0.89 & 0.78 & 0.85 & 0.85 \\
\hline 2 & 4 & 0.87 & 0.75 & 0.88 & 0.86 \\
\hline 3 & 1 & 0.85 & 0.74 & 0.82 & 0.81 \\
\hline 3 & 2 & 0.85 & 0.74 & 0.82 & 0.81 \\
\hline 3 & 3 & 0.88 & 0.78 & 0.81 & 0.82 \\
\hline 3 & 4 & 0.83 & 0.71 & 0.79 & 0.78 \\
\hline 4 & 1 & 0.81 & 0.77 & 0.81 & 0.81 \\
\hline 4 & 2 & 0.81 & 0.77 & 0.80 & 0.81 \\
\hline 4 & 3 & 0.86 & 0.80 & 0.80 & 0.82 \\
\hline 4 & 4 & 0.78 & 0.73 & 0.84 & 0.81 \\
\hline 5 & 1 & 0.87 & 0.74 & - & - \\
\hline 5 & 2 & 0.87 & 0.74 & - & - \\
\hline 5 & 3 & 0.89 & 0.86 & 0.83 & 0.87 \\
\hline 5 & 4 & 0.87 & 0.78 & 0.89 & 0.87 \\
\hline
\end{tabular}

Table D.1.18 the results of calibration and validations based on the maximum width

\begin{tabular}{|c|c|c|c|c|c|}
\hline \multicolumn{2}{|c|}{ Configurations } & \multirow{2}{*}{$\begin{array}{c}\text { Calibration } \\
\text { Kappa } \\
\end{array}$} & \multirow{2}{*}{$\begin{array}{c}\mathbf{1}^{\text {st }} \text { Validation } \\
\text { Kappa } \\
\end{array}$} & \multirow{2}{*}{$\begin{array}{cc}2^{\text {nd }} & \text { Validation } \\
\text { Kappa }\end{array}$} & \multirow{2}{*}{$\begin{array}{c}3^{\text {rd }} \text { Validation } \\
\text { Kappa } \\
\end{array}$} \\
\hline$\varphi_{g}$ & $\Lambda_{l}$ & & & & \\
\hline 1 & 1 & 0.78 & 0.83 & 0.94 & 0.91 \\
\hline 1 & 2 & 0.75 & 0.83 & 0.94 & 0.91 \\
\hline 1 & 3 & 0.73 & 0.81 & 0.92 & 0.89 \\
\hline 1 & 4 & 0.69 & 0.79 & 0.91 & 0.88 \\
\hline 2 & 1 & 0.81 & 0.89 & 0.89 & 0.90 \\
\hline 2 & 2 & 0.80 & 0.87 & 0.88 & 0.89 \\
\hline 2 & 3 & 0.79 & 0.83 & - & - \\
\hline 2 & 4 & 0.80 & 0.87 & 0.88 & 0.88 \\
\hline 3 & 1 & 0.86 & 0.87 & 0.90 & 0.90 \\
\hline 3 & 2 & 0.84 & 0.85 & 0.89 & 0.89 \\
\hline 3 & 3 & 0.84 & - & - & - \\
\hline 3 & 4 & 0.84 & 0.85 & 0.89 & 0.89 \\
\hline 4 & 1 & 0.86 & 0.91 & 0.94 & 0.94 \\
\hline 4 & 2 & 0.85 & 0.84 & - & - \\
\hline 4 & 3 & 0.86 & - & - & - \\
\hline 4 & 4 & 0.85 & 0.84 & - & - \\
\hline 5 & 1 & 0.86 & 0.91 & 0.93 & 0.94 \\
\hline 5 & 2 & 0.85 & - & - & - \\
\hline 5 & 3 & 0.87 & 0.80 & - & - \\
\hline 5 & 4 & 0.85 & - & - & - \\
\hline
\end{tabular}


Table D.1.19 the results of calibration and validations based on the peaks in speed

\begin{tabular}{|c|c|c|c|c|c|}
\hline \multicolumn{2}{|c|}{ Configurations } & \multirow{2}{*}{$\begin{array}{c}\text { Calibration } \\
\text { Kappa }\end{array}$} & \multirow{2}{*}{$\begin{array}{c}\mathbf{1}^{\text {st }} \text { Validation } \\
\text { Kappa }\end{array}$} & \multirow{2}{*}{$\begin{array}{c}2^{\text {nd }} \text { Validation } \\
\text { Kappa }\end{array}$} & \multirow{2}{*}{$\frac{3^{\text {rd }} \text { Validation }}{\text { Kappa }}$} \\
\hline$\varphi_{g}$ & $\Lambda_{l}$ & & & & \\
\hline 1 & 1 & 0.45 & 0.56 & 0.61 & 0.61 \\
\hline 1 & 2 & 0.44 & 0.49 & - & - \\
\hline 1 & 3 & 0.40 & - & - & - \\
\hline 1 & 4 & 0.32 & - & - & - \\
\hline 2 & 1 & 0.54 & 0.58 & 0.65 & 0.64 \\
\hline 2 & 2 & 0.52 & 0.53 & - & - \\
\hline 2 & 3 & 0.48 & - & - & - \\
\hline 2 & 4 & - & - & - & - \\
\hline 3 & 1 & 0.61 & 0.64 & 0.68 & 0.69 \\
\hline 3 & 2 & 0.59 & 0.55 & - & - \\
\hline 3 & 3 & 0.52 & - & - & - \\
\hline 3 & 4 & 0.43 & - & - & - \\
\hline 4 & 1 & 0.64 & 0.64 & 0.66 & 0.69 \\
\hline 4 & 2 & 0.59 & 0.50 & - & - \\
\hline 4 & 3 & 0.50 & - & - & - \\
\hline 4 & 4 & 0.50 & - & - & - \\
\hline 5 & 1 & 0.66 & 0.77 & 0.65 & 0.73 \\
\hline 5 & 2 & 0.62 & - & - & - \\
\hline 5 & 3 & 0.52 & - & - & - \\
\hline 5 & 4 & 0.52 & - & - & - \\
\hline
\end{tabular}

Table D.1.20 the results of calibration and validations based on the effective weighted average frequency

\begin{tabular}{|c|c|c|c|c|c|c|}
\hline \multicolumn{3}{|c|}{ Configurations } & \multirow{2}{*}{$\begin{array}{c}\text { Calibration } \\
\text { Kappa } \\
\end{array}$} & \multirow{2}{*}{$\begin{array}{c}1^{\text {st }} \text { Validation } \\
\text { Kappa }\end{array}$} & \multirow{2}{*}{$\begin{array}{cl}2^{\text {nd }} & \text { Validation } \\
\text { Kappa }\end{array}$} & \multirow{2}{*}{$\frac{3^{\text {rd }} \text { Validation }}{\text { Kappa }}$} \\
\hline$\varphi_{g}$ & $\Lambda_{l}$ & $\Gamma_{n}$ & & & & \\
\hline 1 & 1 & 1 & 0.26 & 0.31 & 0.47 & 0.43 \\
\hline 1 & 1 & 2 & 0.26 & 0.26 & 0.50 & 0.41 \\
\hline 1 & 1 & 3 & 0.26 & 0.32 & 0.46 & 0.43 \\
\hline 1 & 1 & 4 & 0.26 & 0.33 & 0.46 & 0.43 \\
\hline 1 & 1 & 1 & 0.27 & 0.32 & 0.46 & 0.43 \\
\hline 1 & 1 & 6 & 0.28 & 0.26 & 0.51 & 0.41 \\
\hline 1 & 2 & 1 & 0.22 & 0.25 & 0.46 & 0.38 \\
\hline 1 & 2 & 2 & 0.22 & - & - & - \\
\hline 1 & 2 & 3 & 0.23 & 0.23 & 0.48 & 0.38 \\
\hline 1 & 2 & 4 & 0.23 & 0.23 & 0.49 & 0.38 \\
\hline 1 & 2 & 1 & 0.23 & 0.28 & 0.45 & 0.40 \\
\hline 1 & 2 & 6 & 0.24 & - & - & - \\
\hline 1 & 3 & 1 & 0.21 & - & - & - \\
\hline 1 & 3 & 2 & 0.21 & - & - & - \\
\hline 1 & 3 & 3 & 0.21 & 0.23 & 0.46 & 0.69 \\
\hline 1 & 3 & 4 & 0.21 & - & - & - \\
\hline 1 & 3 & 1 & 0.22 & - & - & - \\
\hline 1 & 3 & 6 & 0.23 & - & - & - \\
\hline 1 & 4 & 1 & 0.22 & 0.26 & 0.45 & 0.38 \\
\hline 1 & 4 & 2 & 0.22 & 0.23 & 0.47 & 0.37 \\
\hline 1 & 4 & 3 & 0.23 & 0.24 & 0.47 & 0.37 \\
\hline 1 & 4 & 4 & 0.23 & 0.24 & 0.48 & 0.38 \\
\hline 1 & 4 & 1 & 0.23 & 0.29 & 0.44 & 0.40 \\
\hline 1 & 4 & 6 & 0.24 & - & - & - \\
\hline
\end{tabular}


Table D.1.20 the results of calibration and validations based on the effective weighted average frequency

\begin{tabular}{|c|c|c|c|c|c|c|}
\hline \multicolumn{3}{|c|}{ Configurations } & \multirow{2}{*}{$\begin{array}{c}\text { Calibration } \\
\text { Kappa } \\
\end{array}$} & \multirow{2}{*}{$\frac{\mathbf{1}^{\text {st }} \text { Validation }}{\text { Kappa }}$} & \multirow{2}{*}{$\begin{array}{c}2^{\text {nd }} \text { Validation } \\
\text { Kappa } \\
\end{array}$} & \multirow{2}{*}{$\frac{3^{\text {rd }} \text { Validation }}{\text { Kappa }}$} \\
\hline$\varphi_{g}$ & $\Lambda_{l}$ & $\Gamma_{n}$ & & & & \\
\hline 2 & 1 & 1 & 0.31 & 0.33 & 0.49 & 0.45 \\
\hline 2 & 1 & 2 & 0.32 & 0.33 & 0.47 & 0.44 \\
\hline 2 & 1 & 3 & 0.33 & 0.35 & 0.48 & 0.45 \\
\hline 2 & 1 & 4 & 0.33 & 0.32 & 0.51 & 0.45 \\
\hline 2 & 1 & 1 & 0.34 & 0.34 & 0.48 & 0.45 \\
\hline 2 & 1 & 6 & 0.35 & 0.35 & 0.49 & 0.46 \\
\hline 2 & 2 & 1 & 0.26 & 0.27 & 0.48 & 0.40 \\
\hline 2 & 2 & 2 & 0.27 & - & - & - \\
\hline 2 & 2 & 3 & 0.27 & 0.24 & 0.49 & 0.39 \\
\hline 2 & 2 & 4 & 0.28 & 0.24 & 0.50 & 0.40 \\
\hline 2 & 2 & 1 & 0.28 & 0.30 & 0.46 & 0.42 \\
\hline 2 & 2 & 6 & 0.29 & 0.28 & 0.46 & 0.41 \\
\hline 2 & 3 & 1 & 0.24 & - & - & - \\
\hline 2 & 3 & 2 & 0.26 & - & - & - \\
\hline 2 & 3 & 3 & 0.26 & 0.24 & 0.47 & - \\
\hline 2 & 3 & 4 & 0.26 & - & - & - \\
\hline 2 & 3 & 1 & 0.26 & 0.25 & 0.48 & 0.39 \\
\hline 2 & 3 & 6 & 0.27 & 0.24 & 0.48 & - \\
\hline 2 & 4 & 1 & 0.26 & 0.27 & 0.47 & 0.40 \\
\hline 2 & 4 & 2 & 0.27 & - & - & - \\
\hline 2 & 4 & 3 & 0.27 & 0.24 & 0.49 & 0.39 \\
\hline 2 & 4 & 4 & 0.28 & 0.24 & 0.49 & 0.40 \\
\hline 2 & 4 & 1 & 0.28 & 0.30 & 0.45 & 0.42 \\
\hline 2 & 4 & 6 & 0.29 & 0.28 & 0.46 & 0.41 \\
\hline 3 & 1 & 1 & 0.40 & 0.41 & 0.57 & 0.54 \\
\hline 3 & 1 & 2 & 0.42 & 0.43 & 0.55 & 0.54 \\
\hline 3 & 1 & 3 & 0.44 & 0.43 & 0.55 & 0.55 \\
\hline 3 & 1 & 4 & 0.44 & 0.44 & 0.56 & 0.55 \\
\hline 3 & 1 & 1 & 0.44 & 0.45 & 0.54 & 0.54 \\
\hline 3 & 1 & 6 & 0.44 & 0.44 & 0.54 & 0.54 \\
\hline 3 & 2 & 1 & 0.34 & 0.39 & 0.48 & 0.49 \\
\hline 3 & 2 & 2 & 0.34 & 0.39 & 0.47 & 0.49 \\
\hline 3 & 2 & 3 & 0.35 & 0.36 & 0.53 & 0.49 \\
\hline 3 & 2 & 4 & 0.36 & 0.36 & 0.53 & 0.50 \\
\hline 3 & 2 & 1 & 0.36 & 0.36 & 0.52 & 0.49 \\
\hline 3 & 2 & 6 & 0.36 & 0.38 & 0.50 & 0.50 \\
\hline 3 & 3 & 1 & 0.33 & 0.37 & - & - \\
\hline 3 & 3 & 2 & 0.32 & 0.38 & - & - \\
\hline 3 & 3 & 3 & 0.32 & 0.37 & - & - \\
\hline 3 & 3 & 4 & 0.33 & 0.37 & - & - \\
\hline 3 & 3 & 1 & 0.33 & 0.38 & - & - \\
\hline 3 & 3 & 6 & 0.33 & 0.36 & 0.48 & - \\
\hline 3 & 4 & 1 & 0.34 & 0.39 & 0.48 & 0.49 \\
\hline 3 & 4 & 2 & 0.34 & 0.39 & 0.47 & 0.49 \\
\hline 3 & 4 & 3 & 0.35 & 0.36 & 0.53 & 0.49 \\
\hline 3 & 4 & 4 & 0.36 & 0.36 & 0.53 & 0.50 \\
\hline 3 & 4 & 1 & 0.36 & 0.36 & 0.52 & 0.49 \\
\hline 3 & 4 & 6 & 0.36 & 0.38 & 0.50 & 0.50 \\
\hline 4 & 1 & 1 & 0.54 & 0.49 & 0.67 & 0.65 \\
\hline 4 & 1 & 2 & 0.54 & 0.49 & 0.68 & 0.65 \\
\hline
\end{tabular}


Table D.1.20 the results of calibration and validations based on the effective weighted average frequency

\begin{tabular}{|c|c|c|c|c|c|c|}
\hline \multicolumn{3}{|c|}{ Configurations } & \multirow{2}{*}{$\begin{array}{c}\text { Calibration } \\
\text { Kappa }\end{array}$} & \multirow{2}{*}{$\begin{array}{c}\mathbf{1}^{\text {st }} \text { Validation } \\
\text { Kappa }\end{array}$} & \multirow{2}{*}{$\begin{array}{c}2^{\text {nd }} \text { Validation } \\
\text { Kappa }\end{array}$} & \multirow{2}{*}{$\frac{3^{\text {rd }} \text { Validation }}{\text { Kappa }}$} \\
\hline$\varphi_{g}$ & $\Lambda_{l}$ & $\Gamma_{n}$ & & & & \\
\hline 4 & 1 & 3 & 0.54 & 0.50 & 0.68 & 0.65 \\
\hline 4 & 1 & 4 & 0.54 & 0.50 & 0.66 & 0.65 \\
\hline 4 & 1 & 1 & 0.54 & 0.49 & 0.64 & 0.63 \\
\hline 4 & 1 & 6 & 0.54 & 0.48 & 0.65 & 0.63 \\
\hline 4 & 2 & 1 & 0.49 & 0.47 & 0.62 & 0.61 \\
\hline 4 & 2 & 2 & 0.49 & 0.46 & 0.60 & 0.60 \\
\hline 4 & 2 & 3 & 0.48 & 0.46 & 0.60 & 0.60 \\
\hline 4 & 2 & 4 & 0.47 & 0.45 & 0.61 & 0.60 \\
\hline 4 & 2 & 1 & 0.48 & 0.46 & 0.60 & 0.60 \\
\hline 4 & 2 & 6 & 0.48 & 0.45 & 0.61 & 0.60 \\
\hline 4 & 3 & 1 & 0.47 & 0.44 & 0.59 & 0.59 \\
\hline 4 & 3 & 2 & 0.47 & 0.45 & 0.58 & 0.59 \\
\hline 4 & 3 & 3 & 0.46 & 0.44 & - & - \\
\hline 4 & 3 & 4 & 0.45 & 0.44 & - & - \\
\hline 4 & 3 & 1 & 0.45 & 0.43 & - & - \\
\hline 4 & 3 & 6 & 0.45 & 0.45 & - & - \\
\hline 4 & 4 & 1 & 0.49 & 0.47 & 0.62 & 0.61 \\
\hline 4 & 4 & 2 & 0.49 & 0.46 & 0.60 & 0.60 \\
\hline 4 & 4 & 3 & 0.48 & 0.46 & 0.60 & 0.60 \\
\hline 4 & 4 & 4 & 0.47 & 0.45 & 0.61 & 0.60 \\
\hline 4 & 4 & 1 & 0.48 & 0.46 & 0.60 & 0.60 \\
\hline 4 & 4 & 6 & 0.48 & 0.45 & 0.61 & 0.60 \\
\hline 5 & 1 & 1 & 0.61 & 0.55 & 0.79 & 0.73 \\
\hline 5 & 1 & 2 & 0.60 & 0.56 & 0.80 & 0.74 \\
\hline 5 & 1 & 3 & 0.62 & 0.55 & 0.78 & 0.73 \\
\hline 5 & 1 & 4 & 0.62 & 0.56 & 0.77 & 0.73 \\
\hline 5 & 1 & 1 & 0.61 & 0.55 & 0.73 & 0.70 \\
\hline 5 & 1 & 6 & 0.61 & 0.53 & 0.73 & 0.70 \\
\hline 5 & 2 & 1 & 0.57 & 0.54 & 0.73 & 0.70 \\
\hline 5 & 2 & 2 & 0.56 & 0.53 & 0.71 & 0.69 \\
\hline 5 & 2 & 3 & 0.55 & 0.52 & 0.71 & 0.68 \\
\hline 5 & 2 & 4 & 0.55 & 0.53 & 0.71 & 0.69 \\
\hline 5 & 2 & 1 & 0.55 & 0.53 & 0.70 & 0.68 \\
\hline 5 & 2 & 6 & 0.55 & 0.51 & 0.71 & 0.68 \\
\hline 5 & 3 & 1 & 0.54 & 0.52 & 0.69 & 0.68 \\
\hline 5 & 3 & 2 & 0.54 & 0.52 & - & - \\
\hline 5 & 3 & 3 & 0.53 & 0.51 & - & - \\
\hline 5 & 3 & 4 & 0.53 & 0.50 & - & - \\
\hline 5 & 3 & 1 & 0.51 & 0.51 & - & - \\
\hline 5 & 3 & 6 & 0.51 & 0.51 & - & - \\
\hline 5 & 4 & 1 & 0.57 & 0.54 & 0.73 & 0.70 \\
\hline 5 & 4 & 2 & 0.56 & 0.53 & 0.71 & - \\
\hline 5 & 4 & 3 & 0.55 & 0.52 & 0.71 & 0.68 \\
\hline 5 & 4 & 4 & 0.55 & 0.53 & 0.71 & 0.69 \\
\hline 5 & 4 & 1 & 0.55 & 0.53 & 0.70 & 0.68 \\
\hline 5 & 4 & 6 & 0.55 & 0.51 & 0.71 & 0.68 \\
\hline
\end{tabular}


Table D.1.21 the results of calibration and validations based on the effective frequency

\begin{tabular}{|c|c|c|c|c|c|c|}
\hline \multicolumn{3}{|c|}{ Configurations } & \multirow{2}{*}{$\begin{array}{c}\text { Calibration } \\
\text { Kappa } \\
\end{array}$} & \multirow{2}{*}{$\begin{array}{c}\mathbf{1}^{\text {st }} \text { Validation } \\
\text { Kappa }\end{array}$} & \multirow{2}{*}{$\begin{array}{c}2^{\text {nd }} \text { Validation } \\
\text { Kappa } \\
\end{array}$} & \multirow{2}{*}{$\begin{array}{c}3^{\text {rd }} \text { Validation } \\
\text { Kappa }\end{array}$} \\
\hline$\varphi_{g}$ & $\Lambda_{l}$ & $\eta_{m}$ & & & & \\
\hline 1 & 1 & 1 & 0.14 & 0.11 & 0.15 & 0.11 \\
\hline 1 & 1 & 2 & 0.16 & 0.12 & 0.22 & 0.18 \\
\hline 1 & 1 & 3 & 0.17 & 0.14 & 0.22 & 0.18 \\
\hline 1 & 2 & 1 & - & - & - & - \\
\hline 1 & 2 & 2 & - & - & - & - \\
\hline 1 & 2 & 3 & - & - & - & - \\
\hline 1 & 3 & 1 & - & - & - & - \\
\hline 1 & 3 & 2 & - & - & - & - \\
\hline 1 & 3 & 3 & - & - & - & - \\
\hline 1 & 4 & 1 & - & - & - & - \\
\hline 1 & 4 & 2 & - & - & - & - \\
\hline 1 & 4 & 3 & - & - & - & - \\
\hline 2 & 1 & 1 & 0.16 & 0.11 & 0.16 & 0.12 \\
\hline 2 & 1 & 2 & 0.19 & 0.12 & 0.23 & 0.19 \\
\hline 2 & 1 & 3 & 0.21 & 0.15 & 0.23 & 0.19 \\
\hline 2 & 2 & 1 & - & - & - & - \\
\hline 2 & 2 & 2 & - & - & - & - \\
\hline 2 & 2 & 3 & - & - & - & - \\
\hline 2 & 3 & 1 & - & - & - & - \\
\hline 2 & 3 & 2 & - & - & - & - \\
\hline 2 & 3 & 3 & - & - & - & - \\
\hline 2 & 4 & 1 & - & - & - & - \\
\hline 2 & 4 & 2 & - & - & - & - \\
\hline 2 & 4 & 3 & - & - & - & - \\
\hline 3 & 1 & 1 & 0.21 & 0.26 & - & - \\
\hline 3 & 1 & 2 & 0.33 & 0.17 & 0.30 & 0.26 \\
\hline 3 & 1 & 3 & 0.33 & 0.26 & 0.28 & 0.28 \\
\hline 3 & 2 & 1 & - & - & - & - \\
\hline 3 & 2 & 2 & - & - & - & - \\
\hline 3 & 2 & 3 & - & - & - & - \\
\hline 3 & 3 & 1 & - & - & - & - \\
\hline 3 & 3 & 2 & - & - & - & - \\
\hline 3 & 3 & 3 & - & - & - & - \\
\hline 3 & 4 & 1 & - & - & - & - \\
\hline 3 & 4 & 2 & - & - & - & - \\
\hline 3 & 4 & 3 & - & - & - & - \\
\hline 4 & 1 & 1 & 0.26 & 0.29 & - & - \\
\hline 4 & 1 & 2 & 0.38 & 0.31 & 0.24 & 0.28 \\
\hline 4 & 1 & 3 & 0.45 & 0.39 & 0.26 & 0.33 \\
\hline 4 & 2 & 1 & - & - & - & - \\
\hline 4 & 2 & 2 & - & - & - & - \\
\hline 4 & 2 & 3 & - & - & - & - \\
\hline 4 & 3 & 1 & - & - & - & - \\
\hline 4 & 3 & 2 & - & - & - & - \\
\hline 4 & 3 & 3 & - & - & - & - \\
\hline 4 & 4 & 1 & - & - & - & - \\
\hline 4 & 4 & 2 & - & - & - & - \\
\hline 4 & 4 & 3 & - & - & - & - \\
\hline 5 & 1 & 1 & 0.26 & 0.28 & - & - \\
\hline 5 & 1 & 2 & 0.39 & 0.32 & 0.22 & 0.28 \\
\hline 5 & 1 & 3 & 0.46 & 0.41 & 0.25 & 0.34 \\
\hline
\end{tabular}


Table D.1.21 the results of calibration and validations based on the effective frequency

\begin{tabular}{|c|c|c|c|c|c|c|}
\hline \multicolumn{3}{|c|}{ Configurations } & Calibration & $\mathbf{1}^{\text {st }}$ Validation & $\mathbf{2}^{\text {nd }}$ Validation & $\mathbf{3}^{\text {rd }}$ Validation \\
\hline $\boldsymbol{\varphi}_{\boldsymbol{g}}$ & $\boldsymbol{\Lambda}_{\boldsymbol{l}}$ & $\boldsymbol{\eta}_{\boldsymbol{m}}$ & Kappa & Kappa & Kappa & Kappa \\
\hline 5 & 2 & 1 & - & - & - \\
\hline 5 & 2 & 2 & - & - & - \\
\hline 5 & 2 & 3 & 0.31 & 0.24 & 0.24 & - \\
\hline 5 & 3 & 1 & - & - & - & - \\
\hline 5 & 3 & 2 & - & - & - & - \\
\hline 5 & 3 & 3 & - & - & - & - \\
\hline 5 & 4 & 2 & - & - & - & - \\
\hline 5 & 4 & 3 & - & - & - & - \\
\hline 5 & 4 & & - & & - \\
\hline
\end{tabular}




\section{D.2 The results of calibrations and validations in percentage scale}

Table D.2.1 the results of calibration procedure 1 and its validations.

\begin{tabular}{|c|c|c|c|c|c|c|c|c|c|c|c|}
\hline \multicolumn{4}{|c|}{ Configurations } & \multicolumn{2}{|c|}{ Calibration } & \multicolumn{2}{|c|}{$1^{\text {st }}$ Validation } & \multicolumn{2}{|c|}{$2^{\text {nd }}$ Validation } & \multicolumn{2}{|c|}{$3^{\text {rd }}$ Validation } \\
\hline$\varphi_{g}$ & $\Lambda_{l}$ & $\Gamma_{n}$ & $\eta_{m}$ & Vehicles & Pedestrians & Vehicles & Pedestrians & Vehicles & Pedestrians & Vehicles & Pedestrians \\
\hline 1 & 1 & 1 & 1 & 99 & 91 & 96 & 95 & 91 & 98 & 94 & 97 \\
\hline 1 & 1 & 1 & 2 & 99 & 91 & 96 & 95 & 88 & 97 & 93 & 96 \\
\hline 1 & 1 & 1 & 3 & 99 & 91 & 96 & 95 & 88 & 97 & 93 & 96 \\
\hline 1 & 1 & 2 & 1 & 99 & 91 & 96 & 95 & 90 & 98 & 93 & 97 \\
\hline 1 & 1 & 2 & 2 & 99 & 91 & 96 & 95 & - & - & - & - \\
\hline 1 & 1 & 2 & 3 & 99 & 91 & 96 & 95 & - & - & - & - \\
\hline 1 & 1 & 3 & 1 & 99 & 91 & 96 & 95 & 91 & 97 & 94 & 96 \\
\hline 1 & 1 & 3 & 2 & 99 & 91 & 96 & 95 & - & - & - & - \\
\hline 1 & 1 & 3 & 3 & 99 & 91 & 96 & 95 & - & - & - & - \\
\hline 1 & 1 & 4 & 1 & 99 & 91 & 96 & 95 & 90 & 97 & 93 & 96 \\
\hline 1 & 1 & 4 & 2 & 99 & 91 & 96 & 95 & 89 & 97 & 93 & 96 \\
\hline 1 & 1 & 4 & 3 & 99 & 91 & 96 & 95 & 89 & 97 & 93 & 96 \\
\hline 1 & 1 & 1 & 1 & 99 & 91 & 96 & 95 & 92 & 98 & 94 & 97 \\
\hline 1 & 1 & 1 & 2 & 99 & 91 & 96 & 95 & 90 & 97 & 93 & 96 \\
\hline 1 & 1 & 1 & 3 & 99 & 91 & 96 & 95 & 90 & 97 & 93 & 96 \\
\hline 1 & 1 & 6 & 1 & 99 & 91 & 96 & 95 & 91 & 97 & 94 & 96 \\
\hline 1 & 1 & 6 & 2 & 99 & 91 & 96 & 95 & 89 & 97 & 93 & 96 \\
\hline 1 & 1 & 6 & 3 & 99 & 91 & 96 & 95 & 89 & 97 & 93 & 96 \\
\hline 1 & 2 & 1 & 1 & 99 & 95 & 96 & 96 & 92 & 97 & 94 & 96 \\
\hline 1 & 2 & 1 & 2 & 99 & 95 & 96 & 96 & 92 & 97 & 94 & 96 \\
\hline 1 & 2 & 1 & 3 & 99 & 95 & 96 & 96 & 92 & 97 & 94 & 96 \\
\hline 1 & 2 & 2 & 1 & 99 & 95 & 95 & 96 & 92 & 97 & 94 & 96 \\
\hline 1 & 2 & 2 & 2 & 99 & 95 & 95 & 96 & 92 & 97 & 94 & 96 \\
\hline 1 & 2 & 2 & 3 & 99 & 95 & 95 & 96 & 92 & 97 & 94 & 96 \\
\hline 1 & 2 & 3 & 1 & 99 & 95 & 95 & 96 & 93 & 97 & 94 & 96 \\
\hline 1 & 2 & 3 & 2 & 99 & 95 & 95 & 96 & 93 & 97 & 94 & 96 \\
\hline 1 & 2 & 3 & 3 & 99 & 95 & 95 & 96 & 93 & 97 & 94 & 96 \\
\hline 1 & 2 & 4 & 1 & 99 & 95 & 96 & 96 & 93 & 97 & 94 & 97 \\
\hline 1 & 2 & 4 & 2 & 99 & 95 & 96 & 96 & 93 & 97 & 94 & 97 \\
\hline 1 & 2 & 4 & 3 & 99 & 95 & 96 & 96 & 93 & 97 & 94 & 97 \\
\hline 1 & 2 & 1 & 1 & 99 & 95 & 96 & 96 & 93 & 97 & 94 & 96 \\
\hline 1 & 2 & 1 & 2 & 99 & 95 & 96 & 96 & 93 & 97 & 94 & 96 \\
\hline 1 & 2 & 1 & 3 & 99 & 95 & 96 & 96 & 93 & 97 & 94 & 96 \\
\hline 1 & 2 & 6 & 1 & 99 & 95 & 95 & 96 & 92 & 97 & 94 & 96 \\
\hline
\end{tabular}


Table D.2.1 the results of calibration procedure 1 and its validations.

\begin{tabular}{|c|c|c|c|c|c|c|c|c|c|c|c|}
\hline \multicolumn{4}{|c|}{ Configurations } & \multicolumn{2}{|c|}{ Calibration } & \multicolumn{2}{|c|}{$1^{\text {st }}$ Validation } & \multicolumn{2}{|c|}{$2^{\text {nd }}$ Validation } & \multicolumn{2}{|c|}{$3^{\text {rd }}$ Validation } \\
\hline$\varphi_{g}$ & $\Lambda_{l}$ & $\Gamma_{n}$ & $\eta_{m}$ & Vehicles & Pedestrians & Vehicles & Pedestrians & Vehicles & Pedestrians & Vehicles & Pedestrians \\
\hline 1 & 2 & 6 & 2 & 99 & 95 & 95 & 96 & 92 & 97 & 94 & 96 \\
\hline 1 & 2 & 6 & 3 & 99 & 95 & 95 & 96 & 92 & 97 & 94 & 96 \\
\hline 1 & 3 & 1 & 1 & 99 & 92 & 96 & 90 & - & - & - & - \\
\hline 1 & 3 & 1 & 2 & 99 & 92 & 96 & 90 & - & - & - & - \\
\hline 1 & 3 & 1 & 3 & 99 & 92 & 96 & 90 & - & - & - & - \\
\hline 1 & 3 & 2 & 1 & 99 & 93 & 96 & 90 & - & - & - & - \\
\hline 1 & 3 & 2 & 2 & 99 & 93 & 96 & 90 & - & - & - & - \\
\hline 1 & 3 & 2 & 3 & 99 & 93 & 96 & 90 & - & - & - & - \\
\hline 1 & 3 & 3 & 1 & 99 & 92 & 95 & 90 & - & - & - & - \\
\hline 1 & 3 & 3 & 2 & 99 & 92 & 95 & 90 & - & - & - & - \\
\hline 1 & 3 & 3 & 3 & 99 & 92 & 95 & 90 & - & - & - & - \\
\hline 1 & 3 & 4 & 1 & 99 & 93 & 96 & 90 & - & - & - & - \\
\hline 1 & 3 & 4 & 2 & 99 & 93 & 96 & 90 & - & - & - & - \\
\hline 1 & 3 & 4 & 3 & 99 & 93 & 96 & 90 & - & - & - & - \\
\hline 1 & 3 & 1 & 1 & 99 & 93 & 96 & 90 & - & - & - & - \\
\hline 1 & 3 & 1 & 2 & 99 & 93 & 96 & 90 & - & - & - & - \\
\hline 1 & 3 & 1 & 3 & 99 & 93 & 96 & 90 & - & - & - & - \\
\hline 1 & 3 & 6 & 1 & 99 & 93 & 96 & 90 & - & - & - & - \\
\hline 1 & 3 & 6 & 2 & 99 & 93 & 96 & 90 & - & - & - & - \\
\hline 1 & 3 & 6 & 3 & 99 & 93 & 96 & 90 & - & - & - & - \\
\hline 1 & 4 & 1 & 1 & - & - & - & - & - & - & - & - \\
\hline 1 & 4 & 1 & 2 & - & - & - & - & - & - & - & - \\
\hline 1 & 4 & 1 & 3 & - & - & - & - & - & - & - & - \\
\hline 1 & 4 & 2 & 1 & - & - & - & - & - & - & - & - \\
\hline 1 & 4 & 2 & 2 & - & - & - & - & - & - & - & - \\
\hline 1 & 4 & 2 & 3 & - & - & - & - & - & - & - & - \\
\hline 1 & 4 & 3 & 1 & - & - & - & - & - & - & - & - \\
\hline 1 & 4 & 3 & 2 & - & - & - & - & - & - & - & - \\
\hline 1 & 4 & 3 & 3 & - & - & - & - & - & - & - & - \\
\hline 1 & 4 & 4 & 1 & - & - & - & - & - & - & - & - \\
\hline 1 & 4 & 4 & 2 & - & - & - & - & - & - & - & - \\
\hline 1 & 4 & 4 & 3 & - & - & - & - & - & - & - & - \\
\hline 1 & 4 & 1 & 1 & - & - & - & - & - & - & - & - \\
\hline 1 & 4 & 1 & 2 & - & - & - & - & - & - & - & - \\
\hline 1 & 4 & 1 & 3 & - & - & - & - & - & - & - & - \\
\hline 1 & 4 & 6 & 1 & - & - & - & - & - & - & - & - \\
\hline
\end{tabular}


Table D.2.1 the results of calibration procedure 1 and its validations.

\begin{tabular}{|c|c|c|c|c|c|c|c|c|c|c|c|}
\hline \multicolumn{4}{|c|}{ Configurations } & \multicolumn{2}{|c|}{ Calibration } & \multicolumn{2}{|c|}{$1^{\text {st }}$ Validation } & \multicolumn{2}{|c|}{$2^{\text {nd }}$ Validation } & \multicolumn{2}{|c|}{$3^{\text {rd }}$ Validation } \\
\hline$\varphi_{g}$ & $\Lambda_{l}$ & $\Gamma_{n}$ & $\eta_{m}$ & Vehicles & Pedestrians & Vehicles & Pedestrians & Vehicles & Pedestrians & Vehicles & Pedestrians \\
\hline 1 & 4 & 6 & 2 & - & - & - & - & - & - & - & - \\
\hline 1 & 4 & 6 & 3 & - & - & - & - & - & - & - & - \\
\hline 2 & 1 & 1 & 1 & 99 & 98 & 94 & 93 & 89 & 97 & 92 & 96 \\
\hline 2 & 1 & 1 & 2 & 99 & 98 & 94 & 93 & 89 & 97 & 92 & 96 \\
\hline 2 & 1 & 1 & 3 & 99 & 98 & 94 & 93 & 89 & 97 & 92 & 96 \\
\hline 2 & 1 & 2 & 1 & 99 & 98 & 94 & 93 & 89 & 97 & 92 & 96 \\
\hline 2 & 1 & 2 & 2 & 99 & 98 & 94 & 93 & 89 & 97 & 92 & 96 \\
\hline 2 & 1 & 2 & 3 & 99 & 98 & 94 & 93 & 89 & 97 & 92 & 96 \\
\hline 2 & 1 & 3 & 1 & 99 & 98 & 94 & 93 & 89 & 97 & 92 & 96 \\
\hline 2 & 1 & 3 & 2 & 99 & 98 & 94 & 93 & 89 & 97 & 92 & 96 \\
\hline 2 & 1 & 3 & 3 & 99 & 98 & 94 & 93 & 89 & 97 & 92 & 96 \\
\hline 2 & 1 & 4 & 1 & 99 & 98 & 94 & 91 & 90 & 97 & 92 & 95 \\
\hline 2 & 1 & 4 & 2 & 99 & 98 & 94 & 91 & 90 & 97 & 92 & 95 \\
\hline 2 & 1 & 4 & 3 & 99 & 98 & 94 & 91 & 90 & 97 & 92 & 95 \\
\hline 2 & 1 & 1 & 1 & 99 & 98 & 94 & 92 & 90 & 96 & 92 & 94 \\
\hline 2 & 1 & 1 & 2 & 99 & 98 & 94 & 92 & 90 & 96 & 92 & 94 \\
\hline 2 & 1 & 1 & 3 & 99 & 98 & 94 & 92 & 90 & 96 & 92 & 94 \\
\hline 2 & 1 & 6 & 1 & 99 & 98 & 94 & 93 & 90 & 97 & 92 & 96 \\
\hline 2 & 1 & 6 & 2 & 99 & 98 & 94 & 93 & 90 & 97 & 92 & 96 \\
\hline 2 & 1 & 6 & 3 & 99 & 98 & 94 & 93 & 90 & 97 & 92 & 96 \\
\hline 2 & 2 & 1 & 1 & 99 & 99 & 95 & 96 & - & - & - & - \\
\hline 2 & 2 & 1 & 2 & 99 & 99 & 95 & 96 & - & - & - & - \\
\hline 2 & 2 & 1 & 3 & 99 & 99 & 95 & 96 & - & - & - & - \\
\hline 2 & 2 & 2 & 1 & 99 & 99 & 95 & 96 & - & - & - & - \\
\hline 2 & 2 & 2 & 2 & 99 & 99 & 95 & 96 & - & - & - & - \\
\hline 2 & 2 & 2 & 3 & 99 & 99 & 95 & 96 & - & - & - & - \\
\hline 2 & 2 & 3 & 1 & 99 & 99 & 94 & 99 & - & - & - & - \\
\hline 2 & 2 & 3 & 2 & 99 & 99 & 94 & 99 & - & - & - & - \\
\hline 2 & 2 & 3 & 3 & 99 & 99 & 94 & 99 & - & - & - & - \\
\hline 2 & 2 & 4 & 1 & 99 & 99 & 94 & 97 & - & - & - & - \\
\hline 2 & 2 & 4 & 2 & 99 & 99 & 94 & 97 & - & - & - & - \\
\hline 2 & 2 & 4 & 3 & 99 & 99 & 94 & 97 & - & - & - & - \\
\hline 2 & 2 & 1 & 1 & 99 & 99 & 94 & 97 & - & - & - & - \\
\hline 2 & 2 & 1 & 2 & 99 & 99 & 94 & 97 & - & - & - & - \\
\hline 2 & 2 & 1 & 3 & 99 & 99 & 94 & 97 & - & - & - & - \\
\hline 2 & 2 & 6 & 1 & 99 & 99 & 95 & 97 & - & - & - & - \\
\hline
\end{tabular}


Table D.2.1 the results of calibration procedure 1 and its validations.

\begin{tabular}{|c|c|c|c|c|c|c|c|c|c|c|c|}
\hline \multicolumn{4}{|c|}{ Configurations } & \multicolumn{2}{|c|}{ Calibration } & \multicolumn{2}{|c|}{$1^{\text {st }}$ Validation } & \multicolumn{2}{|c|}{$2^{\text {nd }}$ Validation } & \multicolumn{2}{|c|}{$3^{\text {rd }}$ Validation } \\
\hline$\varphi_{g}$ & $\Lambda_{l}$ & $\Gamma_{n}$ & $\eta_{m}$ & Vehicles & Pedestrians & Vehicles & Pedestrians & Vehicles & Pedestrians & Vehicles & Pedestrians \\
\hline 2 & 2 & 6 & 2 & 99 & 99 & 95 & 97 & - & - & - & - \\
\hline 2 & 2 & 6 & 3 & 99 & 99 & 95 & 97 & - & - & - & - \\
\hline 2 & 3 & 1 & 1 & 99 & 98 & 94 & 94 & 88 & 94 & 91 & 94 \\
\hline 2 & 3 & 1 & 2 & 99 & 98 & 94 & 94 & 88 & 94 & 91 & 94 \\
\hline 2 & 3 & 1 & 3 & 99 & 98 & 94 & 94 & 88 & 94 & 91 & 94 \\
\hline 2 & 3 & 2 & 1 & 99 & 98 & 94 & 94 & 87 & 94 & 91 & 94 \\
\hline 2 & 3 & 2 & 2 & 99 & 98 & 94 & 94 & 87 & 94 & 91 & 94 \\
\hline 2 & 3 & 2 & 3 & 99 & 98 & 94 & 94 & 87 & 94 & 91 & 94 \\
\hline 2 & 3 & 3 & 1 & 99 & 98 & 94 & 94 & 87 & 94 & 91 & 94 \\
\hline 2 & 3 & 3 & 2 & 99 & 98 & 94 & 94 & 87 & 94 & 91 & 94 \\
\hline 2 & 3 & 3 & 3 & 99 & 98 & 94 & 94 & 87 & 94 & 91 & 94 \\
\hline 2 & 3 & 4 & 1 & 99 & 98 & 94 & 94 & 87 & 94 & 91 & 94 \\
\hline 2 & 3 & 4 & 2 & 99 & 98 & 94 & 94 & 87 & 94 & 91 & 94 \\
\hline 2 & 3 & 4 & 3 & 99 & 98 & 94 & 94 & 87 & 94 & 91 & 94 \\
\hline 2 & 3 & 1 & 1 & 99 & 98 & 94 & 94 & 87 & 94 & 91 & 94 \\
\hline 2 & 3 & 1 & 2 & 99 & 98 & 94 & 94 & 87 & 94 & 91 & 94 \\
\hline 2 & 3 & 1 & 3 & 99 & 98 & 94 & 94 & 87 & 94 & 91 & 94 \\
\hline 2 & 3 & 6 & 1 & 99 & 98 & 94 & 94 & 87 & 94 & 91 & 94 \\
\hline 2 & 3 & 6 & 2 & 99 & 98 & 94 & 94 & 87 & 94 & 91 & 94 \\
\hline 2 & 3 & 6 & 3 & 99 & 98 & 94 & 94 & 87 & 94 & 91 & 94 \\
\hline 2 & 4 & 1 & 1 & 99 & 97 & - & - & - & - & - & - \\
\hline 2 & 4 & 1 & 2 & 99 & 97 & - & - & - & - & - & - \\
\hline 2 & 4 & 1 & 3 & 99 & 97 & - & - & - & - & - & - \\
\hline 2 & 4 & 2 & 1 & 99 & 97 & - & - & - & - & - & - \\
\hline 2 & 4 & 2 & 2 & 99 & 97 & - & - & - & - & - & - \\
\hline 2 & 4 & 2 & 3 & 99 & 97 & - & - & - & - & - & - \\
\hline 2 & 4 & 3 & 1 & 99 & 97 & - & - & - & - & - & - \\
\hline 2 & 4 & 3 & 2 & 99 & 97 & - & - & - & - & - & - \\
\hline 2 & 4 & 3 & 3 & 99 & 97 & - & - & - & - & - & - \\
\hline 2 & 4 & 4 & 1 & 99 & 97 & - & - & - & - & - & - \\
\hline 2 & 4 & 4 & 2 & 99 & 97 & - & - & - & - & - & - \\
\hline 2 & 4 & 4 & 3 & 99 & 97 & - & - & - & - & - & - \\
\hline 2 & 4 & 1 & 1 & 99 & 97 & - & - & - & - & - & - \\
\hline 2 & 4 & 1 & 2 & 99 & 97 & - & - & - & - & - & - \\
\hline 2 & 4 & 1 & 3 & 99 & 97 & - & - & - & - & - & - \\
\hline 2 & 4 & 6 & 1 & 99 & 97 & - & - & - & - & - & - \\
\hline
\end{tabular}


Table D.2.1 the results of calibration procedure 1 and its validations.

\begin{tabular}{|c|c|c|c|c|c|c|c|c|c|c|c|}
\hline \multicolumn{4}{|c|}{ Configurations } & \multicolumn{2}{|c|}{ Calibration } & \multicolumn{2}{|c|}{$1^{\text {st }}$ Validation } & \multicolumn{2}{|c|}{$2^{\text {nd }}$ Validation } & \multicolumn{2}{|c|}{$3^{\text {rd }}$ Validation } \\
\hline$\varphi_{g}$ & $\Lambda_{l}$ & $\Gamma_{n}$ & $\eta_{m}$ & Vehicles & Pedestrians & Vehicles & Pedestrians & Vehicles & Pedestrians & Vehicles & Pedestrians \\
\hline 2 & 4 & 6 & 2 & 99 & 97 & - & - & - & - & - & - \\
\hline 2 & 4 & 6 & 3 & 99 & 97 & - & - & - & - & - & - \\
\hline 3 & 1 & 1 & 1 & 99 & 99 & 92 & 94 & - & - & - & - \\
\hline 3 & 1 & 1 & 2 & 99 & 99 & 92 & 94 & - & - & - & - \\
\hline 3 & 1 & 1 & 3 & 99 & 99 & 92 & 94 & - & - & - & - \\
\hline 3 & 1 & 2 & 1 & 99 & 99 & 92 & 94 & - & - & - & - \\
\hline 3 & 1 & 2 & 2 & 99 & 99 & 92 & 94 & - & - & - & - \\
\hline 3 & 1 & 2 & 3 & 99 & 99 & 92 & 94 & - & - & - & - \\
\hline 3 & 1 & 3 & 1 & 99 & 99 & 92 & 96 & - & - & - & - \\
\hline 3 & 1 & 3 & 2 & 99 & 99 & 92 & 96 & - & - & - & - \\
\hline 3 & 1 & 3 & 3 & 99 & 99 & 92 & 96 & - & - & - & - \\
\hline 3 & 1 & 4 & 1 & 99 & 99 & 92 & 94 & - & - & - & - \\
\hline 3 & 1 & 4 & 2 & 99 & 99 & 92 & 94 & - & - & - & - \\
\hline 3 & 1 & 4 & 3 & 99 & 99 & 92 & 94 & - & - & - & - \\
\hline 3 & 1 & 1 & 1 & 99 & 99 & 92 & 94 & - & - & - & - \\
\hline 3 & 1 & 1 & 2 & 99 & 99 & 92 & 94 & - & - & - & - \\
\hline 3 & 1 & 1 & 3 & 99 & 99 & 92 & 94 & - & - & - & - \\
\hline 3 & 1 & 6 & 1 & 99 & 99 & 92 & 97 & - & - & - & - \\
\hline 3 & 1 & 6 & 2 & 99 & 99 & 92 & 97 & - & - & - & - \\
\hline 3 & 1 & 6 & 3 & 99 & 99 & 92 & 97 & - & - & - & - \\
\hline 3 & 2 & 1 & 1 & 99 & 99 & 91 & 96 & 90 & 92 & 91 & 93 \\
\hline 3 & 2 & 1 & 2 & 99 & 99 & 91 & 96 & 90 & 92 & 91 & 93 \\
\hline 3 & 2 & 1 & 3 & 99 & 99 & 91 & 96 & 90 & 92 & 91 & 93 \\
\hline 3 & 2 & 2 & 1 & 99 & 99 & - & - & - & - & - & - \\
\hline 3 & 2 & 2 & 2 & 99 & 99 & - & - & - & - & - & - \\
\hline 3 & 2 & 2 & 3 & 99 & 99 & - & - & - & - & - & - \\
\hline 3 & 2 & 3 & 1 & 100 & 99 & 92 & 96 & 89 & 92 & 90 & 93 \\
\hline 3 & 2 & 3 & 2 & 100 & 99 & 92 & 96 & 89 & 92 & 90 & 93 \\
\hline 3 & 2 & 3 & 3 & 100 & 99 & 92 & 96 & 89 & 92 & 90 & 93 \\
\hline 3 & 2 & 4 & 1 & 100 & 99 & 92 & 96 & 89 & 92 & 90 & 93 \\
\hline 3 & 2 & 4 & 2 & 100 & 99 & 92 & 96 & 89 & 92 & 90 & 93 \\
\hline 3 & 2 & 4 & 3 & 100 & 99 & 92 & 96 & 89 & 92 & 90 & 93 \\
\hline 3 & 2 & 1 & 1 & 100 & 99 & - & - & - & - & - & - \\
\hline 3 & 2 & 1 & 2 & 100 & 99 & - & - & - & - & - & - \\
\hline 3 & 2 & 1 & 3 & 100 & 99 & - & - & - & - & - & - \\
\hline 3 & 2 & 6 & 1 & 99 & 99 & - & - & - & - & - & - \\
\hline
\end{tabular}


Table D.2.1 the results of calibration procedure 1 and its validations.

\begin{tabular}{|c|c|c|c|c|c|c|c|c|c|c|c|}
\hline \multicolumn{4}{|c|}{ Configurations } & \multicolumn{2}{|c|}{ Calibration } & \multicolumn{2}{|c|}{$\mathbf{1}^{\text {st }}$ Validation } & \multicolumn{2}{|c|}{$2^{\text {nd }}$ Validation } & \multicolumn{2}{|c|}{$3^{\text {rd }}$ Validation } \\
\hline$\varphi_{g}$ & $\Lambda_{l}$ & $\Gamma_{n}$ & $\eta_{m}$ & Vehicles & Pedestrians & Vehicles & Pedestrians & Vehicles & Pedestrians & Vehicles & Pedestrians \\
\hline 3 & 2 & 6 & 2 & 99 & 99 & - & - & - & - & - & - \\
\hline 3 & 2 & 6 & 3 & 99 & 99 & - & - & - & - & - & - \\
\hline 3 & 3 & 1 & 1 & 99 & 100 & - & - & - & - & - & - \\
\hline 3 & 3 & 1 & 2 & 99 & 100 & - & - & - & - & - & - \\
\hline 3 & 3 & 1 & 3 & 99 & 100 & - & - & - & - & - & - \\
\hline 3 & 3 & 2 & 1 & 99 & 100 & 92 & 99 & 87 & 96 & 90 & 97 \\
\hline 3 & 3 & 2 & 2 & 99 & 100 & 92 & 99 & 87 & 96 & 90 & 97 \\
\hline 3 & 3 & 2 & 3 & 99 & 100 & 92 & 99 & 87 & 96 & 90 & 97 \\
\hline 3 & 3 & 3 & 1 & 99 & 97 & 95 & 94 & 88 & 91 & 92 & 92 \\
\hline 3 & 3 & 3 & 2 & 99 & 97 & 95 & 94 & 88 & 91 & 92 & 92 \\
\hline 3 & 3 & 3 & 3 & 99 & 97 & 95 & 94 & 88 & 91 & 92 & 92 \\
\hline 3 & 3 & 4 & 1 & 99 & 97 & 95 & 94 & 88 & 91 & 92 & 92 \\
\hline 3 & 3 & 4 & 2 & 99 & 97 & 95 & 94 & 88 & 91 & 92 & 92 \\
\hline 3 & 3 & 4 & 3 & 99 & 97 & 95 & 94 & 88 & 91 & 92 & 92 \\
\hline 3 & 3 & 1 & 1 & 99 & 99 & 91 & 99 & 84 & 94 & 88 & 96 \\
\hline 3 & 3 & 1 & 2 & 99 & 99 & 91 & 99 & 84 & 94 & 88 & 96 \\
\hline 3 & 3 & 1 & 3 & 99 & 99 & 91 & 99 & 84 & 94 & 88 & 96 \\
\hline 3 & 3 & 6 & 1 & 99 & 99 & 91 & 99 & 85 & 94 & 88 & 96 \\
\hline 3 & 3 & 6 & 2 & 99 & 99 & 91 & 99 & 85 & 94 & 88 & 96 \\
\hline 3 & 3 & 6 & 3 & 99 & 99 & 91 & 99 & 85 & 94 & 88 & 96 \\
\hline 3 & 4 & 1 & 1 & 99 & 96 & 96 & 92 & - & - & - & - \\
\hline 3 & 4 & 1 & 2 & 99 & 96 & 96 & 92 & - & - & - & - \\
\hline 3 & 4 & 1 & 3 & 99 & 96 & 96 & 92 & - & - & - & - \\
\hline 3 & 4 & 2 & 1 & 99 & 96 & 96 & 94 & - & - & - & - \\
\hline 3 & 4 & 2 & 2 & 99 & 96 & 96 & 94 & - & - & - & - \\
\hline 3 & 4 & 2 & 3 & 99 & 96 & 96 & 94 & - & - & - & - \\
\hline 3 & 4 & 3 & 1 & 99 & 96 & 96 & 92 & - & - & - & - \\
\hline 3 & 4 & 3 & 2 & 99 & 96 & 96 & 92 & - & - & - & - \\
\hline 3 & 4 & 3 & 3 & 99 & 96 & 96 & 92 & - & - & - & - \\
\hline 3 & 4 & 4 & 1 & 99 & 96 & 96 & 92 & - & - & - & - \\
\hline 3 & 4 & 4 & 2 & 99 & 96 & 96 & 92 & - & - & - & - \\
\hline 3 & 4 & 4 & 3 & 99 & 96 & 96 & 92 & - & - & - & - \\
\hline 3 & 4 & 1 & 1 & 100 & 96 & 96 & 92 & - & - & - & - \\
\hline 3 & 4 & 1 & 2 & 100 & 96 & 96 & 92 & - & - & - & - \\
\hline 3 & 4 & 1 & 3 & 100 & 96 & 96 & 92 & - & - & - & - \\
\hline 3 & 4 & 6 & 1 & 100 & 96 & 96 & 92 & - & - & - & - \\
\hline
\end{tabular}


Table D.2.1 the results of calibration procedure 1 and its validations.

\begin{tabular}{|c|c|c|c|c|c|c|c|c|c|c|c|}
\hline \multicolumn{4}{|c|}{ Configurations } & \multicolumn{2}{|c|}{ Calibration } & \multicolumn{2}{|c|}{$1^{\text {st }}$ Validation } & \multicolumn{2}{|c|}{$2^{\text {nd }}$ Validation } & \multicolumn{2}{|c|}{$3^{\text {rd }}$ Validation } \\
\hline$\varphi_{g}$ & $\Lambda_{l}$ & $\Gamma_{n}$ & $\eta_{m}$ & Vehicles & Pedestrians & Vehicles & Pedestrians & Vehicles & Pedestrians & Vehicles & Pedestrians \\
\hline 3 & 4 & 6 & 2 & 100 & 96 & 96 & 92 & - & - & - & - \\
\hline 3 & 4 & 6 & 3 & 100 & 96 & 96 & 92 & - & - & - & - \\
\hline 4 & 1 & 1 & 1 & 100 & 96 & 96 & 87 & 96 & 100 & 96 & 94 \\
\hline 4 & 1 & 1 & 2 & 100 & 96 & 96 & 87 & 96 & 100 & 96 & 94 \\
\hline 4 & 1 & 1 & 3 & 100 & 96 & 96 & 87 & 96 & 100 & 96 & 94 \\
\hline 4 & 1 & 2 & 1 & 100 & 96 & 96 & 87 & 97 & 100 & 96 & 94 \\
\hline 4 & 1 & 2 & 2 & 100 & 96 & 96 & 87 & 97 & 100 & 96 & 94 \\
\hline 4 & 1 & 2 & 3 & 100 & 96 & 96 & 87 & 97 & 100 & 96 & 94 \\
\hline 4 & 1 & 3 & 1 & 100 & 96 & 96 & 89 & 96 & 100 & 96 & 95 \\
\hline 4 & 1 & 3 & 2 & 100 & 96 & 96 & 89 & 96 & 100 & 96 & 95 \\
\hline 4 & 1 & 3 & 3 & 100 & 96 & 96 & 89 & 96 & 100 & 96 & 95 \\
\hline 4 & 1 & 4 & 1 & 100 & 96 & 96 & 89 & 96 & 100 & 96 & 95 \\
\hline 4 & 1 & 4 & 2 & 100 & 96 & 96 & 89 & 96 & 100 & 96 & 95 \\
\hline 4 & 1 & 4 & 3 & 100 & 96 & 96 & 89 & 96 & 100 & 96 & 95 \\
\hline 4 & 1 & 1 & 1 & 100 & 96 & 96 & 90 & 96 & 100 & 96 & 96 \\
\hline 4 & 1 & 1 & 2 & 100 & 96 & 96 & 89 & 96 & 100 & 96 & 95 \\
\hline 4 & 1 & 1 & 3 & 100 & 96 & 96 & 89 & 96 & 100 & 96 & 95 \\
\hline 4 & 1 & 6 & 1 & 100 & 96 & 96 & 89 & 96 & 100 & 96 & 95 \\
\hline 4 & 1 & 6 & 2 & 100 & 96 & 96 & 89 & 96 & 100 & 96 & 95 \\
\hline 4 & 1 & 6 & 3 & 100 & 96 & 96 & 89 & 96 & 100 & 96 & 95 \\
\hline 4 & 2 & 1 & 1 & 99 & 99 & 92 & 92 & - & - & - & - \\
\hline 4 & 2 & 1 & 2 & 99 & 99 & 92 & 92 & - & - & - & - \\
\hline 4 & 2 & 1 & 3 & 99 & 99 & 92 & 92 & - & - & - & - \\
\hline 4 & 2 & 2 & 1 & 99 & 97 & 92 & 93 & - & - & - & - \\
\hline 4 & 2 & 2 & 2 & 99 & 97 & 92 & 93 & - & - & - & - \\
\hline 4 & 2 & 2 & 3 & 99 & 97 & 92 & 93 & - & - & - & - \\
\hline 4 & 2 & 3 & 1 & 99 & 97 & 92 & 93 & - & - & - & - \\
\hline 4 & 2 & 3 & 2 & 99 & 97 & 92 & 93 & - & - & - & - \\
\hline 4 & 2 & 3 & 3 & 99 & 97 & 92 & 93 & - & - & - & - \\
\hline 4 & 2 & 4 & 1 & 99 & 97 & 92 & 93 & - & - & - & - \\
\hline 4 & 2 & 4 & 2 & 99 & 97 & 92 & 93 & - & - & - & - \\
\hline 4 & 2 & 4 & 3 & 99 & 97 & 92 & 93 & - & - & - & - \\
\hline 4 & 2 & 1 & 1 & 99 & 97 & 92 & 93 & - & - & - & - \\
\hline 4 & 2 & 1 & 2 & 99 & 97 & 92 & 93 & - & - & - & - \\
\hline 4 & 2 & 1 & 3 & 99 & 97 & 92 & 93 & - & - & - & - \\
\hline 4 & 2 & 6 & 1 & 99 & 97 & 92 & 93 & - & - & - & - \\
\hline
\end{tabular}


Table D.2.1 the results of calibration procedure 1 and its validations.

\begin{tabular}{|c|c|c|c|c|c|c|c|c|c|c|c|}
\hline \multicolumn{4}{|c|}{ Configurations } & \multicolumn{2}{|c|}{ Calibration } & \multicolumn{2}{|c|}{$1^{\text {st }}$ Validation } & \multicolumn{2}{|c|}{$2^{\text {nd }}$ Validation } & \multicolumn{2}{|c|}{$3^{\text {rd }}$ Validation } \\
\hline$\varphi_{g}$ & $\Lambda_{l}$ & $\Gamma_{n}$ & $\eta_{m}$ & Vehicles & Pedestrians & Vehicles & Pedestrians & Vehicles & Pedestrians & Vehicles & Pedestrians \\
\hline 4 & 2 & 6 & 2 & 99 & 97 & 92 & 93 & - & - & - & - \\
\hline 4 & 2 & 6 & 3 & 99 & 97 & 92 & 93 & - & - & - & - \\
\hline 4 & 3 & 1 & 1 & 99 & 100 & - & - & - & - & - & - \\
\hline 4 & 3 & 1 & 2 & 99 & 100 & - & - & - & - & - & - \\
\hline 4 & 3 & 1 & 3 & 99 & 100 & - & - & - & - & - & - \\
\hline 4 & 3 & 2 & 1 & 99 & 99 & - & - & - & - & - & - \\
\hline 4 & 3 & 2 & 2 & 99 & 99 & - & - & - & - & - & - \\
\hline 4 & 3 & 2 & 3 & 99 & 99 & - & - & - & - & - & - \\
\hline 4 & 3 & 3 & 1 & 99 & 99 & - & - & - & - & - & - \\
\hline 4 & 3 & 3 & 2 & 99 & 99 & - & - & - & - & - & - \\
\hline 4 & 3 & 3 & 3 & 99 & 99 & - & - & - & - & - & - \\
\hline 4 & 3 & 4 & 1 & 99 & 99 & - & - & - & - & - & - \\
\hline 4 & 3 & 4 & 2 & 99 & 99 & - & - & - & - & - & - \\
\hline 4 & 3 & 4 & 3 & 99 & 99 & - & - & - & - & - & - \\
\hline 4 & 3 & 1 & 1 & 99 & 100 & 90 & 93 & - & - & - & - \\
\hline 4 & 3 & 1 & 2 & 99 & 100 & 90 & 93 & - & - & - & - \\
\hline 4 & 3 & 1 & 3 & 99 & 100 & 90 & 93 & - & - & - & - \\
\hline 4 & 3 & 6 & 1 & 99 & 100 & 90 & 93 & - & - & - & - \\
\hline 4 & 3 & 6 & 2 & 99 & 100 & 90 & 93 & - & - & - & - \\
\hline 4 & 3 & 6 & 3 & 99 & 100 & 90 & 93 & - & - & - & - \\
\hline 4 & 4 & 1 & 1 & 99 & 99 & 91 & 100 & - & - & - & - \\
\hline 4 & 4 & 1 & 2 & 99 & 99 & 91 & 100 & - & - & - & - \\
\hline 4 & 4 & 1 & 3 & 99 & 99 & 91 & 100 & - & - & - & - \\
\hline 4 & 4 & 2 & 1 & 99 & 99 & 91 & 100 & - & - & - & - \\
\hline 4 & 4 & 2 & 2 & 99 & 99 & 91 & 100 & - & - & - & - \\
\hline 4 & 4 & 2 & 3 & 99 & 99 & 91 & 100 & - & - & - & - \\
\hline 4 & 4 & 3 & 1 & 99 & 99 & 91 & 100 & - & - & - & - \\
\hline 4 & 4 & 3 & 2 & 99 & 99 & 91 & 100 & - & - & - & - \\
\hline 4 & 4 & 3 & 3 & 99 & 99 & 91 & 100 & - & - & - & - \\
\hline 4 & 4 & 4 & 1 & 99 & 99 & 91 & 100 & - & - & - & - \\
\hline 4 & 4 & 4 & 2 & 99 & 99 & 91 & 100 & - & - & - & - \\
\hline 4 & 4 & 4 & 3 & 99 & 99 & 91 & 100 & - & - & - & - \\
\hline 4 & 4 & 1 & 1 & 99 & 99 & 91 & 100 & - & - & - & - \\
\hline 4 & 4 & 1 & 2 & 99 & 99 & 91 & 100 & - & - & - & - \\
\hline 4 & 4 & 1 & 3 & 99 & 99 & 91 & 100 & - & - & - & - \\
\hline 4 & 4 & 6 & 1 & 99 & 99 & 91 & 100 & - & - & - & - \\
\hline
\end{tabular}


Table D.2.1 the results of calibration procedure 1 and its validations.

\begin{tabular}{|c|c|c|c|c|c|c|c|c|c|c|c|}
\hline \multicolumn{4}{|c|}{ Configurations } & \multicolumn{2}{|c|}{ Calibration } & \multicolumn{2}{|c|}{$\mathbf{1}^{\text {st }}$ Validation } & \multicolumn{2}{|c|}{$2^{\text {nd }}$ Validation } & \multicolumn{2}{|c|}{$3^{\text {rd }}$ Validation } \\
\hline$\varphi_{g}$ & $\Lambda_{l}$ & $\Gamma_{n}$ & $\eta_{m}$ & Vehicles & Pedestrians & Vehicles & Pedestrians & Vehicles & Pedestrians & Vehicles & Pedestrians \\
\hline 4 & 4 & 6 & 2 & 99 & 99 & 91 & 100 & - & - & - & - \\
\hline 4 & 4 & 6 & 3 & 99 & 99 & 91 & 100 & - & - & - & - \\
\hline 5 & 1 & 1 & 1 & 99 & 100 & 89 & 96 & 84 & 98 & 87 & 97 \\
\hline 5 & 1 & 1 & 2 & 99 & 100 & 89 & 96 & 84 & 98 & 87 & 97 \\
\hline 5 & 1 & 1 & 3 & 99 & 100 & 89 & 96 & 84 & 98 & 87 & 97 \\
\hline 5 & 1 & 2 & 1 & 99 & 100 & 89 & 96 & 84 & 98 & 87 & 97 \\
\hline 5 & 1 & 2 & 2 & 99 & 100 & 89 & 96 & 84 & 98 & 87 & 97 \\
\hline 5 & 1 & 2 & 3 & 99 & 100 & 89 & 96 & 84 & 98 & 87 & 97 \\
\hline 5 & 1 & 3 & 1 & 99 & 100 & 89 & 98 & 83 & 100 & 87 & 99 \\
\hline 5 & 1 & 3 & 2 & 99 & 100 & 89 & 98 & 83 & 98 & 87 & 98 \\
\hline 5 & 1 & 3 & 3 & 99 & 100 & 89 & 98 & 83 & 98 & 87 & 98 \\
\hline 5 & 1 & 4 & 1 & 99 & 100 & 90 & 93 & 84 & 98 & 88 & 96 \\
\hline 5 & 1 & 4 & 2 & 99 & 100 & 90 & 93 & 84 & 98 & 88 & 96 \\
\hline 5 & 1 & 4 & 3 & 99 & 100 & 90 & 93 & 84 & 98 & 88 & 96 \\
\hline 5 & 1 & 1 & 1 & 99 & 100 & 90 & 96 & 84 & 100 & 88 & 98 \\
\hline 5 & 1 & 1 & 2 & 99 & 100 & 90 & 96 & 84 & 100 & 88 & 98 \\
\hline 5 & 1 & 1 & 3 & 99 & 100 & 90 & 96 & 84 & 100 & 88 & 98 \\
\hline 5 & 1 & 6 & 1 & 99 & 100 & 90 & 96 & 84 & 98 & 88 & 97 \\
\hline 5 & 1 & 6 & 2 & 99 & 100 & 90 & 96 & 86 & 98 & 88 & 97 \\
\hline 5 & 1 & 6 & 3 & 99 & 100 & 90 & 96 & 86 & 98 & 88 & 97 \\
\hline 5 & 2 & 1 & 1 & 99 & 98 & 89 & 89 & 83 & 91 & 87 & 90 \\
\hline 5 & 2 & 1 & 2 & 99 & 98 & 89 & 89 & 83 & 91 & 87 & 90 \\
\hline 5 & 2 & 1 & 3 & 99 & 98 & 89 & 89 & 83 & 91 & 87 & 90 \\
\hline 5 & 2 & 2 & 1 & 99 & 98 & 90 & 93 & 83 & 89 & 87 & 91 \\
\hline 5 & 2 & 2 & 2 & 99 & 98 & 90 & 93 & 83 & 89 & 87 & 91 \\
\hline 5 & 2 & 2 & 3 & 99 & 98 & 90 & 93 & 83 & 89 & 87 & 91 \\
\hline 5 & 2 & 3 & 1 & 99 & 98 & 90 & 89 & 83 & 87 & 87 & 88 \\
\hline 5 & 2 & 3 & 2 & 99 & 98 & 90 & 89 & 83 & 87 & 87 & 88 \\
\hline 5 & 2 & 3 & 3 & 99 & 98 & 90 & 89 & 83 & 87 & 87 & 88 \\
\hline 5 & 2 & 4 & 1 & 99 & 98 & 90 & 87 & 83 & 87 & 87 & 87 \\
\hline 5 & 2 & 4 & 2 & 99 & 98 & 90 & 87 & 83 & 87 & 87 & 87 \\
\hline 5 & 2 & 4 & 3 & 99 & 98 & 90 & 87 & 83 & 87 & 87 & 87 \\
\hline 5 & 2 & 1 & 1 & 99 & 98 & 90 & 89 & 83 & 89 & 87 & 89 \\
\hline 5 & 2 & 1 & 2 & 99 & 98 & 90 & 89 & 83 & 89 & 87 & 89 \\
\hline 5 & 2 & 1 & 3 & 99 & 98 & 90 & 89 & 83 & 89 & 87 & 89 \\
\hline 5 & 2 & 6 & 1 & 99 & 100 & 87 & 96 & 83 & 87 & 86 & 91 \\
\hline
\end{tabular}


Table D.2.1 the results of calibration procedure 1 and its validations.

\begin{tabular}{|c|c|c|c|c|c|c|c|c|c|c|c|}
\hline \multicolumn{4}{|c|}{ Configurations } & \multicolumn{2}{|c|}{ Calibration } & \multicolumn{2}{|c|}{$\mathbf{1}^{\text {st }}$ Validation } & \multicolumn{2}{|c|}{$2^{\text {nd }}$ Validation } & \multicolumn{2}{|c|}{$3^{\text {rd }}$ Validation } \\
\hline$\varphi_{g}$ & $\Lambda_{l}$ & $\Gamma_{n}$ & $\eta_{m}$ & Vehicles & Pedestrians & Vehicles & Pedestrians & Vehicles & Pedestrians & Vehicles & Pedestrians \\
\hline 5 & 2 & 6 & 2 & 99 & 100 & 87 & 96 & 83 & 87 & 86 & 91 \\
\hline 5 & 2 & 6 & 3 & 99 & 100 & 87 & 96 & 83 & 87 & 86 & 91 \\
\hline 5 & 3 & 1 & 1 & 99 & 98 & 92 & 91 & 85 & 89 & 89 & 90 \\
\hline 5 & 3 & 1 & 2 & 99 & 98 & 92 & 91 & 85 & 89 & 89 & 90 \\
\hline 5 & 3 & 1 & 3 & 99 & 98 & 92 & 91 & 85 & 89 & 89 & 90 \\
\hline 5 & 3 & 2 & 1 & 99 & 98 & 93 & 93 & 85 & 91 & 90 & 92 \\
\hline 5 & 3 & 2 & 2 & 99 & 98 & 93 & 93 & 85 & 91 & 90 & 92 \\
\hline 5 & 3 & 2 & 3 & 99 & 98 & 93 & 93 & 85 & 91 & 90 & 92 \\
\hline 5 & 3 & 3 & 1 & 99 & 98 & 92 & 91 & 84 & 87 & 89 & 89 \\
\hline 5 & 3 & 3 & 2 & 99 & 98 & 92 & 91 & 84 & 87 & 89 & 89 \\
\hline 5 & 3 & 3 & 3 & 99 & 98 & 92 & 91 & 84 & 87 & 89 & 89 \\
\hline 5 & 3 & 4 & 1 & 99 & 98 & 93 & 93 & 85 & 87 & 90 & 90 \\
\hline 5 & 3 & 4 & 2 & 99 & 98 & 93 & 93 & 85 & 87 & 90 & 90 \\
\hline 5 & 3 & 4 & 3 & 99 & 98 & 93 & 93 & 85 & 87 & 90 & 90 \\
\hline 5 & 3 & 1 & 1 & 99 & 98 & 93 & 96 & 85 & 87 & 90 & 91 \\
\hline 5 & 3 & 1 & 2 & 99 & 98 & 93 & 96 & 85 & 87 & 90 & 91 \\
\hline 5 & 3 & 1 & 3 & 99 & 98 & 93 & 96 & 85 & 87 & 90 & 91 \\
\hline 5 & 3 & 6 & 1 & 99 & 98 & 93 & 93 & 87 & 87 & 91 & 90 \\
\hline 5 & 3 & 6 & 2 & 99 & 98 & 93 & 93 & 87 & 87 & 91 & 90 \\
\hline 5 & 3 & 6 & 3 & 99 & 98 & 93 & 93 & 87 & 87 & 91 & 90 \\
\hline 5 & 4 & 1 & 1 & 97 & 100 & 90 & 93 & 84 & 91 & 88 & 92 \\
\hline 5 & 4 & 1 & 2 & 97 & 100 & 90 & 93 & 82 & 91 & 87 & 92 \\
\hline 5 & 4 & 1 & 3 & 97 & 100 & 90 & 93 & 82 & 91 & 87 & 92 \\
\hline 5 & 4 & 2 & 1 & 97 & 100 & 90 & 93 & 84 & 87 & 88 & 90 \\
\hline 5 & 4 & 2 & 2 & 97 & 100 & 90 & 93 & 82 & 89 & 87 & 91 \\
\hline 5 & 4 & 2 & 3 & 97 & 100 & 90 & 93 & 82 & 89 & 87 & 91 \\
\hline 5 & 4 & 3 & 1 & 97 & 100 & 89 & 93 & 83 & 84 & 87 & 89 \\
\hline 5 & 4 & 3 & 2 & 97 & 100 & 89 & 93 & - & - & - & - \\
\hline 5 & 4 & 3 & 3 & 97 & 100 & 89 & 93 & - & - & - & - \\
\hline 5 & 4 & 4 & 1 & 97 & 100 & 90 & 93 & 83 & 91 & 87 & 92 \\
\hline 5 & 4 & 4 & 2 & 97 & 100 & 90 & 93 & 81 & 91 & 86 & 92 \\
\hline 5 & 4 & 4 & 3 & 97 & 100 & 90 & 93 & 81 & 91 & 86 & 92 \\
\hline 5 & 4 & 1 & 1 & 97 & 100 & 90 & 93 & 83 & 89 & 87 & 91 \\
\hline 5 & 4 & 1 & 2 & 97 & 100 & 90 & 93 & 81 & 89 & 86 & 91 \\
\hline 5 & 4 & 1 & 3 & 97 & 100 & 90 & 93 & 81 & 89 & 86 & 91 \\
\hline 5 & 4 & 6 & 1 & 97 & 100 & 90 & 93 & 82 & 91 & 87 & 92 \\
\hline
\end{tabular}


Table D.2.1 the results of calibration procedure 1 and its validations.

\begin{tabular}{|c|c|c|c|c|c|c|c|c|c|c|c|}
\hline \multicolumn{4}{|c|}{ Configurations } & \multicolumn{2}{|c|}{ Calibration } & \multicolumn{2}{|c|}{$1^{\text {st }}$ Validation } & \multicolumn{2}{|c|}{$2^{\text {nd }}$ Validation } & \multicolumn{2}{|c|}{$3^{\text {rd }}$ Validation } \\
\hline$\varphi_{g}$ & $\Lambda_{l}$ & $\Gamma_{n}$ & $\eta_{m}$ & Vehicles & Pedestrians & Vehicles & Pedestrians & Vehicles & Pedestrians & Vehicles & Pedestrians \\
\hline 5 & 4 & 6 & 2 & 97 & 100 & 90 & 93 & 80 & 91 & 86 & 92 \\
\hline 5 & 4 & 6 & 3 & 97 & 100 & 90 & 93 & 80 & 91 & 86 & 92 \\
\hline
\end{tabular}

Table D.2.2 the results of calibration procedure 2 and its validations.

\begin{tabular}{|c|c|c|c|c|c|c|c|c|c|}
\hline \multicolumn{2}{|c|}{ Configurations } & \multicolumn{2}{|c|}{ Calibration } & \multicolumn{2}{|c|}{$\mathbf{1}^{\text {st }}$ Validation } & \multicolumn{2}{|c|}{$2^{\text {nd }}$ Validation } & \multicolumn{2}{|c|}{$3^{\text {rd }}$ Validation } \\
\hline$\varphi_{g}$ & $\Lambda_{l}$ & Vehicles & Pedestrians & Vehicles & Pedestrians & Vehicles & Pedestrians & Vehicles & Pedestrians \\
\hline 1 & 1 & 96 & 89 & 93 & 96 & 89 & 99 & 91 & 98 \\
\hline 1 & 2 & - & - & - & - & - & - & - & - \\
\hline 1 & 3 & 96 & 90 & 90 & 94 & - & - & - & - \\
\hline 1 & 4 & - & - & - & - & - & - & - & - \\
\hline 2 & 1 & 98 & 91 & - & - & - & - & - & - \\
\hline 2 & 2 & 98 & 96 & 96 & 97 & 86 & 99 & 91 & 98 \\
\hline 2 & 3 & - & - & - & - & - & - & - & - \\
\hline 2 & 4 & - & - & - & - & - & - & - & - \\
\hline 3 & 1 & 98 & 95 & 92 & 97 & 88 & 97 & 90 & 97 \\
\hline 3 & 2 & - & - & - & - & - & - & - & - \\
\hline 3 & 3 & - & - & - & - & - & - & - & - \\
\hline 3 & 4 & 98 & 96 & 91 & 99 & 88 & 98 & 90 & 98 \\
\hline 4 & 1 & 95 & 99 & 86 & 100 & - & - & - & - \\
\hline 4 & 2 & 96 & 97 & 88 & 93 & - & - & - & - \\
\hline 4 & 3 & 98 & 93 & 93 & 87 & - & - & - & - \\
\hline 4 & 4 & 98 & 99 & 90 & 100 & 89 & 100 & 90 & 100 \\
\hline 5 & 1 & 96 & 100 & 88 & 100 & 80 & 91 & 85 & 96 \\
\hline 5 & 2 & 97 & 98 & 86 & 93 & 73 & 98 & 81 & 96 \\
\hline 5 & 3 & 97 & 92 & 88 & 83 & 80 & 98 & 85 & 90 \\
\hline 5 & 4 & 97 & 98 & 90 & 91 & 79 & 98 & 86 & 95 \\
\hline
\end{tabular}


Table D.2.3 the results of calibration and validations based on the average speed

\begin{tabular}{|c|c|c|c|c|c|c|c|c|c|}
\hline \multicolumn{2}{|c|}{ Configurations } & \multicolumn{2}{|c|}{ Calibration } & \multicolumn{2}{|c|}{$1^{\text {st }}$ Validation } & \multicolumn{2}{|c|}{$2^{\text {nd }}$ Validation } & \multicolumn{2}{|c|}{$3^{\text {rd }}$ Validation } \\
\hline$\varphi_{g}$ & $\Lambda_{l}$ & Vehicles & Pedestrians & Vehicles & Pedestrians & Vehicles & Pedestrians & Vehicles & Pedestrians \\
\hline 1 & 1 & 38 & 64 & 34 & 79 & 21 & 85 & 28 & 82 \\
\hline 1 & 2 & 36 & 64 & 34 & 79 & 20 & 85 & 28 & 83 \\
\hline 1 & 3 & 21 & 82 & 18 & 88 & - & - & - & - \\
\hline 1 & 4 & 36 & 63 & 34 & 79 & 20 & 85 & 27 & 83 \\
\hline 2 & 1 & 40 & 63 & 42 & 65 & 29 & 80 & 36 & 75 \\
\hline 2 & 2 & 33 & 70 & 33 & 80 & 20 & 85 & 27 & 83 \\
\hline 2 & 3 & 38 & 62 & 41 & 65 & 29 & 80 & 35 & 74 \\
\hline 2 & 4 & 33 & 70 & 33 & 80 & 20 & 85 & 27 & 83 \\
\hline 3 & 1 & 40 & 61 & 44 & 69 & 30 & 75 & 38 & 73 \\
\hline 3 & 2 & 39 & 61 & 43 & 69 & 30 & 75 & 37 & 73 \\
\hline 3 & 3 & 39 & 61 & 42 & 69 & 30 & 75 & 37 & 73 \\
\hline 3 & 4 & 39 & 61 & 43 & 69 & 30 & 75 & 37 & 73 \\
\hline 4 & 1 & 59 & 36 & 54 & 39 & 46 & 41 & 51 & 40 \\
\hline 4 & 2 & 59 & 36 & 54 & 39 & 46 & 44 & 51 & 42 \\
\hline 4 & 3 & 59 & 36 & 54 & 39 & 46 & 48 & 51 & 44 \\
\hline 4 & 4 & 59 & 36 & 54 & 39 & 46 & 44 & 51 & 42 \\
\hline 5 & 1 & 62 & 46 & 56 & 50 & 44 & 44 & 51 & 47 \\
\hline 5 & 2 & 62 & 46 & 56 & 50 & 44 & 47 & 51 & 48 \\
\hline 5 & 3 & 62 & 48 & 59 & 50 & 44 & 53 & 53 & 52 \\
\hline 5 & 4 & 62 & 46 & 56 & 50 & 44 & 47 & 51 & 48 \\
\hline
\end{tabular}


Table D.2.4 the results of calibration and validations based on the maximum speed

\begin{tabular}{|c|c|c|c|c|c|c|c|c|c|}
\hline \multicolumn{2}{|c|}{ Configurations } & \multicolumn{2}{|c|}{ Calibration } & \multicolumn{2}{|c|}{$1^{\text {st }}$ Validation } & \multicolumn{2}{|c|}{$2^{\text {nd }}$ Validation } & \multicolumn{2}{|c|}{$3^{\text {rd }}$ Validation } \\
\hline$\varphi_{g}$ & $\Lambda_{l}$ & Vehicles & Pedestrians & Vehicles & Pedestrians & Vehicles & Pedestrians & Vehicles & Pedestrians \\
\hline 1 & 1 & 75 & 65 & 75 & 81 & 49 & 93 & 63 & 88 \\
\hline 1 & 2 & 78 & 66 & 77 & 78 & 51 & 95 & 65 & 88 \\
\hline 1 & 3 & 74 & 68 & 73 & 84 & 48 & 97 & 61 & 92 \\
\hline 1 & 4 & 71 & 64 & 73 & 82 & 45 & 93 & 60 & 88 \\
\hline 2 & 1 & 76 & 71 & 75 & 82 & 50 & 93 & 63 & 89 \\
\hline 2 & 2 & 77 & 74 & 75 & 84 & 48 & 97 & 63 & 92 \\
\hline 2 & 3 & 74 & 74 & 73 & 84 & 48 & 97 & 62 & 92 \\
\hline 2 & 4 & 73 & 72 & 73 & 83 & 46 & 93 & 61 & 89 \\
\hline 3 & 1 & 78 & 75 & 78 & 86 & 49 & 93 & 65 & 90 \\
\hline 3 & 2 & 79 & 76 & 80 & 82 & 49 & 96 & 66 & 90 \\
\hline 3 & 3 & 78 & 76 & 77 & 79 & 53 & 95 & 66 & 89 \\
\hline 3 & 4 & 76 & 73 & 78 & 81 & 49 & 92 & 65 & 88 \\
\hline 4 & 1 & 77 & 79 & 80 & 87 & 52 & 93 & 68 & 90 \\
\hline 4 & 2 & 78 & 79 & 79 & 87 & 49 & 97 & 67 & 93 \\
\hline 4 & 3 & 78 & 79 & 77 & 87 & 51 & 97 & 66 & 93 \\
\hline 4 & 4 & 77 & 79 & 78 & 87 & 49 & 93 & 66 & 90 \\
\hline 5 & 1 & 78 & 88 & 81 & 91 & 49 & 98 & 69 & 95 \\
\hline 5 & 2 & 78 & 88 & 78 & 91 & 45 & 98 & 66 & 95 \\
\hline 5 & 3 & 80 & 87 & 78 & 85 & 52 & 98 & 68 & 91 \\
\hline 5 & 4 & 78 & 88 & 78 & 91 & 45 & 98 & 66 & 95 \\
\hline
\end{tabular}


Table D.2.5 the results of calibration and validations based on the average acceleration

\begin{tabular}{|c|c|c|c|c|c|c|c|c|c|}
\hline \multicolumn{2}{|c|}{ Configurations } & \multicolumn{2}{|c|}{ Calibration } & \multicolumn{2}{|c|}{$1^{\text {st }}$ Validation } & \multicolumn{2}{|c|}{$2^{\text {nd }}$ Validation } & \multicolumn{2}{|c|}{$3^{\text {rd }}$ Validation } \\
\hline$\varphi_{g}$ & $\Lambda_{l}$ & Vehicles & Pedestrians & Vehicles & Pedestrians & Vehicles & Pedestrians & Vehicles & Pedestrians \\
\hline 1 & 1 & 51 & 38 & 60 & 32 & 59 & 19 & 60 & 24 \\
\hline 1 & 2 & 63 & 38 & 73 & 32 & 82 & 19 & 77 & 24 \\
\hline 1 & 3 & 64 & 38 & 74 & 30 & 83 & 17 & 78 & 22 \\
\hline 1 & 4 & 54 & 38 & 67 & 32 & 70 & 17 & 68 & 23 \\
\hline 2 & 1 & 50 & 42 & 58 & 36 & 59 & 20 & 59 & 26 \\
\hline 2 & 2 & 51 & 39 & 60 & 34 & 60 & 17 & 60 & 23 \\
\hline 2 & 3 & 59 & 39 & 72 & 33 & 80 & 17 & 76 & 23 \\
\hline 2 & 4 & 51 & 39 & 60 & 34 & 60 & 17 & 60 & 23 \\
\hline 3 & 1 & 53 & 41 & 59 & 39 & 60 & 18 & 60 & 26 \\
\hline 3 & 2 & 53 & 39 & 61 & 38 & 61 & 15 & 61 & 24 \\
\hline 3 & 3 & 57 & 37 & 69 & 36 & 74 & 14 & 72 & 23 \\
\hline 3 & 4 & 53 & 39 & 61 & 38 & 61 & 15 & 61 & 24 \\
\hline 4 & 1 & 55 & 39 & 62 & 36 & 65 & 11 & 63 & 22 \\
\hline 4 & 2 & 74 & 18 & 75 & 11 & 74 & 7 & 74 & 9 \\
\hline 4 & 3 & 74 & 18 & 75 & 11 & 74 & 7 & 74 & 9 \\
\hline 4 & 4 & 67 & 18 & - & - & - & - & - & - \\
\hline 5 & 1 & 56 & 40 & 64 & 28 & 65 & 13 & 64 & 21 \\
\hline 5 & 2 & 55 & 38 & 64 & 30 & 64 & 13 & 64 & 22 \\
\hline 5 & 3 & 60 & 38 & 69 & 28 & 82 & 13 & 74 & 21 \\
\hline 5 & 4 & 55 & 38 & 64 & 30 & 64 & 13 & 64 & 22 \\
\hline
\end{tabular}


Table D.2.6 the results of calibration and validations based on the maximum acceleration

\begin{tabular}{|c|c|c|c|c|c|c|c|c|c|}
\hline \multicolumn{2}{|c|}{ Configurations } & \multicolumn{2}{|c|}{ Calibration } & \multicolumn{2}{|c|}{$1^{\text {st }}$ Validation } & \multicolumn{2}{|c|}{$2^{\text {nd }}$ Validation } & \multicolumn{2}{|c|}{$3^{\text {rd }}$ Validation } \\
\hline$\varphi_{g}$ & $\Lambda_{l}$ & Vehicles & Pedestrians & Vehicles & Pedestrians & Vehicles & Pedestrians & Vehicles & Pedestrians \\
\hline 1 & 1 & 4 & 81 & - & - & - & - & - & - \\
\hline 1 & 2 & 4 & 81 & - & - & - & - & - & - \\
\hline 1 & 3 & 4 & 81 & - & - & - & - & - & - \\
\hline 1 & 4 & 4 & 81 & - & - & - & - & - & - \\
\hline 2 & 1 & 5 & 89 & 0 & 79 & - & - & - & - \\
\hline 2 & 2 & 4 & 89 & - & - & - & - & - & - \\
\hline 2 & 3 & 4 & 89 & - & - & - & - & - & - \\
\hline 2 & 4 & 4 & 89 & - & - & - & - & - & - \\
\hline 3 & 1 & 6 & 92 & 0 & 88 & - & - & - & - \\
\hline 3 & 2 & 5 & 92 & 0 & 85 & - & - & - & - \\
\hline 3 & 3 & 5 & 92 & 0 & 85 & - & - & - & - \\
\hline 3 & 4 & 5 & 92 & 0 & 85 & - & - & - & - \\
\hline 4 & 1 & 6 & 96 & 0 & 90 & - & - & - & - \\
\hline 4 & 2 & 6 & 96 & - & - & - & - & - & - \\
\hline 4 & 3 & 6 & 96 & - & - & - & - & - & - \\
\hline 4 & 4 & 6 & 96 & - & - & - & - & - & - \\
\hline 5 & 1 & 9 & 98 & 1 & 93 & - & - & - & - \\
\hline 5 & 2 & 8 & 98 & - & - & - & - & - & - \\
\hline 5 & 3 & 8 & 98 & - & - & - & - & - & - \\
\hline 5 & 4 & 8 & 98 & - & - & - & - & - & - \\
\hline
\end{tabular}


Table D.2.7 the results of calibration and validations based on the average deceleration

\begin{tabular}{|c|c|c|c|c|c|c|c|c|c|}
\hline \multicolumn{2}{|c|}{ Configurations } & \multicolumn{2}{|c|}{ Calibration } & \multicolumn{2}{|c|}{$1^{\text {st }}$ Validation } & \multicolumn{2}{|c|}{$2^{\text {nd }}$ Validation } & \multicolumn{2}{|c|}{$3^{\text {rd }}$ Validation } \\
\hline$\varphi_{g}$ & $\Lambda_{l}$ & Vehicles & Pedestrians & Vehicles & Pedestrians & Vehicles & Pedestrians & Vehicles & Pedestrians \\
\hline 1 & 1 & 59 & 49 & 61 & 44 & 80 & 35 & 70 & 39 \\
\hline 1 & 2 & 53 & 43 & 55 & 44 & 71 & 35 & 63 & 39 \\
\hline 1 & 3 & 59 & 38 & 62 & 36 & 83 & 23 & 72 & 28 \\
\hline 1 & 4 & 30 & 38 & 28 & 35 & 47 & 23 & 37 & 28 \\
\hline 2 & 1 & 28 & 75 & 26 & 80 & 43 & 67 & 34 & 72 \\
\hline 2 & 2 & 54 & 45 & 56 & 47 & 72 & 34 & 63 & 39 \\
\hline 2 & 3 & 59 & 39 & 61 & 38 & 83 & 22 & 72 & 28 \\
\hline 2 & 4 & 30 & 39 & 29 & 38 & 47 & 22 & 37 & 28 \\
\hline 3 & 1 & 26 & 75 & 24 & 78 & 46 & 71 & 34 & 73 \\
\hline 3 & 2 & 49 & 47 & 52 & 51 & 73 & 33 & 62 & 40 \\
\hline 3 & 3 & 61 & 33 & 69 & 28 & - & - & - & - \\
\hline 3 & 4 & 24 & 41 & 24 & 47 & 39 & 40 & 31 & 43 \\
\hline 4 & 1 & 38 & 52 & 38 & 66 & 58 & 43 & 46 & 53 \\
\hline 4 & 2 & 57 & 34 & 62 & 26 & - & - & - & - \\
\hline 4 & 3 & 61 & 30 & 65 & 23 & - & - & - & - \\
\hline 4 & 4 & 32 & 37 & 29 & 44 & 48 & 35 & 37 & 39 \\
\hline 5 & 1 & 47 & 65 & 51 & 72 & 74 & 53 & 60 & 63 \\
\hline 5 & 2 & 40 & 65 & 40 & 67 & 72 & 53 & 52 & 60 \\
\hline 5 & 3 & 68 & 37 & 74 & 26 & 92 & 13 & - & - \\
\hline 5 & 4 & 40 & 44 & 39 & 33 & 62 & 27 & 47 & 30 \\
\hline
\end{tabular}


Table D.2.8 the results of calibration and validations based on the maximum deceleration

\begin{tabular}{|c|c|c|c|c|c|c|c|c|c|}
\hline \multicolumn{2}{|c|}{ Configurations } & \multicolumn{2}{|c|}{ Calibration } & \multicolumn{2}{|c|}{$1^{\text {st }}$ Validation } & \multicolumn{2}{|c|}{$2^{\text {nd }}$ Validation } & \multicolumn{2}{|c|}{$3^{\text {rd }}$ Validation } \\
\hline$\varphi_{g}$ & $\Lambda_{l}$ & Vehicles & Pedestrians & Vehicles & Pedestrians & Vehicles & Pedestrians & Vehicles & Pedestrians \\
\hline 1 & 1 & 2 & 81 & 0 & 75 & 2 & 56 & 1 & 64 \\
\hline 1 & 2 & 3 & 79 & 1 & 73 & 2 & 54 & 1 & 62 \\
\hline 1 & 3 & 3 & 78 & 1 & 72 & 2 & 54 & 1 & 61 \\
\hline 1 & 4 & 1 & 81 & 0 & 75 & 0 & 57 & 0 & 64 \\
\hline 2 & 1 & 2 & 90 & 0 & 83 & 2 & 57 & 1 & 67 \\
\hline 2 & 2 & 4 & 88 & 1 & 82 & 2 & 55 & 1 & 65 \\
\hline 2 & 3 & 3 & 87 & 1 & 81 & 2 & 54 & 1 & 64 \\
\hline 2 & 4 & 1 & 90 & 0 & 83 & 0 & 57 & 0 & 67 \\
\hline 3 & 1 & 4 & 88 & 1 & 89 & 3 & 60 & 2 & 71 \\
\hline 3 & 2 & 4 & 88 & 1 & 89 & 2 & 60 & 2 & 71 \\
\hline 3 & 3 & 4 & 87 & 1 & 88 & 2 & 59 & 2 & 70 \\
\hline 3 & 4 & 1 & 91 & 0 & 90 & 0 & 63 & 0 & 73 \\
\hline 4 & 1 & 6 & 91 & 1 & 90 & 5 & 59 & 3 & 73 \\
\hline 4 & 2 & 6 & 91 & 1 & 90 & 3 & 59 & 2 & 73 \\
\hline 4 & 3 & 4 & 91 & 1 & 90 & 2 & 59 & 2 & 73 \\
\hline 4 & 4 & 0 & 91 & 0 & 90 & 1 & 59 & 0 & 73 \\
\hline 5 & 1 & 8 & 94 & 1 & 89 & 3 & 64 & 2 & 77 \\
\hline 5 & 2 & 8 & 94 & 1 & 89 & 2 & 64 & 2 & 77 \\
\hline 5 & 3 & 6 & 85 & 1 & 87 & 0 & 56 & 1 & 71 \\
\hline 5 & 4 & 0 & 94 & 0 & 89 & 0 & 64 & 0 & 77 \\
\hline
\end{tabular}


Table D.2.9 the results of calibration and validations based on the average direction

\begin{tabular}{|c|c|c|c|c|c|c|c|c|c|}
\hline \multicolumn{2}{|c|}{ Configurations } & \multicolumn{2}{|c|}{ Calibration } & \multicolumn{2}{|c|}{$1^{\text {st }}$ Validation } & \multicolumn{2}{|c|}{$2^{\text {nd }}$ Validation } & \multicolumn{2}{|c|}{$3^{\text {rd }}$ Validation } \\
\hline$\varphi_{g}$ & $\Lambda_{l}$ & Vehicles & Pedestrians & Vehicles & Pedestrians & Vehicles & Pedestrians & Vehicles & Pedestrians \\
\hline 1 & 1 & 77 & 6 & 69 & 9 & - & - & - & - \\
\hline 1 & 2 & 76 & 6 & 66 & 9 & - & - & - & - \\
\hline 1 & 3 & 66 & 7 & 61 & 9 & - & - & - & - \\
\hline 1 & 4 & 68 & 6 & 61 & 8 & - & - & - & - \\
\hline 2 & 1 & 77 & 4 & 68 & 9 & - & - & - & - \\
\hline 2 & 2 & 76 & 4 & 65 & 8 & - & - & - & - \\
\hline 2 & 3 & 69 & 6 & 61 & 8 & - & - & - & - \\
\hline 2 & 4 & 70 & 4 & 61 & 8 & - & - & - & - \\
\hline 3 & 1 & 78 & 5 & 67 & 10 & - & - & - & - \\
\hline 3 & 2 & 76 & 5 & 64 & 8 & - & - & - & - \\
\hline 3 & 3 & 71 & 5 & 60 & 8 & - & - & - & - \\
\hline 3 & 4 & 73 & 5 & 61 & 8 & 3 & 6 & - & - \\
\hline 4 & 1 & 75 & 0 & 63 & 0 & - & - & - & - \\
\hline 4 & 2 & 75 & 0 & 63 & 0 & - & - & - & - \\
\hline 4 & 3 & 72 & 4 & 60 & 8 & - & - & - & - \\
\hline 4 & 4 & 73 & 4 & 60 & 8 & - & - & - & - \\
\hline 5 & 1 & 74 & 0 & 62 & 0 & 3 & 0 & - & - \\
\hline 5 & 2 & 74 & 0 & 62 & 0 & 3 & 0 & - & - \\
\hline 5 & 3 & 70 & 0 & 52 & 0 & - & - & - & - \\
\hline 5 & 4 & 71 & 0 & 54 & 0 & - & - & - & - \\
\hline
\end{tabular}


Table D.2.10 the results of calibration and validations based on the maximum direction

\begin{tabular}{|c|c|c|c|c|c|c|c|c|c|}
\hline \multicolumn{2}{|c|}{ Configurations } & \multicolumn{2}{|c|}{ Calibration } & \multicolumn{2}{|c|}{$1^{\text {st }}$ Validation } & \multicolumn{2}{|c|}{$2^{\text {nd }}$ Validation } & \multicolumn{2}{|c|}{$3^{\text {rd }}$ Validation } \\
\hline$\varphi_{g}$ & $\Lambda_{l}$ & Vehicles & Pedestrians & Vehicles & Pedestrians & Vehicles & Pedestrians & Vehicles & Pedestrians \\
\hline 1 & 1 & 82 & 94 & 77 & 88 & 44 & 91 & 62 & 90 \\
\hline 1 & 2 & - & - & - & - & - & - & - & - \\
\hline 1 & 3 & - & - & - & - & - & - & - & - \\
\hline 1 & 4 & - & - & - & - & - & - & - & - \\
\hline 2 & 1 & 83 & 96 & 77 & 89 & 44 & 91 & 62 & 90 \\
\hline 2 & 2 & 75 & 99 & 66 & 100 & - & - & - & - \\
\hline 2 & 3 & - & - & - & - & - & - & - & - \\
\hline 2 & 4 & - & - & - & - & - & - & - & - \\
\hline 3 & 1 & 85 & 96 & 77 & 86 & 49 & 94 & 64 & 91 \\
\hline 3 & 2 & 77 & 99 & 65 & 100 & - & - & - & - \\
\hline 3 & 3 & - & - & - & - & - & - & - & - \\
\hline 3 & 4 & - & - & - & - & - & - & - & - \\
\hline 4 & 1 & 85 & 96 & 77 & 84 & - & - & - & - \\
\hline 4 & 2 & 76 & 99 & 64 & 100 & - & - & - & - \\
\hline 4 & 3 & - & - & - & - & - & - & - & - \\
\hline 4 & 4 & 75 & 97 & 62 & 89 & 57 & 91 & 69 & 88 \\
\hline 5 & 1 & 86 & 98 & 78 & 85 & 62 & 89 & 72 & 87 \\
\hline 5 & 2 & - & - & - & - & - & - & - & - \\
\hline 5 & 3 & - & - & - & - & - & - & - & - \\
\hline 5 & 4 & - & - & - & - & - & - & - & - \\
\hline
\end{tabular}


Table D.2.11 the results of calibration and validations based on the average of change in direction

\begin{tabular}{|c|c|c|c|c|c|c|c|c|c|}
\hline \multicolumn{2}{|c|}{ Configurations } & \multicolumn{2}{|c|}{ Calibration } & \multicolumn{2}{|c|}{$1^{\text {st }}$ Validation } & \multicolumn{2}{|c|}{$2^{\text {nd }}$ Validation } & \multicolumn{2}{|c|}{$3^{\text {rd }}$ Validation } \\
\hline$\varphi_{g}$ & $\Lambda_{l}$ & Vehicles & Pedestrians & Vehicles & Pedestrians & Vehicles & Pedestrians & Vehicles & Pedestrians \\
\hline 1 & 1 & 38 & 2 & 29 & 1 & - & - & - & - \\
\hline 1 & 2 & 38 & 2 & 29 & 1 & - & - & - & - \\
\hline 1 & 3 & 38 & 2 & 29 & 1 & - & - & - & - \\
\hline 1 & 4 & 74 & 1 & 58 & 1 & - & - & - & - \\
\hline 2 & 1 & 42 & 0 & 30 & 0 & - & - & - & - \\
\hline 2 & 2 & 42 & 0 & 30 & 0 & - & - & - & - \\
\hline 2 & 3 & 42 & 0 & 30 & 0 & - & - & - & - \\
\hline 2 & 4 & 42 & 0 & 30 & 0 & - & - & - & - \\
\hline 3 & 1 & 71 & 0 & 42 & 0 & - & - & - & - \\
\hline 3 & 2 & 71 & 0 & 42 & 0 & - & - & - & - \\
\hline 3 & 3 & 71 & 0 & 42 & 0 & - & - & - & - \\
\hline 3 & 4 & 71 & 0 & 42 & 0 & - & - & - & - \\
\hline 4 & 1 & 53 & 0 & 32 & 0 & - & - & - & - \\
\hline 4 & 2 & 53 & 0 & 32 & 0 & - & - & - & - \\
\hline 4 & 3 & 53 & 0 & 32 & 0 & - & - & - & - \\
\hline 4 & 4 & 53 & 0 & 32 & 0 & - & - & - & - \\
\hline 5 & 1 & 63 & 0 & 38 & 0 & - & - & - & - \\
\hline 5 & 2 & 63 & 0 & 38 & 0 & - & - & - & - \\
\hline 5 & 3 & 63 & 0 & 38 & 0 & - & - & - & - \\
\hline 5 & 4 & 63 & 0 & 38 & 0 & - & - & - & - \\
\hline
\end{tabular}


Table D.2.12 the results of calibration and validations based on the average of cosine of change in direction

\begin{tabular}{|c|c|c|c|c|c|c|c|c|c|}
\hline \multicolumn{2}{|c|}{ Configurations } & \multicolumn{2}{|c|}{ Calibration } & \multicolumn{2}{|c|}{$1^{\text {st }}$ Validation } & \multicolumn{2}{|c|}{$2^{\text {nd }}$ Validation } & \multicolumn{2}{|c|}{$3^{\text {rd }}$ Validation } \\
\hline$\varphi_{g}$ & $\Lambda_{l}$ & Vehicles & Pedestrians & Vehicles & Pedestrians & Vehicles & Pedestrians & Vehicles & Pedestrians \\
\hline 1 & 1 & 65 & 1 & 64 & 0 & 64 & 0 & 64 & 0 \\
\hline 1 & 2 & 62 & 2 & 63 & 2 & 63 & 0 & 63 & 1 \\
\hline 1 & 3 & 51 & 13 & 54 & 13 & 51 & 7 & 53 & 10 \\
\hline 1 & 4 & 55 & 2 & 56 & 2 & 56 & 0 & 56 & 1 \\
\hline 2 & 1 & 68 & 1 & 64 & 2 & 63 & 0 & 64 & 1 \\
\hline 2 & 2 & 66 & 1 & 63 & 2 & 63 & 0 & 63 & 1 \\
\hline 2 & 3 & 55 & 11 & 54 & 13 & 52 & 7 & 53 & 9 \\
\hline 2 & 4 & 58 & 1 & 56 & 2 & 56 & 0 & 56 & 1 \\
\hline 3 & 1 & 74 & 1 & 69 & 1 & 67 & 0 & 68 & 1 \\
\hline 3 & 2 & 66 & 11 & 61 & 7 & 58 & 4 & 59 & 5 \\
\hline 3 & 3 & 63 & 13 & 59 & 14 & 55 & 6 & 58 & 9 \\
\hline 3 & 4 & 66 & 1 & 61 & 1 & 58 & 0 & 59 & 1 \\
\hline 4 & 1 & 82 & 4 & 71 & 0 & 71 & 0 & 71 & 0 \\
\hline 4 & 2 & 81 & 4 & 71 & 0 & 71 & 0 & 71 & 0 \\
\hline 4 & 3 & 75 & 10 & 65 & 11 & 58 & 7 & 62 & 9 \\
\hline 4 & 4 & 75 & 4 & 65 & 0 & 58 & 0 & 62 & 0 \\
\hline 5 & 1 & 87 & 0 & 77 & 0 & 75 & 0 & 76 & 0 \\
\hline 5 & 2 & 78 & 10 & 67 & 13 & - & - & - & - \\
\hline 5 & 3 & 78 & 10 & 65 & 13 & - & - & - & - \\
\hline 5 & 4 & 81 & 0 & 69 & 0 & 65 & 0 & 67 & 0 \\
\hline
\end{tabular}


Table D.2.13 the results of calibration and validations based on the average area

\begin{tabular}{|c|c|c|c|c|c|c|c|c|c|}
\hline \multicolumn{2}{|c|}{ Configurations } & \multicolumn{2}{|c|}{ Calibration } & \multicolumn{2}{|c|}{$1^{\text {st }}$ Validation } & \multicolumn{2}{|c|}{$2^{\text {nd }}$ Validation } & \multicolumn{2}{|c|}{$3^{\text {rd }}$ Validation } \\
\hline$\varphi_{g}$ & $\Lambda_{l}$ & Vehicles & Pedestrians & Vehicles & Pedestrians & Vehicles & Pedestrians & Vehicles & Pedestrians \\
\hline 1 & 1 & 93 & 96 & 86 & 99 & 83 & 99 & 85 & 99 \\
\hline 1 & 2 & 91 & 96 & 84 & 99 & 83 & 99 & 83 & 99 \\
\hline 1 & 3 & 89 & 97 & 81 & 100 & 80 & 99 & 80 & 100 \\
\hline 1 & 4 & 91 & 92 & 83 & 96 & 81 & 99 & 82 & 98 \\
\hline 2 & 1 & 94 & 98 & 87 & 99 & 84 & 99 & 85 & 99 \\
\hline 2 & 2 & 92 & 98 & 84 & 99 & 82 & 96 & 83 & 97 \\
\hline 2 & 3 & 91 & 98 & 81 & 100 & - & - & - & - \\
\hline 2 & 4 & 92 & 98 & 84 & 99 & 81 & 96 & 83 & 97 \\
\hline 3 & 1 & 96 & 97 & 85 & 99 & 85 & 100 & 85 & 99 \\
\hline 3 & 2 & 94 & 97 & 83 & 99 & 82 & 98 & 82 & 98 \\
\hline 3 & 3 & 92 & 97 & 79 & 100 & - & - & - & - \\
\hline 3 & 4 & 94 & 97 & 83 & 99 & 82 & 98 & 82 & 98 \\
\hline 4 & 1 & 97 & 97 & 86 & 100 & - & - & - & - \\
\hline 4 & 2 & 95 & 99 & 78 & 100 & - & - & - & - \\
\hline 4 & 3 & 94 & 99 & - & - & - & - & - & - \\
\hline 4 & 4 & 95 & 99 & 78 & 100 & 86 & 100 & 86 & 100 \\
\hline 5 & 1 & 96 & 100 & 81 & 100 & 79 & 76 & 81 & 88 \\
\hline 5 & 2 & 95 & 100 & 75 & 100 & 72 & 98 & 74 & 99 \\
\hline 5 & 3 & 94 & 100 & 75 & 100 & 69 & 100 & 73 & 100 \\
\hline 5 & 4 & 95 & 100 & 75 & 100 & 72 & 98 & 74 & 99 \\
\hline
\end{tabular}


Table D.2.14 the results of calibration and validations based on the maximum area

\begin{tabular}{|c|c|c|c|c|c|c|c|c|c|}
\hline \multicolumn{2}{|c|}{ Configurations } & \multicolumn{2}{|c|}{ Calibration } & \multicolumn{2}{|c|}{$1^{\text {st }}$ Validation } & \multicolumn{2}{|c|}{$2^{\text {nd }}$ Validation } & \multicolumn{2}{|c|}{$3^{\text {rd }}$ Validation } \\
\hline$\varphi_{g}$ & $\Lambda_{l}$ & Vehicles & Pedestrians & Vehicles & Pedestrians & Vehicles & Pedestrians & Vehicles & Pedestrians \\
\hline 1 & 1 & 96 & 92 & 95 & 94 & 95 & 96 & 95 & 95 \\
\hline 1 & 2 & 94 & 95 & 93 & 97 & - & - & - & - \\
\hline 1 & 3 & 94 & 95 & 93 & 97 & - & - & - & - \\
\hline 1 & 4 & 94 & 91 & 93 & 94 & - & - & - & - \\
\hline 2 & 1 & 97 & 94 & 94 & 97 & 90 & 90 & 93 & 93 \\
\hline 2 & 2 & 97 & 94 & 94 & 97 & 90 & 90 & 93 & 93 \\
\hline 2 & 3 & 97 & 94 & 94 & 97 & 90 & 90 & 92 & 93 \\
\hline 2 & 4 & 97 & 94 & 94 & 97 & 90 & 90 & 92 & 93 \\
\hline 3 & 1 & 98 & 95 & 91 & 97 & 87 & 82 & 89 & 88 \\
\hline 3 & 2 & 98 & 95 & 91 & 97 & 87 & 82 & 89 & 88 \\
\hline 3 & 3 & 98 & 95 & 91 & 97 & 87 & 82 & 89 & 88 \\
\hline 3 & 4 & 98 & 95 & 91 & 97 & 87 & 82 & 89 & 88 \\
\hline 4 & 1 & 98 & 96 & 92 & 98 & 88 & 87 & 90 & 92 \\
\hline 4 & 2 & 98 & 96 & 92 & 98 & 89 & 87 & 91 & 92 \\
\hline 4 & 3 & 98 & 96 & 92 & 98 & 89 & 87 & 91 & 92 \\
\hline 4 & 4 & 98 & 96 & 92 & 98 & 89 & 87 & 91 & 92 \\
\hline 5 & 1 & 98 & 96 & 93 & 100 & 86 & 82 & 91 & 91 \\
\hline 5 & 2 & 98 & 96 & 93 & 100 & 87 & 82 & 91 & 91 \\
\hline 5 & 3 & 98 & 96 & 93 & 100 & 86 & 82 & 91 & 91 \\
\hline 5 & 4 & 98 & 96 & 93 & 100 & 87 & 82 & 91 & 91 \\
\hline
\end{tabular}


Table D.2.15 the results of calibration and validations based on the average length

\begin{tabular}{|c|c|c|c|c|c|c|c|c|c|}
\hline \multicolumn{2}{|c|}{ Configurations } & \multicolumn{2}{|c|}{ Calibration } & \multicolumn{2}{|c|}{$1^{\text {st }}$ Validation } & \multicolumn{2}{|c|}{$2^{\text {nd }}$ Validation } & \multicolumn{2}{|c|}{$3^{\text {rd }}$ Validation } \\
\hline$\varphi_{g}$ & $\Lambda_{l}$ & Vehicles & Pedestrians & Vehicles & Pedestrians & Vehicles & Pedestrians & Vehicles & Pedestrians \\
\hline 1 & 1 & 96 & 88 & 84 & 88 & 91 & 99 & 87 & 95 \\
\hline 1 & 2 & 95 & 88 & 84 & 88 & 91 & 99 & 87 & 95 \\
\hline 1 & 3 & 94 & 89 & 83 & 89 & 90 & 99 & 86 & 95 \\
\hline 1 & 4 & 95 & 85 & 84 & 85 & 89 & 99 & 86 & 94 \\
\hline 2 & 1 & 96 & 90 & 83 & 88 & 90 & 97 & 86 & 94 \\
\hline 2 & 2 & 96 & 88 & 84 & 85 & 90 & 96 & 87 & 92 \\
\hline 2 & 3 & 95 & 90 & 83 & 87 & 90 & 96 & 86 & 92 \\
\hline 2 & 4 & 96 & 88 & 84 & 85 & 90 & 96 & 87 & 92 \\
\hline 3 & 1 & 97 & 91 & 82 & 85 & 90 & 100 & 86 & 94 \\
\hline 3 & 2 & 96 & 91 & 81 & 86 & 88 & 96 & 84 & 92 \\
\hline 3 & 3 & 96 & 91 & 82 & 85 & 90 & 98 & 85 & 93 \\
\hline 3 & 4 & 96 & 91 & 81 & 86 & 88 & 96 & 84 & 92 \\
\hline 4 & 1 & 97 & 91 & 84 & 84 & 91 & 100 & 87 & 93 \\
\hline 4 & 2 & 96 & 91 & 83 & 85 & 90 & 99 & 86 & 93 \\
\hline 4 & 3 & 97 & 91 & 83 & 84 & 91 & 100 & 87 & 93 \\
\hline 4 & 4 & 96 & 91 & 83 & 85 & 90 & 99 & 86 & 93 \\
\hline 5 & 1 & 99 & 96 & 88 & 89 & 92 & 100 & 89 & 95 \\
\hline 5 & 2 & 98 & 96 & 87 & 91 & 89 & 98 & 88 & 95 \\
\hline 5 & 3 & 98 & 96 & 87 & 91 & 89 & 98 & 88 & 95 \\
\hline 5 & 4 & 98 & 96 & 87 & 91 & 89 & 98 & 88 & 95 \\
\hline
\end{tabular}


Table D.2.16 the results of calibration and validations based on the maximum length

\begin{tabular}{|c|c|c|c|c|c|c|c|c|c|}
\hline \multicolumn{2}{|c|}{ Configurations } & \multicolumn{2}{|c|}{ Calibration } & \multicolumn{2}{|c|}{$1^{\text {st }}$ Validation } & \multicolumn{2}{|c|}{$2^{\text {nd }}$ Validation } & \multicolumn{2}{|c|}{$3^{\text {rd }}$ Validation } \\
\hline$\varphi_{g}$ & $\Lambda_{l}$ & Vehicles & Pedestrians & Vehicles & Pedestrians & Vehicles & Pedestrians & Vehicles & Pedestrians \\
\hline 1 & 1 & 96 & 85 & 94 & 73 & 98 & 99 & 96 & 88 \\
\hline 1 & 2 & 90 & 91 & 86 & 79 & 95 & 99 & - & - \\
\hline 1 & 3 & - & - & - & - & - & - & - & - \\
\hline 1 & 4 & 90 & 88 & 86 & 76 & - & - & - & - \\
\hline 2 & 1 & 95 & 91 & 87 & 76 & 95 & 99 & 90 & 91 \\
\hline 2 & 2 & 92 & 91 & - & - & - & - & - & - \\
\hline 2 & 3 & - & - & - & - & - & - & - & - \\
\hline 2 & 4 & 92 & 91 & 89 & 76 & 94 & 98 & 92 & 90 \\
\hline 3 & 1 & 97 & 88 & 92 & 68 & 97 & 99 & 94 & 87 \\
\hline 3 & 2 & 93 & 91 & 88 & 71 & 96 & 100 & 91 & 89 \\
\hline 3 & 3 & - & - & - & - & - & - & - & - \\
\hline 3 & 4 & 93 & 91 & 88 & 71 & 96 & 100 & 91 & 89 \\
\hline 4 & 1 & 97 & 91 & 92 & 67 & 97 & 100 & 94 & 85 \\
\hline 4 & 2 & 93 & 93 & 84 & 75 & 95 & 100 & 88 & 89 \\
\hline 4 & 3 & - & - & - & - & - & - & - & - \\
\hline 4 & 4 & 93 & 93 & 84 & 75 & 95 & 100 & 88 & 89 \\
\hline 5 & 1 & 98 & 92 & - & - & - & - & - & - \\
\hline 5 & 2 & 95 & 94 & - & - & - & - & - & - \\
\hline 5 & 3 & 93 & 94 & 87 & 76 & 94 & 99 & 90 & 91 \\
\hline 5 & 4 & 95 & 94 & 96 & 74 & 97 & 100 & 96 & 87 \\
\hline
\end{tabular}


Table D.2.17 the results of calibration and validations based on the average width

\begin{tabular}{|c|c|c|c|c|c|c|c|c|c|}
\hline \multicolumn{2}{|c|}{ Configurations } & \multicolumn{2}{|c|}{ Calibration } & \multicolumn{2}{|c|}{$1^{\text {st }}$ Validation } & \multicolumn{2}{|c|}{$2^{\text {nd }}$ Validation } & \multicolumn{2}{|c|}{$3^{\text {rd }}$ Validation } \\
\hline$\varphi_{g}$ & $\Lambda_{l}$ & Vehicles & Pedestrians & Vehicles & Pedestrians & Vehicles & Pedestrians & Vehicles & Pedestrians \\
\hline 1 & 1 & 92 & 90 & 87 & 97 & 81 & 96 & 84 & 96 \\
\hline 1 & 2 & 91 & 91 & 87 & 99 & 82 & 98 & 85 & 98 \\
\hline 1 & 3 & 90 & 92 & 84 & 99 & 80 & 98 & 82 & 98 \\
\hline 1 & 4 & 91 & 88 & 87 & 96 & 80 & 98 & 84 & 97 \\
\hline 2 & 1 & 94 & 93 & 88 & 99 & 82 & 96 & 85 & 97 \\
\hline 2 & 2 & 93 & 94 & 86 & 99 & 78 & 97 & 82 & 98 \\
\hline 2 & 3 & 91 & 94 & 84 & 99 & 77 & 95 & 81 & 97 \\
\hline 2 & 4 & 93 & 94 & 86 & 99 & 77 & 97 & 82 & 98 \\
\hline 3 & 1 & 96 & 93 & 86 & 99 & 82 & 98 & 84 & 98 \\
\hline 3 & 2 & 95 & 93 & 84 & 99 & 77 & 99 & 81 & 99 \\
\hline 3 & 3 & 94 & 93 & 83 & 99 & 76 & 97 & 80 & 98 \\
\hline 3 & 4 & 95 & 93 & 84 & 99 & 77 & 99 & 81 & 99 \\
\hline 4 & 1 & 97 & 93 & 88 & 98 & 84 & 97 & 86 & 98 \\
\hline 4 & 2 & 96 & 93 & 87 & 98 & 83 & 99 & 86 & 99 \\
\hline 4 & 3 & 96 & 93 & 85 & 98 & 83 & 97 & 84 & 98 \\
\hline 4 & 4 & 96 & 93 & 87 & 98 & 83 & 99 & 86 & 99 \\
\hline 5 & 1 & 96 & 94 & 88 & 98 & 85 & 96 & 87 & 97 \\
\hline 5 & 2 & 96 & 94 & 88 & 98 & 84 & 98 & 86 & 98 \\
\hline 5 & 3 & 95 & 94 & 83 & 91 & 84 & 78 & - & - \\
\hline 5 & 4 & 96 & 94 & 88 & 98 & 84 & 98 & 86 & 98 \\
\hline
\end{tabular}


Table D.2.18 the results of calibration and validations based on the maximum width

\begin{tabular}{|c|c|c|c|c|c|c|c|c|c|}
\hline \multicolumn{2}{|c|}{ Configurations } & \multicolumn{2}{|c|}{ Calibration } & \multicolumn{2}{|c|}{$1^{\text {st }}$ Validation } & \multicolumn{2}{|c|}{$2^{\text {nd }}$ Validation } & \multicolumn{2}{|c|}{$3^{\text {rd }}$ Validation } \\
\hline$\varphi_{g}$ & $\Lambda_{l}$ & Vehicles & Pedestrians & Vehicles & Pedestrians & Vehicles & Pedestrians & Vehicles & Pedestrians \\
\hline 1 & 1 & 89 & 92 & 91 & 97 & 81 & 92 & 87 & 94 \\
\hline 1 & 2 & 90 & 90 & 93 & 99 & 86 & 93 & 90 & 95 \\
\hline 1 & 3 & 88 & 95 & 90 & 100 & 78 & 96 & 85 & 98 \\
\hline 1 & 4 & 90 & 87 & 93 & 96 & 84 & 93 & 89 & 94 \\
\hline 2 & 1 & 93 & 96 & 92 & 99 & - & - & - & - \\
\hline 2 & 2 & 95 & 90 & 94 & 99 & 86 & 92 & 90 & 94 \\
\hline 2 & 3 & 93 & 96 & 92 & 99 & 79 & 92 & - & - \\
\hline 2 & 4 & 95 & 90 & 94 & 99 & 85 & 92 & 90 & 94 \\
\hline 3 & 1 & 96 & 95 & 92 & 100 & 78 & 94 & 85 & 96 \\
\hline 3 & 2 & 96 & 95 & 91 & 97 & 78 & 90 & 85 & 93 \\
\hline 3 & 3 & 96 & 95 & 91 & 99 & 78 & 92 & 85 & 95 \\
\hline 3 & 4 & 96 & 95 & 91 & 97 & 78 & 90 & 85 & 93 \\
\hline 4 & 1 & 96 & 96 & 93 & 100 & 81 & 93 & 88 & 96 \\
\hline 4 & 2 & 95 & 99 & 90 & 100 & 79 & 93 & 86 & 96 \\
\hline 4 & 3 & 96 & 96 & 92 & 97 & 81 & 91 & 88 & 93 \\
\hline 4 & 4 & 95 & 99 & 90 & 100 & 79 & 93 & 86 & 96 \\
\hline 5 & 1 & 96 & 100 & 94 & 98 & 82 & 89 & 89 & 93 \\
\hline 5 & 2 & 96 & 100 & 93 & 100 & 82 & 91 & 89 & 96 \\
\hline 5 & 3 & 96 & 100 & 94 & 98 & 82 & 89 & 89 & 93 \\
\hline 5 & 4 & 96 & 100 & 93 & 100 & 82 & 91 & 89 & 96 \\
\hline
\end{tabular}


Table D.2.19 the results of calibration and validations based on the peaks in speed

\begin{tabular}{|c|c|c|c|c|c|c|c|c|c|}
\hline \multicolumn{2}{|c|}{ Configurations } & \multicolumn{2}{|c|}{ Calibration } & \multicolumn{2}{|c|}{$1^{\text {st }}$ Validation } & \multicolumn{2}{|c|}{$2^{\text {nd }}$ Validation } & \multicolumn{2}{|c|}{$3^{\text {rd }}$ Validation } \\
\hline$\varphi_{g}$ & $\Lambda_{l}$ & Vehicles & Pedestrians & Vehicles & Pedestrians & Vehicles & Pedestrians & Vehicles & Pedestrians \\
\hline 1 & 1 & 93 & 26 & 96 & 29 & 97 & 24 & 96 & 26 \\
\hline 1 & 2 & 93 & 22 & 96 & 28 & 97 & 23 & 96 & 25 \\
\hline 1 & 3 & 93 & 18 & 96 & 22 & 98 & 17 & 97 & 19 \\
\hline 1 & 4 & 90 & 20 & 93 & 23 & 94 & 21 & 93 & 22 \\
\hline 2 & 1 & 93 & 31 & 93 & 36 & 95 & 37 & 94 & 37 \\
\hline 2 & 2 & 94 & 21 & 94 & 26 & 95 & 27 & 94 & 26 \\
\hline 2 & 3 & 94 & 17 & 94 & 18 & 95 & 20 & - & - \\
\hline 2 & 4 & 94 & 19 & 94 & 25 & - & - & - & - \\
\hline 3 & 1 & 91 & 37 & 92 & 31 & 93 & 35 & 93 & 33 \\
\hline 3 & 2 & 90 & 28 & 92 & 22 & 93 & 30 & 93 & 27 \\
\hline 3 & 3 & 90 & 27 & 92 & 19 & 93 & 26 & 93 & 23 \\
\hline 3 & 4 & 90 & 20 & 92 & 15 & 93 & 27 & 93 & 22 \\
\hline 4 & 1 & 95 & 34 & 94 & 30 & 95 & 33 & 95 & 32 \\
\hline 4 & 2 & 94 & 25 & 94 & 23 & 95 & 27 & 95 & 25 \\
\hline 4 & 3 & 94 & 24 & 94 & 21 & 95 & 21 & 95 & 21 \\
\hline 4 & 4 & 94 & 18 & 94 & 13 & 95 & 25 & 95 & 20 \\
\hline 5 & 1 & 91 & 37 & 95 & 30 & 95 & 36 & 95 & 33 \\
\hline 5 & 2 & 91 & 29 & 95 & 26 & 95 & 27 & 95 & 26 \\
\hline 5 & 3 & 91 & 25 & 95 & 20 & 95 & 11 & 95 & 15 \\
\hline 5 & 4 & 96 & 17 & 96 & 11 & 96 & 27 & 96 & 19 \\
\hline
\end{tabular}


Table D.2.20 the results of calibration and validations based on the effective weighted average frequency

\begin{tabular}{|c|c|c|c|c|c|c|c|c|c|c|}
\hline \multicolumn{3}{|c|}{ Configurations } & \multicolumn{2}{|c|}{ Calibration } & \multicolumn{2}{|c|}{$1^{\text {st }}$ Validation } & \multicolumn{2}{|c|}{$2^{\text {nd }}$ Validation } & \multicolumn{2}{|c|}{$3^{\text {rd }}$ Validation } \\
\hline$\varphi_{g}$ & $\Lambda_{l}$ & $\Gamma_{n}$, & Vehicles & Pedestrians & Vehicles & Pedestrians & Vehicles & Pedestrians & Vehicles & Pedestrians \\
\hline 1 & 1 & 1 & 46 & 14 & 53 & 13 & 36 & 12 & 45 & 12 \\
\hline 1 & 1 & 2 & 46 & 13 & 54 & 12 & 38 & 12 & 46 & 12 \\
\hline 1 & 1 & 3 & 46 & 13 & 53 & 12 & 39 & 11 & 46 & 11 \\
\hline 1 & 1 & 4 & 43 & 15 & 51 & 15 & 34 & 9 & 43 & 12 \\
\hline 1 & 1 & 1 & 46 & 13 & 54 & 13 & 41 & 15 & 48 & 14 \\
\hline 1 & 1 & 6 & 47 & 13 & 54 & 13 & 41 & 15 & 48 & 14 \\
\hline 1 & 2 & 1 & 37 & 14 & 42 & 13 & - & - & - & - \\
\hline 1 & 2 & 2 & 37 & 14 & 42 & 13 & - & - & - & - \\
\hline 1 & 2 & 3 & 37 & 14 & 42 & 12 & - & - & - & - \\
\hline 1 & 2 & 4 & 36 & 17 & 43 & 11 & - & - & - & - \\
\hline 1 & 2 & 1 & 36 & 15 & 43 & 14 & - & - & - & - \\
\hline 1 & 2 & 6 & 36 & 17 & 43 & 13 & - & - & - & - \\
\hline 1 & 3 & 1 & 31 & 14 & - & - & - & - & - & - \\
\hline 1 & 3 & 2 & 30 & 17 & - & - & - & - & - & - \\
\hline 1 & 3 & 3 & 29 & 17 & - & - & - & - & - & - \\
\hline 1 & 3 & 4 & 30 & 16 & - & - & - & - & - & - \\
\hline 1 & 3 & 1 & 33 & 13 & - & - & - & - & - & - \\
\hline 1 & 3 & 6 & 31 & 17 & - & - & - & - & - & - \\
\hline 1 & 4 & 1 & 37 & 12 & 41 & 10 & 29 & 5 & - & - \\
\hline 1 & 4 & 2 & 37 & 13 & 41 & 12 & - & - & - & - \\
\hline 1 & 4 & 3 & 36 & 13 & 41 & 11 & - & - & - & - \\
\hline 1 & 4 & 4 & 34 & 16 & 42 & 10 & - & - & - & - \\
\hline 1 & 4 & 1 & 36 & 15 & 43 & 11 & - & - & - & - \\
\hline 1 & 4 & 6 & 36 & 16 & 43 & 11 & - & - & - & - \\
\hline 2 & 1 & 1 & 51 & 17 & 55 & 15 & 37 & 13 & 46 & 13 \\
\hline 2 & 1 & 2 & 51 & 15 & 55 & 13 & 40 & 13 & 48 & 13 \\
\hline 2 & 1 & 3 & 51 & 15 & 55 & 13 & 40 & 11 & 48 & 12 \\
\hline 2 & 1 & 4 & 48 & 18 & 52 & 17 & 35 & 10 & 44 & 13 \\
\hline 2 & 1 & 1 & 49 & 18 & 54 & 16 & 37 & 12 & 46 & 13 \\
\hline 2 & 1 & 6 & 52 & 15 & 56 & 15 & 42 & 15 & 49 & 15 \\
\hline 2 & 2 & 1 & 42 & 17 & 43 & 15 & - & - & - & - \\
\hline 2 & 2 & 2 & 41 & 17 & 43 & 15 & - & - & - & - \\
\hline 2 & 2 & 3 & 41 & 17 & 43 & 13 & - & - & - & - \\
\hline 2 & 2 & 4 & 40 & 20 & 44 & 12 & - & - & - & - \\
\hline 2 & 2 & 1 & 40 & 20 & 44 & 13 & - & - & - & - \\
\hline 2 & 2 & 6 & 40 & 20 & 45 & 15 & - & - & - & - \\
\hline
\end{tabular}


Table D.2.20 the results of calibration and validations based on the effective weighted average frequency

\begin{tabular}{|c|c|c|c|c|c|c|c|c|c|c|}
\hline \multicolumn{3}{|c|}{ Configurations } & \multicolumn{2}{|c|}{ Calibration } & \multicolumn{2}{|c|}{$1^{\text {st }}$ Validation } & \multicolumn{2}{|c|}{$2^{\text {nd }}$ Validation } & \multicolumn{2}{|c|}{$3^{\text {rd }}$ Validation } \\
\hline$\varphi_{g}$ & $\Lambda_{l}$ & $\Gamma_{n}$, & Vehicles & Pedestrians & Vehicles & Pedestrians & Vehicles & Pedestrians & Vehicles & Pedestrians \\
\hline 2 & 3 & 1 & 35 & 17 & - & - & - & - & - & - \\
\hline 2 & 3 & 2 & 33 & 20 & - & - & - & - & - & - \\
\hline 2 & 3 & 3 & 33 & 20 & - & - & - & - & - & - \\
\hline 2 & 3 & 4 & 34 & 19 & - & - & - & - & - & - \\
\hline 2 & 3 & 1 & 34 & 19 & - & - & - & - & - & - \\
\hline 2 & 3 & 6 & 35 & 20 & - & - & - & - & - & - \\
\hline 2 & 4 & 1 & 41 & 13 & 42 & 11 & 30 & 6 & - & - \\
\hline 2 & 4 & 2 & 41 & 15 & 43 & 13 & - & - & - & - \\
\hline 2 & 4 & 3 & 40 & 15 & 43 & 12 & - & - & - & - \\
\hline 2 & 4 & 4 & 39 & 18 & 44 & 11 & - & - & - & - \\
\hline 2 & 4 & 1 & 40 & 18 & 44 & 12 & - & - & - & - \\
\hline 2 & 4 & 6 & 40 & 19 & 44 & 12 & - & - & - & - \\
\hline 3 & 1 & 1 & 61 & 20 & 63 & 18 & 42 & 13 & 53 & 15 \\
\hline 3 & 1 & 2 & 61 & 17 & 63 & 17 & 46 & 13 & 55 & 15 \\
\hline 3 & 1 & 3 & 61 & 17 & 63 & 17 & 46 & 13 & 55 & 14 \\
\hline 3 & 1 & 4 & 63 & 13 & 63 & 17 & 46 & 13 & 55 & 15 \\
\hline 3 & 1 & 1 & 58 & 21 & 63 & 19 & 42 & 13 & 53 & 15 \\
\hline 3 & 1 & 6 & 61 & 17 & 64 & 18 & 47 & 17 & 56 & 17 \\
\hline 3 & 2 & 1 & 49 & 20 & 50 & 18 & - & - & - & - \\
\hline 3 & 2 & 2 & 49 & 20 & 50 & 18 & - & - & - & - \\
\hline 3 & 2 & 3 & 49 & 20 & 49 & 17 & - & - & - & - \\
\hline 3 & 2 & 4 & 47 & 24 & 51 & 15 & - & - & - & - \\
\hline 3 & 2 & 1 & 47 & 24 & 51 & 17 & - & - & - & - \\
\hline 3 & 2 & 6 & 48 & 24 & 51 & 18 & - & - & - & - \\
\hline 3 & 3 & 1 & - & - & - & - & - & - & - & - \\
\hline 3 & 3 & 2 & - & - & - & - & - & - & - & - \\
\hline 3 & 3 & 3 & - & - & - & - & - & - & - & - \\
\hline 3 & 3 & 4 & - & - & - & - & - & - & - & - \\
\hline 3 & 3 & 1 & - & - & - & - & - & - & - & - \\
\hline 3 & 3 & 6 & 41 & 24 & - & - & - & - & - & - \\
\hline 3 & 4 & 1 & 49 & 16 & 49 & 14 & 34 & 5 & - & - \\
\hline 3 & 4 & 2 & 49 & 17 & 49 & 17 & - & - & - & - \\
\hline 3 & 4 & 3 & 45 & 21 & 49 & 13 & - & - & - & - \\
\hline 3 & 4 & 4 & 46 & 21 & 51 & 14 & - & - & - & - \\
\hline 3 & 4 & 1 & 47 & 21 & 51 & 15 & - & - & - & - \\
\hline 3 & 4 & 6 & 47 & 23 & 51 & 15 & - & - & - & - \\
\hline
\end{tabular}


Table D.2.20 the results of calibration and validations based on the effective weighted average frequency

\begin{tabular}{|c|c|c|c|c|c|c|c|c|c|c|}
\hline \multicolumn{3}{|c|}{ Configurations } & \multicolumn{2}{|c|}{ Calibration } & \multicolumn{2}{|c|}{$1^{\text {st }}$ Validation } & \multicolumn{2}{|c|}{$2^{\text {nd }}$ Validation } & \multicolumn{2}{|c|}{$3^{\text {rd }}$ Validation } \\
\hline$\varphi_{g}$ & $\Lambda_{l}$ & $\Gamma_{n}$, & Vehicles & Pedestrians & Vehicles & Pedestrians & Vehicles & Pedestrians & Vehicles & Pedestrians \\
\hline 4 & 1 & 1 & 72 & 19 & 69 & 18 & 56 & 15 & 64 & 16 \\
\hline 4 & 1 & 2 & 71 & 19 & 70 & 18 & 56 & 15 & 64 & 16 \\
\hline 4 & 1 & 3 & 70 & 19 & 69 & 18 & 56 & 15 & 64 & 16 \\
\hline 4 & 1 & 4 & 67 & 22 & 69 & 21 & 51 & 13 & 61 & 17 \\
\hline 4 & 1 & 1 & 66 & 24 & 69 & 21 & 52 & 15 & 62 & 18 \\
\hline 4 & 1 & 6 & 70 & 18 & 70 & 20 & 54 & 17 & 63 & 18 \\
\hline 4 & 2 & 1 & 54 & 27 & 56 & 18 & - & - & - & - \\
\hline 4 & 2 & 2 & 55 & 25 & 56 & 18 & - & - & - & - \\
\hline 4 & 2 & 3 & 55 & 27 & 56 & 18 & - & - & - & - \\
\hline 4 & 2 & 4 & 55 & 27 & 56 & 18 & - & - & - & - \\
\hline 4 & 2 & 1 & 55 & 27 & 56 & 20 & - & - & - & - \\
\hline 4 & 2 & 6 & 56 & 27 & 57 & 21 & - & - & - & - \\
\hline 4 & 3 & 1 & - & - & - & - & - & - & - & - \\
\hline 4 & 3 & 2 & - & - & - & - & - & - & - & - \\
\hline 4 & 3 & 3 & - & - & - & - & - & - & - & - \\
\hline 4 & 3 & 4 & - & - & - & - & - & - & - & - \\
\hline 4 & 3 & 1 & - & - & - & - & - & - & - & - \\
\hline 4 & 3 & 6 & - & - & - & - & - & - & - & - \\
\hline 4 & 4 & 1 & 55 & 22 & 55 & 15 & - & - & - & - \\
\hline 4 & 4 & 2 & 54 & 22 & 56 & 15 & - & - & - & - \\
\hline 4 & 4 & 3 & 54 & 24 & 56 & 16 & - & - & - & - \\
\hline 4 & 4 & 4 & 53 & 25 & 56 & 16 & - & - & - & - \\
\hline 4 & 4 & 1 & 55 & 24 & 56 & 18 & - & - & - & - \\
\hline 4 & 4 & 6 & 55 & 25 & 56 & 18 & - & - & - & - \\
\hline 5 & 1 & 1 & 76 & 25 & 75 & 22 & 63 & 20 & 70 & 21 \\
\hline 5 & 1 & 2 & 74 & 25 & 74 & 22 & 64 & 20 & 70 & 21 \\
\hline 5 & 1 & 3 & 73 & 25 & 73 & 22 & 63 & 20 & 69 & 21 \\
\hline 5 & 1 & 4 & 68 & 31 & 70 & 28 & 56 & 18 & 65 & 23 \\
\hline 5 & 1 & 1 & 68 & 31 & 73 & 24 & 59 & 20 & 68 & 22 \\
\hline 5 & 1 & 6 & 65 & 35 & 69 & 30 & 57 & 22 & 65 & 26 \\
\hline 5 & 2 & 1 & 57 & 35 & 62 & 24 & - & - & - & - \\
\hline 5 & 2 & 2 & 56 & 33 & 61 & 24 & - & - & - & - \\
\hline 5 & 2 & 3 & 58 & 35 & 62 & 24 & - & - & - & - \\
\hline 5 & 2 & 4 & 57 & 35 & 61 & 24 & - & - & - & - \\
\hline 5 & 2 & 1 & 56 & 35 & 62 & 26 & - & - & - & - \\
\hline 5 & 2 & 6 & 56 & 35 & 62 & 26 & - & - & - & - \\
\hline
\end{tabular}


Table D.2.20 the results of calibration and validations based on the effective weighted average frequency

\begin{tabular}{|c|c|c|c|c|c|c|c|c|c|c|}
\hline \multicolumn{3}{|c|}{ Configurations } & \multicolumn{2}{|c|}{ Calibration } & \multicolumn{2}{|c|}{$1^{\text {st }}$ Validation } & \multicolumn{2}{|c|}{$2^{\text {nd }}$ Validation } & \multicolumn{2}{|c|}{$3^{\text {rd }}$ Validation } \\
\hline$\varphi_{g}$ & $\Lambda_{l}$ & $\Gamma_{n}$, & Vehicles & Pedestrians & Vehicles & Pedestrians & Vehicles & Pedestrians & Vehicles & Pedestrians \\
\hline 5 & 3 & 1 & - & - & - & - & - & - & - & - \\
\hline 5 & 3 & 2 & - & - & - & - & - & - & - & - \\
\hline 5 & 3 & 3 & - & - & - & - & - & - & - & - \\
\hline 5 & 3 & 4 & - & - & - & - & - & - & - & - \\
\hline 5 & 3 & 1 & - & - & - & - & - & - & - & - \\
\hline 5 & 3 & 6 & - & - & - & - & - & - & - & - \\
\hline 5 & 4 & 1 & 57 & 27 & 61 & 17 & - & - & - & - \\
\hline 5 & 4 & 2 & 56 & 29 & 61 & 20 & - & - & - & - \\
\hline 5 & 4 & 3 & 57 & 31 & 62 & 20 & - & - & - & - \\
\hline 5 & 4 & 4 & 55 & 33 & 61 & 22 & - & - & - & - \\
\hline 5 & 4 & 1 & 56 & 31 & 62 & 22 & - & - & - & - \\
\hline 5 & 4 & 6 & 56 & 33 & 62 & 24 & - & - & - & - \\
\hline
\end{tabular}

Table D.2.21 the results of calibration and validations based on the effective frequency

\begin{tabular}{|c|c|c|c|c|c|c|c|c|c|c|}
\hline \multicolumn{3}{|c|}{ Configurations } & \multicolumn{2}{|c|}{ Calibration } & \multicolumn{2}{|c|}{$\mathbf{1}^{\text {st }}$ Validation } & \multicolumn{2}{|c|}{$2^{\text {nd }}$ Validation } & \multicolumn{2}{|c|}{$3^{\text {rd }}$ Validation } \\
\hline$\varphi_{g}$ & $\Lambda_{l}$ & $\eta_{m}$ & Vehicles & Pedestrians & Vehicles & Pedestrians & Vehicles & Pedestrians & Vehicles & Pedestrians \\
\hline 1 & 1 & 1 & 87 & 7 & 81 & 2 & 95 & 7 & 88 & 5 \\
\hline 1 & 1 & 2 & 80 & 11 & 80 & 10 & 94 & 8 & 86 & 9 \\
\hline 1 & 1 & 3 & 78 & 14 & 78 & 14 & 90 & 9 & 84 & 11 \\
\hline 1 & 2 & 1 & 87 & 6 & 81 & 1 & 95 & 5 & 88 & 4 \\
\hline 1 & 2 & 2 & 79 & 7 & 79 & 5 & 93 & 4 & 86 & 4 \\
\hline 1 & 2 & 3 & 80 & 9 & 79 & 7 & 92 & 3 & 85 & 5 \\
\hline 1 & 3 & 1 & 87 & 3 & 81 & 0 & 95 & 1 & 88 & 0 \\
\hline 1 & 3 & 2 & 79 & 5 & 79 & 4 & 93 & 1 & 86 & 2 \\
\hline 1 & 3 & 3 & 83 & 7 & 80 & 5 & 93 & 3 & 86 & 4 \\
\hline 1 & 4 & 1 & 85 & 3 & 81 & 0 & 93 & 1 & 87 & 0 \\
\hline 1 & 4 & 2 & 82 & 3 & 80 & 4 & 92 & 4 & 86 & 4 \\
\hline 1 & 4 & 3 & 78 & 5 & 78 & 5 & 89 & 4 & 83 & 4 \\
\hline 2 & 1 & 1 & 86 & 2 & 82 & 1 & 95 & 4 & 88 & 3 \\
\hline 2 & 1 & 2 & 74 & 16 & 70 & 15 & 84 & 24 & 77 & 21 \\
\hline 2 & 1 & 3 & 84 & 9 & 80 & 8 & 91 & 8 & 85 & 8 \\
\hline 2 & 2 & 1 & 86 & 2 & 82 & 1 & 95 & 4 & 88 & 3 \\
\hline 2 & 2 & 2 & 74 & 13 & 70 & 11 & 84 & 18 & 77 & 16 \\
\hline 2 & 2 & 3 & 84 & 3 & 80 & 2 & 91 & 5 & 85 & 4 \\
\hline
\end{tabular}


Table D.2.21 the results of calibration and validations based on the effective frequency

\begin{tabular}{|c|c|c|c|c|c|c|c|c|c|c|}
\hline \multicolumn{3}{|c|}{ Configurations } & \multicolumn{2}{|c|}{ Calibration } & \multicolumn{2}{|c|}{$\mathbf{1}^{\text {st }}$ Validation } & \multicolumn{2}{|c|}{$2^{\text {nd }}$ Validation } & \multicolumn{2}{|c|}{$3^{\text {rd }}$ Validation } \\
\hline$\varphi_{g}$ & $\Lambda_{l}$ & $\eta_{m}$ & Vehicles & Pedestrians & Vehicles & Pedestrians & Vehicles & Pedestrians & Vehicles & Pedestrians \\
\hline 2 & 3 & 1 & 86 & 1 & 82 & 0 & - & - & - & - \\
\hline 2 & 3 & 2 & 74 & 8 & 70 & 9 & 84 & 9 & 77 & 9 \\
\hline 2 & 3 & 3 & 84 & 2 & 80 & 1 & 91 & 2 & 85 & 2 \\
\hline 2 & 4 & 1 & 86 & 1 & 82 & 0 & 94 & 2 & 88 & 1 \\
\hline 2 & 4 & 2 & 84 & 2 & 81 & 2 & - & - & - & - \\
\hline 2 & 4 & 3 & 84 & 3 & 80 & 3 & 90 & 2 & 85 & 3 \\
\hline 3 & 1 & 1 & 83 & 4 & 78 & 4 & 94 & 3 & 86 & 3 \\
\hline 3 & 1 & 2 & 83 & 11 & 76 & 10 & 92 & 16 & 84 & 14 \\
\hline 3 & 1 & 3 & 82 & 4 & 78 & 8 & 92 & 13 & 84 & 11 \\
\hline 3 & 2 & 1 & 83 & 4 & 78 & 1 & 94 & 0 & 86 & 1 \\
\hline 3 & 2 & 2 & 83 & 11 & 78 & 8 & 93 & 9 & 85 & 9 \\
\hline 3 & 2 & 3 & 82 & 3 & 78 & 7 & 92 & 13 & 84 & 10 \\
\hline 3 & 3 & 1 & 83 & 3 & 78 & 1 & 94 & 0 & 86 & 1 \\
\hline 3 & 3 & 2 & 83 & 7 & 78 & 4 & 93 & 6 & 85 & 5 \\
\hline 3 & 3 & 3 & 82 & 3 & 78 & 4 & 92 & 7 & 84 & 6 \\
\hline 3 & 4 & 1 & 83 & 0 & 78 & 1 & 94 & 0 & 86 & 1 \\
\hline 3 & 4 & 2 & 83 & 0 & 76 & 0 & 92 & 2 & 84 & 1 \\
\hline 3 & 4 & 3 & 82 & 1 & 78 & 1 & 92 & 0 & 84 & 1 \\
\hline 4 & 1 & 1 & 78 & 6 & 74 & 2 & 91 & 1 & 81 & 1 \\
\hline 4 & 1 & 2 & 78 & 10 & 73 & 8 & 92 & 15 & 81 & 12 \\
\hline 4 & 1 & 3 & 76 & 4 & 75 & 7 & 91 & 5 & 82 & 6 \\
\hline 4 & 2 & 1 & 78 & 3 & 74 & 2 & 91 & 1 & 81 & 1 \\
\hline 4 & 2 & 2 & 78 & 7 & 74 & 7 & 92 & 4 & 82 & 5 \\
\hline 4 & 2 & 3 & 76 & 3 & 74 & 15 & 90 & 7 & 81 & 10 \\
\hline 4 & 3 & 1 & 78 & 3 & 74 & 0 & 91 & 1 & 81 & 1 \\
\hline 4 & 3 & 2 & 78 & 3 & 73 & 2 & 92 & 3 & 81 & 2 \\
\hline 4 & 3 & 3 & 76 & 3 & 75 & 3 & 91 & 3 & 82 & 3 \\
\hline 4 & 4 & 1 & 78 & 1 & 74 & 0 & 91 & 0 & 81 & 0 \\
\hline 4 & 4 & 2 & 77 & 3 & 72 & 2 & 91 & 0 & 80 & 1 \\
\hline 4 & 4 & 3 & 76 & 1 & 75 & 2 & 91 & 0 & 82 & 1 \\
\hline 5 & 1 & 1 & 69 & 8 & 67 & 4 & 84 & 9 & 73 & 7 \\
\hline 5 & 1 & 2 & 69 & 12 & 63 & 7 & 84 & 20 & 71 & 13 \\
\hline 5 & 1 & 3 & 93 & 17 & 92 & 11 & 83 & 16 & 89 & 13 \\
\hline 5 & 2 & 1 & 69 & 6 & 67 & 2 & 84 & 7 & 73 & 4 \\
\hline 5 & 2 & 2 & 68 & 6 & 62 & 4 & 84 & 9 & 70 & 7 \\
\hline 5 & 2 & 3 & 93 & 15 & 92 & 9 & - & - & - & - \\
\hline
\end{tabular}


Table D.2.21 the results of calibration and validations based on the effective frequency

\begin{tabular}{|c|c|c|c|c|c|c|c|c|c|c|}
\hline \multicolumn{3}{|c|}{ Configurations } & \multicolumn{2}{|c|}{ Calibration } & \multicolumn{2}{|c|}{$\mathbf{1}^{\text {st }}$ Validation } & \multicolumn{2}{|c|}{$2^{\text {nd }}$ Validation } & \multicolumn{2}{|c|}{$3^{\text {rd }}$ Validation } \\
\hline$\varphi_{g}$ & $\Lambda_{l}$ & $\eta_{m}$ & Vehicles & Pedestrians & Vehicles & Pedestrians & Vehicles & Pedestrians & Vehicles & Pedestrians \\
\hline 5 & 3 & 1 & 71 & 4 & 67 & 0 & 86 & 2 & 74 & 1 \\
\hline 5 & 3 & 2 & 68 & 4 & 62 & 2 & 84 & 0 & 70 & 1 \\
\hline 5 & 3 & 3 & 95 & 10 & 96 & 2 & 95 & 7 & 96 & 4 \\
\hline 5 & 4 & 1 & 71 & 2 & 67 & 0 & 84 & 0 & 73 & 0 \\
\hline 5 & 4 & 2 & 69 & 4 & 63 & 2 & 84 & 7 & 71 & 4 \\
\hline
\end{tabular}




\section{Appendix E}

\section{The Results of the Calibrations and Validations for the fourth model "Cyclists and pedestrians"}




\section{E.1 The results of calibrations and validations using Kappa statistics}

Table E.1.1 the results of calibration procedure 1 and its validations.

\begin{tabular}{|c|c|c|c|c|c|c|c|}
\hline \multicolumn{4}{|c|}{ Configurations } & \multirow{2}{*}{$\begin{array}{c}\text { Calibration } \\
\text { Kappa } \\
\end{array}$} & \multirow{2}{*}{$\frac{\mathbf{1}^{\text {st }} \text { Validation }}{\text { Kappa }}$} & \multirow{2}{*}{$\begin{array}{c}2^{\text {nd }} \text { Validation } \\
\text { Kappa } \\
\end{array}$} & \multirow{2}{*}{$\frac{3^{\text {rd }} \text { Validation }}{\text { Kappa }}$} \\
\hline$\varphi_{g}$ & $\Lambda_{l}$ & $\Gamma_{n}$ & $\eta_{m}$ & & & & \\
\hline 1 & 1 & 1 & 1 & 0.99 & 0.75 & 0.70 & 0.72 \\
\hline 1 & 1 & 1 & 2 & 0.98 & 0.75 & 0.73 & 0.74 \\
\hline 1 & 1 & 1 & 3 & 0.99 & 0.75 & 0.73 & 0.74 \\
\hline 1 & 1 & 2 & 1 & 0.99 & 0.75 & 0.72 & 0.73 \\
\hline 1 & 1 & 2 & 2 & 0.99 & 0.75 & 0.72 & 0.74 \\
\hline 1 & 1 & 2 & 3 & 0.99 & 0.75 & 0.72 & 0.74 \\
\hline 1 & 1 & 3 & 1 & 0.99 & 0.75 & 0.72 & 0.73 \\
\hline 1 & 1 & 3 & 2 & 0.99 & 0.75 & 0.72 & 0.74 \\
\hline 1 & 1 & 3 & 3 & 0.99 & 0.75 & 0.72 & 0.74 \\
\hline 1 & 1 & 4 & 1 & 0.99 & 0.75 & 0.72 & 0.73 \\
\hline 1 & 1 & 4 & 2 & 0.99 & 0.75 & 0.72 & 0.74 \\
\hline 1 & 1 & 4 & 3 & 0.99 & 0.75 & 0.72 & 0.74 \\
\hline 1 & 1 & 1 & 1 & 0.99 & 0.75 & 0.72 & 0.73 \\
\hline 1 & 1 & 1 & 2 & 0.99 & 0.75 & 0.72 & 0.74 \\
\hline 1 & 1 & 1 & 3 & 0.99 & 0.75 & 0.72 & 0.74 \\
\hline 1 & 1 & 6 & 1 & 0.99 & 0.75 & 0.72 & 0.73 \\
\hline 1 & 1 & 6 & 2 & 0.99 & 0.75 & 0.72 & 0.74 \\
\hline 1 & 1 & 6 & 3 & 0.99 & 0.75 & 0.72 & 0.74 \\
\hline 1 & 2 & 1 & 1 & 1.00 & 0.75 & - & - \\
\hline 1 & 2 & 1 & 2 & 0.99 & 0.75 & - & - \\
\hline 1 & 2 & 1 & 3 & 1.00 & 0.75 & - & - \\
\hline 1 & 2 & 2 & 1 & 1.00 & 0.75 & - & - \\
\hline 1 & 2 & 2 & 2 & 1.00 & 0.77 & - & - \\
\hline 1 & 2 & 2 & 3 & 1.00 & 0.75 & - & - \\
\hline 1 & 2 & 3 & 1 & 1.00 & 0.75 & - & - \\
\hline 1 & 2 & 3 & 2 & 1.00 & 0.77 & - & - \\
\hline 1 & 2 & 3 & 3 & 1.00 & 0.75 & - & - \\
\hline 1 & 2 & 4 & 1 & 1.00 & 0.75 & - & - \\
\hline 1 & 2 & 4 & 2 & 1.00 & 0.77 & - & - \\
\hline 1 & 2 & 4 & 3 & 1.00 & 0.75 & - & - \\
\hline 1 & 2 & 1 & 1 & 1.00 & 0.75 & - & - \\
\hline 1 & 2 & 1 & 2 & 1.00 & 0.77 & - & - \\
\hline 1 & 2 & 1 & 3 & 1.00 & 0.75 & - & - \\
\hline 1 & 2 & 6 & 1 & 1.00 & 0.75 & - & - \\
\hline 1 & 2 & 6 & 2 & 1.00 & 0.77 & - & - \\
\hline 1 & 2 & 6 & 3 & 1.00 & 0.75 & - & - \\
\hline 1 & 3 & 1 & 1 & 1.00 & 0.73 & - & - \\
\hline 1 & 3 & 1 & 2 & 1.00 & 0.73 & - & - \\
\hline 1 & 3 & 1 & 3 & 1.00 & 0.73 & - & - \\
\hline 1 & 3 & 2 & 1 & 1.00 & 0.71 & - & - \\
\hline 1 & 3 & 2 & 2 & 1.00 & 0.73 & - & - \\
\hline 1 & 3 & 2 & 3 & 1.00 & 0.73 & - & - \\
\hline 1 & 3 & 3 & 1 & 1.00 & 0.73 & - & - \\
\hline 1 & 3 & 3 & 2 & 1.00 & 0.74 & - & - \\
\hline 1 & 3 & 3 & 3 & 1.00 & 0.74 & - & - \\
\hline 1 & 3 & 4 & 1 & 1.00 & 0.74 & - & - \\
\hline 1 & 3 & 4 & 2 & 1.00 & 0.75 & - & - \\
\hline 1 & 3 & 4 & 3 & 1.00 & 0.75 & - & - \\
\hline 1 & 3 & 1 & 1 & 1.00 & 0.73 & - & - \\
\hline
\end{tabular}


Table E.1.1 the results of calibration procedure 1 and its validations.

\begin{tabular}{|c|c|c|c|c|c|c|c|}
\hline \multicolumn{4}{|c|}{ Configurations } & \multirow{2}{*}{$\begin{array}{c}\text { Calibration } \\
\text { Kappa } \\
\end{array}$} & \multirow{2}{*}{$\begin{array}{c}\mathbf{1}^{\text {st }} \text { Validation } \\
\text { Kappa } \\
\end{array}$} & \multirow{2}{*}{$\begin{array}{c}2^{\text {nd }} \text { Validation } \\
\text { Kappa } \\
\end{array}$} & \multirow{2}{*}{$\begin{array}{c}\mathbf{3}^{\text {rd }} \text { Validation } \\
\text { Kappa }\end{array}$} \\
\hline$\varphi_{g}$ & $\Lambda_{l}$ & $\Gamma_{n}$ & $\eta_{m}$ & & & & \\
\hline 1 & 3 & 1 & 2 & 1.00 & 0.74 & - & - \\
\hline 1 & 3 & 1 & 3 & 1.00 & 0.74 & - & - \\
\hline 1 & 3 & 6 & 1 & 1.00 & 0.73 & - & - \\
\hline 1 & 3 & 6 & 2 & 1.00 & 0.74 & - & - \\
\hline 1 & 3 & 6 & 3 & 1.00 & 0.74 & - & - \\
\hline 1 & 4 & 1 & 1 & - & - & - & - \\
\hline 1 & 4 & 1 & 2 & - & - & - & - \\
\hline 1 & 4 & 1 & 3 & - & - & - & - \\
\hline 1 & 4 & 2 & 1 & - & - & - & - \\
\hline 1 & 4 & 2 & 2 & - & - & - & - \\
\hline 1 & 4 & 2 & 3 & - & - & - & - \\
\hline 1 & 4 & 3 & 1 & - & - & - & - \\
\hline 1 & 4 & 3 & 2 & - & - & - & - \\
\hline 1 & 4 & 3 & 3 & - & - & - & - \\
\hline 1 & 4 & 4 & 1 & - & - & - & - \\
\hline 1 & 4 & 4 & 2 & - & - & - & - \\
\hline 1 & 4 & 4 & 3 & - & - & - & - \\
\hline 1 & 4 & 1 & 1 & - & - & - & - \\
\hline 1 & 4 & 1 & 2 & - & - & - & - \\
\hline 1 & 4 & 1 & 3 & - & - & - & - \\
\hline 1 & 4 & 6 & 1 & - & - & - & - \\
\hline 1 & 4 & 6 & 2 & - & - & - & - \\
\hline 1 & 4 & 6 & 3 & - & - & - & - \\
\hline 2 & 1 & 1 & 1 & 1.00 & 0.71 & 0.72 & 0.73 \\
\hline 2 & 1 & 1 & 2 & 1.00 & 0.74 & 0.76 & 0.76 \\
\hline 2 & 1 & 1 & 3 & 1.00 & 0.74 & 0.76 & 0.76 \\
\hline 2 & 1 & 2 & 1 & 1.00 & 0.74 & 0.71 & 0.73 \\
\hline 2 & 1 & 2 & 2 & 1.00 & 0.77 & 0.75 & 0.76 \\
\hline 2 & 1 & 2 & 3 & 1.00 & 0.77 & 0.75 & 0.76 \\
\hline 2 & 1 & 3 & 1 & 1.00 & 0.70 & 0.70 & 0.71 \\
\hline 2 & 1 & 3 & 2 & 1.00 & 0.74 & 0.76 & 0.76 \\
\hline 2 & 1 & 3 & 3 & 1.00 & 0.74 & 0.76 & 0.76 \\
\hline 2 & 1 & 4 & 1 & 1.00 & 0.71 & 0.70 & 0.72 \\
\hline 2 & 1 & 4 & 2 & 1.00 & 0.73 & 0.74 & 0.75 \\
\hline 2 & 1 & 4 & 3 & 1.00 & 0.73 & 0.74 & 0.75 \\
\hline 2 & 1 & 1 & 1 & 1.00 & 0.70 & 0.72 & 0.73 \\
\hline 2 & 1 & 1 & 2 & 1.00 & 0.71 & 0.77 & 0.76 \\
\hline 2 & 1 & 1 & 3 & 1.00 & 0.71 & 0.77 & 0.76 \\
\hline 2 & 1 & 6 & 1 & 1.00 & 0.71 & 0.71 & 0.72 \\
\hline 2 & 1 & 6 & 2 & 1.00 & 0.75 & 0.73 & 0.74 \\
\hline 2 & 1 & 6 & 3 & 1.00 & 0.75 & 0.73 & 0.74 \\
\hline 2 & 2 & 1 & 1 & 0.98 & - & - & - \\
\hline 2 & 2 & 1 & 2 & 0.98 & 0.66 & 0.74 & 0.72 \\
\hline 2 & 2 & 1 & 3 & 0.98 & 0.66 & 0.74 & 0.73 \\
\hline 2 & 2 & 2 & 1 & 0.98 & - & - & - \\
\hline 2 & 2 & 2 & 2 & 0.98 & 0.66 & 0.73 & 0.72 \\
\hline 2 & 2 & 2 & 3 & 0.98 & 0.68 & 0.73 & 0.72 \\
\hline 2 & 2 & 3 & 1 & 0.98 & - & - & - \\
\hline 2 & 2 & 3 & 2 & 0.98 & 0.68 & 0.70 & 0.70 \\
\hline 2 & 2 & 3 & 3 & 0.98 & 0.70 & 0.72 & 0.72 \\
\hline 2 & 2 & 4 & 1 & 0.98 & - & - & - \\
\hline
\end{tabular}


Table E.1.1 the results of calibration procedure 1 and its validations.

\begin{tabular}{|c|c|c|c|c|c|c|c|}
\hline \multicolumn{4}{|c|}{ Configurations } & \multirow{2}{*}{$\begin{array}{c}\text { Calibration } \\
\text { Kappa } \\
\end{array}$} & \multirow{2}{*}{$\begin{array}{c}\mathbf{1}^{\text {st }} \text { Validation } \\
\text { Kappa } \\
\end{array}$} & \multirow{2}{*}{$\begin{array}{c}2^{\text {nd }} \text { Validation } \\
\text { Kappa } \\
\end{array}$} & \multirow{2}{*}{$\begin{array}{c}\mathbf{3}^{\text {rd }} \text { Validation } \\
\text { Kappa }\end{array}$} \\
\hline$\varphi_{g}$ & $\Lambda_{l}$ & $\Gamma_{n}$ & $\eta_{m}$ & & & & \\
\hline 2 & 2 & 4 & 2 & 0.98 & - & - & - \\
\hline 2 & 2 & 4 & 3 & 0.98 & 0.65 & 0.73 & 0.71 \\
\hline 2 & 2 & 1 & 1 & 0.98 & - & - & - \\
\hline 2 & 2 & 1 & 2 & 0.98 & 0.66 & 0.70 & 0.70 \\
\hline 2 & 2 & 1 & 3 & 0.98 & 0.68 & 0.70 & 0.70 \\
\hline 2 & 2 & 6 & 1 & 0.98 & - & - & - \\
\hline 2 & 2 & 6 & 2 & 0.98 & 0.67 & 0.71 & 0.70 \\
\hline 2 & 2 & 6 & 3 & 0.98 & 0.68 & 0.72 & 0.71 \\
\hline 2 & 3 & 1 & 1 & 1.00 & - & - & - \\
\hline 2 & 3 & 1 & 2 & 1.00 & - & - & - \\
\hline 2 & 3 & 1 & 3 & 1.00 & - & - & - \\
\hline 2 & 3 & 2 & 1 & 1.00 & - & - & - \\
\hline 2 & 3 & 2 & 2 & 1.00 & - & - & - \\
\hline 2 & 3 & 2 & 3 & 1.00 & - & - & - \\
\hline 2 & 3 & 3 & 1 & 1.00 & - & - & - \\
\hline 2 & 3 & 3 & 2 & 1.00 & - & - & - \\
\hline 2 & 3 & 3 & 3 & 1.00 & - & - & - \\
\hline 2 & 3 & 4 & 1 & 1.00 & - & - & - \\
\hline 2 & 3 & 4 & 2 & 1.00 & - & - & - \\
\hline 2 & 3 & 4 & 3 & 1.00 & - & - & - \\
\hline 2 & 3 & 1 & 1 & 1.00 & - & - & - \\
\hline 2 & 3 & 1 & 2 & 1.00 & - & - & - \\
\hline 2 & 3 & 1 & 3 & 1.00 & - & - & - \\
\hline 2 & 3 & 6 & 1 & 1.00 & - & - & - \\
\hline 2 & 3 & 6 & 2 & 1.00 & - & - & - \\
\hline 2 & 3 & 6 & 3 & 1.00 & - & - & - \\
\hline 2 & 4 & 1 & 1 & 1.00 & - & - & - \\
\hline 2 & 4 & 1 & 2 & 1.00 & - & - & - \\
\hline 2 & 4 & 1 & 3 & 1.00 & - & - & - \\
\hline 2 & 4 & 2 & 1 & 1.00 & 0.63 & - & - \\
\hline 2 & 4 & 2 & 2 & 1.00 & 0.63 & - & - \\
\hline 2 & 4 & 2 & 3 & 1.00 & - & - & - \\
\hline 2 & 4 & 3 & 1 & 1.00 & - & - & - \\
\hline 2 & 4 & 3 & 2 & 1.00 & - & - & - \\
\hline 2 & 4 & 3 & 3 & 1.00 & - & - & - \\
\hline 2 & 4 & 4 & 1 & 1.00 & - & - & - \\
\hline 2 & 4 & 4 & 2 & 1.00 & - & - & - \\
\hline 2 & 4 & 4 & 3 & 1.00 & - & - & - \\
\hline 2 & 4 & 1 & 1 & 1.00 & - & - & - \\
\hline 2 & 4 & 1 & 2 & 1.00 & - & - & - \\
\hline 2 & 4 & 1 & 3 & 1.00 & - & - & - \\
\hline 2 & 4 & 6 & 1 & 1.00 & - & - & - \\
\hline 2 & 4 & 6 & 2 & 1.00 & - & - & - \\
\hline 2 & 4 & 6 & 3 & 1.00 & - & - & - \\
\hline 3 & 1 & 1 & 1 & 1.00 & 0.86 & 0.80 & 0.82 \\
\hline 3 & 1 & 1 & 2 & 1.00 & 0.86 & 0.80 & 0.82 \\
\hline 3 & 1 & 1 & 3 & 1.00 & 0.86 & 0.80 & 0.82 \\
\hline 3 & 1 & 2 & 1 & 1.00 & 0.89 & 0.81 & 0.84 \\
\hline 3 & 1 & 2 & 2 & 1.00 & 0.89 & 0.81 & 0.84 \\
\hline 3 & 1 & 2 & 3 & 1.00 & 0.89 & 0.81 & 0.84 \\
\hline 3 & 1 & 3 & 1 & 1.00 & 0.86 & 0.84 & 0.85 \\
\hline
\end{tabular}


Table E.1.1 the results of calibration procedure 1 and its validations.

\begin{tabular}{|c|c|c|c|c|c|c|c|}
\hline \multicolumn{4}{|c|}{ Configurations } & \multirow{2}{*}{$\begin{array}{c}\text { Calibration } \\
\text { Kappa } \\
\end{array}$} & \multirow{2}{*}{$\begin{array}{c}\mathbf{1}^{\text {st }} \text { Validation } \\
\text { Kappa }\end{array}$} & \multirow{2}{*}{$\begin{array}{c}2^{\text {nd }} \text { Validation } \\
\text { Kappa } \\
\end{array}$} & \multirow{2}{*}{$\begin{array}{c}3^{\text {rd }} \text { Validation } \\
\text { Kappa }\end{array}$} \\
\hline$\varphi_{g}$ & $\Lambda_{l}$ & $\Gamma_{n}$ & $\eta_{m}$ & & & & \\
\hline 3 & 1 & 3 & 2 & 1.00 & 0.86 & 0.84 & 0.85 \\
\hline 3 & 1 & 3 & 3 & 1.00 & 0.86 & 0.84 & 0.85 \\
\hline 3 & 1 & 4 & 1 & 1.00 & 0.86 & 0.82 & 0.84 \\
\hline 3 & 1 & 4 & 2 & 1.00 & 0.86 & 0.82 & 0.84 \\
\hline 3 & 1 & 4 & 3 & 1.00 & 0.86 & 0.82 & 0.84 \\
\hline 3 & 1 & 1 & 1 & 1.00 & 0.86 & 0.83 & 0.85 \\
\hline 3 & 1 & 1 & 2 & 1.00 & 0.86 & 0.84 & 0.85 \\
\hline 3 & 1 & 1 & 3 & 1.00 & 0.86 & 0.84 & 0.85 \\
\hline 3 & 1 & 6 & 1 & 1.00 & 0.87 & 0.82 & 0.85 \\
\hline 3 & 1 & 6 & 2 & 1.00 & 0.87 & 0.82 & 0.85 \\
\hline 3 & 1 & 6 & 3 & 1.00 & 0.87 & 0.82 & 0.85 \\
\hline 3 & 2 & 1 & 1 & 0.98 & - & - & - \\
\hline 3 & 2 & 1 & 2 & 0.98 & - & - & - \\
\hline 3 & 2 & 1 & 3 & 0.98 & - & - & - \\
\hline 3 & 2 & 2 & 1 & 0.98 & - & - & - \\
\hline 3 & 2 & 2 & 2 & 0.98 & - & - & - \\
\hline 3 & 2 & 2 & 3 & 0.98 & - & - & - \\
\hline 3 & 2 & 3 & 1 & 0.98 & - & - & - \\
\hline 3 & 2 & 3 & 2 & 0.98 & - & - & - \\
\hline 3 & 2 & 3 & 3 & 0.98 & - & - & - \\
\hline 3 & 2 & 4 & 1 & 0.98 & - & - & - \\
\hline 3 & 2 & 4 & 2 & 0.98 & - & - & - \\
\hline 3 & 2 & 4 & 3 & 0.98 & - & - & - \\
\hline 3 & 2 & 1 & 1 & 0.98 & - & - & - \\
\hline 3 & 2 & 1 & 2 & 0.98 & - & - & - \\
\hline 3 & 2 & 1 & 3 & 0.98 & - & - & - \\
\hline 3 & 2 & 6 & 1 & 0.98 & - & - & - \\
\hline 3 & 2 & 6 & 2 & 0.98 & - & - & - \\
\hline 3 & 2 & 6 & 3 & 0.98 & - & - & - \\
\hline 3 & 3 & 1 & 1 & 1.00 & - & - & - \\
\hline 3 & 3 & 1 & 2 & 1.00 & - & - & - \\
\hline 3 & 3 & 1 & 3 & 1.00 & - & - & - \\
\hline 3 & 3 & 2 & 1 & 1.00 & - & - & - \\
\hline 3 & 3 & 2 & 2 & 1.00 & - & - & - \\
\hline 3 & 3 & 2 & 3 & 1.00 & - & - & - \\
\hline 3 & 3 & 3 & 1 & 1.00 & - & - & - \\
\hline 3 & 3 & 3 & 2 & 1.00 & - & - & - \\
\hline 3 & 3 & 3 & 3 & 1.00 & - & - & - \\
\hline 3 & 3 & 4 & 1 & 1.00 & - & - & - \\
\hline 3 & 3 & 4 & 2 & 1.00 & - & - & - \\
\hline 3 & 3 & 4 & 3 & 1.00 & - & - & - \\
\hline 3 & 3 & 1 & 1 & 1.00 & - & - & - \\
\hline 3 & 3 & 1 & 2 & 1.00 & - & - & - \\
\hline 3 & 3 & 1 & 3 & 1.00 & - & - & - \\
\hline 3 & 3 & 6 & 1 & 1.00 & - & - & - \\
\hline 3 & 3 & 6 & 2 & 1.00 & - & - & - \\
\hline 3 & 3 & 6 & 3 & 1.00 & - & - & - \\
\hline 3 & 4 & 1 & 1 & 1.00 & - & - & - \\
\hline 3 & 4 & 1 & 2 & 1.00 & - & - & - \\
\hline 3 & 4 & 1 & 3 & 1.00 & - & - & - \\
\hline 3 & 4 & 2 & 1 & 1.00 & - & - & - \\
\hline
\end{tabular}


Table E.1.1 the results of calibration procedure 1 and its validations.

\begin{tabular}{|c|c|c|c|c|c|c|c|}
\hline \multicolumn{4}{|c|}{ Configurations } & \multirow{2}{*}{$\begin{array}{c}\text { Calibration } \\
\text { Kappa } \\
\end{array}$} & \multirow{2}{*}{$\begin{array}{c}\mathbf{1}^{\text {st }} \text { Validation } \\
\text { Kappa } \\
\end{array}$} & \multirow{2}{*}{$\begin{array}{c}2^{\text {nd }} \text { Validation } \\
\text { Kappa } \\
\end{array}$} & \multirow{2}{*}{$\begin{array}{c}3^{\text {rd }} \text { Validation } \\
\text { Kappa } \\
\end{array}$} \\
\hline$\varphi_{g}$ & $\Lambda_{l}$ & $\Gamma_{n}$ & $\eta_{m}$ & & & & \\
\hline 3 & 4 & 2 & 2 & 1.00 & - & - & - \\
\hline 3 & 4 & 2 & 3 & 1.00 & - & - & - \\
\hline 3 & 4 & 3 & 1 & 1.00 & - & - & - \\
\hline 3 & 4 & 3 & 2 & 1.00 & - & - & - \\
\hline 3 & 4 & 3 & 3 & 1.00 & - & - & - \\
\hline 3 & 4 & 4 & 1 & 1.00 & - & - & - \\
\hline 3 & 4 & 4 & 2 & 1.00 & - & - & - \\
\hline 3 & 4 & 4 & 3 & 1.00 & - & - & - \\
\hline 3 & 4 & 1 & 1 & 1.00 & - & - & - \\
\hline 3 & 4 & 1 & 2 & 1.00 & - & - & - \\
\hline 3 & 4 & 1 & 3 & 1.00 & - & - & - \\
\hline 3 & 4 & 6 & 1 & 1.00 & - & - & - \\
\hline 3 & 4 & 6 & 2 & 1.00 & - & - & - \\
\hline 3 & 4 & 6 & 3 & 1.00 & - & - & - \\
\hline 4 & 1 & 1 & 1 & 1.00 & 0.91 & 0.81 & 0.86 \\
\hline 4 & 1 & 1 & 2 & 1.00 & 0.91 & 0.81 & 0.86 \\
\hline 4 & 1 & 1 & 3 & 1.00 & 0.91 & 0.81 & 0.86 \\
\hline 4 & 1 & 2 & 1 & 1.00 & 0.94 & 0.83 & 0.87 \\
\hline 4 & 1 & 2 & 2 & 1.00 & 0.94 & 0.83 & 0.87 \\
\hline 4 & 1 & 2 & 3 & 1.00 & 0.94 & 0.83 & 0.87 \\
\hline 4 & 1 & 3 & 1 & 1.00 & 0.89 & 0.87 & 0.89 \\
\hline 4 & 1 & 3 & 2 & 1.00 & 0.89 & 0.87 & 0.89 \\
\hline 4 & 1 & 3 & 3 & 1.00 & 0.89 & 0.87 & 0.89 \\
\hline 4 & 1 & 4 & 1 & 1.00 & 0.89 & 0.84 & 0.87 \\
\hline 4 & 1 & 4 & 2 & 1.00 & 0.89 & 0.84 & 0.87 \\
\hline 4 & 1 & 4 & 3 & 1.00 & 0.89 & 0.84 & 0.87 \\
\hline 4 & 1 & 1 & 1 & 1.00 & 0.89 & 0.86 & 0.88 \\
\hline 4 & 1 & 1 & 2 & 1.00 & 0.89 & 0.87 & 0.89 \\
\hline 4 & 1 & 1 & 3 & 1.00 & 0.89 & 0.87 & 0.89 \\
\hline 4 & 1 & 6 & 1 & 1.00 & 0.92 & 0.85 & 0.88 \\
\hline 4 & 1 & 6 & 2 & 1.00 & 0.92 & 0.85 & 0.88 \\
\hline 4 & 1 & 6 & 3 & 1.00 & 0.92 & 0.85 & 0.88 \\
\hline 4 & 2 & 1 & 1 & 1.00 & - & - & - \\
\hline 4 & 2 & 1 & 2 & 1.00 & - & - & - \\
\hline 4 & 2 & 1 & 3 & 1.00 & - & - & - \\
\hline 4 & 2 & 2 & 1 & 1.00 & - & - & - \\
\hline 4 & 2 & 2 & 2 & 1.00 & - & - & - \\
\hline 4 & 2 & 2 & 3 & 1.00 & - & - & - \\
\hline 4 & 2 & 3 & 1 & 1.00 & - & - & - \\
\hline 4 & 2 & 3 & 2 & 1.00 & - & - & - \\
\hline 4 & 2 & 3 & 3 & 1.00 & - & - & - \\
\hline 4 & 2 & 4 & 1 & 1.00 & - & - & - \\
\hline 4 & 2 & 4 & 2 & 1.00 & - & - & - \\
\hline 4 & 2 & 4 & 3 & 1.00 & - & - & - \\
\hline 4 & 2 & 1 & 1 & 1.00 & - & - & - \\
\hline 4 & 2 & 1 & 2 & 1.00 & - & - & - \\
\hline 4 & 2 & 1 & 3 & 1.00 & - & - & - \\
\hline 4 & 2 & 6 & 1 & 1.00 & - & - & - \\
\hline 4 & 2 & 6 & 2 & 1.00 & - & - & - \\
\hline 4 & 2 & 6 & 3 & 1.00 & - & - & - \\
\hline 4 & 3 & 1 & 1 & 1.00 & - & - & - \\
\hline
\end{tabular}


Table E.1.1 the results of calibration procedure 1 and its validations.

\begin{tabular}{|c|c|c|c|c|c|c|c|}
\hline \multicolumn{4}{|c|}{ Configurations } & \multirow{2}{*}{$\begin{array}{c}\text { Calibration } \\
\text { Kappa } \\
\end{array}$} & \multirow{2}{*}{$\begin{array}{c}\mathbf{1}^{\text {st }} \text { Validation } \\
\text { Kappa } \\
\end{array}$} & \multirow{2}{*}{$\begin{array}{c}2^{\text {nd }} \text { Validation } \\
\text { Kappa } \\
\end{array}$} & \multirow{2}{*}{$\begin{array}{c}\mathbf{3}^{\text {rd }} \text { Validation } \\
\text { Kappa }\end{array}$} \\
\hline$\varphi_{g}$ & $\Lambda_{l}$ & $\Gamma_{n}$ & $\eta_{m}$ & & & & \\
\hline 4 & 3 & 1 & 2 & 1.00 & - & - & - \\
\hline 4 & 3 & 1 & 3 & 1.00 & - & - & - \\
\hline 4 & 3 & 2 & 1 & 1.00 & - & - & - \\
\hline 4 & 3 & 2 & 2 & 1.00 & - & - & - \\
\hline 4 & 3 & 2 & 3 & 1.00 & - & - & - \\
\hline 4 & 3 & 3 & 1 & 1.00 & - & - & - \\
\hline 4 & 3 & 3 & 2 & 1.00 & - & - & - \\
\hline 4 & 3 & 3 & 3 & 1.00 & - & - & - \\
\hline 4 & 3 & 4 & 1 & 1.00 & - & - & - \\
\hline 4 & 3 & 4 & 2 & 1.00 & - & - & - \\
\hline 4 & 3 & 4 & 3 & 1.00 & - & - & - \\
\hline 4 & 3 & 1 & 1 & 1.00 & - & - & - \\
\hline 4 & 3 & 1 & 2 & 1.00 & - & - & - \\
\hline 4 & 3 & 1 & 3 & 1.00 & - & - & - \\
\hline 4 & 3 & 6 & 1 & 1.00 & - & - & - \\
\hline 4 & 3 & 6 & 2 & 1.00 & - & - & - \\
\hline 4 & 3 & 6 & 3 & 1.00 & - & - & - \\
\hline 4 & 4 & 1 & 1 & 1.00 & - & - & - \\
\hline 4 & 4 & 1 & 2 & 0.98 & - & - & - \\
\hline 4 & 4 & 1 & 3 & 0.98 & - & - & - \\
\hline 4 & 4 & 2 & 1 & 1.00 & - & - & - \\
\hline 4 & 4 & 2 & 2 & 0.98 & - & - & - \\
\hline 4 & 4 & 2 & 3 & 0.98 & - & - & - \\
\hline 4 & 4 & 3 & 1 & 1.00 & - & - & - \\
\hline 4 & 4 & 3 & 2 & 0.98 & - & - & - \\
\hline 4 & 4 & 3 & 3 & 0.98 & - & - & - \\
\hline 4 & 4 & 4 & 1 & 1.00 & - & - & - \\
\hline 4 & 4 & 4 & 2 & 1.00 & - & - & - \\
\hline 4 & 4 & 4 & 3 & 1.00 & - & - & - \\
\hline 4 & 4 & 1 & 1 & 1.00 & - & - & - \\
\hline 4 & 4 & 1 & 2 & 1.00 & - & - & - \\
\hline 4 & 4 & 1 & 3 & 1.00 & - & - & - \\
\hline 4 & 4 & 6 & 1 & 1.00 & - & - & - \\
\hline 4 & 4 & 6 & 2 & 1.00 & - & - & - \\
\hline 4 & 4 & 6 & 3 & 1.00 & - & - & - \\
\hline 5 & 1 & 1 & 1 & 1.00 & 0.89 & 0.72 & 0.80 \\
\hline 5 & 1 & 1 & 2 & 1.00 & 0.89 & 0.76 & 0.82 \\
\hline 5 & 1 & 1 & 3 & 1.00 & 0.89 & 0.76 & 0.82 \\
\hline 5 & 1 & 2 & 1 & 1.00 & 0.92 & 0.82 & 0.87 \\
\hline 5 & 1 & 2 & 2 & 1.00 & 0.92 & 0.82 & 0.87 \\
\hline 5 & 1 & 2 & 3 & 1.00 & 0.92 & 0.82 & 0.87 \\
\hline 5 & 1 & 3 & 1 & 1.00 & 0.84 & 0.83 & 0.85 \\
\hline 5 & 1 & 3 & 2 & 1.00 & 0.84 & 0.83 & 0.85 \\
\hline 5 & 1 & 3 & 3 & 1.00 & 0.84 & 0.83 & 0.85 \\
\hline 5 & 1 & 4 & 1 & 1.00 & 0.84 & 0.83 & 0.85 \\
\hline 5 & 1 & 4 & 2 & 1.00 & 0.84 & 0.83 & 0.85 \\
\hline 5 & 1 & 4 & 3 & 1.00 & 0.84 & 0.83 & 0.85 \\
\hline 5 & 1 & 1 & 1 & 1.00 & 0.87 & 0.83 & 0.86 \\
\hline 5 & 1 & 1 & 2 & 1.00 & 0.87 & 0.83 & 0.86 \\
\hline 5 & 1 & 1 & 3 & 1.00 & 0.87 & 0.83 & 0.86 \\
\hline 5 & 1 & 6 & 1 & 1.00 & 0.84 & 0.81 & 0.84 \\
\hline
\end{tabular}


Table E.1.1 the results of calibration procedure 1 and its validations.

\begin{tabular}{|c|c|c|c|c|c|c|c|}
\hline \multicolumn{4}{|c|}{ Configurations } & \multirow{2}{*}{$\begin{array}{c}\text { Calibration } \\
\text { Kappa } \\
\end{array}$} & \multirow{2}{*}{$\begin{array}{c}\mathbf{1}^{\text {st }} \text { Validation } \\
\text { Kappa }\end{array}$} & \multirow{2}{*}{$\begin{array}{c}2^{\text {nd }} \text { Validation } \\
\text { Kappa } \\
\end{array}$} & \multirow{2}{*}{$\begin{array}{c}3^{\text {rd }} \text { Validation } \\
\text { Kappa }\end{array}$} \\
\hline$\varphi_{g}$ & $\Lambda_{l}$ & $\Gamma_{n}$ & $\eta_{m}$ & & & & \\
\hline 5 & 1 & 6 & 2 & 1.00 & 0.82 & 0.81 & 0.83 \\
\hline 5 & 1 & 6 & 3 & 1.00 & 0.82 & 0.81 & 0.83 \\
\hline 5 & 2 & 1 & 1 & 1.00 & - & - & - \\
\hline 5 & 2 & 1 & 2 & 1.00 & - & - & - \\
\hline 5 & 2 & 1 & 3 & 1.00 & - & - & - \\
\hline 5 & 2 & 2 & 1 & 1.00 & - & - & - \\
\hline 5 & 2 & 2 & 2 & 1.00 & - & - & - \\
\hline 5 & 2 & 2 & 3 & 1.00 & - & - & - \\
\hline 5 & 2 & 3 & 1 & 1.00 & - & - & - \\
\hline 5 & 2 & 3 & 2 & 1.00 & - & - & - \\
\hline 5 & 2 & 3 & 3 & 1.00 & - & - & - \\
\hline 5 & 2 & 4 & 1 & 1.00 & - & - & - \\
\hline 5 & 2 & 4 & 2 & 1.00 & - & - & - \\
\hline 5 & 2 & 4 & 3 & 1.00 & - & - & - \\
\hline 5 & 2 & 1 & 1 & 1.00 & - & - & - \\
\hline 5 & 2 & 1 & 2 & 1.00 & - & - & - \\
\hline 5 & 2 & 1 & 3 & 1.00 & - & - & - \\
\hline 5 & 2 & 6 & 1 & 1.00 & - & - & - \\
\hline 5 & 2 & 6 & 2 & 1.00 & - & - & - \\
\hline 5 & 2 & 6 & 3 & 1.00 & - & - & - \\
\hline 5 & 3 & 1 & 1 & 1.00 & - & - & - \\
\hline 5 & 3 & 1 & 2 & 1.00 & - & - & - \\
\hline 5 & 3 & 1 & 3 & 1.00 & - & - & - \\
\hline 5 & 3 & 2 & 1 & 1.00 & - & - & - \\
\hline 5 & 3 & 2 & 2 & 1.00 & - & - & - \\
\hline 5 & 3 & 2 & 3 & 1.00 & - & - & - \\
\hline 5 & 3 & 3 & 1 & 1.00 & - & - & - \\
\hline 5 & 3 & 3 & 2 & 1.00 & - & - & - \\
\hline 5 & 3 & 3 & 3 & 1.00 & - & - & - \\
\hline 5 & 3 & 4 & 1 & 1.00 & - & - & - \\
\hline 5 & 3 & 4 & 2 & 1.00 & - & - & - \\
\hline 5 & 3 & 4 & 3 & 1.00 & - & - & - \\
\hline 5 & 3 & 1 & 1 & 1.00 & - & - & - \\
\hline 5 & 3 & 1 & 2 & 1.00 & - & - & - \\
\hline 5 & 3 & 1 & 3 & 1.00 & - & - & - \\
\hline 5 & 3 & 6 & 1 & 1.00 & - & - & - \\
\hline 5 & 3 & 6 & 2 & 1.00 & - & - & - \\
\hline 5 & 3 & 6 & 3 & 1.00 & - & - & - \\
\hline 5 & 4 & 1 & 1 & 1.00 & - & - & - \\
\hline 5 & 4 & 1 & 2 & 1.00 & - & - & - \\
\hline 5 & 4 & 1 & 3 & 1.00 & - & - & - \\
\hline 5 & 4 & 2 & 1 & 1.00 & - & - & - \\
\hline 5 & 4 & 2 & 2 & 1.00 & - & - & - \\
\hline 5 & 4 & 2 & 3 & 1.00 & - & - & - \\
\hline 5 & 4 & 3 & 1 & 1.00 & - & - & - \\
\hline 5 & 4 & 3 & 2 & 1.00 & - & - & - \\
\hline 5 & 4 & 3 & 3 & 1.00 & - & - & - \\
\hline 5 & 4 & 4 & 1 & 1.00 & - & - & - \\
\hline 5 & 4 & 4 & 2 & 1.00 & - & - & - \\
\hline 5 & 4 & 4 & 3 & 1.00 & - & - & - \\
\hline 5 & 4 & 1 & 1 & 1.00 & - & - & - \\
\hline
\end{tabular}


Table E.1.1 the results of calibration procedure 1 and its validations.

\begin{tabular}{|c|c|c|c|c|c|c|c|}
\hline \multicolumn{4}{|c|}{ Configurations } & Calibration & $1^{\text {st }}$ Validation & $2^{\text {nd }}$ Validation & $3^{\text {rd }}$ Validation \\
\hline$\varphi_{g}$ & $\Lambda_{l}$ & $\Gamma_{n}$ & $\eta_{m}$ & Kappa & Kappa & Kappa & Kappa \\
\hline 5 & 4 & 1 & 2 & 1.00 & - & - & - \\
\hline 5 & 4 & 1 & 3 & 1.00 & - & - & - \\
\hline 5 & 4 & 6 & 1 & 1.00 & - & - & - \\
\hline 5 & 4 & 6 & 2 & 1.00 & - & - & - \\
\hline 5 & 4 & 6 & 3 & 1.00 & - & - & - \\
\hline
\end{tabular}

Table E.1.2 the results of calibration procedure 2 and its validations.

\begin{tabular}{|c|c|c|c|c|c|c|c|}
\hline \multicolumn{4}{|c|}{ Configurations } & \multirow{2}{*}{$\begin{array}{c}\text { Calibration } \\
\text { Kappa } \\
\end{array}$} & \multirow{2}{*}{$\begin{array}{c}\mathbf{1}^{\text {st }} \text { Validation } \\
\text { Kappa }\end{array}$} & \multirow{2}{*}{$\begin{array}{c}2^{\text {nd }} \text { Validation } \\
\text { Kappa }\end{array}$} & \multirow{2}{*}{$\begin{array}{c}\mathbf{3}^{\text {rd }} \text { Validation } \\
\text { Kappa }\end{array}$} \\
\hline$\varphi_{g}$ & $\Lambda_{l}$ & $\Gamma_{n}$ & $\eta_{m}$ & & & & \\
\hline 1 & 1 & 1 & 1 & 0.88 & 0.94 & 0.90 & 0.92 \\
\hline 1 & 1 & 1 & 2 & 0.88 & 0.94 & 0.90 & 0.92 \\
\hline 1 & 1 & 1 & 3 & 0.88 & 0.94 & 0.90 & 0.92 \\
\hline 1 & 1 & 2 & 1 & 0.88 & 0.94 & 0.90 & 0.92 \\
\hline 1 & 1 & 2 & 2 & 0.88 & 0.94 & 0.90 & 0.92 \\
\hline 1 & 1 & 2 & 3 & 0.88 & 0.94 & 0.90 & 0.92 \\
\hline 1 & 1 & 3 & 1 & 0.88 & 0.94 & 0.90 & 0.92 \\
\hline 1 & 1 & 3 & 2 & 0.88 & 0.94 & 0.90 & 0.92 \\
\hline 1 & 1 & 3 & 3 & 0.88 & 0.94 & 0.90 & 0.92 \\
\hline 1 & 1 & 4 & 1 & 0.88 & 0.94 & 0.90 & 0.92 \\
\hline 1 & 1 & 4 & 2 & 0.88 & 0.94 & 0.90 & 0.92 \\
\hline 1 & 1 & 4 & 3 & 0.88 & 0.94 & 0.90 & 0.92 \\
\hline 1 & 1 & 1 & 1 & 0.88 & 0.94 & 0.90 & 0.92 \\
\hline 1 & 1 & 1 & 2 & 0.88 & 0.94 & 0.90 & 0.92 \\
\hline 1 & 1 & 1 & 3 & 0.88 & 0.94 & 0.90 & 0.92 \\
\hline 1 & 1 & 6 & 1 & 0.88 & 0.94 & 0.90 & 0.92 \\
\hline 1 & 1 & 6 & 2 & 0.88 & 0.94 & 0.90 & 0.92 \\
\hline 1 & 1 & 6 & 3 & 0.88 & 0.94 & 0.90 & 0.92 \\
\hline 1 & 2 & 1 & 1 & 0.82 & - & - & - \\
\hline 1 & 2 & 1 & 2 & 0.82 & - & - & - \\
\hline 1 & 2 & 1 & 3 & 0.82 & - & - & - \\
\hline 1 & 2 & 2 & 1 & 0.82 & - & - & - \\
\hline 1 & 2 & 2 & 2 & 0.82 & - & - & - \\
\hline 1 & 2 & 2 & 3 & 0.82 & - & - & - \\
\hline 1 & 2 & 3 & 1 & 0.82 & - & - & - \\
\hline 1 & 2 & 3 & 2 & 0.82 & - & - & - \\
\hline 1 & 2 & 3 & 3 & 0.82 & - & - & - \\
\hline 1 & 2 & 4 & 1 & 0.82 & - & - & - \\
\hline 1 & 2 & 4 & 2 & 0.82 & - & - & - \\
\hline 1 & 2 & 4 & 3 & 0.82 & - & - & - \\
\hline 1 & 2 & 1 & 1 & 0.82 & - & - & - \\
\hline 1 & 2 & 1 & 2 & 0.82 & - & - & - \\
\hline 1 & 2 & 1 & 3 & 0.82 & - & - & - \\
\hline 1 & 2 & 6 & 1 & 0.82 & - & - & - \\
\hline 1 & 2 & 6 & 2 & 0.82 & - & - & - \\
\hline 1 & 2 & 6 & 3 & 0.82 & - & - & - \\
\hline 1 & 3 & 1 & 1 & 0.85 & - & - & - \\
\hline 1 & 3 & 1 & 2 & 0.85 & - & - & - \\
\hline 1 & 3 & 1 & 3 & 0.85 & - & - & - \\
\hline 1 & 3 & 2 & 1 & 0.85 & - & - & - \\
\hline 1 & 3 & 2 & 2 & 0.85 & - & - & - \\
\hline
\end{tabular}


Table E.1.2 the results of calibration procedure 2 and its validations.

\begin{tabular}{|c|c|c|c|c|c|c|c|}
\hline \multicolumn{4}{|c|}{ Configurations } & \multirow{2}{*}{$\begin{array}{c}\text { Calibration } \\
\text { Kappa } \\
\end{array}$} & \multirow{2}{*}{$\begin{array}{c}\mathbf{1}^{\text {st }} \text { Validation } \\
\text { Kappa }\end{array}$} & \multirow{2}{*}{$\begin{array}{c}2^{\text {nd }} \text { Validation } \\
\text { Kappa } \\
\end{array}$} & \multirow{2}{*}{$\begin{array}{c}3^{\text {rd }} \text { Validation } \\
\text { Kappa }\end{array}$} \\
\hline$\varphi_{g}$ & $\Lambda_{l}$ & $\Gamma_{n}$ & $\eta_{m}$ & & & & \\
\hline 1 & 3 & 2 & 3 & 0.85 & - & - & - \\
\hline 1 & 3 & 3 & 1 & 0.85 & - & - & - \\
\hline 1 & 3 & 3 & 2 & 0.85 & - & - & - \\
\hline 1 & 3 & 3 & 3 & 0.85 & - & - & - \\
\hline 1 & 3 & 4 & 1 & 0.85 & - & - & - \\
\hline 1 & 3 & 4 & 2 & 0.85 & - & - & - \\
\hline 1 & 3 & 4 & 3 & 0.85 & - & - & - \\
\hline 1 & 3 & 1 & 1 & 0.85 & - & - & - \\
\hline 1 & 3 & 1 & 2 & 0.85 & - & - & - \\
\hline 1 & 3 & 1 & 3 & 0.85 & - & - & - \\
\hline 1 & 3 & 6 & 1 & 0.85 & - & - & - \\
\hline 1 & 3 & 6 & 2 & 0.85 & - & - & - \\
\hline 1 & 3 & 6 & 3 & 0.85 & - & - & - \\
\hline 1 & 4 & 1 & 1 & - & - & - & - \\
\hline 1 & 4 & 1 & 2 & - & - & - & - \\
\hline 1 & 4 & 1 & 3 & - & - & - & - \\
\hline 1 & 4 & 2 & 1 & - & - & - & - \\
\hline 1 & 4 & 2 & 2 & - & - & - & - \\
\hline 1 & 4 & 2 & 3 & - & - & - & - \\
\hline 1 & 4 & 3 & 1 & - & - & - & - \\
\hline 1 & 4 & 3 & 2 & - & - & - & - \\
\hline 1 & 4 & 3 & 3 & - & - & - & - \\
\hline 1 & 4 & 4 & 1 & - & - & - & - \\
\hline 1 & 4 & 4 & 2 & - & - & - & - \\
\hline 1 & 4 & 4 & 3 & - & - & - & - \\
\hline 1 & 4 & 1 & 1 & - & - & - & - \\
\hline 1 & 4 & 1 & 2 & - & - & - & - \\
\hline 1 & 4 & 1 & 3 & - & - & - & - \\
\hline 1 & 4 & 6 & 1 & - & - & - & - \\
\hline 1 & 4 & 6 & 2 & - & - & - & - \\
\hline 1 & 4 & 6 & 3 & - & - & - & - \\
\hline 2 & 1 & 1 & 1 & 0.94 & 0.97 & 0.90 & 0.92 \\
\hline 2 & 1 & 1 & 2 & 0.94 & 0.97 & 0.90 & 0.92 \\
\hline 2 & 1 & 1 & 3 & 0.94 & 0.97 & 0.90 & 0.92 \\
\hline 2 & 1 & 2 & 1 & 0.94 & 0.97 & 0.90 & 0.92 \\
\hline 2 & 1 & 2 & 2 & 0.94 & 0.97 & 0.90 & 0.92 \\
\hline 2 & 1 & 2 & 3 & 0.94 & 0.97 & 0.90 & 0.92 \\
\hline 2 & 1 & 3 & 1 & 0.94 & 0.97 & 0.90 & 0.92 \\
\hline 2 & 1 & 3 & 2 & 0.94 & 0.97 & 0.90 & 0.92 \\
\hline 2 & 1 & 3 & 3 & 0.94 & 0.97 & 0.90 & 0.92 \\
\hline 2 & 1 & 4 & 1 & 0.94 & 0.97 & 0.90 & 0.92 \\
\hline 2 & 1 & 4 & 2 & 0.94 & 0.97 & 0.90 & 0.92 \\
\hline 2 & 1 & 4 & 3 & 0.94 & 0.97 & 0.90 & 0.92 \\
\hline 2 & 1 & 1 & 1 & 0.94 & 0.97 & 0.90 & 0.92 \\
\hline 2 & 1 & 1 & 2 & 0.94 & 0.97 & 0.90 & 0.92 \\
\hline 2 & 1 & 1 & 3 & 0.94 & 0.97 & 0.90 & 0.92 \\
\hline 2 & 1 & 6 & 1 & 0.94 & 0.97 & 0.90 & 0.92 \\
\hline 2 & 1 & 6 & 2 & 0.94 & 0.97 & 0.90 & 0.92 \\
\hline 2 & 1 & 6 & 3 & 0.94 & 0.97 & 0.90 & 0.92 \\
\hline 2 & 2 & 1 & 1 & - & - & - & - \\
\hline 2 & 2 & 1 & 2 & - & - & - & - \\
\hline
\end{tabular}


Table E.1.2 the results of calibration procedure 2 and its validations.

\begin{tabular}{|c|c|c|c|c|c|c|c|}
\hline \multicolumn{4}{|c|}{ Configurations } & \multirow{2}{*}{$\begin{array}{c}\text { Calibration } \\
\text { Kappa } \\
\end{array}$} & \multirow{2}{*}{$\begin{array}{c}\mathbf{1}^{\text {st }} \text { Validation } \\
\text { Kappa } \\
\end{array}$} & \multirow{2}{*}{$\begin{array}{c}2^{\text {nd }} \text { Validation } \\
\text { Kappa } \\
\end{array}$} & \multirow{2}{*}{$\begin{array}{c}\mathbf{3}^{\text {rd }} \text { Validation } \\
\text { Kappa } \\
\end{array}$} \\
\hline$\varphi_{g}$ & $\Lambda_{l}$ & $\Gamma_{n}$ & $\eta_{m}$ & & & & \\
\hline 2 & 2 & 1 & 3 & - & - & - & - \\
\hline 2 & 2 & 2 & 1 & - & - & - & - \\
\hline 2 & 2 & 2 & 2 & - & - & - & - \\
\hline 2 & 2 & 2 & 3 & - & - & - & - \\
\hline 2 & 2 & 3 & 1 & - & - & - & - \\
\hline 2 & 2 & 3 & 2 & - & - & - & - \\
\hline 2 & 2 & 3 & 3 & - & - & - & - \\
\hline 2 & 2 & 4 & 1 & - & - & - & - \\
\hline 2 & 2 & 4 & 2 & - & - & - & - \\
\hline 2 & 2 & 4 & 3 & - & - & - & - \\
\hline 2 & 2 & 1 & 1 & - & - & - & - \\
\hline 2 & 2 & 1 & 2 & - & - & - & - \\
\hline 2 & 2 & 1 & 3 & - & - & - & - \\
\hline 2 & 2 & 6 & 1 & - & - & - & - \\
\hline 2 & 2 & 6 & 2 & - & - & - & - \\
\hline 2 & 2 & 6 & 3 & - & - & - & - \\
\hline 2 & 3 & 1 & 1 & 0.91 & - & - & - \\
\hline 2 & 3 & 1 & 2 & 0.91 & - & - & - \\
\hline 2 & 3 & 1 & 3 & 0.91 & - & - & - \\
\hline 2 & 3 & 2 & 1 & 0.91 & - & - & - \\
\hline 2 & 3 & 2 & 2 & 0.91 & - & - & - \\
\hline 2 & 3 & 2 & 3 & 0.91 & - & - & - \\
\hline 2 & 3 & 3 & 1 & 0.91 & - & - & - \\
\hline 2 & 3 & 3 & 2 & 0.91 & - & - & - \\
\hline 2 & 3 & 3 & 3 & 0.91 & - & - & - \\
\hline 2 & 3 & 4 & 1 & 0.91 & - & - & - \\
\hline 2 & 3 & 4 & 2 & 0.91 & - & - & - \\
\hline 2 & 3 & 4 & 3 & 0.91 & - & - & - \\
\hline 2 & 3 & 1 & 1 & 0.91 & - & - & - \\
\hline 2 & 3 & 1 & 2 & 0.91 & - & - & - \\
\hline 2 & 3 & 1 & 3 & 0.91 & - & - & - \\
\hline 2 & 3 & 6 & 1 & 0.91 & - & - & - \\
\hline 2 & 3 & 6 & 2 & 0.91 & - & - & - \\
\hline 2 & 3 & 6 & 3 & 0.91 & - & - & - \\
\hline 2 & 4 & 1 & 1 & 0.95 & - & - & - \\
\hline 2 & 4 & 1 & 2 & 0.95 & - & - & - \\
\hline 2 & 4 & 1 & 3 & 0.95 & - & - & - \\
\hline 2 & 4 & 2 & 1 & 0.95 & - & - & - \\
\hline 2 & 4 & 2 & 2 & 0.95 & - & - & - \\
\hline 2 & 4 & 2 & 3 & 0.95 & - & - & - \\
\hline 2 & 4 & 3 & 1 & 0.95 & - & - & - \\
\hline 2 & 4 & 3 & 2 & 0.95 & - & - & - \\
\hline 2 & 4 & 3 & 3 & 0.95 & - & - & - \\
\hline 2 & 4 & 4 & 1 & 0.95 & - & - & - \\
\hline 2 & 4 & 4 & 2 & 0.95 & - & - & - \\
\hline 2 & 4 & 4 & 3 & 0.95 & - & - & - \\
\hline 2 & 4 & 1 & 1 & 0.95 & - & - & - \\
\hline 2 & 4 & 1 & 2 & 0.95 & - & - & - \\
\hline 2 & 4 & 1 & 3 & 0.95 & - & - & - \\
\hline 2 & 4 & 6 & 1 & 0.95 & - & - & - \\
\hline 2 & 4 & 6 & 2 & 0.95 & - & - & - \\
\hline
\end{tabular}


Table E.1.2 the results of calibration procedure 2 and its validations.

\begin{tabular}{|c|c|c|c|c|c|c|c|}
\hline \multicolumn{4}{|c|}{ Configurations } & \multirow{2}{*}{$\begin{array}{c}\text { Calibration } \\
\text { Kappa } \\
\end{array}$} & \multirow{2}{*}{$\begin{array}{c}\mathbf{1}^{\text {st }} \text { Validation } \\
\text { Kappa }\end{array}$} & \multirow{2}{*}{$\begin{array}{c}2^{\text {nd }} \text { Validation } \\
\text { Kappa } \\
\end{array}$} & \multirow{2}{*}{$\begin{array}{c}3^{\text {rd }} \text { Validation } \\
\text { Kappa }\end{array}$} \\
\hline$\varphi_{g}$ & $\Lambda_{l}$ & $\Gamma_{n}$ & $\eta_{m}$ & & & & \\
\hline 2 & 4 & 6 & 3 & 0.95 & - & - & - \\
\hline 3 & 1 & 1 & 1 & 0.93 & 0.96 & 0.91 & 0.93 \\
\hline 3 & 1 & 1 & 2 & 0.93 & 0.96 & 0.91 & 0.93 \\
\hline 3 & 1 & 1 & 3 & 0.93 & 0.96 & 0.91 & 0.93 \\
\hline 3 & 1 & 2 & 1 & 0.93 & 0.96 & 0.91 & 0.93 \\
\hline 3 & 1 & 2 & 2 & 0.93 & 0.96 & 0.91 & 0.93 \\
\hline 3 & 1 & 2 & 3 & 0.93 & 0.96 & 0.91 & 0.93 \\
\hline 3 & 1 & 3 & 1 & 0.93 & 0.96 & 0.91 & 0.93 \\
\hline 3 & 1 & 3 & 2 & 0.93 & 0.96 & 0.91 & 0.93 \\
\hline 3 & 1 & 3 & 3 & 0.93 & 0.96 & 0.91 & 0.93 \\
\hline 3 & 1 & 4 & 1 & 0.93 & 0.96 & 0.91 & 0.93 \\
\hline 3 & 1 & 4 & 2 & 0.93 & 0.96 & 0.91 & 0.93 \\
\hline 3 & 1 & 4 & 3 & 0.93 & 0.96 & 0.91 & 0.93 \\
\hline 3 & 1 & 1 & 1 & 0.93 & 0.96 & 0.91 & 0.93 \\
\hline 3 & 1 & 1 & 2 & 0.93 & 0.96 & 0.91 & 0.93 \\
\hline 3 & 1 & 1 & 3 & 0.93 & 0.96 & 0.91 & 0.93 \\
\hline 3 & 1 & 6 & 1 & 0.93 & 0.96 & 0.91 & 0.93 \\
\hline 3 & 1 & 6 & 2 & 0.93 & 0.96 & 0.91 & 0.93 \\
\hline 3 & 1 & 6 & 3 & 0.93 & 0.96 & 0.91 & 0.93 \\
\hline 3 & 2 & 1 & 1 & - & - & - & - \\
\hline 3 & 2 & 1 & 2 & - & - & - & - \\
\hline 3 & 2 & 1 & 3 & - & - & - & - \\
\hline 3 & 2 & 2 & 1 & - & - & - & - \\
\hline 3 & 2 & 2 & 2 & - & - & - & - \\
\hline 3 & 2 & 2 & 3 & - & - & - & - \\
\hline 3 & 2 & 3 & 1 & - & - & - & - \\
\hline 3 & 2 & 3 & 2 & - & - & - & - \\
\hline 3 & 2 & 3 & 3 & - & - & - & - \\
\hline 3 & 2 & 4 & 1 & - & - & - & - \\
\hline 3 & 2 & 4 & 2 & - & - & - & - \\
\hline 3 & 2 & 4 & 3 & - & - & - & - \\
\hline 3 & 2 & 1 & 1 & - & - & - & - \\
\hline 3 & 2 & 1 & 2 & - & - & - & - \\
\hline 3 & 2 & 1 & 3 & - & - & - & - \\
\hline 3 & 2 & 6 & 1 & - & - & - & - \\
\hline 3 & 2 & 6 & 2 & - & - & - & - \\
\hline 3 & 2 & 6 & 3 & - & - & - & - \\
\hline 3 & 3 & 1 & 1 & 0.91 & - & - & - \\
\hline 3 & 3 & 1 & 2 & 0.91 & - & - & - \\
\hline 3 & 3 & 1 & 3 & 0.91 & - & - & - \\
\hline 3 & 3 & 2 & 1 & 0.91 & - & - & - \\
\hline 3 & 3 & 2 & 2 & 0.91 & - & - & - \\
\hline 3 & 3 & 2 & 3 & 0.91 & - & - & - \\
\hline 3 & 3 & 3 & 1 & 0.91 & - & - & - \\
\hline 3 & 3 & 3 & 2 & 0.91 & - & - & - \\
\hline 3 & 3 & 3 & 3 & 0.91 & - & - & - \\
\hline 3 & 3 & 4 & 1 & 0.91 & - & - & - \\
\hline 3 & 3 & 4 & 2 & 0.91 & - & - & - \\
\hline 3 & 3 & 4 & 3 & 0.91 & - & - & - \\
\hline 3 & 3 & 1 & 1 & 0.91 & - & - & - \\
\hline 3 & 3 & 1 & 2 & 0.91 & - & - & - \\
\hline
\end{tabular}


Table E.1.2 the results of calibration procedure 2 and its validations.

\begin{tabular}{|c|c|c|c|c|c|c|c|}
\hline \multicolumn{4}{|c|}{ Configurations } & \multirow{2}{*}{$\begin{array}{c}\text { Calibration } \\
\text { Kappa } \\
\end{array}$} & \multirow{2}{*}{$\begin{array}{c}\mathbf{1}^{\text {st }} \text { Validation } \\
\text { Kappa } \\
\end{array}$} & \multirow{2}{*}{$\begin{array}{c}2^{\text {nd }} \text { Validation } \\
\text { Kappa } \\
\end{array}$} & \multirow{2}{*}{$\begin{array}{c}\mathbf{3}^{\text {rd }} \text { Validation } \\
\text { Kappa }\end{array}$} \\
\hline$\varphi_{g}$ & $\Lambda_{l}$ & $\Gamma_{n}$ & $\eta_{m}$ & & & & \\
\hline 3 & 3 & 1 & 3 & 0.91 & - & - & - \\
\hline 3 & 3 & 6 & 1 & 0.91 & - & - & - \\
\hline 3 & 3 & 6 & 2 & 0.91 & - & - & - \\
\hline 3 & 3 & 6 & 3 & 0.91 & - & - & - \\
\hline 3 & 4 & 1 & 1 & 0.96 & - & - & - \\
\hline 3 & 4 & 1 & 2 & 0.96 & - & - & - \\
\hline 3 & 4 & 1 & 3 & 0.96 & - & - & - \\
\hline 3 & 4 & 2 & 1 & 0.96 & - & - & - \\
\hline 3 & 4 & 2 & 2 & 0.96 & - & - & - \\
\hline 3 & 4 & 2 & 3 & 0.96 & - & - & - \\
\hline 3 & 4 & 3 & 1 & 0.96 & - & - & - \\
\hline 3 & 4 & 3 & 2 & 0.96 & - & - & - \\
\hline 3 & 4 & 3 & 3 & 0.96 & - & - & - \\
\hline 3 & 4 & 4 & 1 & 0.96 & - & - & - \\
\hline 3 & 4 & 4 & 2 & 0.96 & - & - & - \\
\hline 3 & 4 & 4 & 3 & 0.96 & - & - & - \\
\hline 3 & 4 & 1 & 1 & 0.96 & - & - & - \\
\hline 3 & 4 & 1 & 2 & 0.96 & - & - & - \\
\hline 3 & 4 & 1 & 3 & 0.96 & - & - & - \\
\hline 3 & 4 & 6 & 1 & 0.96 & - & - & - \\
\hline 3 & 4 & 6 & 2 & 0.96 & - & - & - \\
\hline 3 & 4 & 6 & 3 & 0.96 & - & - & - \\
\hline 4 & 1 & 1 & 1 & 0.96 & 0.98 & 0.87 & 0.92 \\
\hline 4 & 1 & 1 & 2 & 0.96 & 0.98 & 0.87 & 0.92 \\
\hline 4 & 1 & 1 & 3 & 0.96 & 0.98 & 0.87 & 0.92 \\
\hline 4 & 1 & 2 & 1 & 0.96 & 0.98 & 0.87 & 0.92 \\
\hline 4 & 1 & 2 & 2 & 0.96 & 0.98 & 0.87 & 0.92 \\
\hline 4 & 1 & 2 & 3 & 0.96 & 0.98 & 0.87 & 0.92 \\
\hline 4 & 1 & 3 & 1 & 0.96 & 0.98 & 0.87 & 0.92 \\
\hline 4 & 1 & 3 & 2 & 0.96 & 0.98 & 0.87 & 0.92 \\
\hline 4 & 1 & 3 & 3 & 0.96 & 0.98 & 0.87 & 0.92 \\
\hline 4 & 1 & 4 & 1 & 0.96 & 0.98 & 0.87 & 0.92 \\
\hline 4 & 1 & 4 & 2 & 0.96 & 0.98 & 0.87 & 0.92 \\
\hline 4 & 1 & 4 & 3 & 0.96 & 0.98 & 0.87 & 0.92 \\
\hline 4 & 1 & 1 & 1 & 0.96 & 0.98 & 0.87 & 0.92 \\
\hline 4 & 1 & 1 & 2 & 0.96 & 0.98 & 0.87 & 0.92 \\
\hline 4 & 1 & 1 & 3 & 0.96 & 0.98 & 0.87 & 0.92 \\
\hline 4 & 1 & 6 & 1 & 0.96 & 0.98 & 0.87 & 0.92 \\
\hline 4 & 1 & 6 & 2 & 0.96 & 0.98 & 0.87 & 0.92 \\
\hline 4 & 1 & 6 & 3 & 0.96 & 0.98 & 0.87 & 0.92 \\
\hline 4 & 2 & 1 & 1 & 0.90 & 0.94 & 0.84 & 0.88 \\
\hline 4 & 2 & 1 & 2 & 0.90 & 0.94 & 0.84 & 0.88 \\
\hline 4 & 2 & 1 & 3 & 0.90 & 0.94 & 0.84 & 0.88 \\
\hline 4 & 2 & 2 & 1 & 0.90 & 0.94 & 0.84 & 0.88 \\
\hline 4 & 2 & 2 & 2 & 0.90 & 0.94 & 0.84 & 0.88 \\
\hline 4 & 2 & 2 & 3 & 0.90 & 0.94 & 0.84 & 0.88 \\
\hline 4 & 2 & 3 & 1 & 0.90 & 0.94 & 0.84 & 0.88 \\
\hline 4 & 2 & 3 & 2 & 0.90 & 0.94 & 0.84 & 0.88 \\
\hline 4 & 2 & 3 & 3 & 0.90 & 0.94 & 0.84 & 0.88 \\
\hline 4 & 2 & 4 & 1 & 0.90 & 0.94 & 0.84 & 0.88 \\
\hline 4 & 2 & 4 & 2 & 0.90 & 0.94 & 0.84 & 0.88 \\
\hline
\end{tabular}


Table E.1.2 the results of calibration procedure 2 and its validations.

\begin{tabular}{|c|c|c|c|c|c|c|c|}
\hline \multicolumn{4}{|c|}{ Configurations } & \multirow{2}{*}{$\begin{array}{c}\text { Calibration } \\
\text { Kappa } \\
\end{array}$} & \multirow{2}{*}{$\begin{array}{c}\mathbf{1}^{\text {st }} \text { Validation } \\
\text { Kappa }\end{array}$} & \multirow{2}{*}{$\begin{array}{c}2^{\text {nd }} \text { Validation } \\
\text { Kappa } \\
\end{array}$} & \multirow{2}{*}{$\begin{array}{c}3^{\text {rd }} \text { Validation } \\
\text { Kappa }\end{array}$} \\
\hline$\varphi_{g}$ & $\Lambda_{l}$ & $\Gamma_{n}$ & $\eta_{m}$ & & & & \\
\hline 4 & 2 & 4 & 3 & 0.90 & 0.94 & 0.84 & 0.88 \\
\hline 4 & 2 & 1 & 1 & 0.90 & 0.94 & 0.84 & 0.88 \\
\hline 4 & 2 & 1 & 2 & 0.90 & 0.94 & 0.84 & 0.88 \\
\hline 4 & 2 & 1 & 3 & 0.90 & 0.94 & 0.84 & 0.88 \\
\hline 4 & 2 & 6 & 1 & 0.90 & 0.94 & 0.84 & 0.88 \\
\hline 4 & 2 & 6 & 2 & 0.90 & 0.94 & 0.84 & 0.88 \\
\hline 4 & 2 & 6 & 3 & 0.90 & 0.94 & 0.84 & 0.88 \\
\hline 4 & 3 & 1 & 1 & 0.92 & - & - & - \\
\hline 4 & 3 & 1 & 2 & 0.92 & - & - & - \\
\hline 4 & 3 & 1 & 3 & 0.92 & - & - & - \\
\hline 4 & 3 & 2 & 1 & 0.92 & - & - & - \\
\hline 4 & 3 & 2 & 2 & 0.92 & - & - & - \\
\hline 4 & 3 & 2 & 3 & 0.92 & - & - & - \\
\hline 4 & 3 & 3 & 1 & 0.92 & - & - & - \\
\hline 4 & 3 & 3 & 2 & 0.92 & - & - & - \\
\hline 4 & 3 & 3 & 3 & 0.92 & - & - & - \\
\hline 4 & 3 & 4 & 1 & 0.92 & - & - & - \\
\hline 4 & 3 & 4 & 2 & 0.92 & - & - & - \\
\hline 4 & 3 & 4 & 3 & 0.92 & - & - & - \\
\hline 4 & 3 & 1 & 1 & 0.92 & - & - & - \\
\hline 4 & 3 & 1 & 2 & 0.92 & - & - & - \\
\hline 4 & 3 & 1 & 3 & 0.92 & - & - & - \\
\hline 4 & 3 & 6 & 1 & 0.92 & - & - & - \\
\hline 4 & 3 & 6 & 2 & 0.92 & - & - & - \\
\hline 4 & 3 & 6 & 3 & 0.92 & - & - & - \\
\hline 4 & 4 & 1 & 1 & 0.98 & - & - & - \\
\hline 4 & 4 & 1 & 2 & 0.98 & - & - & - \\
\hline 4 & 4 & 1 & 3 & 0.98 & - & - & - \\
\hline 4 & 4 & 2 & 1 & 0.98 & - & - & - \\
\hline 4 & 4 & 2 & 2 & 0.98 & - & - & - \\
\hline 4 & 4 & 2 & 3 & 0.98 & - & - & - \\
\hline 4 & 4 & 3 & 1 & 0.98 & - & - & - \\
\hline 4 & 4 & 3 & 2 & 0.98 & - & - & - \\
\hline 4 & 4 & 3 & 3 & 0.98 & - & - & - \\
\hline 4 & 4 & 4 & 1 & 0.98 & - & - & - \\
\hline 4 & 4 & 4 & 2 & 0.98 & - & - & - \\
\hline 4 & 4 & 4 & 3 & 0.98 & - & - & - \\
\hline 4 & 4 & 1 & 1 & 0.98 & - & - & - \\
\hline 4 & 4 & 1 & 2 & 0.98 & - & - & - \\
\hline 4 & 4 & 1 & 3 & 0.98 & - & - & - \\
\hline 4 & 4 & 6 & 1 & 0.98 & - & - & - \\
\hline 4 & 4 & 6 & 2 & 0.98 & - & - & - \\
\hline 4 & 4 & 6 & 3 & 0.98 & - & - & - \\
\hline 5 & 1 & 1 & 1 & 0.97 & 0.97 & 0.93 & 0.95 \\
\hline 5 & 1 & 1 & 2 & 0.97 & 0.97 & 0.93 & 0.95 \\
\hline 5 & 1 & 1 & 3 & 0.97 & 0.97 & 0.93 & 0.95 \\
\hline 5 & 1 & 2 & 1 & 0.97 & 0.97 & 0.93 & 0.95 \\
\hline 5 & 1 & 2 & 2 & 0.97 & 0.97 & 0.93 & 0.95 \\
\hline 5 & 1 & 2 & 3 & 0.97 & 0.97 & 0.93 & 0.95 \\
\hline 5 & 1 & 3 & 1 & 0.97 & 0.97 & 0.93 & 0.95 \\
\hline 5 & 1 & 3 & 2 & 0.97 & 0.97 & 0.93 & 0.95 \\
\hline
\end{tabular}


Table E.1.2 the results of calibration procedure 2 and its validations.

\begin{tabular}{|c|c|c|c|c|c|c|c|}
\hline \multicolumn{4}{|c|}{ Configurations } & \multirow{2}{*}{$\begin{array}{c}\text { Calibration } \\
\text { Kappa } \\
\end{array}$} & \multirow{2}{*}{$\begin{array}{c}\mathbf{1}^{\text {st }} \text { Validation } \\
\text { Kappa }\end{array}$} & \multirow{2}{*}{$\begin{array}{c}2^{\text {nd }} \text { Validation } \\
\text { Kappa } \\
\end{array}$} & \multirow{2}{*}{$\begin{array}{c}3^{\text {rd }} \text { Validation } \\
\text { Kappa }\end{array}$} \\
\hline$\varphi_{g}$ & $\Lambda_{l}$ & $\Gamma_{n}$ & $\eta_{m}$ & & & & \\
\hline 5 & 1 & 3 & 3 & 0.97 & 0.97 & 0.93 & 0.95 \\
\hline 5 & 1 & 4 & 1 & 0.97 & 0.97 & 0.93 & 0.95 \\
\hline 5 & 1 & 4 & 2 & 0.97 & 0.97 & 0.93 & 0.95 \\
\hline 5 & 1 & 4 & 3 & 0.97 & 0.97 & 0.93 & 0.95 \\
\hline 5 & 1 & 1 & 1 & 0.97 & 0.97 & 0.93 & 0.95 \\
\hline 5 & 1 & 1 & 2 & 0.97 & 0.97 & 0.93 & 0.95 \\
\hline 5 & 1 & 1 & 3 & 0.97 & 0.97 & 0.93 & 0.95 \\
\hline 5 & 1 & 6 & 1 & 0.97 & 0.97 & 0.93 & 0.95 \\
\hline 5 & 1 & 6 & 2 & 0.97 & 0.97 & 0.93 & 0.95 \\
\hline 5 & 1 & 6 & 3 & 0.97 & 0.97 & 0.93 & 0.95 \\
\hline 5 & 2 & 1 & 1 & - & - & - & - \\
\hline 5 & 2 & 1 & 2 & - & - & - & - \\
\hline 5 & 2 & 1 & 3 & - & - & - & - \\
\hline 5 & 2 & 2 & 1 & - & - & - & - \\
\hline 5 & 2 & 2 & 2 & - & - & - & - \\
\hline 5 & 2 & 2 & 3 & - & - & - & - \\
\hline 5 & 2 & 3 & 1 & - & - & - & - \\
\hline 5 & 2 & 3 & 2 & - & - & - & - \\
\hline 5 & 2 & 3 & 3 & - & - & - & - \\
\hline 5 & 2 & 4 & 1 & - & - & - & - \\
\hline 5 & 2 & 4 & 2 & - & - & - & - \\
\hline 5 & 2 & 4 & 3 & - & - & - & - \\
\hline 5 & 2 & 1 & 1 & - & - & - & - \\
\hline 5 & 2 & 1 & 2 & - & - & - & - \\
\hline 5 & 2 & 1 & 3 & - & - & - & - \\
\hline 5 & 2 & 6 & 1 & - & - & - & - \\
\hline 5 & 2 & 6 & 2 & - & - & - & - \\
\hline 5 & 2 & 6 & 3 & - & - & - & - \\
\hline 5 & 3 & 1 & 1 & - & - & - & - \\
\hline 5 & 3 & 1 & 2 & - & - & - & - \\
\hline 5 & 3 & 1 & 3 & - & - & - & - \\
\hline 5 & 3 & 2 & 1 & - & - & - & - \\
\hline 5 & 3 & 2 & 2 & - & - & - & - \\
\hline 5 & 3 & 2 & 3 & - & - & - & - \\
\hline 5 & 3 & 3 & 1 & - & - & - & - \\
\hline 5 & 3 & 3 & 2 & - & - & - & - \\
\hline 5 & 3 & 3 & 3 & - & - & - & - \\
\hline 5 & 3 & 4 & 1 & - & - & - & - \\
\hline 5 & 3 & 4 & 2 & - & - & - & - \\
\hline 5 & 3 & 4 & 3 & - & - & - & - \\
\hline 5 & 3 & 1 & 1 & - & - & - & - \\
\hline 5 & 3 & 1 & 2 & - & - & - & - \\
\hline 5 & 3 & 1 & 3 & - & - & - & - \\
\hline 5 & 3 & 6 & 1 & - & - & - & - \\
\hline 5 & 3 & 6 & 2 & - & - & - & - \\
\hline 5 & 3 & 6 & 3 & - & - & - & - \\
\hline 5 & 4 & 1 & 1 & 1.00 & - & - & - \\
\hline 5 & 4 & 1 & 2 & 1.00 & - & - & - \\
\hline 5 & 4 & 1 & 3 & 1.00 & - & - & - \\
\hline 5 & 4 & 2 & 1 & 1.00 & - & - & - \\
\hline 5 & 4 & 2 & 2 & 1.00 & - & - & - \\
\hline
\end{tabular}


Table E.1.2 the results of calibration procedure 2 and its validations.

\begin{tabular}{|c|c|c|c|c|c|c|c|}
\hline \multicolumn{4}{|c|}{ Configurations } & \multirow{2}{*}{$\begin{array}{c}\text { Calibration } \\
\text { Kappa } \\
\end{array}$} & \multirow{2}{*}{$\begin{array}{c}\mathbf{1}^{\text {st }} \text { Validation } \\
\text { Kappa }\end{array}$} & \multirow{2}{*}{$\begin{array}{c}2^{\text {nd }} \text { Validation } \\
\text { Kappa } \\
\end{array}$} & \multirow{2}{*}{$\begin{array}{c}3^{\text {rd }} \text { Validation } \\
\text { Kappa }\end{array}$} \\
\hline$\varphi_{g}$ & $\Lambda_{l}$ & $\Gamma_{n}$ & $\eta_{m}$ & & & & \\
\hline 5 & 4 & 2 & 3 & 1.00 & - & - & - \\
\hline 5 & 4 & 3 & 1 & 1.00 & - & - & - \\
\hline 5 & 4 & 3 & 2 & 1.00 & - & - & - \\
\hline 5 & 4 & 3 & 3 & 1.00 & - & - & - \\
\hline 5 & 4 & 4 & 1 & 1.00 & - & - & - \\
\hline 5 & 4 & 4 & 2 & 1.00 & - & - & - \\
\hline 5 & 4 & 4 & 3 & 1.00 & - & - & - \\
\hline 5 & 4 & 1 & 1 & 1.00 & - & - & - \\
\hline 5 & 4 & 1 & 2 & 1.00 & - & - & - \\
\hline 5 & 4 & 1 & 3 & 1.00 & - & - & - \\
\hline 5 & 4 & 6 & 1 & 1.00 & - & - & - \\
\hline 5 & 4 & 6 & 2 & 1.00 & - & - & - \\
\hline 5 & 4 & 6 & 3 & 1.00 & - & - & - \\
\hline
\end{tabular}

Table E.1.3 the results of calibration and validations based on the average speed

\begin{tabular}{|c|c|c|c|c|c|}
\hline \multicolumn{2}{|c|}{ Configurations } & \multirow{2}{*}{$\begin{array}{c}\text { Calibration } \\
\text { Kappa } \\
\end{array}$} & \multirow{2}{*}{$\begin{array}{c}\mathbf{1}^{\text {st }} \text { Validation } \\
\text { Kappa } \\
\end{array}$} & \multirow{2}{*}{$\begin{array}{cc}2^{\text {nd }} & \text { Validation } \\
\text { Kappa } \\
\end{array}$} & \multirow{2}{*}{$\begin{array}{c}3^{\text {rd }} \text { Validation } \\
\text { Kappa }\end{array}$} \\
\hline$\varphi_{g}$ & $\Lambda_{l}$ & & & & \\
\hline 1 & 1 & 0.88 & 0.94 & 0.90 & 0.92 \\
\hline 1 & 2 & 0.82 & - & - & - \\
\hline 1 & 3 & - & - & - & - \\
\hline 1 & 4 & - & - & - & - \\
\hline 2 & 1 & 0.94 & 0.97 & 0.90 & 0.92 \\
\hline 2 & 2 & - & - & - & - \\
\hline 2 & 3 & - & - & - & - \\
\hline 2 & 4 & - & - & - & - \\
\hline 3 & 1 & 0.93 & 0.96 & 0.91 & 0.93 \\
\hline 3 & 2 & 0.86 & - & - & - \\
\hline 3 & 3 & - & - & - & - \\
\hline 3 & 4 & 0.84 & - & - & - \\
\hline 4 & 1 & 0.96 & 0.98 & 0.87 & 0.92 \\
\hline 4 & 2 & 0.90 & 0.94 & 0.84 & 0.88 \\
\hline 4 & 3 & - & - & - & - \\
\hline 4 & 4 & 0.88 & 0.92 & 0.84 & 0.88 \\
\hline 5 & 1 & 0.97 & 0.97 & 0.93 & 0.95 \\
\hline 5 & 2 & 0.93 & 0.95 & - & - \\
\hline 5 & 3 & - & - & - & - \\
\hline 5 & 4 & 0.93 & 0.92 & - & - \\
\hline
\end{tabular}


Table E.1.4 the results of calibration and validations based on the maximum speed

\begin{tabular}{|c|c|c|c|c|c|}
\hline \multicolumn{2}{|c|}{ Configurations } & \multirow{2}{*}{$\begin{array}{c}\text { Calibration } \\
\text { Kappa }\end{array}$} & \multirow{2}{*}{$\begin{array}{c}\mathbf{1}^{\text {st }} \text { Validation } \\
\text { Kappa }\end{array}$} & \multirow{2}{*}{$\begin{array}{c}2^{\text {nd }} \text { Validation } \\
\text { Kappa }\end{array}$} & \multirow{2}{*}{$\frac{3^{\text {rd }} \text { Validation }}{\text { Kappa }}$} \\
\hline$\varphi_{g}$ & $\Lambda_{l}$ & & & & \\
\hline 1 & 1 & 0.84 & 0.80 & 0.83 & 0.83 \\
\hline 1 & 2 & 0.75 & 0.74 & - & - \\
\hline 1 & 3 & - & - & - & - \\
\hline 1 & 4 & - & - & - & - \\
\hline 2 & 1 & 0.87 & 0.82 & 0.83 & 0.83 \\
\hline 2 & 2 & 0.77 & 0.75 & - & - \\
\hline 2 & 3 & - & - & - & - \\
\hline 2 & 4 & - & - & - & - \\
\hline 3 & 1 & 0.89 & 0.89 & 0.89 & 0.89 \\
\hline 3 & 2 & 0.84 & 0.78 & - & - \\
\hline 3 & 3 & - & - & - & - \\
\hline 3 & 4 & 0.77 & - & - & - \\
\hline 4 & 1 & 0.91 & 0.89 & 0.86 & 0.88 \\
\hline 4 & 2 & 0.88 & - & - & - \\
\hline 4 & 3 & - & - & - & - \\
\hline 4 & 4 & 0.80 & - & - & - \\
\hline 5 & 1 & 0.90 & 0.87 & 0.76 & 0.82 \\
\hline 5 & 2 & 0.88 & 0.76 & 0.70 & 0.74 \\
\hline 5 & 3 & 0.80 & 0.76 & 0.76 & 0.78 \\
\hline 5 & 4 & 0.81 & 0.77 & 0.73 & 0.77 \\
\hline
\end{tabular}

Table E.1.5 the results of calibration and validations based on the average acceleration

\begin{tabular}{|c|c|c|c|c|c|}
\hline \multicolumn{2}{|c|}{ Configurations } & \multirow{2}{*}{$\begin{array}{c}\text { Calibration } \\
\text { Kappa } \\
\end{array}$} & \multirow{2}{*}{$\begin{array}{c}\mathbf{1}^{\text {st }} \text { Validation } \\
\text { Kappa } \\
\end{array}$} & \multirow{2}{*}{$\begin{array}{cc}2^{\text {nd }} & \text { Validation } \\
\text { Kappa } \\
\end{array}$} & \multirow{2}{*}{$\begin{array}{c}\mathbf{3}^{\text {rd }} \text { Validation } \\
\text { Kappa } \\
\end{array}$} \\
\hline$\varphi_{g}$ & $\Lambda_{l}$ & & & & \\
\hline 1 & 1 & 0.06 & 0.09 & 0.08 & 0.09 \\
\hline 1 & 2 & 0.05 & - & - & - \\
\hline 1 & 3 & 0.04 & - & - & - \\
\hline 1 & 4 & 0.06 & 0.02 & - & - \\
\hline 2 & 1 & 0.11 & - & - & - \\
\hline 2 & 2 & 0.10 & - & - & - \\
\hline 2 & 3 & 0.08 & - & - & - \\
\hline 2 & 4 & 0.06 & - & - & - \\
\hline 3 & 1 & 0.10 & 0.10 & - & - \\
\hline 3 & 2 & 0.10 & - & - & - \\
\hline 3 & 3 & 0.06 & 0.01 & - & - \\
\hline 3 & 4 & 0.07 & 0.01 & - & - \\
\hline 4 & 1 & 0.13 & 0.12 & - & - \\
\hline 4 & 2 & 0.12 & - & - & - \\
\hline 4 & 3 & 0.09 & - & - & - \\
\hline 4 & 4 & 0.09 & 0.03 & - & - \\
\hline 5 & 1 & 0.16 & 0.11 & - & - \\
\hline 5 & 2 & 0.15 & - & - & - \\
\hline 5 & 3 & 0.11 & - & - & - \\
\hline 5 & 4 & 0.11 & - & - & - \\
\hline
\end{tabular}


Table E.1.6 the results of calibration and validations based on the maximum acceleration

\begin{tabular}{|c|c|c|c|c|c|}
\hline \multicolumn{2}{|c|}{ Configurations } & \multirow{2}{*}{$\begin{array}{c}\text { Calibration } \\
\text { Kappa } \\
\end{array}$} & \multirow{2}{*}{$\begin{array}{c}\mathbf{1}^{\text {st }} \text { Validation } \\
\text { Kappa }\end{array}$} & \multirow{2}{*}{$\begin{array}{c}2^{\text {nd }} \text { Validation } \\
\text { Kappa } \\
\end{array}$} & \multirow{2}{*}{$\begin{array}{c}3^{\text {rd }} \text { Validation } \\
\text { Kappa }\end{array}$} \\
\hline$\varphi_{g}$ & $\Lambda_{l}$ & & & & \\
\hline 1 & 1 & 0.11 & - & - & - \\
\hline 1 & 2 & 0.11 & - & - & - \\
\hline 1 & 3 & 0.10 & 0.02 & - & - \\
\hline 1 & 4 & 0.10 & 0.02 & - & - \\
\hline 2 & 1 & 0.18 & - & - & - \\
\hline 2 & 2 & 0.18 & - & - & - \\
\hline 2 & 3 & 0.17 & - & - & - \\
\hline 2 & 4 & 0.16 & 0.01 & - & - \\
\hline 3 & 1 & 0.20 & 0.08 & - & - \\
\hline 3 & 2 & 0.20 & 0.09 & - & - \\
\hline 3 & 3 & 0.18 & 0.10 & - & - \\
\hline 3 & 4 & 0.20 & 0.09 & - & - \\
\hline 4 & 1 & 0.17 & 0.05 & - & - \\
\hline 4 & 2 & 0.17 & 0.08 & - & - \\
\hline 4 & 3 & 0.16 & 0.08 & - & - \\
\hline 4 & 4 & 0.16 & 0.07 & - & - \\
\hline 5 & 1 & 0.23 & 0.04 & - & - \\
\hline 5 & 2 & 0.24 & 0.05 & - & - \\
\hline 5 & 3 & 0.21 & 0.06 & - & - \\
\hline 5 & 4 & 0.21 & 0.06 & - & - \\
\hline
\end{tabular}

Table E.1.7 the results of calibration and validations based on the average deceleration

\begin{tabular}{|c|c|c|c|c|c|}
\hline \multicolumn{2}{|c|}{ Configurations } & \multirow{2}{*}{$\begin{array}{c}\text { Calibration } \\
\text { Kappa } \\
\end{array}$} & \multirow{2}{*}{$\begin{array}{c}\mathbf{1}^{\text {st }} \text { Validation } \\
\text { Kappa } \\
\end{array}$} & \multirow{2}{*}{$\begin{array}{cc}2^{\text {nd }} & \text { Validation } \\
\text { Kappa } \\
\end{array}$} & \multirow{2}{*}{$\begin{array}{c}3^{\text {rd }} \text { Validation } \\
\text { Kappa } \\
\end{array}$} \\
\hline$\varphi_{g}$ & $\Lambda_{l}$ & & & & \\
\hline 1 & 1 & 0.13 & 0.03 & 0.14 & 0.11 \\
\hline 1 & 2 & 0.13 & 0.02 & 0.16 & 0.12 \\
\hline 1 & 3 & 0.12 & 0.03 & 0.11 & 0.09 \\
\hline 1 & 4 & 0.12 & - & - & - \\
\hline 2 & 1 & 0.10 & 0.02 & 0.15 & 0.12 \\
\hline 2 & 2 & 0.10 & 0.01 & 0.17 & 0.13 \\
\hline 2 & 3 & 0.10 & 0.02 & 0.15 & 0.12 \\
\hline 2 & 4 & 0.10 & - & - & - \\
\hline 3 & 1 & 0.13 & 0.04 & 0.14 & 0.12 \\
\hline 3 & 2 & 0.13 & 0.02 & 0.15 & 0.13 \\
\hline 3 & 3 & 0.13 & 0.04 & 0.13 & 0.12 \\
\hline 3 & 4 & 0.13 & - & - & - \\
\hline 4 & 1 & 0.17 & 0.02 & 0.15 & 0.13 \\
\hline 4 & 2 & 0.17 & - & - & - \\
\hline 4 & 3 & 0.17 & - & - & - \\
\hline 4 & 4 & 0.16 & - & - & - \\
\hline 5 & 1 & 0.19 & - & - & - \\
\hline 5 & 2 & 0.19 & - & - & - \\
\hline 5 & 3 & 0.19 & - & - & - \\
\hline 5 & 4 & 0.19 & - & - & - \\
\hline
\end{tabular}


Table E.1.8 the results of calibration and validations based on the maximum deceleration

\begin{tabular}{|c|c|c|c|c|c|}
\hline \multicolumn{2}{|c|}{ Configurations } & \multirow{2}{*}{$\begin{array}{c}\text { Calibration } \\
\text { Kappa }\end{array}$} & \multirow{2}{*}{$\begin{array}{c}\mathbf{1}^{\text {st }} \text { Validation } \\
\text { Kappa }\end{array}$} & \multirow{2}{*}{$\frac{2^{\text {nd }} \text { Validation }}{\text { Kappa }}$} & \multirow{2}{*}{$\frac{3^{\text {rd }} \text { Validation }}{\text { Kappa }}$} \\
\hline$\varphi_{g}$ & $\Lambda_{l}$ & & & & \\
\hline 1 & 1 & 0.12 & 0.02 & - & - \\
\hline 1 & 2 & 0.14 & 0.03 & - & - \\
\hline 1 & 3 & 0.12 & 0.06 & - & - \\
\hline 1 & 4 & 0.13 & 0.04 & - & - \\
\hline 2 & 1 & 0.20 & 0.08 & - & - \\
\hline 2 & 2 & 0.20 & 0.05 & - & - \\
\hline 2 & 3 & 0.18 & 0.07 & - & - \\
\hline 2 & 4 & 0.19 & 0.09 & - & - \\
\hline 3 & 1 & 0.16 & 0.13 & - & - \\
\hline 3 & 2 & 0.15 & 0.14 & - & - \\
\hline 3 & 3 & 0.14 & 0.11 & - & - \\
\hline 3 & 4 & 0.14 & 0.12 & - & - \\
\hline 4 & 1 & 0.20 & 0.17 & - & - \\
\hline 4 & 2 & 0.19 & 0.17 & - & - \\
\hline 4 & 3 & 0.17 & 0.14 & - & - \\
\hline 4 & 4 & 0.18 & 0.15 & - & - \\
\hline 5 & 1 & 0.28 & 0.17 & - & - \\
\hline 5 & 2 & 0.26 & 0.19 & - & - \\
\hline 5 & 3 & 0.24 & 0.13 & - & - \\
\hline 5 & 4 & 0.26 & 0.15 & - & - \\
\hline
\end{tabular}

Table E.1.9 the results of calibration and validations based on the average direction

\begin{tabular}{|c|c|c|c|c|c|}
\hline \multicolumn{2}{|c|}{ Configurations } & \multirow{2}{*}{$\begin{array}{c}\text { Calibration } \\
\text { Kappa }\end{array}$} & \multirow{2}{*}{$\begin{array}{c}\mathbf{1}^{\text {st }} \text { Validation } \\
\text { Kappa }\end{array}$} & \multirow{2}{*}{$\begin{array}{c}2^{\text {nd }} \text { Validation } \\
\text { Kappa }\end{array}$} & \multirow{2}{*}{$\begin{array}{c}3^{\text {rd }} \text { Validation } \\
\text { Kappa }\end{array}$} \\
\hline$\varphi_{g}$ & $\Lambda_{l}$ & & & & \\
\hline 1 & 1 & 0.31 & 0.33 & 0.23 & 0.26 \\
\hline 1 & 2 & 0.31 & 0.27 & 0.23 & 0.25 \\
\hline 1 & 3 & 0.31 & 0.27 & 0.23 & 0.25 \\
\hline 1 & 4 & 0.30 & 0.27 & 0.23 & 0.25 \\
\hline 2 & 1 & 0.34 & 0.25 & 0.22 & 0.22 \\
\hline 2 & 2 & 0.32 & 0.25 & 0.22 & 0.22 \\
\hline 2 & 3 & 0.33 & 0.29 & 0.22 & 0.24 \\
\hline 2 & 4 & 0.32 & 0.25 & 0.22 & 0.22 \\
\hline 3 & 1 & 0.32 & 0.30 & 0.21 & 0.23 \\
\hline 3 & 2 & 0.30 & 0.30 & 0.21 & 0.23 \\
\hline 3 & 3 & 0.31 & 0.25 & 0.22 & 0.22 \\
\hline 3 & 4 & 0.30 & 0.30 & 0.21 & 0.23 \\
\hline 4 & 1 & 0.36 & 0.30 & 0.18 & 0.20 \\
\hline 4 & 2 & 0.34 & 0.30 & 0.18 & 0.20 \\
\hline 4 & 3 & 0.35 & 0.26 & 0.18 & 0.19 \\
\hline 4 & 4 & 0.34 & 0.30 & 0.18 & 0.20 \\
\hline 5 & 1 & 0.38 & 0.32 & 0.13 & 0.18 \\
\hline 5 & 2 & 0.36 & 0.32 & 0.13 & 0.18 \\
\hline 5 & 3 & 0.36 & 0.32 & 0.14 & 0.18 \\
\hline 5 & 4 & 0.36 & 0.32 & 0.13 & 0.18 \\
\hline
\end{tabular}


Table E.1.10 the results of calibration and validations based on the maximum direction

\begin{tabular}{|c|c|c|c|c|c|}
\hline \multicolumn{2}{|c|}{ Configurations } & \multirow{2}{*}{$\begin{array}{c}\text { Calibration } \\
\text { Kappa }\end{array}$} & \multirow{2}{*}{$\begin{array}{c}\mathbf{1}^{\text {st }} \text { Validation } \\
\text { Kappa }\end{array}$} & \multirow{2}{*}{$\begin{array}{c}2^{\text {nd }} \text { Validation } \\
\text { Kappa }\end{array}$} & \multirow{2}{*}{$\frac{3^{\text {rd }} \text { Validation }}{\text { Kappa }}$} \\
\hline$\varphi_{g}$ & $\Lambda_{l}$ & & & & \\
\hline 1 & 1 & 0.25 & 0.19 & 0.19 & 0.19 \\
\hline 1 & 2 & 0.25 & 0.19 & 0.19 & 0.19 \\
\hline 1 & 3 & 0.25 & 0.19 & 0.19 & 0.19 \\
\hline 1 & 4 & 0.25 & 0.20 & 0.19 & 0.20 \\
\hline 2 & 1 & 0.27 & 0.18 & 0.19 & 0.19 \\
\hline 2 & 2 & 0.27 & 0.16 & 0.19 & 0.19 \\
\hline 2 & 3 & 0.27 & 0.17 & 0.19 & 0.19 \\
\hline 2 & 4 & 0.27 & 0.16 & 0.19 & 0.19 \\
\hline 3 & 1 & 0.26 & 0.18 & 0.18 & 0.19 \\
\hline 3 & 2 & 0.26 & 0.18 & 0.18 & 0.19 \\
\hline 3 & 3 & 0.26 & 0.18 & 0.18 & 0.19 \\
\hline 3 & 4 & 0.26 & 0.18 & 0.18 & 0.19 \\
\hline 4 & 1 & 0.26 & 0.15 & 0.14 & 0.16 \\
\hline 4 & 2 & 0.26 & 0.15 & 0.14 & 0.16 \\
\hline 4 & 3 & 0.26 & 0.15 & 0.14 & 0.16 \\
\hline 4 & 4 & 0.26 & 0.15 & 0.14 & 0.16 \\
\hline 5 & 1 & 0.26 & 0.18 & 0.10 & 0.13 \\
\hline 5 & 2 & 0.26 & 0.18 & 0.10 & 0.13 \\
\hline 5 & 3 & 0.26 & 0.18 & 0.10 & 0.13 \\
\hline 5 & 4 & 0.26 & 0.18 & 0.10 & 0.13 \\
\hline
\end{tabular}

Table E.1.11 the results of calibration and validations based on the average of change in direction

\begin{tabular}{|c|c|c|c|c|c|}
\hline \multicolumn{2}{|c|}{ Configurations } & \multirow{2}{*}{$\begin{array}{c}\text { Calibration } \\
\text { Kappa } \\
\end{array}$} & \multirow{2}{*}{$\begin{array}{c}\mathbf{1}^{\text {st }} \text { Validation } \\
\text { Kappa }\end{array}$} & \multirow{2}{*}{$\begin{array}{c}2^{\text {nd }} \text { Validation } \\
\text { Kappa } \\
\end{array}$} & \multirow{2}{*}{$\begin{array}{c}3^{\text {rd }} \text { Validation } \\
\text { Kappa }\end{array}$} \\
\hline$\varphi_{g}$ & $\Lambda_{l}$ & & & & \\
\hline 1 & 1 & 0.24 & 0.19 & 0.11 & 0.14 \\
\hline 1 & 2 & 0.26 & 0.21 & 0.14 & 0.16 \\
\hline 1 & 3 & 0.30 & 0.22 & 0.14 & 0.17 \\
\hline 1 & 4 & 0.25 & 0.14 & 0.11 & 0.13 \\
\hline 2 & 1 & 0.31 & 0.18 & 0.11 & 0.14 \\
\hline 2 & 2 & 0.33 & 0.26 & 0.14 & 0.18 \\
\hline 2 & 3 & 0.38 & 0.29 & 0.14 & 0.19 \\
\hline 2 & 4 & 0.28 & 0.20 & 0.11 & 0.14 \\
\hline 3 & 1 & 0.26 & 0.12 & 0.14 & 0.14 \\
\hline 3 & 2 & 0.28 & 0.21 & 0.17 & 0.19 \\
\hline 3 & 3 & 0.34 & 0.24 & 0.18 & 0.20 \\
\hline 3 & 4 & 0.26 & 0.14 & 0.14 & 0.16 \\
\hline 4 & 1 & 0.28 & 0.19 & 0.20 & 0.23 \\
\hline 4 & 2 & 0.29 & 0.20 & 0.17 & 0.19 \\
\hline 4 & 3 & 0.32 & 0.16 & 0.15 & 0.17 \\
\hline 4 & 4 & 0.26 & 0.11 & 0.14 & 0.16 \\
\hline 5 & 1 & 0.31 & 0.25 & 0.15 & 0.22 \\
\hline 5 & 2 & 0.32 & 0.27 & 0.13 & 0.19 \\
\hline 5 & 3 & 0.37 & 0.20 & 0.11 & 0.15 \\
\hline 5 & 4 & 0.28 & 0.19 & 0.11 & 0.16 \\
\hline
\end{tabular}


Table E.1.12 the results of calibration and validations based on the average of cosine of change in direction

\begin{tabular}{|c|c|c|c|c|c|}
\hline \multicolumn{2}{|c|}{ Configurations } & \multirow{2}{*}{$\begin{array}{c}\text { Calibration } \\
\text { Kappa }\end{array}$} & \multirow{2}{*}{$\begin{array}{c}\mathbf{1}^{\text {st }} \text { Validation } \\
\text { Kappa }\end{array}$} & \multirow{2}{*}{$\begin{array}{c}2^{\text {nd }} \text { Validation } \\
\text { Kappa }\end{array}$} & \multirow{2}{*}{$\begin{array}{c}\mathbf{3}^{\text {rd }} \text { Validation } \\
\text { Kappa }\end{array}$} \\
\hline$\varphi_{g}$ & $\Lambda_{l}$ & & & & \\
\hline 1 & 1 & 0.11 & 0.11 & 0.10 & 0.10 \\
\hline 1 & 2 & 0.11 & 0.11 & 0.10 & 0.10 \\
\hline 1 & 3 & 0.11 & 0.11 & 0.06 & 0.08 \\
\hline 1 & 4 & 0.09 & 0.11 & 0.10 & 0.10 \\
\hline 2 & 1 & 0.11 & 0.13 & 0.10 & 0.11 \\
\hline 2 & 2 & 0.11 & 0.13 & 0.10 & 0.11 \\
\hline 2 & 3 & 0.11 & 0.14 & 0.09 & 0.10 \\
\hline 2 & 4 & 0.11 & 0.13 & 0.10 & 0.11 \\
\hline 3 & 1 & 0.10 & 0.13 & 0.08 & 0.10 \\
\hline 3 & 2 & 0.10 & 0.13 & 0.08 & 0.10 \\
\hline 3 & 3 & 0.15 & 0.20 & 0.02 & 0.06 \\
\hline 3 & 4 & 0.10 & 0.13 & 0.08 & 0.10 \\
\hline 4 & 1 & 0.10 & 0.12 & 0.08 & 0.10 \\
\hline 4 & 2 & 0.10 & 0.12 & 0.08 & 0.10 \\
\hline 4 & 3 & 0.17 & 0.21 & 0.01 & 0.05 \\
\hline 4 & 4 & 0.10 & 0.12 & 0.08 & 0.10 \\
\hline 5 & 1 & 0.11 & 0.12 & 0.08 & 0.09 \\
\hline 5 & 2 & 0.16 & 0.11 & 0.03 & 0.05 \\
\hline 5 & 3 & 0.28 & 0.27 & 0.04 & 0.08 \\
\hline 5 & 4 & 0.13 & 0.12 & 0.02 & 0.04 \\
\hline
\end{tabular}

Table E.1.13 the results of calibration and validations based on the average area

\begin{tabular}{|c|c|c|c|c|c|}
\hline \multicolumn{2}{|c|}{ Configurations } & \multirow{2}{*}{$\begin{array}{c}\text { Calibration } \\
\text { Kappa } \\
\end{array}$} & \multirow{2}{*}{$\begin{array}{c}\mathbf{1}^{\text {st }} \text { Validation } \\
\text { Kappa }\end{array}$} & \multirow{2}{*}{$\begin{array}{c}2^{\text {nd }} \text { Validation } \\
\text { Kappa } \\
\end{array}$} & \multirow{2}{*}{$\begin{array}{c}\mathbf{3}^{\text {rd }} \text { Validation } \\
\text { Kappa }\end{array}$} \\
\hline$\varphi_{g}$ & $\Lambda_{l}$ & & & & \\
\hline 1 & 1 & 0.44 & 0.45 & 0.12 & 0.28 \\
\hline 1 & 2 & 0.39 & 0.40 & 0.11 & 0.25 \\
\hline 1 & 3 & 0.34 & 0.34 & 0.10 & 0.23 \\
\hline 1 & 4 & 0.30 & - & - & - \\
\hline 2 & 1 & 0.52 & 0.48 & 0.11 & 0.28 \\
\hline 2 & 2 & 0.44 & 0.41 & 0.11 & 0.26 \\
\hline 2 & 3 & 0.41 & 0.35 & 0.11 & 0.24 \\
\hline 2 & 4 & 0.39 & 0.31 & - & - \\
\hline 3 & 1 & 0.50 & 0.50 & 0.10 & 0.29 \\
\hline 3 & 2 & 0.44 & 0.44 & 0.10 & 0.27 \\
\hline 3 & 3 & 0.40 & 0.38 & 0.09 & 0.26 \\
\hline 3 & 4 & 0.39 & 0.32 & - & - \\
\hline 4 & 1 & 0.51 & 0.55 & 0.17 & 0.38 \\
\hline 4 & 2 & 0.45 & 0.48 & 0.17 & 0.36 \\
\hline 4 & 3 & 0.42 & 0.42 & 0.16 & 0.34 \\
\hline 4 & 4 & 0.40 & 0.36 & - & - \\
\hline 5 & 1 & 0.55 & 0.59 & 0.29 & 0.48 \\
\hline 5 & 2 & 0.51 & 0.56 & 0.29 & 0.47 \\
\hline 5 & 3 & 0.48 & 0.47 & 0.28 & 0.43 \\
\hline 5 & 4 & 0.45 & 0.41 & - & - \\
\hline
\end{tabular}


Table E.1.14 the results of calibration and validations based on the maximum area

\begin{tabular}{|c|c|c|c|c|c|}
\hline \multicolumn{2}{|c|}{ Configurations } & \multirow{2}{*}{$\begin{array}{c}\text { Calibration } \\
\text { Kappa }\end{array}$} & \multirow{2}{*}{$\begin{array}{c}\mathbf{1}^{\text {st }} \text { Validation } \\
\text { Kappa }\end{array}$} & \multirow{2}{*}{$\begin{array}{c}2^{\text {nd }} \text { Validation } \\
\text { Kappa }\end{array}$} & \multirow{2}{*}{$\frac{3^{\text {rd }} \text { Validation }}{\text { Kappa }}$} \\
\hline$\varphi_{g}$ & $\Lambda_{l}$ & & & & \\
\hline 1 & 1 & 0.15 & 0.08 & 0.01 & 0.04 \\
\hline 1 & 2 & 0.09 & 0.04 & - & - \\
\hline 1 & 3 & 0.05 & 0.01 & - & - \\
\hline 1 & 4 & 0.06 & 0.03 & - & - \\
\hline 2 & 1 & 0.19 & 0.12 & 0.02 & 0.06 \\
\hline 2 & 2 & 0.11 & 0.03 & - & - \\
\hline 2 & 3 & 0.05 & 0.01 & - & - \\
\hline 2 & 4 & 0.08 & 0.04 & - & - \\
\hline 3 & 1 & 0.17 & 0.18 & 0.01 & 0.06 \\
\hline 3 & 2 & 0.11 & 0.05 & - & - \\
\hline 3 & 3 & 0.05 & - & - & - \\
\hline 3 & 4 & 0.07 & 0.05 & - & - \\
\hline 4 & 1 & 0.20 & 0.23 & 0.02 & 0.09 \\
\hline 4 & 2 & 0.14 & 0.09 & - & - \\
\hline 4 & 3 & 0.06 & - & - & - \\
\hline 4 & 4 & 0.08 & - & - & - \\
\hline 5 & 1 & 0.20 & 0.15 & 0.04 & 0.10 \\
\hline 5 & 2 & 0.15 & 0.09 & 0.04 & 0.06 \\
\hline 5 & 3 & 0.07 & - & - & - \\
\hline 5 & 4 & 0.09 & 0.05 & 0.03 & 0.04 \\
\hline
\end{tabular}

Table E.1.15 the results of calibration and validations based on the average length

\begin{tabular}{|c|c|c|c|c|c|}
\hline \multicolumn{2}{|c|}{ Configurations } & \multirow{2}{*}{$\begin{array}{c}\text { Calibration } \\
\text { Kappa }\end{array}$} & \multirow{2}{*}{$\begin{array}{c}\mathbf{1}^{\text {st }} \text { Validation } \\
\text { Kappa }\end{array}$} & \multirow{2}{*}{$\begin{array}{c}2^{\text {nd }} \text { Validation } \\
\text { Kappa }\end{array}$} & \multirow{2}{*}{$\begin{array}{c}3^{\text {rd }} \text { Validation } \\
\text { Kappa }\end{array}$} \\
\hline$\varphi_{g}$ & $\Lambda_{l}$ & & & & \\
\hline 1 & 1 & 0.11 & 0.08 & 0.01 & 0.04 \\
\hline 1 & 2 & 0.13 & 0.04 & 0.01 & 0.03 \\
\hline 1 & 3 & 0.12 & 0.04 & 0.01 & 0.03 \\
\hline 1 & 4 & 0.10 & 0.09 & 0.01 & 0.05 \\
\hline 2 & 1 & 0.11 & 0.06 & 0.01 & 0.03 \\
\hline 2 & 2 & 0.13 & 0.02 & 0.01 & 0.03 \\
\hline 2 & 3 & 0.11 & 0.01 & 0.01 & 0.02 \\
\hline 2 & 4 & 0.10 & 0.05 & 0.01 & 0.03 \\
\hline 3 & 1 & 0.18 & 0.03 & 0.02 & 0.03 \\
\hline 3 & 2 & 0.20 & - & - & - \\
\hline 3 & 3 & 0.17 & - & - & - \\
\hline 3 & 4 & 0.16 & 0.02 & 0.01 & 0.02 \\
\hline 4 & 1 & 0.20 & - & - & - \\
\hline 4 & 2 & 0.21 & - & - & - \\
\hline 4 & 3 & 0.18 & - & - & - \\
\hline 4 & 4 & 0.17 & - & - & - \\
\hline 5 & 1 & 0.23 & 0.01 & 0.03 & 0.05 \\
\hline 5 & 2 & 0.23 & - & - & - \\
\hline 5 & 3 & 0.21 & 0.02 & 0.02 & 0.05 \\
\hline 5 & 4 & 0.20 & 0.01 & 0.02 & 0.04 \\
\hline
\end{tabular}


Table E.1.16 the results of calibration and validations based on the maximum length

\begin{tabular}{|c|c|c|c|c|c|}
\hline \multicolumn{2}{|c|}{ Configurations } & \multirow{2}{*}{$\begin{array}{c}\text { Calibration } \\
\text { Kappa } \\
\end{array}$} & \multirow{2}{*}{$\begin{array}{c}\mathbf{1}^{\text {st }} \text { Validation } \\
\text { Kappa }\end{array}$} & \multirow{2}{*}{$\begin{array}{c}2^{\text {nd }} \text { Validation } \\
\text { Kappa } \\
\end{array}$} & \multirow{2}{*}{$\begin{array}{c}3^{\text {rd }} \text { Validation } \\
\text { Kappa }\end{array}$} \\
\hline$\varphi_{g}$ & $\Lambda_{l}$ & & & & \\
\hline 1 & 1 & 0.20 & 0.14 & - & - \\
\hline 1 & 2 & 0.25 & 0.14 & 0.01 & 0.01 \\
\hline 1 & 3 & 0.24 & 0.18 & - & - \\
\hline 1 & 4 & 0.15 & 0.10 & - & - \\
\hline 2 & 1 & 0.24 & 0.13 & - & - \\
\hline 2 & 2 & 0.26 & 0.09 & 0.01 & - \\
\hline 2 & 3 & 0.21 & 0.09 & - & - \\
\hline 2 & 4 & 0.19 & 0.10 & - & - \\
\hline 3 & 1 & 0.26 & 0.11 & - & - \\
\hline 3 & 2 & 0.28 & 0.03 & 0.02 & - \\
\hline 3 & 3 & 0.23 & 0.02 & - & - \\
\hline 3 & 4 & 0.20 & 0.10 & - & - \\
\hline 4 & 1 & 0.26 & 0.05 & 0.01 & 0.01 \\
\hline 4 & 2 & 0.30 & - & - & - \\
\hline 4 & 3 & 0.24 & 0.16 & 0.02 & 0.07 \\
\hline 4 & 4 & 0.21 & 0.07 & 0.01 & 0.04 \\
\hline 5 & 1 & 0.32 & 0.12 & 0.02 & 0.03 \\
\hline 5 & 2 & 0.31 & 0.18 & 0.04 & 0.07 \\
\hline 5 & 3 & 0.26 & 0.22 & 0.02 & 0.09 \\
\hline 5 & 4 & 0.25 & 0.10 & 0.02 & 0.06 \\
\hline
\end{tabular}

Table E.1.17 the results of calibration and validations based on the average width

\begin{tabular}{|c|c|c|c|c|c|}
\hline \multicolumn{2}{|c|}{ Configurations } & \multirow{2}{*}{$\begin{array}{c}\text { Calibration } \\
\text { Kappa } \\
\end{array}$} & \multirow{2}{*}{$\begin{array}{c}\mathbf{1}^{\text {st }} \text { Validation } \\
\text { Kappa } \\
\end{array}$} & \multirow{2}{*}{$\begin{array}{c}\text { Validation } 2 \\
\text { Kappa } \\
\end{array}$} & \multirow{2}{*}{$\begin{array}{c}3^{\text {rd }} \text { Validation } \\
\text { Kappa } \\
\end{array}$} \\
\hline$\varphi_{g}$ & $\Lambda_{l}$ & & & & \\
\hline 1 & 1 & 0.48 & 0.40 & 0.25 & 0.32 \\
\hline 1 & 2 & 0.45 & 0.33 & - & - \\
\hline 1 & 3 & 0.34 & 0.27 & - & - \\
\hline 1 & 4 & - & - & - & - \\
\hline 2 & 1 & 0.61 & 0.46 & 0.20 & 0.29 \\
\hline 2 & 2 & 0.61 & 0.38 & - & - \\
\hline 2 & 3 & 0.47 & 0.32 & - & - \\
\hline 2 & 4 & - & - & - & - \\
\hline 3 & 1 & 0.60 & 0.50 & 0.16 & 0.29 \\
\hline 3 & 2 & 0.63 & 0.39 & 0.04 & - \\
\hline 3 & 3 & 0.50 & 0.34 & - & - \\
\hline 3 & 4 & - & - & - & - \\
\hline 4 & 1 & 0.62 & 0.56 & 0.20 & 0.35 \\
\hline 4 & 2 & 0.65 & 0.44 & 0.08 & - \\
\hline 4 & 3 & 0.55 & 0.39 & - & - \\
\hline 4 & 4 & - & - & - & - \\
\hline 5 & 1 & 0.63 & 0.58 & 0.21 & 0.37 \\
\hline 5 & 2 & 0.64 & 0.48 & 0.09 & 0.25 \\
\hline 5 & 3 & 0.56 & 0.47 & - & - \\
\hline 5 & 4 & - & - & - & - \\
\hline
\end{tabular}


Table E.1.18 the results of calibration and validations based on the maximum width

\begin{tabular}{|c|c|c|c|c|c|}
\hline \multicolumn{2}{|c|}{ Configurations } & \multirow{2}{*}{$\begin{array}{c}\text { Calibration } \\
\text { Kappa } \\
\end{array}$} & \multirow{2}{*}{$\begin{array}{c}\mathbf{1}^{\text {st }} \text { Validation } \\
\text { Kappa }\end{array}$} & \multirow{2}{*}{$\begin{array}{c}2^{\text {nd }} \text { Validation } \\
\text { Kappa }\end{array}$} & \multirow{2}{*}{$\frac{3^{\text {rd }} \text { Validation }}{\text { Kappa }}$} \\
\hline$\varphi_{g}$ & $\Lambda_{l}$ & & & & \\
\hline 1 & 1 & 0.39 & 0.43 & 0.50 & 0.50 \\
\hline 1 & 2 & 0.35 & 0.37 & 0.42 & 0.42 \\
\hline 1 & 3 & - & - & - & - \\
\hline 1 & 4 & - & - & - & - \\
\hline 2 & 1 & 0.50 & 0.43 & 0.49 & 0.49 \\
\hline 2 & 2 & 0.49 & 0.43 & 0.42 & 0.45 \\
\hline 2 & 3 & 0.35 & 0.35 & - & - \\
\hline 2 & 4 & - & - & - & - \\
\hline 3 & 1 & 0.49 & 0.51 & 0.54 & 0.56 \\
\hline 3 & 2 & 0.50 & 0.50 & 0.47 & 0.51 \\
\hline 3 & 3 & 0.36 & 0.43 & - & - \\
\hline 3 & 4 & - & - & - & - \\
\hline 4 & 1 & 0.47 & 0.59 & 0.56 & 0.60 \\
\hline 4 & 2 & 0.50 & 0.57 & 0.54 & 0.60 \\
\hline 4 & 3 & 0.35 & 0.52 & 0.46 & 0.52 \\
\hline 4 & 4 & - & - & - & - \\
\hline 5 & 1 & 0.47 & 0.35 & - & - \\
\hline 5 & 2 & 0.50 & 0.51 & 0.54 & 0.59 \\
\hline 5 & 3 & 0.34 & 0.50 & 0.49 & 0.54 \\
\hline 5 & 4 & - & - & - & - \\
\hline
\end{tabular}

Table E.1.19 the results of calibration and validations based on the peaks in speed

\begin{tabular}{|c|c|c|c|c|c|}
\hline \multicolumn{2}{|c|}{ Configurations } & \multirow{2}{*}{$\begin{array}{c}\text { Calibration } \\
\text { Kappa }\end{array}$} & \multirow{2}{*}{$\begin{array}{c}\mathbf{1}^{\text {st }} \text { Validation } \\
\text { Kappa }\end{array}$} & \multirow{2}{*}{$\begin{array}{cl}2^{\text {nd }} & \text { Validation } \\
& \text { Kappa }\end{array}$} & \multirow{2}{*}{$\frac{3^{\text {rd }} \text { Validation }}{\text { Kappa }}$} \\
\hline$\varphi_{g}$ & $\Lambda_{l}$ & & & & \\
\hline 1 & 1 & 0.16 & 0.13 & 0.07 & 0.09 \\
\hline 1 & 2 & 0.15 & 0.11 & 0.15 & 0.14 \\
\hline 1 & 3 & 0.15 & 0.11 & 0.14 & 0.13 \\
\hline 1 & 4 & 0.15 & 0.10 & 0.10 & 0.10 \\
\hline 2 & 1 & 0.15 & 0.15 & 0.06 & 0.09 \\
\hline 2 & 2 & 0.15 & 0.12 & 0.14 & 0.14 \\
\hline 2 & 3 & 0.14 & 0.13 & 0.13 & 0.13 \\
\hline 2 & 4 & 0.14 & 0.12 & 0.09 & 0.10 \\
\hline 3 & 1 & 0.13 & 0.12 & 0.04 & 0.07 \\
\hline 3 & 2 & 0.12 & 0.12 & 0.05 & 0.07 \\
\hline 3 & 3 & 0.12 & 0.11 & 0.08 & 0.09 \\
\hline 3 & 4 & 0.12 & 0.11 & 0.08 & 0.09 \\
\hline 4 & 1 & 0.12 & 0.10 & 0.02 & 0.05 \\
\hline 4 & 2 & 0.11 & 0.10 & 0.03 & 0.05 \\
\hline 4 & 3 & 0.12 & 0.12 & 0.01 & 0.05 \\
\hline 4 & 4 & 0.12 & 0.09 & 0.06 & 0.08 \\
\hline 5 & 1 & 0.12 & 0.15 & 0.01 & 0.06 \\
\hline 5 & 2 & 0.11 & 0.15 & 0.02 & 0.06 \\
\hline 5 & 3 & 0.12 & 0.17 & 0.03 & 0.08 \\
\hline 5 & 4 & 0.12 & 0.14 & 0.04 & 0.08 \\
\hline
\end{tabular}


Table E.1.20 the results of calibration and validations based on the effective weighted average frequency

\begin{tabular}{|c|c|c|c|c|c|c|}
\hline \multicolumn{3}{|c|}{ Configurations } & \multirow{2}{*}{$\begin{array}{c}\text { Calibration } \\
\text { Kappa } \\
\end{array}$} & \multirow{2}{*}{$\frac{\mathbf{1}^{\text {st }} \text { Validation }}{\text { Kappa }}$} & \multirow{2}{*}{$\begin{array}{cc}2^{\text {nd }} \text { Validation } \\
\text { Kappa } \\
\end{array}$} & \multirow{2}{*}{$\begin{array}{c}\mathbf{3}^{\text {rd }} \text { Validation } \\
\text { Kappa }\end{array}$} \\
\hline$\varphi_{g}$ & $\Lambda_{l}$ & $\Gamma_{n}$ & & & & \\
\hline 1 & 1 & 1 & 0.15 & 0.13 & 0.15 & 0.15 \\
\hline 1 & 1 & 2 & 0.14 & 0.11 & 0.15 & 0.14 \\
\hline 1 & 1 & 3 & 0.15 & 0.13 & 0.15 & 0.15 \\
\hline 1 & 1 & 4 & 0.16 & 0.11 & 0.14 & 0.14 \\
\hline 1 & 1 & 1 & 0.16 & 0.12 & 0.15 & 0.15 \\
\hline 1 & 1 & 6 & 0.16 & 0.12 & 0.16 & 0.15 \\
\hline 1 & 2 & 1 & 0.13 & 0.11 & 0.15 & 0.14 \\
\hline 1 & 2 & 2 & 0.12 & 0.09 & 0.12 & 0.11 \\
\hline 1 & 2 & 3 & 0.13 & 0.11 & 0.14 & 0.14 \\
\hline 1 & 2 & 4 & 0.13 & 0.11 & 0.15 & 0.14 \\
\hline 1 & 2 & 1 & 0.12 & 0.09 & 0.17 & 0.15 \\
\hline 1 & 2 & 6 & 0.13 & 0.12 & 0.13 & 0.13 \\
\hline 1 & 3 & 1 & 0.11 & 0.07 & 0.07 & 0.08 \\
\hline 1 & 3 & 2 & 0.12 & 0.06 & 0.11 & 0.10 \\
\hline 1 & 3 & 3 & 0.12 & 0.09 & 0.10 & 0.11 \\
\hline 1 & 3 & 4 & 0.11 & 0.10 & 0.14 & 0.13 \\
\hline 1 & 3 & 1 & 0.11 & 0.09 & 0.11 & 0.12 \\
\hline 1 & 3 & 6 & 0.11 & 0.09 & 0.06 & 0.08 \\
\hline 1 & 4 & 1 & 0.12 & 0.12 & 0.11 & 0.12 \\
\hline 1 & 4 & 2 & 0.13 & 0.09 & 0.13 & 0.12 \\
\hline 1 & 4 & 3 & 0.13 & 0.11 & 0.12 & 0.13 \\
\hline 1 & 4 & 4 & 0.13 & 0.10 & 0.13 & 0.13 \\
\hline 1 & 4 & 1 & 0.13 & 0.11 & 0.13 & 0.13 \\
\hline 1 & 4 & 6 & 0.13 & 0.12 & 0.13 & 0.13 \\
\hline 2 & 1 & 1 & 0.22 & 0.18 & 0.16 & 0.17 \\
\hline 2 & 1 & 2 & 0.22 & 0.16 & 0.15 & 0.16 \\
\hline 2 & 1 & 3 & 0.23 & 0.14 & 0.17 & 0.17 \\
\hline 2 & 1 & 4 & 0.24 & 0.15 & 0.16 & 0.16 \\
\hline 2 & 1 & 1 & 0.24 & 0.15 & 0.16 & 0.17 \\
\hline 2 & 1 & 6 & 0.24 & 0.15 & 0.17 & 0.17 \\
\hline 2 & 2 & 1 & 0.19 & 0.15 & 0.15 & 0.16 \\
\hline 2 & 2 & 2 & 0.18 & 0.15 & 0.13 & 0.15 \\
\hline 2 & 2 & 3 & 0.19 & 0.15 & 0.14 & 0.15 \\
\hline 2 & 2 & 4 & 0.19 & 0.16 & 0.13 & 0.15 \\
\hline 2 & 2 & 1 & 0.19 & 0.15 & 0.14 & 0.15 \\
\hline 2 & 2 & 6 & 0.20 & 0.16 & 0.14 & 0.15 \\
\hline 2 & 3 & 1 & 0.17 & 0.11 & 0.07 & 0.09 \\
\hline 2 & 3 & 2 & 0.17 & 0.11 & 0.09 & 0.10 \\
\hline 2 & 3 & 3 & 0.17 & 0.11 & 0.10 & 0.10 \\
\hline 2 & 3 & 4 & 0.17 & 0.11 & 0.08 & 0.09 \\
\hline 2 & 3 & 1 & 0.17 & 0.11 & 0.06 & 0.09 \\
\hline 2 & 3 & 6 & 0.17 & 0.12 & 0.06 & 0.09 \\
\hline 2 & 4 & 1 & 0.18 & 0.14 & 0.12 & 0.14 \\
\hline 2 & 4 & 2 & 0.18 & 0.14 & 0.13 & 0.14 \\
\hline 2 & 4 & 3 & 0.19 & 0.13 & 0.11 & 0.12 \\
\hline 2 & 4 & 4 & 0.19 & 0.13 & 0.11 & 0.12 \\
\hline 2 & 4 & 1 & 0.20 & 0.14 & 0.14 & 0.14 \\
\hline 2 & 4 & 6 & 0.20 & 0.14 & 0.14 & 0.15 \\
\hline 3 & 1 & 1 & 0.26 & 0.23 & 0.17 & 0.21 \\
\hline 3 & 1 & 2 & 0.26 & 0.21 & 0.17 & 0.20 \\
\hline
\end{tabular}


Table E.1.20 the results of calibration and validations based on the effective weighted average frequency

\begin{tabular}{|c|c|c|c|c|c|c|}
\hline \multicolumn{3}{|c|}{ Configurations } & \multirow{2}{*}{$\begin{array}{c}\text { Calibration } \\
\text { Kappa } \\
\end{array}$} & \multirow{2}{*}{$\begin{array}{c}\mathbf{1}^{\text {st }} \text { Validation } \\
\text { Kappa } \\
\end{array}$} & \multirow{2}{*}{$\begin{array}{c}2^{\text {nd }} \text { Validation } \\
\text { Kappa } \\
\end{array}$} & \multirow{2}{*}{$\begin{array}{c}\mathbf{3}^{\text {rd }} \text { Validation } \\
\text { Kappa }\end{array}$} \\
\hline$\varphi_{g}$ & $\Lambda_{l}$ & $\Gamma_{n}$ & & & & \\
\hline 3 & 1 & 3 & 0.27 & 0.18 & 0.19 & 0.20 \\
\hline 3 & 1 & 4 & 0.28 & 0.19 & 0.17 & 0.19 \\
\hline 3 & 1 & 1 & 0.29 & 0.20 & 0.18 & 0.20 \\
\hline 3 & 1 & 6 & 0.29 & 0.20 & 0.19 & 0.21 \\
\hline 3 & 2 & 1 & 0.22 & 0.20 & 0.17 & 0.20 \\
\hline 3 & 2 & 2 & 0.22 & 0.20 & 0.15 & 0.18 \\
\hline 3 & 2 & 3 & 0.23 & 0.19 & 0.16 & 0.18 \\
\hline 3 & 2 & 4 & 0.23 & 0.21 & 0.14 & 0.18 \\
\hline 3 & 2 & 1 & 0.23 & 0.19 & 0.15 & 0.18 \\
\hline 3 & 2 & 6 & 0.24 & 0.20 & 0.15 & 0.18 \\
\hline 3 & 3 & 1 & 0.20 & 0.13 & 0.08 & 0.12 \\
\hline 3 & 3 & 2 & 0.20 & 0.13 & 0.10 & 0.12 \\
\hline 3 & 3 & 3 & 0.20 & 0.12 & 0.06 & - \\
\hline 3 & 3 & 4 & 0.20 & 0.13 & 0.08 & 0.11 \\
\hline 3 & 3 & 1 & 0.20 & 0.15 & 0.06 & 0.11 \\
\hline 3 & 3 & 6 & 0.21 & 0.15 & 0.06 & 0.11 \\
\hline 3 & 4 & 1 & 0.22 & 0.18 & 0.14 & 0.17 \\
\hline 3 & 4 & 2 & 0.22 & 0.18 & 0.14 & 0.17 \\
\hline 3 & 4 & 3 & 0.23 & 0.16 & 0.12 & 0.15 \\
\hline 3 & 4 & 4 & 0.23 & 0.16 & 0.11 & 0.14 \\
\hline 3 & 4 & 1 & 0.24 & 0.18 & 0.15 & 0.17 \\
\hline 3 & 4 & 6 & 0.24 & 0.18 & 0.16 & 0.18 \\
\hline 4 & 1 & 1 & 0.30 & 0.25 & 0.23 & 0.27 \\
\hline 4 & 1 & 2 & 0.30 & 0.23 & 0.21 & 0.25 \\
\hline 4 & 1 & 3 & 0.30 & 0.21 & 0.21 & 0.24 \\
\hline 4 & 1 & 4 & 0.31 & 0.21 & 0.23 & 0.25 \\
\hline 4 & 1 & 1 & 0.32 & 0.22 & 0.23 & 0.25 \\
\hline 4 & 1 & 6 & 0.32 & 0.22 & 0.26 & 0.27 \\
\hline 4 & 2 & 1 & 0.25 & 0.22 & 0.22 & 0.25 \\
\hline 4 & 2 & 2 & 0.25 & 0.22 & 0.18 & 0.22 \\
\hline 4 & 2 & 3 & 0.26 & 0.20 & 0.19 & 0.22 \\
\hline 4 & 2 & 4 & 0.26 & 0.23 & 0.18 & 0.23 \\
\hline 4 & 2 & 1 & 0.26 & 0.21 & 0.20 & 0.23 \\
\hline 4 & 2 & 6 & 0.27 & 0.22 & 0.19 & 0.23 \\
\hline 4 & 3 & 1 & 0.23 & 0.16 & 0.12 & 0.16 \\
\hline 4 & 3 & 2 & 0.23 & 0.16 & 0.12 & 0.16 \\
\hline 4 & 3 & 3 & 0.23 & 0.16 & 0.12 & 0.16 \\
\hline 4 & 3 & 4 & 0.23 & 0.16 & 0.10 & 0.15 \\
\hline 4 & 3 & 1 & 0.23 & 0.17 & 0.08 & 0.15 \\
\hline 4 & 3 & 6 & 0.24 & 0.18 & 0.08 & 0.15 \\
\hline 4 & 4 & 1 & 0.25 & 0.20 & 0.17 & 0.21 \\
\hline 4 & 4 & 2 & 0.25 & 0.20 & 0.17 & 0.21 \\
\hline 4 & 4 & 3 & 0.26 & 0.19 & 0.15 & 0.19 \\
\hline 4 & 4 & 4 & 0.26 & 0.18 & 0.14 & 0.18 \\
\hline 4 & 4 & 1 & 0.26 & 0.19 & 0.19 & 0.22 \\
\hline 4 & 4 & 6 & 0.27 & 0.20 & 0.20 & 0.23 \\
\hline 5 & 1 & 1 & 0.40 & 0.34 & 0.35 & 0.38 \\
\hline 5 & 1 & 2 & 0.40 & 0.31 & 0.33 & 0.35 \\
\hline 5 & 1 & 3 & 0.40 & 0.28 & 0.33 & 0.35 \\
\hline 5 & 1 & 4 & 0.40 & 0.25 & 0.34 & 0.33 \\
\hline
\end{tabular}


Table E.1.20 the results of calibration and validations based on the effective weighted average frequency

\begin{tabular}{|c|c|c|c|c|c|c|}
\hline \multicolumn{3}{|c|}{ Configurations } & Calibration & $1^{\text {st }}$ Validation & $2^{\text {nd }}$ Validation & $3^{\text {rd }}$ Validation \\
\hline$\varphi_{g}$ & $\Lambda_{l}$ & $\Gamma_{n}$ & Kappa & Kappa & Kappa & Kappa \\
\hline 5 & 1 & 1 & 0.41 & 0.26 & 0.34 & 0.34 \\
\hline 5 & 1 & 6 & 0.41 & 0.24 & 0.33 & 0.32 \\
\hline 5 & 2 & 1 & 0.34 & 0.29 & 0.32 & 0.34 \\
\hline 5 & 2 & 2 & 0.33 & 0.27 & 0.30 & 0.32 \\
\hline 5 & 2 & 3 & 0.35 & 0.27 & 0.30 & 0.32 \\
\hline 5 & 2 & 4 & 0.35 & 0.30 & 0.29 & 0.33 \\
\hline 5 & 2 & 1 & 0.36 & 0.26 & 0.30 & 0.32 \\
\hline 5 & 2 & 6 & 0.37 & 0.28 & 0.30 & 0.32 \\
\hline 5 & 3 & 1 & 0.30 & 0.24 & 0.18 & 0.25 \\
\hline 5 & 3 & 2 & 0.30 & 0.27 & 0.23 & 0.29 \\
\hline 5 & 3 & 3 & 0.30 & 0.25 & 0.23 & 0.28 \\
\hline 5 & 3 & 4 & 0.30 & 0.24 & 0.23 & 0.27 \\
\hline 5 & 3 & 1 & 0.30 & 0.26 & 0.22 & 0.27 \\
\hline 5 & 3 & 6 & 0.31 & 0.25 & 0.15 & 0.24 \\
\hline 5 & 4 & 1 & 0.33 & 0.28 & 0.28 & 0.32 \\
\hline 5 & 4 & 2 & 0.33 & 0.27 & 0.28 & 0.30 \\
\hline 5 & 4 & 3 & 0.34 & 0.26 & 0.23 & 0.28 \\
\hline 5 & 4 & 4 & 0.34 & 0.25 & 0.29 & 0.30 \\
\hline 5 & 4 & 1 & 0.35 & 0.24 & 0.30 & 0.31 \\
\hline 5 & 4 & 6 & 0.36 & 0.24 & 0.32 & 0.31 \\
\hline
\end{tabular}

Table E.1.21 the results of calibration and validations based on the effective frequency

\begin{tabular}{|c|c|c|c|c|c|c|}
\hline \multicolumn{3}{|c|}{ Configurations } & \multirow{2}{*}{$\begin{array}{c}\text { Calibration } \\
\text { Kappa } \\
\end{array}$} & \multirow{2}{*}{$\begin{array}{c}\mathbf{1}^{\text {st }} \text { Validation } \\
\text { Kappa }\end{array}$} & \multirow{2}{*}{$\begin{array}{cc}2^{\text {nd }} & \text { Validation } \\
\text { Kappa }\end{array}$} & \multirow{2}{*}{$\frac{3^{\text {rd }} \text { Validation }}{\text { Kappa }}$} \\
\hline$\varphi_{g}$ & $\Lambda_{l}$ & $\eta_{m}$ & & & & \\
\hline 1 & 1 & 1 & 0.10 & 0.07 & 0.06 & 0.06 \\
\hline 1 & 1 & 2 & 0.12 & 0.14 & 0.07 & 0.10 \\
\hline 1 & 1 & 3 & 0.14 & 0.07 & 0.07 & 0.07 \\
\hline 1 & 2 & 1 & 0.12 & 0.06 & 0.05 & 0.05 \\
\hline 1 & 2 & 2 & 0.08 & 0.13 & - & - \\
\hline 1 & 2 & 3 & 0.09 & - & - & - \\
\hline 1 & 3 & 1 & 0.12 & 0.06 & 0.05 & 0.05 \\
\hline 1 & 3 & 2 & 0.09 & 0.11 & 0.03 & 0.06 \\
\hline 1 & 3 & 3 & 0.08 & - & - & - \\
\hline 1 & 4 & 1 & 0.11 & 0.08 & 0.05 & 0.06 \\
\hline 1 & 4 & 2 & 0.08 & 0.12 & 0.03 & 0.06 \\
\hline 1 & 4 & 3 & 0.07 & - & - & - \\
\hline 2 & 1 & 1 & 0.11 & 0.15 & 0.01 & 0.05 \\
\hline 2 & 1 & 2 & 0.15 & 0.17 & 0.08 & 0.11 \\
\hline 2 & 1 & 3 & 0.18 & 0.09 & 0.08 & 0.08 \\
\hline 2 & 2 & 1 & 0.12 & 0.09 & 0.06 & 0.07 \\
\hline 2 & 2 & 2 & 0.09 & 0.12 & - & - \\
\hline 2 & 2 & 3 & 0.11 & - & - & - \\
\hline 2 & 3 & 1 & 0.11 & 0.09 & 0.05 & 0.06 \\
\hline 2 & 3 & 2 & 0.09 & 0.12 & - & - \\
\hline 2 & 3 & 3 & 0.10 & - & - & - \\
\hline 2 & 4 & 1 & 0.11 & 0.09 & 0.05 & 0.06 \\
\hline 2 & 4 & 2 & 0.09 & 0.12 & - & - \\
\hline 2 & 4 & 3 & 0.08 & - & - & - \\
\hline 3 & 1 & 1 & 0.15 & 0.22 & 0.07 & 0.10 \\
\hline
\end{tabular}


Table E.1.21 the results of calibration and validations based on the effective frequency

\begin{tabular}{|c|c|c|c|c|c|c|}
\hline \multicolumn{3}{|c|}{ Configurations } & Calibration & $\mathbf{1}^{\text {st }}$ Validation & $2^{\text {nd }}$ Validation & $3^{\text {rd }}$ Validation \\
\hline$\varphi_{g}$ & $\Lambda_{l}$ & $\eta_{m}$ & Kappa & Kappa & Kappa & Kappa \\
\hline 3 & 1 & 2 & 0.19 & 0.23 & 0.12 & 0.15 \\
\hline 3 & 1 & 3 & 0.25 & 0.12 & 0.12 & 0.12 \\
\hline 3 & 2 & 1 & 0.10 & 0.10 & 0.05 & - \\
\hline 3 & 2 & 2 & 0.10 & - & - & - \\
\hline 3 & 2 & 3 & 0.15 & - & - & - \\
\hline 3 & 3 & 1 & 0.10 & 0.10 & 0.05 & - \\
\hline 3 & 3 & 2 & - & - & - & - \\
\hline 3 & 3 & 3 & 0.12 & - & - & - \\
\hline 3 & 4 & 1 & 0.10 & 0.10 & 0.05 & - \\
\hline 3 & 4 & 2 & - & - & - & - \\
\hline 3 & 4 & 3 & 0.11 & - & - & - \\
\hline 4 & 1 & 1 & 0.15 & 0.24 & 0.08 & 0.11 \\
\hline 4 & 1 & 2 & 0.22 & 0.17 & 0.07 & 0.09 \\
\hline 4 & 1 & 3 & 0.32 & 0.16 & 0.14 & 0.15 \\
\hline 4 & 2 & 1 & - & - & - & - \\
\hline 4 & 2 & 2 & - & - & - & - \\
\hline 4 & 2 & 3 & 0.19 & 0.07 & 0.07 & - \\
\hline 4 & 3 & 1 & - & - & - & - \\
\hline 4 & 3 & 2 & - & - & - & - \\
\hline 4 & 3 & 3 & 0.15 & 0.07 & - & - \\
\hline 4 & 4 & 1 & - & - & - & - \\
\hline 4 & 4 & 2 & - & - & - & - \\
\hline 4 & 4 & 3 & - & - & - & - \\
\hline 5 & 1 & 1 & 0.18 & 0.16 & 0.08 & 0.11 \\
\hline 5 & 1 & 2 & 0.28 & 0.25 & 0.07 & 0.11 \\
\hline 5 & 1 & 3 & 0.38 & 0.22 & 0.14 & 0.17 \\
\hline 5 & 2 & 1 & - & - & - & - \\
\hline 5 & 2 & 2 & 0.17 & - & - & - \\
\hline 5 & 2 & 3 & 0.26 & 0.11 & 0.10 & 0.11 \\
\hline 5 & 3 & 1 & - & - & - & - \\
\hline 5 & 3 & 2 & - & - & - & - \\
\hline 5 & 3 & 3 & 0.17 & 0.09 & 0.04 & - \\
\hline 5 & 4 & 1 & - & - & - & - \\
\hline 5 & 4 & 2 & - & - & - & - \\
\hline 5 & 4 & 3 & - & - & - & - \\
\hline
\end{tabular}




\section{E.2 The results of calibrations and validations in percentage scale}

Table E.2.1 the results of calibration procedure 1 and its validations.

\begin{tabular}{|c|c|c|c|c|c|c|c|c|c|c|c|}
\hline \multicolumn{4}{|c|}{ Configurations } & \multicolumn{2}{|c|}{ Calibration } & \multicolumn{2}{|c|}{$1^{\text {st }}$ Validation } & \multicolumn{2}{|c|}{$2^{\text {nd }}$ Validation } & \multicolumn{2}{|c|}{$3^{\text {rd }}$ Validation } \\
\hline$\varphi_{g}$ & $\Lambda_{l}$ & $\Gamma_{n}$ & $\eta_{m}$ & Cyclists & Pedestrians & Cyclists & Pedestrians & Cyclists & Pedestrians & Cyclists & Pedestrians \\
\hline 1 & 1 & 1 & 1 & 99 & 100 & 91 & 84 & 87 & 84 & 88 & 84 \\
\hline 1 & 1 & 1 & 2 & 98 & 100 & 91 & 84 & 87 & 86 & 88 & 86 \\
\hline 1 & 1 & 1 & 3 & 99 & 100 & 91 & 84 & 87 & 86 & 88 & 86 \\
\hline 1 & 1 & 2 & 1 & 99 & 100 & 91 & 84 & 86 & 86 & 88 & 85 \\
\hline 1 & 1 & 2 & 2 & 99 & 100 & 91 & 84 & 86 & 86 & 88 & 86 \\
\hline 1 & 1 & 2 & 3 & 99 & 100 & 91 & 84 & 86 & 86 & 88 & 86 \\
\hline 1 & 1 & 3 & 1 & 99 & 100 & 91 & 84 & 86 & 86 & 88 & 85 \\
\hline 1 & 1 & 3 & 2 & 99 & 100 & 91 & 84 & 86 & 86 & 88 & 86 \\
\hline 1 & 1 & 3 & 3 & 99 & 100 & 91 & 84 & 86 & 86 & 88 & 86 \\
\hline 1 & 1 & 4 & 1 & 99 & 100 & 91 & 84 & 86 & 86 & 88 & 85 \\
\hline 1 & 1 & 4 & 2 & 99 & 100 & 91 & 84 & 86 & 86 & 88 & 86 \\
\hline 1 & 1 & 4 & 3 & 99 & 100 & 91 & 84 & 86 & 86 & 88 & 86 \\
\hline 1 & 1 & 1 & 1 & 99 & 100 & 91 & 84 & 86 & 86 & 88 & 85 \\
\hline 1 & 1 & 1 & 2 & 99 & 100 & 91 & 84 & 86 & 86 & 88 & 86 \\
\hline 1 & 1 & 1 & 3 & 99 & 100 & 91 & 84 & 86 & 86 & 88 & 86 \\
\hline 1 & 1 & 6 & 1 & 99 & 100 & 91 & 84 & 86 & 86 & 88 & 85 \\
\hline 1 & 1 & 6 & 2 & 99 & 100 & 91 & 84 & 86 & 86 & 88 & 86 \\
\hline 1 & 1 & 6 & 3 & 99 & 100 & 91 & 84 & 86 & 86 & 88 & 86 \\
\hline 1 & 2 & 1 & 1 & 100 & 100 & 86 & 90 & - & - & - & - \\
\hline 1 & 2 & 1 & 2 & 99 & 100 & 86 & 90 & - & - & - & - \\
\hline 1 & 2 & 1 & 3 & 100 & 100 & 86 & 90 & - & - & - & - \\
\hline 1 & 2 & 2 & 1 & 100 & 100 & 86 & 90 & - & - & - & - \\
\hline 1 & 2 & 2 & 2 & 100 & 100 & 88 & 90 & - & - & - & - \\
\hline 1 & 2 & 2 & 3 & 100 & 100 & 86 & 90 & - & - & - & - \\
\hline 1 & 2 & 3 & 1 & 100 & 100 & 86 & 90 & - & - & - & - \\
\hline 1 & 2 & 3 & 2 & 100 & 100 & 88 & 90 & - & - & - & - \\
\hline 1 & 2 & 3 & 3 & 100 & 100 & 86 & 90 & - & - & - & - \\
\hline 1 & 2 & 4 & 1 & 100 & 100 & 86 & 90 & - & - & - & - \\
\hline 1 & 2 & 4 & 2 & 100 & 100 & 88 & 90 & - & - & - & - \\
\hline 1 & 2 & 4 & 3 & 100 & 100 & 86 & 90 & - & - & - & - \\
\hline 1 & 2 & 1 & 1 & 100 & 100 & 86 & 90 & - & - & - & - \\
\hline 1 & 2 & 1 & 2 & 100 & 100 & 88 & 90 & - & - & - & - \\
\hline 1 & 2 & 1 & 3 & 100 & 100 & 86 & 90 & - & - & - & - \\
\hline 1 & 2 & 6 & 1 & 100 & 100 & 86 & 90 & - & - & - & - \\
\hline
\end{tabular}


Table E.2.1 the results of calibration procedure 1 and its validations.

\begin{tabular}{|c|c|c|c|c|c|c|c|c|c|c|c|}
\hline \multicolumn{4}{|c|}{ Configurations } & \multicolumn{2}{|c|}{ Calibration } & \multicolumn{2}{|c|}{$1^{\text {st }}$ Validation } & \multicolumn{2}{|c|}{$2^{\text {nd }}$ Validation } & \multicolumn{2}{|c|}{$3^{\text {rd }}$ Validation } \\
\hline$\varphi_{g}$ & $\Lambda_{l}$ & $\Gamma_{n}$ & $\eta_{m}$ & Cyclists & Pedestrians & Cyclists & Pedestrians & Cyclists & Pedestrians & Cyclists & Pedestrians \\
\hline 1 & 2 & 6 & 2 & 100 & 100 & 88 & 90 & - & - & - & - \\
\hline 1 & 2 & 6 & 3 & 100 & 100 & 86 & 90 & - & - & - & - \\
\hline 1 & 3 & 1 & 1 & 100 & 100 & 89 & 84 & - & - & - & - \\
\hline 1 & 3 & 1 & 2 & 100 & 100 & 89 & 84 & - & - & - & - \\
\hline 1 & 3 & 1 & 3 & 100 & 100 & 89 & 84 & - & - & - & - \\
\hline 1 & 3 & 2 & 1 & 100 & 100 & 90 & 81 & - & - & - & - \\
\hline 1 & 3 & 2 & 2 & 100 & 100 & 90 & 83 & - & - & - & - \\
\hline 1 & 3 & 2 & 3 & 100 & 100 & 90 & 83 & - & - & - & - \\
\hline 1 & 3 & 3 & 1 & 100 & 100 & 90 & 83 & - & - & - & - \\
\hline 1 & 3 & 3 & 2 & 100 & 100 & 90 & 84 & - & - & - & - \\
\hline 1 & 3 & 3 & 3 & 100 & 100 & 90 & 84 & - & - & - & - \\
\hline 1 & 3 & 4 & 1 & 100 & 100 & 90 & 84 & - & - & - & - \\
\hline 1 & 3 & 4 & 2 & 100 & 100 & 90 & 86 & - & - & - & - \\
\hline 1 & 3 & 4 & 3 & 100 & 100 & 90 & 86 & - & - & - & - \\
\hline 1 & 3 & 1 & 1 & 100 & 100 & 89 & 84 & - & - & - & - \\
\hline 1 & 3 & 1 & 2 & 100 & 100 & 89 & 86 & - & - & - & - \\
\hline 1 & 3 & 1 & 3 & 100 & 100 & 89 & 86 & - & - & - & - \\
\hline 1 & 3 & 6 & 1 & 100 & 100 & 90 & 83 & - & - & - & - \\
\hline 1 & 3 & 6 & 2 & 100 & 100 & 90 & 84 & - & - & - & - \\
\hline 1 & 3 & 6 & 3 & 100 & 100 & 90 & 84 & - & - & - & - \\
\hline 1 & 4 & 1 & 1 & - & - & - & - & - & - & - & - \\
\hline 1 & 4 & 1 & 2 & - & - & - & - & - & - & - & - \\
\hline 1 & 4 & 1 & 3 & - & - & - & - & - & - & - & - \\
\hline 1 & 4 & 2 & 1 & - & - & - & - & - & - & - & - \\
\hline 1 & 4 & 2 & 2 & - & - & - & - & - & - & - & - \\
\hline 1 & 4 & 2 & 3 & - & - & - & - & - & - & - & - \\
\hline 1 & 4 & 3 & 1 & - & - & - & - & - & - & - & - \\
\hline 1 & 4 & 3 & 2 & - & - & - & - & - & - & - & - \\
\hline 1 & 4 & 3 & 3 & - & - & - & - & - & - & - & - \\
\hline 1 & 4 & 4 & 1 & - & - & - & - & - & - & - & - \\
\hline 1 & 4 & 4 & 2 & - & - & - & - & - & - & - & - \\
\hline 1 & 4 & 4 & 3 & - & - & - & - & - & - & - & - \\
\hline 1 & 4 & 1 & 1 & - & - & - & - & - & - & - & - \\
\hline 1 & 4 & 1 & 2 & - & - & - & - & - & - & - & - \\
\hline 1 & 4 & 1 & 3 & - & - & - & - & - & - & - & - \\
\hline 1 & 4 & 6 & 1 & - & - & - & - & - & - & - & - \\
\hline
\end{tabular}


Table E.2.1 the results of calibration procedure 1 and its validations.

\begin{tabular}{|c|c|c|c|c|c|c|c|c|c|c|c|}
\hline \multicolumn{4}{|c|}{ Configurations } & \multicolumn{2}{|c|}{ Calibration } & \multicolumn{2}{|c|}{$1^{\text {st }}$ Validation } & \multicolumn{2}{|c|}{$2^{\text {nd }}$ Validation } & \multicolumn{2}{|c|}{$3^{\text {rd }}$ Validation } \\
\hline$\varphi_{g}$ & $\Lambda_{l}$ & $\Gamma_{n}$ & $\eta_{m}$ & Cyclists & Pedestrians & Cyclists & Pedestrians & Cyclists & Pedestrians & Cyclists & Pedestrians \\
\hline 1 & 4 & 6 & 2 & - & - & - & - & - & - & - & - \\
\hline 1 & 4 & 6 & 3 & - & - & - & - & - & - & - & - \\
\hline 2 & 1 & 1 & 1 & 100 & 100 & 97 & 71 & 88 & 84 & 91 & 81 \\
\hline 2 & 1 & 1 & 2 & 100 & 100 & 97 & 75 & 91 & 86 & 93 & 83 \\
\hline 2 & 1 & 1 & 3 & 100 & 100 & 97 & 75 & 91 & 86 & 93 & 83 \\
\hline 2 & 1 & 2 & 1 & 100 & 100 & 98 & 73 & 87 & 84 & 91 & 82 \\
\hline 2 & 1 & 2 & 2 & 100 & 100 & 98 & 76 & 90 & 86 & 93 & 84 \\
\hline 2 & 1 & 2 & 3 & 100 & 100 & 98 & 76 & 90 & 86 & 93 & 84 \\
\hline 2 & 1 & 3 & 1 & 100 & 100 & 94 & 73 & 86 & 84 & 89 & 82 \\
\hline 2 & 1 & 3 & 2 & 100 & 100 & 96 & 76 & 91 & 86 & 93 & 84 \\
\hline 2 & 1 & 3 & 3 & 100 & 100 & 96 & 76 & 91 & 86 & 93 & 84 \\
\hline 2 & 1 & 4 & 1 & 100 & 100 & 96 & 73 & 86 & 85 & 90 & 82 \\
\hline 2 & 1 & 4 & 2 & 100 & 100 & 94 & 76 & 89 & 86 & 91 & 84 \\
\hline 2 & 1 & 4 & 3 & 100 & 100 & 94 & 76 & 89 & 86 & 91 & 84 \\
\hline 2 & 1 & 1 & 1 & 100 & 100 & 94 & 73 & 89 & 84 & 91 & 82 \\
\hline 2 & 1 & 1 & 2 & 100 & 100 & 94 & 75 & 92 & 86 & 93 & 83 \\
\hline 2 & 1 & 1 & 3 & 100 & 100 & 94 & 75 & 92 & 86 & 93 & 83 \\
\hline 2 & 1 & 6 & 1 & 100 & 100 & 94 & 75 & 88 & 83 & 91 & 81 \\
\hline 2 & 1 & 6 & 2 & 100 & 100 & 94 & 78 & 90 & 84 & 92 & 82 \\
\hline 2 & 1 & 6 & 3 & 100 & 100 & 94 & 78 & 90 & 84 & 92 & 82 \\
\hline 2 & 2 & 1 & 1 & 99 & 100 & - & - & - & - & - & - \\
\hline 2 & 2 & 1 & 2 & 99 & 100 & 89 & 76 & 89 & 85 & 89 & 83 \\
\hline 2 & 2 & 1 & 3 & 99 & 100 & 89 & 76 & 88 & 86 & 88 & 84 \\
\hline 2 & 2 & 2 & 1 & 99 & 100 & - & - & - & - & - & - \\
\hline 2 & 2 & 2 & 2 & 99 & 100 & 88 & 78 & 88 & 85 & 88 & 84 \\
\hline 2 & 2 & 2 & 3 & 99 & 100 & 88 & 80 & 87 & 87 & 87 & 85 \\
\hline 2 & 2 & 3 & 1 & 99 & 100 & - & - & - & - & - & - \\
\hline 2 & 2 & 3 & 2 & 99 & 100 & 88 & 80 & 88 & 83 & 88 & 82 \\
\hline 2 & 2 & 3 & 3 & 99 & 100 & 88 & 82 & 87 & 85 & 88 & 84 \\
\hline 2 & 2 & 4 & 1 & 99 & 100 & - & - & - & - & - & - \\
\hline 2 & 2 & 4 & 2 & 99 & 100 & - & - & - & - & - & - \\
\hline 2 & 2 & 4 & 3 & 99 & 100 & 87 & 78 & 86 & 87 & 86 & 85 \\
\hline 2 & 2 & 1 & 1 & 99 & 100 & - & - & - & - & - & - \\
\hline 2 & 2 & 1 & 2 & 99 & 100 & 89 & 76 & 85 & 85 & 87 & 83 \\
\hline 2 & 2 & 1 & 3 & 99 & 100 & 89 & 78 & 85 & 85 & 87 & 84 \\
\hline 2 & 2 & 6 & 1 & 99 & 100 & - & - & - & - & - & - \\
\hline
\end{tabular}


Table E.2.1 the results of calibration procedure 1 and its validations.

\begin{tabular}{|c|c|c|c|c|c|c|c|c|c|c|c|}
\hline \multicolumn{4}{|c|}{ Configurations } & \multicolumn{2}{|c|}{ Calibration } & \multicolumn{2}{|c|}{$1^{\text {st }}$ Validation } & \multicolumn{2}{|c|}{$2^{\text {nd }}$ Validation } & \multicolumn{2}{|c|}{$3^{\text {rd }}$ Validation } \\
\hline$\varphi_{g}$ & $\Lambda_{l}$ & $\Gamma_{n}$ & $\eta_{m}$ & Cyclists & Pedestrians & Cyclists & Pedestrians & Cyclists & Pedestrians & Cyclists & Pedestrians \\
\hline 2 & 2 & 6 & 2 & 99 & 100 & 87 & 80 & 88 & 84 & 88 & 83 \\
\hline 2 & 2 & 6 & 3 & 99 & 100 & 87 & 82 & 87 & 85 & 87 & 84 \\
\hline 2 & 3 & 1 & 1 & 100 & 100 & - & - & - & - & - & - \\
\hline 2 & 3 & 1 & 2 & 100 & 100 & - & - & - & - & - & - \\
\hline 2 & 3 & 1 & 3 & 100 & 100 & - & - & - & - & - & - \\
\hline 2 & 3 & 2 & 1 & 100 & 100 & - & - & - & - & - & - \\
\hline 2 & 3 & 2 & 2 & 100 & 100 & - & - & - & - & - & - \\
\hline 2 & 3 & 2 & 3 & 100 & 100 & - & - & - & - & - & - \\
\hline 2 & 3 & 3 & 1 & 100 & 100 & - & - & - & - & - & - \\
\hline 2 & 3 & 3 & 2 & 100 & 100 & - & - & - & - & - & - \\
\hline 2 & 3 & 3 & 3 & 100 & 100 & - & - & - & - & - & - \\
\hline 2 & 3 & 4 & 1 & 100 & 100 & - & - & - & - & - & - \\
\hline 2 & 3 & 4 & 2 & 100 & 100 & - & - & - & - & - & - \\
\hline 2 & 3 & 4 & 3 & 100 & 100 & - & - & - & - & - & - \\
\hline 2 & 3 & 1 & 1 & 100 & 100 & - & - & - & - & - & - \\
\hline 2 & 3 & 1 & 2 & 100 & 100 & - & - & - & - & - & - \\
\hline 2 & 3 & 1 & 3 & 100 & 100 & - & - & - & - & - & - \\
\hline 2 & 3 & 6 & 1 & 100 & 100 & - & - & - & - & - & - \\
\hline 2 & 3 & 6 & 2 & 100 & 100 & - & - & - & - & - & - \\
\hline 2 & 3 & 6 & 3 & 100 & 100 & - & - & - & - & - & - \\
\hline 2 & 4 & 1 & 1 & 100 & 100 & - & - & - & - & - & - \\
\hline 2 & 4 & 1 & 2 & 100 & 100 & - & - & - & - & - & - \\
\hline 2 & 4 & 1 & 3 & 100 & 100 & - & - & - & - & - & - \\
\hline 2 & 4 & 2 & 1 & 100 & 100 & 89 & 73 & - & - & - & - \\
\hline 2 & 4 & 2 & 2 & 100 & 100 & 89 & 73 & - & - & - & - \\
\hline 2 & 4 & 2 & 3 & 100 & 100 & - & - & - & - & - & - \\
\hline 2 & 4 & 3 & 1 & 100 & 100 & - & - & - & - & - & - \\
\hline 2 & 4 & 3 & 2 & 100 & 100 & - & - & - & - & - & - \\
\hline 2 & 4 & 3 & 3 & 100 & 100 & - & - & - & - & - & - \\
\hline 2 & 4 & 4 & 1 & 100 & 100 & - & - & - & - & - & - \\
\hline 2 & 4 & 4 & 2 & 100 & 100 & - & - & - & - & - & - \\
\hline 2 & 4 & 4 & 3 & 100 & 100 & - & - & - & - & - & - \\
\hline 2 & 4 & 1 & 1 & 100 & 100 & - & - & - & - & - & - \\
\hline 2 & 4 & 1 & 2 & 100 & 100 & - & - & - & - & - & - \\
\hline 2 & 4 & 1 & 3 & 100 & 100 & - & - & - & - & - & - \\
\hline 2 & 4 & 6 & 1 & 100 & 100 & - & - & - & - & - & - \\
\hline
\end{tabular}


Table E.2.1 the results of calibration procedure 1 and its validations.

\begin{tabular}{|c|c|c|c|c|c|c|c|c|c|c|c|}
\hline \multicolumn{4}{|c|}{ Configurations } & \multicolumn{2}{|c|}{ Calibration } & \multicolumn{2}{|c|}{$1^{\text {st }}$ Validation } & \multicolumn{2}{|c|}{$2^{\text {nd }}$ Validation } & \multicolumn{2}{|c|}{$3^{\text {rd }}$ Validation } \\
\hline$\varphi_{g}$ & $\Lambda_{l}$ & $\Gamma_{n}$ & $\eta_{m}$ & Cyclists & Pedestrians & Cyclists & Pedestrians & Cyclists & Pedestrians & Cyclists & Pedestrians \\
\hline 2 & 4 & 6 & 2 & 100 & 100 & - & - & - & - & - & - \\
\hline 2 & 4 & 6 & 3 & 100 & 100 & - & - & - & - & - & - \\
\hline 3 & 1 & 1 & 1 & 100 & 100 & 100 & 83 & 92 & 89 & 95 & 88 \\
\hline 3 & 1 & 1 & 2 & 100 & 100 & 100 & 83 & 91 & 90 & 95 & 88 \\
\hline 3 & 1 & 1 & 3 & 100 & 100 & 100 & 83 & 91 & 90 & 95 & 88 \\
\hline 3 & 1 & 2 & 1 & 100 & 100 & 100 & 87 & 93 & 90 & 96 & 89 \\
\hline 3 & 1 & 2 & 2 & 100 & 100 & 100 & 87 & 91 & 91 & 95 & 90 \\
\hline 3 & 1 & 2 & 3 & 100 & 100 & 100 & 87 & 91 & 91 & 95 & 90 \\
\hline 3 & 1 & 3 & 1 & 100 & 100 & 99 & 85 & 96 & 90 & 97 & 89 \\
\hline 3 & 1 & 3 & 2 & 100 & 100 & 99 & 85 & 94 & 91 & 96 & 90 \\
\hline 3 & 1 & 3 & 3 & 100 & 100 & 99 & 85 & 94 & 91 & 96 & 90 \\
\hline 3 & 1 & 4 & 1 & 100 & 100 & 99 & 85 & 94 & 90 & 96 & 89 \\
\hline 3 & 1 & 4 & 2 & 100 & 100 & 99 & 85 & 92 & 91 & 95 & 90 \\
\hline 3 & 1 & 4 & 3 & 100 & 100 & 99 & 85 & 92 & 91 & 95 & 90 \\
\hline 3 & 1 & 1 & 1 & 100 & 100 & 97 & 87 & 96 & 90 & 96 & 89 \\
\hline 3 & 1 & 1 & 2 & 100 & 100 & 97 & 87 & 95 & 91 & 96 & 90 \\
\hline 3 & 1 & 1 & 3 & 100 & 100 & 97 & 87 & 95 & 91 & 96 & 90 \\
\hline 3 & 1 & 6 & 1 & 100 & 100 & 99 & 87 & 95 & 90 & 96 & 89 \\
\hline 3 & 1 & 6 & 2 & 100 & 100 & 99 & 87 & 93 & 91 & 95 & 90 \\
\hline 3 & 1 & 6 & 3 & 100 & 100 & 99 & 87 & 93 & 91 & 95 & 90 \\
\hline 3 & 2 & 1 & 1 & 99 & 100 & - & - & - & - & - & - \\
\hline 3 & 2 & 1 & 2 & 99 & 100 & - & - & - & - & - & - \\
\hline 3 & 2 & 1 & 3 & 99 & 100 & - & - & - & - & - & - \\
\hline 3 & 2 & 2 & 1 & 99 & 100 & - & - & - & - & - & - \\
\hline 3 & 2 & 2 & 2 & 99 & 100 & - & - & - & - & - & - \\
\hline 3 & 2 & 2 & 3 & 99 & 100 & - & - & - & - & - & - \\
\hline 3 & 2 & 3 & 1 & 99 & 100 & - & - & - & - & - & - \\
\hline 3 & 2 & 3 & 2 & 99 & 100 & - & - & - & - & - & - \\
\hline 3 & 2 & 3 & 3 & 99 & 100 & - & - & - & - & - & - \\
\hline 3 & 2 & 4 & 1 & 99 & 100 & - & - & - & - & - & - \\
\hline 3 & 2 & 4 & 2 & 99 & 100 & - & - & - & - & - & - \\
\hline 3 & 2 & 4 & 3 & 99 & 100 & - & - & - & - & - & - \\
\hline 3 & 2 & 1 & 1 & 99 & 100 & - & - & - & - & - & - \\
\hline 3 & 2 & 1 & 2 & 99 & 100 & - & - & - & - & - & - \\
\hline 3 & 2 & 1 & 3 & 99 & 100 & - & - & - & - & - & - \\
\hline 3 & 2 & 6 & 1 & 99 & 100 & - & - & - & - & - & - \\
\hline
\end{tabular}


Table E.2.1 the results of calibration procedure 1 and its validations.

\begin{tabular}{|c|c|c|c|c|c|c|c|c|c|c|c|}
\hline \multicolumn{4}{|c|}{ Configurations } & \multicolumn{2}{|c|}{ Calibration } & \multicolumn{2}{|c|}{$1^{\text {st }}$ Validation } & \multicolumn{2}{|c|}{$2^{\text {nd }}$ Validation } & \multicolumn{2}{|c|}{$3^{\text {rd }}$ Validation } \\
\hline$\varphi_{g}$ & $\Lambda_{l}$ & $\Gamma_{n}$ & $\eta_{m}$ & Cyclists & Pedestrians & Cyclists & Pedestrians & Cyclists & Pedestrians & Cyclists & Pedestrians \\
\hline 3 & 2 & 6 & 2 & 99 & 100 & - & - & - & - & - & - \\
\hline 3 & 2 & 6 & 3 & 99 & 100 & - & - & - & - & - & - \\
\hline 3 & 3 & 1 & 1 & 100 & 100 & - & - & - & - & - & - \\
\hline 3 & 3 & 1 & 2 & 100 & 100 & - & - & - & - & - & - \\
\hline 3 & 3 & 1 & 3 & 100 & 100 & - & - & - & - & - & - \\
\hline 3 & 3 & 2 & 1 & 100 & 100 & - & - & - & - & - & - \\
\hline 3 & 3 & 2 & 2 & 100 & 100 & - & - & - & - & - & - \\
\hline 3 & 3 & 2 & 3 & 100 & 100 & - & - & - & - & - & - \\
\hline 3 & 3 & 3 & 1 & 100 & 100 & - & - & - & - & - & - \\
\hline 3 & 3 & 3 & 2 & 100 & 100 & - & - & - & - & - & - \\
\hline 3 & 3 & 3 & 3 & 100 & 100 & - & - & - & - & - & - \\
\hline 3 & 3 & 4 & 1 & 100 & 100 & - & - & - & - & - & - \\
\hline 3 & 3 & 4 & 2 & 100 & 100 & - & - & - & - & - & - \\
\hline 3 & 3 & 4 & 3 & 100 & 100 & - & - & - & - & - & - \\
\hline 3 & 3 & 1 & 1 & 100 & 100 & - & - & - & - & - & - \\
\hline 3 & 3 & 1 & 2 & 100 & 100 & - & - & - & - & - & - \\
\hline 3 & 3 & 1 & 3 & 100 & 100 & - & - & - & - & - & - \\
\hline 3 & 3 & 6 & 1 & 100 & 100 & - & - & - & - & - & - \\
\hline 3 & 3 & 6 & 2 & 100 & 100 & - & - & - & - & - & - \\
\hline 3 & 3 & 6 & 3 & 100 & 100 & - & - & - & - & - & - \\
\hline 3 & 4 & 1 & 1 & 100 & 100 & - & - & - & - & - & - \\
\hline 3 & 4 & 1 & 2 & 100 & 100 & - & - & - & - & - & - \\
\hline 3 & 4 & 1 & 3 & 100 & 100 & - & - & - & - & - & - \\
\hline 3 & 4 & 2 & 1 & 100 & 100 & - & - & - & - & - & - \\
\hline 3 & 4 & 2 & 2 & 100 & 100 & - & - & - & - & - & - \\
\hline 3 & 4 & 2 & 3 & 100 & 100 & - & - & - & - & - & - \\
\hline 3 & 4 & 3 & 1 & 100 & 100 & - & - & - & - & - & - \\
\hline 3 & 4 & 3 & 2 & 100 & 100 & - & - & - & - & - & - \\
\hline 3 & 4 & 3 & 3 & 100 & 100 & - & - & - & - & - & - \\
\hline 3 & 4 & 4 & 1 & 100 & 100 & - & - & - & - & - & - \\
\hline 3 & 4 & 4 & 2 & 100 & 100 & - & - & - & - & - & - \\
\hline 3 & 4 & 4 & 3 & 100 & 100 & - & - & - & - & - & - \\
\hline 3 & 4 & 1 & 1 & 100 & 100 & - & - & - & - & - & - \\
\hline 3 & 4 & 1 & 2 & 100 & 100 & - & - & - & - & - & - \\
\hline 3 & 4 & 1 & 3 & 100 & 100 & - & - & - & - & - & - \\
\hline 3 & 4 & 6 & 1 & 100 & 100 & - & - & - & - & - & - \\
\hline
\end{tabular}


Table E.2.1 the results of calibration procedure 1 and its validations.

\begin{tabular}{|c|c|c|c|c|c|c|c|c|c|c|c|}
\hline \multicolumn{4}{|c|}{ Configurations } & \multicolumn{2}{|c|}{ Calibration } & \multicolumn{2}{|c|}{$1^{\text {st }}$ Validation } & \multicolumn{2}{|c|}{$2^{\text {nd }}$ Validation } & \multicolumn{2}{|c|}{$3^{\text {rd }}$ Validation } \\
\hline$\varphi_{g}$ & $\Lambda_{l}$ & $\Gamma_{n}$ & $\eta_{m}$ & Cyclists & Pedestrians & Cyclists & Pedestrians & Cyclists & Pedestrians & Cyclists & Pedestrians \\
\hline 3 & 4 & 6 & 2 & 100 & 100 & - & - & - & - & - & - \\
\hline 3 & 4 & 6 & 3 & 100 & 100 & - & - & - & - & - & - \\
\hline 4 & 1 & 1 & 1 & 100 & 100 & 100 & 90 & 91 & 92 & 95 & 92 \\
\hline 4 & 1 & 1 & 2 & 100 & 100 & 100 & 90 & 89 & 93 & 94 & 92 \\
\hline 4 & 1 & 1 & 3 & 100 & 100 & 100 & 90 & 89 & 93 & 94 & 92 \\
\hline 4 & 1 & 2 & 1 & 100 & 100 & 100 & 92 & 91 & 93 & 95 & 93 \\
\hline 4 & 1 & 2 & 2 & 100 & 100 & 100 & 92 & 88 & 95 & 93 & 94 \\
\hline 4 & 1 & 2 & 3 & 100 & 100 & 100 & 92 & 88 & 95 & 93 & 94 \\
\hline 4 & 1 & 3 & 1 & 100 & 100 & 98 & 90 & 95 & 94 & 96 & 93 \\
\hline 4 & 1 & 3 & 2 & 100 & 100 & 98 & 90 & 92 & 95 & 95 & 94 \\
\hline 4 & 1 & 3 & 3 & 100 & 100 & 98 & 90 & 92 & 95 & 95 & 94 \\
\hline 4 & 1 & 4 & 1 & 100 & 100 & 98 & 90 & 92 & 93 & 95 & 93 \\
\hline 4 & 1 & 4 & 2 & 100 & 100 & 98 & 90 & 89 & 95 & 93 & 94 \\
\hline 4 & 1 & 4 & 3 & 100 & 100 & 98 & 90 & 89 & 95 & 93 & 94 \\
\hline 4 & 1 & 1 & 1 & 100 & 100 & 97 & 92 & 95 & 93 & 96 & 93 \\
\hline 4 & 1 & 1 & 2 & 100 & 100 & 97 & 92 & 93 & 95 & 95 & 94 \\
\hline 4 & 1 & 1 & 3 & 100 & 100 & 97 & 92 & 93 & 95 & 95 & 94 \\
\hline 4 & 1 & 6 & 1 & 100 & 100 & 98 & 92 & 93 & 93 & 96 & 93 \\
\hline 4 & 1 & 6 & 2 & 100 & 100 & 98 & 92 & 91 & 95 & 94 & 94 \\
\hline 4 & 1 & 6 & 3 & 100 & 100 & 98 & 92 & 91 & 95 & 94 & 94 \\
\hline 4 & 2 & 1 & 1 & 100 & 100 & - & - & - & - & - & - \\
\hline 4 & 2 & 1 & 2 & 100 & 100 & - & - & - & - & - & - \\
\hline 4 & 2 & 1 & 3 & 100 & 100 & - & - & - & - & - & - \\
\hline 4 & 2 & 2 & 1 & 100 & 100 & - & - & - & - & - & - \\
\hline 4 & 2 & 2 & 2 & 100 & 100 & - & - & - & - & - & - \\
\hline 4 & 2 & 2 & 3 & 100 & 100 & - & - & - & - & - & - \\
\hline 4 & 2 & 3 & 1 & 100 & 100 & - & - & - & - & - & - \\
\hline 4 & 2 & 3 & 2 & 100 & 100 & - & - & - & - & - & - \\
\hline 4 & 2 & 3 & 3 & 100 & 100 & - & - & - & - & - & - \\
\hline 4 & 2 & 4 & 1 & 100 & 100 & - & - & - & - & - & - \\
\hline 4 & 2 & 4 & 2 & 100 & 100 & - & - & - & - & - & - \\
\hline 4 & 2 & 4 & 3 & 100 & 100 & - & - & - & - & - & - \\
\hline 4 & 2 & 1 & 1 & 100 & 100 & - & - & - & - & - & - \\
\hline 4 & 2 & 1 & 2 & 100 & 100 & - & - & - & - & - & - \\
\hline 4 & 2 & 1 & 3 & 100 & 100 & - & - & - & - & - & - \\
\hline 4 & 2 & 6 & 1 & 100 & 100 & - & - & - & - & - & - \\
\hline
\end{tabular}


Table E.2.1 the results of calibration procedure 1 and its validations.

\begin{tabular}{|c|c|c|c|c|c|c|c|c|c|c|c|}
\hline \multicolumn{4}{|c|}{ Configurations } & \multicolumn{2}{|c|}{ Calibration } & \multicolumn{2}{|c|}{$1^{\text {st }}$ Validation } & \multicolumn{2}{|c|}{$2^{\text {nd }}$ Validation } & \multicolumn{2}{|c|}{$3^{\text {rd }}$ Validation } \\
\hline$\varphi_{g}$ & $\Lambda_{l}$ & $\Gamma_{n}$ & $\eta_{m}$ & Cyclists & Pedestrians & Cyclists & Pedestrians & Cyclists & Pedestrians & Cyclists & Pedestrians \\
\hline 4 & 2 & 6 & 2 & 100 & 100 & - & - & - & - & - & - \\
\hline 4 & 2 & 6 & 3 & 100 & 100 & - & - & - & - & - & - \\
\hline 4 & 3 & 1 & 1 & 100 & 100 & - & - & - & - & - & - \\
\hline 4 & 3 & 1 & 2 & 100 & 100 & - & - & - & - & - & - \\
\hline 4 & 3 & 1 & 3 & 100 & 100 & - & - & - & - & - & - \\
\hline 4 & 3 & 2 & 1 & 100 & 100 & - & - & - & - & - & - \\
\hline 4 & 3 & 2 & 2 & 100 & 100 & - & - & - & - & - & - \\
\hline 4 & 3 & 2 & 3 & 100 & 100 & - & - & - & - & - & - \\
\hline 4 & 3 & 3 & 1 & 100 & 100 & - & - & - & - & - & - \\
\hline 4 & 3 & 3 & 2 & 100 & 100 & - & - & - & - & - & - \\
\hline 4 & 3 & 3 & 3 & 100 & 100 & - & - & - & - & - & - \\
\hline 4 & 3 & 4 & 1 & 100 & 100 & - & - & - & - & - & - \\
\hline 4 & 3 & 4 & 2 & 100 & 100 & - & - & - & - & - & - \\
\hline 4 & 3 & 4 & 3 & 100 & 100 & - & - & - & - & - & - \\
\hline 4 & 3 & 1 & 1 & 100 & 100 & - & - & - & - & - & - \\
\hline 4 & 3 & 1 & 2 & 100 & 100 & - & - & - & - & - & - \\
\hline 4 & 3 & 1 & 3 & 100 & 100 & - & - & - & - & - & - \\
\hline 4 & 3 & 6 & 1 & 100 & 100 & - & - & - & - & - & - \\
\hline 4 & 3 & 6 & 2 & 100 & 100 & - & - & - & - & - & - \\
\hline 4 & 3 & 6 & 3 & 100 & 100 & - & - & - & - & - & - \\
\hline 4 & 4 & 1 & 1 & 100 & 100 & - & - & - & - & - & - \\
\hline 4 & 4 & 1 & 2 & 99 & 100 & - & - & - & - & - & - \\
\hline 4 & 4 & 1 & 3 & 99 & 100 & - & - & - & - & - & - \\
\hline 4 & 4 & 2 & 1 & 100 & 100 & - & - & - & - & - & - \\
\hline 4 & 4 & 2 & 2 & 99 & 100 & - & - & - & - & - & - \\
\hline 4 & 4 & 2 & 3 & 99 & 100 & - & - & - & - & - & - \\
\hline 4 & 4 & 3 & 1 & 100 & 100 & - & - & - & - & - & - \\
\hline 4 & 4 & 3 & 2 & 99 & 100 & - & - & - & - & - & - \\
\hline 4 & 4 & 3 & 3 & 99 & 100 & - & - & - & - & - & - \\
\hline 4 & 4 & 4 & 1 & 100 & 100 & - & - & - & - & - & - \\
\hline 4 & 4 & 4 & 2 & 100 & 100 & - & - & - & - & - & - \\
\hline 4 & 4 & 4 & 3 & 100 & 100 & - & - & - & - & - & - \\
\hline 4 & 4 & 1 & 1 & 100 & 100 & - & - & - & - & - & - \\
\hline 4 & 4 & 1 & 2 & 100 & 100 & - & - & - & - & - & - \\
\hline 4 & 4 & 1 & 3 & 100 & 100 & - & - & - & - & - & - \\
\hline 4 & 4 & 6 & 1 & 100 & 100 & - & - & - & - & - & - \\
\hline
\end{tabular}


Table E.2.1 the results of calibration procedure 1 and its validations.

\begin{tabular}{|c|c|c|c|c|c|c|c|c|c|c|c|}
\hline \multicolumn{4}{|c|}{ Configurations } & \multicolumn{2}{|c|}{ Calibration } & \multicolumn{2}{|c|}{$1^{\text {st }}$ Validation } & \multicolumn{2}{|c|}{$2^{\text {nd }}$ Validation } & \multicolumn{2}{|c|}{$3^{\text {rd }}$ Validation } \\
\hline$\varphi_{g}$ & $\Lambda_{l}$ & $\Gamma_{n}$ & $\eta_{m}$ & Cyclists & Pedestrians & Cyclists & Pedestrians & Cyclists & Pedestrians & Cyclists & Pedestrians \\
\hline 4 & 4 & 6 & 2 & 100 & 100 & - & - & - & - & - & - \\
\hline 4 & 4 & 6 & 3 & 100 & 100 & - & - & - & - & - & - \\
\hline 5 & 1 & 1 & 1 & 100 & 100 & 100 & 88 & 96 & 86 & 98 & 86 \\
\hline 5 & 1 & 1 & 2 & 100 & 100 & 100 & 88 & 96 & 88 & 98 & 88 \\
\hline 5 & 1 & 1 & 3 & 100 & 100 & 100 & 88 & 96 & 88 & 98 & 88 \\
\hline 5 & 1 & 2 & 1 & 100 & 100 & 100 & 91 & 91 & 93 & 96 & 93 \\
\hline 5 & 1 & 2 & 2 & 100 & 100 & 100 & 91 & 91 & 93 & 96 & 93 \\
\hline 5 & 1 & 2 & 3 & 100 & 100 & 100 & 91 & 91 & 93 & 96 & 93 \\
\hline 5 & 1 & 3 & 1 & 100 & 100 & 96 & 88 & 93 & 93 & 95 & 92 \\
\hline 5 & 1 & 3 & 2 & 100 & 100 & 96 & 88 & 93 & 93 & 95 & 92 \\
\hline 5 & 1 & 3 & 3 & 100 & 100 & 96 & 88 & 93 & 93 & 95 & 92 \\
\hline 5 & 1 & 4 & 1 & 100 & 100 & 96 & 88 & 96 & 93 & 96 & 92 \\
\hline 5 & 1 & 4 & 2 & 100 & 100 & 96 & 88 & 96 & 93 & 96 & 92 \\
\hline 5 & 1 & 4 & 3 & 100 & 100 & 96 & 88 & 96 & 93 & 96 & 92 \\
\hline 5 & 1 & 1 & 1 & 100 & 100 & 96 & 91 & 96 & 93 & 96 & 92 \\
\hline 5 & 1 & 1 & 2 & 100 & 100 & 96 & 91 & 96 & 93 & 96 & 92 \\
\hline 5 & 1 & 1 & 3 & 100 & 100 & 96 & 91 & 96 & 93 & 96 & 92 \\
\hline 5 & 1 & 6 & 1 & 100 & 100 & 93 & 91 & 96 & 91 & 95 & 91 \\
\hline 5 & 1 & 6 & 2 & 100 & 100 & 91 & 91 & 96 & 91 & 93 & 91 \\
\hline 5 & 1 & 6 & 3 & 100 & 100 & 91 & 91 & 96 & 91 & 93 & 91 \\
\hline 5 & 2 & 1 & 1 & 100 & 100 & - & - & - & - & - & - \\
\hline 5 & 2 & 1 & 2 & 100 & 100 & - & - & - & - & - & - \\
\hline 5 & 2 & 1 & 3 & 100 & 100 & - & - & - & - & - & - \\
\hline 5 & 2 & 2 & 1 & 100 & 100 & - & - & - & - & - & - \\
\hline 5 & 2 & 2 & 2 & 100 & 100 & - & - & - & - & - & - \\
\hline 5 & 2 & 2 & 3 & 100 & 100 & - & - & - & - & - & - \\
\hline 5 & 2 & 3 & 1 & 100 & 100 & - & - & - & - & - & - \\
\hline 5 & 2 & 3 & 2 & 100 & 100 & - & - & - & - & - & - \\
\hline 5 & 2 & 3 & 3 & 100 & 100 & - & - & - & - & - & - \\
\hline 5 & 2 & 4 & 1 & 100 & 100 & - & - & - & - & - & - \\
\hline 5 & 2 & 4 & 2 & 100 & 100 & - & - & - & - & - & - \\
\hline 5 & 2 & 4 & 3 & 100 & 100 & - & - & - & - & - & - \\
\hline 5 & 2 & 1 & 1 & 100 & 100 & - & - & - & - & - & - \\
\hline 5 & 2 & 1 & 2 & 100 & 100 & - & - & - & - & - & - \\
\hline 5 & 2 & 1 & 3 & 100 & 100 & - & - & - & - & - & - \\
\hline 5 & 2 & 6 & 1 & 100 & 100 & - & - & - & - & - & - \\
\hline
\end{tabular}


Table E.2.1 the results of calibration procedure 1 and its validations.

\begin{tabular}{|c|c|c|c|c|c|c|c|c|c|c|c|}
\hline \multicolumn{4}{|c|}{ Configurations } & \multicolumn{2}{|c|}{ Calibration } & \multicolumn{2}{|c|}{$1^{\text {st }}$ Validation } & \multicolumn{2}{|c|}{$2^{\text {nd }}$ Validation } & \multicolumn{2}{|c|}{$3^{\text {rd }}$ Validation } \\
\hline$\varphi_{g}$ & $\Lambda_{l}$ & $\Gamma_{n}$ & $\eta_{m}$ & Cyclists & Pedestrians & Cyclists & Pedestrians & Cyclists & Pedestrians & Cyclists & Pedestrians \\
\hline 5 & 2 & 6 & 2 & 100 & 100 & - & - & - & - & - & - \\
\hline 5 & 2 & 6 & 3 & 100 & 100 & - & - & - & - & - & - \\
\hline 5 & 3 & 1 & 1 & 100 & 100 & - & - & - & - & - & - \\
\hline 5 & 3 & 1 & 2 & 100 & 100 & - & - & - & - & - & - \\
\hline 5 & 3 & 1 & 3 & 100 & 100 & - & - & - & - & - & - \\
\hline 5 & 3 & 2 & 1 & 100 & 100 & - & - & - & - & - & - \\
\hline 5 & 3 & 2 & 2 & 100 & 100 & - & - & - & - & - & - \\
\hline 5 & 3 & 2 & 3 & 100 & 100 & - & - & - & - & - & - \\
\hline 5 & 3 & 3 & 1 & 100 & 100 & - & - & - & - & - & - \\
\hline 5 & 3 & 3 & 2 & 100 & 100 & - & - & - & - & - & - \\
\hline 5 & 3 & 3 & 3 & 100 & 100 & - & - & - & - & - & - \\
\hline 5 & 3 & 4 & 1 & 100 & 100 & - & - & - & - & - & - \\
\hline 5 & 3 & 4 & 2 & 100 & 100 & - & - & - & - & - & - \\
\hline 5 & 3 & 4 & 3 & 100 & 100 & - & - & - & - & - & - \\
\hline 5 & 3 & 1 & 1 & 100 & 100 & - & - & - & - & - & - \\
\hline 5 & 3 & 1 & 2 & 100 & 100 & - & - & - & - & - & - \\
\hline 5 & 3 & 1 & 3 & 100 & 100 & - & - & - & - & - & - \\
\hline 5 & 3 & 6 & 1 & 100 & 100 & - & - & - & - & - & - \\
\hline 5 & 3 & 6 & 2 & 100 & 100 & - & - & - & - & - & - \\
\hline 5 & 3 & 6 & 3 & 100 & 100 & - & - & - & - & - & - \\
\hline 5 & 4 & 1 & 1 & 100 & 100 & - & - & - & - & - & - \\
\hline 5 & 4 & 1 & 2 & 100 & 100 & - & - & - & - & - & - \\
\hline 5 & 4 & 1 & 3 & 100 & 100 & - & - & - & - & - & - \\
\hline 5 & 4 & 2 & 1 & 100 & 100 & - & - & - & - & - & - \\
\hline 5 & 4 & 2 & 2 & 100 & 100 & - & - & - & - & - & - \\
\hline 5 & 4 & 2 & 3 & 100 & 100 & - & - & - & - & - & - \\
\hline 5 & 4 & 3 & 1 & 100 & 100 & - & - & - & - & - & - \\
\hline 5 & 4 & 3 & 2 & 100 & 100 & - & - & - & - & - & - \\
\hline 5 & 4 & 3 & 3 & 100 & 100 & - & - & - & - & - & - \\
\hline 5 & 4 & 4 & 1 & 100 & 100 & - & - & - & - & - & - \\
\hline 5 & 4 & 4 & 2 & 100 & 100 & - & - & - & - & - & - \\
\hline 5 & 4 & 4 & 3 & 100 & 100 & - & - & - & - & - & - \\
\hline 5 & 4 & 1 & 1 & 100 & 100 & - & - & - & - & - & - \\
\hline 5 & 4 & 1 & 2 & 100 & 100 & - & - & - & - & - & - \\
\hline 5 & 4 & 1 & 3 & 100 & 100 & - & - & - & - & - & - \\
\hline 5 & 4 & 6 & 1 & 100 & 100 & - & - & - & - & - & - \\
\hline
\end{tabular}


Table E.2.1 the results of calibration procedure 1 and its validations.

\begin{tabular}{|c|c|c|c|c|c|c|c|c|c|c|c|}
\hline \multicolumn{4}{|c|}{ Configurations } & \multicolumn{2}{|c|}{ Calibration } & \multicolumn{2}{|c|}{$\mathbf{1}^{\text {st }}$ Validation } & \multicolumn{2}{|c|}{$2^{\text {nd }}$ Validation } & \multicolumn{2}{|c|}{$3^{\text {rd }}$ Validation } \\
\hline$\varphi_{g}$ & $\Lambda_{l}$ & $\Gamma_{n}$ & $\eta_{m}$ & Cyclists & Pedestrians & Cyclists & Pedestrians & Cyclists & Pedestrians & Cyclists & Pedestrians \\
\hline 5 & 4 & 6 & 2 & 100 & 100 & - & - & - & - & - & - \\
\hline 5 & 4 & 6 & 3 & 100 & 100 & - & - & - & - & - & - \\
\hline
\end{tabular}

Table E.2.2 the results of calibration procedure 2 and its validations.

\begin{tabular}{|c|c|c|c|c|c|c|c|c|c|c|c|}
\hline \multicolumn{4}{|c|}{ Configurations } & \multicolumn{2}{|c|}{ Calibration } & \multicolumn{2}{|c|}{$\mathbf{1}^{\text {st }}$ Validation } & \multicolumn{2}{|c|}{$2^{\text {nd }}$ Validation } & \multicolumn{2}{|c|}{$3^{\text {rd }}$ Validation } \\
\hline$\varphi_{g}$ & $\Lambda_{l}$ & $\Gamma_{n}$ & $\eta_{m}$ & Cyclists & Pedestrians & Cyclists & Pedestrians & Cyclists & Pedestrians & Cyclists & Pedestrians \\
\hline 1 & 1 & 1 & 1 & 95.19 & 92.65 & 98.00 & 95.24 & 91.95 & 97.81 & 94.38 & 97.15 \\
\hline 1 & 1 & 1 & 2 & 95.19 & 92.65 & 98.00 & 95.24 & 91.95 & 97.81 & 94.38 & 97.15 \\
\hline 1 & 1 & 1 & 3 & 95.19 & 92.65 & 98.00 & 95.24 & 91.95 & 97.81 & 94.38 & 97.15 \\
\hline 1 & 1 & 2 & 1 & 95.19 & 92.65 & 98.00 & 95.24 & 91.95 & 97.81 & 94.38 & 97.15 \\
\hline 1 & 1 & 2 & 2 & 95.19 & 92.65 & 98.00 & 95.24 & 91.95 & 97.81 & 94.38 & 97.15 \\
\hline 1 & 1 & 2 & 3 & 95.19 & 92.65 & 98.00 & 95.24 & 91.95 & 97.81 & 94.38 & 97.15 \\
\hline 1 & 1 & 3 & 1 & 95.19 & 92.65 & 98.00 & 95.24 & 91.95 & 97.81 & 94.38 & 97.15 \\
\hline 1 & 1 & 3 & 2 & 95.19 & 92.65 & 98.00 & 95.24 & 91.95 & 97.81 & 94.38 & 97.15 \\
\hline 1 & 1 & 3 & 3 & 95.19 & 92.65 & 98.00 & 95.24 & 91.95 & 97.81 & 94.38 & 97.15 \\
\hline 1 & 1 & 4 & 1 & 95.19 & 92.65 & 98.00 & 95.24 & 91.95 & 97.81 & 94.38 & 97.15 \\
\hline 1 & 1 & 4 & 2 & 95.19 & 92.65 & 98.00 & 95.24 & 91.95 & 97.81 & 94.38 & 97.15 \\
\hline 1 & 1 & 4 & 3 & 95.19 & 92.65 & 98.00 & 95.24 & 91.95 & 97.81 & 94.38 & 97.15 \\
\hline 1 & 1 & 1 & 1 & 95.19 & 92.65 & 98.00 & 95.24 & 91.95 & 97.81 & 94.38 & 97.15 \\
\hline 1 & 1 & 1 & 2 & 95.19 & 92.65 & 98.00 & 95.24 & 91.95 & 97.81 & 94.38 & 97.15 \\
\hline 1 & 1 & 1 & 3 & 95.19 & 92.65 & 98.00 & 95.24 & 91.95 & 97.81 & 94.38 & 97.15 \\
\hline 1 & 1 & 6 & 1 & 95.19 & 92.65 & 98.00 & 95.24 & 91.95 & 97.81 & 94.38 & 97.15 \\
\hline 1 & 1 & 6 & 2 & 95.19 & 92.65 & 98.00 & 95.24 & 91.95 & 97.81 & 94.38 & 97.15 \\
\hline 1 & 1 & 6 & 3 & 95.19 & 92.65 & 98.00 & 95.24 & 91.95 & 97.81 & 94.38 & 97.15 \\
\hline 1 & 2 & 1 & 1 & 86.54 & 97.06 & - & - & - & - & - & - \\
\hline 1 & 2 & 1 & 2 & 86.54 & 97.06 & - & - & - & - & - & - \\
\hline 1 & 2 & 1 & 3 & 86.54 & 97.06 & - & - & - & - & - & - \\
\hline 1 & 2 & 2 & 1 & 86.54 & 97.06 & - & - & - & - & - & - \\
\hline 1 & 2 & 2 & 2 & 86.54 & 97.06 & - & - & - & - & - & - \\
\hline 1 & 2 & 2 & 3 & 86.54 & 97.06 & - & - & - & - & - & - \\
\hline 1 & 2 & 3 & 1 & 86.54 & 97.06 & - & - & - & - & - & - \\
\hline 1 & 2 & 3 & 2 & 86.54 & 97.06 & - & - & - & - & - & - \\
\hline 1 & 2 & 3 & 3 & 86.54 & 97.06 & - & - & - & - & - & - \\
\hline 1 & 2 & 4 & 1 & 86.54 & 97.06 & - & - & - & - & - & - \\
\hline
\end{tabular}


Table E.2.2 the results of calibration procedure 2 and its validations.

\begin{tabular}{|c|c|c|c|c|c|c|c|c|c|c|c|}
\hline \multicolumn{4}{|c|}{ Configurations } & \multicolumn{2}{|c|}{ Calibration } & \multicolumn{2}{|c|}{$\mathbf{1}^{\text {st }}$ Validation } & \multicolumn{2}{|c|}{$2^{\text {nd }}$ Validation } & \multicolumn{2}{|c|}{$3^{\text {rd }}$ Validation } \\
\hline$\varphi_{g}$ & $\Lambda_{l}$ & $\Gamma_{n}$ & $\eta_{m}$ & Cyclists & Pedestrians & Cyclists & Pedestrians & Cyclists & Pedestrians & Cyclists & Pedestrians \\
\hline 1 & 2 & 4 & 2 & 86.54 & 97.06 & - & - & - & - & - & - \\
\hline 1 & 2 & 4 & 3 & 86.54 & 97.06 & - & - & - & - & - & - \\
\hline 1 & 2 & 1 & 1 & 86.54 & 97.06 & - & - & - & - & - & - \\
\hline 1 & 2 & 1 & 2 & 86.54 & 97.06 & - & - & - & - & - & - \\
\hline 1 & 2 & 1 & 3 & 86.54 & 97.06 & - & - & - & - & - & - \\
\hline 1 & 2 & 6 & 1 & 86.54 & 97.06 & - & - & - & - & - & - \\
\hline 1 & 2 & 6 & 2 & 86.54 & 97.06 & - & - & - & - & - & - \\
\hline 1 & 2 & 6 & 3 & 86.54 & 97.06 & - & - & - & - & - & - \\
\hline 1 & 3 & 1 & 1 & 88.46 & 98.53 & - & - & - & - & - & - \\
\hline 1 & 3 & 1 & 2 & 88.46 & 98.53 & - & - & - & - & - & - \\
\hline 1 & 3 & 1 & 3 & 88.46 & 98.53 & - & - & - & - & - & - \\
\hline 1 & 3 & 2 & 1 & 88.46 & 98.53 & - & - & - & - & - & - \\
\hline 1 & 3 & 2 & 2 & 88.46 & 98.53 & - & - & - & - & - & - \\
\hline 1 & 3 & 2 & 3 & 88.46 & 98.53 & - & - & - & - & - & - \\
\hline 1 & 3 & 3 & 1 & 88.46 & 98.53 & - & - & - & - & - & - \\
\hline 1 & 3 & 3 & 2 & 88.46 & 98.53 & - & - & - & - & - & - \\
\hline 1 & 3 & 3 & 3 & 88.46 & 98.53 & - & - & - & - & - & - \\
\hline 1 & 3 & 4 & 1 & 88.46 & 98.53 & - & - & - & - & - & - \\
\hline 1 & 3 & 4 & 2 & 88.46 & 98.53 & - & - & - & - & - & - \\
\hline 1 & 3 & 4 & 3 & 88.46 & 98.53 & - & - & - & - & - & - \\
\hline 1 & 3 & 1 & 1 & 88.46 & 98.53 & - & - & - & - & - & - \\
\hline 1 & 3 & 1 & 2 & 88.46 & 98.53 & - & - & - & - & - & - \\
\hline 1 & 3 & 1 & 3 & 88.46 & 98.53 & - & - & - & - & - & - \\
\hline 1 & 3 & 6 & 1 & 88.46 & 98.53 & - & - & - & - & - & - \\
\hline 1 & 3 & 6 & 2 & 88.46 & 98.53 & - & - & - & - & - & - \\
\hline 1 & 3 & 6 & 3 & 88.46 & 98.53 & - & - & - & - & - & - \\
\hline 1 & 4 & 1 & 1 & - & - & - & - & - & - & - & - \\
\hline 1 & 4 & 1 & 2 & - & - & - & - & - & - & - & - \\
\hline 1 & 4 & 1 & 3 & - & - & - & - & - & - & - & - \\
\hline 1 & 4 & 2 & 1 & - & - & - & - & - & - & - & - \\
\hline 1 & 4 & 2 & 2 & - & - & - & - & - & - & - & - \\
\hline 1 & 4 & 2 & 3 & - & - & - & - & - & - & - & - \\
\hline 1 & 4 & 3 & 1 & - & - & - & - & - & - & - & - \\
\hline 1 & 4 & 3 & 2 & - & - & - & - & - & - & - & - \\
\hline 1 & 4 & 3 & 3 & - & - & - & - & - & - & - & - \\
\hline
\end{tabular}


Table E.2.2 the results of calibration procedure 2 and its validations.

\begin{tabular}{|c|c|c|c|c|c|c|c|c|c|c|c|}
\hline \multicolumn{4}{|c|}{ Configurations } & \multicolumn{2}{|c|}{ Calibration } & \multicolumn{2}{|c|}{$\mathbf{1}^{\text {st }}$ Validation } & \multicolumn{2}{|c|}{$2^{\text {nd }}$ Validation } & \multicolumn{2}{|c|}{$3^{\text {rd }}$ Validation } \\
\hline$\varphi_{g}$ & $\Lambda_{l}$ & $\Gamma_{n}$ & $\eta_{m}$ & Cyclists & Pedestrians & Cyclists & Pedestrians & Cyclists & Pedestrians & Cyclists & Pedestrians \\
\hline 1 & 4 & 4 & 1 & - & - & - & - & - & - & - & - \\
\hline 1 & 4 & 4 & 2 & - & - & - & - & - & - & - & - \\
\hline 1 & 4 & 4 & 3 & - & - & - & - & - & - & - & - \\
\hline 1 & 4 & 1 & 1 & - & - & - & - & - & - & - & - \\
\hline 1 & 4 & 1 & 2 & - & - & - & - & - & - & - & - \\
\hline 1 & 4 & 1 & 3 & - & - & - & - & - & - & - & - \\
\hline 1 & 4 & 6 & 1 & - & - & - & - & - & - & - & - \\
\hline 1 & 4 & 6 & 2 & - & - & - & - & - & - & - & - \\
\hline 1 & 4 & 6 & 3 & - & - & - & - & - & - & - & - \\
\hline 2 & 1 & 1 & 1 & 95.51 & 100.00 & 97.75 & 100.00 & 91.61 & 97.81 & 93.97 & 98.32 \\
\hline 2 & 1 & 1 & 2 & 95.51 & 100.00 & 97.75 & 100.00 & 91.61 & 97.81 & 93.97 & 98.32 \\
\hline 2 & 1 & 1 & 3 & 95.51 & 100.00 & 97.75 & 100.00 & 91.61 & 97.81 & 93.97 & 98.32 \\
\hline 2 & 1 & 2 & 1 & 95.51 & 100.00 & 97.75 & 100.00 & 91.61 & 97.81 & 93.97 & 98.32 \\
\hline 2 & 1 & 2 & 2 & 95.51 & 100.00 & 97.75 & 100.00 & 91.61 & 97.81 & 93.97 & 98.32 \\
\hline 2 & 1 & 2 & 3 & 95.51 & 100.00 & 97.75 & 100.00 & 91.61 & 97.81 & 93.97 & 98.32 \\
\hline 2 & 1 & 3 & 1 & 95.51 & 100.00 & 97.75 & 100.00 & 91.61 & 97.81 & 93.97 & 98.32 \\
\hline 2 & 1 & 3 & 2 & 95.51 & 100.00 & 97.75 & 100.00 & 91.61 & 97.81 & 93.97 & 98.32 \\
\hline 2 & 1 & 3 & 3 & 95.51 & 100.00 & 97.75 & 100.00 & 91.61 & 97.81 & 93.97 & 98.32 \\
\hline 2 & 1 & 4 & 1 & 95.51 & 100.00 & 97.75 & 100.00 & 91.61 & 97.81 & 93.97 & 98.32 \\
\hline 2 & 1 & 4 & 2 & 95.51 & 100.00 & 97.75 & 100.00 & 91.61 & 97.81 & 93.97 & 98.32 \\
\hline 2 & 1 & 4 & 3 & 95.51 & 100.00 & 97.75 & 100.00 & 91.61 & 97.81 & 93.97 & 98.32 \\
\hline 2 & 1 & 1 & 1 & 95.51 & 100.00 & 97.75 & 100.00 & 91.61 & 97.81 & 93.97 & 98.32 \\
\hline 2 & 1 & 1 & 2 & 95.51 & 100.00 & 97.75 & 100.00 & 91.61 & 97.81 & 93.97 & 98.32 \\
\hline 2 & 1 & 1 & 3 & 95.51 & 100.00 & 97.75 & 100.00 & 91.61 & 97.81 & 93.97 & 98.32 \\
\hline 2 & 1 & 6 & 1 & 95.51 & 100.00 & 97.75 & 100.00 & 91.61 & 97.81 & 93.97 & 98.32 \\
\hline 2 & 1 & 6 & 2 & 95.51 & 100.00 & 97.75 & 100.00 & 91.61 & 97.81 & 93.97 & 98.32 \\
\hline 2 & 1 & 6 & 3 & 95.51 & 100.00 & 97.75 & 100.00 & 91.61 & 97.81 & 93.97 & 98.32 \\
\hline 2 & 2 & 1 & 1 & - & - & - & - & - & - & - & - \\
\hline 2 & 2 & 1 & 2 & - & - & - & - & - & - & - & - \\
\hline 2 & 2 & 1 & 3 & - & - & - & - & - & - & - & - \\
\hline 2 & 2 & 2 & 1 & - & - & - & - & - & - & - & - \\
\hline 2 & 2 & 2 & 2 & - & - & - & - & - & - & - & - \\
\hline 2 & 2 & 2 & 3 & - & - & - & - & - & - & - & - \\
\hline 2 & 2 & 3 & 1 & - & - & - & - & - & - & - & - \\
\hline 2 & 2 & 3 & 2 & - & - & - & - & - & - & - & - \\
\hline
\end{tabular}


Table E.2.2 the results of calibration procedure 2 and its validations.

\begin{tabular}{|c|c|c|c|c|c|c|c|c|c|c|c|}
\hline \multicolumn{4}{|c|}{ Configurations } & \multicolumn{2}{|c|}{ Calibration } & \multicolumn{2}{|c|}{$\mathbf{1}^{\text {st }}$ Validation } & \multicolumn{2}{|c|}{$2^{\text {nd }}$ Validation } & \multicolumn{2}{|c|}{$3^{\text {rd }}$ Validation } \\
\hline$\varphi_{g}$ & $\Lambda_{l}$ & $\Gamma_{n}$ & $\eta_{m}$ & Cyclists & Pedestrians & Cyclists & Pedestrians & Cyclists & Pedestrians & Cyclists & Pedestrians \\
\hline 2 & 2 & 3 & 3 & - & - & - & - & - & - & - & - \\
\hline 2 & 2 & 4 & 1 & - & - & - & - & - & - & - & - \\
\hline 2 & 2 & 4 & 2 & - & - & - & - & - & - & - & - \\
\hline 2 & 2 & 4 & 3 & - & - & - & - & - & - & - & - \\
\hline 2 & 2 & 1 & 1 & - & - & - & - & - & - & - & - \\
\hline 2 & 2 & 1 & 2 & - & - & - & - & - & - & - & - \\
\hline 2 & 2 & 1 & 3 & - & - & - & - & - & - & - & - \\
\hline 2 & 2 & 6 & 1 & - & - & - & - & - & - & - & - \\
\hline 2 & 2 & 6 & 2 & - & - & - & - & - & - & - & - \\
\hline 2 & 2 & 6 & 3 & - & - & - & - & - & - & - & - \\
\hline 2 & 3 & 1 & 1 & 95.51 & 95.92 & - & - & - & - & - & - \\
\hline 2 & 3 & 1 & 2 & 95.51 & 95.92 & - & - & - & - & - & - \\
\hline 2 & 3 & 1 & 3 & 95.51 & 95.92 & - & - & - & - & - & - \\
\hline 2 & 3 & 2 & 1 & 95.51 & 95.92 & - & - & - & - & - & - \\
\hline 2 & 3 & 2 & 2 & 95.51 & 95.92 & - & - & - & - & - & - \\
\hline 2 & 3 & 2 & 3 & 95.51 & 95.92 & - & - & - & - & - & - \\
\hline 2 & 3 & 3 & 1 & 95.51 & 95.92 & - & - & - & - & - & - \\
\hline 2 & 3 & 3 & 2 & 95.51 & 95.92 & - & - & - & - & - & - \\
\hline 2 & 3 & 3 & 3 & 95.51 & 95.92 & - & - & - & - & - & - \\
\hline 2 & 3 & 4 & 1 & 95.51 & 95.92 & - & - & - & - & - & - \\
\hline 2 & 3 & 4 & 2 & 95.51 & 95.92 & - & - & - & - & - & - \\
\hline 2 & 3 & 4 & 3 & 95.51 & 95.92 & - & - & - & - & - & - \\
\hline 2 & 3 & 1 & 1 & 95.51 & 95.92 & - & - & - & - & - & - \\
\hline 2 & 3 & 1 & 2 & 95.51 & 95.92 & - & - & - & - & - & - \\
\hline 2 & 3 & 1 & 3 & 95.51 & 95.92 & - & - & - & - & - & - \\
\hline 2 & 3 & 6 & 1 & 95.51 & 95.92 & - & - & - & - & - & - \\
\hline 2 & 3 & 6 & 2 & 95.51 & 95.92 & - & - & - & - & - & - \\
\hline 2 & 3 & 6 & 3 & 95.51 & 95.92 & - & - & - & - & - & - \\
\hline 2 & 4 & 1 & 1 & 96.63 & 100.00 & - & - & - & - & - & - \\
\hline 2 & 4 & 1 & 2 & 96.63 & 100.00 & - & - & - & - & - & - \\
\hline 2 & 4 & 1 & 3 & 96.63 & 100.00 & - & - & - & - & - & - \\
\hline 2 & 4 & 2 & 1 & 96.63 & 100.00 & - & - & - & - & - & - \\
\hline 2 & 4 & 2 & 2 & 96.63 & 100.00 & - & - & - & - & - & - \\
\hline 2 & 4 & 2 & 3 & 96.63 & 100.00 & - & - & - & - & - & - \\
\hline 2 & 4 & 3 & 1 & 96.63 & 100.00 & - & - & - & - & - & - \\
\hline
\end{tabular}


Table E.2.2 the results of calibration procedure 2 and its validations.

\begin{tabular}{|c|c|c|c|c|c|c|c|c|c|c|c|}
\hline \multicolumn{4}{|c|}{ Configurations } & \multicolumn{2}{|c|}{ Calibration } & \multicolumn{2}{|c|}{$\mathbf{1}^{\text {st }}$ Validation } & \multicolumn{2}{|c|}{$2^{\text {nd }}$ Validation } & \multicolumn{2}{|c|}{$3^{\text {rd }}$ Validation } \\
\hline$\varphi_{g}$ & $\Lambda_{l}$ & $\Gamma_{n}$ & $\eta_{m}$ & Cyclists & Pedestrians & Cyclists & Pedestrians & Cyclists & Pedestrians & Cyclists & Pedestrians \\
\hline 2 & 4 & 3 & 2 & 96.63 & 100.00 & - & - & - & - & - & - \\
\hline 2 & 4 & 3 & 3 & 96.63 & 100.00 & - & - & - & - & - & - \\
\hline 2 & 4 & 4 & 1 & 96.63 & 100.00 & - & - & - & - & - & - \\
\hline 2 & 4 & 4 & 2 & 96.63 & 100.00 & - & - & - & - & - & - \\
\hline 2 & 4 & 4 & 3 & 96.63 & 100.00 & - & - & - & - & - & - \\
\hline 2 & 4 & 1 & 1 & 96.63 & 100.00 & - & - & - & - & - & - \\
\hline 2 & 4 & 1 & 2 & 96.63 & 100.00 & - & - & - & - & - & - \\
\hline 2 & 4 & 1 & 3 & 96.63 & 100.00 & - & - & - & - & - & - \\
\hline 2 & 4 & 6 & 1 & 96.63 & 100.00 & - & - & - & - & - & - \\
\hline 2 & 4 & 6 & 2 & 96.63 & 100.00 & - & - & - & - & - & - \\
\hline 2 & 4 & 6 & 3 & 96.63 & 100.00 & - & - & - & - & - & - \\
\hline 3 & 1 & 1 & 1 & 94.67 & 100.00 & 98.61 & 97.87 & 91.96 & 97.80 & 94.57 & 97.82 \\
\hline 3 & 1 & 1 & 2 & 94.67 & 100.00 & 98.61 & 97.87 & 91.96 & 97.80 & 94.57 & 97.82 \\
\hline 3 & 1 & 1 & 3 & 94.67 & 100.00 & 98.61 & 97.87 & 91.96 & 97.80 & 94.57 & 97.82 \\
\hline 3 & 1 & 2 & 1 & 94.67 & 100.00 & 98.61 & 97.87 & 91.96 & 97.80 & 94.57 & 97.82 \\
\hline 3 & 1 & 2 & 2 & 94.67 & 100.00 & 98.61 & 97.87 & 91.96 & 97.80 & 94.57 & 97.82 \\
\hline 3 & 1 & 2 & 3 & 94.67 & 100.00 & 98.61 & 97.87 & 91.96 & 97.80 & 94.57 & 97.82 \\
\hline 3 & 1 & 3 & 1 & 94.67 & 100.00 & 98.61 & 97.87 & 91.96 & 97.80 & 94.57 & 97.82 \\
\hline 3 & 1 & 3 & 2 & 94.67 & 100.00 & 98.61 & 97.87 & 91.96 & 97.80 & 94.57 & 97.82 \\
\hline 3 & 1 & 3 & 3 & 94.67 & 100.00 & 98.61 & 97.87 & 91.96 & 97.80 & 94.57 & 97.82 \\
\hline 3 & 1 & 4 & 1 & 94.67 & 100.00 & 98.61 & 97.87 & 91.96 & 97.80 & 94.57 & 97.82 \\
\hline 3 & 1 & 4 & 2 & 94.67 & 100.00 & 98.61 & 97.87 & 91.96 & 97.80 & 94.57 & 97.82 \\
\hline 3 & 1 & 4 & 3 & 94.67 & 100.00 & 98.61 & 97.87 & 91.96 & 97.80 & 94.57 & 97.82 \\
\hline 3 & 1 & 1 & 1 & 94.67 & 100.00 & 98.61 & 97.87 & 91.96 & 97.80 & 94.57 & 97.82 \\
\hline 3 & 1 & 1 & 2 & 94.67 & 100.00 & 98.61 & 97.87 & 91.96 & 97.80 & 94.57 & 97.82 \\
\hline 3 & 1 & 1 & 3 & 94.67 & 100.00 & 98.61 & 97.87 & 91.96 & 97.80 & 94.57 & 97.82 \\
\hline 3 & 1 & 6 & 1 & 94.67 & 100.00 & 98.61 & 97.87 & 91.96 & 97.80 & 94.57 & 97.82 \\
\hline 3 & 1 & 6 & 2 & 94.67 & 100.00 & 98.61 & 97.87 & 91.96 & 97.80 & 94.57 & 97.82 \\
\hline 3 & 1 & 6 & 3 & 94.67 & 100.00 & 98.61 & 97.87 & 91.96 & 97.80 & 94.57 & 97.82 \\
\hline 3 & 2 & 1 & 1 & - & - & - & - & - & - & - & - \\
\hline 3 & 2 & 1 & 2 & - & - & - & - & - & - & - & - \\
\hline 3 & 2 & 1 & 3 & - & - & - & - & - & - & - & - \\
\hline 3 & 2 & 2 & 1 & - & - & - & - & - & - & - & - \\
\hline 3 & 2 & 2 & 2 & - & - & - & - & - & - & - & - \\
\hline 3 & 2 & 2 & 3 & - & - & - & - & - & - & - & - \\
\hline
\end{tabular}


Table E.2.2 the results of calibration procedure 2 and its validations.

\begin{tabular}{|c|c|c|c|c|c|c|c|c|c|c|c|}
\hline \multicolumn{4}{|c|}{ Configurations } & \multicolumn{2}{|c|}{ Calibration } & \multicolumn{2}{|c|}{$\mathbf{1}^{\text {st }}$ Validation } & \multicolumn{2}{|c|}{$2^{\text {nd }}$ Validation } & \multicolumn{2}{|c|}{$3^{\text {rd }}$ Validation } \\
\hline$\varphi_{g}$ & $\Lambda_{l}$ & $\Gamma_{n}$ & $\eta_{m}$ & Cyclists & Pedestrians & Cyclists & Pedestrians & Cyclists & Pedestrians & Cyclists & Pedestrians \\
\hline 3 & 2 & 3 & 1 & - & - & - & - & - & - & - & - \\
\hline 3 & 2 & 3 & 2 & - & - & - & - & - & - & - & - \\
\hline 3 & 2 & 3 & 3 & - & - & - & - & - & - & - & - \\
\hline 3 & 2 & 4 & 1 & - & - & - & - & - & - & - & - \\
\hline 3 & 2 & 4 & 2 & - & - & - & - & - & - & - & - \\
\hline 3 & 2 & 4 & 3 & - & - & - & - & - & - & - & - \\
\hline 3 & 2 & 1 & 1 & - & - & - & - & - & - & - & - \\
\hline 3 & 2 & 1 & 2 & - & - & - & - & - & - & - & - \\
\hline 3 & 2 & 1 & 3 & - & - & - & - & - & - & - & - \\
\hline 3 & 2 & 6 & 1 & - & - & - & - & - & - & - & - \\
\hline 3 & 2 & 6 & 2 & - & - & - & - & - & - & - & - \\
\hline 3 & 2 & 6 & 3 & - & - & - & - & - & - & - & - \\
\hline 3 & 3 & 1 & 1 & 94.67 & 97.56 & - & - & - & - & - & - \\
\hline 3 & 3 & 1 & 2 & 94.67 & 97.56 & - & - & - & - & - & - \\
\hline 3 & 3 & 1 & 3 & 94.67 & 97.56 & - & - & - & - & - & - \\
\hline 3 & 3 & 2 & 1 & 94.67 & 97.56 & - & - & - & - & - & - \\
\hline 3 & 3 & 2 & 2 & 94.67 & 97.56 & - & - & - & - & - & - \\
\hline 3 & 3 & 2 & 3 & 94.67 & 97.56 & - & - & - & - & - & - \\
\hline 3 & 3 & 3 & 1 & 94.67 & 97.56 & - & - & - & - & - & - \\
\hline 3 & 3 & 3 & 2 & 94.67 & 97.56 & - & - & - & - & - & - \\
\hline 3 & 3 & 3 & 3 & 94.67 & 97.56 & - & - & - & - & - & - \\
\hline 3 & 3 & 4 & 1 & 94.67 & 97.56 & - & - & - & - & - & - \\
\hline 3 & 3 & 4 & 2 & 94.67 & 97.56 & - & - & - & - & - & - \\
\hline 3 & 3 & 4 & 3 & 94.67 & 97.56 & - & - & - & - & - & - \\
\hline 3 & 3 & 1 & 1 & 94.67 & 97.56 & - & - & - & - & - & - \\
\hline 3 & 3 & 1 & 2 & 94.67 & 97.56 & - & - & - & - & - & - \\
\hline 3 & 3 & 1 & 3 & 94.67 & 97.56 & - & - & - & - & - & - \\
\hline 3 & 3 & 6 & 1 & 94.67 & 97.56 & - & - & - & - & - & - \\
\hline 3 & 3 & 6 & 2 & 94.67 & 97.56 & - & - & - & - & - & - \\
\hline 3 & 3 & 6 & 3 & 94.67 & 97.56 & - & - & - & - & - & - \\
\hline 3 & 4 & 1 & 1 & 97.33 & 100.00 & - & - & - & - & - & - \\
\hline 3 & 4 & 1 & 2 & 97.33 & 100.00 & - & - & - & - & - & - \\
\hline 3 & 4 & 1 & 3 & 97.33 & 100.00 & - & - & - & - & - & - \\
\hline 3 & 4 & 2 & 1 & 97.33 & 100.00 & - & - & - & - & - & - \\
\hline 3 & 4 & 2 & 2 & 97.33 & 100.00 & - & - & - & - & - & - \\
\hline
\end{tabular}


Table E.2.2 the results of calibration procedure 2 and its validations.

\begin{tabular}{|c|c|c|c|c|c|c|c|c|c|c|c|}
\hline \multicolumn{4}{|c|}{ Configurations } & \multicolumn{2}{|c|}{ Calibration } & \multicolumn{2}{|c|}{$\mathbf{1}^{\text {st }}$ Validation } & \multicolumn{2}{|c|}{$2^{\text {nd }}$ Validation } & \multicolumn{2}{|c|}{$3^{\text {rd }}$ Validation } \\
\hline$\varphi_{g}$ & $\Lambda_{l}$ & $\Gamma_{n}$ & $\eta_{m}$ & Cyclists & Pedestrians & Cyclists & Pedestrians & Cyclists & Pedestrians & Cyclists & Pedestrians \\
\hline 3 & 4 & 2 & 3 & 97.33 & 100.00 & - & - & - & - & - & - \\
\hline 3 & 4 & 3 & 1 & 97.33 & 100.00 & - & - & - & - & - & - \\
\hline 3 & 4 & 3 & 2 & 97.33 & 100.00 & - & - & - & - & - & - \\
\hline 3 & 4 & 3 & 3 & 97.33 & 100.00 & - & - & - & - & - & - \\
\hline 3 & 4 & 4 & 1 & 97.33 & 100.00 & - & - & - & - & - & - \\
\hline 3 & 4 & 4 & 2 & 97.33 & 100.00 & - & - & - & - & - & - \\
\hline 3 & 4 & 4 & 3 & 97.33 & 100.00 & - & - & - & - & - & - \\
\hline 3 & 4 & 1 & 1 & 97.33 & 100.00 & - & - & - & - & - & - \\
\hline 3 & 4 & 1 & 2 & 97.33 & 100.00 & - & - & - & - & - & - \\
\hline 3 & 4 & 1 & 3 & 97.33 & 100.00 & - & - & - & - & - & - \\
\hline 3 & 4 & 6 & 1 & 97.33 & 100.00 & - & - & - & - & - & - \\
\hline 3 & 4 & 6 & 2 & 97.33 & 100.00 & - & - & - & - & - & - \\
\hline 3 & 4 & 6 & 3 & 97.33 & 100.00 & - & - & - & - & - & - \\
\hline 4 & 1 & 1 & 1 & 97.01 & 100.00 & 100.00 & 97.44 & 88.00 & 97.63 & 93.38 & 97.60 \\
\hline 4 & 1 & 1 & 2 & 97.01 & 100.00 & 100.00 & 97.44 & 88.00 & 97.63 & 93.38 & 97.60 \\
\hline 4 & 1 & 1 & 3 & 97.01 & 100.00 & 100.00 & 97.44 & 88.00 & 97.63 & 93.38 & 97.60 \\
\hline 4 & 1 & 2 & 1 & 97.01 & 100.00 & 100.00 & 97.44 & 88.00 & 97.63 & 93.38 & 97.60 \\
\hline 4 & 1 & 2 & 2 & 97.01 & 100.00 & 100.00 & 97.44 & 88.00 & 97.63 & 93.38 & 97.60 \\
\hline 4 & 1 & 2 & 3 & 97.01 & 100.00 & 100.00 & 97.44 & 88.00 & 97.63 & 93.38 & 97.60 \\
\hline 4 & 1 & 3 & 1 & 97.01 & 100.00 & 100.00 & 97.44 & 88.00 & 97.63 & 93.38 & 97.60 \\
\hline 4 & 1 & 3 & 2 & 97.01 & 100.00 & 100.00 & 97.44 & 88.00 & 97.63 & 93.38 & 97.60 \\
\hline 4 & 1 & 3 & 3 & 97.01 & 100.00 & 100.00 & 97.44 & 88.00 & 97.63 & 93.38 & 97.60 \\
\hline 4 & 1 & 4 & 1 & 97.01 & 100.00 & 100.00 & 97.44 & 88.00 & 97.63 & 93.38 & 97.60 \\
\hline 4 & 1 & 4 & 2 & 97.01 & 100.00 & 100.00 & 97.44 & 88.00 & 97.63 & 93.38 & 97.60 \\
\hline 4 & 1 & 4 & 3 & 97.01 & 100.00 & 100.00 & 97.44 & 88.00 & 97.63 & 93.38 & 97.60 \\
\hline 4 & 1 & 1 & 1 & 97.01 & 100.00 & 100.00 & 97.44 & 88.00 & 97.63 & 93.38 & 97.60 \\
\hline 4 & 1 & 1 & 2 & 97.01 & 100.00 & 100.00 & 97.44 & 88.00 & 97.63 & 93.38 & 97.60 \\
\hline 4 & 1 & 1 & 3 & 97.01 & 100.00 & 100.00 & 97.44 & 88.00 & 97.63 & 93.38 & 97.60 \\
\hline 4 & 1 & 6 & 1 & 97.01 & 100.00 & 100.00 & 97.44 & 88.00 & 97.63 & 93.38 & 97.60 \\
\hline 4 & 1 & 6 & 2 & 97.01 & 100.00 & 100.00 & 97.44 & 88.00 & 97.63 & 93.38 & 97.60 \\
\hline 4 & 1 & 6 & 3 & 97.01 & 100.00 & 100.00 & 97.44 & 88.00 & 97.63 & 93.38 & 97.60 \\
\hline 4 & 2 & 1 & 1 & 92.54 & 100.00 & 95.08 & 100.00 & 82.67 & 98.22 & 88.24 & 98.56 \\
\hline 4 & 2 & 1 & 2 & 92.54 & 100.00 & 95.08 & 100.00 & 82.67 & 98.22 & 88.24 & 98.56 \\
\hline 4 & 2 & 1 & 3 & 92.54 & 100.00 & 95.08 & 100.00 & 82.67 & 98.22 & 88.24 & 98.56 \\
\hline 4 & 2 & 2 & 1 & 92.54 & 100.00 & 95.08 & 100.00 & 82.67 & 98.22 & 88.24 & 98.56 \\
\hline
\end{tabular}


Table E.2.2 the results of calibration procedure 2 and its validations.

\begin{tabular}{|c|c|c|c|c|c|c|c|c|c|c|c|}
\hline \multicolumn{4}{|c|}{ Configurations } & \multicolumn{2}{|c|}{ Calibration } & \multicolumn{2}{|c|}{$\mathbf{1}^{\text {st }}$ Validation } & \multicolumn{2}{|c|}{$2^{\text {nd }}$ Validation } & \multicolumn{2}{|c|}{$3^{\text {rd }}$ Validation } \\
\hline$\varphi_{g}$ & $\Lambda_{l}$ & $\Gamma_{n}$ & $\eta_{m}$ & Cyclists & Pedestrians & Cyclists & Pedestrians & Cyclists & Pedestrians & Cyclists & Pedestrians \\
\hline 4 & 2 & 2 & 2 & 92.54 & 100.00 & 95.08 & 100.00 & 82.67 & 98.22 & 88.24 & 98.56 \\
\hline 4 & 2 & 2 & 3 & 92.54 & 100.00 & 95.08 & 100.00 & 82.67 & 98.22 & 88.24 & 98.56 \\
\hline 4 & 2 & 3 & 1 & 92.54 & 100.00 & 95.08 & 100.00 & 82.67 & 98.22 & 88.24 & 98.56 \\
\hline 4 & 2 & 3 & 2 & 92.54 & 100.00 & 95.08 & 100.00 & 82.67 & 98.22 & 88.24 & 98.56 \\
\hline 4 & 2 & 3 & 3 & 92.54 & 100.00 & 95.08 & 100.00 & 82.67 & 98.22 & 88.24 & 98.56 \\
\hline 4 & 2 & 4 & 1 & 92.54 & 100.00 & 95.08 & 100.00 & 82.67 & 98.22 & 88.24 & 98.56 \\
\hline 4 & 2 & 4 & 2 & 92.54 & 100.00 & 95.08 & 100.00 & 82.67 & 98.22 & 88.24 & 98.56 \\
\hline 4 & 2 & 4 & 3 & 92.54 & 100.00 & 95.08 & 100.00 & 82.67 & 98.22 & 88.24 & 98.56 \\
\hline 4 & 2 & 1 & 1 & 92.54 & 100.00 & 95.08 & 100.00 & 82.67 & 98.22 & 88.24 & 98.56 \\
\hline 4 & 2 & 1 & 2 & 92.54 & 100.00 & 95.08 & 100.00 & 82.67 & 98.22 & 88.24 & 98.56 \\
\hline 4 & 2 & 1 & 3 & 92.54 & 100.00 & 95.08 & 100.00 & 82.67 & 98.22 & 88.24 & 98.56 \\
\hline 4 & 2 & 6 & 1 & 92.54 & 100.00 & 95.08 & 100.00 & 82.67 & 98.22 & 88.24 & 98.56 \\
\hline 4 & 2 & 6 & 2 & 92.54 & 100.00 & 95.08 & 100.00 & 82.67 & 98.22 & 88.24 & 98.56 \\
\hline 4 & 2 & 6 & 3 & 92.54 & 100.00 & 95.08 & 100.00 & 82.67 & 98.22 & 88.24 & 98.56 \\
\hline 4 & 3 & 1 & 1 & 94.03 & 100.00 & - & - & - & - & - & - \\
\hline 4 & 3 & 1 & 2 & 94.03 & 100.00 & - & - & - & - & - & - \\
\hline 4 & 3 & 1 & 3 & 94.03 & 100.00 & - & - & - & - & - & - \\
\hline 4 & 3 & 2 & 1 & 94.03 & 100.00 & - & - & - & - & - & - \\
\hline 4 & 3 & 2 & 2 & 94.03 & 100.00 & - & - & - & - & - & - \\
\hline 4 & 3 & 2 & 3 & 94.03 & 100.00 & - & - & - & - & - & - \\
\hline 4 & 3 & 3 & 1 & 94.03 & 100.00 & - & - & - & - & - & - \\
\hline 4 & 3 & 3 & 2 & 94.03 & 100.00 & - & - & - & - & - & - \\
\hline 4 & 3 & 3 & 3 & 94.03 & 100.00 & - & - & - & - & - & - \\
\hline 4 & 3 & 4 & 1 & 94.03 & 100.00 & - & - & - & - & - & - \\
\hline 4 & 3 & 4 & 2 & 94.03 & 100.00 & - & - & - & - & - & - \\
\hline 4 & 3 & 4 & 3 & 94.03 & 100.00 & - & - & - & - & - & - \\
\hline 4 & 3 & 1 & 1 & 94.03 & 100.00 & - & - & - & - & - & - \\
\hline 4 & 3 & 1 & 2 & 94.03 & 100.00 & - & - & - & - & - & - \\
\hline 4 & 3 & 1 & 3 & 94.03 & 100.00 & - & - & - & - & - & - \\
\hline 4 & 3 & 6 & 1 & 94.03 & 100.00 & - & - & - & - & - & - \\
\hline 4 & 3 & 6 & 2 & 94.03 & 100.00 & - & - & - & - & - & - \\
\hline 4 & 3 & 6 & 3 & 94.03 & 100.00 & - & - & - & - & - & - \\
\hline 4 & 4 & 1 & 1 & 98.51 & 100.00 & - & - & - & - & - & - \\
\hline 4 & 4 & 1 & 2 & 98.51 & 100.00 & - & - & - & - & - & - \\
\hline 4 & 4 & 1 & 3 & 98.51 & 100.00 & - & - & - & - & - & - \\
\hline
\end{tabular}


Table E.2.2 the results of calibration procedure 2 and its validations.

\begin{tabular}{|c|c|c|c|c|c|c|c|c|c|c|c|}
\hline \multicolumn{4}{|c|}{ Configurations } & \multicolumn{2}{|c|}{ Calibration } & \multicolumn{2}{|c|}{$\mathbf{1}^{\text {st }}$ Validation } & \multicolumn{2}{|c|}{$2^{\text {nd }}$ Validation } & \multicolumn{2}{|c|}{$3^{\text {rd }}$ Validation } \\
\hline$\varphi_{g}$ & $\Lambda_{l}$ & $\Gamma_{n}$ & $\eta_{m}$ & Cyclists & Pedestrians & Cyclists & Pedestrians & Cyclists & Pedestrians & Cyclists & Pedestrians \\
\hline 4 & 4 & 2 & 1 & 98.51 & 100.00 & - & - & - & - & - & - \\
\hline 4 & 4 & 2 & 2 & 98.51 & 100.00 & - & - & - & - & - & - \\
\hline 4 & 4 & 2 & 3 & 98.51 & 100.00 & - & - & - & - & - & - \\
\hline 4 & 4 & 3 & 1 & 98.51 & 100.00 & - & - & - & - & - & - \\
\hline 4 & 4 & 3 & 2 & 98.51 & 100.00 & - & - & - & - & - & - \\
\hline 4 & 4 & 3 & 3 & 98.51 & 100.00 & - & - & - & - & - & - \\
\hline 4 & 4 & 4 & 1 & 98.51 & 100.00 & - & - & - & - & - & - \\
\hline 4 & 4 & 4 & 2 & 98.51 & 100.00 & - & - & - & - & - & - \\
\hline 4 & 4 & 4 & 3 & 98.51 & 100.00 & - & - & - & - & - & - \\
\hline 4 & 4 & 1 & 1 & 98.51 & 100.00 & - & - & - & - & - & - \\
\hline 4 & 4 & 1 & 2 & 98.51 & 100.00 & - & - & - & - & - & - \\
\hline 4 & 4 & 1 & 3 & 98.51 & 100.00 & - & - & - & - & - & - \\
\hline 4 & 4 & 6 & 1 & 98.51 & 100.00 & - & - & - & - & - & - \\
\hline 4 & 4 & 6 & 2 & 98.51 & 100.00 & - & - & - & - & - & - \\
\hline 4 & 4 & 6 & 3 & 98.51 & 100.00 & - & - & - & - & - & - \\
\hline 5 & 1 & 1 & 1 & 98.08 & 100.00 & 100.00 & 96.97 & 95.56 & 97.81 & 97.80 & 97.65 \\
\hline 5 & 1 & 1 & 2 & 98.08 & 100.00 & 100.00 & 96.97 & 95.56 & 97.81 & 97.80 & 97.65 \\
\hline 5 & 1 & 1 & 3 & 98.08 & 100.00 & 100.00 & 96.97 & 95.56 & 97.81 & 97.80 & 97.65 \\
\hline 5 & 1 & 2 & 1 & 98.08 & 100.00 & 100.00 & 96.97 & 95.56 & 97.81 & 97.80 & 97.65 \\
\hline 5 & 1 & 2 & 2 & 98.08 & 100.00 & 100.00 & 96.97 & 95.56 & 97.81 & 97.80 & 97.65 \\
\hline 5 & 1 & 2 & 3 & 98.08 & 100.00 & 100.00 & 96.97 & 95.56 & 97.81 & 97.80 & 97.65 \\
\hline 5 & 1 & 3 & 1 & 98.08 & 100.00 & 100.00 & 96.97 & 95.56 & 97.81 & 97.80 & 97.65 \\
\hline 5 & 1 & 3 & 2 & 98.08 & 100.00 & 100.00 & 96.97 & 95.56 & 97.81 & 97.80 & 97.65 \\
\hline 5 & 1 & 3 & 3 & 98.08 & 100.00 & 100.00 & 96.97 & 95.56 & 97.81 & 97.80 & 97.65 \\
\hline 5 & 1 & 4 & 1 & 98.08 & 100.00 & 100.00 & 96.97 & 95.56 & 97.81 & 97.80 & 97.65 \\
\hline 5 & 1 & 4 & 2 & 98.08 & 100.00 & 100.00 & 96.97 & 95.56 & 97.81 & 97.80 & 97.65 \\
\hline 5 & 1 & 4 & 3 & 98.08 & 100.00 & 100.00 & 96.97 & 95.56 & 97.81 & 97.80 & 97.65 \\
\hline 5 & 1 & 1 & 1 & 98.08 & 100.00 & 100.00 & 96.97 & 95.56 & 97.81 & 97.80 & 97.65 \\
\hline 5 & 1 & 1 & 2 & 98.08 & 100.00 & 100.00 & 96.97 & 95.56 & 97.81 & 97.80 & 97.65 \\
\hline 5 & 1 & 1 & 3 & 98.08 & 100.00 & 100.00 & 96.97 & 95.56 & 97.81 & 97.80 & 97.65 \\
\hline 5 & 1 & 6 & 1 & 98.08 & 100.00 & 100.00 & 96.97 & 95.56 & 97.81 & 97.80 & 97.65 \\
\hline 5 & 1 & 6 & 2 & 98.08 & 100.00 & 100.00 & 96.97 & 95.56 & 97.81 & 97.80 & 97.65 \\
\hline 5 & 1 & 6 & 3 & 98.08 & 100.00 & 100.00 & 96.97 & 95.56 & 97.81 & 97.80 & 97.65 \\
\hline 5 & 2 & 1 & 1 & - & - & - & - & - & - & - & - \\
\hline 5 & 2 & 1 & 2 & - & - & - & - & - & - & - & - \\
\hline
\end{tabular}


Table E.2.2 the results of calibration procedure 2 and its validations.

\begin{tabular}{|c|c|c|c|c|c|c|c|c|c|c|c|}
\hline \multicolumn{4}{|c|}{ Configurations } & \multicolumn{2}{|c|}{ Calibration } & \multicolumn{2}{|c|}{$\mathbf{1}^{\text {st }}$ Validation } & \multicolumn{2}{|c|}{$2^{\text {nd }}$ Validation } & \multicolumn{2}{|c|}{$3^{\text {rd }}$ Validation } \\
\hline$\varphi_{g}$ & $\Lambda_{l}$ & $\Gamma_{n}$ & $\eta_{m}$ & Cyclists & Pedestrians & Cyclists & Pedestrians & Cyclists & Pedestrians & Cyclists & Pedestrians \\
\hline 5 & 2 & 1 & 3 & - & - & - & - & - & - & - & - \\
\hline 5 & 2 & 2 & 1 & - & - & - & - & - & - & - & - \\
\hline 5 & 2 & 2 & 2 & - & - & - & - & - & - & - & - \\
\hline 5 & 2 & 2 & 3 & - & - & - & - & - & - & - & - \\
\hline 5 & 2 & 3 & 1 & - & - & - & - & - & - & - & - \\
\hline 5 & 2 & 3 & 2 & - & - & - & - & - & - & - & - \\
\hline 5 & 2 & 3 & 3 & - & - & - & - & - & - & - & - \\
\hline 5 & 2 & 4 & 1 & - & - & - & - & - & - & - & - \\
\hline 5 & 2 & 4 & 2 & - & - & - & - & - & - & - & - \\
\hline 5 & 2 & 4 & 3 & - & - & - & - & - & - & - & - \\
\hline 5 & 2 & 1 & 1 & - & - & - & - & - & - & - & - \\
\hline 5 & 2 & 1 & 2 & - & - & - & - & - & - & - & - \\
\hline 5 & 2 & 1 & 3 & - & - & - & - & - & - & - & - \\
\hline 5 & 2 & 6 & 1 & - & - & - & - & - & - & - & - \\
\hline 5 & 2 & 6 & 2 & - & - & - & - & - & - & - & - \\
\hline 5 & 2 & 6 & 3 & - & - & - & - & - & - & - & - \\
\hline 5 & 3 & 1 & 1 & - & - & - & - & - & - & - & - \\
\hline 5 & 3 & 1 & 2 & - & - & - & - & - & - & - & - \\
\hline 5 & 3 & 1 & 3 & - & - & - & - & - & - & - & - \\
\hline 5 & 3 & 2 & 1 & - & - & - & - & - & - & - & - \\
\hline 5 & 3 & 2 & 2 & - & - & - & - & - & - & - & - \\
\hline 5 & 3 & 2 & 3 & - & - & - & - & - & - & - & - \\
\hline 5 & 3 & 3 & 1 & - & - & - & - & - & - & - & - \\
\hline 5 & 3 & 3 & 2 & - & - & - & - & - & - & - & - \\
\hline 5 & 3 & 3 & 3 & - & - & - & - & - & - & - & - \\
\hline 5 & 3 & 4 & 1 & - & - & - & - & - & - & - & - \\
\hline 5 & 3 & 4 & 2 & - & - & - & - & - & - & - & - \\
\hline 5 & 3 & 4 & 3 & - & - & - & - & - & - & - & - \\
\hline 5 & 3 & 1 & 1 & - & - & - & - & - & - & - & - \\
\hline 5 & 3 & 1 & 2 & - & - & - & - & - & - & - & - \\
\hline 5 & 3 & 1 & 3 & - & - & - & - & - & - & - & - \\
\hline 5 & 3 & 6 & 1 & - & - & - & - & - & - & - & - \\
\hline 5 & 3 & 6 & 2 & - & - & - & - & - & - & - & - \\
\hline 5 & 3 & 6 & 3 & - & - & - & - & - & - & - & - \\
\hline 5 & 4 & 1 & 1 & 100.00 & 100.00 & - & - & - & - & - & - \\
\hline
\end{tabular}


Table E.2.2 the results of calibration procedure 2 and its validations.

\begin{tabular}{|c|c|c|c|c|c|c|c|c|c|c|c|}
\hline \multicolumn{4}{|c|}{ Configurations } & \multicolumn{2}{|c|}{ Calibration } & \multicolumn{2}{|c|}{$\mathbf{1}^{\text {st }}$ Validation } & \multicolumn{2}{|c|}{$2^{\text {nd }}$ Validation } & \multicolumn{2}{|c|}{$3^{\text {rd }}$ Validation } \\
\hline$\varphi_{g}$ & $\Lambda_{l}$ & $\Gamma_{n}$ & $\eta_{m}$ & Cyclists & Pedestrians & Cyclists & Pedestrians & Cyclists & Pedestrians & Cyclists & Pedestrians \\
\hline 5 & 4 & 1 & 2 & 100.00 & 100.00 & - & - & - & - & - & - \\
\hline 5 & 4 & 1 & 3 & 100.00 & 100.00 & - & - & - & - & - & - \\
\hline 5 & 4 & 2 & 1 & 100.00 & 100.00 & - & - & - & - & - & - \\
\hline 5 & 4 & 2 & 2 & 100.00 & 100.00 & - & - & - & - & - & - \\
\hline 5 & 4 & 2 & 3 & 100.00 & 100.00 & - & - & - & - & - & - \\
\hline 5 & 4 & 3 & 1 & 100.00 & 100.00 & - & - & - & - & - & - \\
\hline 5 & 4 & 3 & 2 & 100.00 & 100.00 & - & - & - & - & - & - \\
\hline 5 & 4 & 3 & 3 & 100.00 & 100.00 & - & - & - & - & - & - \\
\hline 5 & 4 & 4 & 1 & 100.00 & 100.00 & - & - & - & - & - & - \\
\hline 5 & 4 & 4 & 2 & 100.00 & 100.00 & - & - & - & - & - & - \\
\hline 5 & 4 & 4 & 3 & 100.00 & 100.00 & - & - & - & - & - & - \\
\hline 5 & 4 & 1 & 1 & 100.00 & 100.00 & - & - & - & - & - & - \\
\hline 5 & 4 & 1 & 2 & 100.00 & 100.00 & - & - & - & - & - & - \\
\hline 5 & 4 & 1 & 3 & 100.00 & 100.00 & - & - & - & - & - & - \\
\hline 5 & 4 & 6 & 1 & 100.00 & 100.00 & - & - & - & - & - & - \\
\hline 5 & 4 & 6 & 2 & 100.00 & 100.00 & - & - & - & - & - & - \\
\hline 5 & 4 & 6 & 3 & 100.00 & 100.00 & - & - & - & - & - & - \\
\hline
\end{tabular}


Table E.2.3 the results of calibration and validations based on the average speed

\begin{tabular}{|c|c|c|c|c|c|c|c|c|c|}
\hline \multicolumn{2}{|c|}{ Configurations } & \multicolumn{2}{|c|}{ Calibration } & \multicolumn{2}{|c|}{$1^{\text {st }}$ Validation } & \multicolumn{2}{|c|}{$2^{\text {nd }}$ Validation } & \multicolumn{2}{|c|}{$3^{\text {rd }}$ Validation } \\
\hline$\varphi_{g}$ & $\Lambda_{l}$ & Cyclists & Pedestrians & Cyclists & Pedestrians & Cyclists & Pedestrians & Cyclists & Pedestrians \\
\hline 1 & 1 & 95 & 93 & 98 & 95 & 92 & 98 & 94 & 97 \\
\hline 1 & 2 & 87 & 97 & - & - & - & - & - & - \\
\hline 1 & 3 & - & - & - & - & - & - & - & - \\
\hline 1 & 4 & - & - & - & - & - & - & - & - \\
\hline 2 & 1 & 96 & 100 & 98 & 100 & 92 & 98 & 94 & 98 \\
\hline 2 & 2 & - & - & - & - & - & - & - & - \\
\hline 2 & 3 & - & - & - & - & - & - & - & - \\
\hline 2 & 4 & - & - & - & - & - & - & - & - \\
\hline 3 & 1 & 95 & 100 & 99 & 98 & 92 & 98 & 95 & 98 \\
\hline 3 & 2 & 89 & 100 & - & - & - & - & - & - \\
\hline 3 & 3 & - & - & - & - & - & - & - & - \\
\hline 3 & 4 & 88 & 100 & - & - & - & - & - & - \\
\hline 4 & 1 & 97 & 100 & 100 & 97 & 88 & 98 & 93 & 98 \\
\hline 4 & 2 & 93 & 100 & 95 & 100 & 83 & 98 & 88 & 99 \\
\hline 4 & 3 & - & - & - & - & - & - & - & - \\
\hline 4 & 4 & 91 & 100 & 93 & 100 & 83 & 98 & 88 & 99 \\
\hline 5 & 1 & 98 & 100 & 100 & 97 & 96 & 98 & 98 & 98 \\
\hline 5 & 2 & 94 & 100 & 96 & 100 & - & - & - & - \\
\hline 5 & 3 & - & - & - & - & - & - & - & - \\
\hline 5 & 4 & 94 & 100 & 93 & 100 & - & - & - & - \\
\hline
\end{tabular}


Table E.2.4 the results of calibration and validations based on the maximum speed

\begin{tabular}{|c|c|c|c|c|c|c|c|c|c|}
\hline \multicolumn{2}{|c|}{ Configurations } & \multicolumn{2}{|c|}{ Calibration } & \multicolumn{2}{|c|}{$1^{\text {st }}$ Validation } & \multicolumn{2}{|c|}{$2^{\text {nd }}$ Validation } & \multicolumn{2}{|c|}{$3^{\text {rd }}$ Validation } \\
\hline$\varphi_{g}$ & $\Lambda_{l}$ & Cyclists & Pedestrians & Cyclists & Pedestrians & Cyclists & Pedestrians & Cyclists & Pedestrians \\
\hline 1 & 1 & 95 & 87 & 94 & 83 & 91 & 92 & 92 & 90 \\
\hline 1 & 2 & 88 & 88 & 86 & 89 & - & - & - & - \\
\hline 1 & 3 & - & - & - & - & - & - & - & - \\
\hline 1 & 4 & - & - & - & - & - & - & - & - \\
\hline 2 & 1 & 96 & 90 & 96 & 84 & 90 & 92 & 92 & 90 \\
\hline 2 & 2 & 89 & 90 & 85 & 91 & - & - & - & - \\
\hline 2 & 3 & - & - & - & - & - & - & - & - \\
\hline 2 & 4 & - & - & - & - & - & - & - & - \\
\hline 3 & 1 & 97 & 90 & 99 & 89 & 96 & 94 & 97 & 93 \\
\hline 3 & 2 & 92 & 93 & 89 & 89 & - & - & - & - \\
\hline 3 & 3 & - & - & - & - & - & - & - & - \\
\hline 3 & 4 & 88 & 90 & - & - & - & - & - & - \\
\hline 4 & 1 & 100 & 89 & 100 & 87 & 95 & 93 & 97 & 92 \\
\hline 4 & 2 & 96 & 92 & - & - & - & - & - & - \\
\hline 4 & 3 & - & - & - & - & - & - & - & - \\
\hline 4 & 4 & 91 & 89 & - & - & - & - & - & - \\
\hline 5 & 1 & 98 & 91 & 98 & 88 & 89 & 91 & 93 & 90 \\
\hline 5 & 2 & 96 & 91 & 87 & 88 & 80 & 91 & 84 & 90 \\
\hline 5 & 3 & 88 & 91 & 83 & 94 & 78 & 96 & 80 & 95 \\
\hline 5 & 4 & 92 & 88 & 83 & 94 & 76 & 93 & 79 & 94 \\
\hline
\end{tabular}


Table E.2.5 the results of calibration and validations based on the average acceleration

\begin{tabular}{|c|c|c|c|c|c|c|c|c|c|}
\hline \multicolumn{2}{|c|}{ Configurations } & \multicolumn{2}{|c|}{ Calibration } & \multicolumn{2}{|c|}{$\mathbf{1}^{\text {st }}$ Validation } & \multicolumn{2}{|c|}{$2^{\text {nd }}$ Validation } & \multicolumn{2}{|c|}{$3^{\text {rd }}$ Validation } \\
\hline$\varphi_{g}$ & $\Lambda_{l}$ & Cyclists & Pedestrians & Cyclists & Pedestrians & Cyclists & Pedestrians & Cyclists & Pedestrians \\
\hline 1 & 1 & 65 & 9 & 80 & 8 & 81 & 5 & 80 & 6 \\
\hline 1 & 2 & 38 & 43 & - & - & - & - & - & - \\
\hline 1 & 3 & 12 & 19 & - & - & - & - & - & - \\
\hline 1 & 4 & 43 & 10 & 53 & 6 & - & - & - & - \\
\hline 2 & 1 & 45 & 67 & - & - & - & - & - & - \\
\hline 2 & 2 & 44 & 67 & - & - & - & - & - & - \\
\hline 2 & 3 & 37 & 67 & - & - & - & - & - & - \\
\hline 2 & 4 & 13 & 47 & - & - & - & - & - & - \\
\hline 3 & 1 & 81 & 24 & 90 & 17 & - & - & - & - \\
\hline 3 & 2 & 56 & 51 & - & - & - & - & - & - \\
\hline 3 & 3 & 39 & 63 & 36 & 60 & - & - & - & - \\
\hline 3 & 4 & 44 & 17 & 58 & 9 & - & - & - & - \\
\hline 4 & 1 & 84 & 24 & 90 & 18 & - & - & - & - \\
\hline 4 & 2 & 55 & 54 & - & - & - & - & - & - \\
\hline 4 & 3 & 43 & 49 & - & - & - & - & - & - \\
\hline 4 & 4 & 46 & 16 & 57 & 10 & - & - & - & - \\
\hline 5 & 1 & 85 & 28 & 89 & 18 & - & - & - & - \\
\hline 5 & 2 & 58 & 56 & - & - & - & - & - & - \\
\hline 5 & 3 & 46 & 50 & - & - & - & - & - & - \\
\hline 5 & 4 & 40 & 28 & - & - & - & - & - & - \\
\hline
\end{tabular}


Table E.2.6 the results of calibration and validations based on the maximum acceleration

\begin{tabular}{|c|c|c|c|c|c|c|c|c|c|}
\hline \multicolumn{2}{|c|}{ Configurations } & \multicolumn{2}{|c|}{ Calibration } & \multicolumn{2}{|c|}{$1^{\text {st }}$ Validation } & \multicolumn{2}{|c|}{$2^{\text {nd }}$ Validation } & \multicolumn{2}{|c|}{$3^{\text {rd }}$ Validation } \\
\hline$\varphi_{g}$ & $\Lambda_{l}$ & Cyclists & Pedestrians & Cyclists & Pedestrians & Cyclists & Pedestrians & Cyclists & Pedestrians \\
\hline 1 & 1 & 65 & 35 & - & - & - & - & - & - \\
\hline 1 & 2 & 49 & 47 & - & - & - & - & - & - \\
\hline 1 & 3 & 44 & 47 & 38 & 44 & - & - & - & - \\
\hline 1 & 4 & 49 & 44 & 41 & 44 & - & - & - & - \\
\hline 2 & 1 & 74 & 41 & - & - & - & - & - & - \\
\hline 2 & 2 & 58 & 59 & - & - & - & - & - & - \\
\hline 2 & 3 & 55 & 59 & - & - & - & - & - & - \\
\hline 2 & 4 & 55 & 55 & 45 & 45 & - & - & - & - \\
\hline 3 & 1 & 63 & 56 & 54 & 47 & - & - & - & - \\
\hline 3 & 2 & 63 & 56 & 54 & 51 & - & - & - & - \\
\hline 3 & 3 & 53 & 56 & 47 & 49 & - & - & - & - \\
\hline 3 & 4 & 59 & 56 & 51 & 49 & - & - & - & - \\
\hline 4 & 1 & 64 & 51 & 57 & 41 & - & - & - & - \\
\hline 4 & 2 & 64 & 51 & 57 & 46 & - & - & - & - \\
\hline 4 & 3 & 60 & 51 & 54 & 46 & - & - & - & - \\
\hline 4 & 4 & 60 & 51 & 54 & 44 & - & - & - & - \\
\hline 5 & 1 & 71 & 50 & 61 & 36 & - & - & - & - \\
\hline 5 & 2 & 71 & 50 & 61 & 39 & - & - & - & - \\
\hline 5 & 3 & 65 & 50 & 57 & 39 & - & - & - & - \\
\hline 5 & 4 & 65 & 50 & 57 & 39 & - & - & - & - \\
\hline
\end{tabular}


Table E.2.7 the results of calibration and validations based on the average deceleration

\begin{tabular}{|c|c|c|c|c|c|c|c|c|c|}
\hline \multicolumn{2}{|c|}{ Configurations } & \multicolumn{2}{|c|}{ Calibration } & \multicolumn{2}{|c|}{$1^{\text {st }}$ Validation } & \multicolumn{2}{|c|}{$2^{\text {nd }}$ Validation } & \multicolumn{2}{|c|}{$3^{\text {rd }}$ Validation } \\
\hline$\varphi_{g}$ & $\Lambda_{l}$ & Cyclists & Pedestrians & Cyclists & Pedestrians & Cyclists & Pedestrians & Cyclists & Pedestrians \\
\hline 1 & 1 & 70 & 26 & 71 & 19 & 79 & 32 & 76 & 28 \\
\hline 1 & 2 & 70 & 25 & 70 & 19 & 79 & 33 & 75 & 29 \\
\hline 1 & 3 & 75 & 22 & 79 & 14 & 81 & 26 & 80 & 23 \\
\hline 1 & 4 & 67 & 25 & - & - & - & - & - & - \\
\hline 2 & 1 & 76 & 22 & 73 & 20 & 80 & 32 & 77 & 29 \\
\hline 2 & 2 & 76 & 22 & 72 & 20 & 80 & 33 & 77 & 30 \\
\hline 2 & 3 & 76 & 22 & 73 & 20 & 80 & 31 & 77 & 28 \\
\hline 2 & 4 & 69 & 22 & - & - & - & - & - & - \\
\hline 3 & 1 & 77 & 24 & 74 & 19 & 80 & 31 & 78 & 29 \\
\hline 3 & 2 & 77 & 24 & 72 & 19 & 80 & 32 & 77 & 30 \\
\hline 3 & 3 & 77 & 24 & 74 & 19 & 80 & 30 & 78 & 28 \\
\hline 3 & 4 & 68 & 24 & - & - & - & - & - & - \\
\hline 4 & 1 & 85 & 27 & 79 & 15 & 89 & 30 & 85 & 27 \\
\hline 4 & 2 & 85 & 27 & - & - & - & - & - & - \\
\hline 4 & 3 & 78 & 27 & - & - & - & - & - & - \\
\hline 4 & 4 & 67 & 27 & - & - & - & - & - & - \\
\hline 5 & 1 & 77 & 25 & - & - & - & - & - & - \\
\hline 5 & 2 & 77 & 25 & - & - & - & - & - & - \\
\hline 5 & 3 & 77 & 25 & - & - & - & - & - & - \\
\hline 5 & 4 & 77 & 25 & - & - & - & - & - & - \\
\hline
\end{tabular}


Table E.2.8 the results of calibration and validations based on the maximum deceleration

\begin{tabular}{|c|c|c|c|c|c|c|c|c|c|}
\hline \multicolumn{2}{|c|}{ Configurations } & \multicolumn{2}{|c|}{ Calibration } & \multicolumn{2}{|c|}{$1^{\text {st }}$ Validation } & \multicolumn{2}{|c|}{$2^{\text {nd }}$ Validation } & \multicolumn{2}{|c|}{$3^{\text {rd }}$ Validation } \\
\hline$\varphi_{g}$ & $\Lambda_{l}$ & Cyclists & Pedestrians & Cyclists & Pedestrians & Cyclists & Pedestrians & Cyclists & Pedestrians \\
\hline 1 & 1 & 81 & 22 & 75 & 22 & - & - & - & - \\
\hline 1 & 2 & 78 & 24 & 71 & 22 & - & - & - & - \\
\hline 1 & 3 & 70 & 26 & 65 & 30 & - & - & - & - \\
\hline 1 & 4 & 76 & 22 & 69 & 22 & - & - & - & - \\
\hline 2 & 1 & 88 & 31 & 79 & 27 & - & - & - & - \\
\hline 2 & 2 & 87 & 29 & 80 & 20 & - & - & - & - \\
\hline 2 & 3 & 80 & 31 & 73 & 27 & - & - & - & - \\
\hline 2 & 4 & 84 & 29 & 76 & 27 & - & - & - & - \\
\hline 3 & 1 & 89 & 24 & 89 & 21 & - & - & - & - \\
\hline 3 & 2 & 85 & 24 & 86 & 21 & - & - & - & - \\
\hline 3 & 3 & 80 & 24 & 82 & 21 & - & - & - & - \\
\hline 3 & 4 & 83 & 24 & 83 & 21 & - & - & - & - \\
\hline 4 & 1 & 93 & 24 & 90 & 23 & - & - & - & - \\
\hline 4 & 2 & 88 & 24 & 87 & 23 & - & - & - & - \\
\hline 4 & 3 & 82 & 24 & 82 & 23 & - & - & - & - \\
\hline 4 & 4 & 85 & 24 & 84 & 23 & - & - & - & - \\
\hline 5 & 1 & 96 & 28 & 89 & 24 & - & - & - & - \\
\hline 5 & 2 & 92 & 28 & 87 & 24 & - & - & - & - \\
\hline 5 & 3 & 85 & 28 & 78 & 24 & - & - & - & - \\
\hline 5 & 4 & 88 & 28 & 80 & 24 & - & - & - & - \\
\hline
\end{tabular}


Table E.2.9 the results of calibration and validations based on the average direction

\begin{tabular}{|c|c|c|c|c|c|c|c|c|c|}
\hline \multicolumn{2}{|c|}{ Configurations } & \multicolumn{2}{|c|}{ Calibration } & \multicolumn{2}{|c|}{$\mathbf{1}^{\text {st }}$ Validation } & \multicolumn{2}{|c|}{$2^{\text {nd }}$ Validation } & \multicolumn{2}{|c|}{$3^{\text {rd }}$ Validation } \\
\hline$\varphi_{g}$ & $\Lambda_{l}$ & Cyclists & Pedestrians & Cyclists & Pedestrians & Cyclists & Pedestrians & Cyclists & Pedestrians \\
\hline 1 & 1 & 96 & 10 & 99 & 14 & 96 & 9 & 97 & 10 \\
\hline 1 & 2 & 97 & 9 & 99 & 8 & 97 & 8 & 98 & 8 \\
\hline 1 & 3 & 97 & 9 & 99 & 8 & 97 & 8 & 98 & 8 \\
\hline 1 & 4 & 93 & 9 & 97 & 8 & 97 & 8 & 97 & 8 \\
\hline 2 & 1 & 89 & 22 & 83 & 24 & 93 & 11 & 89 & 14 \\
\hline 2 & 2 & 89 & 20 & 83 & 24 & 93 & 11 & 89 & 14 \\
\hline 2 & 3 & 91 & 18 & 87 & 24 & 94 & 11 & 91 & 14 \\
\hline 2 & 4 & 89 & 20 & 83 & 24 & 93 & 11 & 89 & 14 \\
\hline 3 & 1 & 89 & 20 & 85 & 28 & 96 & 11 & 92 & 14 \\
\hline 3 & 2 & 89 & 17 & 85 & 28 & 96 & 11 & 92 & 14 \\
\hline 3 & 3 & 88 & 20 & 78 & 30 & 96 & 12 & 89 & 15 \\
\hline 3 & 4 & 89 & 17 & 85 & 28 & 96 & 11 & 92 & 14 \\
\hline 4 & 1 & 93 & 22 & 85 & 28 & 96 & 11 & 91 & 14 \\
\hline 4 & 2 & 93 & 19 & 85 & 28 & 96 & 11 & 91 & 14 \\
\hline 4 & 3 & 91 & 22 & 79 & 31 & 96 & 12 & 88 & 15 \\
\hline 4 & 4 & 93 & 19 & 85 & 28 & 96 & 11 & 91 & 14 \\
\hline 5 & 1 & 94 & 22 & 89 & 24 & 93 & 12 & 91 & 15 \\
\hline 5 & 2 & 94 & 19 & 89 & 24 & 93 & 12 & 91 & 15 \\
\hline 5 & 3 & 92 & 22 & 87 & 27 & 93 & 13 & 90 & 16 \\
\hline 5 & 4 & 94 & 19 & 89 & 24 & 93 & 12 & 91 & 15 \\
\hline
\end{tabular}


Table E.2.10 the results of calibration and validations based on the maximum direction

\begin{tabular}{|c|c|c|c|c|c|c|c|c|c|}
\hline \multicolumn{2}{|c|}{ Configurations } & \multicolumn{2}{|c|}{ Calibration } & \multicolumn{2}{|c|}{$1^{\text {st }}$ Validation } & \multicolumn{2}{|c|}{$2^{\text {nd }}$ Validation } & \multicolumn{2}{|c|}{$3^{\text {rd }}$ Validation } \\
\hline$\varphi_{g}$ & $\Lambda_{l}$ & Cyclists & Pedestrians & Cyclists & Pedestrians & Cyclists & Pedestrians & Cyclists & Pedestrians \\
\hline 1 & 1 & 93 & 1 & 86 & 3 & 91 & 1 & 89 & 2 \\
\hline 1 & 2 & 93 & 1 & 86 & 3 & 91 & 1 & 89 & 2 \\
\hline 1 & 3 & 93 & 1 & 86 & 3 & 91 & 1 & 89 & 2 \\
\hline 1 & 4 & 90 & 1 & 85 & 3 & 91 & 1 & 88 & 2 \\
\hline 2 & 1 & 94 & 2 & 87 & 0 & 91 & 1 & 89 & 1 \\
\hline 2 & 2 & 94 & 2 & 84 & 0 & 91 & 1 & 88 & 1 \\
\hline 2 & 3 & 94 & 2 & 85 & 0 & 91 & 1 & 89 & 1 \\
\hline 2 & 4 & 94 & 2 & 84 & 0 & 91 & 1 & 88 & 1 \\
\hline 3 & 1 & 96 & 0 & 86 & 0 & 92 & 1 & 90 & 1 \\
\hline 3 & 2 & 96 & 0 & 86 & 0 & 92 & 1 & 90 & 1 \\
\hline 3 & 3 & 96 & 0 & 86 & 0 & 92 & 1 & 90 & 1 \\
\hline 3 & 4 & 96 & 0 & 86 & 0 & 92 & 1 & 90 & 1 \\
\hline 4 & 1 & 96 & 0 & 84 & 0 & 88 & 1 & 86 & 1 \\
\hline 4 & 2 & 96 & 0 & 84 & 0 & 88 & 1 & 86 & 1 \\
\hline 4 & 3 & 96 & 0 & 84 & 0 & 88 & 1 & 86 & 1 \\
\hline 4 & 4 & 96 & 0 & 84 & 0 & 88 & 1 & 86 & 1 \\
\hline 5 & 1 & 98 & 0 & 85 & 0 & 84 & 1 & 85 & 1 \\
\hline 5 & 2 & 98 & 0 & 85 & 0 & 84 & 1 & 85 & 1 \\
\hline 5 & 3 & 98 & 0 & 85 & 0 & 84 & 1 & 85 & 1 \\
\hline 5 & 4 & 98 & 0 & 85 & 0 & 84 & 1 & 85 & 1 \\
\hline
\end{tabular}


Table E.2.11 the results of calibration and validations based on the average of change in direction

\begin{tabular}{|c|c|c|c|c|c|c|c|c|c|}
\hline \multicolumn{2}{|c|}{ Configurations } & \multicolumn{2}{|c|}{ Calibration } & \multicolumn{2}{|c|}{$1^{\text {st }}$ Validation } & \multicolumn{2}{|c|}{$2^{\text {nd }}$ Validation } & \multicolumn{2}{|c|}{$3^{\text {rd }}$ Validation } \\
\hline$\varphi_{g}$ & $\Lambda_{l}$ & Cyclists & Pedestrians & Cyclists & Pedestrians & Cyclists & Pedestrians & Cyclists & Pedestrians \\
\hline 1 & 1 & 83 & 32 & 80 & 33 & 77 & 31 & 78 & 32 \\
\hline 1 & 2 & 82 & 38 & 78 & 38 & 75 & 37 & 76 & 37 \\
\hline 1 & 3 & 84 & 43 & 80 & 40 & 77 & 37 & 78 & 38 \\
\hline 1 & 4 & 85 & 21 & 83 & 14 & 81 & 20 & 82 & 18 \\
\hline 2 & 1 & 93 & 22 & 88 & 16 & 82 & 20 & 84 & 19 \\
\hline 2 & 2 & 90 & 37 & 84 & 38 & 76 & 37 & 79 & 37 \\
\hline 2 & 3 & 92 & 41 & 87 & 40 & 78 & 37 & 81 & 38 \\
\hline 2 & 4 & 90 & 27 & 84 & 24 & 75 & 28 & 78 & 27 \\
\hline 3 & 1 & 92 & 22 & 86 & 11 & 88 & 19 & 88 & 17 \\
\hline 3 & 2 & 88 & 34 & 85 & 32 & 81 & 36 & 83 & 35 \\
\hline 3 & 3 & 91 & 39 & 86 & 36 & 84 & 37 & 85 & 37 \\
\hline 3 & 4 & 85 & 24 & 83 & 13 & 79 & 21 & 81 & 19 \\
\hline 4 & 1 & 85 & 38 & 82 & 33 & 73 & 48 & 77 & 45 \\
\hline 4 & 2 & 90 & 35 & 85 & 31 & 83 & 37 & 84 & 36 \\
\hline 4 & 3 & 93 & 35 & 87 & 23 & 85 & 33 & 86 & 31 \\
\hline 4 & 4 & 87 & 24 & 84 & 10 & 81 & 22 & 82 & 20 \\
\hline 5 & 1 & 88 & 38 & 85 & 36 & 71 & 47 & 78 & 45 \\
\hline 5 & 2 & 94 & 34 & 89 & 33 & 80 & 37 & 85 & 36 \\
\hline 5 & 3 & 98 & 34 & 89 & 24 & 82 & 32 & 86 & 31 \\
\hline 5 & 4 & 88 & 28 & 83 & 24 & 71 & 31 & 77 & 29 \\
\hline
\end{tabular}


Table E.2.12 the results of calibration and validations based on the average of cosine of change in direction

\begin{tabular}{|c|c|c|c|c|c|c|c|c|c|}
\hline \multicolumn{2}{|c|}{ Configurations } & \multicolumn{2}{|c|}{ Calibration } & \multicolumn{2}{|c|}{$1^{\text {st }}$ Validation } & \multicolumn{2}{|c|}{$2^{\text {nd }}$ Validation } & \multicolumn{2}{|c|}{$3^{\text {rd }}$ Validation } \\
\hline$\varphi_{g}$ & $\Lambda_{l}$ & Cyclists & Pedestrians & Cyclists & Pedestrians & Cyclists & Pedestrians & Cyclists & Pedestrians \\
\hline 1 & 1 & 1 & 54 & 0 & 57 & 0 & 43 & 0 & 47 \\
\hline 1 & 2 & 1 & 54 & 0 & 57 & 0 & 43 & 0 & 47 \\
\hline 1 & 3 & 50 & 7 & 47 & 5 & 47 & 1 & 47 & 2 \\
\hline 1 & 4 & 1 & 47 & 0 & 56 & 0 & 43 & 0 & 46 \\
\hline 2 & 1 & 0 & 53 & 0 & 62 & 0 & 42 & 0 & 47 \\
\hline 2 & 2 & 0 & 53 & 0 & 62 & 0 & 42 & 0 & 47 \\
\hline 2 & 3 & 0 & 51 & 0 & 60 & 0 & 38 & 0 & 43 \\
\hline 2 & 4 & 0 & 53 & 0 & 62 & 0 & 42 & 0 & 47 \\
\hline 3 & 1 & 0 & 51 & 0 & 55 & 0 & 35 & 0 & 39 \\
\hline 3 & 2 & 0 & 51 & 0 & 55 & 0 & 35 & 0 & 39 \\
\hline 3 & 3 & 68 & 20 & 74 & 15 & 73 & 7 & 73 & 9 \\
\hline 3 & 4 & 0 & 51 & 0 & 55 & 0 & 35 & 0 & 39 \\
\hline 4 & 1 & 0 & 49 & 0 & 54 & 0 & 36 & 0 & 39 \\
\hline 4 & 2 & 0 & 49 & 0 & 54 & 0 & 36 & 0 & 39 \\
\hline 4 & 3 & 72 & 22 & 75 & 18 & 75 & 5 & 75 & 8 \\
\hline 4 & 4 & 0 & 49 & 0 & 54 & 0 & 36 & 0 & 39 \\
\hline 5 & 1 & 0 & 47 & 0 & 48 & 0 & 33 & 0 & 36 \\
\hline 5 & 2 & 52 & 34 & 57 & 18 & 62 & 12 & 59 & 14 \\
\hline 5 & 3 & 83 & 25 & 89 & 15 & 87 & 5 & 88 & 7 \\
\hline 5 & 4 & 42 & 25 & 50 & 15 & 44 & 4 & 47 & 6 \\
\hline
\end{tabular}


Table E.2.13 the results of calibration and validations based on the average area

\begin{tabular}{|c|c|c|c|c|c|c|c|c|c|}
\hline \multicolumn{2}{|c|}{ Configurations } & \multicolumn{2}{|c|}{ Calibration } & \multicolumn{2}{|c|}{$\mathbf{1}^{\text {st }}$ Validation } & \multicolumn{2}{|c|}{$2^{\text {nd }}$ Validation } & \multicolumn{2}{|c|}{$3^{\text {rd }}$ Validation } \\
\hline$\varphi_{g}$ & $\Lambda_{l}$ & Cyclists & Pedestrians & Cyclists & Pedestrians & Cyclists & Pedestrians & Cyclists & Pedestrians \\
\hline 1 & 1 & 81 & 62 & 72 & 75 & 16 & 94 & 39 & 89 \\
\hline 1 & 2 & 71 & 60 & 63 & 75 & 15 & 90 & 34 & 86 \\
\hline 1 & 3 & 65 & 63 & 56 & 76 & 15 & 94 & 31 & 89 \\
\hline 1 & 4 & 65 & 47 & - & - & - & - & - & - \\
\hline 2 & 1 & 85 & 65 & 74 & 75 & 15 & 90 & 38 & 86 \\
\hline 2 & 2 & 76 & 65 & 65 & 75 & 15 & 90 & 34 & 86 \\
\hline 2 & 3 & 70 & 67 & 57 & 76 & 15 & 94 & 31 & 90 \\
\hline 2 & 4 & 73 & 59 & 58 & 64 & - & - & - & - \\
\hline 3 & 1 & 85 & 63 & 76 & 74 & 14 & 90 & 39 & 86 \\
\hline 3 & 2 & 77 & 63 & 68 & 74 & 14 & 90 & 35 & 86 \\
\hline 3 & 3 & 72 & 63 & 61 & 74 & 13 & 94 & 32 & 90 \\
\hline 3 & 4 & 76 & 56 & 63 & 60 & - & - & - & - \\
\hline 4 & 1 & 87 & 62 & 79 & 77 & 20 & 90 & 46 & 88 \\
\hline 4 & 2 & 79 & 62 & 70 & 77 & 20 & 90 & 43 & 88 \\
\hline 4 & 3 & 75 & 62 & 62 & 77 & 19 & 93 & 38 & 90 \\
\hline 4 & 4 & 78 & 54 & 64 & 64 & - & - & - & - \\
\hline 5 & 1 & 90 & 63 & 76 & 85 & 31 & 91 & 54 & 89 \\
\hline 5 & 2 & 85 & 63 & 72 & 85 & 31 & 91 & 52 & 89 \\
\hline 5 & 3 & 81 & 63 & 61 & 85 & 29 & 93 & 45 & 92 \\
\hline 5 & 4 & 85 & 53 & 63 & 70 & - & - & - & - \\
\hline
\end{tabular}


Table E.2.14 the results of calibration and validations based on the maximum area

\begin{tabular}{|c|c|c|c|c|c|c|c|c|c|}
\hline \multicolumn{2}{|c|}{ Configurations } & \multicolumn{2}{|c|}{ Calibration } & \multicolumn{2}{|c|}{$1^{\text {st }}$ Validation } & \multicolumn{2}{|c|}{$2^{\text {nd }}$ Validation } & \multicolumn{2}{|c|}{$3^{\text {rd }}$ Validation } \\
\hline$\varphi_{g}$ & $\Lambda_{l}$ & Cyclists & Pedestrians & Cyclists & Pedestrians & Cyclists & Pedestrians & Cyclists & Pedestrians \\
\hline 1 & 1 & 66 & 22 & 56 & 24 & 25 & 9 & 37 & 13 \\
\hline 1 & 2 & 38 & 22 & 31 & 24 & - & - & - & - \\
\hline 1 & 3 & 20 & 21 & 17 & 24 & - & - & - & - \\
\hline 1 & 4 & 21 & 16 & 18 & 17 & - & - & - & - \\
\hline 2 & 1 & 74 & 24 & 61 & 25 & 27 & 9 & 40 & 13 \\
\hline 2 & 2 & 48 & 37 & 36 & 35 & - & - & - & - \\
\hline 2 & 3 & 15 & 24 & 16 & 25 & - & - & - & - \\
\hline 2 & 4 & 25 & 20 & 20 & 20 & - & - & - & - \\
\hline 3 & 1 & 76 & 22 & 71 & 28 & 28 & 9 & 45 & 13 \\
\hline 3 & 2 & 51 & 34 & 43 & 32 & - & - & - & - \\
\hline 3 & 3 & 28 & 41 & - & - & - & - & - & - \\
\hline 3 & 4 & 27 & 15 & 24 & 19 & - & - & - & - \\
\hline 4 & 1 & 78 & 24 & 74 & 33 & 35 & 9 & 52 & 14 \\
\hline 4 & 2 & 72 & 24 & 66 & 33 & - & - & - & - \\
\hline 4 & 3 & 21 & 35 & - & - & - & - & - & - \\
\hline 4 & 4 & 27 & 16 & - & - & - & - & - & - \\
\hline 5 & 1 & 77 & 16 & 65 & 24 & 33 & 12 & 49 & 14 \\
\hline 5 & 2 & 58 & 22 & 46 & 33 & 38 & 12 & 42 & 16 \\
\hline 5 & 3 & 33 & 34 & - & - & - & - & - & - \\
\hline 5 & 4 & 33 & 9 & 24 & 21 & 22 & 10 & 23 & 12 \\
\hline
\end{tabular}


Table E.2.15 the results of calibration and validations based on the average length

\begin{tabular}{|c|c|c|c|c|c|c|c|c|c|}
\hline \multicolumn{2}{|c|}{ Configurations } & \multicolumn{2}{|c|}{ Calibration } & \multicolumn{2}{|c|}{$1^{\text {st }}$ Validation } & \multicolumn{2}{|c|}{$2^{\text {nd }}$ Validation } & \multicolumn{2}{|c|}{$3^{\text {rd }}$ Validation } \\
\hline$\varphi_{g}$ & $\Lambda_{l}$ & Cyclists & Pedestrians & Cyclists & Pedestrians & Cyclists & Pedestrians & Cyclists & Pedestrians \\
\hline 1 & 1 & 64 & 21 & 60 & 19 & 11 & 0 & 31 & 5 \\
\hline 1 & 2 & 73 & 21 & 68 & 16 & 15 & 0 & 36 & 4 \\
\hline 1 & 3 & 73 & 18 & 69 & 14 & 15 & 0 & 37 & 4 \\
\hline 1 & 4 & 71 & 4 & 67 & 13 & 15 & 0 & 36 & 3 \\
\hline 2 & 1 & 62 & 22 & 56 & 20 & 6 & 0 & 25 & 5 \\
\hline 2 & 2 & 80 & 22 & 67 & 18 & 14 & 0 & 34 & 4 \\
\hline 2 & 3 & 79 & 18 & 67 & 16 & 14 & 0 & 34 & 4 \\
\hline 2 & 4 & 62 & 18 & 56 & 16 & 6 & 0 & 25 & 4 \\
\hline 3 & 1 & 75 & 27 & 61 & 19 & 12 & 0 & 31 & 4 \\
\hline 3 & 2 & 83 & 27 & - & - & - & - & - & - \\
\hline 3 & 3 & 81 & 22 & - & - & - & - & - & - \\
\hline 3 & 4 & 64 & 22 & 57 & 15 & 7 & 0 & 27 & 3 \\
\hline 4 & 1 & 79 & 27 & - & - & - & - & - & - \\
\hline 4 & 2 & 82 & 27 & - & - & - & - & - & - \\
\hline 4 & 3 & 84 & 22 & - & - & - & - & - & - \\
\hline 4 & 4 & 67 & 22 & - & - & - & - & - & - \\
\hline 5 & 1 & 85 & 25 & 65 & 15 & 22 & 0 & 44 & 3 \\
\hline 5 & 2 & 87 & 25 & - & - & - & - & - & - \\
\hline 5 & 3 & 81 & 19 & 65 & 12 & 18 & 0 & 42 & 2 \\
\hline 5 & 4 & 79 & 19 & 63 & 12 & 18 & 0 & 41 & 2 \\
\hline
\end{tabular}


Table E.2.16 the results of calibration and validations based on the maximum length

\begin{tabular}{|c|c|c|c|c|c|c|c|c|c|}
\hline \multicolumn{2}{|c|}{ Configurations } & \multicolumn{2}{|c|}{ Calibration } & \multicolumn{2}{|c|}{$\mathbf{1}^{\text {st }}$ Validation } & \multicolumn{2}{|c|}{$2^{\text {nd }}$ Validation } & \multicolumn{2}{|c|}{$3^{\text {rd }}$ Validation } \\
\hline$\varphi_{g}$ & $\Lambda_{l}$ & Cyclists & Pedestrians & Cyclists & Pedestrians & Cyclists & Pedestrians & Cyclists & Pedestrians \\
\hline 1 & 1 & 63 & 31 & 49 & 33 & - & - & - & - \\
\hline 1 & 2 & 82 & 41 & 70 & 41 & 97 & 4 & 86 & 14 \\
\hline 1 & 3 & 95 & 25 & 91 & 22 & - & - & - & - \\
\hline 1 & 4 & 60 & 12 & 48 & 5 & - & - & - & - \\
\hline 2 & 1 & 67 & 39 & 49 & 36 & - & - & - & - \\
\hline 2 & 2 & 88 & 35 & 74 & 31 & 95 & 1 & - & - \\
\hline 2 & 3 & 96 & 16 & 90 & 7 & - & - & - & - \\
\hline 2 & 4 & 67 & 16 & 49 & 5 & - & - & - & - \\
\hline 3 & 1 & 69 & 41 & 53 & 36 & - & - & - & - \\
\hline 3 & 2 & 88 & 37 & 68 & 30 & 97 & 1 & - & - \\
\hline 3 & 3 & 87 & 17 & 67 & 6 & - & - & - & - \\
\hline 3 & 4 & 69 & 17 & 53 & 6 & - & - & - & - \\
\hline 4 & 1 & 72 & 41 & 48 & 38 & 17 & 2 & 31 & 9 \\
\hline 4 & 2 & 88 & 38 & - & - & - & - & - & - \\
\hline 4 & 3 & 96 & 16 & 85 & 21 & 37 & 1 & 59 & 4 \\
\hline 4 & 4 & 72 & 19 & 48 & 8 & 17 & 1 & 31 & 2 \\
\hline 5 & 1 & 77 & 41 & 54 & 42 & 27 & 2 & 41 & 10 \\
\hline 5 & 2 & 94 & 28 & 80 & 30 & 51 & 1 & 66 & 6 \\
\hline 5 & 3 & 90 & 16 & 83 & 24 & 29 & 1 & 56 & 5 \\
\hline 5 & 4 & 77 & 19 & 54 & 9 & 27 & 1 & 41 & 2 \\
\hline
\end{tabular}


Table E.2.17 the results of calibration and validations based on the average width

\begin{tabular}{|c|c|c|c|c|c|c|c|c|c|}
\hline \multicolumn{2}{|c|}{ Configurations } & \multicolumn{2}{|c|}{ Calibration } & \multicolumn{2}{|c|}{$1^{\text {st }}$ Validation } & \multicolumn{2}{|c|}{$2^{\text {nd }}$ Validation } & \multicolumn{2}{|c|}{$3^{\text {rd }}$ Validation } \\
\hline$\varphi_{g}$ & $\Lambda_{l}$ & Cyclists & Pedestrians & Cyclists & Pedestrians & Cyclists & Pedestrians & Cyclists & Pedestrians \\
\hline 1 & 1 & 87 & 54 & 76 & 59 & 49 & 62 & 60 & 61 \\
\hline 1 & 2 & 68 & 71 & 54 & 75 & - & - & - & - \\
\hline 1 & 3 & 54 & 71 & 48 & 76 & - & - & - & - \\
\hline 1 & 4 & - & - & - & - & - & - & - & - \\
\hline 2 & 1 & 96 & 61 & 81 & 64 & 57 & 61 & 66 & 62 \\
\hline 2 & 2 & 82 & 80 & 60 & 80 & - & - & - & - \\
\hline 2 & 3 & 62 & 84 & 51 & 82 & - & - & - & - \\
\hline 2 & 4 & - & - & - & - & - & - & - & - \\
\hline 3 & 1 & 95 & 61 & 81 & 68 & 49 & 62 & 61 & 63 \\
\hline 3 & 2 & 81 & 83 & 58 & 81 & 32 & 63 & - & - \\
\hline 3 & 3 & 65 & 83 & 53 & 81 & - & - & - & - \\
\hline 3 & 4 & - & - & - & - & - & - & - & - \\
\hline 4 & 1 & 96 & 62 & 85 & 69 & 56 & 62 & 69 & 63 \\
\hline 4 & 2 & 82 & 84 & 62 & 82 & 37 & 63 & - & - \\
\hline 4 & 3 & 70 & 84 & 57 & 82 & - & - & - & - \\
\hline 4 & 4 & - & - & - & - & - & - & - & - \\
\hline 5 & 1 & 96 & 63 & 85 & 70 & 51 & 60 & 68 & 62 \\
\hline 5 & 2 & 83 & 81 & 63 & 85 & 38 & 65 & 51 & 69 \\
\hline 5 & 3 & 73 & 81 & 63 & 85 & - & - & - & - \\
\hline 5 & 4 & - & - & - & - & - & - & - & - \\
\hline
\end{tabular}


Table E.2.18 the results of calibration and validations based on the maximum width

\begin{tabular}{|c|c|c|c|c|c|c|c|c|c|}
\hline \multicolumn{2}{|c|}{ Configurations } & \multicolumn{2}{|c|}{ Calibration } & \multicolumn{2}{|c|}{$1^{\text {st }}$ Validation } & \multicolumn{2}{|c|}{$2^{\text {nd }}$ Validation } & \multicolumn{2}{|c|}{$3^{\text {rd }}$ Validation } \\
\hline$\varphi_{g}$ & $\Lambda_{l}$ & Cyclists & Pedestrians & Cyclists & Pedestrians & Cyclists & Pedestrians & Cyclists & Pedestrians \\
\hline 1 & 1 & 83 & 49 & 73 & 67 & 58 & 89 & 64 & 83 \\
\hline 1 & 2 & 63 & 65 & 59 & 78 & 42 & 95 & 49 & 91 \\
\hline 1 & 3 & - & - & - & - & - & - & - & - \\
\hline 1 & 4 & - & - & - & - & - & - & - & - \\
\hline 2 & 1 & 90 & 57 & 74 & 67 & 59 & 86 & 65 & 82 \\
\hline 2 & 2 & 73 & 76 & 65 & 80 & 43 & 95 & 52 & 92 \\
\hline 2 & 3 & 55 & 76 & 52 & 85 & - & - & - & - \\
\hline 2 & 4 & - & - & - & - & - & - & - & - \\
\hline 3 & 1 & 93 & 51 & 81 & 68 & 64 & 86 & 71 & 82 \\
\hline 3 & 2 & 77 & 71 & 74 & 77 & 46 & 95 & 57 & 91 \\
\hline 3 & 3 & 60 & 71 & 60 & 85 & - & - & - & - \\
\hline 3 & 4 & - & - & - & - & - & - & - & - \\
\hline 4 & 1 & 94 & 49 & 87 & 69 & 68 & 85 & 76 & 82 \\
\hline 4 & 2 & 79 & 68 & 80 & 77 & 52 & 95 & 65 & 91 \\
\hline 4 & 3 & 61 & 68 & 67 & 87 & 41 & 95 & 53 & 94 \\
\hline 4 & 4 & - & - & - & - & - & - & - & - \\
\hline 5 & 1 & 88 & 53 & 83 & 39 & - & - & - & - \\
\hline 5 & 2 & 83 & 63 & 80 & 70 & 49 & 96 & 65 & 91 \\
\hline 5 & 3 & 62 & 63 & 65 & 85 & 40 & 96 & 53 & 94 \\
\hline 5 & 4 & - & - & - & - & - & - & - & - \\
\hline
\end{tabular}


Table E.2.19 the results of calibration and validations based on the peaks in speed

\begin{tabular}{|c|c|c|c|c|c|c|c|c|c|}
\hline \multicolumn{2}{|c|}{ Configurations } & \multicolumn{2}{|c|}{ Calibration } & \multicolumn{2}{|c|}{$1^{\text {st }}$ Validation } & \multicolumn{2}{|c|}{$2^{\text {nd }}$ Validation } & \multicolumn{2}{|c|}{$3^{\text {rd }}$ Validation } \\
\hline$\varphi_{g}$ & $\Lambda_{l}$ & Cyclists & Pedestrians & Cyclists & Pedestrians & Cyclists & Pedestrians & Cyclists & Pedestrians \\
\hline 1 & 1 & 18 & 60 & 20 & 60 & 19 & 50 & 20 & 53 \\
\hline 1 & 2 & 15 & 63 & 10 & 68 & 11 & 72 & 10 & 71 \\
\hline 1 & 3 & 12 & 63 & 12 & 65 & 14 & 64 & 13 & 64 \\
\hline 1 & 4 & 12 & 60 & 8 & 57 & 13 & 50 & 11 & 52 \\
\hline 2 & 1 & 13 & 65 & 18 & 64 & 17 & 50 & 18 & 53 \\
\hline 2 & 2 & 10 & 67 & 10 & 67 & 13 & 65 & 12 & 66 \\
\hline 2 & 3 & 9 & 67 & 11 & 67 & 13 & 63 & 13 & 64 \\
\hline 2 & 4 & 9 & 65 & 8 & 64 & 12 & 50 & 10 & 53 \\
\hline 3 & 1 & 5 & 63 & 10 & 62 & 13 & 51 & 11 & 53 \\
\hline 3 & 2 & 4 & 63 & 10 & 62 & 12 & 51 & 11 & 53 \\
\hline 3 & 3 & 4 & 63 & 7 & 62 & 10 & 58 & 9 & 59 \\
\hline 3 & 4 & 4 & 63 & 3 & 62 & 9 & 51 & 7 & 53 \\
\hline 4 & 1 & 3 & 62 & 5 & 59 & 5 & 51 & 5 & 52 \\
\hline 4 & 2 & 1 & 62 & 5 & 59 & 5 & 51 & 5 & 53 \\
\hline 4 & 3 & 3 & 59 & 5 & 56 & 4 & 48 & 4 & 50 \\
\hline 4 & 4 & 1 & 62 & 0 & 59 & 1 & 51 & 1 & 52 \\
\hline 5 & 1 & 2 & 63 & 4 & 67 & 7 & 50 & 5 & 54 \\
\hline 5 & 2 & 0 & 63 & 4 & 67 & 7 & 51 & 5 & 54 \\
\hline 5 & 3 & 2 & 63 & 4 & 73 & 7 & 58 & 5 & 61 \\
\hline 5 & 4 & 0 & 63 & 0 & 67 & 0 & 50 & 0 & 54 \\
\hline
\end{tabular}


Table E.2.20 the results of calibration and validations based on the effective weighted average frequency

\begin{tabular}{|c|c|c|c|c|c|c|c|c|c|c|}
\hline \multicolumn{3}{|c|}{ Configurations } & \multicolumn{2}{|c|}{ Calibration } & \multicolumn{2}{|c|}{$\mathbf{1}^{\text {st }}$ Validation } & \multicolumn{2}{|c|}{$2^{\text {nd }}$ Validation } & \multicolumn{2}{|c|}{$3^{\text {rd }}$ Validation } \\
\hline$\varphi_{g}$ & $\Lambda_{l}$ & $\Gamma_{n}$ & Cyclists & Pedestrians & Cyclists & Pedestrians & Cyclists & Pedestrians & Cyclists & Pedestrians \\
\hline 1 & 1 & 1 & 24 & 87 & 19 & 94 & 14 & 99 & 16 & 98 \\
\hline 1 & 1 & 2 & 23 & 74 & 19 & 79 & 15 & 88 & 17 & 86 \\
\hline 1 & 1 & 3 & 24 & 75 & 18 & 81 & 15 & 87 & 16 & 86 \\
\hline 1 & 1 & 4 & 26 & 84 & 20 & 89 & 19 & 93 & 20 & 92 \\
\hline 1 & 1 & 1 & 27 & 84 & 21 & 89 & 19 & 95 & 20 & 93 \\
\hline 1 & 1 & 6 & 27 & 84 & 21 & 89 & 19 & 95 & 20 & 93 \\
\hline 1 & 2 & 1 & 19 & 87 & 16 & 94 & 13 & 100 & 14 & 98 \\
\hline 1 & 2 & 2 & 19 & 75 & 15 & 81 & 9 & 91 & 11 & 88 \\
\hline 1 & 2 & 3 & 19 & 75 & 14 & 83 & 13 & 89 & 13 & 87 \\
\hline 1 & 2 & 4 & 20 & 69 & 16 & 75 & 12 & 89 & 14 & 85 \\
\hline 1 & 2 & 1 & 14 & 66 & 13 & 70 & 17 & 82 & 15 & 79 \\
\hline 1 & 2 & 6 & 22 & 84 & 18 & 92 & 13 & 98 & 15 & 96 \\
\hline 1 & 3 & 1 & 18 & 87 & 11 & 94 & 6 & 100 & 8 & 98 \\
\hline 1 & 3 & 2 & 18 & 75 & 11 & 81 & 8 & 91 & 9 & 88 \\
\hline 1 & 3 & 3 & 18 & 75 & 11 & 83 & 8 & 90 & 9 & 88 \\
\hline 1 & 3 & 4 & 15 & 69 & 13 & 75 & 9 & 89 & 11 & 85 \\
\hline 1 & 3 & 1 & 18 & 68 & 12 & 75 & 5 & 88 & 8 & 85 \\
\hline 1 & 3 & 6 & 19 & 85 & 13 & 94 & 5 & 99 & 8 & 98 \\
\hline 1 & 4 & 1 & 18 & 87 & 13 & 94 & 8 & 99 & 10 & 98 \\
\hline 1 & 4 & 2 & 19 & 74 & 12 & 81 & 8 & 90 & 10 & 88 \\
\hline 1 & 4 & 3 & 20 & 75 & 12 & 83 & 8 & 89 & 10 & 87 \\
\hline 1 & 4 & 4 & 20 & 69 & 12 & 75 & 7 & 89 & 9 & 85 \\
\hline 1 & 4 & 1 & 19 & 84 & 16 & 89 & 13 & 95 & 14 & 93 \\
\hline 1 & 4 & 6 & 20 & 84 & 17 & 89 & 13 & 95 & 14 & 93 \\
\hline 2 & 1 & 1 & 28 & 100 & 21 & 100 & 15 & 99 & 17 & 100 \\
\hline 2 & 1 & 2 & 28 & 100 & 21 & 98 & 16 & 97 & 18 & 97 \\
\hline 2 & 1 & 3 & 28 & 100 & 21 & 95 & 21 & 95 & 21 & 95 \\
\hline 2 & 1 & 4 & 29 & 100 & 22 & 95 & 20 & 93 & 21 & 94 \\
\hline 2 & 1 & 1 & 30 & 100 & 24 & 95 & 20 & 95 & 21 & 95 \\
\hline 2 & 1 & 6 & 30 & 100 & 24 & 95 & 20 & 95 & 22 & 95 \\
\hline 2 & 2 & 1 & 22 & 100 & 18 & 100 & 13 & 100 & 15 & 100 \\
\hline 2 & 2 & 2 & 21 & 100 & 18 & 100 & 13 & 98 & 15 & 99 \\
\hline 2 & 2 & 3 & 24 & 100 & 17 & 100 & 13 & 99 & 15 & 99 \\
\hline 2 & 2 & 4 & 24 & 100 & 19 & 100 & 13 & 98 & 15 & 99 \\
\hline 2 & 2 & 1 & 24 & 100 & 19 & 98 & 15 & 97 & 16 & 97 \\
\hline 2 & 2 & 6 & 25 & 100 & 20 & 98 & 14 & 98 & 16 & 98 \\
\hline
\end{tabular}


Table E.2.20 the results of calibration and validations based on the effective weighted average frequency

\begin{tabular}{|c|c|c|c|c|c|c|c|c|c|c|}
\hline \multicolumn{3}{|c|}{ Configurations } & \multicolumn{2}{|c|}{ Calibration } & \multicolumn{2}{|c|}{$\mathbf{1}^{\text {st }}$ Validation } & \multicolumn{2}{|c|}{$2^{\text {nd }}$ Validation } & \multicolumn{2}{|c|}{$3^{\text {rd }}$ Validation } \\
\hline$\varphi_{g}$ & $\Lambda_{l}$ & $\Gamma_{n}$ & Cyclists & Pedestrians & Cyclists & Pedestrians & Cyclists & Pedestrians & Cyclists & Pedestrians \\
\hline 2 & 3 & 1 & 21 & 100 & 12 & 100 & 6 & 100 & 9 & 100 \\
\hline 2 & 3 & 2 & 21 & 100 & 12 & 100 & 8 & 100 & 10 & 100 \\
\hline 2 & 3 & 3 & 21 & 92 & 12 & 91 & 8 & 90 & 10 & 90 \\
\hline 2 & 3 & 4 & 21 & 100 & 12 & 100 & 7 & 99 & 9 & 100 \\
\hline 2 & 3 & 1 & 21 & 100 & 13 & 100 & 6 & 99 & 9 & 100 \\
\hline 2 & 3 & 6 & 22 & 100 & 15 & 100 & 6 & 99 & 9 & 100 \\
\hline 2 & 4 & 1 & 21 & 100 & 16 & 98 & 9 & 99 & 12 & 99 \\
\hline 2 & 4 & 2 & 21 & 100 & 16 & 98 & 11 & 97 & 13 & 97 \\
\hline 2 & 4 & 3 & 24 & 100 & 13 & 100 & 8 & 98 & 10 & 98 \\
\hline 2 & 4 & 4 & 24 & 100 & 13 & 98 & 8 & 98 & 10 & 98 \\
\hline 2 & 4 & 1 & 22 & 100 & 18 & 95 & 13 & 95 & 15 & 95 \\
\hline 2 & 4 & 6 & 24 & 100 & 19 & 95 & 13 & 95 & 16 & 95 \\
\hline 3 & 1 & 1 & 33 & 100 & 26 & 100 & 15 & 99 & 20 & 100 \\
\hline 3 & 1 & 2 & 33 & 100 & 26 & 98 & 17 & 97 & 21 & 97 \\
\hline 3 & 1 & 3 & 33 & 100 & 26 & 94 & 22 & 95 & 24 & 94 \\
\hline 3 & 1 & 4 & 35 & 100 & 28 & 94 & 21 & 93 & 24 & 93 \\
\hline 3 & 1 & 1 & 36 & 100 & 29 & 94 & 21 & 95 & 24 & 95 \\
\hline 3 & 1 & 6 & 36 & 100 & 29 & 94 & 21 & 95 & 24 & 95 \\
\hline 3 & 2 & 1 & 27 & 100 & 22 & 100 & 13 & 100 & 17 & 100 \\
\hline 3 & 2 & 2 & 25 & 100 & 22 & 100 & 13 & 98 & 17 & 99 \\
\hline 3 & 2 & 3 & 28 & 100 & 21 & 100 & 14 & 99 & 17 & 99 \\
\hline 3 & 2 & 4 & 28 & 100 & 24 & 100 & 13 & 98 & 17 & 99 \\
\hline 3 & 2 & 1 & 28 & 100 & 24 & 98 & 15 & 97 & 18 & 97 \\
\hline 3 & 2 & 6 & 29 & 100 & 25 & 98 & 14 & 98 & 18 & 98 \\
\hline 3 & 3 & 1 & 25 & 100 & 15 & 100 & 6 & 100 & 10 & 100 \\
\hline 3 & 3 & 2 & 25 & 100 & 15 & 100 & 8 & 100 & 11 & 100 \\
\hline 3 & 3 & 3 & 25 & 93 & 15 & 94 & 8 & 90 & - & - \\
\hline 3 & 3 & 4 & 25 & 100 & 15 & 100 & 7 & 99 & 10 & 100 \\
\hline 3 & 3 & 1 & 25 & 100 & 17 & 100 & 5 & 99 & 10 & 100 \\
\hline 3 & 3 & 6 & 27 & 100 & 18 & 100 & 5 & 99 & 10 & 100 \\
\hline 3 & 4 & 1 & 25 & 100 & 19 & 98 & 9 & 99 & 13 & 99 \\
\hline 3 & 4 & 2 & 25 & 100 & 19 & 98 & 11 & 97 & 14 & 97 \\
\hline 3 & 4 & 3 & 28 & 100 & 17 & 100 & 8 & 98 & 11 & 98 \\
\hline 3 & 4 & 4 & 28 & 100 & 17 & 98 & 7 & 98 & 11 & 98 \\
\hline 3 & 4 & 1 & 27 & 100 & 22 & 94 & 13 & 95 & 17 & 95 \\
\hline 3 & 4 & 6 & 28 & 100 & 24 & 94 & 14 & 95 & 18 & 95 \\
\hline
\end{tabular}


Table E.2.20 the results of calibration and validations based on the effective weighted average frequency

\begin{tabular}{|c|c|c|c|c|c|c|c|c|c|c|}
\hline \multicolumn{3}{|c|}{ Configurations } & \multicolumn{2}{|c|}{ Calibration } & \multicolumn{2}{|c|}{$\mathbf{1}^{\text {st }}$ Validation } & \multicolumn{2}{|c|}{$2^{\text {nd }}$ Validation } & \multicolumn{2}{|c|}{$3^{\text {rd }}$ Validation } \\
\hline$\varphi_{g}$ & $\Lambda_{l}$ & $\Gamma_{n}$ & Cyclists & Pedestrians & Cyclists & Pedestrians & Cyclists & Pedestrians & Cyclists & Pedestrians \\
\hline 4 & 1 & 1 & 37 & 100 & 30 & 100 & 19 & 99 & 24 & 100 \\
\hline 4 & 1 & 2 & 37 & 100 & 30 & 97 & 20 & 97 & 24 & 97 \\
\hline 4 & 1 & 3 & 37 & 100 & 30 & 95 & 20 & 97 & 24 & 97 \\
\hline 4 & 1 & 4 & 37 & 100 & 31 & 92 & 27 & 93 & 29 & 93 \\
\hline 4 & 1 & 1 & 39 & 100 & 33 & 92 & 24 & 95 & 28 & 94 \\
\hline 4 & 1 & 6 & 39 & 100 & 33 & 92 & 27 & 95 & 29 & 94 \\
\hline 4 & 2 & 1 & 30 & 100 & 25 & 100 & 16 & 100 & 20 & 100 \\
\hline 4 & 2 & 2 & 28 & 100 & 25 & 100 & 15 & 98 & 19 & 99 \\
\hline 4 & 2 & 3 & 31 & 100 & 23 & 100 & 16 & 99 & 19 & 99 \\
\hline 4 & 2 & 4 & 31 & 100 & 26 & 100 & 16 & 98 & 21 & 99 \\
\hline 4 & 2 & 1 & 31 & 100 & 26 & 97 & 19 & 97 & 22 & 97 \\
\hline 4 & 2 & 6 & 33 & 100 & 28 & 97 & 17 & 98 & 22 & 98 \\
\hline 4 & 3 & 1 & 28 & 100 & 18 & 100 & 8 & 100 & 13 & 100 \\
\hline 4 & 3 & 2 & 28 & 100 & 18 & 100 & 9 & 100 & 13 & 100 \\
\hline 4 & 3 & 3 & 28 & 100 & 18 & 100 & 9 & 99 & 13 & 100 \\
\hline 4 & 3 & 4 & 28 & 100 & 18 & 100 & 8 & 99 & 13 & 100 \\
\hline 4 & 3 & 1 & 28 & 100 & 20 & 100 & 7 & 99 & 13 & 100 \\
\hline 4 & 3 & 6 & 30 & 100 & 21 & 100 & 7 & 99 & 13 & 100 \\
\hline 4 & 4 & 1 & 28 & 100 & 23 & 97 & 9 & 99 & 15 & 99 \\
\hline 4 & 4 & 2 & 28 & 100 & 21 & 97 & 12 & 97 & 16 & 97 \\
\hline 4 & 4 & 3 & 31 & 100 & 20 & 100 & 9 & 98 & 14 & 98 \\
\hline 4 & 4 & 4 & 31 & 100 & 20 & 97 & 8 & 98 & 13 & 98 \\
\hline 4 & 4 & 1 & 30 & 100 & 25 & 92 & 16 & 95 & 20 & 94 \\
\hline 4 & 4 & 6 & 31 & 100 & 26 & 92 & 17 & 95 & 21 & 94 \\
\hline 5 & 1 & 1 & 46 & 100 & 37 & 100 & 27 & 100 & 32 & 100 \\
\hline 5 & 1 & 2 & 46 & 100 & 37 & 97 & 29 & 97 & 33 & 97 \\
\hline 5 & 1 & 3 & 46 & 100 & 37 & 94 & 29 & 97 & 33 & 96 \\
\hline 5 & 1 & 4 & 46 & 100 & 35 & 91 & 36 & 93 & 35 & 93 \\
\hline 5 & 1 & 1 & 48 & 100 & 37 & 91 & 33 & 95 & 35 & 94 \\
\hline 5 & 1 & 6 & 48 & 100 & 35 & 91 & 31 & 96 & 33 & 95 \\
\hline 5 & 2 & 1 & 38 & 100 & 30 & 100 & 22 & 100 & 26 & 100 \\
\hline 5 & 2 & 2 & 38 & 100 & 28 & 100 & 22 & 99 & 25 & 99 \\
\hline 5 & 2 & 3 & 40 & 100 & 28 & 100 & 24 & 99 & 26 & 99 \\
\hline 5 & 2 & 4 & 40 & 100 & 33 & 100 & 24 & 98 & 29 & 98 \\
\hline 5 & 2 & 1 & 40 & 100 & 30 & 97 & 27 & 97 & 29 & 97 \\
\hline 5 & 2 & 6 & 42 & 100 & 33 & 97 & 27 & 97 & 30 & 97 \\
\hline
\end{tabular}


Table E.2.20 the results of calibration and validations based on the effective weighted average frequency

\begin{tabular}{|c|c|c|c|c|c|c|c|c|c|c|}
\hline \multicolumn{3}{|c|}{ Configurations } & \multicolumn{2}{|c|}{ Calibration } & \multicolumn{2}{|c|}{$1^{\text {st }}$ Validation } & \multicolumn{2}{|c|}{$2^{\text {nd }}$ Validation } & \multicolumn{2}{|c|}{$3^{\text {rd }}$ Validation } \\
\hline$\varphi_{g}$ & $\Lambda_{l}$ & $\Gamma_{n}$, & Cyclists & Pedestrians & Cyclists & Pedestrians & Cyclists & Pedestrians & Cyclists & Pedestrians \\
\hline 5 & 3 & 1 & 35 & 100 & 26 & 100 & 11 & 100 & 19 & 100 \\
\hline 5 & 3 & 2 & 35 & 100 & 28 & 100 & 16 & 100 & 22 & 100 \\
\hline 5 & 3 & 3 & 35 & 100 & 26 & 100 & 18 & 99 & 22 & 99 \\
\hline 5 & 3 & 4 & 35 & 100 & 26 & 100 & 18 & 99 & 22 & 99 \\
\hline 5 & 3 & 1 & 35 & 100 & 28 & 100 & 18 & 99 & 23 & 99 \\
\hline 5 & 3 & 6 & 37 & 100 & 28 & 100 & 11 & 99 & 20 & 99 \\
\hline 5 & 4 & 1 & 37 & 100 & 30 & 97 & 16 & 99 & 23 & 99 \\
\hline 5 & 4 & 2 & 37 & 100 & 28 & 97 & 20 & 97 & 24 & 97 \\
\hline 5 & 4 & 3 & 38 & 100 & 26 & 100 & 16 & 98 & 21 & 98 \\
\hline 5 & 4 & 4 & 38 & 100 & 30 & 91 & 24 & 95 & 27 & 94 \\
\hline 5 & 4 & 1 & 38 & 100 & 30 & 91 & 24 & 95 & 27 & 94 \\
\hline 5 & 4 & 6 & 40 & 100 & 30 & 91 & 27 & 95 & 29 & 94 \\
\hline
\end{tabular}

Table E.2.21 the results of calibration and validations based on the effective frequency

\begin{tabular}{|c|c|c|c|c|c|c|c|c|c|c|}
\hline \multicolumn{3}{|c|}{ Configurations } & \multicolumn{2}{|c|}{ Calibration } & \multicolumn{2}{|c|}{$1^{\text {st }}$ Validation } & \multicolumn{2}{|c|}{$2^{\text {nd }}$ Validation } & \multicolumn{2}{|c|}{$3^{\text {rd }}$ Validation } \\
\hline$\varphi_{g}$ & $\Lambda_{l}$ & $\eta_{m}$ & Cyclists & Pedestrians & Cyclists & Pedestrians & Cyclists & Pedestrians & Cyclists & Pedestrians \\
\hline 1 & 1 & 1 & 6 & 49 & 1 & 48 & 5 & 31 & 3 & 35 \\
\hline 1 & 1 & 2 & 55 & 13 & 61 & 8 & 51 & 17 & 55 & 15 \\
\hline 1 & 1 & 3 & 60 & 18 & 47 & 11 & 54 & 14 & 51 & 13 \\
\hline 1 & 2 & 1 & 5 & 53 & 0 & 48 & 4 & 31 & 2 & 35 \\
\hline 1 & 2 & 2 & 5 & 43 & 7 & 52 & - & - & - & - \\
\hline 1 & 2 & 3 & 34 & 18 & - & - & - & - & - & - \\
\hline 1 & 3 & 1 & 4 & 53 & 0 & 48 & 3 & 31 & 2 & 35 \\
\hline 1 & 3 & 2 & 3 & 47 & 3 & 52 & 4 & 29 & 4 & 35 \\
\hline 1 & 3 & 3 & 21 & 21 & - & - & - & - & - & - \\
\hline 1 & 4 & 1 & 5 & 47 & 0 & 48 & 4 & 31 & 2 & 35 \\
\hline 1 & 4 & 2 & 3 & 38 & 4 & 52 & 5 & 22 & 4 & 30 \\
\hline 1 & 4 & 3 & 19 & 16 & - & - & - & - & - & - \\
\hline 2 & 1 & 1 & 71 & 6 & 71 & 9 & 64 & 3 & 66 & 5 \\
\hline 2 & 1 & 2 & 64 & 18 & 69 & 9 & 53 & 17 & 59 & 15 \\
\hline 2 & 1 & 3 & 70 & 24 & 53 & 13 & 56 & 14 & 55 & 14 \\
\hline 2 & 2 & 1 & 2 & 51 & 1 & 47 & 4 & 31 & 3 & 34 \\
\hline 2 & 2 & 2 & 2 & 53 & 3 & 64 & - & - & - & - \\
\hline 2 & 2 & 3 & 39 & 24 & - & - & - & - & - & - \\
\hline
\end{tabular}


Table E.2.21 the results of calibration and validations based on the effective frequency

\begin{tabular}{|c|c|c|c|c|c|c|c|c|c|c|}
\hline \multicolumn{3}{|c|}{ Configurations } & \multicolumn{2}{|c|}{ Calibration } & \multicolumn{2}{|c|}{$\mathbf{1}^{\text {st }}$ Validation } & \multicolumn{2}{|c|}{$2^{\text {nd }}$ Validation } & \multicolumn{2}{|c|}{$3^{\text {rd }}$ Validation } \\
\hline$\varphi_{g}$ & $\Lambda_{l}$ & $\eta_{m}$ & Cyclists & Pedestrians & Cyclists & Pedestrians & Cyclists & Pedestrians & Cyclists & Pedestrians \\
\hline 2 & 3 & 1 & 1 & 51 & 0 & 47 & 2 & 31 & 1 & 34 \\
\hline 2 & 3 & 2 & 2 & 53 & 3 & 64 & - & - & - & - \\
\hline 2 & 3 & 3 & 25 & 29 & - & - & - & - & - & - \\
\hline 2 & 4 & 1 & 1 & 49 & 0 & 47 & 3 & 31 & 2 & 34 \\
\hline 2 & 4 & 2 & 2 & 53 & 3 & 64 & - & - & - & - \\
\hline 2 & 4 & 3 & 22 & 22 & - & - & - & - & - & - \\
\hline 3 & 1 & 1 & 84 & 7 & 88 & 11 & 81 & 3 & 84 & 5 \\
\hline 3 & 1 & 2 & 73 & 22 & 83 & 11 & 67 & 17 & 73 & 16 \\
\hline 3 & 1 & 3 & 81 & 29 & 64 & 15 & 69 & 14 & 67 & 14 \\
\hline 3 & 2 & 1 & 0 & 49 & 0 & 47 & 0 & 36 & - & - \\
\hline 3 & 2 & 2 & 39 & 15 & - & - & - & - & - & - \\
\hline 3 & 2 & 3 & 47 & 29 & - & - & - & - & - & - \\
\hline 3 & 3 & 1 & 0 & 49 & 0 & 47 & 0 & 36 & - & - \\
\hline 3 & 3 & 2 & - & - & - & - & - & - & - & - \\
\hline 3 & 3 & 3 & 29 & 34 & - & - & - & - & - & - \\
\hline 3 & 4 & 1 & 0 & 49 & 0 & 47 & 0 & 36 & - & - \\
\hline 3 & 4 & 2 & - & - & - & - & - & - & - & - \\
\hline 3 & 4 & 3 & 27 & 27 & - & - & - & - & - & - \\
\hline 4 & 1 & 1 & 85 & 8 & 92 & 13 & 92 & 4 & 92 & 5 \\
\hline 4 & 1 & 2 & 78 & 16 & 72 & 3 & 71 & 2 & 71 & 2 \\
\hline 4 & 1 & 3 & 88 & 30 & 74 & 15 & 81 & 14 & 78 & 14 \\
\hline 4 & 2 & 1 & - & - & - & - & - & - & - & - \\
\hline 4 & 2 & 2 & - & - & - & - & - & - & - & - \\
\hline 4 & 2 & 3 & 52 & 32 & 41 & 18 & 36 & 15 & - & - \\
\hline 4 & 3 & 1 & - & - & - & - & - & - & - & - \\
\hline 4 & 3 & 2 & - & - & - & - & - & - & - & - \\
\hline 4 & 3 & 3 & 33 & 38 & 25 & 23 & - & - & - & - \\
\hline 4 & 4 & 1 & - & - & - & - & - & - & - & - \\
\hline 4 & 4 & 2 & - & - & - & - & - & - & - & - \\
\hline 4 & 4 & 3 & - & - & - & - & - & - & - & - \\
\hline 5 & 1 & 1 & 92 & 0 & 91 & 0 & 93 & 1 & 92 & 1 \\
\hline 5 & 1 & 2 & 87 & 19 & 91 & 3 & 80 & 3 & 86 & 3 \\
\hline 5 & 1 & 3 & 92 & 34 & 87 & 18 & 87 & 17 & 87 & 17 \\
\hline 5 & 2 & 1 & - & - & - & - & - & - & - & - \\
\hline 5 & 2 & 2 & 56 & 19 & - & - & - & - & - & - \\
\hline 5 & 2 & 3 & 65 & 34 & 54 & 21 & 53 & 19 & 54 & 19 \\
\hline
\end{tabular}


Table E.2.21 the results of calibration and validations based on the effective frequency

\begin{tabular}{|c|c|c|c|c|c|c|c|c|c|c|}
\hline \multicolumn{3}{|c|}{ Configurations } & \multicolumn{2}{|c|}{ Calibration } & \multicolumn{2}{|c|}{$\mathbf{1}^{\text {st }}$ Validation } & \multicolumn{2}{|c|}{$2^{\text {nd }}$ Validation } & \multicolumn{2}{|c|}{$3^{\text {rd }}$ Validation } \\
\hline$\varphi_{g}$ & $\Lambda_{l}$ & $\eta_{m}$ & Cyclists & Pedestrians & Cyclists & Pedestrians & Cyclists & Pedestrians & Cyclists & Pedestrians \\
\hline 5 & 3 & 1 & - & - & - & - & - & - & - & - \\
\hline 5 & 3 & 2 & - & - & - & - & - & - & - & - \\
\hline 5 & 3 & 3 & 40 & 38 & 33 & 27 & 18 & 22 & - & - \\
\hline 5 & 4 & 1 & - & - & - & - & - & - & - & - \\
\hline 5 & 4 & 2 & - & - & - & - & - & - & - & - \\
\hline
\end{tabular}

